\title{
CONTROL AND INTIMATE PARTNER AGGRESSION
}

\author{
FIONA LOUISE DEMPSEY
}

\author{
A thesis \\ Submitted to Victoria University of Wellington \\ In fulfilment of the requirements for the degree of \\ Doctor of Philosophy in Forensic Psychology
}

Victoria University of Wellington

2021 


\begin{abstract}
Control is fundamental to theoretical conceptualizations of intimate partner aggression (IPA). In particular, it has been instrumental in the development of typologies of IPA, where control has been associated with more frequent and serious IPA carried out by men against women. Consequently, the concept of control has heavily influenced the design of treatment and legislation targeting partner violence. However, there is considerable theoretical divergence as to how control should be conceptualized, operationalized, and measured. This thesis comprises a series of studies designed to test the validity of some of the key theoretical assumptions that inform the common conceptualizations of control by examining control as a behavior, as a motivation, and as an outcome. Chapter 1 provides an overview of the thesis rationale and objectives. Chapter 2 investigated the theoretical assumption that non-physical 'controlling behaviors' (e.g., restricting access to money; threatening harm) comprise a unique form of IPA. Exploratory $(N=561)$ and confirmatory $(N=424)$ factor analyses on 54 measures used across the IPA literature identified three forms of aggression: Eclectic Aggression, Direct Psychological Aggression, Monitoring Acts. There was no evidence for a distinct form of 'controlling behaviors'. Chapter 3 systematically reviewed the literature on motivations for physical and psychological IPA. The review aimed to appraise the quality of the literature and ascertain which motivations had the largest effect sizes. A meta-analysis of the motivations for physical IPA suggested self-defense, retaliation for emotional hurt, and communication difficulties had larger effect sizes than control. Chapter 4 investigated the assumptions that control motivations are associated with more severe and frequent IPA and IPA perpetrated by men. Categorical principal and latent class analyses $(N=1166)$ found considerable heterogeneity in motivations for IPA for both genders, but no evidence of distinct patterns or profiles of controlling motivations for either men or women. Chapter 5 investigated the
\end{abstract}


assumptions that coercive control is experienced exclusively by women and is related to experiencing specific types and more frequent IPA $(N=1174)$. Evidence did not support a "coercive control" pattern or profile in people who experienced IPA, or that coercive control outcomes were gendered, or associated with the type or the frequency of IPA behaviors used. Regressing the item-average of coercive control outcomes on experiences of IPA in a path analysis provided some evidence that gender and experiences of physical and psychological aggression predicted feelings of coercive control. Collectively, the results of the thesis identified considerable heterogeneity in the patterns of behaviors, motivations and outcomes for IPA. The evidence challenges existing conceptualizations of control as a distinct and gendered construct and indicates the need for the development of a theoretical explanation of control, that is both gender-inclusive and multifactorial, to guide future research. 


\section{ACKNOWLEDGMENTS}

I would like to express my sincere and enduring thanks to my supervisors, Louise Dixon and Matt Hammond. I could not have imagined a better supervisory team, both in terms of your respective expertise and your care and support of me as an individual. The song, Mountain At My Gates, was my PhD song.

“...I see a mountain in my way

It's looming larger by the day

I see a darkness in my fate

I'll drive my car without the brakes

Oh, gimme some time

Show me the foothold from which I can climb

Yeah, when I feel low

You show me a signpost for where I should go" (Foals, 2015)

For me, the first verse encapsulates those times of feeling overwhelmed and uncertain during some parts of my $\mathrm{PhD}$ journey. The chorus, however, reminds me of your support and constant enthusiasm for my research; I would leave our meetings with a renewed drive and a clear sense of direction. Thank you for facilitating and guiding me through the PhD process and for making it such a positive experience.

Thank you to my friends in the School of Psychology. I have been incredibly lucky to have shared my postgraduate experience with so many intelligent and kind people, and I greatly value your friendship, support, and advice over the years.

Thank you most importantly to my mother, Neroli. Your belief in me and continued encouragement and support has been invaluable and has meant I have been able to undertake and complete this research. I will be forever grateful to you. 


\section{TABLE OF CONTENTS}

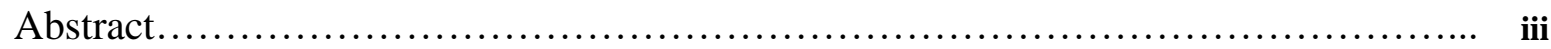

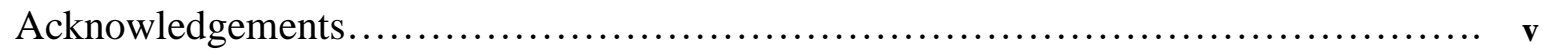

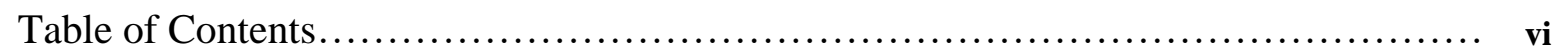

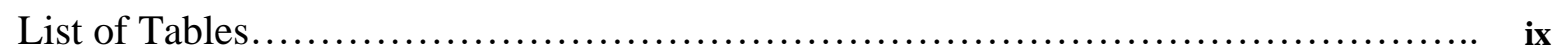

List of Figures...................

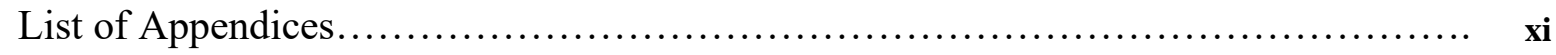

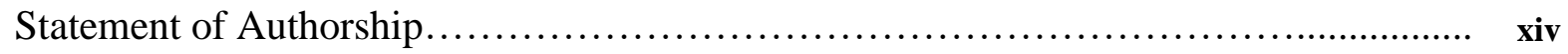

Chapter 1: Introduction...................................................... 1

Intimate Partner Aggression: Definitions, Prevalence, and Consequences. 1

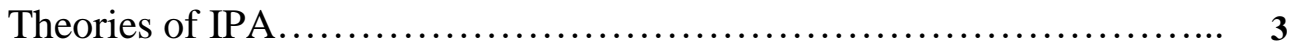

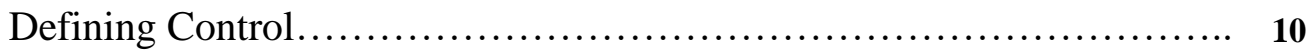

Existing Operationalizations and Measurement of Control............ 11

Aims and Structure of the Thesis............................... 16

Chapter 2: The Discriminance of Controlling Behaviors.......................... 19

Introduction................................................. 19

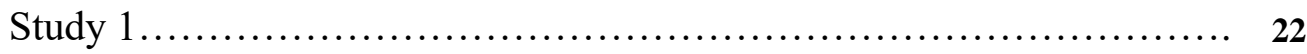

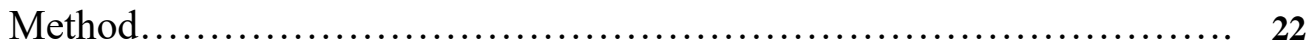

Results................................................... 25

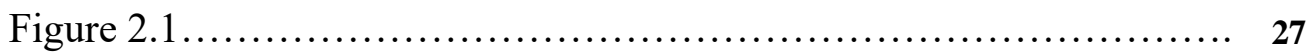

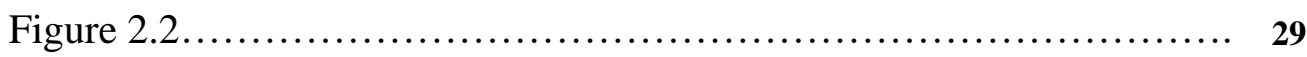

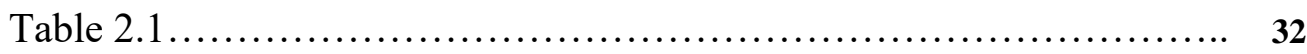

Study 2 A..................................................... 33

Method.......................................................... 33

Results......................................................... 36

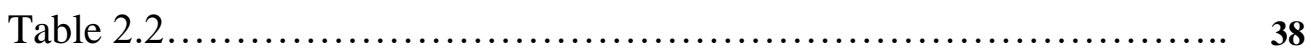

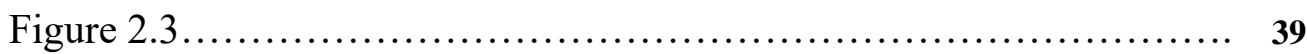

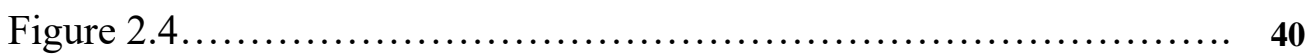

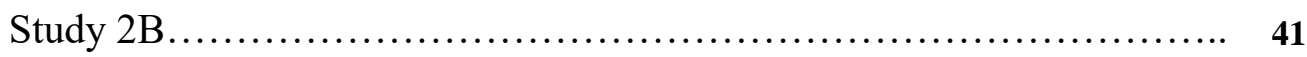

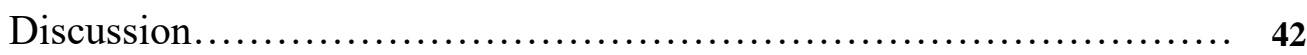


Chapter 3: Motivations for Physical and Psychological Intimate Partner Aggression: A Systematic Review............................................................. 48

Introduction.................................................. 48

Method ............................................................. 51

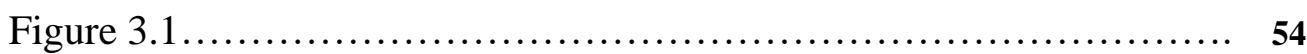

Results.................................................... 57

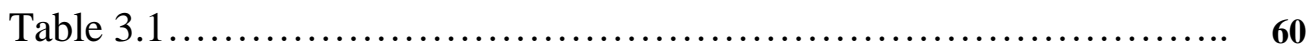

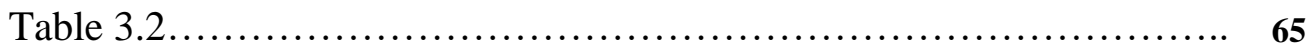

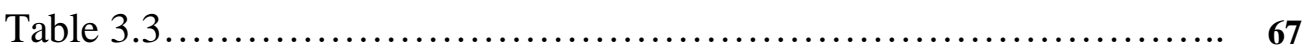

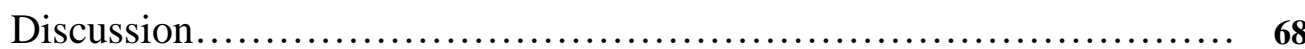

Chapter 4: $\quad$ Motivations for Intimate Partner Aggression........................ 76

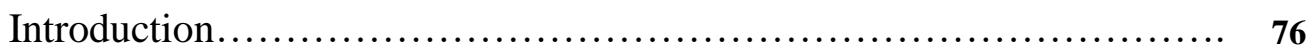

Method............................................................. 80

Results........................................................ 83

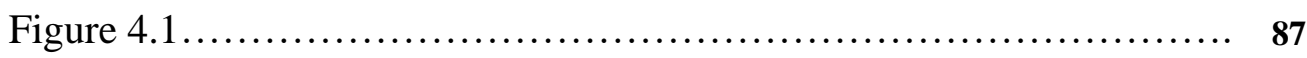

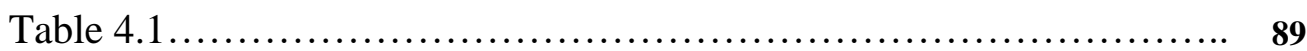

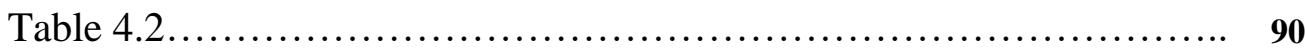

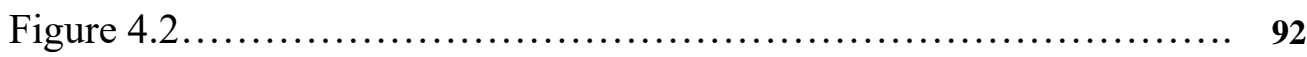

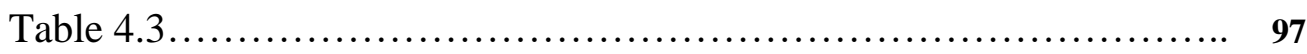

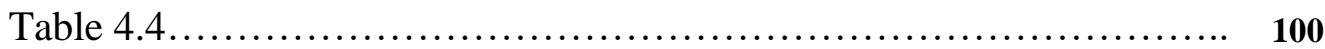

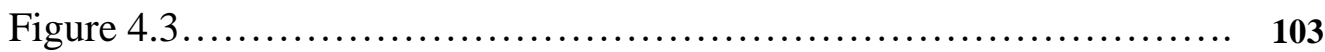

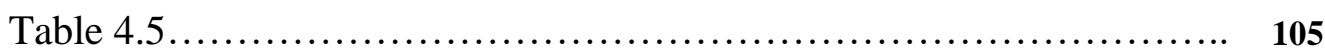

Discussion.................................................... 107

Chapter 5: Examining Coercive Control as an Outcome of Intimate Partner Aggression......................................................................................................................... 114

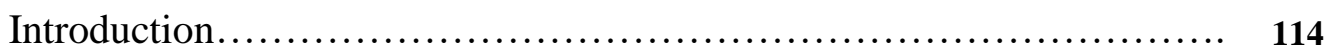

Method.......................................................... 117

Results........................................................ 119

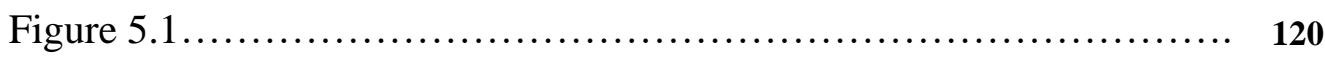

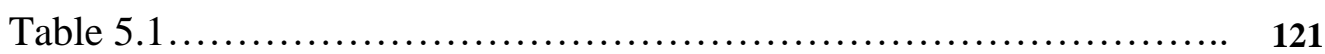

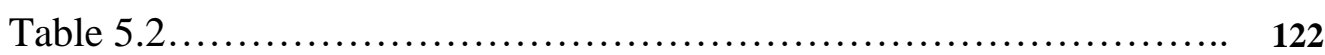

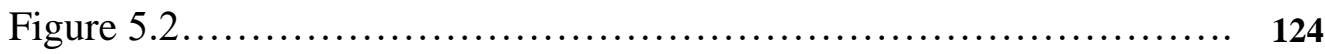

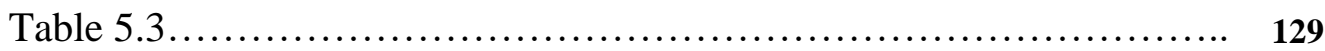

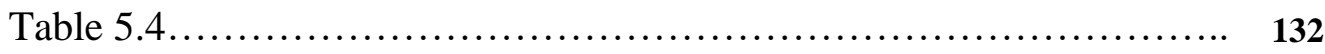

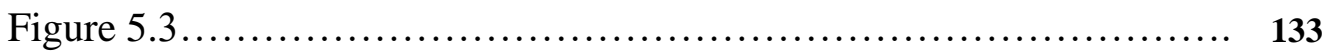




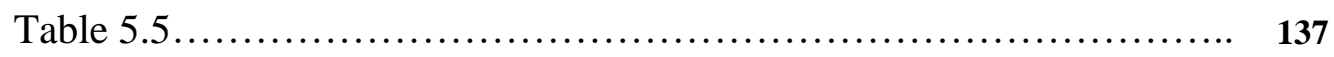

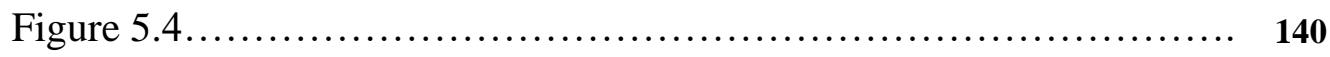

Discussion................................................ 141

Chapter 6: $\quad$ Discussion....................................................... 147

Summary of Key Findings and Contributions to the Literature.......... 147

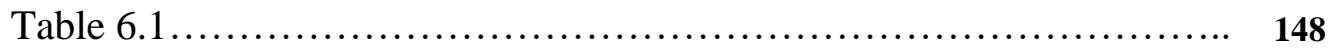

Implications for Theory, Practice, Policy, and Legislation............. 151

Limitations and Future Research.............................. 156

Conclusion................................................... 160

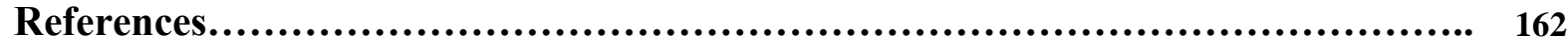

Appendices.................................................................. 182 


\section{LIST OF TABLES}

Table

Title

Page

Table 2.1 Descriptive statistics and intercorrelations for experienced and perpetrated behavior scales

Table 2.2 Fit indices for null, configural, and metric models for experienced and perpetrated behavior.

Table 3.1 Descriptive information of studies included in the systematic review

Table 3.2 Effect sizes for motivational-categories for physical IPA for males and females

Table 3.3 Summary of meta-analytic results for physical IPA for males and females.

Table 4.1 Frequencies of motivations for IPA endorsed (total and by gender)...

Table 4.2 Gender comparison of endorsement of motivations for IPA 90

Table 4.3 Motivations endorsed for perpetrated acts by type

Table 4.4 Model fit for the difference class solutions of the Latent Class Analysis

Table 4.5 Four-class solution one-way ANOVAs predicting mean level differences in outcome variables.

Table 5.1 Frequencies of outcomes for IPA endorsed (total and by gender)......

Table 5.2 Gender comparison of endorsement of outcomes for IPA....

Table 5.3 Outcomes endorsed for perpetrated acts by type

Table 5.4 Model fit for the difference class solutions of the Latent Class Analysis.

Table 5.5 Four-class solution one-way ANOVAs predicting mean level differences in outcome variables.

Table 6.1 Summary of main findings by chapter. 


\section{LIST OF FIGURES}

Figure

Title

Page

Figure 2.1 Scree plots produced by exploratory factor and parallel analyses

(experienced behavior).

Figure 2.2 Scree plots produced by exploratory factor and parallel analyses (perpetrated behavior)

Figure 2.3 Four factor model (experienced behavior) showing the standardized estimates for the factor correlations and loadings.

Figure 2.4 Three factor model (perpetrated behavior) showing the standardized estimates for the factor correlations and loadings

Figure 3.1 PRISMA flow diagram outlining the number of studies identified, screened, and deemed (in)eligible at each stage of the review process

Figure 4.1 Frequency of reported IPA perpetration by type

Figure 4.2 Scree plot from the categorical principal components analysis (CATPCA)

Figure 4.3 Four-class solution of motivations for IPA.

Figure 5.1 Frequency of experienced IPA by type

Figure 5.2 Scree plot from the categorical principal components analysis (CATPCA)

Figure 5.3 Four-class solution of outcomes for IPA.

Figure 5.4 Manifest variable model showing the standardized parameter estimates 


\section{LIST OF APPENDICES}

\section{Appendix}

Appendix A Copyright permission obtained from John Wiley and Sons

Appendix B Pattern matrix for the experienced behavior scale (Chapter 2) ........

Appendix C Pattern matrix for the perpetrated behavior scale (Chapter 2)..........

Appendix D List of parcelled items included in the confirmatory factor analysis for the perpetrated and experienced models....

Appendix E Standardized and unstandardized coefficients for the four-factor model (experienced behavior; Chapter 2)

2) $\ldots \ldots \ldots \ldots \ldots \ldots \ldots \ldots \ldots$

Appendix F Standardized and unstandardized coefficients for the three-factor

Appendix G Factor correlations for the experienced behavior model (Chapter 2)

Appendix H Factor correlations for the perpetrated behavior model (Chapter 2).

Appendix I Factor loadings for the experienced behavior model (Chapter 2).....

Appendix $\mathbf{J} \quad$ Factor loadings for the perpetrated behavior model (Chapter 2)......

Appendix K Data Extraction Form (Chapter 3).

Appendix L Quality Assessment Form (Chapter 3)....

Appendix M Coding of Motivations (Chapter 3)

Appendix N

Summary s
(Chapter 3)

Appendix $\mathrm{O}$

Summary statistics and forest plot for communication difficulties

(Chapter 3)....

Appendix P Summary statistics and forest plot for control (Chapter 3)

Appendix Q Summary statistics and forest plot for emotional regulation difficulties (Chapter 3)

Appendix R Summary statistics and forest plot for jealousy (Chapter 3)

Appendix S Summary statistics and forest plot for retaliation for emotional hurt (Chapter 3).
Page

182

201

202

203

204

205

304 
Appendix T Summary statistics and forest plot for self-defense (Chapter 3)..... 308

Appendix U Summary statistics and forest plot for to express anger (Chapter 3) 309

Appendix V Summary statistics and forest plot for to get attention (Chapter 3).. 310

Appendix W Funnel plot of Standard Error by Standard difference in means for

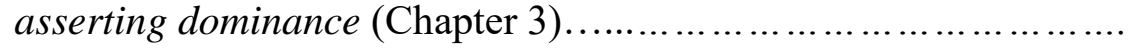

Appendix X Funnel plot of Standard Error by Standard difference in means for communication difficulties (Chapter 3 ).

Appendix Y Funnel plot of Standard Error by Standard difference in means for control (Chapter 3)

Appendix Z Funnel plot of Standard Error by Standard difference in means for emotional regulation difficulties (Chapter 3$)$.........................

Appendix AA Funnel plot of Standard Error by Standard difference in means for jealousy (Chapter 3).

Appendix BB Funnel plot of Standard Error by Standard difference in means for retaliation for emotional hurt (Chapter 3 ).

Appendix CC Funnel plot of Standard Error by Standard difference in means for self-defense (Chapter 3)

Appendix DD Funnel plot of Standard Error by Standard difference in means for

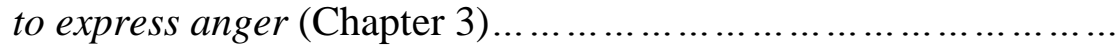

Appendix EE Funnel plot of Standard Error by Standard difference in means for to get attention (Chapter 3)....

Appendix FF Items included in categorical principal components and latent class analyses (Chapter 4)

Appendix GG Intercorrelations for the three-component solution produced by the CATPCA (Chapter 4)

Appendix HH Items removed from the CATPCA that were either non-significant or cross-loaded (Chapter 4).

Appendix II Pattern matrix for the three-component solution (Chapter 4).........

Appendix JJ Comparison of the fit statistics between the freely estimated and constrained models (Chapter 4)

Appendix KK Items included in categorical principal components, latent class, and manifest variable path analyses (Chapter 5). 
Appendix LL Items removed from the CATPCA that were either non-significant

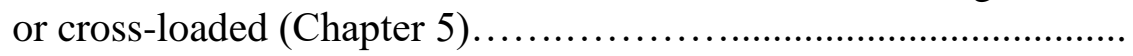

Appendix MM Pattern matrix for the three-component solution (Chapter 5).........

Appendix NN Comparison of the fit statistics between the freely estimated and constrained models (Chapter 5)

Appendix OO Parameter estimates of the manifest variable path model (Chapter

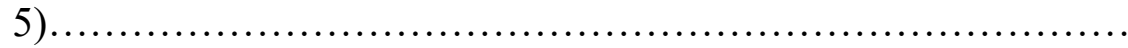




\section{STATEMENT OF ORIGINAL AUTHORSHIP}

I confirm that the work presented in this thesis in this thesis is my own and that I am the lead author in the published articles. Where information has been derived from other sources, I confirm that this has been indicated.

\section{PUBLICATIONS INCLUDED IN TEXT}

Dempsey, F., Hammond, M., \& Dixon, L. (2020). Investigating whether controlling and aggressive relationship behaviors are discriminant. Aggressive Behavior (46), 437-448. https://doi.org/10.1002/ab.21905

Permission was obtained from John Wiley and Sons for the inclusion of the paper published in Aggressive Behavior. Refer to Appendix A for copyright permission. 


\section{Chapter 1: INTRODUCTION}

\section{Intimate Partner Aggression: Definitions, Prevalence, and Consequences}

Intimate partner aggression (IPA) is the use of physical, sexual, and psychological aggression, and controlling behaviors towards a current or ex-partner (see Dixon \& GrahamKevan, 2011; 2020; National Center for Injury Prevention and Control, 2020; World Health Organization, 2010). While there is a consensus on the types of behaviors that constitute IPA, there is considerable disagreement with regards to who perpetrates and experiences IPA. While some researchers have adopted a gender-inclusive approach others consider IPA to be an issue of men's violence against women (Garcia-Moreno \& Watts, 2011; Pence \& Paymar, 1993). These underlying theoretical differences affect sampling decisions and survey design, which impact the estimates of the prevalence of IPA (Esquivel-Santoveña \& Dixon, 2012).

IPA has historically been understood as a problem of men's violence towards women (Hamel, 2007; e.g., Dobash \& Dobash, 1979). This understanding was reflected in an early research focus on female victimization that utilized selected samples from agency and emergency departments. Estimates of IPA from such research yielded high rates of male to female IPA. This sampling strategy was critiqued by researchers, such as Straus and Gelles (1999), who argued these samples were not representative and, thus, these estimates provided an inaccurate picture of the prevalence of IPA (Esquivel-Santoveña \& Dixon, 2012).

In response, Straus conducted the National Family Violence Survey in $1975(N=2,143)$ and $1985(N=3,520$; see Straus \& Gelles, 1986). These were large, nationally representative, gender-inclusive surveys, which showed high levels of bi-directional violence and gender symmetric rates of IPA. However, the Conflict Tactics Scale (Straus, 1979) was used in these 
studies to measure familial aggression. This scale has been critiqued as acontextual and for its focus on discrete acts (see Dobash \& Dobash, 2004).

Other large community representative studies, such as 'violence against women' surveys (e.g., National Violence Against Women Survey [NVAW]: see Tjaden \& Thoennes, 2000) and crime surveys (e.g., New Zealand Crime \& Victims Survey: Ministry of Justice, 2020) typically report higher rates of IPA for women than men (Esquivel-Santoveña \& Dixon, 2012). The framing of these surveys with either their explicit focus on female victimization or crime may account for the disagreement in the incidence of female-male aggression. While crime surveys are representative and survey the experiences of men and women, they prime participants to respond to questions within the context of criminal behavior and are, therefore, thought to underestimate the incidence of IPA, particularly men's victimization (EsquivelSantoveña \& Dixon). Similarly, 'violence against women' surveys, by the very way they are advertised, may prime individuals and affect gender prevalence rates. Research into how survey methodology affects the responses of men and women has been recommended (Tjaden \& Thoennes).

Estimates of the prevalence of IPA are also affected by differing operationalizations. For example, the National Intimate Partner and Sexual Violence Survey conducted by the Centers for Disease Control (CDC) estimate $25 \%$ of women and $10 \%$ of men experience IPA in their lifetime. CDC defines IPA as contact sexual violence, physical violence, and/ or stalking (National Center for Injury Prevention and Control, 2020). The inclusion of sexual violence in definitions of IPA may skew the results by gender, as women are reported to experience this type of violence at higher rates than men (World Health Organization, 2010). In contrast, the New Zealand Crime and Victims Survey, which is an annual, nation-wide crime survey $(N=8038)$, adopts a much wider definition of IPA that includes robbery, harassment, and threatening behavior, and property damage, in addition to physical and sexual assault 
(Ministry of Justice, 2020). The lifetime prevalence rate of IPA in this survey was $16 \%$. Differences in operationalizations and survey methodology also make cross-cultural comparisons on the prevalence of IPA unfeasible (Esquivel-Santoveña \& Dixon, 2012).

Despite the challenges in estimating rates of IPA, it is widely accepted that IPA is a major social problem (Krug et al., 2002) and has been recognized as a worldwide public health issue by the World Health Organisation (see Garcia-Moreno \& Watts, 2011). Indeed, a review of studies examining the effects of IPA on women's health reported both acute and chronic health consequences, including cardiovascular and respiratory disorders, diabetes, gynecological symptoms, gastro-intestinal problems, higher rates of human immunodeficiency virus, and lower self-perceived physical health (Dillon et al., 2013). Similarly, male victims of partner violence report significantly higher levels of depression, post-traumatic stress disorder, associated physical symptoms, and poor health scores compared to controls (Hines \& Douglas, 2018). Higher levels of mental illness such as depression, anxiety, and substance use have also been associated with experiencing IPA for both men and women (see Coker et al., 2002; Hines \& Douglas, 2010).

\section{Theories of IPA}

Differences in gender prevalence rates have lent support for different theories of IPA. Two conceptualizations have arguably dominated the field of IPA research: The gendered and family violence perspectives.

The gendered perspective. This explanation of IPA began in the 1970s (e.g., Dobash \& Dobash, 1979) and gained popularity when accounts of female victims of IPA were subsumed within the wider feminist movement, thereby transforming IPA into a gendered issue (Hamel, 2007). The gendered theory of IPA considers men's violence towards women as a product of sexual inequality and societal patriarchal norms that maintain men's dominance over 
women (Bell \& Naugle, 2008). Women's aggression towards men is understood as taking place within this context of power and control and is therefore viewed as self-defensive (see Dobash \& Dobash, 2004).

However, research, utilizing community and agency samples have demonstrated women's use of IPA is not limited to aggression perpetrated in self-defense. Whitaker et al. (2007) analyzed the data from the 2001 National Longitudinal Study of Adolescent Health to examine the prevalence of reciprocal and non-reciprocal physical IPA in young adults aged 18-28 years from the United States $(N=11,370)$. Violence was reported in $23.9 \%$ of relationships, of which $50.3 \%$ was characterized as non-reciprocal violence. The perpetrator of this violence was reported to be female in $70.7 \%$ of these non-reciprocal violent relationships by both men $(74.9 \%)$ and women $(67.7 \%)$. These findings challenge the interpretation of women's aggression as a response to men's violence and self-defense as an explanation for female perpetrated IPA. Indeed, Hines et al. (2007) analyzed the content of calls from male victims $(N=190)$ to a domestic abuse helpline and found all the men experienced physical violence from their female partners and 94.8\% experienced behavior described as 'controlling'. 'Controlling behaviors' were coded as per the control tactics on the Power and Control Wheel of the Duluth Model (Pence \& Paymar, 1993) and included intimidation, coercion and threats, and emotional abuse. The results from this study clearly illustrate the aggression experienced by these men was not perpetrated in self-defense and is analogous to the experiences of female victims in agency samples.

Research investigating gender differences in motivations for IPA has found inconsistent results, with both men and women endorsing control and self-defense as motivations for their use of IPA (see Langhinrichsen-Rohling et al., 2012 for a review). While some correlational research has found evidence of a relationship between men's patriarchal beliefs and their perpetration of IPA (see Leonard \& Senchak, 1996; Smith, 1990), other studies, including a 
meta-analysis, have failed to find evidence of this (see Coleman \& Straus, 1986; Dutton, 1995a; Sugarman \& Frankel, 1996).

Despite this lack of consistent empirical support (Bell \& Naugle, 2008), the gendered perspective of IPA has been highly influential with regards to the treatment of IPA (Dixon et al., 2012). For example, the Duluth Model adopts a psychoeducational approach, which is informed by the gendered perspective. This model considers IPA a form of 'power and control' perpetrated exclusively by men (Dutton, 2006a). The Duluth Model is the most widely implemented treatment approach for male perpetrated IPA in the western world. Indeed, the Duluth model is legislated as mandatory in most American states and Canadian provinces, and where not mandated or implemented in its pure form, substantially influences other approaches (Dutton). Despite the wide-spread implementation of the Duluth Model since it was first established in 1981 (Dutton), research into treatment outcomes shows recidivism rates of greater than 30\% (see Babcock et al., 2004; Shepard, 1992), questioning the efficacy of this approach.

As a single-factor, macro-level theory, the gendered perspective cannot account for why all men do not aggress against their female partners, for women's IPA, and the presence of IPA in the relationships of sexual and gender minorities (Dutton, 1995a). Patriarchy as an explanation for IPA and the gendered perspective has, therefore, been criticized as inadequate (Dutton) and heterosexist (Hassouneh \& Glass, 2008). Prevalence rates of IPA in same-sex relationships are difficult to ascertain, as research has employed both small, selected samples and differing methodologies (Hassouneh \& Glass). However, higher rates of IPA have been found in samples identifying as non-heterosexual, relative to those identifying as heterosexual (see Dank et al., 2014), necessitating an increased research focus. 
The family violence perspective. The family violence perspective of IPA is based on conflict theory (Graham-Kevan, 2007). This theory states that conflict is a process that facilitates social change and the establishment of group identity through the shared experiences of conflict of interest (Coser, 1957). Conflict of interest is considered ubiquitous and is experienced, not only by social groups but by families and individuals in intimate relationships (Carlson \& Jones, 2010). The methods used to forward self-interest and resolve conflict may involve the perpetration of IPA (Straus, 1979). The underlying motive for the use of these methods is the need to maintain or regain control of a situation and is what characterizes them as conflict tactics (Graham-Kevan, 2007). Based on this theory, Straus (1979) developed the Conflict Tactics Scale, designed to measure the tactics of three methods of relationship conflict resolution: reasoning, verbal aggression, and physical violence. This scale and the Revised Conflict Tactics Scale (CTS2: Straus et al., 1996) are the most widely used measures to assess IPA (Straus \& Douglas, 2004).

In response to the qualitative and socio-political nature of gendered research, researchers from the family violence perspective obtained data from men and women in large nationally representative sample surveys, as touched upon earlier in this introduction. Results from the $1975(N=2,143)$ and $1985(N=3,520)$ National Family Violence Surveys (see Straus \& Gelles, 1986) showed physical assault was perpetrated by men and women at similar rates (see Archer, 2018; Hamel, 2007; Straus et al., 1980). Following criticism of gender bias in reporting, the results from the National Family Violence Survey of married and cohabiting couples in 1985 were re-calculated so that only the information reported by women was analyzed. Women's assault rate (.124) was slightly higher than men's assault rate (.122) though not at a statistically significant level, for both minor and severe assaults (Straus, 1997). The finding of gender symmetry in the perpetration of physical IPA is well supported by hundreds of empirical studies conducted around the western world (Archer, 2018) including meta- 
analyses (Archer, 2000; Desmarais et al., 2012). It should be noted, however, that sex differences in IPA emerge with an increase in the severity of violence and with homicide (Archer, 2018). Meta-analyses show men perpetrate more severe forms of IPA (e.g., "beat up"; $d=.06 ; 32$ studies; $N=23,121$; Archer, 2002), although these effect sizes are small. However, the estimates for the effect sizes for partner homicide are comparatively high at between $d=$ 1.05 and 1.38 (Archer, 2018).

The weight of evidence for gender symmetric rates of aggression in heterosexual relationships coupled with a lack of evidence for single-factor theories (e.g., gendered theory or psychological dysfunction) led family violence researchers to propose the need for multifactorial theories of IPA (Dutton, 2006a). Dutton's (1995b) nested ecological framework adopts a 'bottom-up', gender-inclusive, multi-system approach, which accounts for characteristics of the individual, their relationships, and wider social context, and the interactions between them. A model, rather than a theory, it has nevertheless, been influential in the assessment and treatment of IPA (Hamel, 2014). Empirical research employing nested ecological approaches combine risk factors for IPA from multiple ecological levels (e.g., employment, relationship/ marital satisfaction, depression) to predict the amount of variance explained by hypothesized models (e.g., Stith et al., 2004). They are, therefore, able to account for equifinality with regard to IPA (Dixon \& Wride, 2020). For example, approximately 50\% of the variance was explained by the risk factors predicting men's and women's perpetration of IPA in a study conducted by O'Leary et al. (2007).

Johnson's typology. Attempting to reconcile the conflicting findings from the gendered and family violence perspectives, Johnson (1995) proposed a typology that suggests these perspectives highlight qualitatively different forms of violence and result from differing sampling strategies (Langhinrichsen-Rohling, 2010). Johnson claimed four types of violence could be differentiated by the presence or absence of control in the relationship, which 
consequently characterized the frequency and severity of aggression in that relationship (Johnson, 2006). He claimed studies using representative/ community samples over-select individuals, whose relationships are not characterized by control, rationalizing that the partners of those individuals, whose relationships are characterized by control, would be too afraid of their partners to participate in research. In contrast, he has stated agency samples over-select those individuals experiencing control in their intimate relationships (1995). The type of violence identified by Johnson that is most often reported in studies using clinical and agency samples is 'intimate terrorism' (later re-labeled coercive controlling violence; 2008), characterized by one partner's use of unidirectional controlling behavior used in conjunction with physical violence. 'Situational couple violence' is purported to be mostly seen in community samples and describes relationships where the violence is bi-directional and is the result of an escalation of conflict as opposed to control motivations. 'Violent resistance' is used to describe the violent response of one partner to an 'intimate terrorist', while 'mutual violent control' describes the situation in which both partners use violence and control in the relationship.

Johnson tested his theory by analyzing pre-existing interviews with an agency and matched community samples of women (2006). The items he created to measure the existence and/ or extent of control in the relationships of these women were designed to be analogous to the control tactics on the Power and Control Wheel of the Duluth Model (Pence \& Paymar, 1993). He claimed 'intimate terrorism' is perpetrated almost exclusively by men (97\% of his sample), while 'violent resistance' was typified almost exclusively by women (96\%). 'Situational couple violence' and 'mutual violent control' were found to be gender symmetric, as men comprised $56 \%$ of the 'situational couple violence' group and $50 \%$ of the 'mutual violent control' group, and women comprising the remaining $44 \%$ and $50 \%$ respectively (2006). However, Johnson's development and testing of his theory utilized samples with high 
rates of female victimization and male perpetration, such as those from women's shelters, their male partners, or men who were involved in the criminal justice system (Straus \& Gozjolko, 2014). This ultimately resulted in sampling bias, and therefore, questions the generalisability of his findings. Indeed, his research did not set out to secure a sample of men who had experienced harm from their female partner, so it is not possible to conclude that men do not experience 'intimate terrorism', as this was not tested (Dixon \& Graham-Kevan, 2020). Indeed, little research has examined male victimization from intimate terrorism in any detail (Hines \& Douglas, 2010). Research that has investigated Johnson's typology in non-selected samples has not found support for the gendered nature of 'intimate terrorism'. Ehrensaft et al. (2004), Laroche (2005), and Straus and Gozjolko found no gender difference in the number of individuals in community samples, who could be classed as 'intimate terrorists' (i.e., using high levels of control and aggression). Notably, Bates et al. (2014) found women were significantly more likely to be classed as 'high control', while men were significantly more likely to be categorized as 'low control' in a large university sample. In another study investigating the controlling behaviors of married couples, Stets and Hammond (2002) found wives engaged in more monitoring behaviors and restricted the activities of their spouse of which they did not approve more frequently, than did their husbands. This finding has been corroborated in the New Zealand Crime and Safety Survey (Ministry of Justice, 2014) with $17 \%$ of men versus $14 \%$ of women experiencing controlling behaviors in their relationships. These studies provide evidence that both men and women can use high levels of control and aggression in their intimate relationships.

Coercive control. While there is a nascent body of evidence demonstrating men's and women's use of control in their intimate relationships, the current conceptualization of coercive control is heavily influenced by the gendered perspective to the point where the two have almost become synonymous. Coercive control is, therefore, most frequently defined by the 
entrapment, isolation, the restriction of liberty and autonomy of women, which is facilitated by sexual inequality on both micro and macro levels (Stark, 2007). The political underpinnings of this construct differentiate coercive control from that of control, although the two are frequently conflated and used interchangeably in the IPA literature (Walker et al., 2017).

This thesis adopts a gender-inclusive approach to explore the accuracy of the gendered and family violence theories of control in relation to gender differences.

\section{Defining Control}

Despite a consensus in the legal, research, and practice literature regarding the theorized importance of control to understanding IPA, there is no commonly accepted definition (Hamberger et al., 2017). Control has most frequently been defined in terms of how it is operationalized, rather than as an abstract concept.

Behavioral definitions of control tend to be tautological, involving the use of 'controlling' behaviors or tactics to control an intimate partner (e.g., Beck et al., 2009; Johnson, 1999). The emphasis on specific behaviors as opposed to defining the construct itself has resulted in the frequent conflation of control with psychological abuse (Graham-Kevan, 2007). While these constructs share common features (e.g., intent to harm, adverse effect on victims; McHugh et al. 2013), not all psychological abuse is considered controlling and vice versa (Hamberger et al., 2017).

Adding to this ambiguity is the inclusion of coercion in 'definitions' of control. Although coercion (Oxford University Press, 2020a) can be distinguished from control (Oxford University Press, 2020b) by the use of force, the terms are used synonymously, particularly in legislation criminalizing “controlling or coercive behavior" (Serious Crimes Act 2015 [UK], s 76). Definitions of coercive control typically focus on the outcome or impact on the victim, including terminology such as isolation, domination, and subjugation (see Stark, 2007). 
This thesis considers control an abstract concept and, consequently, defines control as attempts to compel or constrain an intimate partner's behavior. The omission of behavioral references recognizes that control tactics are often specific to a relationship (Hamberger et al., 2017) and ensures the definition is gender-inclusive.

\section{Existing Operationalizations and Measurement of Control}

A lack of consensus regarding how control is defined and conceptualized has resulted in ambiguity surrounding how control should best be operationalized and measured (Hamberger et al., 2017). Control has been operationalized in the literature as a behavior, a motivation, and a consequence or outcome of IPA (Hamberger et al.).

Control as a behavior. Control is most frequently defined in the literature as the use of non-violent behaviors enacted to gain or maintain dominance in an intimate relationship (Stark, 2007). Thus, most commonly, control and coercive control has been measured using controlling behavior scales (Follingstad, 2007), which assess the frequency of these non-violent behaviors. Examples of these behaviors include deliberately preventing your partner from sleeping, deliberately keeping your partner short of money, restricting their activities and their access to friends and family, belittling your partner in public, gaslighting (deliberately distorting someone's reality), verbal abuse such as shouting, swearing, overt criticism, and threatening to harm your partner and/or people/ pets they care about.

Controlling behavior scales (e.g., Relationship Behavior Rating Scale-Revised: Beck et al., 2013; Checklist of Controlling Behaviors: Lehmann et al., 2012; Psychological Maltreatment of Women Inventory: Tolman, 1989) are widely used in studies investigating the relationship between control and IPA (e.g., Bates et al., 2014; Graham-Kevan \& Archer, 2009). Their use as correlates and predictors of IPA suggests "controlling behaviors" are considered distinct from other forms of IPA, such as physical aggression, despite there being no theoretical or statistical basis for this. However, some gendered researchers suggest that the presence of 
control in intimate relationships is not limited to the use of "controlling behaviors". Instead, maintaining that men use a range of tactics, such as threats, physical violence, and sexual aggression, to dominate and control their female partners (Stark, 2006).

The identification of the underlying motivation of an aggressive act by simply observing that act is considered problematic. One behavioral act (e.g., punch) can have diverse motivations (e.g., express anger or punish partner), and conversely, one motivation (e.g., wanting partner to listen to you) can impel different behavioral acts (e.g., yelled at or slapped partner; Bushman \& Anderson, 2001). This difficulty is recognized in the general aggression literature and, thus, motivations (e.g., instrumental aggression; hostile aggression), rather than behaviors, have been used to understand aggression (Bushman \& Anderson). This approach has extended to the functional assessment of violent offenders, which focuses on why a particular behavior is occurring, as opposed to what behavior is occurring, as behavior is influenced by context (Ireland, 2009).

Indeed, the rationale for the Psychological Maltreatment of Women Inventory (Tolman, 1989), which is the most widely used measure of control in intimate relationships (Hamberger et al., 2017), demonstrates how non-physical behaviors are in fact IPA modified by social context. This scale was initially designed to measure the psychologically abusive behaviors in intimate relationships of men undergoing treatment for IPA. Tolman (1989) proposed these men would refrain from committing physical acts of violence against their wives/ partners while under the surveillance of the treatment program in which they were participating, and instead find alternative, non-physical means by which to control them.

Despite their wide-spread use, there are multiple issues regarding the use of controlling behavior scales. The arguably favored gendered theory of IPA has meant much of the data that has informed the development of controlling behavior measures has been collected from women's refuges or advocacy services (McHugh et al., 2013). Consequently, our current 
conceptualizations of control and the measures that continue to inform our understanding of control are based upon the experiences of non-representative samples. The severe and escalatory nature of the violence experienced by these women means the items developed from these experiences are likely to form different and rare patterns of associations to those in a general population (DeVellis, 2012). This makes the generalisability of these measures, and potentially their validity, more questionable; a phenomenon referred to as the clinical fallacy (Straus, 1997).

Indeed, IPA scales were developed to understand women's experiences of relationship violence (Follingstad \& Rogers, 2013). Thus, the use of female victim experiences to inform the development of measures of controlling behaviors has resulted in the conflation of behavioral acts with the perceived motivation of control. However, the motivation for the use of these "controlling behaviors" has not been directly tested or measured (Hamberger et al., 2017), and thus the assumption they are enacted with the intent to control an intimate partner persists.

Furthermore, the divergent validity of controlling behaviors from other types of IPA has not been investigated, and, thus, the question as to whether "controlling behaviors" are distinct based on the underlying motivation of control remains. The construct validity of the scales developed to measure control in intimate relationships and of their continued use is, therefore, spurious. The questionable validity of controlling behavioral scales is concerning considering the widely accepted association between the frequency of IPA and control has been established using these measures. This necessitates the investigation into the underlying motivations for behaviors currently labeled in the literature as "controlling".

Control as a motivation. The examination of motivation is necessary to contextualize human behavior and give it meaning, thus, providing a deeper understanding of a particular behavior (Scheer, 2001). Motivations are theorized as causal and as explanations for behavior 
(see Ward, 2017) and are, thus, considered an important area of research (LanghinrichsenRohling et al., 2012), which informs the assessment and treatment of aggressive behavior (Ireland, 2009).

Much of the research that has been conducted on motivations for IPA has been heavily influenced by the gendered perspective (Dutton, 2006a). This influence has resulted in the concentrated study of control as a motivation for male perpetrated IPA and self-defence as a motivation for female perpetrated IPA. Indeed, control and self-defence were the most (76\%), and the third most (61\%) frequently studied motivations in a systematic review of 75 studies of motivations for physical IPA (Langhinrichsen-Rohling et al., 2012). Existing reviews (see Bair-Merritt et al., 2010; Langhinrichsen-Rohling et al.) have simply focussed on the frequency with motivations for IPA have been researched. To date, no study has statistically examined the effect sizes of motivations to ascertain which of these have the largest effect sizes and would, therefore, make the most appropriate and effective treatment targets. Thus, although control has a well-established and important relationship with IPA from a theoretical perspective, the strength of this association, relative to alternative motivations for IPA, has not been tested. This is important given the assumptions of the gendered perspective and its influence on treatment programs (Dutton, 2006a) and legislation (Hester et al., 2017).

Despite the apparent importance of control as a research topic, some research investigating motivations for IPA has found that alternative motivations (e.g., retaliation, emotional dysregulation, communication difficulties) are endorsed more frequently than control (see Elmquist et al., 2014; Follingstad et al., 1991; Harned, 2001; Kelley et al., 2015; Ross, 2011). However, there is considerable inconsistency in study findings, which has been attributed to how studies have operationalized motivations (Langhinrichsen-Rohling et al., 2012). Quantitative measures have been developed to assess a variety of motivations for the perpetration of IPA (e.g., Motivations and Effects Questionnaire: Follingstad et al.; Reasons 
for Violence Scale: Stuart et al., 2006). However, these measures include risk factors and emotions, neither of which current psychological theory considers causal (see Heffernan et al., 2019; Ward \& Carter, 2018). In addition, such measures have infrequently been subject to scale development and, therefore, lack validity.

While the consideration of motivations for IPA is useful from a treatment perspective (Ireland, 2009), the operationalization of control as a motivation does not account for how successful an individual's attempts at control might be. Thus, examining control from the victim's perspective by operationalizing control as an outcome of IPA may present an arguably more accurate alternative to motivations.

Control as an outcome. Control, and especially coercive control, is frequently conceptualized as a pattern of harm (see Smith, Tessaro, \& Earp, 1995; Stark, 2007). However, controlling behavior scales, which are most frequently employed in studies investigating the relationship between (coercive) control and IPA, measure discrete events. This approach fails to account for the frequency with which these behaviors have been used and, thus, does not provide information regarding the chronicity of abuse considered characteristic of (coercive) control (see Stark, 2007). Researchers have, therefore, suggested that behavioral measures, particularly those that count the number of discrete acts of 'controlling behaviors' experienced by participants, are an inaccurate way of measuring (coercive) control (Hardesty et al., 2015). In addition, the acontextual nature of behavioral measures fails to capture the meaning and impact that IPA has on individuals' lives (Smith, Earp, \& DeVillis, 1995) and to ascertain whether the acts themselves are experienced as controlling (Hamberger et al., 2017). The consideration of victim experiences and the operationalization of control as an outcome of IPA may, therefore, result in increased validity and accuracy in the measurement of control.

However, there are comparatively few studies that have empirically investigated the outcomes of IPA. Much of the existing research that has operationalized control as an outcome 
is qualitative, has focussed on women, and has been influenced by the gendered perspective (e.g., Crossman \& Hardesty, 2018; Landenburger, 1989; Smith, Tessaro, \& Earp, 1995). The assumption that the consequences of IPA are more deleterious for women than for men has therefore become accepted. There is, however, some empirical evidence to support this supposition, as research has found women are more fearful than men, as a result of IPA (see Hamberger \& Larsen, 2015 for a review). Though, gender differences in fear have been attributed to socialization (Langhinrichsen-Rohling, 2010), trauma (Kernsmith, 2006), and survey methodology (Hamberger \& Larsen). The influence of the gendered perspective on the development of measurement tools (e.g., Women's Experiences with Battering Scale: Smith, Earp, \& DeVellis, 1995) as previously mentioned, has led to a bias in the way in which control is measured (McHugh et al., 2013), such as the focus on fear. This raises questions regarding the validity of research findings and our depth of understanding of men's and women's experiences of control.

\section{Aims and Structure of the Thesis}

The subjective nature and complexity of control make the construct difficult to conceptualize, define, and measure (McHugh et al., 2013). Contributing to this problem has been a lack of consensus regarding the operationalization of control (Hamberger et al., 2017). This thesis aims to test the validity of the commonly accepted conceptualizations of control by examining control as a behavior, motivation, and an outcome to determine how this construct can best be conceptualized and measured. Approval from the Victoria University School of Psychology Human Ethics Committee was obtained for each of the studies included in this thesis to minimise the risk of potential harm to participants was minimised and ensure their

confidentiality (Chapter 2: \# 25918; Chapters 4 and 5: \# 27163). The professional codes of New Zealand Psychological Society and New Zealand Psychologists Board were also adhered to. 
Controlling behaviors are included in definitions and legislation and comprise measurement tools designed to assess the level of control in intimate relationships. These behaviors are typically non-physical, are considered a distinct sub-type of IPA, and are assumed to be motivated by control. However, the validity of these assumptions has not been tested. Chapter 2 presents the first study in the thesis (Study 1), which aims to clarify whether behaviors, previously labeled in the IPA literature as "controlling" are statistically distinct from other aggressive acts perpetrated against and experienced by individuals in intimate relationships. Exploratory and confirmatory factor analyses in online and student samples were used to investigate the divergent validity of "controlling behaviors".

The assumption that IPA perpetrated by men is motivated by control and IPA perpetrated by women is motivated by self-defence informs treatment. However, there is considerable inconsistency in the findings of research investigating motivations for IPA and gender. Chapter 3 systematically reviews the published literature on motivations for physical and psychological IPA perpetrated by men and women to objectively ascertain which motivations would make the most appropriate targets for intervention. This includes an appraisal of the quality of research and meta-analysis of the included studies.

Chapter 4 (Study 2) aims to investigate the relationship between a range of motivations for IPA and the type and frequency of aggressive acts used by men and women in intimate relationships. The accepted association between control and IPA has been established using proxy behavioral measures as opposed to directly assessing motivations. In an online sample, Study 2 examines whether the association between control motivations and the type and frequency of IPA is stronger than for non-control motivations and whether these associations are stronger for men than for women. This study also tests the validity of Johnson's typology (1995; 2006), which differentiates on the presence of control motivations, characterizes types 
by the frequency of violence, and maintains men predominantly comprise the most controlling profile.

Coercive control is fundamental to the gendered conceptualization of IPA and is considered the most deleterious form of IPA, resulting in the entrapment and subjugation of women (Stark, 2007). Coercive control has been used to differentiate between types of IPA (Johnson, 1995; 2006) and to inform policy and legislation (see Hester et al., 2017). Chapter 5 (Study 3) tests the current conceptualization of coercive control, in an online sample, by investigating whether coercive control outcomes are associated with specific types of IPA, with an increased frequency of IPA, and are gendered.

Chapter 6 provides a summary of the previous chapters and a general discussion of the main findings of the thesis, in addition to study limitations, the theoretical and practical implications of the research, and how the findings could direct future research. 


\section{Chapter 2: THE DISCRIMINANCE OF CONTROLLING BEHAVIORS}

Intimate partner aggression (IPA) is recognized as a worldwide public health issue with severe individual, community, and societal consequences (Krug et al., 2002). As such, attempts to explain why aggression in relationships occurs have dominated the literature. Control is widely considered central in explaining IPA, particularly in relation to why men engage in IPA toward women (Hamberger et al., 2017), and in predicting more severe types of IPA (Johnson, 1995).

Much of the emphasis that has been placed on control in the IPA literature is a result of the gendered perspective of IPA (Bates et al., 2014; Dobash \& Dobash, 1979; Felson \& Outlaw, 2007); the main assumption of which is that IPA is a patriarchal paradigm in which men's violence towards women takes place within the context of control (Bates et al., 2014). In contrast, the Family Violence perspective argues that rates of IPA are gender symmetric (Hamel, 2007). Data obtained from the National Family Violence Survey of married and cohabiting couples in 1975 and 1985 showed the rate of physical assault was similar for both males and females, with the female to male assault rate slightly higher, though not at a statistically significant level (Straus, 1997). These findings are in accordance with those reported by Archer (2000) in a meta-analysis of studies on marital and dating violence in which he found women hit their partners significantly more frequently than men, but also had a higher rate of injury requiring medical attention, with a small effect size of .08 in the male direction (Archer, 2018)

Johnson (1995) proposed a typology to reconcile the conflicting research findings from the gendered and family violence perspectives, suggesting the findings highlighted

This chapter is published as "Investigating whether controlling and aggressive relationship behaviors are discriminant" in Aggressive Behavior (46), 437-448. https://doi.org/10.1002/ab.21905 
qualitatively different forms of violence. Johnson categorized different types of IPA by distinguishing those which take place within the context of control from those within a context of conflict between couples. Johnson (2006) developed his typology by measuring the use of physical aggression and control reported by the female member of a couple ( $N=274$ women). By recruiting women from a shelter/court referred sample and a matched community sample he demonstrated that men and women used physical aggression in the absence of control at approximately equal rates and that the violence used was typically of low severity (termed 'situational couple violence'). Men were shown to primarily use severe physical aggression in the context of control (termed 'intimate terrorism').

To inform his typology, Johnson (2006) used a range of non-physical "control tactics" (e.g., restricting access to money, isolating from friends/family, being overly critical, using threats of harm) thought to identify the latent construct of control, used to gain power over an intimate partner, and that correspond to the tactics of Pence and Paymar's (1993) Power and Control Wheel. Johnson's work has been influential in explaining IPA and prioritizing family violence resources to address violence against women and children (Dixon et al., 2020). Furthermore, Johnson's (2006) and Pence and Paymar's (1993) work has been extended to the development of laws that recognize controlling behavior in an intimate relationship to be a criminal offense (e.g., Domestic Violence Act 2018 (Ireland), s 39; Serious Crimes Act 2015 (UK), s 76) and in the design of male perpetrator intervention programs, with many statefunded programs necessitating the re-education of men regarding their need for control over women and their use of specific behaviors to achieve this (Dutton, 2006a).

Thus, 'controlling behaviors' have become an accepted way to identify control in relationships. 'Controlling behaviors' are also an integral part of definitions of IPA proposed by governments and organizations responsible for family violence policy and practice (e.g., Family Violence Death Review Committee, 2016; Home Office, 2013). Such descriptions 
endorse 'controlling behaviors' as a specific subset of actions distinct from other types of IPA and have been defined as

".. a range of acts designed to make a person subordinate and/or dependent by isolating them from sources of support, exploiting their resources and capacities for personal gain, depriving them of the means needed for independence, resistance and escape and regulating their everyday behavior" (Serious Crimes Act 2015 (UK), s 76).

Although control is most frequently conceptualized in the literature as the use of nonviolent behaviors (Stark, 2007), researchers have also suggested that perpetrators use a range of behaviors including physical and sexual violence to control their partners, not just those behaviors that are traditionally seen as "controlling" (Family Violence Death Review Committee, 2016; Pence \& Paymer, 1993; Stark). Adding to this lack of clarity, the definition of control and what constitutes controlling behavior is particularly confounded by the considerable overlap between behaviors that previous research has labeled as "controlling" and psychological aggression (Ehrensaft et al., 1999). Consequently, the subjective nature and complexity of control make the construct difficult to define and measure (McHugh et al., 2013).

In spite of this, the centrality of 'controlling behaviors' in legal and political domains has resulted in the development of psychometric tools to measure them (e.g., Relationship Behavior Rating Scale-Revised: Beck et al, 2013; Controlling Behaviors Scale: Graham-Kevan \& Archer, 2003; Controlling and Abusive Tactics Questionnaire: Hamel et al., 2015; Checklist of Controlling Behaviors: Lehmann et al., 2012). The tools have been developed based on the assumption that attempts to gain power and control over an intimate partner can be measured by a quantifiable set of non-physical behavioral acts. Thus, 'controlling behaviors' are assumed to be distinct from other types of IPA. As the measurement of control is deemed important in identifying the likelihood of severe physical and psychological harm and 
escalation of physical injury, development of laws, policy, and practice (Johnson, 2006) it is imperative such assumptions are validated.

The current research investigates the extent to which acts identified as 'controlling behaviors' in the IPA literature are distinct from other aggressive relationship acts. In Study 1, we reviewed the IPA and relationship literatures to identify 1397 items involving "controlling", physical, sexual, and psychologically aggressive acts perpetrated and/or experienced by an intimate partner. One hundred and one items were retained following the application of exclusion criteria, and these items formed our online questionnaire. We then surveyed the use and experiences of a range of aggressive acts in intimate relationships in an online community sample, employing exploratory factor analysis. We hypothesize that behaviors previously conceptualized as 'controlling behaviors' will be statistically distinct, forming a single factor. Study 2 A recruited a student sample and employed a confirmatory factor analysis to replicate the factor structure found in Study 1. Finally, we conducted measurement invariance to investigate whether the structure and meaning of identified constructs were equivalent between the community and student samples (Study 2B).

\section{STUDY 1}

\section{Method}

Participants. Participants were a sample of Amazon Mechanical Turk (MTurk) workers. MTurk workers sign up for the service voluntarily and select to complete surveys that are advertised on MTurk at their own discretion. Although the quality of the data obtained from MTurk samples has been questioned in the literature (Hauser \& Schwarz, 2016), research has shown that the data collected from MTurk populations is of equal or better quality (Chandler et al., 2014; Hauser \& Schwarz), and is more demographically diverse (Buhrmester et al., 2011) than student samples. 
Eligible users were 18 years or older, in the United States of America or Canada, and were involved in a romantic relationship lasting at least one month in the last year $(N=561$; $56 \%$ male, $44 \%$ female, $\left.M_{\text {age }}=34.7, S D=10.6\right)$. Sixty-seven percent $(n=376)$ of the sample identified as White, 10.9\% $(n=61)$ identified as African American, 7\% $(n=39)$ identified as Latinx, 5.5\% identified as Asian $(n=31), 1.8 \%$ identified as Native American $(n=10), 7.8 \%$ were of another ethnicity $(n=44)$, and $8.2 \%$ identified with more than one ethnicity $(n=46)$. Participants were excluded if they did not consent to participate $(0.3 \%, n=2)$, did not meet the relationship criteria $(4.2 \%, n=27)$, or did not identify as either male or female $(1.2 \%, n=8)$. Those who completed the survey unreasonably quickly (under 281 seconds) were also removed $(4.5 \%, n=29)$, as were those who showed clear patterns of non-responding $(3.4 \%, n=22)$, leaving the sample described above.

Procedure. The information and consent sheet provided participants with a link directing them to the online survey site (Qualtrics) to complete the survey. The study was described as "a survey about people's experiences of aggression in their intimate relationships". Upon completion, participants were debriefed and given details of helplines and support organizations specific to their geographical region. Participants received \$1.35 (USD) in appreciation of their time. The study received approval from the Victoria University School of Psychology Human Ethics Committee.

\section{Materials.}

Selection of candidate items. We identified behavioral acts relating to "controlling" or stalking behaviors, or psychological, physical, and sexual abuse from five reviews of measures of IPA (Graham-Kevan, 2007; Hamberger et al., 2017; O'Leary, 1999; Strauchler et al., 2004; Thompson et al., 2006). This resulted in 46 papers for review, and 1227 items for consideration. We also reviewed the non-aggression relationship literature to identify items involving 
behaviors that people employ to influence or regulate their intimate partners. The sites searched were: Google Scholar, PsycINFO, Sage Journals, ScienceDirect. The search terms used were: "communication styles and intimate relationships", "communication strategies and intimate relationships", "conflict strategies and intimate relationships", “interpersonal influence and intimate relationships", "power strategies and intimate relationships", and "partner regulation". This search identified eight papers, with a total of 170 partner-regulation items. Only items that came from scales that had been published in peer-reviewed journals or books, had data on their psychometric properties, and were designed for research and survey purposes were included, resulting in a review of 54 scales, and a total of 1397 items for consideration. The searches of the aggression and non-aggression literature took place between mid-December 2017 to late January 2018. Our inclusion criteria for items were that the items (1) related to behavior rather than intention or outcome, and (2) had acceptable face validity. Of the 1397 items, 321 items were excluded because they described feelings, intention or motivation, or other outcomes associated with behavior (e.g., "Drove recklessly to frighten the other person"). We excluded 295 items that contained subjective terms (e.g., "Showing up at places in threatening ways") or complex language (e.g., "regulatory harassment"), or were double-barrelled items (e.g., "Punched, shoved or grabbed me"). The removal of such items reduces ambiguity and improves clarity (DeVellis, 2012). We then removed 680 items that contained similar wording and/or content to another item, leaving a total of 101 items. Finally, we formed response category pairs (e.g., "My partner slapped me"; "I slapped my partner") to obtain 202 items assessing both perpetrated behaviors and experiences of these behaviors.

Questionnaire format. The online questionnaire first asked for demographic information then presented the 202 items described above. Participants had to have been in a romantic relationship lasting at least one month in the past year. They were instructed to respond with regards to this relationship, or their most volatile relationship if they had been in 
more than one in the past 12 months. They were also asked to disregard or ignore any experiences of behaviors that may have occurred within the context of consensual sexual activity, to differentiate BDSM behaviors from IPA. A five-point Likert scale was selected as the response range as this is considered satisfactory to provide sufficient variance (Hinkin, 1998). A numerical response format (e.g., Never, 1-2 times, 3-5 times, 6-10 times, more than 10 times) was chosen as the items related to discrete events, and to avoid the potential ambiguity of response options such as 'occasionally', and ‘sometimes' (DeVellis, 2012). Items were presented in a randomized order to reduce response sets and increase participant engagement (see Straus et al., 1996).

\section{Treatment of data.}

Missingness. Little's MCAR test for missingness was conducted (Little \& Rubin, 2002) with $1 \%$ of any one item missing and a total of $.009 \%$ of data found to be missing $\chi 2(1201)=$ 2191.86, $p<.001$. Although this test was significant, there is currently no test for establishing whether the data is MNAR or MAR and, due to the fact the amount of missing data was so small, the decision to proceed with further analyses was made. Exploratory factor analysis ran in SPSS provides listwise or pairwise deletion or replacement with the mean as options for imputing missing data. However, these methods are known to introduce bias into the data set and reduce statistical power (Field, 2009). Consequently, data were imputed using Expectation Maximisation (EM), a maximum likelihood technique that provides less biased estimates than other techniques including listwise deletion (Kline, 2005).

\section{Results}

We tested the extent to which measurement of aggressive acts considered 'controlling behaviors' would form a single factor, separate from other forms of IPA, using exploratory factor analysis (EFA). Analyses were conducted in IBM Statistics for Windows (Version 25.0; 
IBM Corp., 2017). We specified the Maximum Likelihood extraction method as the data were continuous (Gaskin \& Happell, 2014). A Promax rotation method with a Kaiser Normalisation was chosen because we assumed the factors would be correlated (Field, 2009; Gaskin \& Happell).

As recommended by Raîche et al. (2013), multiple methods of estimation for determining the number of factors to retain were employed to ameliorate the limitations associated with individual methods (e.g., overestimation of the number factors using Cattell's (1966) scree plot). The procedures for determining the number of factors to be retained included Cattell's scree plot, and Horn's (1965) parallel analysis using the 95th percentile rule (Courtney, 2013). A comparison of the scree plots produced by the EFA (actual data) and the parallel analysis (simulated data) was used to determine the number of factors to retain. The number of factors that precede the point where the two lines intersect indicate the number of factors in the solution (Preacher \& MacCallum, 2003). Items with minimum loadings of .4 (Stevens, 1986) and items that cross-loaded between two or more factors with a difference of greater than .2 between primary and secondary factor loadings were retained (see Howard, 2016).

"Experienced Behaviors" model structure. An EFA was used to identify the factor structure among items in the questionnaire from which a clear four-factor structure emerged (see Appendix Bfor the full list of items, factor loadings, Eigenvalues, and percentage of variance explained by the factors). The results from the comparative scree plot (see Figure 2.1) confirmed a four-factor solution, which was consistent with the non-graphical methods employed . 


\section{Figure 2.1.}

Scree plots produced by exploratory factor and parallel analyses (experienced behavior).

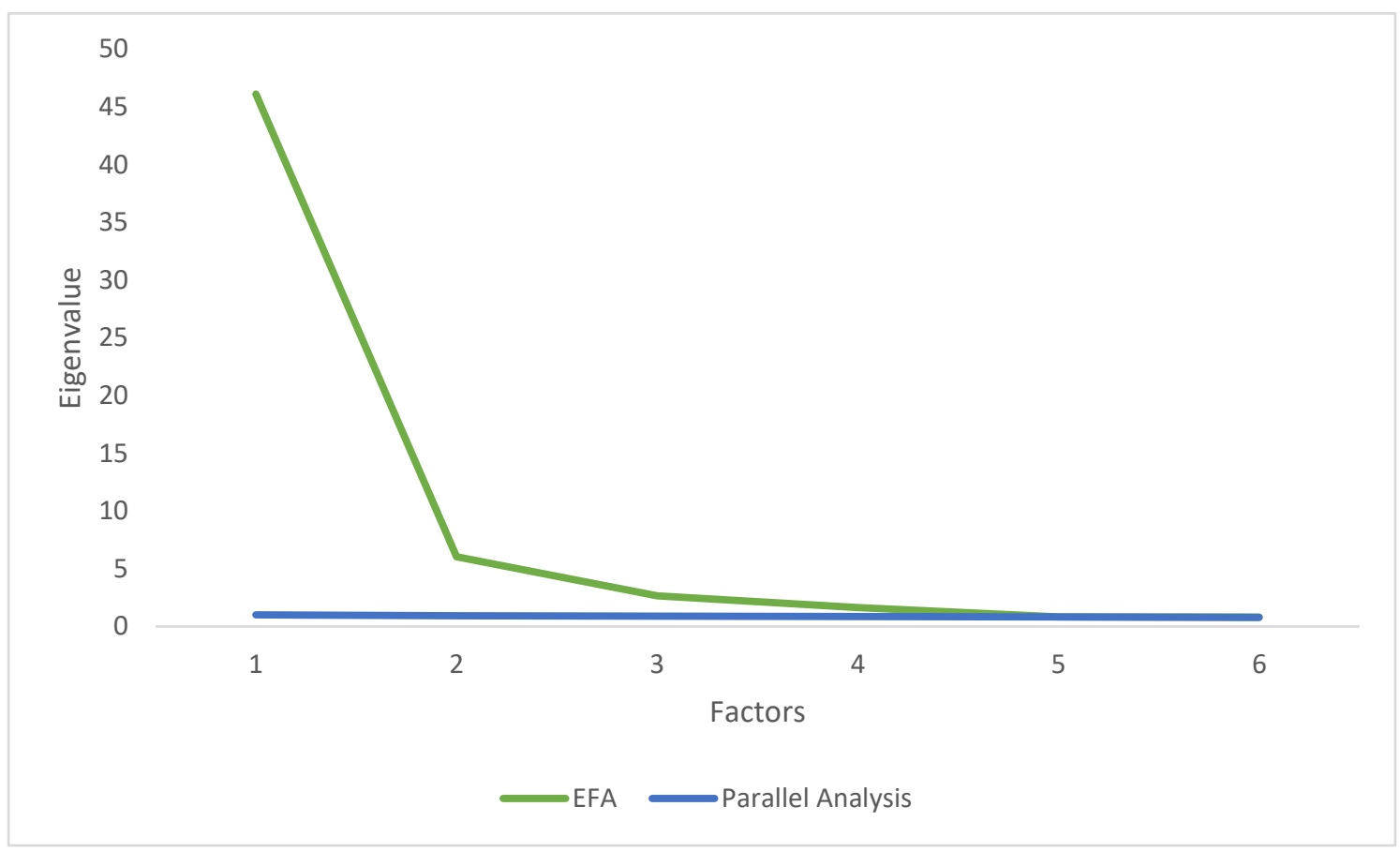

Factor 1 in the experienced behavior model consisted of 46 items and accounted for $55.6 \%$ of the variance with an Eigenvalue of 46.12. This factor included a wide range of items from measures that prior research has labeled physical violence, sexual violence and coercion, physical threats and threats to reputation, restricting access to transport, family and money, and 'controlling behaviors'. We labeled this factor "Eclectic Aggression" because it contained a wide range of direct and indirect aggressive acts. Severe physical and sexual violence items had the highest factor loadings, indicating that these items were more characteristic of the factor (Giles, 2002). Forty-six percent of items in the "Eclectic Aggression" factor were obtained from scales or subscales expressly designed to measure control (See Appendix B).

Factor 2 comprised 23 items, accounting for $7.2 \%$ of the variance, with an Eigenvalue of 6.04. It included non-physical violent behaviors that were directly enacted toward the participant and would be described in the literature as direct psychological aggression (e.g., yelling and criticizing both the partner and their family) and items that prior research has 
considered 'controlling behaviors' (52\%; e.g., "My partner told me my feelings were irrational or crazy”). Accordingly, we labeled this factor "Direct Psychological Aggression".

Factor 3 had nine items and explained $2.6 \%$ of the total variance, with an Eigenvalue of 2.66. This factor included monitoring behaviors, which were mostly covert in nature, such as listening to telephone conversations and reading e/mail, with obvious undertones of jealousy (e.g., "My partner accused me of being unfaithful or flirting with others"). This factor was therefore labeled "Monitoring Acts". One hundred percent of the items in the Monitoring Acts factor were taken from scales or subscales used to determine the presence or absence of control in intimate relationships.

Factor 4 had four items and accounted for $1.5 \%$ of the total variance, with an Eigenvalue of 1.63. Factor 4 was labeled "Prosocial Relationship Behaviors" because it contained positive relationship behaviors (e.g., "My partner showed concern about my feelings"). None of the items in this factor originated from measures used to assess control.

In sum, the Monitoring Acts factor was exclusively comprised of behaviors that have been previously conceptualized as "controlling". These results would appear to support the original hypothesis that such behaviors are statistically distinct from other forms of IPA.

"Perpetrated Behaviors" model structure. A three-factor structure was found for items in the perpetrated behavior model. (see Appendix $\mathrm{C}$ for the full list of items, factor loadings, Eigenvalues, and percentage of variance explained for the factors in this model). The scree plots produced by exploratory factor and parallel analyses confirmed the three-factor solution (see Figure 2.2). 


\section{Figure 2.2.}

Scree plots produced by exploratory factor and parallel analyses (perpetrated behavior).

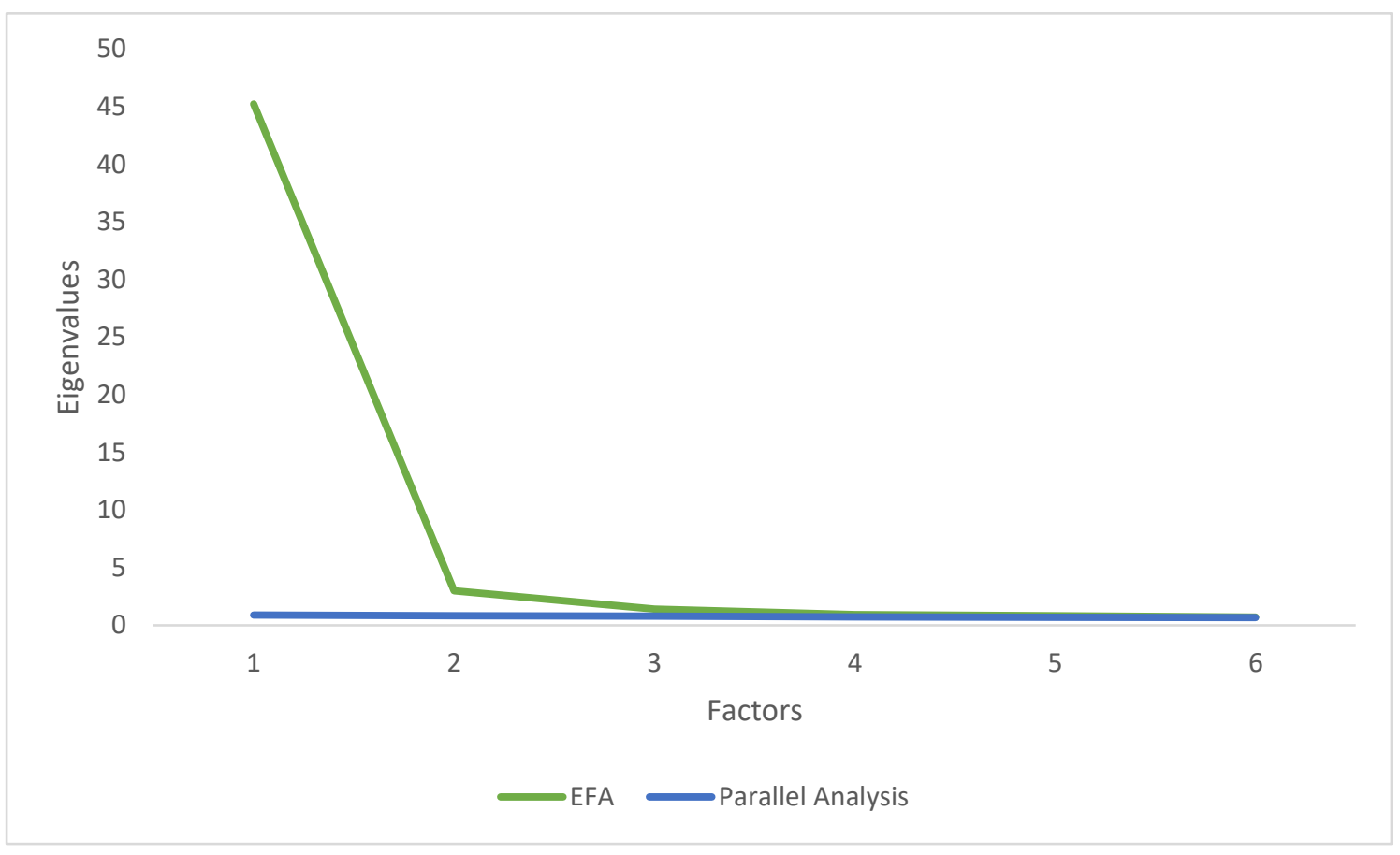

Factor 1 consisted of 51 items and accounted for $65.9 \%$ of the variance, with an Eigenvalue of 45.24. Factor 1 was similar to that of the experienced behavior model in that it included both the majority of the items and the same range of behaviors, including traditionally considered 'controlling behaviors', and for this reason, was also labeled "Eclectic Aggression". Forty-nine percent of the items in this factor are taken from scales or subscales specifically designed to assess control in intimate relationships (see Appendix C).

Factor 2 contained eight items, accounting for 3.9\% of the variance, with an Eigenvalue of 3.00. These items included non-physical violent behaviors that were directly enacted toward the participant, and thus it was appropriate to also label this factor "Direct Psychological Aggression". Thirty-eight percent of the items in this factor were obtained from control (sub)scales (e.g., "I purposely withheld affection or sex"; "I deliberately ignored my partner").

Factor 3 contained nine items, explaining $1.6 \%$ of the variance, with an Eigenvalue of 1.41. It contained some of the Monitoring Acts items identified in the reported experiences 
EFA, however, it also contained four items that loaded on the experienced "Direct Psychological Aggression" factor. Based on the overriding similarities, pertaining to Monitoring Acts and psychologically aggressive behavior, this factor was also labeled "Monitoring Acts". It too contained behaviors that prior research has deemed 'controlling behaviors' $(67 \%)$.

Unlike the items relating to experienced behaviors, no single factor was comprised solely of items that previous research has labeled as "controlling". Rather, these types of behaviors were distributed across the three factors. These results did not support the original hypothesis.

Descriptive statistics. Means, standard deviations, Cronbach's alphas, and zero-order correlations between the factors are presented in Table 2.1 (experienced behavior and perpetrated behavior).

Experienced behavior. Strong, positive, and statistically significant correlations were found between Eclectic Aggression and Direct Psychological Aggression (.72, $p<.001)$, Eclectic Aggression and Monitoring Acts $(.82, p<.001)$, and Direct Psychological Aggression and Monitoring Acts $(.81, p<.001)$. The correlations between Prosocial Relationship Behavior and Eclectic Aggression (-.19, $p<.001)$, Prosocial Relationship Behavior and Direct Psychological Aggression (-.20, $p<.001)$, and Prosocial Relationship Behavior and Monitoring Acts $(-.23, p<.001)$ were negative and weak, but statistically significant.

Perpetrated behavior. The correlations between Eclectic Aggression and Direct Psychological Aggression (.68, $p<.001)$, Eclectic Aggression and Monitoring Acts $(.85, p<$ $.001)$, and Direct Psychological Aggression and Monitoring Acts $(.75, p<.001)$ were strong, positive and statistically significant. 
In sum, the high correlations between factors in both the experienced behavior and perpetrated behavior models indicate the items may not be measuring distinct constructs as we hypothesized and would be expected based on prior research. 
Table 2.1

Descriptive statistics and intercorrelations for experienced and perpetrated behavior scales

\begin{tabular}{|c|c|c|c|c|c|c|c|}
\hline Variable & $\begin{array}{l}\text { Eclectic } \\
\text { Aggression }\end{array}$ & $\begin{array}{c}\text { Direct } \\
\text { Psychological } \\
\text { Aggression }\end{array}$ & $\begin{array}{l}\text { Monitoring } \\
\text { Acts }\end{array}$ & $\begin{array}{l}\text { Prosocial } \\
\text { Relationship } \\
\text { Behavior }\end{array}$ & $M$ & $S D$ & $\alpha$ \\
\hline \multicolumn{8}{|c|}{ Experienced behavior scale } \\
\hline Eclectic Aggression & - & - & - & - & 1.60 & .92 & .99 \\
\hline $\begin{array}{l}\text { Direct Psychological } \\
\text { Aggression }\end{array}$ & $.72 * *$ & - & - & - & 2.18 & .99 & .97 \\
\hline Monitoring Acts & $.82 * *$ & $.81 * *$ & - & - & 1.92 & 1.05 & .94 \\
\hline $\begin{array}{l}\text { Prosocial Relationship } \\
\text { Behavior }\end{array}$ & $-.19 * *$ & $-.20 * *$ & $-.23 * *$ & - & 3.61 & 1.07 & .79 \\
\hline \multicolumn{8}{|c|}{ Perpetrated behavior scale } \\
\hline Eclectic Aggression & - & - & - & - & 1.54 & .91 & .99 \\
\hline $\begin{array}{l}\text { Direct Psychological } \\
\text { Aggression }\end{array}$ & $.68 * *$ & - & - & - & 2.10 & .94 & .90 \\
\hline Monitoring Acts & $.85^{* *}$ & $.75^{* *}$ & - & - & 1.88 & .95 & .93 \\
\hline
\end{tabular}




\section{STUDY 2A}

\section{Method}

Participants. Participants were a convenience sample of undergraduate psychology students at a New Zealand university $\left(N=424 ; 26 \%\right.$ male, $74 \%$ female, $\left.M_{\text {age }}=19.5, S D=3.4\right)$. The eligibility criteria for the study were the same as Study 1 aside from location. The ethnic breakdown of this sample was as follows: $54.2 \%(n=230)$ identified as New Zealand European, 9.4\% ( $n=40)$ identified as Māori, 4\% $(n=17)$ identified as of Pacific Nations descent, 9\% (n $=38)$ identified as Asian, $17 \%(n=72)$ were of another ethnicity, and 6.6\% $(n=28)$ did not report their ethnicity. Twenty-three percent of participants identified with more than one ethnicity $(n=96)$. Exclusion criteria were identical to Study 1: Participants were removed if they did not consent to participate $(1 \%, n=5)$, did not meet the relationship criteria $(8.7 \%, n=$ $43)$, or did not identify as either male or female $(3.2 \%, n=16)$. Those who completed the survey in less than 281 seconds were also removed $(0.2 \%, n=1)$, as were those who showed clear patterns of non-responding $(1 \%, n=5)$.

Procedure and materials. Students were first-year psychology students recruited through Sona System software. The procedure and measures of relationship behaviors were identical to Study 1 except that students received credit towards a mandatory course research component as thanks for their participation.

\section{Treatment of data.}

Missingness. Little's MCAR test for missingness was conducted (Little \& Rubin, 2002) with $0.5 \%$ of any one item missing and a total of $.004 \%$ of data found to be missing $\chi 2(1382)$ $=691.06, p<.001$. As with the community data, this amount was deemed to be inconsequential and EM was employed to impute the missing data. 
Item selection. Results from Study 1 identified that Factor 1 (for both perpetrated behavior and experienced behavior items) and Factor 2 for perpetrated behavior contained a relatively large number of items (46 and 51, and 23 respectively). In Study 2, we reduced the numbers of items to remove redundancy without compromising the latent factor. Reducing the number of items in each factor also reduced the amount of measurement error, thereby improving model fit (Matsunaga, 2008). Accordingly, we selected the 20 highest-loading and most frequently endorsed items on each factor that appeared in both the experienced behavior and perpetrated behavior models (See Appendix D for a full list of items).

Parceling. We parceled items for the factors due to the large and varied number of items across factors. Parceling is a technique by which groups of items are combined and averaged to form 'parcels', which are then used as indicators of the latent factors (Cattell \& Burdsal, 1975). This reduces the number of items in the model and improves the model fit by enhancing communalities and reducing random error (Matsunaga, 2008). Three parcels were created per factor as recommended by Bandalos (2002) and Rogers and Schmitt (2004). Items were randomly assigned to parcels (See Appendix D for the list of items comprising each parcel).

\section{Data analysis.}

Confirmatory Factor Analysis (CFA). CFA was used to verify the factor structure of the experienced and perpetrated models tentatively established in Study 1. All analyses were conducted in IBM Amos (Version 25.0; Arbuckle, 2017). To assess the acceptability of the fit of the model produced by a CFA, three key areas should be examined: An evaluation of the strength and significance of the parameter estimates; an investigation into potential areas of localized strain or ill fit; and the model fit statistics (Brown, 2014).

We followed recommendations for reporting model fit by Kline (2005) and Brown (2014): the chi-square statistic, associated degrees of freedom and $p$-value (CMIN/DF, $p$ ), and 
three approximate fit indices: Root Mean Square Error of Approximation (RMSEA), Standardized Root Mean-square Residual (SRMR), and the Comparative Fit Index (CFI). The CMIN/ DF, which is the chi-square degrees of freedom ratio statistic should be close to five or below with a non-significant $p$-value for the model to be considered a good fit for the data. However, chi-square is known to be sensitive to both the size of the correlations between factors and large sample sizes, which results in the failure of the exact-fit test and a significant $p$-value (Kline). Values $\leq .08$ are indicative of good model fit for the SRMR and $\leq .06$ for the RMSEA (Brown; Kline). However, RMSEA values of $\leq .08$ are still considered to be adequate, and should not result in rejection unless they are $\geq .1$ (Browne \& Cudeck, 1992; MacCallum et al., 1996). For the CFI, values $\geq .95$ are considered a good model fit (Kline). All analyses were performed using IBM Amos statistical software using a Maximum Likelihood estimation (Version 25.0; Arbuckle, 2017)

\section{Results}

\section{Experienced Behavior model.}

Model fit. The fit indices were within the suggested parameters recommended by Kline (2005) and Brown (2014) described above. These as mentioned above (CMIN/DF $=3.42, p<$ $.001 ; \mathrm{RMSEA}=.08[.06, .09] ; \mathrm{SRMR}=.05, \mathrm{CFI}=.97$; refer to Table 2.2). These results indicate the model is an acceptable fit for the data, with the exception of the $p$-value for the chi-square statistic: The model failed the exact-fit test, producing a significant result. This was expected and disregarded due to our sample size $(N=424$; Kline). The model fit indices were supportive of the model, which we, therefore, accepted, allowing subsequent analyses to be conducted. The model is graphically represented in Figure 2.1.

Parameter Estimates and Standard Errors (SES). The standardized and unstandardized coefficients are reported in Appendix E. No standardized coefficients were larger than 1.96, indicating no evidence for localized strain or specific areas of ill-fit within the model (Brown, 
2014). All coefficients were significant $(p<.001)$, indicating their importance to their respective latent factors and the model generally (Brown).

The factor loadings for Eclectic Aggression, Direct Psychological Aggression, and Monitoring Acts were very high, particularly for Direct Psychological Aggression $(\geq .90$; see Appendix I). Loadings of $\geq .60$ are considered strong, and evidence of a common underlying factor, and good convergent validity (Cabrera-Nguyen, 2010). However, high loadings (e.g., > .90) are indications of poor discriminant validity (Brown, 2014). One of the factors for Prosocial Relationship Behavior had a loading of .31 , which although relatively low, is still considered "salient" in the context of applied research (Brown, p. 115).

We examined the factor correlations to assess the discriminant validity of the model (see Appendix G). High factor correlations above .80 in applied research, such as those found between Eclectic Aggression and Monitoring Acts (.80) and Direct Psychological Aggression and Monitoring Acts (.85), are likely indications of poor discriminant validity (Brown, 2014).

The high factor loadings and correlations suggest that these constructs are not clearly distinct from one another as our hypothesis and prior research would predict.

\section{Perpetrated Behavior model.}

Model fit. The fit statistics indicated the model for the perpetrated behavior model was an acceptable fit for the data $(\mathrm{CMIN} / \mathrm{DF}=3.86, p<.001 ; \mathrm{RMSEA}=.08[.07, .10]$; SRMR $=$ $.04, \mathrm{CFI}=.97$; refer to Table 2.2). The significant result for the chi-square was again disregarded due to the size of the correlations between factors and the sample size (Kline, 2005). The model was consequently accepted, validating the structure of the perpetrated behavior model tentatively established by the EFA in Study 1 . The model is graphically represented in Figure 2.2.

Parameter Estimates and Standard Errors (SES). The standardized and unstandardized coefficients are reported in Appendix F. An examination of the standardized coefficients 
showed no areas of localized strain in that none were greater than 1.96 (Brown, 2014). Similar to the experienced behavior model, all coefficients were significant $(p<.001)$, demonstrating their importance to their respective latent constructs and the perpetrated behavior model (Brown).

The factor loadings indicated good convergent validity for all factors with loadings between .73 and .89 (see Appendix J).

The factor correlations in the perpetrated model were in the range of .47 and .79, thereby demonstrating acceptable discriminant validity (refer to Appendix $\mathrm{H}$ for factor correlation results). These results indicate the items may, therefore, be measuring distinct constructs and are supportive of our hypothesis. 
Table 2.2

Fit indices for null, configural, and metric models for experienced and perpetrated behavior

\begin{tabular}{|c|c|c|c|c|c|c|c|c|}
\hline Model & $\chi^{2}$ & $d f$ & $p$ & CMIN/DF & RMSEA $(90 \%$ CI) & SRMR & CFI & $\Delta \chi^{2}$ \\
\hline \multicolumn{9}{|c|}{ Experienced Behavior model } \\
\hline Community sample & 200.64 & 48 & $<.001$ & 4.18 & $.08(.07, .09)$ & .03 & .98 & \\
\hline Student sample & 164.23 & 48 & $<.001$ & 3.42 & $.08(.06, .09)$ & .05 & .97 & \\
\hline Configural & 364.87 & 96 & $<.001$ & 3.80 & $.05(.05, .06)$ & .05 & .98 & \\
\hline Metric & 583.43 & 104 & $<.001$ & 5.61 & $.07(.06, .07)$ & .07 & .96 & $<.001$ \\
\hline \multicolumn{9}{|c|}{ Perpetrated Behavior model } \\
\hline Community sample & 95.09 & 24 & $<.001$ & 3.96 & $.07(.06, .09)$ & .03 & .99 & \\
\hline Student sample & 92.64 & 24 & $<.001$ & 3.86 & $.08(.07, .10)$ & .04 & .97 & \\
\hline Configural & 187.74 & 48 & $<.001$ & 3.91 & $.05(.05, .06)$ & .04 & .98 & \\
\hline Metric & 287.68 & 54 & $<.001$ & 5.33 & $.07(.06, .07)$ & .04 & .97 & $<.001$ \\
\hline
\end{tabular}


Figure 2.3.

Four factor model (experienced behavior) showing the standardized estimates for the factor correlations and loadings

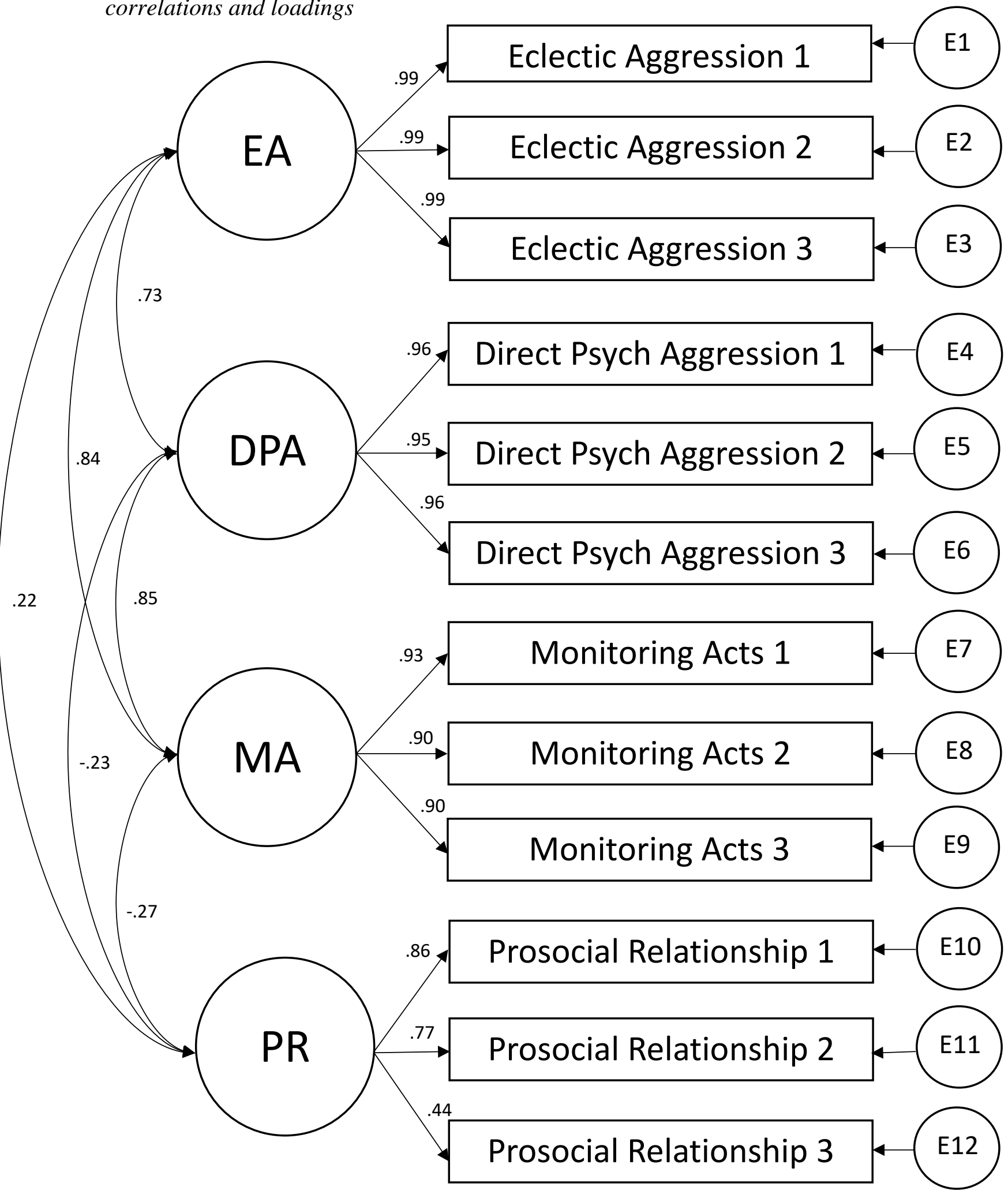

Note. EA = Eclectic Aggression; DPA = Direct Psychological Aggression; MA = Monitoring Acts; $\mathrm{E}=$ Error .

The first indicator path for each latent factor has been constrained to 1 . 
Figure 2.4.

Three factor model (perpetrated behavior) showing the standardized estimates for the factor correlations and loadings

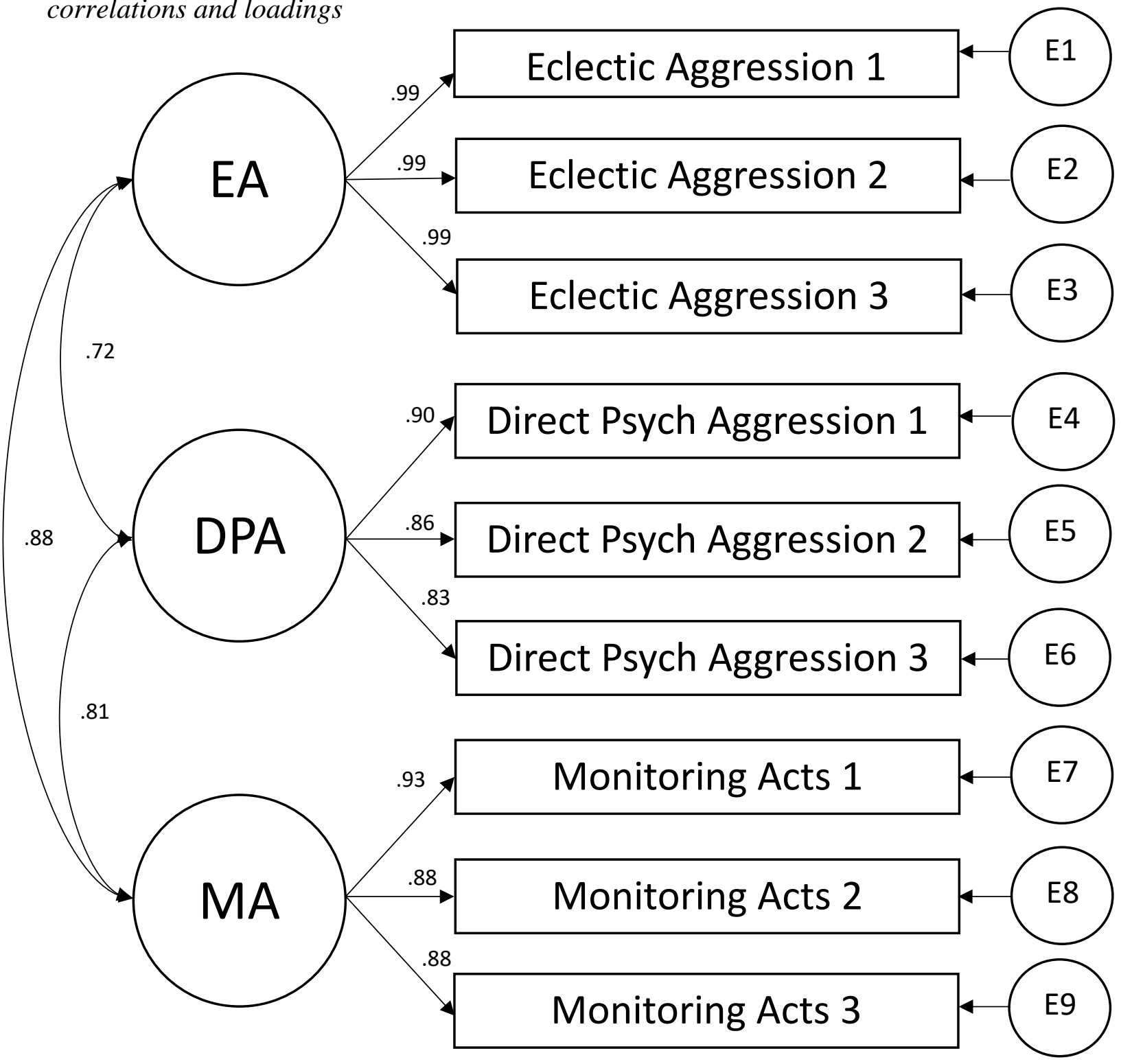

Note. $\mathrm{EA}=$ Eclectic Aggression; DPA = Direct Psychological Aggression; $\mathrm{MA}=$ Monitoring Acts; $\mathrm{E}=$ Error . The first indicator path for each latent factor has been constrained to 1. 


\section{STUDY 2B}

\section{Measurement Invariance}

The results of the CFA performed in Study 2A replicated those of the EFA conducted in Study 1, establishing the individual factors as latent constructs. As separate samples are required for EFAs and CFAs (Cabrera-Nguyen, 2010), Study 2B aimed to investigate whether the structure and meaning of these constructs were equivalent between the community and student samples. This test of the psychometric equivalence of constructs between the samples is called measurement invariance (Putnick \& Bornstein, 2016).

Measurement invariance was tested using a structural equation modeling framework in IBM Amos (Version 25.0; Arbuckle, 2017). This involves a stepwise procedure using CFA, which produces model fit indices, the results of which can be seen in Table 2.2. These indices are assessed to evaluate how well the hypothesized model fits the observed data (Putnick \& Bornstein, 2016). The first step in testing for measurement invariance is to establish whether the model fits well for both groups. CFAs were run separately for the student and the community sample using the parcelled items, with the results showing the data was an acceptable fit for both groups (refer Table 2.2).

Configural invariance. Configural invariance tests whether the latent structure is equivalent across groups, by running the model for both samples simultaneously and constraining the factorial structure to equivalence across samples (Putnick \& Bornstein, 2016). The model fit indices were indicative of a good fit and configural invariance was achieved for the experienced behavior model $(\mathrm{CMIN} / \mathrm{DF}=3.80, p<.001 ; \mathrm{RMSEA}=.05[.05, .06] ; \mathrm{SRMR}$ $=.05, \mathrm{CFI}=.98)$ and for the perpetrated behavior model $(\mathrm{CMIN} / \mathrm{DF}=3.91, p<.001 ; \mathrm{RMSEA}$ $=.05[.05, .06] ; \mathrm{SRMR}=.04, \mathrm{CFI}=.98)$. This provides evidence that the same factor structure is present in both the community and student samples, and that they conceptualize the latent 
constructs in the same way (Milfont \& Fischer, 2010). This indicates the appropriateness of testing for metric invariance (Putnick \& Bornstein).

Metric invariance. This was assessed by constraining the factor loadings to equivalence across both groups. The chi-square value and the degrees of freedom produced for the configural model are then compared to that of the metric model. The difference between these statistics was tested in a chi-square difference test to ascertain whether constrained (metric) model was a significantly worse fit than the unconstrained (configural) model, the result of which was significant for the experienced behavior model $\left(X^{2}\right.$ difference $=218.56, d f$ difference $=8, p<.001)$ and the perpetrated behavior model $\left(X^{2}\right.$ difference $=99.94, d f$ difference $=6, p<.001 ;$ refer Table 2.2). These results indicated metric invariance had not been achieved and that there were significant group differences in the underlying latent constructs (Milfont \& Fischer, 2010).

\section{Discussion}

Control has been placed at the center of theoretical explanations for intimate partner aggression (IPA) and at the center of laws that make IPA a criminal offense. Accordingly, current psychometric tools assume that 'controlling behaviors' are discrete non-physical behavioral acts that are distinct from other types of IPA. This study set out to investigate the divergent validity of 'controlling behaviors' against other forms of IPA using exploratory (EFA) and confirmatory (CFA) factor analyses. It was hypothesized that 'controlling behaviors' (as identified by past research) would comprise a single factor in both the experienced and perpetrated behavior models. However, EFA analysis of community sample data and CFA analysis of student data found that 'controlling behaviors' were not distinct from other aggressive acts. Instead, 'controlling behavior' items were dispersed across three factors in both the experienced and perpetrated behavior models in the exploratory and confirmatory factor analyses (Studies 1 and 2A respectively). 
The EFA with the community sample identified clear factor structures for the experienced behavior and perpetrated behavior models, containing four and three factors respectively. Both models had factors that were labeled as "Eclectic Aggression", "Direct Psychological Aggression", and "Monitoring Acts". The experienced behavior model also contained an additional factor that was labeled "Prosocial Relationship Behaviors". Only the "Monitoring Acts" factor identified in the experienced behavior model contained only behaviors that had been previously identified as 'controlling behaviors' in the literature. However, the high correlations between these factors indicated that these factors may not be measuring distinct constructs. The results of the CFA replicated those of the EFA for both the experienced and perpetrated behavior models. Again, previously labeled 'controlling behaviors' were distributed across the factors of experienced and perpetrated behavior models to such an extent that none of the factors were characterized by 'controlling behaviors'. Furthermore, an examination of the parameter estimates indicated poor discriminant validity for the experienced behavior model. Therefore, the results of this study show no statistical difference between behavioral acts that have previously been labeled in the literature as "controlling" and other types of IPA, such as psychological aggression, sexual coercion, and physical assault.

The findings show that we cannot reliably identify control using a discrete set of nonphysical controlling behavioral acts. This is in line with theoretical perspectives that assert aggressors use a range of behaviors, including physical and sexual violence, to achieve power and control in relationships (Johnson, 1999; Stark, 2007), rather than a specific subset of behaviors specifically intended to obtain control. Furthermore, it is necessary to measure an aggressive behavioral act separately to its motivation because the same act can be driven by different goals for different people on different occasions (e.g., Archer \& Browne, 1989; Campbell et al., 1985; Straus et al., 1996). Existing tools that measure controlling behaviors 
have relied on previous literature that has associated control motivations with a subset of nonphysical tactics, rather than testing if a relationship between them and control motivations exist. For example, the behavioral acts in the Controlling Behaviors Scale-Revised (Graham-Kevan \& Archer, 2003) were informed by the literature from Pence \& Paymar's Domestic Abuse Intervention Project, which was developed from a small sample ( $N=9$; Dutton, 2006a) with no empirical investigation of the relationship between these acts and motivations held by the perpetrators (also see Checklist of Controlling Behaviors: Lehmann et al., 2012; CTS2: Straus et al., 1996; Psychological Maltreatment of Women Inventory: Tolman, 1989). Together these findings suggest there is a need to determine better ways to measure control in intimate relationships than identifying self-reported non-physical behavioral acts that are assumed to indicate control.

\section{Strengths, Limitations, and Future Directions}

The factorial structure of the models in the current study diverge considerably from those found in much of the existing literature. Not only did this study fail to find evidence of a distinct sub-group of previously identified 'controlling behaviors', but it also did not find delineated groups of acts of physical and sexual aggression that have commonly formed tools used to identify rates of IPA (e.g., CTS2: Straus et al., 1996). One possible explanation for these different results could be attributable to the factor rotation method employed. Orthogonal rotation methods, such as Varimax, have commonly been used in the development of IPA measures (e.g., Checklist of Controlling Behaviors: Lehmann et al., 2012; Psychological Maltreatment of Women Inventory: Tolman, 1989). This type of factor rotation constrains the latent factors to be uncorrelated (Brown, 2014). However, there is considerable empirical evidence demonstrating subtypes of IPA, such as physical and psychological aggression, are correlated (Hou et al., 2011; O’Leary \& Smith Slep, 2003; Sears et al., 2007). On the basis of this evidence, this study employed an oblique rotational method, which allows latent factors to 
correlate (Brown). The high factor correlations in the current study, therefore, cast doubt upon the results of studies that have utilized orthogonal rotation methods. These methods have likely led to distorted factorial solutions (Brown) and thus the underlying validity of the measures designed to assess 'controlling behaviors' in intimate relationships is questionable.

It was hypothesized that controlling behaviors would be statistically distinct from other forms of IPA, and thus form a single factor comprised of these items. One hundred percent of the items in the Monitoring Acts factor in the experienced behavior model were obtained from scales or subscales that were specifically designed to measure 'controlling behavior' (SeeAppendix B), suggesting these items would make likely candidates for what could be considered 'controlling behavior'. However, Kline (2005) and Borsboom (2008) warn against reifying factors and assuming modeled latent constructs are truly representative of, and correspond to, observed phenomena. Latent constructs are artifacts of the results of a study, and therefore, require multi-method replication to establish their validity. Their advice should be considered when interpreting these results, particularly in relation to the Monitoring Acts factor in the experienced behavior model.

These results have important theoretical implications as they suggest the current conceptualization of 'controlling behavior' is likely too heterogeneous, resulting in the labeling of behaviors that are seemingly normatively associated with control but are in fact related to a separate construct. Indeed, there is a discrepancy between the types of items in the Monitoring Acts factor in the experienced behavior model (e.g., My partner called me or text messaged me constantly; My partner accused me of being unfaithful or flirting with others) and the type of behavior used in definitions of control in the IPA literature. The items in this factor are more characteristic of relationship anxiety behaviors, rather than embodying the deprivation of autonomy, entrapment and exploitation of an intimate partner highlighted by current definitions of control in the literature (e.g., Ehrensaft et al., 1999; Stark, 2007) and in legislation (Domestic 
Violence Act (Ireland), s 39; Scottish Government, 2015; Serious Crimes Act 2015 (UK), s 76). The lack of a clear theoretical conceptualization or definition of control (Hamberger et al., 2017) has resulted in the poor validity of current measures that identify control, and in ambiguity regarding the types of behaviors that can be considered 'controlling' (GrahamKevan, 2007).

Future research should, therefore, focus on refining the concept of 'controlling behavior' to make it more homogenous and more clearly defined. The importance of considering the relationship between the data patterns designed to measure control and the construct itself should not be overlooked (Borsboom, 2008), and thus, further research involving measurement models should be undertaken to assist in the clarification of control and 'controlling behavior'.

Some features of this study limit the conclusions that can be drawn from the results. These include the use of a self-report design, which can result in social desirability bias. However, social desirability in participants' reports of their aggressive behaviors toward others was ameliorated in the current research by participants' anonymity (Durant et al., 2002), and the use of an internet-based measure (Joinson, 1999). We also identified similar patterns of results in the assessment of participants' experienced aggressive behaviors, which further minimizes desirability biases (see Sugarman \& Hotaling, 1997). Nonetheless, the use of a scale to assess participants' levels of social desirability bias (e.g., Balanced Inventory of Desirable Responding: Paulhus, 1991) could also be employed.

\section{Conclusion}

This study found no evidence that previously identified controlling behaviors are statistically distinct from other IPA acts. This finding challenges research to confirm whether a set of discrete non-physical behavioral acts can be used to accurately identify control in relationships and questions the necessity of tools that adopt this methodology. This is 
important considering the real-world impact that the measurement of control in intimate relationships carries. A logical step forward is to turn to the study of general aggression which has successfully identified a variety of types of aggressive behaviors and robust measurement tools. Learning from this field it is evident that a key component is to identify the intentional behavioral act and the motivation accompanying that act separately (Archer \& Browne, 1989; Campbell et al., 1985). We, therefore, propose future research should consider the motivation of the perpetrator to identify the presence of control in intimate relationships. This could be achieved by investigating whether certain motivations, such as control, are distinctly related to specific types of IPA, and whether these relationships differ by gender (refer Chapter 4) 


\section{Chapter 3: MOTIVATIONS FOR PHYSICAL AND PSYCHOLOGICAL INTIMATE PARTNER AGGRESSION: A SYSTEMATIC REVIEW}

The high rates of intimate partner aggression (IPA) are of great concern to politicians, researchers, practitioners, and policymakers. Indeed, the prevention of this public health issue has received much scientific inquiry and is a priority of the World Health Organization (see World Health Organization, 2019). It is necessary to specify the relationships between causal mechanisms of IPA (Ward \& Hudson, 1998) to guide interventions that can reduce the social problem (Loseke et al., 2005). Motivations are part of causal processes that produce behavior (see Ward, 2017), and serve as important targets in the assessment and treatment of aggressive behavior, shedding light on the function of that behavior (Ireland, 2009). Thus, to thoroughly understand and address the perpetration of IPA, motivations, or goals are considered an important area of research (Langhinrichsen-Rohling et al., 2012).

Treatment programs that aim to reduce IPA recidivism have been heavily influenced by gendered theory (Dutton, 2006a). This theory considers IPA a consequence of male privilege and patriarchy and therefore poses that the motivations of male power and control and women's self-defense are central to understanding IPA and in progressing treatment (Bloomfield \& Dixon, 2015; Pence \& Paymar, 1993). In line with this, much research that has explored the motivations associated with IPA has been guided by the gendered perspective (Hamberger et al., 2017; Wagers, 2015). For example, of the 75 studies included in a systematic review of motivations for physical IPA, 57 (76\%) focused on power/ control and $46(61 \%)$ on selfdefense, making these the most and third most frequently measured motivations respectively in the review (Langhinrichsen-Rohling et al., 2012). However, existing research findings on "control" are inconsistent. Of eight studies that conducted statistical gender comparisons of self-reported power/ control as a motivation for IPA three found males were more motivated by power/ control than females (Barnett et al., 1997; Ehrensaft et al., 1999; Shorey et al., 2010), 
one found females were more motivated by power/control than males (Follingstad et al., 1991), three found no significant gender differences in power/control (Harned, 2001; Kernsmith, 2005; Ross, 2011), and one study found mixed results (Makepeace, 1986). Furthermore, while some research has found significant gender differences in self-defense motives for IPA (Foshee, 1996; Harned; Makepeace), others have not (Cascardi \& Vivian, 1995; Perry \& Fromuth, 2005; Shorey et al.).

Other research has demonstrated a range of alternative gender-inclusive motivations are frequently endorsed by both men and women, such as the desire to express anger or emotional dysregulation (see Elmquist et al., 2014; Follingstad et al., 1991; Harned, 2001). Such motivations can be described by alternative theoretical perspectives to gendered theory (e.g., male sexual proprietariness: Buss, 2002; attachment style: Dutton, 1995a; Holtzworth-Munroe \& Stuart, 1994). Despite such inconsistencies in findings and challenges to the gendered explanation of IPA, treatment programs have continued to be heavily guided by this approach. However, effect sizes for these programs are small (Babcock et al., 2004; Ford \& Regoli, 1993; Gannon et al., 2019), suggesting alternative approaches are required.

In addition to the above problems, prior reviews on IPA motivation have also questioned whether the inconsistency in study results is attributable to operationalization of motivations (Langhinrichsen-Rohling et al., 2012). For example, some studies have failed to define selfdefense (Makepeace, 1986), while others have failed to adequately differentiate between selfdefense and retaliatory actions (Harned, 2001; Saunders, 1986). A lack of conceptual clarity is also regarded as an issue, with research frequently conflating emotions with motivations (see Langhinrichsen-Rohling et al.; e.g., Belus et al., 2014; Hettrich \& O'Leary, 2007). For example, anger and jealousy are emotions that have frequently been included in prior research on motivations (e.g., Follingstad et al., 1991; Shorey et al., 2010; Stuart et al., 2006). However, current theory operationalizes emotions as context-specific states that motivate an individual to 
pursue a particular goal, including elevating or demoting goals, but are not motivations in-andof themselves (Cosmides \& Tooby, 2000; Ward \& Carter, 2018). Accordingly, theory states that motivation requires an intention that results in a behavior (Scheer, 2001; Ward, 2017). Despite difficulties in operationalizing emotions as motivations (see Langhinrichsen-Rohling et al.), emotions have been included in the current review given the frequency with which they are used in prior research.

Reviews of the IPA motivation literature have also questioned whether inconsistencies are attributable to measurement of motivations (Langhinrichsen-Rohling et al., 2012). Many of the tools developed to measure motivations for the perpetration of IPA lack a theoretical basis and appropriate scale development techniques such as exploratory factor analysis (e.g., Babcock et al., 2003; Carrado et al., 1996; Follingstad et al., 1991; Makepeace, 1986; Stuart et al., 2006). Assessment and treatment of IPA are informed by measures of motivations, and thus the validity and reliability of these tools are important to ensure intervention efforts are effective (Langhinrichsen-Rohling et al.).

In summary, previous reviews have examined the range and frequency of motivations associated with physical IPA (Bair-Merritt et al., 2010; Langhinrichsen-Rohling et al., 2012). However, they have not provided clear definitions of motivation, critically appraised the quality of research methodology, or examined the effect sizes associated with perpetration and gendered perpetration. These steps are necessary in order to objectively ascertain which motivations have the strongest associations with IPA and would, therefore, make the most appropriate treatment targets. As effect sizes are independent of sample size (Card, 2012), a comparison of effect size resolves the issue of differences in statistical significance between studies and will, therefore, be able to identify the key motivations for the use of IPA for males and females. This information will contribute to the understanding of IPA and guide treatment design and allocation. This is particularly salient for women in treatment for IPA as current 
programs are often reliant on an understanding of male-perpetrated IPA (Kernsmith, 2005; Stewart et al., 2014).

This review therefore aims to appraise the quality of research and effect sizes associated with the motivations of physical and psychological IPA perpetrated by men and women. A systematic review and meta-analysis of the published literature will be conducted to achieve this aim. Specifically, three pre-registered research questions will be examined.

1. How has empirical research measured motivations for physical and psychological IPA?

2. What are the largest effect sizes of motivations for the use of physical and psychological IPA by men and women?

3. Does the quality of research impact the effect sizes of motivations for the perpetration of physical and psychological IPA?

\section{Method}

\section{Protocol}

The method for this systematic review was pre-registered in a document that was uploaded to the Open Science Framework in December 2019 (https://osf.io/rsj2p/).

\section{Information Sources and Search Strategy}

Database search. PsycINFO, Sage Journals, and Web of Science databases were searched in July 2019. The keywords used in the search were: "intimate partner violence" OR "domestic violence" AND intent* OR motiv*. The search in PsycINFO was limited to the abstracts of quantitative, journal articles, published in English. No date restriction was imposed. This resulted in 242 articles.

The search in Sage Journals was limited to the abstracts of journal articles date with no date restriction, resulting in 212 articles. The search in Web of Science was unrestricted by date 
but limited to articles published in English. The search terms were searched for in the titles of the journals as the abstract was not available as a field code on this database. This search resulted in 35 articles. Overall, the database search identified 489 articles relating to motivations for the use of physical and/ or psychological IPA. These database searches were replicated in March 2020 to ensure no recently published papers were omitted from the review prior to its submission for publication. The dates searched were from January 2019 to March 2020. This search yielded an additional 11 articles from PsycINFO, seven from Web of Science, and two from Sage Journals.

Additional resources. A request was made via blog post to the Association of Domestic Violence Intervention Providers for unpublished data. However, there was no response to this request. Langhinrichsen-Rohling et al. (2012) conducted a systematic review of motivations for the use of physical aggression in intimate relationships. The 74 studies included in their review were included in the current review due to their highly relevant nature. Two additional records were identified by the authors, resulting in a total of 76 additional resources.

\section{Study Inclusion and Exclusion Criteria}

Five hundred and eighty-five articles were imported into Covidence, an online software program specifically designed to facilitate the systematic review process. This software was used to remove duplicates ( 71 excluded) and for screening 514 title/ abstracts (446 excluded) and 68 full-text articles (44 excluded). Twenty-four articles were deemed by the first author and two post-graduate research assistants to fulfill the review criteria $(\kappa=.46)$. Figure 3.1 shows the PRISMA flow diagram, which includes information regarding the identification, screening, and exclusion and inclusion of studies.

The focus of this review was on studies investigating self-reported motivations for physical and psychological IPA. Physical and psychological IPA were defined as acts of 
physical and non-physical aggression, occurring within the context of an intimate relationship. Sexual IPA was excluded from the review as research has suggested the motives for this form of IPA are different from physical and psychological IPA (Neal et al., 2015).

These studies had to be quantitative, use self-reported measures of aggression and motivations for the use of this aggression, and a continuous/ ordinal measure of aggression. The studies sampled heterosexual, forensic/clinical, community, and student populations and included both adolescent and adult age groups.

This study defined motivation or motives as a preceding act or psychological or physiological state which influences an individual's behavior. This definition was deliberately broad to encompass a range of items that have been labeled in previous literature as 'motivations'. Motivations were not, however, considered to be personality characteristics (e.g., attachment style, impulsivity) or risk factors for IPA (e.g., substance use, attitudes towards violence).

Studies were excluded due to a focus on risk factors or personality characteristics as opposed to motivations for aggression, the use of hypothetical scenarios (e.g., vignettes), and motivations that were inferred by the victim or by the researcher (i.e., were not self-report). One of the goals of the study was to investigate how motivations were measured in the IPA literature. As a result, only quantitative studies were included. 
Figure 3.1

PRISMA flow diagram outlining the number of studies identified, screened and deemed (in)eligible at each stage of the review process

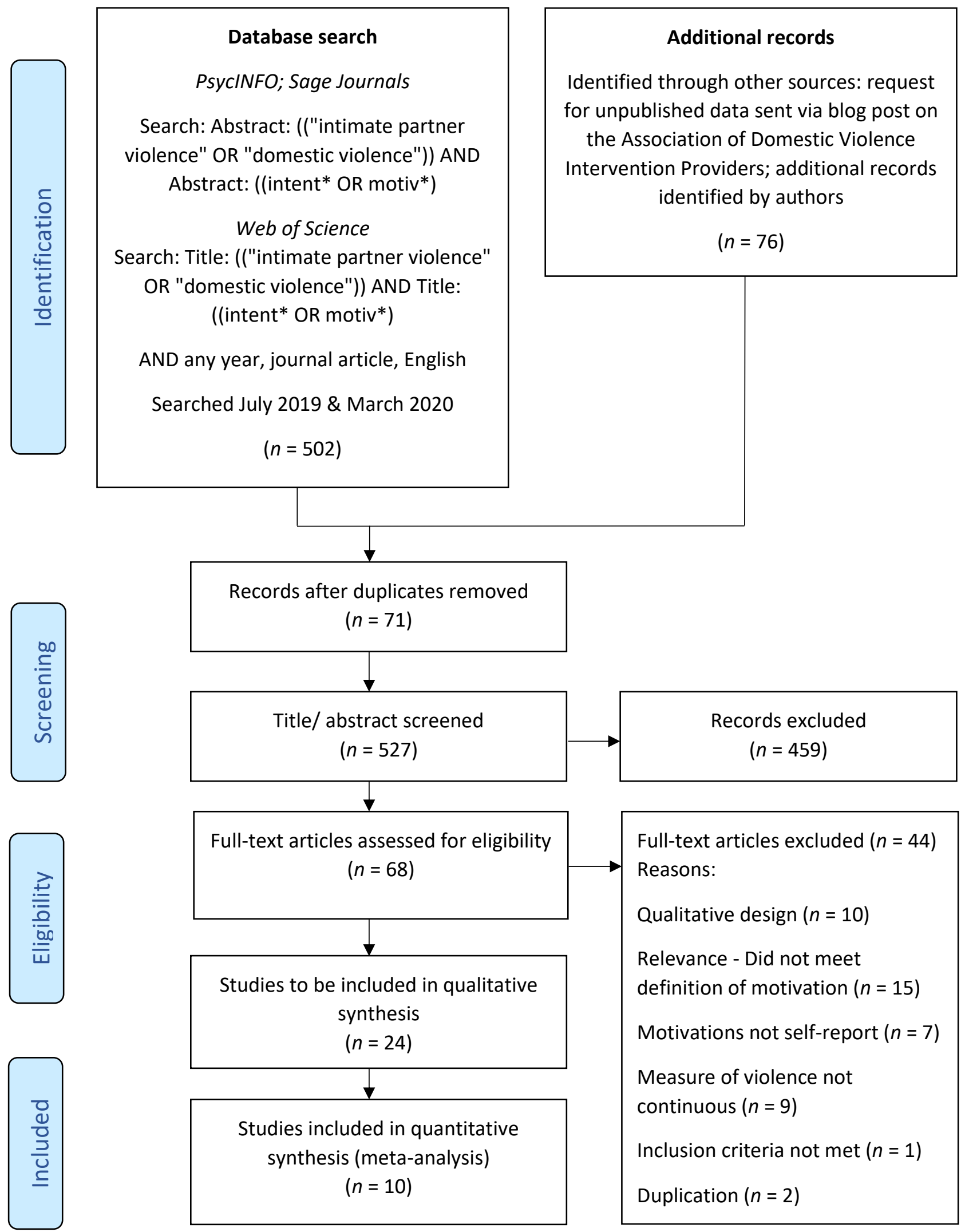




\section{Review Protocol and Quality Assessment}

The review was conducted in line with the PRISMA guidelines (Moher et al., 2009) and was informed by the AMSTAR systematic review quality guide (Shea et al., 2009).

Data were extracted to facilitate the appraisal of the quality of 24 articles, which were assessed according to criteria based on the Mixed Methods Appraisal Tool (Version 2018: Hong

et al.) and those recommended by Hawker et al. (2002; see Appendix K for the data extraction document and Appendix L for the quality appraisal document). The specific quality criteria were selected to assess the methodological rigor of the studies, and thus, determine the degree of confidence we can have in the results and conclusions drawn by the authors (Braverman \& Arnold, 2008). These criteria included sample size and composition, conceptualization and measurement of constructs, and the appropriateness of the analysis to answer specific research questions. As part of the quality appraisal procedure, G*Power (Faul et al., 2009) was used to calculate whether each of the studies had $\geq 80 \%$ power to detect an effect size of $r=.20$. This was calculated as an a priori analysis to determine whether the studies had the requisite sample size to achieve this effect.

In the process of data extraction, one further study was excluded as it was found it did not meet the review's inclusion criteria. Thus, the total number of studies included in the review was 23.

The quality scores were converted to percentages as some of the criteria did not apply to all of the studies (e.g., the studies conducted descriptive analysis and so the criteria regarding the appropriateness of the analysis and the power to detect an effect were not relevant) allowing the studies to be directly compared with one another. The purpose of appraising the quality of each study was to facilitate a sensitivity analysis: An assessment of the effects of including or excluding low-quality studies on meta-analytic results (Harden \& Gough, 2012). 


\section{Treatment of data}

Coding. Items from the included studies were coded by the first author and two postgraduate research assistants to facilitate the entry of the data into the Comprehensive MetaAnalysis (CMA) software program $(\kappa=.60$; see Appendix M). Some items from the measures used in some studies did not meet the definition of a motivation as mentioned previously (e.g., risk factors), and were thus excluded. Items, which have been labeled in the existing literature as 'motivations', were coded into 17 descriptive "motivational-categories", given that the list includes emotions that are theoretically considered emotional states rather than motivations (see Cosmides \& Tooby, 2000; Ward \& Carter, 2018). Eight motivational-categories were assessed in either one or two studies, and as CMA requires a minimum of three studies to assess for publication bias, only those categories that were measured by three or more studies were entered into CMA and were subject to analysis (Version 2.2.064; Borenstein et al., 2011). Publication bias is the selective publication of studies with results that are significant and in the expected direction, resulting in a body of literature that may not be representative of the studies conducted in that field (Card, 2012).

The final nine motivational-categories were: Asserting dominance (e.g., "to feel more powerful); communication difficulties (e.g., "show feelings that cannot be expressed in words"); control (e.g., "to get control over the other person"); emotional regulation difficulties (e.g., "because of stress"); jealousy (e.g., "because of jealousy"); retaliation for emotional hurt (e.g., "to get back at or to retaliate for being emotionally hurt by your partner); self-defense (e.g., "to

protect self"); to express anger (e.g., "show anger"); to get attention (e.g., "trying to get partner's attention). 


\section{Results}

\section{Research Question 1: How has empirical research measured motivations for physical and psychological IPA?}

The quality of 23 articles that fulfilled the a priori criteria of the systematic review was appraised. The results from this process can be found in Appendix K (data extraction) and Appendix L (quality appraisal).

Quality scores. There was a wide range in the quality of the studies, with scores ranging from $27-84 \%$. Possible patterns in the data were explored in IBM SPSS Statistics for Windows (Version 26.0; IBM Corp., 2019a). Relationships between the quality score, the year the study was conducted, and the sample size were examined. Scatterplots suggested the relationship between these variables was non-linear, thus, indicating Spearman's correlations were the most appropriate analysis choice. Results showed a significant positive association between the year in which the study was conducted and quality score $(r s(21)=.74, p<.001)$. The significant relationship between year and quality score could be attributable to improved reporting of research findings and greater adherence to APA guidelines. For example, the studies with the lowest scores were also those that omitted important details such as definitions, psychometric information, and study limitations (e.g., Fiebert \& Gonzalez, 1997 [28\%]; Makepeace, 1986 [27\%]). The relationship between quality score and sample size was not significant $(p>.05)$.

Samples. Eight studies sampled forensic populations, three sampled community populations, and 12 sampled student populations (see Table 3.1). Basic demographic details were generally well-reported with all studies reporting the sex of their participants and 20 studies reporting the age and ethnic composition of their samples. However, only 14 studies provided an explicit rationale for researching their specific population. For example, studies with student samples frequently failed to discuss the increased rate of IPA in relation to the 
general population (see Straus, 2004), the adverse effects specific to student populations (see Stith et al., 1992), or the risk of enduring patterns of IPA that persist into adulthood (see O'Leary et al., 2014).

None of the studies conducted an a priori power analysis. [In our post-hoc power analysis] only five of the 23 studies had $80 \%$ or greater power to detect a medium-sized correlation $(r=.20)$. Thus, the majority of the samples we identified $(78 \%)$ had inadequate sample sizes to detect even moderate associations between motivations and IPA.

Measurement. Only two out of 23 studies measured motivations for IPA using scales previously validated in studies using factor analysis (Belus et al., 2014; Kelley et al., 2015). Ten studies created their own measure of motivations. Of these, only two studies (Caldwell et al., 2009; Fernández-Fuertes \& Fuertes, 2010) performed exploratory factor analyses which are considered essential for scale development (see DeVellis, 2012).

Six studies used the Motives and Effects Questionnaire (Follingstad et al., 1991), two of which modified the scale either by adding and deleting items (Leisring, 2013) or by combining similar items (Neal et al., 2015). Three studies performed principal component analyses that resulted in different solutions (Harned, 2001; Kernsmith, 2005; Walley-Jean \& Swan, 2009).

In sum, measurement of IPA motivations displayed substantial heterogeneity across all of the studies. The most commonly used scales (Reasons for Violence Scale: Stuart et al, 2006; Motives and Effects Questionnaire: Follingstad et al., 1991) were used in different forms across studies and lacked consistent validation using confirmatory factor analysis.

Thirteen of the 23 studies failed to provide data or report results sufficient for calculation of effect sizes (see Table 3.1). Nine studies reported means or mean percentages without corresponding standard deviations. Two studies reported percentage bands (e.g., $5-40 \%$ ), and 
two studies reported the mean and standard deviation or standard error but as they only sampled a female population, an effect size could not be calculated using a difference between means. Thus, the following meta-analyses were only able to include 10 studies.

Physical and psychological motivations. The purpose of the review was to investigate the motivations for physical and psychological IPA. However, 5 of the 10 studies in the metaanalyses only focused on motivations for physical IPA. In addition, while one of the studies that investigated motivations for both for physical and psychological IPA clearly distinguished between the two in the reporting of their results (Fernández-Fuertes \& Fuertes, 2010), the remaining four either summed the physical and psychological aggression scores (Barnett et al., 1997) or were not explicitly clear as to whether their results related to psychological IPA (Caldwell et al., 2009; Ross, 2011; Walley-Jean \& Swan, 2009). Therefore, our subsequent analyses focus on motivations for physical IPA.

Statistical analyses. All analyses were conducted using Comprehensive Meta-Analysis (CMA) software program (Version 2.2.064, Borenstein et al., 2011).

Model. Random effects models were used for all analyses to allow for the true effect sizes to differ. Random effects models were used as the systematic review includes studies from different populations, utilizing a range of sample sizes, and a variety of measures. Due to this variability, our expectation was there would be substantial variation across the true effects making the random effects model the most appropriate to use (Borenstein et al., 2010). Thus, while some fixed effects results are reported in Table 3.2, the results from the random-effects model are reported in Tables 3.2 and 3.3, appendices, and are discussed in text. 
Table 3.1

Descriptive information of studies included in the systematic review

\begin{tabular}{|c|c|c|c|c|c|c|c|c|}
\hline Study & Year & $N$ & Country & Gender & Sample & $\begin{array}{c}\text { Number of } \\
\text { motivations measured }\end{array}$ & $\begin{array}{l}\text { Quality score } \\
(\%)\end{array}$ & Included in meta \\
\hline Babcock et al. & 2003 & 52 & USA & Female & Forensic & 11 & 48 & No \\
\hline Barnett et al. & 1997 & 64 & USA & Both & Forensic/ shelter & 8 & 52 & Yes \\
\hline Belus et al. & 2014 & 431 & USA & Both & Student & 2 & 84 & Yes \\
\hline Caldwell et al. & 2008 & 412 & USA & Female & Community & 5 & 84 & Yes \\
\hline Carrado et al. & 1996 & 191 & UK & Both & Community & 8 & 37 & No \\
\hline DeKeseredy et al. & 1997 & 1061 & Canada & Female & Community & 2 & 50 & No \\
\hline Elmquist et al. & 2014 & 177 & USA & Both & Forensic & 29 & 56 & Yes \\
\hline Fernandez-Fuertes \& Fuertes & 2010 & 567 & Spain & Both & Student & 1 & 65 & Yes \\
\hline Fiebert \& Gonzalez & 1997 & 285 & USA & Female & Student & 10 & 28 & No \\
\hline Follingstad et al. & 1991 & 115 & USA & Both & Student & 13 & 44 & No \\
\hline Harned & 2001 & 134 & USA & Both & Student & 12 & 70 & Yes \\
\hline Hettrich \& O'Leary & 2001 & 124 & USA & Female & Student & 12 & 39 & No \\
\hline Kelley et al. & 2015 & 221 & USA & Both & Student & 19 & 81 & Yes \\
\hline Kernsmith & 2005 & 125 & USA & Both & Forensic & 16 & 33 & No \\
\hline Leisring & 2013 & 125 & USA & Female & Student & 15 & 57 & No \\
\hline Makepeace & 1986 & 2338 & USA & Both & Student & 6 & 27 & No \\
\hline Neal et al. & 2015 & 484 & USA & Female & Student & 11 & 78 & No \\
\hline Ross & 2011 & 86 & USA & Both & Forensic & 29 & 52 & Yes \\
\hline Saunders & 1986 & 52 & USA & Female & Forensic & 2 & 44 & No \\
\hline Shorey et al. & 2011 & 60 & USA & Both & Student & 29 & 69 & Yes \\
\hline Stuart et al. & 2006 & 87 & USA & Female & Forensic & 29 & 58 & No \\
\hline Swan \& Snow & 2003 & 95 & USA & Female & Forensic & 3 & 56 & No \\
\hline Walley-Jean \& Swan & 2009 & 42 & USA & Female & Student & 13 & 52 & Yes \\
\hline
\end{tabular}


Composite scores. Several studies used measures that contained similar items, which when coded, were coded as the same motivation (e.g., control), resulting in multiples of a motivation within a study. This data could not be entered into CMA separately as participant's scores would not be independent of one another. This lack of independence would result in an overestimation of the precision of the summary effect and an underestimation of the variance (Borenstein et al., 2009). The multiple motivations within a study were therefore combined to make one for the respective study using the mean of the selected outcome. This method results in the software assuming the correlation between the motivations was 1 , which results in an underestimation of the precision of the summary effect. This approach was selected as the motivations were at least normatively associated and are therefore likely to be moderately correlated with one another. Further, this composite approach is considered to be the more conservative of the computational options available and one that is recommended by Borenstein et al.

Subgroups. Subgroups were combined using a random-effects model and a common among-study variance component was assumed.

Meta-analyses. We statistically compared the effect sizes of the nine motivationalcategories. Effect sizes were calculated depending on the type of data reported in the respective studies. We then converted these to Hedge's $g$ to facilitate their comparison and the calculation of a summary effect: the association between motivations and physical IPA, averaged across studies. We evaluated the weighted effect sizes using Cohen's (1969) classification of \pm .10 , $.30, .50$ as small, medium, and large effect sizes. We calculated Cochran's $Q$-statistic, which is a statistical significance test of heterogeneity used to ascertain whether genuine differences, such as methodological characteristics, are responsible for variation in study results, or whether this can be attributed to random error (Card, 2012). We also calculated the $I^{2}$ statistic, which indicates the proportion of heterogeneity and thus the consistency of findings across studies 
(Borenstein et al., 2009). $I^{2}$ values of $25 \%, 50 \%$, and $75 \%$ are considered to have low, medium, and high heterogeneity (Higgins et al., 2003). Summary statistics and forest plots for the nine motivational-categories can be found in Appendices $\mathrm{N}-\mathrm{V}$.

Effect sizes. The motivational-categories for physical IPA with the largest relative effect sizes were for self-defense (-.33), retaliation for emotional hurt (-0.28), and communication difficulties (-0.24). These would be classed as medium-sized effect sizes. The remaining six motivational-categories had small effect sizes ranging from -.01 to .19 (refer Table 3.2). There was a considerable range in the effect sizes for several of the motivational-categories, such as self-defense, asserting dominance, and to express anger (refer Table 3.2). Furthermore, the estimates showed differing directions of effect for all categories, with the exception of retaliation for emotional hurt, which were all in the female direction (refer Appendices M-V ).

Heterogeneity. Five motivational-categories, self-defense, asserting dominance, jealousy, to express anger, and emotional regulation difficulties showed significant heterogeneity as evidenced by Cochran's $Q$-statistic, which indicated the studies in their respective analyses did not share a common effect size (Borenstein et al., 2009). Retaliation for emotional hurt, communication difficulties, control and to get attention had non-significant $p$ values. However, these results were most likely a consequence of low statistical power due to large within-study variance and the small number of studies in the analyses. Thus, the nonsignificant $Q$-statistic should not be seen as evidence of consistency in these effect sizes (Borenstein et al.).

Self-defense, asserting dominance, jealousy, emotional regulation difficulties had high heterogeneity $\left(I^{2}>75 \%\right)$, and to express anger had medium heterogeneity $\left(I^{2}>50 \%\right)$ indicating that the effect sizes are not consistent across the studies in their respective analyses. $I^{2}$ values close to zero, such as that of control $\left(I^{2}=7.8 \%\right)$, suggest that the observed variance is due to random error as opposed to study characteristics (Borenstein et al., 2009). Retaliation for 
emotional hurt, communication difficulties, and to get attention had $I^{2}$ values of zero, suggestive of consistency in effect size. However, evaluations of the forest plots reveal the precision of the estimates to be very poor, as these analyses were frequently comprised of studies that had small sample sizes, some of which had samples of less than 100 participants (e.g., Shorey et al., 2010; Wally-Jean \& Swan, 2009). This lack of precision potentially obscured significant heterogeneity, resulting in the $I^{2}$ statistic values of zero (Borenstein et al.). Thus, it is not possible to comment with any degree of certainty on the consistency of the effect sizes for retaliation for emotional hurt, communication difficulties, and to get attention.

Due to the different sample types (i.e., three forensic/clinical samples, one community sample, and six student samples) as well as the range of measures used, we expected heterogeneity across the estimates (see Higgins et al., 2003). The amount of heterogeneity as evidenced by the imprecise estimates of the effect (wide confidence intervals), overlapping confidence intervals, and differing directions of the effect indicated large amounts of dispersion, and thus, inconsistency. This has implications for the conclusions that can be drawn from the results of the meta-analyses, as the consistency of the findings of the studies in a meta-analysis determines whether the findings from the meta-analysis are generalizable (Higgins et al.).

Although self-defense, retaliation for emotional hurt, and communication difficulties had the largest relative effect sizes of the motivational-categories in the review, due to high heterogeneity and large amounts of dispersion, these results should be considered spurious and not conclusive evidence for the importance of these motivational-categories for physical IPA. More specific analyses using sample type as a moderating variable were not viable due to the small number of samples.

Publication bias. Publication bias analyses were conducted for each of the motivational-categories. Evaluations of the funnel plots of the random effects and observed studies (refer Appendices W-EE) were inconclusive for all motivational-categories, as due to 
the small number of studies in each analysis, it was not possible to judge whether the funnels were asymmetric. Indeed, funnel plots are considered inaccurate methods of evaluating publication bias in analyses where the number of studies is less than ten (Sedgwick, 2013).

The trim-and-fill method for detecting publication bias was not performed as the direction of the effect of the missing studies must be specified and eight of the nine motivational-categories had estimates of the effect in differing directions (Shi \& Lin, 2019). Furthermore, empirical evidence has shown that high levels of heterogeneity adversely affect the power of the trim-and-fill method, thereby making this an inappropriate method for detecting publication bias in this review (Shi \& Lin).

Kendall's rank correlation and Egger's linear regression are bias indicators that estimate the asymmetry of data. These analyses were not conducted, however, as their power to detect bias with small numbers of studies is low (Egger et al., 1997).

We calculated the Fail-safe $N$ test, which computes the number of studies that would need to be added to the analysis to cause the overall effect size to become statistically insignificant (Borenstein et al., 2009). Larger Fail-safe $N$ values indicate greater robustness of the significance of the overall effect size (Borenstein et al.). To get attention, to express anger, emotional regulation difficulties, and control had Fail-safe $N$ values of zero. Retaliation for emotional hurt and communication difficulties had values of less than five, and jealousy and asserting dominance had Fail-safe $N$ values of less than ten (refer Table 3.3). Self-defense was the only motivational-category that yielded a Fail-safe $N$ value that could be considered adequately high at 40 (Card, 2012). However, Fail-safe $N$ does not account for heterogeneity, which previous results have shown greatly affect the studies in these analyses (Card), particularly, those of self-defense. Thus, given the low number of studies and high heterogeneity in each analysis, it is not possible to say whether or how likely it is publication bias had some impact on the findings of this review. 


\section{Table 3.2.}

Effect sizes for motivational-categories for physical IPA for males and females.

\begin{tabular}{|c|c|c|c|c|c|c|c|c|c|c|c|}
\hline & \multicolumn{2}{|c|}{ Fixed Effects Model } & \multicolumn{3}{|c|}{ Random Effects Model } & \multirow[b]{2}{*}{$p$} & \multirow[b]{2}{*}{$Q$} & \multirow[b]{2}{*}{$I^{2}(\%)$} & \multirow[b]{2}{*}{$k$} & \multirow[b]{2}{*}{$N$} & \multirow[b]{2}{*}{$\%$ Female } \\
\hline & $g$ & $95 \% \mathrm{CI}$ & $g$ & Range & $95 \% \mathrm{CI}$ & & & & & & \\
\hline Self-defense & -0.38 & $-0.51,-0.26$ & -0.33 & $.18,-1.16$ & $-0.66,-0.03$ & .032 & $31.79 * *$ & 81.13 & 7 & 1154 & 71.83 \\
\hline Retaliation for emotional hurt & -0.28 & $-0.49,-0.07$ & -0.28 & $-.10,-.41$ & $-0.49,-0.07$ & .011 & 0.68 & 0.00 & 4 & 365 & 56.16 \\
\hline Communication difficulties & -0.24 & $-0.44,-0.04$ & -0.24 & $.00,-.33$ & $-0.44,-0.04$ & .022 & 0.89 & 0.00 & 4 & 413 & 64.65 \\
\hline Asserting dominance & 0.27 & $0.14,0.40$ & 0.19 & $-.18, .66$ & $-0.13,0.52$ & .248 & $23.05 * *$ & 78.31 & 6 & 977 & 75.95 \\
\hline Control & 0.17 & $0.19,0.31$ & 0.16 & $-.07, .34$ & $0.03,0.32$ & .046 & 3.25 & 7.58 & 4 & 783 & 81.35 \\
\hline Jealousy & 0.19 & $0.09,0.29$ & 0.13 & $-.20, .53$ & $-0.20,0.46$ & .448 & $38.06 * *$ & 89.49 & 5 & 1647 & 55.00 \\
\hline To get attention & 0.10 & $-0.15,0.34$ & 0.10 & $-.01, .27$ & $-0.15,0.34$ & .435 & 0.90 & 0.00 & 4 & 300 & 70.00 \\
\hline To express anger & 0.09 & $-0.26,0.08$ & 0.03 & $-.12, .76$ & $-0.35,0.41$ & .880 & $9.99 *$ & 69.97 & 4 & 667 & 72.86 \\
\hline Emotional regulation difficulties & -0.05 & $-0.08,0.18$ & -0.01 & $-.24,-.50$ & $-0.42,0.41$ & .969 & $41.89 *$ & 88.06 & 6 & 1020 & 72.25 \\
\hline
\end{tabular}

Note. ${ }^{* *} p<.001 ; *<.05$; Effect sizes in the negative direction indicate the relationship between the motivational-category and physical IPA was stronger for women relative 


\section{Research Question 2: What are the largest effect sizes of motivations for the use of physical IPA by men and women?}

This question could not be answered because eight of the ten studies in the review made gender comparisons, seven of these reported the results as means and standard deviations, the difference in which was used to calculate an effect size. This meant males and females could not be entered as subgroups and gender could not act as a moderator.

However, the direction of the effect for each motivational-category is reported (refer Table 3.2). This indicated that self-defense, retaliation for emotional hurt, communication difficulties, emotional regulation difficulties were stronger for women relative to men, and asserting dominance, control, jealousy, to get attention, to express anger were stronger for men relative to women. With the exception of retaliation for emotional hurt, in which the studies were all in the female direction, estimates show differences in the direction of the effect within motivational-categories, making these results inconclusive. While the results for the studies for retaliation for emotional hurt were consistent, the analysis contained four studies, and thus the evidence that gender may be an important factor to consider in future research is only suggestive. Although the direction of the effect is reported, due to the large range in effect sizes within each category, and the inconsistency in the results we cannot say with any degree of certainty that a particular motivational-category is more important for either gender. 


\section{Table 3.3}

Summary of meta-analytic results for physical IPA for males and females

\begin{tabular}{|c|c|c|c|c|}
\hline Motivational-category & Summary effect & Heterogeneity & Moderator analysis & Publication bias \\
\hline Self-defense & $\begin{array}{c}g=-.33 ; p=.037 \\
-0.64,0.02(95 \% \text { CI })\end{array}$ & $\begin{array}{l}Q(6)=31.79, p<.001 \\
I^{2}=81.13\end{array}$ & $\begin{array}{l}\text { Quality score: } Q(6)=7.26 \\
p=.297\end{array}$ & $\begin{array}{l}\text { Funnel plot: Inconclusive } \\
\text { Fail-safe } N \text { : } 40\end{array}$ \\
\hline Retaliation for emotional hurt & $\begin{array}{c}g=-.28 ; p=.011 \\
-0.49,-0.07(95 \% \mathrm{CI})\end{array}$ & $\begin{array}{l}Q(3)=.68, p=.878 \\
I^{2}=.00\end{array}$ & $\begin{array}{l}\text { Quality score: } Q(3)=.68 \\
p=.880\end{array}$ & $\begin{array}{l}\text { Funnel plot: Inconclusive } \\
\text { Fail-safe } N: 2\end{array}$ \\
\hline Communication difficulties & $\begin{array}{c}g=-.24 ; p=.022 \\
-0.44,-0.04(95 \% \mathrm{CI})\end{array}$ & $\begin{array}{l}Q(3)=.89, p=.827 \\
I^{2}=.00\end{array}$ & $\begin{array}{l}\text { Quality score: } Q(3)=.89 \\
p=.827\end{array}$ & $\begin{array}{l}\text { Funnel plot: Inconclusive } \\
\text { Fail-safe } N: 1\end{array}$ \\
\hline Asserting dominance & $\begin{array}{c}g=.19 ; p=.248 \\
-0.13,0.52(95 \% \mathrm{CI})\end{array}$ & $\begin{array}{l}Q(5)=23.05, p<.001 \\
I^{2}=.78 .31\end{array}$ & $\begin{array}{l}\text { Quality score: } Q(5)=4.64 \\
p=.461\end{array}$ & $\begin{array}{l}\text { Funnel plot: Inconclusive } \\
\text { Fail-safe } N: 8\end{array}$ \\
\hline Control & $\begin{array}{c}g=.16 ; p=.046 \\
0.03,0.32(95 \% \mathrm{CI})\end{array}$ & $\begin{array}{l}Q(3)=3.25, p=.355 \\
I^{2}=7.58\end{array}$ & $\begin{array}{l}\text { Quality score: } Q(3)=3.25 \\
p=.355\end{array}$ & $\begin{array}{l}\text { Funnel plot: Inconclusive } \\
\text { Fail-safe } N \text { : } 0\end{array}$ \\
\hline Jealousy & $\begin{array}{c}g=.13 ; p=.448 \\
-0.20,0.46(95 \% \mathrm{CI})\end{array}$ & $\begin{array}{l}Q(4)=38.06, p<.001 \\
I^{2}=89.49\end{array}$ & $\begin{array}{l}\text { Quality score: } Q(4)=2.55 \\
p=.635\end{array}$ & $\begin{array}{l}\text { Funnel plot: Inconclusive } \\
\text { Fail-safe } N: 6\end{array}$ \\
\hline To get attention & $\begin{array}{c}g=.10 ; p=.435 \\
-0.15,0.34(95 \% \mathrm{CI})\end{array}$ & $\begin{array}{l}Q(3)=.90, p=.825 \\
I^{2}=.00\end{array}$ & $\begin{array}{l}\text { Quality score: } Q(3)=.90 \\
p=.825\end{array}$ & $\begin{array}{l}\text { Funnel plot: Inconclusive } \\
\text { Fail-safe } N \text { : } 0\end{array}$ \\
\hline To express anger & $\begin{array}{c}g=.03 ; p=.880 \\
-0.35,0.41(95 \% \mathrm{CI})\end{array}$ & $\begin{array}{l}Q(3)=9.99, p<.05 \\
I^{2}=69.97\end{array}$ & $\begin{array}{l}\text { Quality score: } Q(3)=3.30 \\
p=.348\end{array}$ & $\begin{array}{l}\text { Funnel plot: Inconclusive } \\
\text { Fail-safe } N \text { : } 0\end{array}$ \\
\hline Emotional regulation difficulties & $\begin{array}{c}g=-.01 ; p=.969 \\
-0.42,0.41(95 \% \text { CI })\end{array}$ & $\begin{array}{l}Q(5)=41.89, p<.001 \\
I^{2}=88.06\end{array}$ & $\begin{array}{l}\text { Quality score: } Q(5)=2.86 \\
p=.722\end{array}$ & $\begin{array}{l}\text { Funnel plot: Inconclusive } \\
\text { Fail-safe } N \text { : } 0\end{array}$ \\
\hline
\end{tabular}




\section{Research Question 3: Does the quality of research impact the effect sizes of motivations for the perpetration of physical IPA?}

A sensitivity analysis could not be conducted due to the low number of studies that were adequately reported in the review $(n=13)$. A moderation analysis was instead conducted using the quality score assigned to each study during the appraisal process. As the moderator was continuous it was performed within a regression framework (meta-regression), using a randomeffects model (method of moments), with the quality score entered as the predictor. Table 3.3 shows there was no evidence that the quality of the studies in the review affected any of the results. This could be attributable to the fact that the lower quality studies, which had neglected to report their results in a way that enabled them to be included in the meta-analyses, were also subsequently excluded from this analysis. Indeed, an independent samples $t$-test revealed the quality scores of the studies included in the meta-analyses had a significantly higher mean $(M$ $=66.50, S D=13.27)$ than those studies excluded from the meta-analyses $(M=46.08, S D=$ $14.14 ; t(22)=3.52, p<.05)$. However, it should also be noted that Borenstein et al. (2009) suggest a ratio of 10 studies per covariate for a meta-regression. Thus, the small number of studies in each analysis may have affected the results of the meta-regression.

\section{Discussion}

Understanding motivations for IPA is considered important to inform treatment and prevention efforts (Langhinrichsen-Rohling et al., 2012). This systematic review set out to expand on the aims of previous systematic reviews that have focussed on the frequency of motivations (e.g., Bair-Merritt et al., 2010; Langhinrichsen-Rohling et al.), by comparing the effect sizes of a range of motivations using meta-analysis and critically appraising the quality of the studies conducted. This is necessary to identify motivations for the perpetration of IPA that are most strongly supported by robust research to ensure research and clinical practice can 
be guided by a sound evidence base. Three questions were considered and the results of each are summarised below in turn.

The first research question examined how motivations for men's and women's use of physical and psychological IPA has been measured. The quality of 23 studies was appraised, revealing a general failure to define key variables and inconsistency in measurement. Only ten of these studies contained data in a form that enabled them to be included in meta-analyses. Only five of these 10 studies focused on both physical and psychological IPA, and only one of these presented their results in a way that would have enabled psychological IPA to be included in subsequent analyses. Thus, motivations for physical IPA became the primary focus of the meta-analyses.

The meta-analytic results indicated self-defense, retaliation for emotional hurt, and communication difficulties had the largest relative effect sizes. However, these results showed high levels of heterogeneity and dispersion and should, therefore, be regarded as inconclusive. The small number of studies in each analysis meant the results of the publication bias and metaregression analyses are similarly questionable.

The second and third research questions were unable to be investigated due to insufficient information in the published research. The second question investigated which motivations had the largest relative effect sizes for men and women. However, the way in which data was reported in seven of the eight studies that made gender comparisons meant the effect size had to be calculated as a difference between means before being converted to Hedge's $g$. This meant gender could not be entered into the analyses as a moderator and that this research question could not be answered. The third research question investigated whether the quality of the research had an impact on the effect sizes of motivations for the perpetration of IPA by conducting a meta-regression using the quality score as the predictor. The results demonstrated 
that the quality of the studies did not significantly impact the effect sizes for any of the motivational-categories.

\section{Explanations and Implications of Findings}

Review \& Analyses (Research Question 1). Eighty-seven percent of the studies in this review did not define motivation. This lack of clarity resulted in the frequent interchange of motivations and reasons for IPA within papers and within scales (e.g., Reasons for Violence Scale: Stuart et al., 2006). This is problematic as reasons, such as substance use, while correlated with IPA perpetration and with offending generally, are not explanatory, and thus make inappropriate treatment targets (Heffernan et al., 2019).

Furthermore, emotions (e.g., jealousy, anger) were commonly conflated with motivations. While current theory suggests emotions motivate goal-directed behavior by coordinating physiological, cognitive, and behavioral responses (Ward \& Carter, 2018), they do not directly drive behavior (Prinz, 2004). The indirect relationship between emotions and behavior means emotions should be viewed as motives (Prinz). For example, an individual may become angry (emotion/ motive) at seeing their partner talking to an attractive stranger. This perceived threat triggers an attachment-related need (motivation) to preserve the relationship (goal). It is important for researchers to understand and make the distinction between motives and motivations to ensure treatment for IPA is effectively targeting the underlying cause of perpetration.

In addition to the general failure to define variables, there was also a lack of consistency in operationalizing motivations despite the frequent use of the same measures (e.g., Reasons for Violence Scale: Stuart et al., 2006; Motives and Effects Questionnaire: Follingstad et al., 1991). Contributing to this problem was the use of exploratory factor (EFA) and principal component analyses (PCA) that resulted in the inclusion of divergent items within the same factor/ component. In one such example, Kernsmith (2005) included self-defense items (e.g., 
'to protect yourself') with retaliation items (e.g., 'to get back at your partner for hurting you emotionally'; 'to show anger'). In a further example, Harned's (2001) study has a factor labeled anger/ jealousy. While the constructs in these examples may have some shared characteristics and may even co-occur, they are very different, and theoretical as well as statistical consideration should have been given to their inclusion in the same factor/ component.

The studies included in this review were cross-sectional and retrospective. The use of this methodology has limitations as it necessitates participants' ability to critically reflect, accurately identify and recall their motivation(s) for a specific act; a relationship that is not always linear (Ireland, 2009). Furthermore, measuring motivations at one time-point can be seen as problematic due to the transient nature of motivations. Future research should, therefore, employ different designs to investigate this complex phenomenon.

The nine motivational-categories included in this analysis were the same as those in both the Bair-Merritt et al. (2010) and Langhinrichsen-Rohling et al. (2012) reviews. While these reviews investigated the frequency with which motivations were studied, this review statistically compared the strength of the relationship between the motivational-categories for physical IPA for men and women. The motivational-categories with the largest relative effect sizes were self-defense, retaliation for emotional hurt, and communication difficulties. These effect sizes were significant and medium-sized. The remaining six motivational-categories had small effect sizes and were all non-significant, with the exception of control.

Although the effect size for control was significant and stronger for males relative to females, it was small (.16). These findings have implications for both control theory and the treatment of IPA, suggesting that control is perhaps undeserving of the hegemonic position it currently occupies in treatment and legislative spheres, and other motivations may make more effective treatment targets 
The small number of studies able to be included in the meta-analyses limits the degree of certainty we can have when interpreting the results. For example, the low statistical power directly impacted on Cochran's $Q$ statistic, while the small numbers in each analysis also affected the publication bias analyses (funnel plot, Fail-safe $N$ test) and meta-regression analyses. This issue could be resolved by the reporting of data that would facilitate metaanalyses. Specifically, researchers should be reporting effect sizes for their data as per American Psychological Association recommendations (American Psychological Association, 2001).

The Gender Question (Research Question 2). While we were unable to use gender as a moderating variable in our analyses, the motivational-categories for self-defense, retaliation for emotional hurt, communication difficulties, and emotional regulation difficulties were stronger for women relative to men, whereas asserting dominance, control, jealousy, to get attention, and to express anger were stronger for men relative to women,According to the gendered theory of IPA, control and self-defense are central motivations for the use of men's and women's IPA, respectively (Dutton, 2006a). The results of this review offer limited support for this theory as the effect size for control (.16) wasstronger for men. However, asserting dominance was also stronger for men and had a larger effect size than control. Given the small number of studies in the meta-analysis for control, one of which was assigned a weighting of over $50 \%$, and the differing directions of the effect of the estimates, the evidence is far from conclusive.

The largest effect size for women was for self-defense, however, this motivational category also showed differing directions of the effect of the estimates. Retaliation for emotional hurt was the only motivational-category that showed consistency in the estimates of the direction of the effect (female), and thus may be the only motivational-category for which we have suggestive evidence that gender is important to consider in future research. 
The meta-analyses allowed us to objectively evaluate the evidence for gendered theory by comparing the standardized effect sizes of the motivations for IPA. Unfortunately, due to the small number of studies in each analysis and the differing directions of the effects, the results cannot provide definitive evidence to either support or counter this theory nor determine the most important motivations for males and females with any certainty. However, they do suggest that treatment for IPA should emphasize a range of motivations, rather than exclusively focusing on control.

Quality of Research (Research Question 3). We were unable to conduct a sensitivity analysis due to the small number of studies in the review, however, a meta-regression showed that there was no evidence that the quality of research was related to the results of any of the analyses. Although the quality of the studies included in the meta-regression did not impact the effect sizes of motivations for the perpetration of IPA, poor reporting meant $57 \%$ of the studies in the systematic review had to be excluded from the meta-analyses, limiting the conclusions we can draw from the analyses. Thus, the quality of the studies could be said to have indirectly affected the results of this review. Similarly, in the Langhinrichsen-Rohling et al. (2012) review, not all the studies subjected their data to statistical analysis. For example, four of the 12 studies that made gendered comparisons for the motivation of power/control did not statistically analyze the results. This highlights the importance of ensuring the research conducted is methodologically robust and data is reported in a format that is able to be used by future researchers.

\section{Limitations and Future Research}

This review did not include any studies that focused exclusively on men's motivations for the use of IPA. This absence echoes the findings of the Langhinrichsen-Rohling et al. (2012) review, in which only eight percent of studies used exclusively male samples. LanghinrichsenRohling et al. suggest the neglect of males as research subjects is perhaps attributable to the 
gendered assumption that men's use of IPA is motivated by control. A further similarity with the Langhinrichsen-Rohling et al. review was the small number of studies using community samples ( $16 \%$ compared to $13 \%$ in the current review). The continued exclusion of these populations from the research literature will result in knowledge gaps and the distortion of our understanding of IPA generally. Thus, future researchers should aim to address these deficits by actively engaging with these populations.

To effectively investigate motivations for the use of IPA and determine which of these have the largest effect sizes, these motivations must be clearly defined, and these definitions consistently applied. For example, the definitions of self-defense in this review were varied and as previously mentioned, were frequently conflated with retaliation. This is an issue that has previously been raised by multiple researchers (e.g., Babcock et al., 2019; LanghinrichsenRohling et al., 2012; Leisring, 2013). Therefore, we recommend future researchers use the legal definition for self-defense to bring consistency to this construct (see Garner \& Black, 2014, p. $1565)$.

Future research should also address the lack of theoretically based and appropriately developed scales for assessing motivations for IPA. As part of the validation process, these scales should also be subject to invariance testing to ensure their use is germane for both males and females.

\section{Conclusion}

This is the first review that statistically compares the relative importance of motivations for the perpetration of physical IPA for men and women. It also provides a much-needed critique of the methodological quality of research that examines motivations associated with IPA. While the results of the meta-analyses are inconclusive and highlight the need for future research to improve and develop, they indicate there is limited statistical evidence to support the hegemonic position that control has occupied as a motivation for IPA for the last 40 years. 
Rather, the importance of alternative motivations that demonstrate larger effect sizes than control is highlighted as necessitating closer consideration. Therefore, moving beyond theories and measurements centered on control is important if effective treatment of IPA is to be realized. 


\section{Chapter 4: MOTIVATIONS FOR INTIMATE PARTNER} AGGRESSION

Michael Johnson's (1995) seminal paper Patriarchal Terrorism and Common Couple Violence and his subsequent work have guided a generation of researchers and practitioners working in the field of intimate partner aggression (IPA; Dutton, 2006a). His theoretical accounts aimed to reconcile the conflict between feminist and family violence researchers, suggesting their findings highlighted qualitatively different forms of violence. In summary, he proposed that 'intimate terrorism' (later termed 'coercive controlling violence') is characterized primarily by men's severe and frequent physical aggression toward women, driven by patriarchy, power, and control motivations. In contrast, 'common couple violence' (later labeled 'situational couple violence') describes low level (infrequent and low severity), bidirectional violence born out of conflict with an absence of control motivations. Johnson hypothesized that men who are highly controlling are unlikely to let their female partner participate in research, and therefore argued that 'intimate terrorism' would be best identified in studies that worked with clinical samples (e.g., women attending courts, or shelters), and 'common couple violence' in studies with couples residing in the community.

Johnson's (2006) analysis of 274 existing interviews with women, who had been in contact with shelters or courts, and a matched sample of women residing in the same neighborhood, found support for his assertions. Gender asymmetry characterized the behavior of those classified as 'intimate terrorists', with $97 \%$ categorized as male, and gender symmetry characterized those classified by 'situational couple violence' (56\% male; $44 \%$ female). However, matching groups based on neighborhood arguably increased the likelihood that the male partners knew each other, and as pro-criminal peers are a risk factor for general crime (Bonta \& Andrews, 2017), this could have over selected for male criminality and aggressiveness. Importantly, men who had experienced victimization were not sampled, 
making it impossible to infer conclusions about men's experiences of victimization. Such limitations invalidate the conclusion that women can be rarely classified as 'intimate terrorists' (Dixon \& Graham Kevan, 2020; Dutton, 2006b; Graham-Kevan, 2007; Hines \& Douglas, 2019). Indeed, research that has tested the typology using population-based samples has found high numbers of 'intimate terrorists' in the community (Bates \& Graham-Kevan, 2016; Laroche, 2005) and higher numbers of women classified as 'intimate terrorists' than men (Bates et al., 2014; Hines \& Douglas; Straus \& Gozjolko, 2016). However, gender-inclusive investigations into 'intimate terrorism' are rare.

Despite such criticisms, Johnson's typology (1995; 2006) has continued to influence research and policy (e.g., Hardesty et al., 2015; Hines \& Douglas, 2010). For example, the need to distinguish between different types of violence has been recognized in law, whereby control has been included in recent legislation in the United Kingdom (Serious Crimes Act 2015 [UK], s 76), Scotland (Scottish Government, 2015), and Ireland (Domestic Violence Act 2018 [Ireland]). This inclusion is likely due to the positive relationship between increases in levels of control and frequency of physical aggression for men and women, for which there is some evidence (e.g., Bates et al., 2014). However, studies that have found this association have frequently identified control through the use of self/ victim report tools that measure controlling behaviors. Controlling behaviors can be understood as a specific set of non-physical aggressive acts widely assumed to be associated with an instrumental motivation of control (e.g., Controlling Behaviors Scale-Revised: Graham-Kevan \& Archer, 2003; Checklist of Controlling Behaviors: Lehmann et al., 2012; Scottish Government, 2015; Serious Crimes Act 2015 [UK], s 76).

Dempsey et al. (2020) empirically tested the statistical validity of 'controlling behaviors' by investigating whether these behaviors formed a distinct factor when their cooccurrence with other forms of intimate partner aggression (IPA), such as physical and 
psychological aggressive acts, was explored in community and student samples of men and women. Measures of controlling behaviors were taken from (sub)scales of tools that had been specifically designed to measure such acts (e.g., Controlling Behaviors Scale-Revised: GrahamKevan \& Archer, 2003; Controlling and Abusive Tactics Questionnaire: Hamel et al., 2015; Checklist of Controlling Behaviors: Lehmann et al., 2012). Factor analyses showed that 'controlling behaviors' did not form a distinct factor but rather were distributed across three factors of two models, each of which explored IPA from the perspective of either the victims or perpetrators of IPA. The largest factor for both models was labeled Eclectic Aggression, which incorporated a diverse range of behaviors including physical and sexual aggression, threats, and 'controlling behaviors'. The second factor, labeled Direct Psychological Aggression, was typified by non-physical aggressive behaviors that have been described in the literature as psychological aggression (e.g., overt criticism, shouting) and also contained items previously defined as controlling behaviors (e.g., "My partner deliberately ignored me"; "My partner told me my feelings were irrational or crazy"). The third factor, labeled Monitoring Acts, was solely comprised of items such as the checking of partner's social media pages and keeping track of their telephone/ cell phone usage. These items did not meet the study's definition of control (attempts to compel or constrain an intimate partner's behavior), and it was thus concluded they were more reflective of relationship anxiety, rather than a desire to influence or control an intimate partner. The lack of statistical distinction between controlling behaviors and other aggressive relationship acts suggested the validity of behavioral measures to assess for the presence of control in relationships is questionable. It was posed that other factors, such as the motivations driving those behavioral acts, would be more helpful in determining whether control is present in a relationship, as individuals who commit the same aggressive act can have disparate motivations for behaving in this way (Bushman \& Anderson, 2001). 
This study aims to adopt a gender-inclusive approach to study the relationship between control motivations and the type and frequency of behavioral acts used by men and women in intimate relationships, as first noted by Johnson (1995). We are mindful that a review of the literature has identified there is no validated way to measure motivations for IPA acts (BairMerritt et al., 2010; Langhinrichsen-Rohling et al., 2012). Existing measures are not theoretically derived and have methodological problems, including a lack of operational definitions for commonly measured motivations, such as self-defense, and poor construct validity (Langhinrichsen-Rohling et al.). Furthermore, previous research investigating the relationship between control and IPA have operationalized control as a series of non-violent ‘controlling behaviors' (e.g., Bates \& Graham-Kevan, 2016; Straus \& Gozjolko, 2014; 2016). Therefore, this study will use a measure of motivations developed from control theory and general aggression theories and a review of the qualitative IPA literature. Research conducted by Belus et al. (2014), Caldwell et al. (2009), Cousins and Gangestad (2007), Follingstad et al. (1991), and Hamberger and Guse (2002) has demonstrated that a wide range of motivations are associated with IPA. Indeed, motivations such as retaliation, communication difficulties, emotional dysregulation, and self-defense are endorsed with a greater frequency by both genders for the perpetration of physical IPA than control (Elmquist et al., 2014; Follingstad et al.; Harned, 2001; Kelley et al., 2015; Ross, 2011).

Specifically, the following hypothesis will be tested:

H1: If patterns of control motivations are distinct from those of general aggression motivations, then control motivations will be more strongly associated with the perpetration of Eclectic Aggression, Direct Psychological Aggression, and Monitoring Acts than general aggression motivations.

$\mathrm{H} 2$ : If patterns of control motivations are distinct from those of general aggression motivations, then control motivations will be more strongly associated with an increased 
frequency of perpetrating Eclectic Aggression, Direct Psychological Aggression, and Monitoring Acts than general aggression motivations.

H3: If patterns of control motivations are distinct from those of general aggression motivations, then the association between control motivations and the perpetration of Eclectic Aggression, Direct Psychological Aggression, and Monitoring Acts will be stronger for men than women.

A categorical principal component analysis will be conducted to test Hypotheses 1 and 3, followed by a latent class analysis to test Hypotheses 1, 2, and 3. The use of both variable and person-centered analyses results in a more robust examination of these hypotheses.

\section{Method}

\section{Participants and Procedure}

The project was advertised on Amazon's Mechanical Turk (MTurk) to users from North America (the United States and Canada) as a "research survey about aggression in your current or a recent relationship". Participants were required to speak English, be at least 18 years old, and have been in an intimate/dating relationship that has lasted at least one month in the last 12 months. Participants received \$1.20 (USD). Data were collected between 6th and 9th December 2019 from 1915 individuals. Those who had completed less than $90 \%$ of the survey were removed $(n=653)$. The sample distribution of time was inspected to identify and remove those participants who had completed the survey unfeasibly quickly ( $n=16$; under 508 seconds). As one of the hypotheses directly investigates binary gender differences, participants who identified as non-binary were also removed from the dataset $(n=6)$. This data was collected for two studies, which examine both individuals' use and experiences of aggression. As the current study was only concerned with individuals' motivations for the use of aggression, 74 people, who reported exclusive experiences of aggression were also removed. The remaining 
1166 individuals had consented to participate in the study and met both the relationship and perpetration criteria ( $45.5 \%$ male, $54.5 \%$ female, $M_{\text {age }}=36.14, S D=10.23$ ).

The ethnic composition of the sample was: $13.2 \%(n=154)$ African American, $5.7 \%(n$ = 67) Asian American, 65.5\% $(n=764)$ European American, 5.9\% $(n=69)$ Latinx, $0.8 \%(n=$ 9) Native American, and 2.9\% $(n=34)$ Other, such as Middle Eastern or other European. Six percent of the sample did not provide any information regarding their ethnicity $(n=69)$, while $10.4 \%$ identified with more than one ethnic group $(n=121)$. The vast majority of the sample identified as heterosexual $(85.5 \%, n=997)$, with $11.2 \%$ identifying as bisexual $(n=131)$. The remaining $3.2 \%$ identified as either gay $(1.3 \%, n=15)$, lesbian $(.6 \%, n=7)$, pansexual $(.7 \%, n$ $=8)$, asexual $(.3 \%, n=4)$, or 'other' $(.3 \%, n=4)$. The method for this study was prespecified in a document that was uploaded to the Open Science Framework in December 2019 (https://osf.io/jdnw9/).

\section{Materials}

Format of questionnaire. Two screening questions established the eligibility of people to participate in the study. These relate to (1) participant's relationship status and (2) the presence and recency of aggression in their intimate relationship. A negative response to either one of these questions made an individual ineligible and directed them to the end of the survey.

The survey included sections on demographics, participant's use and motivations for their use of aggression towards their intimate partner, and social desirability. For each question that participants indicated they had perpetrated an aggressive behavioral act, a list of possible motivations for this act would appear. All behavioral items were randomized within their respective sections to increase participant engagement (see Straus et al., 1996).

Participants were asked to answer each question in relation to their current or most recent relationship. They were also asked to ignore any of the behaviors that occurred within the context of consensual sexual activity. 
Perpetrated IPA. The questions relating to perpetrated behavioral acts were items derived from a previous study conducted by the authors (Dempsey et al., 2020). These 45 items had been endorsed by more than $20 \%$ of the participants in this study and were drawn from each of the three factors identified using exploratory (EFA) and confirmatory factor analyses (CFA) with data collected from community and student samples. These factors were labeled: Eclectic Aggression (18 items; $\alpha=.98$; e.g., "I choked, strangled, or suffocated my partner"), Direct Psychological Aggression (14 items; $\alpha=.94$; e.g., "I deliberately acted in a hurtful way towards my partner"), and Monitoring Acts (13 items; $\alpha=.95$; e.g., "I checked my partner's social network page[s]").

In the current study, the frequency of the items was assessed using a numerical format (e.g., Never, 1-2 times, 3-5 times, 6-10 times, more than 10 times), allowing participants to easily discriminate between response options and avoid the ambiguity of text options (e.g., “sometimes", "seldom"; DeVellis, 2012).

Motivations for IPA. The questions relating to the motivations for the use of the aggressive acts were informed by theories of general aggression, other theories such as control theory (Dobash \& Dobash, 1979) and attachment theory (Dutton, 2006a), and a review of the qualitative literature on the motivations for the use of IPA. Examples of these items include: "I wanted to protect myself from them physically harming me in that moment", "I wanted to express my anger" and "I wanted them to listen to me". A free-text box was also provided to allow participants to state motivations that were not already listed.

Participants could select up to three motivations for a specific act, which allowed for a degree of complexity of motivation. Although some studies have not placed restrictions on the number of motivations participants can select (e.g., Shorey et al., 2010; Stuart et al., 2006), we were interested in what individual's considered to be the most salient motivations for their use of IPA. This section was binary scored $($ Endorsed $=1$; Not endorsed $=0$ ). 
Social desirability bias. When conducting research in the field of IPA, it is considered important to account for social desirability response bias (Arias \& Beach, 1987). The Balanced Inventory of Desirable Responding Short Form (BIDR-16: Paulhus, 1991) was used to assess social desirability in this study. The 7 -point scale $(1=$ Not true, $7=$ True $)$ has 16 items, which assess self-deception enhancement and impression management. Higher scores on this scale are indicative of socially desirable responding. The short form has been found to have equivalent structure, validity, and reliability to the original 40 item BIDR scale (Hart et al., 2015; Paulhus). The Cronbach's alpha in the current study was considered acceptable $(\alpha=.73)$.

\section{Treatment of data}

To allow us to differentiate between types of missing data, questions that had been skipped were coded as '9' and questions that did not apply (i.e., participants had answered 'never' to the behavioral questions, therefore the motivation question was not applicable) were coded as ' 8 '.

Missing value analysis conducted in IBM SPSS Statistics for Windows (Version 26.0; IBM Corp., 2019a) showed none of the behavioral data was missing, but $.023 \%$ of the data on motivations was incomplete. Little's MCAR test for missingness (Little \& Rubin, 2002) was non-significant, demonstrating this data was missing completely at random (MCAR) $\chi 2(1680)$ $=1347.51, p=1.00)$. Due to this result and the small amount of missingness in the data set, an amount considered ignorable (Schafer, 1999), the decision was made not to impute.

\section{Results}

\section{Power Analysis}

A Markov Chain Monte Carlo simulation with a Metropolis-Hastings algorithm was conducted a priori using the R package 'bindata' (Leisch et al., 2012) in RStudio (Version 1.1.456; R Core Team, 2013) to determine the appropriate sample size to produce reliable factorial solutions. The Markov chain Monte Carlo (MCMC) is a class of methods for sampling 
from a probability distribution that is useful in the analysis of complex models (Robert \& Casella, 2004).

The simulation was based on survey data from a pilot study investigating participants' motivations for their use of physical IPA (A'Court, 2020). We used the estimated probability of selecting a given motivation and the correlations between motivations to run the simulation. The R package 'BayesFM' (Piatek, 2017) was used to conduct Bayesian exploratory factor analysis (BEFA). We considered sample sizes of 100, 200, 300, 400, 500, and 600. We used a burn-in of 2000 iterations and saved a further 10,000 iterations for posterior inference. Burn-in is the process by which the initial samples are discarded (van Ravenzwaaij et al., 2018).

Latent factors are identified by calculating the correlations between items. However, as some of the motivations in the dataset were less frequently endorsed than others, the correlations were too low to identify latent factors and, therefore, to estimate a consistent factor structure. Therefore, based on the results of the simulation, we could not be confident regarding the estimate of the sample size.

There is a lack of consensus in the literature as to how to determine a priori sample size, with various heuristics recommended (DeVellis, 2012; Kyriazos, 2018). This study used the sample size to parameter ratio of 20:1 as recommended by Kline (2005) as a rough guide. Employing this heuristic would, therefore, indicate a required sample of 340 participants for the BEFA.

Although MCMC methods are not reliant on asymptotic normality and are therefore not dependent on large sample sizes (McNeish, 2016), ordinal data, such as that in the current study, requires relatively larger samples than continuous data (Kline, 2005). In addition, data that is not normally distributed requires an increased sample size (Kyriazos, 2018), and it is expected, based both on previous work and existing literature (e.g., Cascardi et al., 2019), that the data 
collected will be (slightly) positively skewed. Furthermore, smaller correlations, as found during the MCMC simulation, mean larger sample sizes are required (Kyriazos).

A sample of 500 participants for factor analysis is regarded as 'very good' according to the guidelines attributed to Comrey (1973) and cited by Tinsley and Tinsley (1987) and DeVellis (2012). Consequently, we planned to collect data from approximately 1200 participants and then randomly divide the sample into two equal sub-samples, allowing $\approx 600$ participants for use in the BEFA and the remaining $\approx 600$ participants for use in the Bayesian confirmatory factor analysis.

\section{Statistical Analysis}

We conducted a Bayesian exploratory factor analysis (BEFA; Piatek, 2017) in RStudio (Version 1.1.456; R Core Team, 2013). Despite increasing the number of iterations to $1,000,000$, the chain was not mixing well and was extremely slow to converge. Therefore, due to time constraints, the decision was made to move away from Bayesian analysis and adopt a frequentist approach.

We experienced considerable difficulties in performing the exploratory factor analysis (EFA). This was attributable to the large number of nominal variables (900) and the small correlations between them. Although the dataset had been randomly split to facilitate performing EFA and confirmatory factor analysis on independent samples, the combined dataset was used in an attempt to get the model to run. The Kaiser-Meyer-Olkin (KMO; Kaiser, 1974) Measure of Sampling Adequacy indicates the appropriateness of conducting an EFA based on the proportion of common variance in the dataset, with values of above .60 considered desirable (Howard, 2016). The KMO value for the current analysis was .38, suggesting the correlation matrix was not factorable and thus EFA was not an appropriate method of analyzing the data (see Hair, 2010). Indeed, less than one percent of the correlations $(.93 \%)$ were $\geq .30$. 
We simplified the model by reducing the number of items by looking at the frequency of the behaviors and removing one-third of the lowest frequency items (by \%) for each of the three factors found in the previous study conducted by the authors (Dempsey et al., 2020). The total number of behavioural items included in the analysis was therefore 30 ('Eclectic Aggression': 12 items; 'Direct Psychological Aggression': Nine items; 'Monitoring Acts': Nine items: see Appendix FF for a full list of items included in this analysis). Principal components analysis is a data reduction technique (Linting et al., 2007). We, therefore, performed a categorical principal component analysis (CATPCA) to further reduce the number of variables to a smaller number of components (Linting et al., 2011). A standard principal component analysis assumes linear relationships between variables and numerical levels of measurement (e.g., interval or ratio). However, as the data relating to motivations was nominal, a CATPCA, which accounts for non-linearity and non-numeric measurement (Linting \& van der Kooij, 2012), was employed. In addition to reducing the number of items, the CATPCA explores the relationships between variables, thus, making it an appropriate choice of analysis to investigate possible associations between motivations and types of IPA.

Descriptive statistics. All analyses were conducted in IBM SPSS Statistics for Windows (Version 26.0; IBM Corp., 2019a). Zero-order correlations between the components are presented in Appendix GG.

Perpetrated IPA. Participants reported perpetrating approximately ten IPA acts, at least once, in the past year $(M=9.92, S D=3.71)$. Descriptive statistics showed this variable was highly positively skewed and the Mann-Whitney U test, the non-parametric equivalent of the independent samples $t$-test was used to make gender comparisons. This test showed a statistically significant, though small, gender difference in perpetration, with men $(M d n=9.00)$ reporting slightly more acts than women $(M d n=9.00) U\left(N_{\text {women }}=635, N_{\text {men }}=531\right)=$ 150571.50, $z=-3.21, p<.001, d=.18$. Figure 4.1 shows the frequency of IPA ('Eclectic 
Aggression'; 'Direct Psychological Aggression'; 'Monitoring Acts') by the percentage of the sample who reported perpetrating those acts.

\section{Figure 4.1}

Frequency of reported IPA perpetration by type

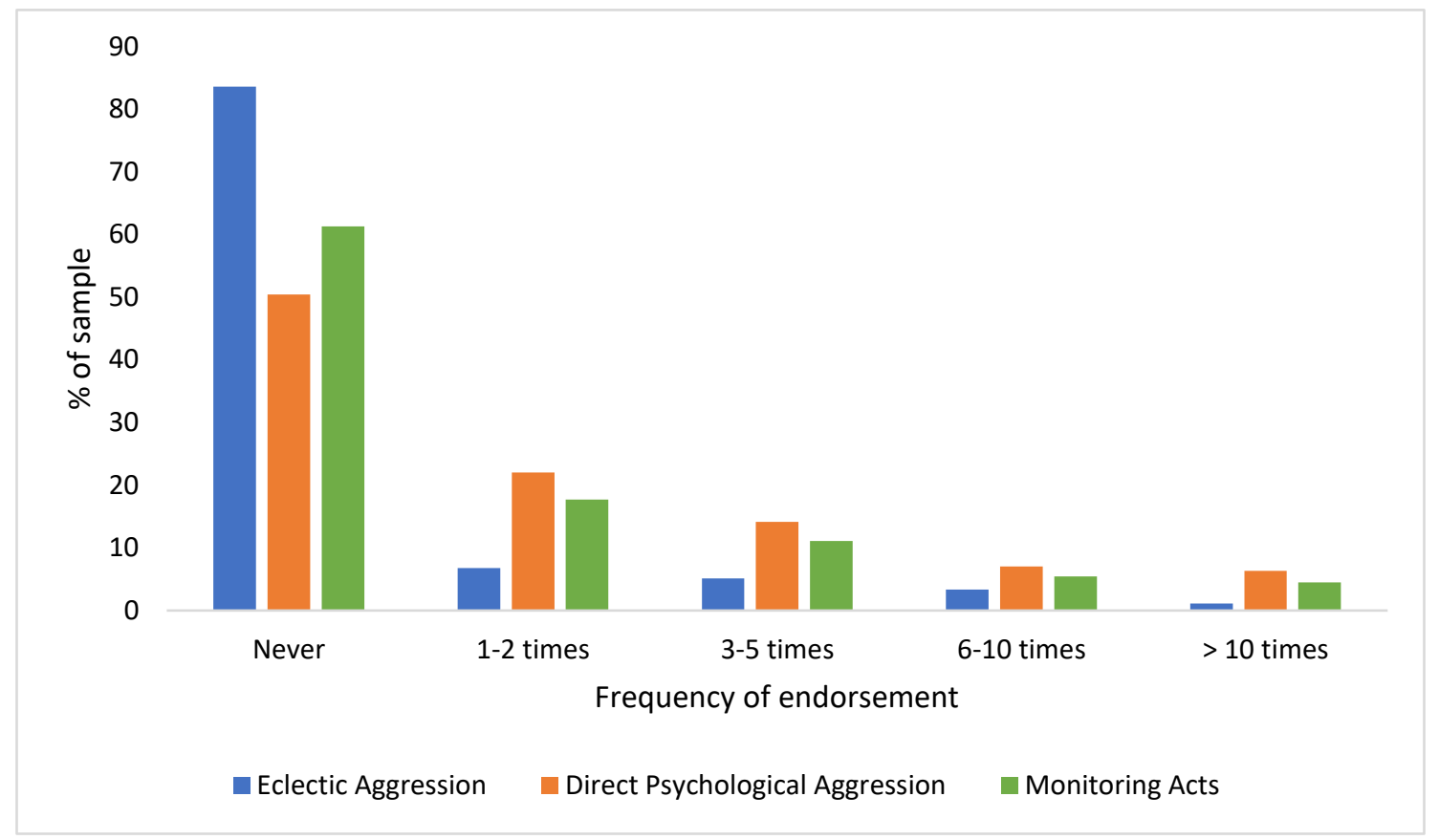

Motivations for IPA. Table 4.1 shows the number of times each of the motivations was endorsed, by the entire sample, and by gender. "I wanted to express my anger" (782 times), "I wanted to express my frustration" (760 times), "I wanted to get their attention" (617 times), and "I wanted them to listen to me" (617 times) were the most frequently endorsed motivations in the study. Independent samples Mann-Whitney U tests were conducted to investigate gender differences in motivations, the results of which can be seen in Table 4.2. The largest effect sizes were for "I wanted to feel powerful" $(d=.27)$, "I wanted to escape from them" $(d=.25)$, "I wanted to make them do something for me" $(d=.25)$, and "I wanted to protect myself from them physically harming me in that moment" $(d=.22)$. The estimates of the effect were all in the male direction. Five of the six motivations that were derived from control theory showed 
significant gender differences, and that men endorsed these motivations more frequently than women, with effect sizes ranging from .18 to .27 . 


\section{Table 4.1}

Frequencies of motivations for IPA endorsed (total and by gender)

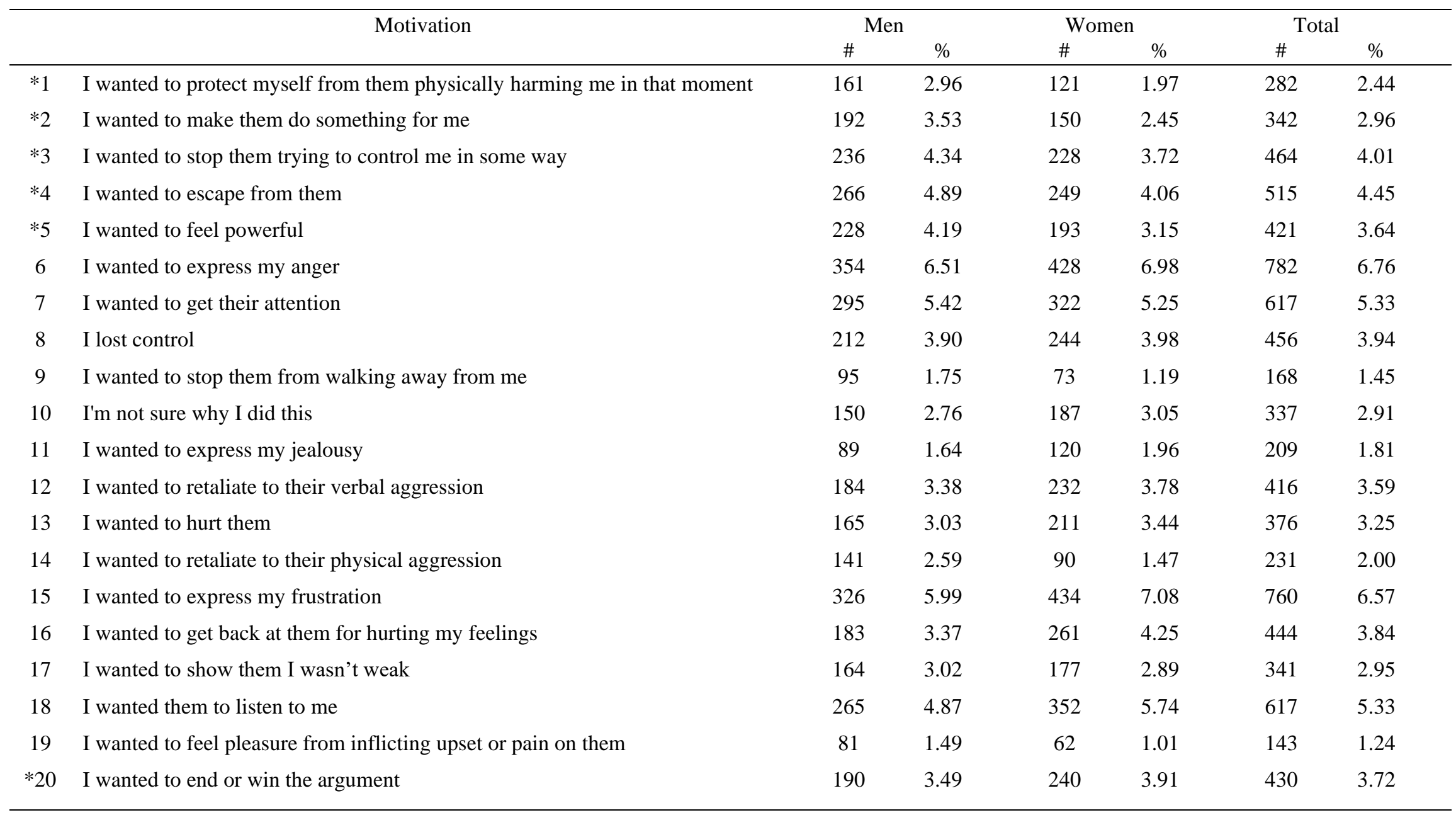

Note. Percentages do not add up to $100 \%$ because participants could endorse up to three motivations per act; ${ }^{*}$ Motivations derived from control theory 
Table 4.2.

Gender comparison of endorsement of motivations for IPA

\begin{tabular}{|c|c|c|c|c|c|c|}
\hline & Motivations & $\begin{array}{c}\text { Men } \\
M \text { Rank }\end{array}$ & $\begin{array}{l}\text { Women } \\
M \text { Rank }\end{array}$ & $z$ & $p$ & $d$ \\
\hline 1 & I wanted to protect myself from them physically harming me in that moment & 623.37 & 550.16 & -4.93 & $<.001$ & .22 \\
\hline 2 & I wanted to make them do something for me & 629.77 & 544.81 & -5.35 & $<.001$ & .25 \\
\hline 3 & I wanted to stop them trying to control me in some way & 616.99 & 555.49 & -3.53 & $<.001$ & .18 \\
\hline 4 & I wanted to escape from them & 628.45 & 545.91 & -4.62 & $<.001$ & .25 \\
\hline 5 & I wanted to feel powerful & 633.32 & 541.84 & -5.38 & $<.001$ & .27 \\
\hline 6 & I wanted to express my anger & 580.91 & 585.67 & .25 & .805 & .01 \\
\hline 7 & I wanted to get their attention & 606.46 & 564.30 & -2.27 & $<.05$ & .13 \\
\hline 8 & I lost control & 589.66 & 578.35 & -.65 & .513 & .03 \\
\hline 9 & I wanted to stop them from walking away from me & 604.45 & 565.98 & -3.18 & $<.001$ & .11 \\
\hline 10 & I'm not sure why I did this & 582.16 & 584.62 & .16 & .876 & .01 \\
\hline 11 & I wanted to express my jealousy & 577.65 & 588.39 & .81 & .416 & .03 \\
\hline 12 & I wanted to retaliate to their verbal aggression & 579.37 & 586.95 & .45 & .654 & .02 \\
\hline 13 & I wanted to hurt them & 581.54 & 585.14 & .22 & .826 & .01 \\
\hline 14 & I wanted to retaliate to their physical aggression & 623.63 & 549.94 & -5.35 & $<.001$ & .22 \\
\hline 15 & I wanted to express my frustration & 561.65 & 601.77 & 2.09 & $<.05$ & .12 \\
\hline 16 & I wanted to get back at them for hurting my feelings & 569.50 & 595.21 & 1.49 & .135 & .08 \\
\hline 17 & I wanted to show them I wasn't weak & 595.93 & 573.11 & -1.44 & .150 & .07 \\
\hline 18 & I wanted them to listen to me & 563.16 & 600.51 & 2.02 & $<.05$ & .11 \\
\hline 19 & I wanted to feel pleasure from inflicting upset or pain on them & 601.74 & 568.25 & -2.97 & $<.05$ & .10 \\
\hline 20 & I wanted to end or win the argument & 573.40 & 591.94 & 1.09 & .274 & .06 \\
\hline
\end{tabular}

Note. $M$ Rank $=$ mean rank; $z=$ standardized test statistic; $d=$ standardized effect size 
Categorical principal component analysis (CATPCA). We conducted a CATPCA to ascertain whether patterns of control motivations were distinct from those of general aggression motivations, and if so, whether control motivations were more strongly associated with the perpetration of Eclectic Aggression, Direct Psychological Aggression, and Monitoring Acts than general aggression motivations. This analysis was conducted in IBM SPSS Statistics for Windows (Version 26.0; IBM Corp., 2019a). We performed a spline nominal transformation using a first-degree polynomial function. The nominal analysis produces a step function, which can result in irregular quantification, affecting stability (Linting \& van der Kooij, 2012). However, the addition of the spline smooths the transformation (Linting et al., 2007) and increases the amount of variance that can be extracted for each component by optimizing the relationships between variables (Cascardi et al., 2019).

The number of knots and degrees of polynomials that make up the spline dictates the shape and smoothness of the transformation (see Linting \& van der Kooij, 2012 for further information). We used a variable principal normalization, a method that also maximizes the associations between variables, with balanced bootstrapping, using 1000 samples, and a Procrustes rotation, as recommended by Linting et al (2007). Missing data points $(0.023 \%)$ were excluded from the analysis using Passive, which is the default option for missing data in CATPCA.

We examined the scree plots for solutions with up to 8 dimensions to determine the appropriate number of components to retain. These were suggestive of either a three or four component solution (see Figure 4.2). Due to the subjective nature of the scree plot (Raîche et al., 2013), we also used the 'Parallel Analysis and Other Non-Graphical Solutions to the Cattell Scree Test' package in R (Raiche, 2020) to inform our decision. 


\section{Figure 4.2.}

Scree plot from the categorical principal components analysis (CATPCA)

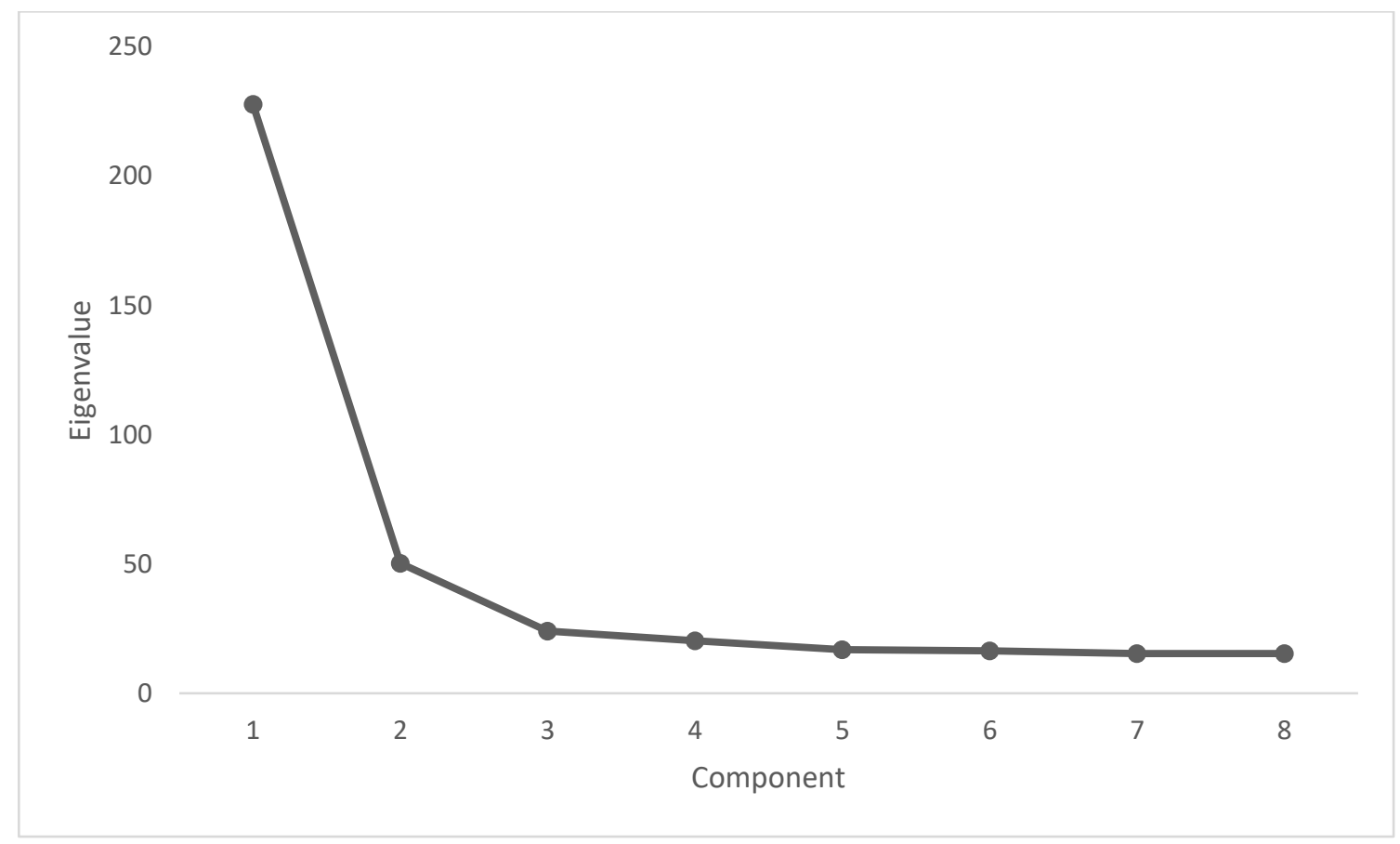

We employed three methods of estimation, all of which use eigenvalues, the amount of variance explained by each component (Linting et al., 2011), to calculate the number of components in the solution. The Cattell-Nelson-Gorsuch CNG indices (nCng) suggested a three-component solution, however the multiple regression procedure to determine the number of components/ factors (nMreg) and the Bartlett, Anderson, and Lawley procedures to determine the number of components/ factors (nBartlett) suggested four-component solutions, and thus the four-component solution was selected. The significance of the item loadings determined by an examination of the bootstrapped confidence intervals was used as a variable selection criterion. Items that were non-significant and were therefore not contributing to the solution were excluded from subsequent analyses (54 items; see Appendix HH; Linting \& van der Kooij, 2012). These items were exclusively related to motivations from general theories of aggression (motivations 10-20) for behaviors such as "I made my partner dependent on me for money" (seven items), "I threatened to withhold money from my partner" (10 items), "I 
purposely withheld affection or sex" (eight items), "I kept my partner from leaving the house" (six items), and "I slapped my partner" (six items).

The four-component solution was rotated using a Promax rotation and a Kaiser normalization, which is an oblique rotation method recommended for large data sets (IBM Corp., 2019b). Promax allows the components to be correlated with one another, thereby making it the most appropriate choice for social science research (Gaskin \& Happell, 2014). To increase the distinction between the components, items that cross-loaded on two or more components with a difference of less than .20 (131 items; see Appendix HH; see Howard, 2016) were removed. All variables had communalities of greater than .50 , which is considered excellent (Comrey, 1973), and minimum loadings of .40 (Stevens, 1986). See Appendix II for the full list of items, Eigenvalues, and percentage of variance explained by the components.

Component 1: Eclectic Aggression. Component one consisted of 208 items, with an Eigenvalue of 153.91 , which accounted for $37.09 \%$ of the variance. This component was characterized by behaviors relating to physical aggression, physical threats and threats to reputation, sexual coercion, destruction of property, and restricting finance and freedom of movement. These behaviors were derived exclusively from the 'Eclectic Aggression' factor found in the previous study and due to this similarity, we labeled this the 'Eclectic Aggression Component'. There was a lack of distinction in the range of motivations endorsed for the majority of behaviors in this component with all 20 motivations endorsed for seven of the 12 behavioral acts. The motivations that were not endorsed for specific behaviors were derived from a variety of theories. The least frequently endorsed motivations were derived from theories of attachment (motivation 11), hostile aggression and the status inconsistency hypothesis (motivations 12 and 14), control theory (motivations 17 and 20), and proactive aggression (motivation 19). Table 4.3 provides a full summary of the motivations selected for each behavioral act. 
Component 2: Direct Psychological Aggression. This component was comprised of 114 items, with an Eigenvalue of 81.63, explaining $19.67 \%$ of the variance. This component contained items relating to deliberately ignoring, criticizing, and being unpleasant towards your partner, one of which was derived from the 'Monitoring Acts' factor and five from the 'Direct Psychological Aggression' factor. This component was labeled the 'Direct Psychological Aggression Component'. With few exceptions, all motivations were endorsed for these six behaviors (see Table 4.3).

Component 3: Monitoring Acts. Component three consisted of 55 items, with an Eigenvalue of 87.33 , explaining $21.04 \%$ of the variance. These items represented behaviors associated with relationship anxiety and the monitoring of an intimate partner. These behaviors were exclusively derived from the 'Monitoring Acts' factor and thus, this component was labeled the 'Monitoring Acts Component'. As with components one and two, the majority of motivations were endorsed, with only five motivations either non-significant or cross-loading (see Table 4.3).

Component 4. This component explained $15.35 \%$ of the variance, with an Eigenvalue of 63.72 and contained 38 items that related to two psychologically aggressive behaviors (I changed the subject on purpose when my partner was trying to discuss a problem; I criticized my partner's friends, family, or co-workers). However, there was no apparent distinction between these behaviors and those comprising component two, nor was there obvious communality that would explain why these behaviors had formed the fourth component. As this component was not interpretable it was excluded from further analysis, and the solution was rerun with three components.

The exclusion of these items did not affect the loadings of the other three principal components but altered their respective rotated eigenvalues. This increased the percentage of variance explained by the 'Eclectic Aggression Component' (increase of 2.67\%) and the 'Direct 
Psychological Aggression Component' (increase of 2.71\%) but decreased the percentage of variance accounted for by the 'Monitoring Acts Component' (decrease of.21\%).

Component solutions by gender. Gender was entered as a supplementary variable in the analysis to ascertain whether the component solution varied by gender. An evaluation of the category points bi-plot indicated clear separation between the centroid coordinates along dimensions one and two, but relatively less distinction along dimension three. Statistical comparisons for each of the component scores for men and women were therefore conducted to provide an objective interpretation.

'Eclectic Aggression Component' scores of women $(M d n=.53)$ were higher than those of men $(M d n=.38)$. A Mann-Whitney test indicated this difference was statistically significant $U\left(N_{\text {women }}=635, N_{\text {men }}=531\right)=200310.00, z=5.54, p<.001$. Similarly, 'Direct Psychological Aggression Component' scores of women $(M d n=-.18)$ were also significantly higher than those of men $(M d n=.16) U\left(N_{\text {women }}=635, N_{\text {men }}=531\right)=153636.00, z=-2.61, p<.009$. However, an independent samples $t$-test demonstrated there was no significant difference between men $(M=.01, S D=1.02)$ and women $(M=-.01, S D=.99)$ on 'Monitoring Acts Component' scores $t(1164)=.33, p=.743$.

Summary. We hypothesized (Hypothesis 1) that if patterns of control motivations were distinct from those of general aggression motivations, then control motivations would be more strongly associated with the perpetration of Eclectic Aggression, Direct Psychological Aggression, and Monitoring Acts than general aggression motivations. However, the theory underlying the motivations (control or general aggression) did not contribute to the structure of the component solution. Rather, the vast majority of motivations were endorsed for a variety of behaviors, demonstrating that there was very little distinction between motivations for physical, psychological, and monitoring acts. In addition, the results do not support Hypothesis 3 regarding gender, as there was no significant gender difference in component scores for 
Monitoring Acts and the component scores of women were significantly higher than those of men for Eclectic Aggression and Direct Psychological Aggression. 


\section{Table 4.3.}

Motivations endorsed for perpetrated acts by type

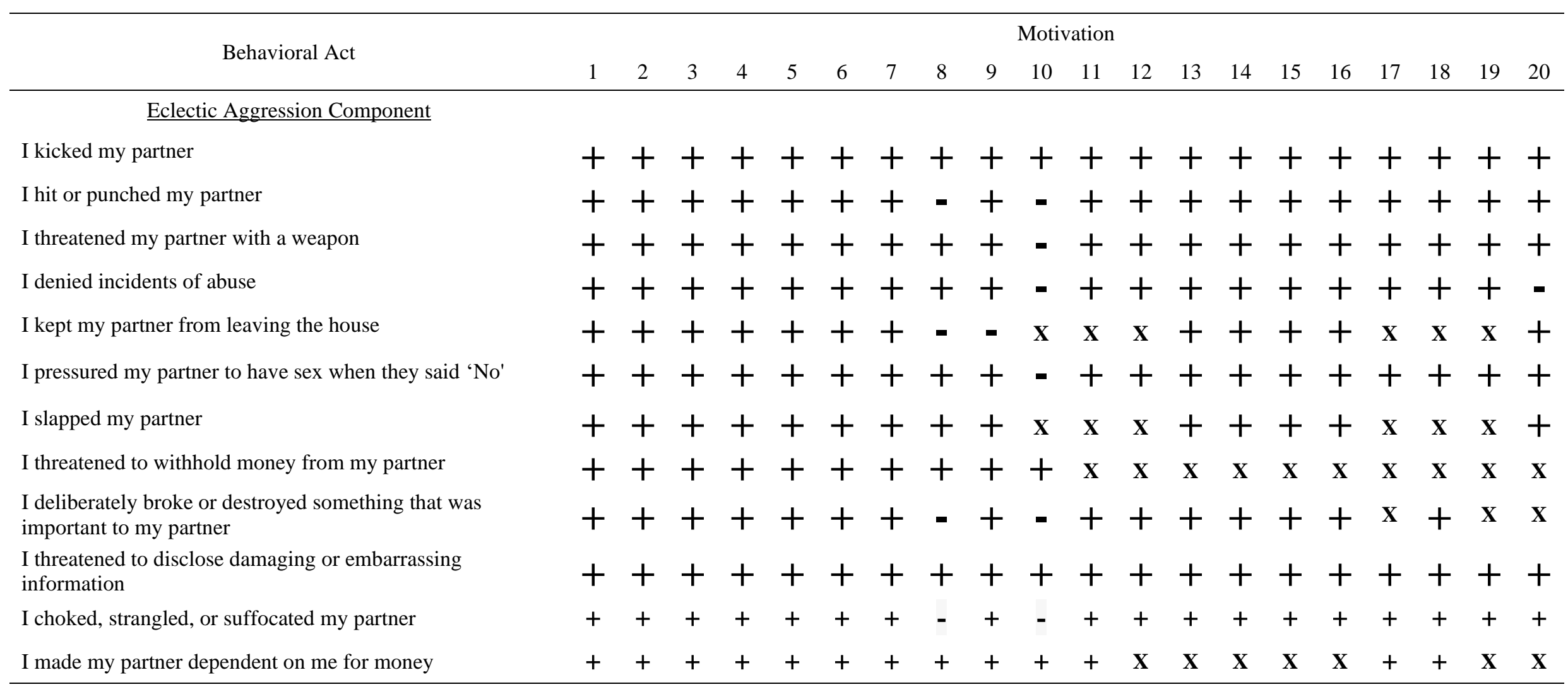

Note $+\mathbf{+}=$ positive item loading $\geq .7 ;-=$ negative item loading $\geq .7 ;+=$ positive item loading $\leq .6 ;-=$ negative item loading $\leq .6 ; \mathbf{X}=$ non-loading items; $1=\mid$ wanted to protect $\mathrm{myself}$ from them physically harming me in that moment; $2=1$ wanted to make them do something for me; $3=1$ wanted to stop them trying to control me in some way; $4=1$ wanted to escape from them; $5=1$ wanted to feel powerful; $6=\mid$ wanted to express my anger; $7=\mid$ wanted to get their attention; $8=\mid$ lost control; $9=\mid$ wanted to stop them from walking away from me; $10=\mid$ ' $m$ not sure why $\mid$ did this; $11=\mid$ wanted to express my jealousy; $12=\mid$ wanted to retaliate to their verbal aggression; $13=\mid$ wanted to hurt them; $14=\mid$ wanted to retaliate to their physical aggression; $15=\mid$ wanted to express my frustration; 16 = I wanted to get back at them for hurting my feelings; $17=\mid$ wanted to show them I wasn't weak; $18=\mid$ wanted them to listen to me; $19=\mid$ wanted to feel pleasure from inflicting upset or pain on them; $20=1$ wanted to end or win the argument. 
Table 4.3 cont

\begin{tabular}{|c|c|c|c|c|c|c|c|c|c|c|c|c|c|c|c|c|c|c|c|c|}
\hline \multirow{2}{*}{ Behavioral Act } & \multicolumn{20}{|c|}{ Motivation } \\
\hline & 1 & 2 & 3 & 4 & 5 & 6 & 7 & 8 & 9 & 10 & 11 & 12 & 13 & 14 & 15 & 16 & 17 & 18 & 19 & 20 \\
\hline \multicolumn{21}{|l|}{ Direct Psychological Aggression Component } \\
\hline I was critical or unpleasant toward my partner & + & + & & + & + & + & + & 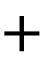 & + & + & + & + & + & + & + & + & + & + & + & + \\
\hline I screamed or yelled at my partner & + & + & + & + & + & + & + & + & + & + & + & + & + & + & + & + & + & + & + & + \\
\hline I pouted or acted upset when I didn't get my way & + & + & + & + & + & + & + & + & + & - & - & + & + & + & + & + & + & + & + & + \\
\hline $\begin{array}{l}\text { I stomped out of the house or room during a disagreement } \\
\text { with my partner }\end{array}$ & + & + & + & + & + & + & + & + & - & - & + & + & + & + & $\mathbf{X}$ & + & + & + & + & $\mathbf{X}$ \\
\hline I deliberately acted in a hurtful way towards my partner & + & + & + & + & + & + & + & + & + & - & + & + & + & + & + & + & + & + & + & + \\
\hline I deliberately ignored my partner & - & + & + & + & + & + & + & + & + & $\mathbf{X}$ & $\mathbf{X}$ & + & + & $\mathbf{X}$ & $\mathbf{X}$ & + & + & + & + & + \\
\hline \multicolumn{21}{|l|}{ Monitoring Acts Component } \\
\hline $\begin{array}{l}\text { I accused my partner of being unfaithful or flirting with } \\
\text { others }\end{array}$ & + & & & 1 & + & + & + & + & L & 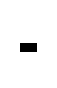 & + & + & + & + & + & + & + & + & + & F \\
\hline I checked my partner's social network page(s) & + & + & + & + & + & + & + & + & 十 & + & + & + & + & + & + & + & + & + & + & + \\
\hline $\begin{array}{l}\text { I insisted on knowing where my partner went and who they } \\
\text { spoke to when we were not together }\end{array}$ & + & + & + & + & + & + & + & + & + & $\mathbf{X}$ & $\mathbf{X}$ & $\mathbf{X}$ & + & $\mathbf{X}$ & + & + & + & $\mathbf{X}$ & + & + \\
\hline
\end{tabular}

Note. $+=$ positive item loading $\geq .7 ;-=$ negative item loading $\geq .7 ;+=$ positive item loading $\leq .6 ;-=$ negative item loading $\leq .6 ; \mathbf{X}=$ non-loading items; $1=$ I wanted to protect $\mathrm{myself}$ from them physically harming me in that moment; $2=$ I wanted to make them do something for me; $3=$ I wanted to stop them trying to control me in some way; $4=I$ wanted to escape from them; $5=1$ wanted to feel powerful; $6=$ I wanted to express my anger; $7=\mid$ wanted to get their attention; $8=\mid$ lost control; $9=\mid$ wanted to stop them from walking away from me; $10=\mid$ ' $m$ not sure why I did this; $11=\mid$ wanted to express my jealousy; $12=\mid$ wanted to retaliate to their verbal aggression; $13=\mid$ wanted to hurt them; $14=\mid$ wanted to retaliate to their physical aggression; $15=\mid$ wanted to express my frustration; 16 = I wanted to get back at them for hurting my feelings; 17 = I wanted to show them I wasn't weak; $18=\mid \mathrm{wanted}$ them to listen to me; 19 = I wanted to feel pleasure from inflicting upset or pain on them; $20=$ I wanted to end or win the argument. 
Latent class analysis (LCA) on motivations of IPA. Next, an LCA was conducted using Mplus (Version 8; Muthén \& Muthén, 1998-2017a). The same 20 motivation and 30 behavioral variables as were used in the CATPCA were used in the LCA (see Appendix FF). LCA is a type of mixture modeling whereby class membership explains the association between individuals' patterns of responding to observed variables (Muthén \& Muthén, 1998-2017b). LCA was used in the current study to ascertain whether profiles, created by the categorical motivational variables, were associated with the frequency of IPA. Specifically, we aimed to investigate the existence of a controlling profile (Hypothesis 1), whether this profile perpetrated IPA with greater frequency than non-controlling profiles (Hypothesis 2), and whether this profile was predominantly comprised of men (Hypothesis 3).

Model selection. Previous IPA typology research has found evidence of perpetrator subtypes characterized by control or emotional dysregulation (see Gottman et al., 1995; Holtzworth-Munroe \& Stuart, 1994; Johnson, 2006). We, therefore, began by running a twoclass solution and continued to increase the number of classes, evaluating the relative fit statistics and interpretability of the classes to determine the most appropriate number to retain (see Table 4.4). We compared the Akaike Information Criterion (AIC), Bayesian Information Criterion (BIC), entropy, and the Bootstrap Likelihood Ratio (BLRT) and the Vuong-LoMendell-Rubin (VLMRLRT; Lo et al., 2001) test statistics from the competing models. AIC and BIC values represent the balance between model fit and parsimony with smaller values indicating a more optimal balance (Collins \& Lanza, 2010), while entropy is a measure of classification accuracy with values close to one indicating high levels of accuracy (Geiser, 2012). Non-significant values on the BLRT and VLMRLRT tests indicate the estimated model would be a better fit for the data with one less class (Geiser).

We considered solutions for up to 12 classes. These results showed continued decreases in the AIC and BIC and very high entropy for all solutions (> .99), suggesting solutions with 
increasingly higher numbers of classes were a better fit for the data. The VLMRLRT test indicated that a ten-class solution performed significantly better than an eleven-class solution. However, subsequent evaluation of the 10-class solution revealed a lack of both parsimony and interpretability, factors that are considered very important in deciding the number of classes to retain (Porcu \& Giambona, 2017).

\section{Table 4.4.}

Model fit for the difference class solutions of the Latent Class Analysis

\begin{tabular}{lccccc}
\hline Classes & AIC & BIC & VLMRLRT & BLRT & Entropy \\
\hline Two classes & 42577.06 & 42890.86 & 0.000 & 0.000 & 1.000 \\
Three classes & 38504.08 & 38959.60 & 0.000 & 0.000 & 1.000 \\
Four classes & 34399.77 & 34997.01 & 0.000 & 0.000 & .989 \\
Five classes & 31445.13 & 32184.08 & 0.000 & 0.000 & .989 \\
Six classes & 30042.64 & 30923.31 & 0.000 & 0.000 & .994 \\
Seven classes & 28968.28 & 29990.67 & 0.001 & 0.000 & .995 \\
Eight classes & 27568.16 & 28732.26 & 0.000 & 0.000 & .989 \\
Nine classes & 26350.30 & 27656.13 & 0.000 & 0.000 & .992 \\
Ten classes & 25609.31 & 27056.85 & 0.004 & 0.000 & .995 \\
Eleven classes & 24683.27 & 26272.53 & 0.391 & 0.000 & .995 \\
Twelve classes & 23952.35 & 25683.32 & 0.000 & 0.666 & .994 \\
\hline
\end{tabular}

Note: AIC = Akaike Information Criterion; BIC = Bayesian Information Criterion; VLMRLRT = Vuong-Lo-MendellRubin Likelihood Ratio Test; BLRT = Bootstrap Likelihood Ratio Test.

Indeed, latent class models should be interpretable, theoretically meaningful, and informed by existing research, with fit statistics comprising just one of the criteria in model selection (Collins \& Lanza, 2010; Geiser, 2012).

We subsequently selected the four-class solution as the preferred model as this had the largest decrease in AIC and BIC values (difference of 4104.31 between the 3 and 4-class solutions), and had higher entropy, and was more parsimonious than solutions with more than 
five classes. Furthermore, the four-class solution was theoretically interpretable and provided clearer distinctions of the classes than the two and three-class solutions.

Class solutions by gender. To assess the effect of gender on the model, we constrained the item-response probabilities to be equal across classes and compared the model fit statistics of the constrained model to those of the freely estimated model (discussed above; Lanza et al., 2013). The imposed parameter restrictions should substantially worsen the fit (Collins \& Lanza, 2010), thereby indicating the importance of gender to the model. However, the constrained model demonstrated smaller AIC and BIC values than the unconstrained model and continued to improve up to 12 classes (see Appendix JJ), suggesting measurement invariance by class across gender. The $G^{2}$ and chi-square difference tests are frequently employed to statistically compare two nested models, however, due to their sensitivity to sample size, neither of these tests is appropriate in this study (Lanza et al.).

Description of classes. Figure 4.3 illustrates the differences in mean scores of motivations for the perpetration of IPA for the four-class solution. Each class has been labeled according to their patterns of reported motivations for perpetrating IPA.

Class 1: Emotional/ Communication Problems. The aggression reported by the largest class ( $N=501 ; 44 \%$ male; $56 \%$ female) was motivated by high levels of "wanting to express anger' and 'wanting to express frustration' and poor communication as evidenced by moderate levels of 'wanting to get their partners attention' and 'wanting their partner to listen to them' and was thus labeled the 'Emotional/ Communication Problems' class. There was almost a complete absence of motivations derived from control theory for this class, with the exception of 'wanting to escape from their partner', which was reported at a low level.

Class 2: Reactive Violence. The second class was comprised of 262 individuals (47\% male; $53 \%$ female), who highly endorsed some control theory motivations for their use of 
aggression (e.g., 'wanting to stop their partner controlling them'; 'wanting to escape from their partner'; 'wanting to end or win an argument'; 'wanting to feel powerful') but also highly endorsed motivations derived from theories of general aggression (e.g., 'wanting to express anger'; 'wanting to get their partners attention'; 'loss of control'; wanting to retaliate to verbal and emotional aggression'; 'wanting to hurt their partner'; 'wanting to express frustration'; 'wanting their partner to listen to them'). These motivations suggest a pattern of responding or reacting to relationship conflict and this class was consequently labeled as the 'Reactive Violence' class.

Class 3: Heterogenous. Class three was the smallest class, comprised of 168 individuals (42\% male; $58 \%$ female). This class endorsed all motivations at a very high level, displaying a variety of motivations for different behaviors, and was thus labeled the 'Heterogenous' class.

Class 4: Homogenous. In contrast, class $4(N=235 ; 49 \%$ male; $51 \%$ female $)$ appeared not to endorse any of the motivations and due to this uniformity were labeled the 'Homogenous' class.

Class differences on demographic variables. One-way ANOVAs with Games-Howell post hoc analyses were conducted in IBM SPSS Statistics for Windows (Version 26.0; IBM Corp., 2019a) to explore demographic differences in the four classes. These revealed significant differences in relationship status $(F(3)=4.44, p<.05)$ between the 'Reactive Violence' and the 'Emotional/ Communication Problem' and 'Homogenous' classes, and the 'Emotional/ Communication Problem' and 'Homogenous' classes. These results could be attributable to the relatively high numbers of individuals who respectively identified as married, cohabiting, or de-facto in the 'Emotional/ Communication Problem' class. 


\section{Figure 4.3.}

Four-class solution of motivations for IPA.

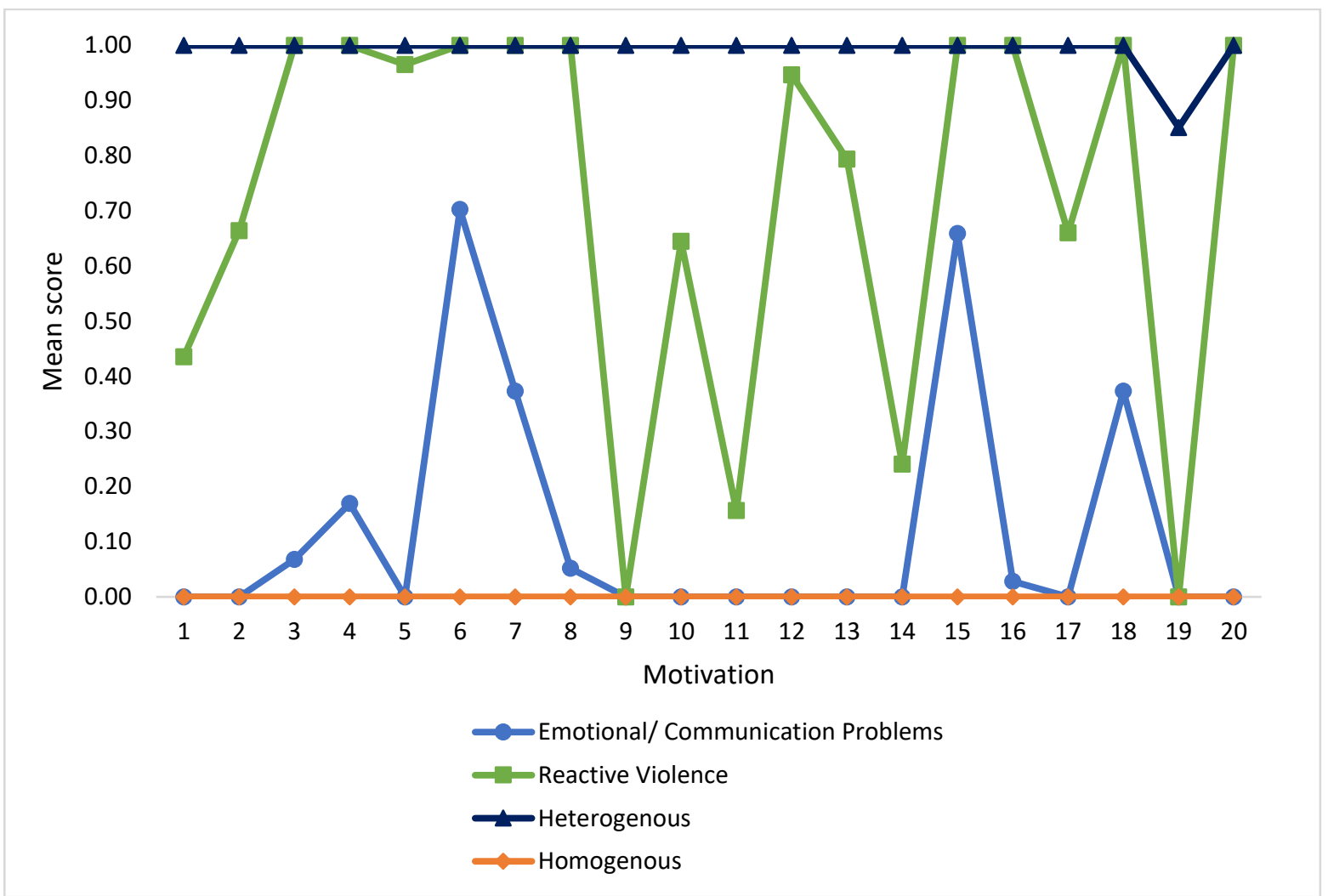

Note. 1 = I wanted to protect myself from them physically harming me in that moment; 2 = I wanted to make them do something for me; 3 = I wanted to stop them trying to control me in some way; 4 = I wanted to escape from them; 5 = I wanted to feel powerful; 6 = I wanted to express my anger; 7 = I wanted to get their attention; $8=$ I lost control; $9=$ I wanted to stop them from walking away from me; $10=I$ 'm not sure why I did this; $11=I$ wanted to express my jealousy; 12 = I wanted to retaliate to their verbal aggression; 13 = I wanted to hurt them; 14 = I wanted to retaliate to their physical aggression; 15 = I wanted to express my frustration; $16=$ I wanted to get back at them for hurting my feelings; 17 = I wanted to show them I wasn't weak; $18=$ I wanted them to listen to me; 19 = I wanted to feel pleasure from inflicting upset or pain on them; $20=$ I wanted to end or win the argument.

Differences in education level were also significant $(F(3)=4.58, p=.003)$ between the 'Homogenous' class and other classes, and the 'Emotional/ Communication Problem' and 'Heterogenous' classes. Differences in employment level were not significant $(F(3)=.53, p=$ .664). Welch's robust of equality of means tests were conducted for those variables that violated the assumption of homogeneity of variance. 
The results showed there was no significant difference in age $(F(3,510.11)=2.41, p=$ $.066)$ or gender $(F(3,496.84)=.97, p=.408)$ across the four classes. There was a significant difference between classes in sexual orientation $(F(3,448.39)=10.11, p<.001)$ with a GamesHowell post hoc analyses showing these differences were between the 'Emotional/ Communication Problem' and 'Reactive Violence' and 'Homogenous' classes. This is likely attributable to the lower proportion of individuals, who identified as bi-sexual in the 'Emotional/ Communication Problem' class (5.8\% versus $16.4 \%$ and $18.7 \%$ respectively). Differences in ethnicity could not be statistically investigated as one of the classes ('Emotional/ Communication Problems') had zero variance.

Class differences on outcome variables. One-way ANOVAs using Welch's robust test of equality of means were used to investigate mean differences in 'Eclectic Aggression', 'Direct Psychological Aggression', Monitoring Acts', the total number of aggressive acts, and social desirability in the four classes (see Table 4.5). Games-Howell post hoc analyses revealed the classes were significantly different from one another on each type of IPA and the total number of perpetrated acts.

The 'Heterogenous' class reported the most 'Eclectic Aggression', 'Direct Psychological Aggression', and total number of aggressive acts, while the 'Homogenous' class reported perpetrating the most Monitoring Acts', in addition to high levels of other forms of IPA. There were no significant differences between the 'Emotional/ Communication Problem' and 'Heterogenous' classes or between the 'Reactive Violence' and 'Homogenous' classes on either the Self-Deceptive Enhancement or Impression Management sub-scales measuring social desirability. Furthermore, there was no significant difference between the 'Heterogenous' and 'Reactive Violence' classes on the Self-Deceptive Enhancement sub-scale. 
Table 4.5.

Four-class solution one-way ANOVAs predicting mean level differences in outcome variables

\begin{tabular}{|c|c|c|c|c|c|c|c|c|c|}
\hline & \multicolumn{2}{|c|}{$\begin{array}{c}\text { Emotional/ } \\
\text { Communication } \\
\text { Problems } \\
(N=501 ; 43 \%) \\
\end{array}$} & \multicolumn{2}{|c|}{$\begin{array}{l}\text { Reactive Violence } \\
\underline{(N=262 ; 22.5 \%)}\end{array}$} & \multicolumn{2}{|c|}{$\begin{array}{l}\text { Heterogenous } \\
(N=168 ; 14.4 \%) \\
\end{array}$} & \multicolumn{2}{|c|}{$\begin{array}{c}\text { Homogenous } \\
(N=235 ; 20.1 \%) \\
\end{array}$} & \multirow{2}{*}{$\begin{array}{c}\text { Welch's } \\
\quad F \\
\end{array}$} \\
\hline & $M$ & $S D$ & $M$ & $S D$ & $M$ & $S D$ & $M$ & $S D$ & \\
\hline Eclectic Aggression & $.01^{\mathrm{abc}}$ & .02 & $.02^{\text {ade }}$ & .06 & $.99^{\mathrm{bdf}}$ & .50 & $.85^{\mathrm{cef}}$ & .52 & $423.03 *$ \\
\hline $\begin{array}{l}\text { Direct Psychological } \\
\text { Aggression }\end{array}$ & $.56^{\mathrm{abc}}$ & .14 & $.90^{\text {ade }}$ & .22 & $1.84^{\text {bdf }}$ & .34 & $1.30^{\mathrm{cef}}$ & .33 & $1126.48 *$ \\
\hline Monitoring Acts & $.37^{\mathrm{abc}}$ & .28 & $.41^{\text {ade }}$ & .21 & $1.30^{\text {bdf }}$ & .33 & $1.51^{\mathrm{cef}}$ & .42 & $805.97 *$ \\
\hline Total number of behaviors & $7.16^{\mathrm{abc}}$ & 2.04 & $8.21^{\text {ade }}$ & 1.38 & $14.87^{\mathrm{bdf}}$ & .53 & $14.20^{\text {cef }}$ & 1.35 & $3166.57^{*}$ \\
\hline $\begin{array}{l}\text { Social Desirability } \\
\text { (Impression Management) }\end{array}$ & $4.13^{\mathrm{a}}$ & .84 & $4.43^{\mathrm{b}}$ & .89 & $4.03^{\mathrm{bc}}$ & .93 & $4.48^{\mathrm{ac}}$ & 1.07 & $13.82 *$ \\
\hline $\begin{array}{l}\text { Social Desirability (Self- } \\
\text { Deceptive Enhancement) }\end{array}$ & $4.15^{\mathrm{ab}}$ & .78 & $4.45^{\mathrm{ac}}$ & .88 & $4.31^{\mathrm{cd}}$ & .76 & $4.66^{\mathrm{bd}}$ & 1.00 & $18.96^{*}$ \\
\hline
\end{tabular}

Note. $M=$ mean; $S D=$ standard deviation; ${ }^{*} p<.001$; Means with the same superscript differed significantly $(p<.05)$ 
To assess whether social desirability bias was high in the sample as a whole, the mean scores of self-deceptive enhancement and impression management were compared to those from a study designed to validate the BIDR-16, conducted by Hart et al. (2015; see Study 2, N $=670$ ). The sample was recruited from research websites and data was collected from multiple countries around the world.

A Welch two samples $t$-test demonstrated there was no significant difference in mean scores between the current sample $(M=4.34, S D=.87)$ and the Hart et al. sample $(M=4.30$, $S D=1.14)$ on self-deceptive enhancement $t(1119.69)=.79, p=.432, d=.04$. However, the Hart et al. sample $(M=4.50, S D=1.24)$ was significantly higher than the current sample $(M=$ $4.25, S D=.93)$ on impression management scores $t(1105.06)=5.54, p<.001, d=.24$. All analyses were conducted in IBM SPSS Statistics for Windows (Version 26.0; IBM Corp., 2019a).

Summary. An LCA was conducted to ascertain whether there was a class of individuals who were uniquely motivated by control (Hypothesis 1) and whether these motivations were associated with perpetrating more frequent IPA (Hypothesis 2). Model fit statistics for up to 12 classes were evaluated and showed continued decreases in the AIC and BIC, as per the results of the CATPCA, demonstrating that motivations for aggression are heterogeneous. This necessitated a greater emphasis on theory and prior knowledge of the IPA literature in the selection of the number of classes to retain. Despite increasing the number of classes, there was still no evidence of a class that primarily endorsed motivations of control that could be characterized as 'controlling'. Classes, who endorsed motivations derived from control theory, also endorsed motivations derived from theories of general aggression. Thus, the results of the LCA did not support Hypothesis 1. Support for Hypothesis 2 is contingent on the existence of a controlling profile, and, thus, there was also a lack of evidence to support an increased frequency of IPA in individuals that primarily endorsed motivations of control. 
Similarly, there was no evidence to support Hypothesis 3, as gender was found to have no effect on the four-class solution when item-response probabilities were constrained, and the constrained and unconstrained models were compared. Furthermore, Welch's robust of equality of means test showed there was no significant difference in gender across the four classes and the percentage of men and women in each class was fairly symmetric, with a higher percentage of women in each.

\section{Discussion}

This study aimed to adopt a gender-inclusive approach to explore the relationship between control motivations and the types and frequencies of behavioral acts used by men and women in intimate relationships. In doing so it examines the validity of Johnson's $(1995,2006)$ typology which has postulated that 'intimate terrorism' is characterized by control motivations, frequent and severe levels of physical harm potential, and is primarily gendered. Three hypotheses were tested to achieve this aim. Specifically, whether patterns of control motivations are distinct from those of general aggression motivations by testing if (Hypothesis 1) control motivations are more strongly associated with the perpetration of Eclectic Aggression, Direct Psychological Aggression, and Monitoring Acts than general aggression motivations; (Hypothesis 2) if control motivations are more strongly associated with an increased frequency of perpetrating Eclectic Aggression, Direct Psychological Aggression, and Monitoring Acts than general aggression motivations; and (Hypothesis 3) if the association between control motivations and the perpetration of Eclectic Aggression, Direct Psychological Aggression, and Monitoring Acts are stronger for men than women. Results found that control motivations were not more strongly associated with the perpetration of types of IPA, gendered perpetration, or level of frequency of perpetration. A detailed exploration of the results of each hypothesis is provided below. 


\section{Hypothesis 1 \& 2: Control and Type and Frequency of IPA}

We conducted a CATPCA to test Hypothesis 1, the results of which did not reveal a distinct pattern of control. Motivations relating to a particular behavior loaded almost exclusively on the same component, highlighting the heterogeneous nature of motivations for different types of IPA. The three-component solution was instead defined by Eclectic Aggression, Direct Psychological Aggression, and Monitoring Acts, replicating the factor structure of that which was found in a previous study (see Dempsey et al., 2020). Therefore, we did not find evidence of specific behaviors that were uniquely associated with controlling motivations as hypothesized.

Similarly, in the latent class analysis, which we conducted to test Hypotheses 1 and 2, there was no evidence of a profile characterized by controlling motivations. The 'Emotional/ Communication Problems' class constituted $43 \%$ of the sample and reported the lowest levels of aggression across all types, which was predominantly motivated by high levels of frustration and anger. This class was also characterized by an absence of both control and retaliatory violence motivations.

The 'Reactive Violence' class also displayed low levels of aggression across all types, although these were higher than in the 'Emotional/ Communication Problems' class. The low level of aggression and retaliatory nature of motivations (e.g., 'I wanted to retaliate to their verbal aggression'; 'I wanted to get back at them for hurting my feelings') that characterize this class are suggestive of Johnson's 'situational couple violence' $(1995,2006)$. However, the 'Reactive Violence' class also endorsed control motivations, which is inconsistent with Johnson's theory that classifies 'situational couple violence' as non-controlling. In addition, Johnson theorizes that 'situational couple violence' should be the most prevalent type of IPA in a community sample, however, the 'Reactive Violence' class comprised less than one quarter $(22.5 \%)$ of the sample. 
The 'Heterogenous' class (14.4\%) reported the highest frequencies of Eclectic Aggression, Direct Psychological Aggression, and total number of aggressive behaviors, and the second-highest frequency of Monitoring Acts. This class could be considered to engage in Johnson's 'mutual violent control' (2006) as they reported high levels of aggression and were the only class to endorse control theory motivations relating to both controlling their partners (e.g., 'I wanted to make them do something for me') and experiencing control (e.g., 'I wanted to stop them trying to control me in some way'). However, this class equally endorsed motivations from theories of general aggression, such as 'wanting to express their anger' and 'wanting to retaliate to their verbal aggression' and the reactive nature of these motivations are inconsistent with 'mutual violent control'.

The 'Homogenous' class (20.1\%) reported the highest frequency of Monitoring Acts and the second-highest frequencies of Eclectic Aggression, Direct Psychological Aggression, and total number of aggressive behaviors. Despite their high levels of aggression, they endorsed motivations at such low levels that the mean scores for each of the motivations were zero.

In sum, the results do not provide evidence in support of Hypotheses 1 and 2 regarding patterns of control motivations and their association with the frequency at which aggression is perpetrated. Rather, higher frequencies of aggression were associated with a diverse range of motivations in the current study. Furthermore, the absence of a pattern of controlling motivations and the configuration of the profiles created by the motivational variables are inconsistent with Johnson's typology. Not only did this study fail to find evidence of a controlling profile similar to Johnson's (2006) 'intimate terrorist' or 'mutual violent control', but also failed to find evidence of a profile with an absence of control motivations similar to his 'situational couple violence'. Instead, while the 'Reactive Violence' class reported lowlevel IPA, which was motivated by retaliation, contrary to Johnson's 'situational couple violence', they also featured control motivations. Furthermore, although ostensibly similar to 
Johnson's 'mutual violent control' due to the high levels of aggression and endorsement of control theory motivations, the 'Heterogenous' class was not gendered and was also high on retaliatory motivations.

\section{Hypothesis 3: Control and Gender}

The results do not support our hypothesis regarding a stronger association between control motivations and IPA for men, than for women, as not only were patterns of control motivations were not evident in the CATPCA, but women had higher scores on both the 'Eclectic Aggression' and 'Direct Psychological Aggression Components'. There was no significant gender difference for the 'Monitoring Acts Component'. Similarly, gender did not have an effect on the latent class analysis solution, nor was there a significant difference between the four classes on gender. Thus, neither the CATPCA nor the latent class analysis provided evidence that would support our hypothesis or Johnson's typology (1995; 2006).

\section{Strengths, Limitations, and Future Directions}

The patterns of motivations endorsed by the 'Heterogenous' and 'Homogenous' classes could be attributed to method bias, although efforts to ameliorate this during survey design were undertaken. These included: differential response formats for the behavior, motivation, and social desirability questions; the use of a number format to reduce ambiguity (e.g., 3-5 times); the piloting of the survey; the randomization of behavioral items so that more severe aggression items were balanced with more socially acceptable ones (see Podsakoff et al., 2012). The 'Homogenous' classs' patterns of responding could alternatively be explained by social desirability response bias. This class had the highest mean scores on both the impression management and self-deceptive enhancement subscales, although their mean scores were not significantly different from those of the 'Reactive Violence' class.

Although method bias is a possible explanation for the lack of discrimination in motivation for the 'Heterogenous' and 'Homogenous' classes, their patterns of responding 
could alternatively be seen as a lack of awareness and/or the ability to differentiate between motivations owing to the high levels of critical reflection and recall required by this task. If this is indeed the case, it suggests the way in which motivations are currently measured in empirical research may need to be revised to account for individuals' cognitive biases.

Some features of this study limit the conclusions that can be drawn from the results, the first of which is the use of a cross-sectional, survey design. Motivations are complex phenomena, which do not necessarily have a linear relationship with the subsequent aggressive act and can change over time (Ireland, 2009). Future research could build on this study by using latent transitional analysis to investigate how and why motivations develop over time, both of which are important to understand for assessment and treatment.

A second limitation of the study was the need to reduce the number of behavioral items. One-third of the lowest frequency items (by \%) were removed from the Eclectic Aggression, Direct Psychological Aggression, and Monitoring Acts factors to simplify the model and ensure that it would run. This, therefore, reduced the variability of behavior and removed some of the more severe items, which were less frequently endorsed, from the analyses. However, the analyses did include items that would be considered as severely aggressive such as 'I choked, strangled, or suffocated my partner' and 'I threatened my partner with a weapon'.

A third limitation is the use of an MTurk sample. These samples are not considered to be representative of the United States population, in that they consist of disproportionately larger numbers of White Americans and lower numbers of Latinx and Black Americans. However, MTurk samples are considered to be more representative than student samples (Stewart et al., 2017). MTurk also facilitates the gathering of large samples (Stewart et al.), something that is a strength of this study. Indeed, a recent systematic review of physical and psychological motivations for IPA found $78 \%$ of studies in the review were underpowered (had $80 \%$ or greater power to detect a medium-sized effect of $r=.20$; Chapter 3 ). A further strength 
of this study was the examination of motivations for specific aggressive acts, as opposed to asking about motivations for physical or psychological aggression generally. This design enabled us to see the considerable overlap of motivations for a diverse range of aggressive relationship behaviors.

\section{Conclusion}

This research builds on the work of Dempsey et al. (2020) to further show that a specific set of behavioral acts characterized by control motivations do not exist. Rather, results show that individuals endorse a wide range of motivations for the perpetration of different types of IPA (Eclectic Aggression; Direct Psychological Aggression; Monitoring Acts). This study therefore further questions the validity of tools that claim to measure 'controlling behaviors' from the perpetration of behavioral acts alone. Research should therefore consider alternative ways to measure control than the typically used controlling behavior scales.

In addition, no evidence was found to show that control motivations were associated with gender or the frequency of IPA behaviors used. These findings have implications for Johnson's typology $(1995 ; 2006)$, which differentiates between types of IPA on the basis that control motivated IPA will be gendered and result in a greater frequency and severity of aggression ('intimate terrorism') and that gender-inclusive 'situational couple violence' occurs in the absence of control. This study adds to the growing number of publications that have failed to find evidence of the 'situational couple violence' and 'intimate terrorism' categories described by Johnson (e.g., Bates et al., 2014; Hines \& Douglas, 2019; Straus \& Gozjolko, 2016). In particular, results found that the majority of high frequency controlling IPA is not gendered and that low-level violence featured control motivations. Research should further explore the nature of reciprocal IPA from a gender-inclusive perspective and motivations outside of control. 
Finally, the finding that controlling motivations were not more strongly associated with IPA than general aggression has implications for the design of treatment programs. The treatment for IPA has been heavily influenced by the gendered perspective in western countries for over 40 years (Hamel, 2007). The results of this study, therefore, undermine the validity of programs that primarily address control. The heterogeneity and idiosyncrasy of motivations in this study demonstrate how important the inclusion of alternative motivations in treatment design and implementation is if we are to effectively address IPA (Dixon \& Wride, 2020). Furthermore, without an emphasis on control as a primary motivation for IPA, the rationale for regarding IPA as a distinct subtype of aggression, warranting specialized treatment, is also debatable. 


\section{Chapter 5: EXAMINING COERCIVE CONTROL AS AN OUTCOME OF INTIMATE PARTNER AGGRESSION}

The intimate partner aggression (IPA) literature has long proposed that aggression characterized by coercive control is the most pervasive, serious, and gendered form of partner aggression (e.g., Pence \& Paymar, 1993), and is “conceptually distinct from episodic physical assault" (Smith, Tessaro, \& Earp, 1995, p. 275). Coercive control has therefore become a key feature in explaining men's aggression toward female intimate partners and has been defined as a pattern of aggression, intimidation, isolation, and entrapment, which both results from and perpetuates gender inequality (Stark, 2010).

In an attempt to reconcile data that suggested the existence of different forms of IPA, Johnson (1995) described two types of IPA characterized by the presence or absence of coercive control. 'Patriarchal terrorism' ('intimate terrorism'; 2006) is proposed to be "a product of patriarchal traditions of men's right to control their women" (1995, p. 284), characterized by coercive control and unidirectional, male to female aggression that is both frequent and severe. 'Situational couple violence' is defined by the absence of control and the presence of infrequent, bi-directional aggression of low severity. The presence of coercive control in relationships has been empirically shown to increase the frequency with which IPA is experienced (see Bates \& Graham-Kevan, 2016), the negative impact of IPA on victims (see Anderson, 2008), and the risk of intimate partner homicide (see Campbell et al., 2000). Such evidence has impacted the development of IPA legislation in numerous jurisdictions across the world (see Hester et al., 2017; e.g., Domestic Violence Act [Ireland] s 39; Scottish Government, 2015; Serious Crimes Act 2015 [UK], s 76). Although Johnson's test of his typology (2006) found 97\% of 'intimate terrorists' were men and that 'situational couple violence' was characterized by gender symmetry (56\% men; 44\% women), his methodology has been heavily criticized. Specifically, criticism has focused on its reliance on non-random female self-reports and for not including 
men who had experienced victimization from women, thus overemphasizing the female victim experience (see Dixon \& Graham Kevan, 2020). Indeed, other research has since demonstrated higher rates of 'intimate terrorism' in women, suggesting it is not a predominantly gendered phenomenon (Bates et al., 2014). Johnson revised his typology to account for the role of female perpetration by re-labeling 'patriarchal terrorism' as 'intimate terrorism' and later as 'coercive controlling violence', in recognition "that not all coercive control was rooted in patriarchal structures and attitudes, nor perpetrated exclusively by men” (Kelly \& Johnson, 2008, p. 479). However, Johnson still maintains rates of 'coercive controlling violence' are predominantly gender asymmetric (Kelly \& Johnson). Other researchers, such as Stark (2007), consider gender a defining feature of coercive control, experienced exclusively by women. Stark views coercive control as men's response to women's increasing social equality and its use as the need to subvert this process on a personal level, within the context of their intimate relationships.

The lack of consistent empirical evidence supporting a gendered conceptualization of coercive control has resulted in theoretical divergence and a lack of consensus about how it should be conceptualized (Hamberger et al., 2017). Despite this, the proposed serious nature of coercive and controlling IPA has resulted in attempts to measure this phenomenon to identify it and prevent harm (Kelly \& Johnson, 2008). The most frequent method of measurement is through the use of 'controlling behaviors' (Hamberger et al.; e.g., Checklist of Controlling Behaviors: Lehmann et al., 2012; Psychological Maltreatment of Women Inventory: Tolman, 1989). Such measures are comprised of non-physical acts (e.g., restricting access to family/ friends, money) that are considered to be a distinct subtype of IPA uniquely motivated by the desire to control an intimate partner. However, Dempsey et al. (2020) questioned the validity of 'controlling behaviors' as a unique subtype of IPA and whether they could be used to proxy the use of control and/ or coercive control in relationships. Exploratory and confirmatory factor analyses found that items derived from (sub)scales developed to measure control were not 
statistically distinct from items measuring other forms of IPA (e.g., physical, sexual, psychological aggression; Dempsey et al.) and were therefore not a good proxy for identifying coercive controlling IPA. In addition, the lack of context inherent in discrete acts of aggression has led researchers investigating coercive control to consider the outcomes or consequences of IPA as an alternative to behavioral measures (Hamberger et al.). Qualitative research with female victims has suggested the distinctiveness of coercive control is attributable to the adverse outcomes experienced, such as disempowerment, entrapment, and fear (Smith, Tessaro, \& Earp, 1995). Therefore, the operationalization of coercive control as an outcome and the examination of the meaning individuals attribute to their experiences of IPA, is perhaps a more theoretically sound method to investigate this construct, than behavioral measures.

This study explores whether coercive control can be best identified by measuring the outcomes reported by people who have experienced IPA victimization. Popular definitions of coercive control that have lent support to the gendered theory of IPA have described it as feelings of entrapment, isolation, and a loss of autonomy, caused by patterns of abuse (see Stark, 2007). This study, therefore, operationalized coercive control by measuring the presence of such outcomes and testing if the gendered assumptions of the commonly accepted conceptualization were supported using this measurement. By adopting an open investigation into the effects of gender we are adopting a gender-inclusive approach and considering diversity issues. Three hypotheses were tested:

H1: If patterns of coercive control outcomes are distinct from other outcomes associated with IPA, then these outcomes will be more strongly associated with experiencing IPA (Eclectic Aggression, Direct Psychological Aggression, or Monitoring Acts) than other outcomes associated with IPA.

$\mathrm{H} 2$ : If profiles characterized by coercive control outcomes are distinct from profiles characterized by other outcomes associated with IPA, then these profiles will be associated with 
experiencing more frequent IPA (Eclectic Aggression, Direct Psychological Aggression, and Monitoring) than the other profiles.

H3: If patterns of coercive control outcomes are distinct from other outcomes associated with IPA, then these outcomes will be more strongly associated with experiencing IPA (Eclectic Aggression, Direct Psychological Aggression, and Monitoring Acts) for women than men.

\section{Method}

\section{Participants and Procedure}

Participants were recruited from Amazon's Mechanical Turk (MTurk), a crowdsourcing website, advertised to users from the United States and Canada as a "research survey about aggression in your current or a recent relationship" between 6th and 9th December 2019. Participants received \$1.20 (USD). Eligible individuals were English speaking, at least 18 years old, and had been in an intimate/dating relationship for at least one month in the last 12 months. The current data were collected for two studies to examine individuals' use and experiences of aggression. The method for this study was pre-registered on the Open Science Framework in December 2019 (https://osf.io/jdnw9/). The study received approval from the Victoria University School of Psychology Human Ethics Committee. The survey was started by 1915 people. The final dataset excluded people who completed less than $90 \%$ of the survey $(n=105)$, completed the survey unreasonably quickly ( $n=16$; under 508 seconds), or had not experienced any form of aggression $(n=548)$. The current study focused on testing individuals' experiences of aggression (66 people who exclusively reported perpetrating IPA were removed) and testing claims from the gendered perspective (six people who identified as non-binary were removed).

The final sample comprised 1174 individuals (55\% women, $45 \%$ men; $M_{\text {age }}=36.12$, $S D=10.13)$. The ethnic composition of the sample was: $65.9 \%(n=774)$ European American, 12.6\% $(n=148)$ African American, 5.5\% $(n=64)$ Asian American, 6.3\% $(n=74)$ Latinx, $0.9 \%$ 
$(n=10)$ Native American, and 2.8\% $(n=33)$ Other. Six percent $(n=71)$ of the sample omitted this question, while $10.2 \%$ identified with more than one ethnicity $(n=120)$. The sample predominantly identified as heterosexual $(85.3 \%, n=1001)$, with $11.3 \%$ identifying as bisexual $(n=133)$. The remaining participants identified as either gay $(1.4 \%, n=16))$, lesbian $(0.6 \%, n$ $=7)$, pansexual $(0.9 \%, n=10)$, asexual $(0.3 \%, n=4)$, or 'other' $(0.3 \%, n=3)$.

\section{Materials}

Experienced IPA. Participants completed thirty questions investigating their experiences of IPA, which comprised three factors identified by Dempsey et al. (2020): Eclectic Aggression (12 items; $\alpha=.93$; e.g., "My partner hit or punched me"), Direct Psychological Aggression (9 items; $\alpha=.88$; e.g., "My partner treated me like I was inferior to them") and Monitoring Acts (9 items; $\alpha=.88$; e.g., "My partner called or text messaged me constantly"). The frequency of experiences was assessed using a numerical response format (e.g., Never, 12 times, 3-5 times, 6-10 times, more than 10 times), avoiding the ambiguity of text options (see DeVellis, 2012). Participants were instructed to answer each question concerning their current or most recent relationship and disregard any acts that occurred within the context of consensual sexual activity.

Outcomes of IPA. For each aggressive behavioral act participants indicated they had experienced, a randomized list of possible outcomes for this aggressive behavior appeared (also see Straus et al., 1996). We generated a list of possible experienced outcomes from research from the gendered perspective (e.g., Stark, 2006, 2007; Women's Experience with Battering Scale: Smith, Earp, \& DeVillis, 1995) and the general IPA literature (e.g., Motivations and Effects Questionnaire: Follingstad et al., 1991; the Intimate Violence and Traumatic Affects Scale: Troisi, 2018; Hamberger \& Guse, 2005).

Eleven items indexed experienced "coercive control", including "I felt isolated from friends and family", "I felt afraid for my physical safety", and "I felt intimidated". Nine other 
items were derived from the general IPA literature, including "I felt angry" and "I felt emotionally hurt". For each act of violence that participants experienced, they were given the option to select up to three outcomes to describe how that act affected them (Binary scored: 1 $=$ Experienced $; 0=$ Not Experienced $=0)$.

Social desirability bias. To assess potential social desirability in participants' responses to IPA questions (see Arias \& Beach, 1987), participants completed 16 items of the Balanced Inventory of Desirable Responding Short Form $(1=$ Not true, $7=$ True; Paulhus, 1991). The short-form scale has equivalent structure, validity, and reliability to the original 40-item BIDR scale (Hart et al., 2015; Paulhus, 1991). Items were averaged such that higher scores indicated more socially desirable responding (Cronbach's $\alpha=.71$ ).

\section{Results}

\section{Power Analysis}

A Markov Chain Monte Carlo simulation with a Metropolis-Hastings algorithm was conducted a priori. The results of this analysis proved to be inconclusive, and a heuristic recommended by Kline (2005), suggesting a minimum of 600 participants was required. In the current sample, 1174 participants reported experiencing at least one act of IPA (number of IPA acts experienced in the past year $M=12.24, S D=8.20$ ), indicating sufficient power for the following analyses.

\section{Descriptive statistics}

Figure 5.1 shows the percentage of the sample by the frequency with which they experienced Eclectic Aggression, Direct Psychological Aggression, and Monitoring Acts. The total number of experienced aggressive acts was moderately positively skewed, so we tested for gender differences with a Mann-Whitney U test. Results did not identify significant 
differences between men's $(M d n=11.00)$ and women's experienced aggression $(M d n=10.00)$ $U\left(N_{\text {women }}=646, N_{\text {men }}=528\right)=165615.00, z=-0.85, p=.393$.

\section{Figure 5.1}

Frequency of experienced IPA by type

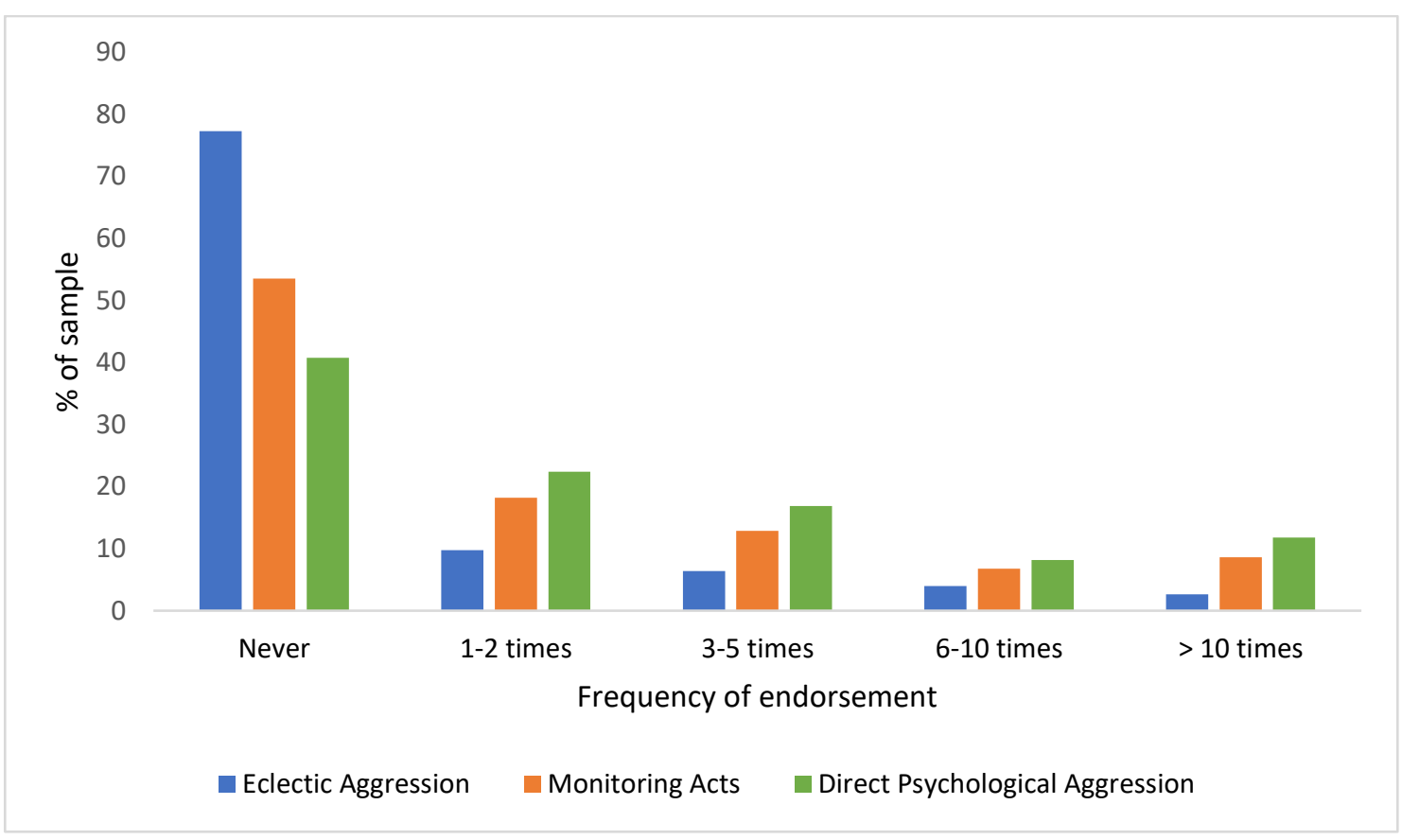




\section{Table 5.1}

Frequencies of outcomes for IPA endorsed (total and by gender)

\begin{tabular}{|c|c|c|c|c|c|c|c|}
\hline & Outoom & & & & & & \\
\hline & Uutcome & \# & $\%$ & \# & $\%$ & \# & $\%$ \\
\hline$* 1$ & I felt intimidated & 230 & 3.44 & 347 & 4.52 & 577 & 4.02 \\
\hline 2 & I felt ashamed & 280 & 4.18 & 343 & 4.47 & 623 & 4.34 \\
\hline$* 3$ & I felt afraid for my physical safety & 169 & 2.53 & 190 & 2.47 & 359 & 2.50 \\
\hline$* 4$ & I felt isolated from friends and family & 199 & 2.97 & 226 & 2.94 & 425 & 2.96 \\
\hline 5 & I felt frustrated & 422 & 6.31 & 569 & 7.41 & 991 & 6.90 \\
\hline * 6 & I felt controlled & 355 & 5.31 & 428 & 5.57 & 783 & 5.45 \\
\hline 7 & It didn't affect me in any way & 206 & 3.08 & 181 & 2.36 & 387 & 2.69 \\
\hline 8 & I found it funny & 229 & 3.42 & 168 & 2.19 & 397 & 2.76 \\
\hline * 9 & I felt trapped & 249 & 3.72 & 325 & 4.23 & 574 & 3.99 \\
\hline 10 & I felt angry & 359 & 5.37 & 492 & 6.41 & 851 & 5.92 \\
\hline * 11 & I felt like I wasn't able to make decisions for myself & 109 & 1.63 & 157 & 2.04 & 266 & 1.85 \\
\hline 12 & I felt guilty & 289 & 4.32 & 284 & 3.70 & 573 & 3.99 \\
\hline * 13 & I felt like I wasn't able to do what I wanted to do & 247 & 3.69 & 314 & 4.09 & 561 & 3.90 \\
\hline * 14 & I felt afraid I was going to make them angry & 227 & 3.39 & 280 & 3.65 & 507 & 3.53 \\
\hline 15 & I felt sad & 340 & 5.08 & 457 & 5.95 & 797 & 5.55 \\
\hline * 16 & I felt afraid I was going to set them off shouting or criticizing me & 213 & 3.18 & 224 & 2.92 & 437 & 3.04 \\
\hline 17 & I felt sexually aroused & 139 & 2.08 & 72 & .94 & 211 & 1.47 \\
\hline * 18 & I felt helpless & 283 & 4.23 & 366 & 4.77 & 649 & 4.52 \\
\hline * 19 & I felt dependent on my partner & 155 & 2.32 & 190 & 2.47 & 345 & 2.40 \\
\hline 20 & I felt emotionally hurt & 305 & 4.56 & 472 & 6.15 & 777 & 5.41 \\
\hline
\end{tabular}

Note. Percentages do not add up to $100 \%$ because participants could endorse up to three outcomes per act; ${ }^{*}$ Outcomes derived from the gendered perspective literature 


\section{Table 5.2}

Gender comparison of endorsement of outcomes for IPA

\begin{tabular}{|c|c|c|c|c|c|c|c|}
\hline \multirow[b]{2}{*}{$*$} & & Outcomes & \multirow{2}{*}{$\begin{array}{c}\begin{array}{c}\text { Men } \\
M \text { Rank }\end{array} \\
553.72\end{array}$} & $\begin{array}{l}\text { Women } \\
M \text { Rank }\end{array}$ & \multirow{2}{*}{$\begin{array}{c}z \\
3.33\end{array}$} & \multirow{2}{*}{$\frac{p}{<.001}$} & \multirow{2}{*}{$\frac{d}{.18}$} \\
\hline & 1 & I felt intimidated & & 615.11 & & & \\
\hline & 2 & I felt ashamed & 593.97 & 582.21 & -.63 & .530 & .03 \\
\hline * & 3 & I felt afraid for my physical safety & 600.95 & 576.50 & -1.51 & .132 & .07 \\
\hline$*$ & 4 & I felt isolated from friends and family & 604.64 & 573.49 & -1.82 & .068 & .09 \\
\hline & 5 & I felt frustrated & 570.95 & 601.03 & 1.52 & .128 & .09 \\
\hline * & 6 & I felt controlled & 584.49 & 589.96 & .28 & .779 & .02 \\
\hline & 7 & It didn't affect me in any way & 628.73 & 553.80 & -4.52 & $<.001$ & .22 \\
\hline & 8 & I found it funny & 651.54 & 535.16 & -6.96 & $<.001$ & .35 \\
\hline * & 9 & I felt trapped & 572.52 & 599.75 & 1.48 & .140 & .08 \\
\hline & 10 & I felt angry & 545.59 & 621.76 & 3.89 & $<.001$ & .23 \\
\hline * & 11 & I felt like I wasn't able to make decisions for myself & 574.88 & 597.82 & 1.58 & .115 & .07 \\
\hline & 12 & I felt guilty & 624.31 & 557.41 & -3.64 & $<.001$ & .20 \\
\hline * & 13 & I felt like I wasn't able to do what I wanted to do & 586.05 & 588.68 & .14 & .886 & .01 \\
\hline * & 14 & I felt afraid I was going to make them angry & 593.51 & 582.59 & -.61 & .542 & .03 \\
\hline & 15 & I felt sad & 561.79 & 608.52 & 2.41 & .016 & .14 \\
\hline * & 16 & I felt afraid I was going to set them off shouting or criticizing me & 610.48 & 568.72 & -2.43 & .015 & .12 \\
\hline & 17 & I felt sexually aroused & 637.01 & 547.04 & -6.76 & $<.001$ & .27 \\
\hline * & 18 & I felt helpless & 570.11 & 601.72 & 1.67 & .094 & .09 \\
\hline * & 19 & I felt dependent on my partner & 588.63 & 586.58 & -.13 & .898 & .01 \\
\hline & 20 & I felt emotionally hurt & 515.64 & 646.23 & 6.73 & $<.001$ & .39 \\
\hline
\end{tabular}

Note. $M$ Rank = mean rank; $z$ = standardized test statistic; $d=$ standardized effect size; $*$ Outcomes derived from the gendered perspective literature 
Experienced outcomes of IPA. Table 5.1 shows the number of times each of the outcomes was endorsed, by the entire sample, and by gender. "I felt frustrated" (991 times), "I felt angry" (851 times), and "I felt sad" (797 times) were the most frequently endorsed outcomes for IPA in the study. Due to the asymmetric distribution of the data, Mann-Whitney U tests were conducted to investigate gender differences in outcomes (see Table 5.2). The largest effect sizes occurred in which females reported moderately higher levels than males for the outcome "I felt emotionally hurt", and males reported moderately higher levels than females for the outcomes "I found it funny", and "I felt sexually aroused". Nine of the 20 outcomes showed statistically significant gender differences, with effect sizes ranging from .39 to .12. Five estimates of the effect were in the male direction ("I found it funny"; "I felt sexually aroused"; "It didn't affect me in any way"; "I felt guilty"; "I felt afraid I was going to set them off shouting or criticizing me") and four in the female direction ("I felt emotionally hurt"; "I felt angry"; "I felt intimidated"; "I felt sad").

\section{Categorical Principal Component Analysis (CATPCA)}

The data for this study was a sub-sample of that used in Chapter 4 of this thesis. As such, the same issues regarding the large number of nominal variables, low correlations between items, and low KMO score also affected this study, thereby making the exploratory factor analysis, which was initially planned, an inappropriate choice of analysis (refer to the results section of Chapter 4, p. 84 for more detail). ACATPCA in IBM SPSS Statistics for Windows (Version 26.0; IBM Corp., 2019a) was, therefore, performed to investigate whether patterns of coercive control outcomes were distinct from other outcomes associated with IPA, and if so, whether these outcomes have a stronger association with experiencing Eclectic Aggression, Direct Psychological Aggression, and Monitoring Acts than other outcomes associated with IPA. Thirty behavioral items comprising 'Eclectic Aggression' (12 items), 
'Direct Psychological Aggression' (Nine items), and 'Monitoring Acts' (Nine items) and 20 outcome items were included in this analysis (see Appendix KK for the full list of items).

We performed a spline nominal transformation using a first-degree polynomial function using a variable principal normalization, with balanced bootstrapping, using 1000 samples, and a Procrustes rotation, as recommended by Linting et al. (2007). The spline and number of degrees dictate the shape and smoothness of the transformation, increasing the amount of variance that can be extracted for each component by optimizing the relationships between variables (see Linting \& van der Kooij, 2012 for further information). The variable principal normalization similarly maximizes the associations between variables (Linting et al., 2007).

Scree plots for up to eight dimensions were examined and suggested the appropriate number of components to retain was three (see Figure 5.2).

\section{Figure 5.2.}

Scree plot from the categorical principal components analysis (CATPCA)

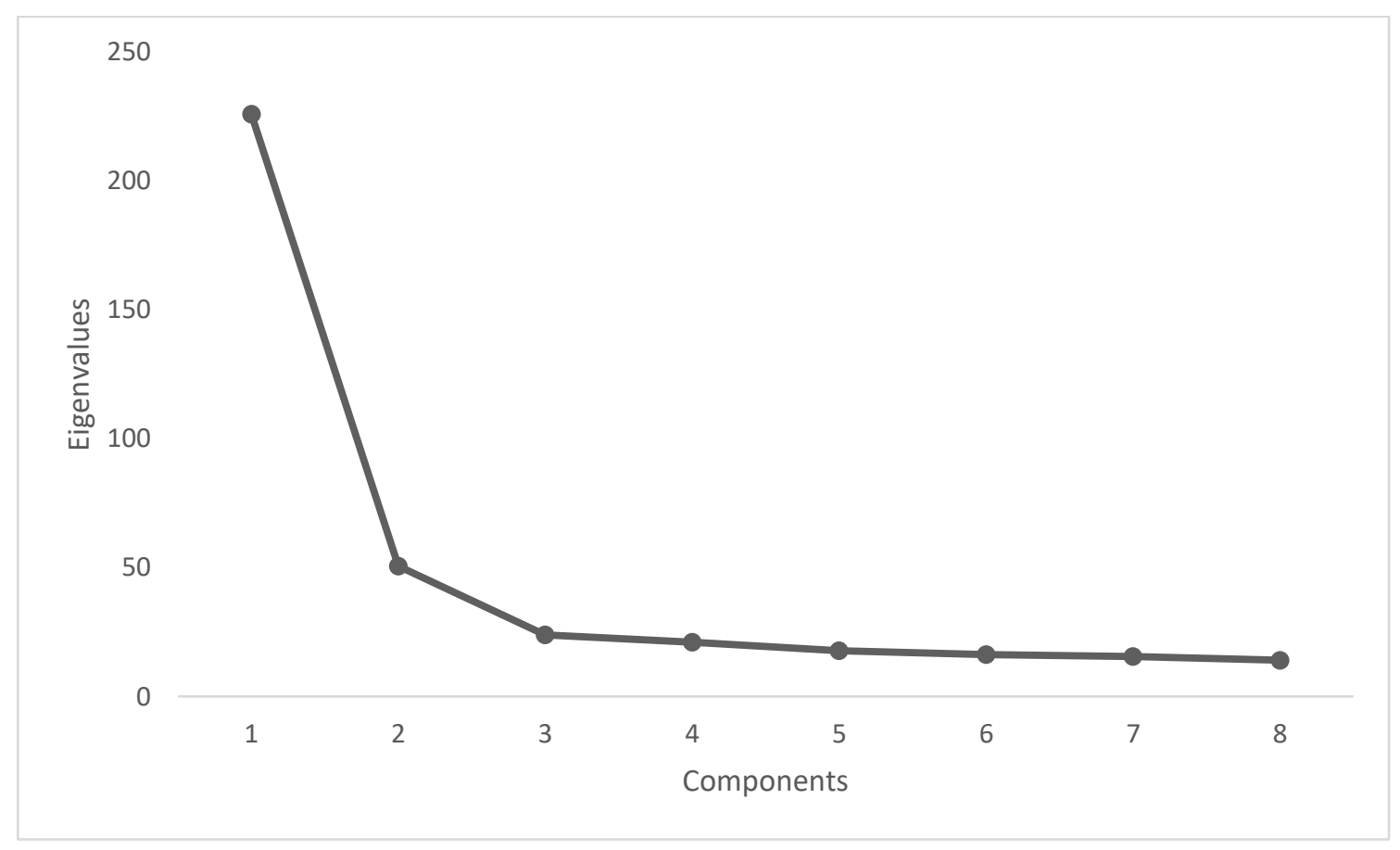


To avoid the subjectivity inherent with scree plots (Raîche et al., 2013), the 'Parallel Analysis and Other Non-Graphical Solutions to the Cattell Scree Test' package in R (Raîche, 2020) was used to objectively determine the number of retained components. We used three methods of estimation, which use eigenvalues, the amount of variance explained by each component (Linting et al., 2011), to calculate the number of components in the solution. The multiple regression procedure to determine the number of components/ factors (nMreg) and the Bartlett, Anderson, and Lawley procedures to determine the number of components/ factors (nBartlett) suggested four-component solutions, while the Cattell-Nelson-Gorsuch CNG indices (nCng) suggested a three-component solution. We selected the consensus result of the four-component solution.

We selected variables according to the significance of the bootstrapped item loadings by examining the lower and upper confidence intervals, and, thus excluded 157 non-significant items (see Appendix LL; Linting \& van der Kooij, 2012). The excluded items were related to outcomes from the gendered and general IPA literature (outcomes 11-20) for a variety of behaviors from the Eclectic Aggression (six items), Monitoring Acts (eight items), and Direct Psychological Aggression (six items) components.

The four-component solution was rotated using a Promax rotation and a Kaiser normalization. Items that cross-loaded on two or more components with a difference of less than .20 were removed (128 items; Appendix LL; see Howard, 2016). All variables had communalities of >.50 (see Comrey, 1973), and minimum loadings of .40 (see Stevens, 1986). See Appendix MMfor the full list of items, Eigenvalues, and percentage of variance explained by the components.

Component 1: Eclectic Aggression. Component one contained 186 items, with an Eigenvalue of 127.97 , accounting for $43.23 \%$ of the variance in the solution. This component contained items relating to physical aggression (e.g., slapping; punching), threats: to physically 
harm, to withhold money, and to reputation, and some psychologically aggressive items (e.g., denying incidents of abuse). These items were exclusively derived from the 'Eclectic Aggression' factor found in Dempsey et al. (2020), and this component was consequently labeled as the 'Eclectic Aggression component'. Table 5.3 shows a simplified representation of the strength and direction of the item loadings for each outcome (see Appendix MM for the pattern matrix).

Component 2: Monitoring Acts. This component comprised 53 items, with an Eigenvalue of 79.64 , explaining $26.9 \%$ of the variance. The items in this component were consistent with the items from the 'Monitoring Acts' factor (e.g., "My partner accused me of being unfaithful or flirting with others"; "My partner called or text messaged me constantly"; see Dempsey et al., 2020) and this component was subsequently labeled the 'Monitoring Acts component'.

Component 3: Direct Psychological Aggression. This component was labeled the 'Direct Psychological Aggression component' due to the similarities between this and the "Direct Psychological Aggression" factor found in the previous and aforementioned research (Dempsey et al., 2020). This component contained 57 items, with an Eigenvalue of 59.94, which explained $20.25 \%$ of the variance. Behaviors in this component included "My partner deliberately ignored me", "My partner treated me like I was inferior to them" and "My partner changed the subject on purpose when I was trying to discuss a problem".

Component 4. The fourth component was comprised of items relating to only one behavior ("My partner screamed or yelled at me"). This is considered to be a psychologically aggressive behavior and should theoretically have been included in Component 3, however, this item was the most frequently endorsed behavior in the study (71\%) and it is possible it formed its own component based on the high response rate rather than the item content. As the interpretability of the solution is considered an important criterion in determining the number 
of components to retain (Linting et al., 2011), the items relating to this behavior were excluded and the analysis run as a three-component solution. The items in each of the three original components were identical in the resulting solution.

Component solutions by gender. To ascertain whether the component solutions varied by gender, this was entered as a supplementary variable in the analysis. The category points biplot suggested some distinction between the centroid coordinates along all three dimensions. Welch two sample $t$-tests were conducted to test gender differences in mean component scores. These indicated men $(M=.12, S D=1.05)$ had significantly higher 'Monitoring Acts component' scores than women $(M=-.10, S D=.95) t(1079.30)=14.08, p<.001, d=.22$, although the difference would be described as small (Cohen, 1969). A medium effect size was found for the difference between genders on 'Eclectic Aggression component' scores $t(947.99)$ $=30.88, p<.001, d=.33$ with women $(M=.15, S D=.83)$ experiencing significantly more Eclectic Aggression than men $(M=-.18, S D=1.15)$. However, there was no significant difference between men's $(M=.03, S D=1.02)$ and women's $(M=-.03, S D=.98)$ 'Direct Psychological Aggression component' scores $t(1113.78)=1.12, p<.05, d=.06$.

We hypothesized that if distinct patterns of coercive control were evident, they would be more strongly associated with female's experiences of types of IPA. However, we did not find evidence of a pattern of control experiences, for which support for Hypothesis 3 is contingent. Rather, the patterns of outcomes formed in relation to the type of aggression experienced, for which significant gender differences were found for Eclectic Aggression and Monitoring Acts.

Summary. It was hypothesized that if patterns of coercive control outcomes are distinct from other outcomes associated with IPA, then these outcomes will be more strongly associated with experiencing Eclectic Aggression, Direct Psychological Aggression, or Monitoring Acts than other outcomes associated with IPA (Hypothesis 1). However, no such patterns were 
evident, and the results instead demonstrated, not only heterogeneity for a variety of IPA acts, but strong and positive associations between these outcomes and a diverse range of aggressive relationship behaviors (see Table 5.3). Furthermore, the outcomes that most frequently failed to load onto the components were derived from the gendered perspective literature (Outcomes 11-20 for the Eclectic Aggression and 12-20 for the Monitoring Acts components). 


\section{Table 5.3}

Outcomes endorsed for perpetrated acts by type

\begin{tabular}{|c|c|c|c|c|c|c|c|c|c|c|c|c|c|c|c|c|c|c|c|c|}
\hline \multirow{2}{*}{ Behavioral Act } & \multicolumn{20}{|c|}{ Outcome } \\
\hline & 1 & 2 & 3 & 4 & 5 & 6 & 7 & 8 & 9 & 10 & 11 & 12 & 13 & 14 & 15 & 16 & 17 & 18 & 19 & 20 \\
\hline \multicolumn{21}{|l|}{ Eclectic Aggression Component } \\
\hline My partner slapped me & + & + & + & + & + & + & + & + & + & + & $\mathbf{X}$ & $\mathbf{X}$ & $\mathbf{X}$ & $\mathbf{X}$ & $\mathbf{X}$ & $\mathbf{X}$ & $\mathbf{X}$ & $\mathbf{X}$ & $\mathbf{X}$ & - \\
\hline My partner hit or punched me & + & + & + & + & + & + & + & + & + & + & - & + & + & + & + & + & + & + & + & + \\
\hline $\begin{array}{l}\text { My partner tried to turn my family, friends, and children } \\
\text { against me }\end{array}$ & + & + & + & + & + & + & + & + & + & + & - & + & + & + & + & + & + & + & + & - \\
\hline My partner kept me from leaving the house & + & + & + & + & + & + & + & + & + & - & + & + & + & + & + & + & + & + & + & + \\
\hline My partner slammed or held me against a wall & + & + & + & + & + & + & + & + & + & - & + & + & + & + & + & + & + & + & + & + \\
\hline My partner threatened me with a weapon & + & + & + & + & + & + & + & + & 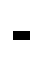 & - & $\mathbf{X}$ & $\mathbf{X}$ & $\mathbf{X}$ & $\mathbf{X}$ & $\mathbf{X}$ & $\mathbf{X}$ & $\mathbf{X}$ & $\mathbf{X}$ & $\mathbf{X}$ & $\mathbf{X}$ \\
\hline My partner denied incidents of abuse & + & + & + & + & + & + & + & + & $\mathbf{-}$ & + & $\mathbf{X}$ & + & + & + & + & $\mathbf{X}$ & + & + & + & - \\
\hline My partner pressured me to have sex when I said 'No' & + & + & + & + & + & + & + & + & - & + & - & - & + & + & $\mathbf{X}$ & $\mathbf{X}$ & + & + & + & $\mathbf{X}$ \\
\hline My partner threatened to withhold money from me & + & + & + & + & + & + & + & + & - & - & $\mathbf{X}$ & $\mathbf{X}$ & $\mathbf{X}$ & $\mathbf{X}$ & $\mathbf{X}$ & $\mathbf{X}$ & $\mathbf{X}$ & $\mathbf{X}$ & $\mathbf{X}$ & $\mathbf{X}$ \\
\hline $\begin{array}{l}\text { My partner deliberately broke or destroyed something that } \\
\text { was important to me }\end{array}$ & + & + & + & + & + & + & + & + & + & + & - & + & + & + & + & + & + & + & + & + \\
\hline $\begin{array}{l}\text { My partner threatened to disclose damaging or } \\
\text { embarrassing information }\end{array}$ & + & + & + & + & + & + & + & + & - & - & + & + & + & + & + & + & $\mathbf{X}$ & + & $\mathbf{X}$ & + \\
\hline
\end{tabular}

Note. $+=$ positive item loading $\geq .7 ;-=$ negative item loading $\geq .7 ;+=$ positive item loading $\leq .6 ;-=$ negative item loading $\leq .6 ; \mathbf{X}=$ non-loading items

1 = I felt intimidated; 2 = I felt ashamed; $3=\mid$ felt afraid for my physical safety; $4=\mid$ felt isolated from friends and family; $5=\mid$ felt frustrated; $6=\mid$ felt controlled; $7=\mid \mathrm{t}$ didn't affect me in any way; 8 = I found it funny; $9=$ I felt trapped; 10 = I felt angry; $11=$ I felt like I wasn't able to make decisions for myself; $12=\mid$ felt guilty; $13=\mid$ felt like I wasn't able to do what I wanted to do; 14 = I felt afraid I was going to make them angry; 15 = I felt sad; $16=$ I felt afraid I was going to set them off shouting or criticizing me; $17=$ I felt sexually aroused; $18=$ I felt helpless; $19=$ I felt dependent on my partner; $20=$ I felt emotionally hurt 
Table 5.3 cont.

\begin{tabular}{|c|c|c|c|c|c|c|c|c|c|c|c|c|c|c|c|c|c|c|c|c|}
\hline \multirow{2}{*}{ Behavioral Act } & \multicolumn{20}{|c|}{ Outcome } \\
\hline & 1 & 2 & 3 & 4 & 5 & 6 & 7 & 8 & 9 & 10 & 11 & 12 & 13 & 14 & 15 & 16 & 17 & 18 & 19 & 20 \\
\hline \multicolumn{21}{|l|}{ Monitoring Acts Component } \\
\hline $\begin{array}{l}\text { My partner insisted on knowing where I went and who I } \\
\text { spoke to when we were not together }\end{array}$ & + & + & + & + & + & + & - & + & + & + & + & + & + & + & + & + & + & + & + & + \\
\hline $\begin{array}{l}\text { My partner accused me of being unfaithful or flirting with } \\
\text { others }\end{array}$ & + & + & + & + & + & + & - & + & + & + & - & $\mathbf{X}$ & $\mathbf{X}$ & $\mathbf{X}$ & + & $\mathbf{X}$ & $\mathbf{X}$ & $\mathbf{X}$ & $\mathbf{X}$ & + \\
\hline My partner checked my social network page(s) & + & + & + & + & + & + & - & + & + & + & - & $\mathbf{X}$ & $\mathbf{X}$ & $\mathbf{X}$ & $\mathbf{X}$ & $\mathbf{X}$ & $\mathbf{X}$ & $\mathbf{X}$ & $\mathbf{X}$ & $\mathbf{X}$ \\
\hline My partner called or text messaged me constantly & + & + & $\mathbf{X}$ & + & + & + & - & + & + & + & $\mathbf{X}$ & $\mathbf{X}$ & $\mathbf{X}$ & $\mathbf{X}$ & $\mathbf{X}$ & $\mathbf{X}$ & $\mathbf{X}$ & $\mathbf{X}$ & $\mathbf{X}$ & $\mathbf{X}$ \\
\hline \multicolumn{21}{|l|}{ Direct Psychological Aggression Component } \\
\hline My partner deliberately ignored me & + & + & + & + & + & + & + & - & + & + & + & + & + & + & + & + & - & + & + & + \\
\hline My partner treated me like I was inferior to them & + & + & + & + & + & + & + & + & + & + & + & + & + & + & + & + & + & + & + & + \\
\hline $\begin{array}{l}\text { My partner changed the subject on purpose when I was } \\
\text { trying to discuss a problem }\end{array}$ & + & + & + & + & + & + & - & - & + & + & + & $\mathbf{X}$ & + & + & + & + & $\mathbf{X}$ & + & $\mathbf{X}$ & + \\
\hline
\end{tabular}

Note. $+=$ positive item loading $\geq .7 ;-=$ negative item loading $\geq .7 ;+=$ positive item loading $\leq .6 ;-=$ negative item loading $\leq .6 ; \mathbf{X}=$ non-loading items

1 = I felt intimidated; 2 = I felt ashamed; 3 = I felt afraid for my physical safety; 4 = I felt isolated from friends and family; $5=\mid$ felt frustrated; 6 = I felt controlled; 7 = It didn't affect me in any way; $8=$ I found it funny; $9=$ I felt trapped; $10=$ I felt angry; $11=$ I felt like I wasn't able to make decisions for myself; $12=\mid$ felt guilty; $13=$ I felt like I wasn't able to do what I wanted to do; 14 = I felt afraid I was going to make them angry; 15 = I felt sad; $16=$ I felt afraid I was going to set them off shouting or criticizing me; 17 = I felt sexually aroused; $18=$ I felt helpless; $19=$ I felt dependent on my partner; $20=$ I felt emotionally hurt 


\section{Latent Class Analysis on Outcomes of IPA}

Given that the item level analyses found no evidence for a unique pattern of coercive control or a unique link to IPA, we tested Hypothesis 1 in an alternative manner by considering whether the construct of coercive control existed as a typology at the person level. Specifically, we conducted a latent class analysis (LCA) on the experienced outcomes of IPA using Mplus (Version 8; Muthén \& Muthén, 1998-2017a). This analysis also allowed us to investigate what proportion of individuals who fit a controlled typology (if one existed) were women (Hypothesis 3). Following this, we tested Hypothesis 2 using ANOVAs to compare the typologies on the frequency of their experiences of Eclectic Aggression, Direct Psychological Aggression, and Monitoring Acts. The same 20 outcome and 30 behavioral variables as were used in the CATPCA were used in the LCA (see Appendix KK).

Model selection. Some researchers from the gendered perspective, such as Stark (2007), would suggest the appropriate number of classes to retain would be two, as dictated by men's and women's differential experiences of coercive control. We began with evaluating the fit statistics and interpretability of a two-class solution and then subsequently increased the number of classes until the results plateaued. Better model fit is indicated by lower Akaike Information Criterion (AIC) and Bayesian Information Criterion (BIC) values, higher entropy, and significant Bootstrap Likelihood Ratio (BLRT) and the Vuong-Lo-Mendell-Rubin (VLMRLRT; Lo et al., 2001) test values. We selected the four-class solution as the best fit for the data due to high entropy, superior AIC/ BIC values than the three-class solution, and significant BLRT and VLMR values (see Table 5.4). The five-class solution showed nonsignificant values on the BLRT and VLMR tests, indicating the estimated model would be a better fit for the data with one less class (Geiser, 2012). 


\section{Table 5.4}

Model fit for the difference class solutions of the Latent Class Analysis

\begin{tabular}{lccccc}
\hline Classes & AIC & BIC & Entropy & VLMRLRT & BLRT \\
\hline Two classes & 60801.39 & 61115.62 & .93 & 0.000 & 0.000 \\
Three classes & 582.70 .85 & 58726.99 & .93 & 0.000 & 0.000 \\
Four classes & 57249.90 & 57847.95 & .91 & 0.000 & 0.000 \\
Five classes & 56723.64 & 57463.59 & .93 & 0.060 & 0.000 \\
Six classes & 56214.35 & 57096.22 & .92 & 0.547 & 0.000 \\
\hline Note. AIC = Akaike Information Criterion; BIC = Bayesian Information Criterion; VLMR = Vuong-Lo-Mendell- \\
Rubin Likelihood Ratio Test; BLRT = Bootstrap Likelihood Ratio Test
\end{tabular}

Class solutions by gender. The item-response probabilities were constrained to be equal across classes to enable us to assess the possible effect of gender on the model. The model fit statistics from the freely estimated model (discussed above) were compared to those of the constrained model (see Appendix NN). An expected worsening in the fit statistics due to the parameter restrictions would indicate an effect of gender on the model. However, the constrained model had lower AIC and BIC values than those of the freely estimated model, indicating a superior fit for the data, and comparable entropy values $\left(G^{2}\right.$ and chi-square difference tests were not suitable due to the large sample size; see Lanza et al., 2013). Thus, we concluded that gender did not explain substantial variance in the class solutions, providing no support for Hypothesis 3.

Description of classes. Figure 5.3 illustrates the differences in mean scores of outcomes for the perpetration of IPA for the four-class solution. Each class has been labeled according to their patterns of reported outcomes of experiencing IPA. 


\section{Figure 5.3}

Four-class solution of outcomes for IPA

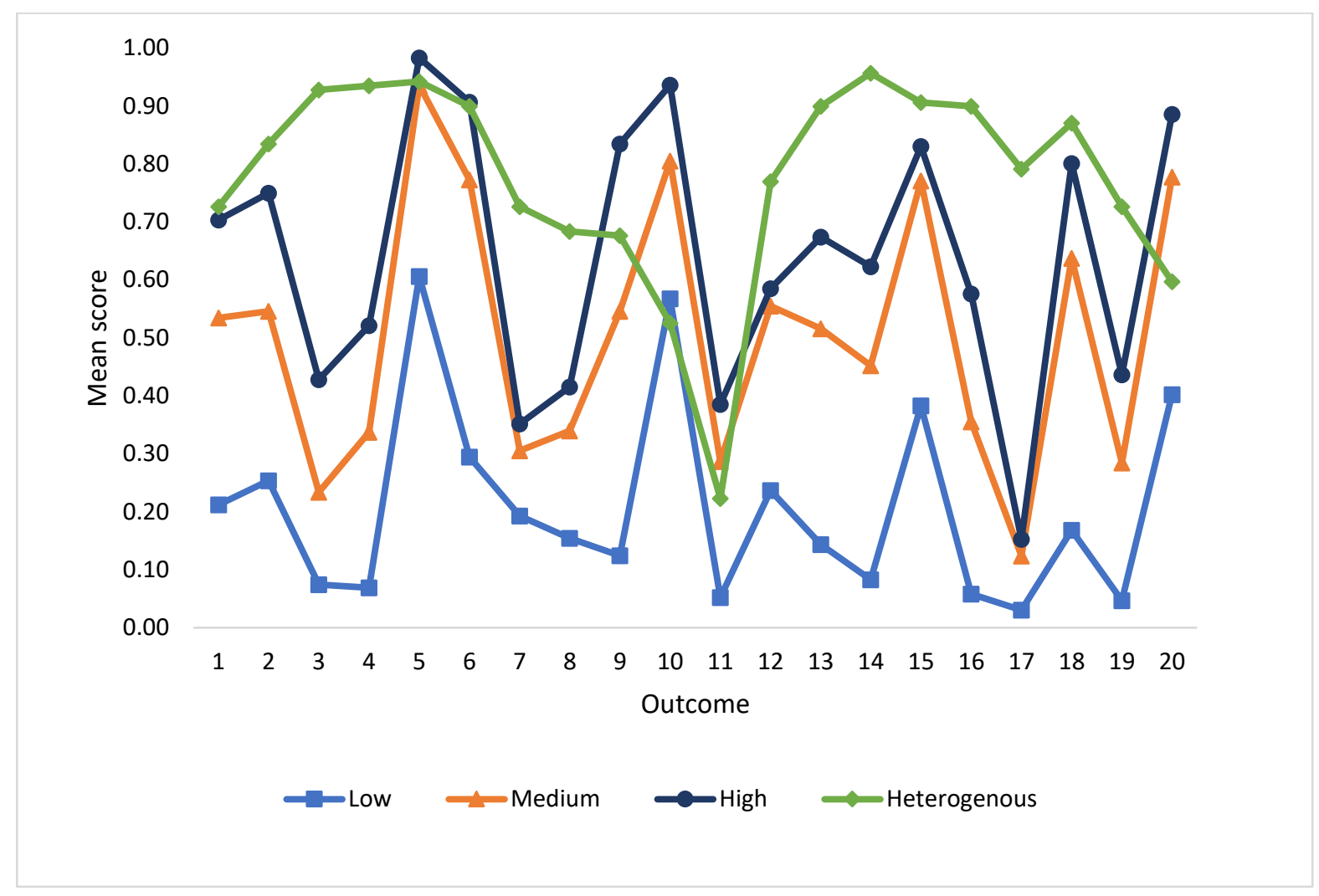

Note. 1 = I felt intimidated; 2 = I felt ashamed; $3=\mid$ felt afraid for my physical safety; 4 = I felt isolated from friends and family; $5=$ I felt frustrated; $6=$ I felt controlled; $7=$ It didn't affect me in any way; $8=I$ found it funny; 9 = I felt trapped; 10 = I felt angry; 11 = I felt like I wasn't able to make decisions for myself; 12 = I felt guilty; 13 = I felt like I wasn't able to do what I wanted to do; 14 = I felt afraid I was going to make them angry; 15 = I felt sad; 16 = I felt afraid I was going to set them off shouting or criticizing me; 17 = I felt sexually aroused; 18 = I felt helpless; 19 = I felt dependent on my partner; 20 = I felt emotionally hurt

Class 1: Low. This class contained 363 individuals, of which $45.5 \%$ were men $(n=165)$ and $54.5 \%$ were women $(n=198)$. They reported the lowest mean scores of the four classes for all outcomes, and due to this pattern of low mean scores, this class was labeled 'Low'. They reported their highest scores on outcomes relating to frustration, anger, sadness, and emotional hurt. However, a sense of autonomy and an absence of fear is what distinguishes this class from the other three. This is evidenced by very low scores on "I felt afraid for my physical safety", "I felt afraid I was going to set them off shouting or criticizing me" and "I felt afraid I was 
going to make them angry", in addition to "I felt isolated from friends and family", "I felt like I wasn't able to make decisions by myself", and "I felt dependent on my partner".

Class 2: Medium. This was the largest class $(N=436)$, comprised of 192 men $(44 \%)$ and 244 women (56\%). Although this class had higher mean scores than the 'Low class for all outcomes, their patterns of responding were similar in that they were relatively high and low on those same outcomes. This class was consequently labeled the 'Medium' class. Like the 'Low' class, the 'Medium' class also reported their highest mean scores on frustration, anger, sadness, and emotional hurt, however, they additionally reported high feelings of helplessness and feeling controlled.

Class 3: High. This class of 93 men (39.4\%) and 143 women (60.6\%) was labeled the 'High' class as they exhibited the same response pattern as seen in the 'Low' and 'Medium' classes but had relatively higher mean scores for all outcomes. This class had the highest mean scores of all classes on outcomes relating to feeling frustrated, trapped, controlled, angry, sad, and emotionally hurt.

Class 4: Heterogenous. The smallest class $(N=139)$ of 78 men $(57.4 \%)$ and 61 women (42.6\%) was labeled the 'Heterogenous' class as their responses were characterized by a mixture of both high and low mean scores for a variety of outcomes. This class had the highest mean scores for "I felt intimidated"; "I felt ashamed" "I felt afraid for my physical safety" "I felt isolated from friends and family"; "It didn't affect me in any way"; "I found it funny"; "I felt guilty" "I felt like I wasn't able to do what I wanted to do"; "I felt afraid I was going to make them angry"; "I felt sad"; "I felt afraid I was going to set them off shouting or criticizing me"; "I felt sexually aroused"; "I felt helpless"; "I felt dependent on my partner". Conversely, they had the lowest or second-lowest mean score for "I felt angry"; "I felt like I wasn't able to make decisions for myself"; "I felt emotionally hurt". 
Class differences on demographic variables. One-way ANOVAs with Games-Howell post hoc analyses were conducted in IBM SPSS Statistics for Windows (Version 26.0; IBM Corp., 2019a) to explore demographic differences in the four classes. Significant Levene's test values indicated the assumption of homogeneity of variance had been violated for all variables and Welch's robust of equality of means tests were conducted to assess group differences. The results showed there were no significant differences in employment status or ethnicity across the four classes. However, there were significant differences between the classes for gender $\left.F(3,473.20)=3.39, p<.05, \eta^{2}=.01\right)$, age $F(3,483.39)=4.51, p<.05, \eta^{2}$ $=.01)$, relationship status $\left.F(3,468.22)=2.81, p<.05, \eta^{2}=.09\right)$ sexual orientation $F(3$, $\left.445.30)=13.38, p<.001, \eta^{2}=.06\right)$, and for education level $F(3,484.76)=24.00, p<.001, \eta^{2}$ $=.05)$. Post hoc analyses revealed the 'Heterogenous' class had significantly higher mean scores than the 'High' class for gender, age, and relationship status. The 'Heterogenous' class also had significantly higher mean scores than the 'Low', Medium', and 'High' classes for sexual orientation and education level. In addition, the 'Low' class had significantly higher mean scores on education level than the 'Medium' and the 'High' classes.

Class differences on aggression variables. One-way ANOVAs using Welch's robust test of equality of means were used to investigate mean differences in 'Eclectic Aggression', 'Direct Psychological Aggression', Monitoring Acts', and the total number of experienced acts in the four classes (see Table 5.5). Games-Howell post hoc analyses revealed the classes were significantly different from one another on the total number of experienced acts and each type of IPA, with the exception of Direct Psychological Aggression as the difference between the mean scores for the 'High' and 'Heterogenous' classes was non-significant. The 'Heterogenous' class reported experiencing the largest total number of aggressive acts and the most 'Eclectic Aggression', and 'Monitoring Acts'. The 'High' class experienced the greatest amount of 'Direct Psychological Aggression'. The amount of aggression experienced by the 
'Low', 'Medium', and 'High' classes directly related to the labels given to each class, suggesting a positive relationship between the frequency of experiencing IPA and the frequency of associated outcomes.

Class differences in social desirability. One-way ANOVAs using Welch's robust test of equality of means and Games-Howell post hoc analyses were conducted to assess group differences in the mean scores of self-deceptive enhancement and impression management (sub-scales of the BIDR-16; see Table 5.5). The results showed significant differences between classes on self-deceptive enhancement: the 'Heterogenous' and 'Low, 'Medium' and 'High classes, and between the 'Low' and 'Medium' and 'Low' and 'High' classes. There were also significant differences between the 'Heterogenous' and 'Low, 'Medium' and 'High classes on impression management.

The sub-scale means were statistically compared to those from Hart et al. (2015) to gauge the level of social desirability response bias in the sample. The sample in the Hart et al. study (Study 2; $N=670$ ) was recruited online and participated in the research to validate the BIDR-16. A Welch two samples $t$-test demonstrated there was no significant difference in mean scores between the current sample $(M=4.24, S D=.91)$ and the Hart et al. sample $(M=4.30$, $S D=1.14)$ on self-deceptive enhancement $t(1156)=1.17, p=.243, d=.06$. However, there was a significant difference in mean scores between the current sample $(M=4.34, S D=.85)$ and the Hart et al. sample $(M=4.50, S D=1.24)$ on impression management $t(1033)=2.97, p$ $<.05, d=.16$. 
Table 5.5.

Four-class solution one-way ANOVAs predicting mean level differences in outcome variables

\begin{tabular}{|c|c|c|c|c|c|c|c|c|c|}
\hline & \multicolumn{2}{|c|}{$\begin{array}{c}\text { Low } \\
(N=363) \\
\end{array}$} & \multicolumn{2}{|c|}{$\begin{array}{l}\text { Medium } \\
(N=436) \\
\end{array}$} & \multicolumn{2}{|c|}{$\begin{array}{c}\text { High } \\
(N=236) \\
\end{array}$} & \multicolumn{2}{|c|}{$\begin{array}{l}\text { Heterogenous } \\
\qquad(N=139)\end{array}$} & \multirow{2}{*}{$\begin{array}{c}\text { Welch's } \\
F\end{array}$} \\
\hline & $M$ & $S D$ & $M$ & $S D$ & $M$ & $S D$ & $M$ & $S D$ & \\
\hline Eclectic Aggression & $.04^{\mathrm{abc}}$ & .10 & $.17^{\text {ade }}$ & .23 & $.61^{\mathrm{bdf}}$ & .42 & $2.12^{\mathrm{cef}}$ & .64 & $1518.80 *$ \\
\hline Monitoring Acts & $.23^{\mathrm{abc}}$ & .26 & $.71^{\text {ade }}$ & .43 & $1.91^{\text {bdf }}$ & .768 & $2.28^{\mathrm{cef}}$ & .74 & $816.07 *$ \\
\hline $\begin{array}{l}\text { Direct Psychological } \\
\text { Aggression }\end{array}$ & $.43^{\mathrm{abc}}$ & .37 & $1.10^{\text {ade }}$ & .60 & $2.33^{\mathrm{bd}}$ & .74 & $2.27^{\mathrm{ce}}$ & .72 & $645.60 *$ \\
\hline Total number of behaviors & $4.41^{\mathrm{abc}}$ & 2.44 & $10.26^{\text {ade }}$ & 2.97 & $18.52^{\text {bdf }}$ & 3.61 & $28.24^{\mathrm{cef}}$ & 2.69 & $2675.44^{*}$ \\
\hline $\begin{array}{l}\text { Social Desirability } \\
\text { (Self-Deceptive Enhancement) }\end{array}$ & $4.06^{\mathrm{abc}}$ & .89 & $4.19^{\mathrm{ad}}$ & .84 & $4.17^{\text {be }}$ & .81 & $5.02^{\text {cde }}$ & 1.01 & $54.96^{*}$ \\
\hline $\begin{array}{l}\text { Social Desirability (Impression } \\
\text { Management) }\end{array}$ & $4.09^{\mathrm{a}}$ & .80 & $4.34^{\mathrm{b}}$ & .75 & $4.29^{c}$ & .74 & $5.11^{\mathrm{abc}}$ & 1.03 & $43.63^{*}$ \\
\hline
\end{tabular}

Note. $M=$ mean $S D=$ standard deviation; $* p<.001 ;$ Means with similar superscripts were significant $p<.05$ 


\section{Latent Variable Path Model}

Our prior analyses indicated no evidence for a unique construct of coercive control at the item-level or a unique typology at the person-level. However, although none of the classes were uniquely characterized by "coercive control" outcomes, the typology that included higher levels of controlling experiences also reported more frequent IPA. Our final model tested Hypothesis 2 and Hypothesis 3 under the assumption that a construct of "coercive control" existed (i.e., assuming Hypothesis 1 to be true). Specifically, we conducted a final model with maximum likelihood estimation in Mplus (Version 8; Muthén \& Muthén, 1998-2017a) to identify the links between people's experiences of IPA and coercive control using the same variables as were used in the previous two analyses (see Appendix KK). An exploratory measurement model for a latent factor of coercive control indicated very poor fit $(\chi 2=2182.44$; $\mathrm{df}=44 ; p<.001 ; \mathrm{RMSEA}=.20[.19, .21] ; \mathrm{SRMR}=.11 ; \mathrm{CFI}=.66)$, so we modelled coercive control as the average of 11-items from the extant literature on coercive control described in Table 5.2 (Cronbach's $\alpha=.85$ ).

We conducted a structural equation model that regressed feeling controlled on experiences of Eclectic Aggression, Direct Psychological Aggression, and Monitoring Acts (testing Hypothesis 2). Each of these constructs was the sum of 15 items identified by Dempsey et al. (2020). To assess the discriminant links between feeling controlled and experienced IPA, we simultaneously included three other outcomes based on negative emotions in the PANAS$X$ framework (Watson \& Clark, 1994): Hostility ("I felt angry"; "I felt frustrated"), guilt (“I felt guilty"; "I felt ashamed"), and sadness ("I felt sad"; "I felt emotionally hurt"). We included interaction terms between all predictors and gender to test whether any of the associations differed between men and women, testing Hypothesis 3 . 
Results from the model are displayed in Appendix OO. Parameter estimates showed Eclectic Aggression significantly and positively predicted 'coercive control' $B=0.083, S E=$ $0.008, \beta=.60,95 \% \mathrm{CI}[.07, .10], \mathrm{z}=10.81, p<.001$, and 'guilt' $B=0.091, S E=0.015, \beta=$ $.54,95 \% \mathrm{CI}[.06, .12], \mathrm{z}=5.97, p<.001$. Eclectic Aggression also significantly, but negatively predicted 'hostility' $B=-0.108, S E=0.016, \beta=-.40,95 \%$ CI $[-.14,-.08], \mathrm{z}=-6.60, p<.001$ (see Figure 5.4 [p. 140], and Appendix OO for the parameter estimates for the model).

Direct Psychological Aggression significantly and positively predicted 'coercive control' $B=0.034, S E=0.008, \beta=.26,95 \%$ CI $[.02, .05], \mathrm{z}=4.34, p<.001$, 'hostility' $B=$ $0.167, S E=0.020, \beta=.64,95 \%$ CI $[.13, .20], \mathrm{z}=8.45, p<.001$ and 'sadness' $B=0.009, S E=$ $0.018, \beta=.52,95 \%$ CI $[.07, .13], \mathrm{z}=5.47, p<.001$. However, Monitoring Acts did not significantly predict any of the outcome variables.

The results demonstrating that coercive control outcomes are positively predicted by Eclectic Aggression and Direct Psychological Aggression provide support for our first hypothesis. Constraining the model to produce a parameter estimate of the difference in the two predictors further revealed Eclectic Aggression to be more strongly predictive of 'coercive control' than Direct Psychological Aggression $(B=0.049, S E=0.011, \mathrm{z}=4.36, p<.001)$.

Gender significantly predicted 'coercive control' $B=-0.138, S E=0.006, \beta=-.04,95 \%$ CI [-.24, -.04], $\mathrm{z}=-2.23, p<.001$ and 'guilt' $B=-0.213, S E=0.102, \beta=-.08,95 \%$ CI [-.48, $.14], \mathrm{z}=-3.06, p<.05$, with men reporting higher levels of both outcomes. However, there was no significant gender interactions for Eclectic Aggression, Direct Psychological Aggression, or Monitoring Acts. Although gender was found to predict both coercive control and guilt, men had higher scores on both variables, and, thus, our hypothesis that this association would be stronger for women was not supported (Hypothesis 3). 


\section{Figure 5.4}

Manifest variable model showing the standardized parameter estimates

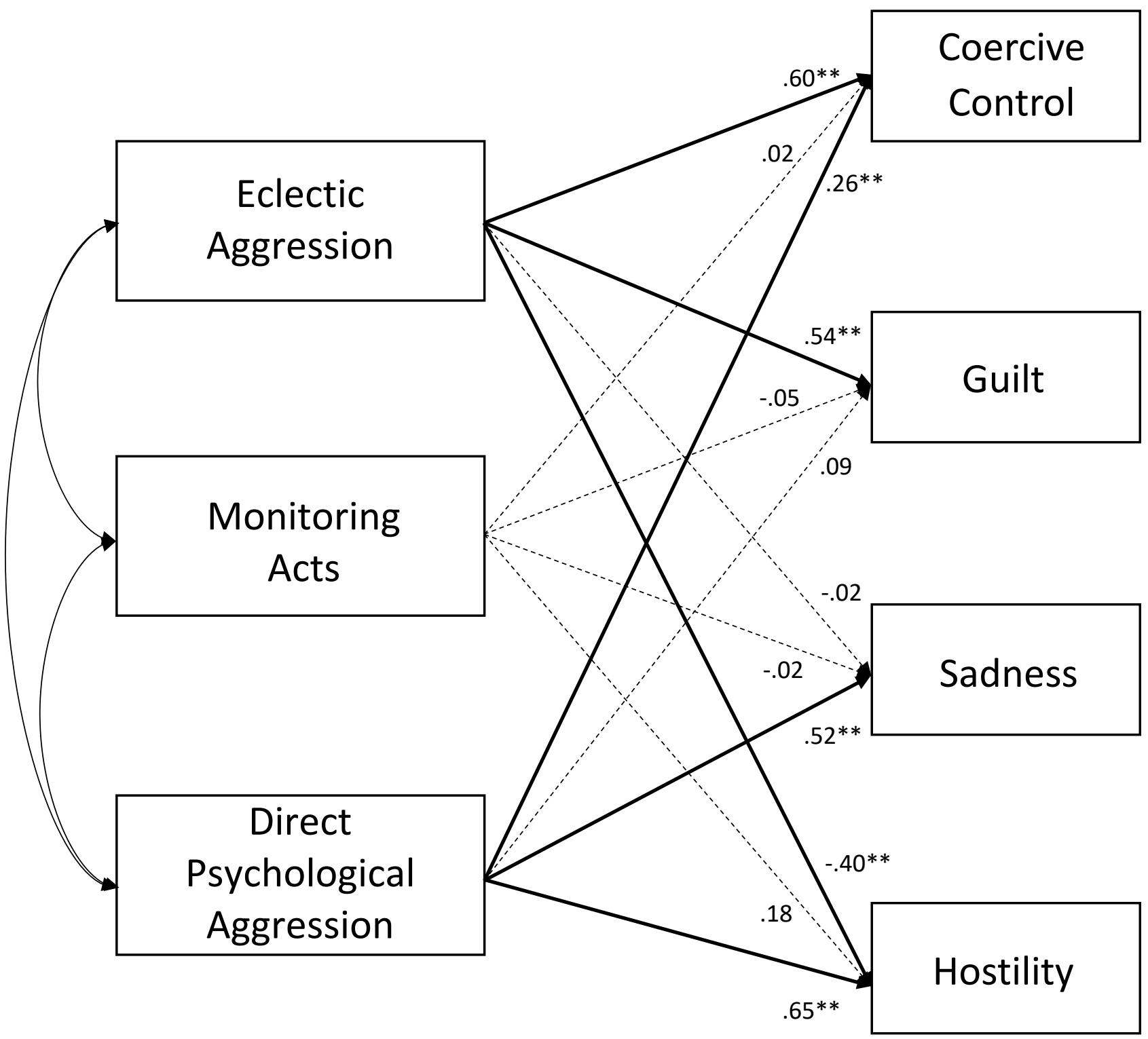

Note. Solid lines indicate significant coefficients, dashed lines indicate non-significant coefficients; ${ }^{* *}=p$ $<.001$; Gender interactions are not displayed but gender was included as a covariate and controlled for in the model. All gender interactions were non-significant. 
The R squared values (see Appendix OO) show that between $22-61 \%$ of the variance is explained by this model. 'Coercive control' accounted for the largest proportion of this (.61), demonstrating the importance of this outcome to IPA relative to the other outcomes included in the model ('hostility' = .39; 'guilt' = .26; 'sadness' = .22).

\section{Discussion}

Coercive control has proved to be an important concept in informing research, practice, and legislation. However, a lack of consensus about how this phenomenon should be conceptualized (e.g., as gendered or gender-inclusive) has resulted in ambiguity about what it is and how it can be identified (Hamberger et al., 2017). This study aimed to test if coercive control could be best identified through measuring the outcomes reported by people who have experienced IPA and if the gendered assumptions of the commonly accepted conceptualization could be upheld using outcomes. Three hypotheses explored the relationships between coercive control and the type and frequency of IPA experienced by men and women using categorical principal analysis (CATPCA), latent class analysis (LCA), and manifest variable path analysis. Results found an association between types of IPA and coercive control, supporting Hypothesis 1. However, no associations were found between coercive control and the frequency of experienced IPA (Hypothesis 2), or gender (Hypothesis 3).

\section{Summary of Results}

Hypothesis 1. The results from the CATPCA and LCA did not support our hypothesis that patterns of coercive control outcomes would be more strongly associated with Eclectic Aggression, Direct Psychological Aggression, or Monitoring Acts than other outcomes associated with IPA, as no evidence of a pattern of coercive control was found in either analysis. However, the manifest variable path analysis provided some support for this hypothesis, as when a pattern of coercive control was manipulated for the purposes of this analysis, Eclectic Aggression and Direct Psychological Aggression were both found to 
positively predict 'coercive control'. As Eclectic Aggression is comprised of items relating to physical aggression and threats of harm to an individual's body and reputation, and Direct Psychological Aggression is comprised of psychologically aggressive tactics, it suggests that either a combination of physical and psychological aggression or psychological aggression alone can result in feelings of 'coercive control'. However, as Eclectic Aggression had a larger beta coefficient, the relationship between physical aggression and experiencing 'coercive control' appears to be stronger. The association found between Eclectic Aggression and 'coercive control' is therefore consistent with Stark's definition of coercion, which he describes as "the use of force or threats to compel or dispel a particular response" (2007, p. 228). Violence is considered a tactic of 'coercive controlling violence', and, thus, the significant relationship found between 'coercive control' and Eclectic Aggression is also consistent with Johnson's typology $(1995,2006)$. The significant relationship found between 'coercive control' and Direct Psychological Aggression is also consistent with Johnson's proposed 'incipient coercive controlling violence' in which no physical aggression is present in the relationship (2008). Experiencing Monitoring Acts did not significantly predict 'coercive control', despite the fact the items in this component were all derived from 'controlling behavior' (sub)scales (see Dempsey et al., 2020). The lack of evidence of an association between items previously labeled as 'controlling' and 'coercive control 'adds weight to the argument questioning their validity and continued use in assessing coercive control in intimate relationships.

Hypothesis 2. The absence of a coercively controlled profile in the LCA resulted in a lack of support for Hypothesis 2. Although the 'Medium', 'High', and 'Heterogenous' classes reported similarly high levels of outcomes associated with coercive control, the outcomes that were most frequently and highly endorsed were derived from general IPA literature (e.g., high levels of anger and frustration, sadness, and emotional hurt). Despite a lack of evidence of a coercively controlled profile, the results from the LCA demonstrate that classes with relatively 
higher mean scores on outcomes, including the coercive control outcomes, experienced IPA at a significantly higher frequency. In the absence of such a profile, the results are, therefore, inconsistent with Johnson's typology that suggests coercive control is associated with more frequent IPA.

Hypothesis 3. Despite conducting both variable (CATPCA) and person-centred (LCA) analyses, we did not find evidence to support our hypothesis that an association between patterns of coercive control outcomes and experiencing IPA would be stronger for women than for men. In addition, a comparison of the model fit of the freely estimated and constrained LCA models revealed gender did not contribute a meaningful amount of variance to the model. In the manifest variable path analysis, gender positively predicted 'coercive control' and 'guilt', however, these associations were found to be stronger for men, than for women. There were no significant gender interactions for any type of IPA. Coercive control, as conceptualized by Stark (2007) is gender-specific and is experienced exclusively by women, and, thus, the lack of evidence for a pattern of coercive control in the CATPCA and LCA and the stronger association for 'coercive control' and 'guilt' for men, rather than women in the manifest variable path analysis, is inconsistent with this. Similarly, the fairly-even proportion of men and women in the four classes in the LCA is inconsistent with Johnson's conceptualization of coercive control, in which a greater proportion of women would be expected to report experiences of 'coercive controlling violence' (2006).

\section{Theoretical and Practical Implications}

The findings from the PCA and the LCA did not support the premise that coercive control can be identified by measuring the outcomes for people who have experienced IPA. Nor did they find evidence to support the assumptions outlined by Johnson and Stark that coercive control is gendered and associated with more frequent levels of IPA. Similarly, although the items comprising the purported 'coercive control' factor were taken directly from 
definitions of coercive control (e.g., Stark, 2007), the Women's Experience with Battering Scale (Smith, Earp, \& DeVillis, 1995) and the qualitative literature, they did not form a coherent factor, as evidenced by the global fit statistics in the latent variable path analysis. While the items in the 'coercive control' factor were normatively associated and would be considered statistically reliable as part of a hypothetical scale, the lack of construct validity suggests the indicators were not theoretically sound and were not measuring coercive control, thus implying an inadequate understanding of what is widely considered to be, and currently conceptualized as, coercive control. The results of this study highlight the need for a theoretically informed, coherent, and clear conceptualization of coercive control from which valid measurement tools can be developed.

In addition, the findings have practical implications. Despite research showing that coercive control cannot be accurately identified using behavioral or outcome measures, Stark's work has been used as a framework to create new legislation that criminalizes coercive or controlling behaviors (e.g., Scottish Government, 2015). It is imperative that legislation that outlines and criminalizes any behavior is informed by clear conceptualizations of coherent constructs, which can be measured and supported by empirical evidence. Furthermore, Johnson's work has proposed that coercive control is gendered and associated with more frequent and serious harm (2006). This has led to the assumption that coercive controlling IPA against women is the type of IPA that resources need to be dedicated to reducing (see Stark, 2010), and that whilst 'situational couple violence' is "regrettable" it is not where the prevention focus should be placed (Dobash \& Dobash, 2004, p. 344).

Thus, resources and legislation have most commonly been dedicated to reducing violence against women and to the development of gender-based treatment programs, such as those informed by the Duluth Model (Pence \& Paymar, 1993), which are based upon a gendered conceptualization of coercive control. However, the effect sizes of treatment 
programs developed from this perspective are small (see Babcock, Green, \& Robie, 2004) and therefore it is imperative that evidence informs the development of future treatment and prevention programs.

\section{Strengths, Limitations, and Future Directions}

Limitations of this research include a cross-sectional design. Behavioral acts were assumed to precede an outcome, however, responses were measured simultaneously. Thus, if an individual was feeling controlled, they may report more IPA as a result. The design of future studies could be altered to account for this, by measuring the frequency of aggression and outcomes at different time points.

A further limitation of this study is the focus on events that occurred in the past year. As coercive control is said to be about patterns of abuse (Stark, 2007), asking about individual's experiences over their life-course to examine the development of the behavior of the perpetrator and victim's responses to that, over time (Dutton, 1999), maybe more beneficial to our understanding of coercive control.

This study chose to focus on the frequency, rather than severity, of aggression in relation to coercive control, as this is consistent with Stark's conceptualization (see Stark \& Hester, 2019). However, the investigation into the relationship between severe violence and coercive control would further our understanding of this construct and is an area for future research to consider.

The measurement approach we employed could be considered a strength of the study as the use of outcomes tie directly to current legislation that has criminalized coercive controlling behavior (e.g., feeling dependent, isolated, controlled, restricted: Domestic Abuse Act 2018 [Scotland]; fear: Serious Crimes Act 2015 [UK], s 76). Individuals reported the frequency with which they experienced an aggressive act and the impact that had on them. This 
aspect of the methodology may help to differentiate between assault and abuse, the latter of which includes tactics of coercive control (Stark, 2007). However, the measure we used was comprised of items taken from the literature and existing measures and was not validated. Future researchers could, therefore, look to develop and validate a scale that focuses on the outcomes of IPA.

\section{Conclusion}

This study found no evidence that the current gendered conceptualization of coercive control, that informs legislation, practice, and policy, is a coherent construct. These findings challenge research to revise the conceptualization of coercive control to produce a valid construct that can contribute to the measurement of this phenomenon. We, therefore, propose research reconsider the current hypothetico-deductive approach in favor of one, which focuses on abductive methods of theory generation. The abductive theory of method framework seeks to explain phenomena, rather than viewing them as predictors of a given theory (see Haig, 2005). This would facilitate the development of causal explanations for coercive control and ultimately contribute to a more comprehensive, gender-inclusive understanding of IPA. 


\section{Chapter 6: DISCUSSION}

The concept of control features heavily in how scholars, practitioners, policymakers, and legislators understand, assess, and respond to intimate partner aggression (IPA). However, commonly accepted conceptualizations and measurements of control remain largely untested. Control is most frequently measured using behavioral scales or victim outcomes and is widely considered a gendered phenomenon. A victim-focused gendered conceptualization has prevailed despite much empirical research suggesting IPA is a gender-inclusive phenomenon (Dutton, 2006a). The wider aggression literature suggests that control might best be measured by examining people's motivations (e.g., Ireland, 2009), whereas gendered theory proposes control motivations are driven by patriarchal societal norms (e.g., Dobash \& Dobash, 1979; Stark, 2007). This thesis presented three studies and a review investigating the best way to conceptualize and measure control. Specifically, we tested the validity of some of the core underlying assumptions of the common conceptualizations of control. This chapter reviews the key findings of each of the studies (see Table 6.1) and their contributions to the field, the limitations and implications of the thesis, and areas for future research.

\section{Summary of Key Findings and Contributions to the Literature}

Chapter 2: The discriminance of controlling behaviors. Controlling behavior scales have been developed and used to assess the presence of control in intimate relationships (e.g., Relationship Behavior Rating Scale-Revised: Beck et al., 2013; Checklist of Controlling Behaviors: Lehmann et al., 2012). Current research (e.g., Bates \& Graham-Kevan, 2016, Hamel et al., 2015) assumes that the distinct behavioral acts assessed in these scales are perpetrated with the intention to control an intimate partner and they are, thus, considered a distinct subtype of IPA. 


\section{Table 6.1}

Summary of main findings by chapter

\begin{tabular}{|c|c|c|c|}
\hline Chapter & Assumption & References & Main finding(s) from chapter \\
\hline 2 & Control is associated with a type of behaviour & $\begin{array}{l}\text { Bates \& Graham-Kevan (2016); Hamel et al. } \\
\text { (2015); Johnson (2006); Tolman (1989) }\end{array}$ & $\begin{array}{l}\text { Behaviors labeled in the literature as controlling } \\
\text { are not statistically distinct from other types of } \\
\text { IPA }\end{array}$ \\
\hline 3 & Control is gendered & Johnson (2006); DeKeseredy et al. (1997) & $\begin{array}{l}\text { Control was not the largest effect size for men. } \\
\text { Self-defense was the largest effect size for } \\
\text { women, and overall. }\end{array}$ \\
\hline \multirow[t]{3}{*}{4} & Control is associated with a type of behaviour & $\begin{array}{l}\text { Bates \& Graham-Kevan (2016); Hamel et al. } \\
\text { (2015); Johnson (2006); Tolman (1989) }\end{array}$ & $\begin{array}{l}\text { Control motivations are not uniquely associated } \\
\text { with a type of behavior }\end{array}$ \\
\hline & $\begin{array}{l}\text { Control is associated with an increased frequency } \\
\text { of IPA }\end{array}$ & Johnson (2006); Kelly \& Johnson (2008) & $\begin{array}{l}\text { Control motivations are not uniquely associated } \\
\text { with an increased frequency of IPA }\end{array}$ \\
\hline & Control is gendered & Johnson (2006); Myhill (2015) & Control motivations are not gender specific \\
\hline \multirow[t]{3}{*}{5} & $\begin{array}{l}\text { Coercive control is associated with a type of } \\
\text { behaviour }\end{array}$ & Hardesty et al, (2015); Johnson (2006) & $\begin{array}{l}\text { Coercive control outcomes are not uniquely } \\
\text { associated with a type of behavior }\end{array}$ \\
\hline & $\begin{array}{l}\text { Coercive control is associated with an increased } \\
\text { frequency of IPA }\end{array}$ & Dichter et al. (2018); Kelly \& Johnson (2008) & $\begin{array}{l}\text { Coercive control outcomes are not uniquely } \\
\text { associated with an increased frequency of IPA }\end{array}$ \\
\hline & Coercive control is gendered & $\begin{array}{l}\text { Johnson (2006); Kelly \& Johnson (2008); Stark } \\
\text { (2007) }\end{array}$ & $\begin{array}{l}\text { Some evidence for men experiencing coercive } \\
\text { control more strongly than women }\end{array}$ \\
\hline
\end{tabular}


Chapter 2 investigated the divergent validity of acts previously labeled in the literature as "controlling behaviors" against other aggressive acts and partner regulation strategies used in relationships. Exploratory factor analysis identified a three-factor solution in both the perpetrated and experienced behavior models: Eclectic Aggression; Direct Psychological Aggression; Monitoring Acts. The behaviors derived from (sub)scales developed to measure control did not form a distinct factor and were instead dispersed across the three factors. Confirmatory factor analysis replicated the factor structure of both models produced by the exploratory factor analysis. The high correlations between factors in the experienced model indicated these factors may not be measuring distinct constructs. The findings of Chapter 2 challenge the assertion that controlling behaviors are a unique subtype of IPA as there was no evidence of a statistical distinction between items from 'controlling behavior' (sub)scales and other aggressive acts. The findings, therefore, suggest current behavioral measures of control lack construct validity and are, thus, unable to assess the presence of control dynamics in intimate relationships.

\section{Chapter 3: Motivations for physical and psychological intimate partner} aggression: A systematic review. The gendered explanation of IPA has been highly influential in informing both public policy and perceptions (Bates et al., 2014; Hamel, 2007), resulting in an emphasis on research into controlling motivations (Dutton, 2006a). While previous reviews have investigated the frequency with which motivations have been studied, this was the first meta-analysis of motivations for physical IPA to be conducted, providing objective evidence of the relative importance of control as a motivation for IPA. The review identified that the majority of studies were of low quality, which reduced the number of studies suitable for inclusion in the analyses. The results of the meta-analysis were inconclusive due to a small number of studies and large amounts of dispersion and heterogeneity. However, the results suggest self-defense, retaliation for emotional hurt, and communication difficulties had the 
largest effect sizes overall, while dominance and self-defense had the largest effect sizes for men and women, respectively. We found no evidence for control and weak evidence for selfdefense as motivations for IPA. We should, therefore, consider other motivations for IPA, such as those underlying retaliation, as treatment targets. The results suggest control motivations, as they are currently measured, are not gendered and gender-inclusive conceptualizations of control should, therefore, be considered.

Chapter 4: Motivations for intimate partner aggression. Chapter 4 built on Chapter 2 by testing the assumption that behaviors previously labeled in the literature as 'controlling' are associated with control motivations. In addition, assumptions regarding the relationships between control motivations and the frequency of IPA, and gender, which underscore Johnson's typology (1995; 2006), were examined. We conducted categorical principal component and latent class analyses to test these assumptions. Control was operationalized as a motivation for IPA perpetration. Four distinct profiles were identified, which showed that a higher frequency of IPA was associated with a diverse range of motivations, and considerable heterogeneity in the motivations for different types of IPA, for men and women. There was no evidence of a unique pattern of control motivations linked to a specific subset of behavioral acts, that control motivations were gendered or were more strongly associated with increased perpetration of IPA. These results lend weight to the argument for a gender-inclusive conceptualization of control and further question the validity of 'controlling behavior' measures.

\section{Chapter 5: Examining coercive control as an outcome of intimate partner}

aggression. Coercive control is considered the most serious form of IPA and as the social isolation, domination, and subjugation of women by their male partners (Stark, 2007). Coercive control has been associated with specific types and an increased frequency of IPA (see Johnson, 2006). Chapter 5 tested the conceptualization of coercive control by operationalizing it as an outcome experienced by male and female victims of IPA. Categorical principal components 
and latent class analyses were conducted to test the assumptions of gender specificity, an association between coercive control outcomes and an increased frequency of IPA, and an association between coercive control outcomes and types of behaviors. There was no evidence of unique associations between coercive controlling outcomes and an increased frequency of IPA or types of behaviors, or evidence to support the gendered conceptualization of coercive control. Structural equation modeling further tested the conceptualization. Coercive control items, which were derived from research informed by the gendered perspective, failed to form a coherent construct in a latent variable path analysis. The average of these items was, therefore, regressed on experiences of IPA in a manifest variable path analysis. Experiences of Eclectic Aggression and Direct Psychological Aggression significantly predicted feelings of 'coercive control', with Eclectic Aggression more strongly predictive. Gender also predicted 'coercive control' though, this association was stronger for men than for women. These results lend further weight to the argument for a gender-inclusive conceptualization of control and suggest coercive control, as it is currently conceptualized, cannot be identified using victim experiences.

\section{Implications for Theory, Practice, Policy, and Legislation}

Assumptions about control have guided common assessment practices, prevention practices, intervention practices, legislation, and research (see Dutton, 2006a; Hamel, 2007). Evidence was not found to support key assumptions about control that typically guide the family violence field. Specifically, we found no evidence that control can be measured using specific non-physical acts labeled 'controlling behaviors', that control is gendered, or that control is uniquely and distinctly associated with an increased frequency of physical IPA (e.g., Johnson, 2006). In this section, I describe how the results of this thesis have important implications for theory, research, policy, practice, and legislation.

Theory. Gendered theory maintains IPA is a contraction of patriarchal values and should be viewed as a representation of the wider social structures that maintain men's control 
over women (Bell \& Naugle, 2008; Hamberger et al., 2017). The findings from this research are inconsistent with this conceptualization as neither control motivations (Chapters 3 and 4) nor experiences of coercive control (Chapter 5) were found to be gender-specific. Indeed, although gender significantly predicted 'coercive control' in Chapter 5, this association was found to be stronger for men, than for women, and, therefore, does not support gendered theory. As a single factor, macro-level theory, gendered theory is unable to account for female's use of control motivated IPA or men's experiences of coercive control. These findings highlight the empirical inadequacy of gendered theory, its lack of depth as a theoretical explanation of IPA, and the need for the development of multi-level theories of IPA, which are currently lacking in the field (Dixon \& Wride, 2020). The results from this thesis also have implications for the conceptualization of coercive control and the typology work of Johnson (1995; 2006), which are predicated on gendered theory. The results from this thesis indicate research should consider both men's and women's experiences of IPA in constructing theories of control. Such theories would then align with current gender-inclusive definitions of IPA (see Dixon \& Graham-Kevan, 2011; National Center for Injury Prevention and Control, 2020).

'Controlling behaviors' are considered a distinct subset of non-violent behaviors by the family violence perspective and some gendered researchers, unique from other types of IPA because of their assumed underlying motivation to control. However, factor analytic results from Chapter 2 showed these 'controlling behaviors' did not form a unique factor and were, instead, dispersed across the factors of the experienced and perpetrated behavior models. In addition, no pattern of behaviors was found to be associated with motivations of control (Chapter 4) or with outcomes of coercive control (Chapter 5). It is suggested the operationalization of control has been conflated with its conceptualization, as with few exceptions (e.g., Dutton et al., 2005), 'controlling behavior' scales are descriptive, rather than theoretically or conceptually based. Consequently, control is not adequately defined, resulting 
in poor construct clarity and considerable overlap with other forms of IPA, particularly psychological aggression. This lack of clarity is most evident from the results in Chapter 2, but the results of Chapters 4 and 5 further suggest the construct of control has been poorly conceptualized and lacks validity. For example, the items that comprised the 'Monitoring Acts' factor (experienced model) in Chapter 2 were exclusively derived from (sub)scales designed to measure control in intimate relationships. It was concluded that this factor was not consistent with common definitions of control, including the thesis definition, and was more reflective of relationship anxiety. This conclusion was supported by evidence that the 'Monitoring Acts' component was not uniquely or strongly associated with control, either as a motivation (Chapter 4) or as an outcome (Chapter 5). Similarly, the poor global fit statistics produced in the latent variable path analysis (Chapter 5) are evidence of incoherence. In sum, the results from Chapters 2, 4, and 5 indicate poor construct validity and a lack of understanding as to the nature of (coercive) control and how it should be defined. The development of a clear theoretical conceptualization and definition of control should therefore be a priority for IPA researchers. However, conceptual agreement and research co-ordination are necessary to achieve meaningful progress in this area (Sullivan, 2019).

Practice and policy. The considerable influence of the gendered perspective has resulted in the centrality of control to our understanding of IPA (Hamberger et al., 2017). Current treatment for IPA is informed by a gendered understanding of partner violence (Dutton, 2006a; Hamel, 2007). The findings from this research, therefore, have implications for treatment design. The Power and Control Wheel, which is the graphic representation of the Duluth Model, is comprised of non-physical tactics (e.g., using isolation; intimidation) used by men to control and subordinate their female partners (Pence \& Paymar, 1993). However, Chapter 4 found that controlling motivations were not more strongly associated with types of IPA than those derived from theories of general aggression, or with gendered perpetration. The 
lack of evidence for a subset of behaviors that are uniquely motivated by control and for the gender specificity of control motivations undermine the validity of the Power and Control Wheel and the central premise of the Duluth Model. Similarly, the results from the metaanalysis provide suggestive evidence that control is not the primary motivation for men's use of IPA. The cumulative results from this research indicate the preeminent role control maintains in treatment programs is seemingly underserved. Given the small treatment effect sizes (see Babcock et al., 2004), interventions should, therefore, focus on alternative motivations for IPA to improve the applicability of program content and lower recidivism rates.

The gendered perspective considers IPA a distinct form of aggression due to the patriarchal context in which it occurs (see Dobash \& Dobash, 1992; Koss et al., 1994). The lack of evidence for control as the key motivation for men's use of IPA not only questions the content of current treatment programs but whether specific treatment programs are, indeed, necessary. This finding has implications for women's treatment programs, as much as men's, as current programs for women are often reliant on an understanding of male-perpetrated IPA (Kernsmith, 2005; Stewart et al., 2014). Furthermore, the small effect sizes of the gender comparisons of motivations for IPA (refer Table 2; Chapter 4) suggest men and women have similar motivations for perpetrating IPA (see also Langhinrichsen-Rohling et al., 2012) and genderinclusive, functional approaches to treatment should be considered.

Johnson (1995; 2006) proposed control motivations could differentiate between types of IPA, which were further distinguished by the frequency of IPA and by gender. Johnson's typological approach to explaining IPA has been influential in tailoring treatment design (Kelly \& Johnson, 2008) and informing social policy (Carlson \& Jones, 2010). However, the latent class analysis conducted in Chapter 4 did not find evidence of a profile, predominantly male or otherwise, that was uniquely characterized by control motivations, thereby equating to what has been described as 'coercive controlling violence/ intimate terrorism' (see Johnson 2006; Stark, 
2010). In addition, motivations of control were not associated with an increase in the frequency of IPA or with gender. These findings challenge the assumption that "male partner abuse is different than female partner abuse and therefore merits different forms of policy and intervention" (Stark, p. 209). Furthermore, the lack of evidence for control as a primary motivation for IPA perpetrated by men negates the rationale for the current focus on 'coercive controlling violence/ intimate terrorism'. The heterogeneity in motivations for IPA reported by men and women (Chapter 4) would suggest a public health approach to partner violence would be a more effective strategy to reduce IPA. Public health approaches are primary prevention focussed and are most successful when interventions are multifaceted and community-based, thus, ensuring greater involvement and accountability (Rosenberg et al., 1992). Such approaches reach individuals who would not normally come to the attention of the criminal justice system (Rosenberg et al.) and would, therefore, encompass aggression classed as 'situational couple violence', which has previously been overlooked due to the influence of the gendered perspective.

Legislation. The gendered conceptualization of IPA, with its inherent emphasis on control, has informed legislation targeting domestic violence and the recent criminalization of controlling behaviors in western countries (e.g., England; Scotland; Ireland). Such legislation relies on victims' experiences of IPA as the standard of proof on which the crimes are contingent (e.g., feeling dependent, isolated, controlled, restricted: Domestic Abuse Act 2018 [Scotland]). While the Scottish Domestic Abuse Act 2018 explicitly takes a gendered approach, the Serious Crimes Act 2015 (UK: s 76) does not specifically mention gender but uses "fear" as the standard of proof for conviction, which research has shown is reported significantly more by women who experience IPA than men who experience IPA (Barnett et al. 1997; Hamberger 2005; Langhinrichsen-Rohling et al. 1995). The results from this research, however, demonstrate that control, as it is currently conceptualized, is not a gendered phenomenon. 
Rather, both men and women perpetrated IPA that was motivated by control and experienced feelings of coercive control resulting from their partner's use of IPA. These findings, therefore, highlight the danger in basing legislation on untested assumptions, which discriminate on the basis of gender. The findings from this thesis indicate a need for gender-inclusive legislation that is based on empirical evidence.

\section{Limitations and Future Research}

The following limitations have been considered in the previous chapters but are addressed here as limitations that characterize the thesis as a whole. The first such limitation is the use of self-report surveys, which have been shown to affect measurement validity by introducing self-report and method biases (Follingstad \& Rogers, 2013). Despite the inherent challenges with this methodology, the investigation into individuals' motivations and experiences of IPA in the current research necessitated the use of self-report measures. Research has demonstrated individuals are more likely to over-report IPA perpetrated by their partner and under-report their own aggression (see Follingstad \& Rogers). However, under-reporting of IPA has been found to relate to severity rather than the frequency of occurrence of IPA (see Heckert and Gondolf, 2000), so may not have impacted the results of this thesis. The severity of IPA in relation to control was not measured in the current study but is another area of inquiry for future research to explore. There are, however, multiple issues relating to the self-reported severity of violence, particularly with regards to what is considered severe. This is highly subjective due to individual personality characteristics that affect perception and the type of act itself. More objective measures, such as emergency department admissions, are also problematic as there are multiple reasons why people choose not to seek help (e.g., people simply may not live within a feasible distance of a hospital). Nevertheless, the severity of violence in relation to control is something for future research to investigate further, in spite of the methodological challenges. In the current research several steps were taken to ameliorate 
the impact of method bias, as outlined in Chapter 4 (refer p. 106; see also Podsakoff et al., 2012) and Chapters 4 and 5 included a measure of social desirability response bias. The mean subscale scores for the sample were the same or significantly lower than those in a study in which participants were not asked about socially undesirable behaviors (Hart et al., 2015).

A second limitation of this research is the use of cross-sectional data. Behavioral acts were assumed to precede both motivations and outcomes and were measured simultaneously. While the assumption that motivation precedes behavior aligns with current motivational theory (e.g., see Ward, 2017), this process is less certain for outcomes. Therefore, reporting feelings of helplessness may make experiences of IPA more salient, resulting in a potential increase in the types and frequency of IPA reported. A related limitation concerns the time-period surveyed in the research. Coercive control is currently conceptualized as the cumulative outcome of a pattern of abusive behaviors (Stark, 2010). Chapter 4 did not find evidence of a profile equating to Johnson’s (1995; 2006) 'coercive controlling violence' type. Similarly, Chapter 5 did not find evidence to support the existence of coercive control as it is currently conceptualized, however, both studies concentrated on participants' experiences of IPA in the past 12 months. This, therefore, raises the question as to whether this time-period is too narrow to account for a 'sufficient' amount of IPA to have occurred to equate to coercive control? The narrow time interval must, therefore, be considered a limitation of this research, despite the increased accuracy (Jaccard et al., 2004) and predictive validity (Nunnally \& Bernstein, 1994) attributed to shorter timeframes. Future research could employ a longitudinal design, in which the outcome is measured at a different time-point to the behavior and include measures of trauma to assess the effects of IPA on participants. This may result in more accurate reporting of IPA and facilitate the investigation into the development of coercive control.

A third limitation of this research is the use of MTurk samples. These samples are not nationally representative (Stewart et al., 2017), which limits the generalizability of the findings. 
Although the number of individuals identifying as Latinx $(6.4 \%)$ in the current research is considerably smaller than what is reported in recent population statistics (18.5\%; United States Census Bureau, 2019), the proportions of African Americans (12.2\%), Asian Americans (5.6\%), and Native Americans (1.2\%) in the MTurk samples were comparable to that of the United States population (13.4\%, 5.9\%, 1.3\% respectively; United States Census Bureau). A higher number of individuals identified as European American (66.1\%) and with more than one ethnic group (9.6\%) in this research than is reported in recent census data $(2019 ; 60.1 \%$ and $2.8 \%$ respectively). However, another crowdsourcing platform, Prolific Academic, currently offers data collection from nationally representative samples in the United Kingdom and United States (Prolific, 2019). Future research sampling these populations could, therefore, employ this platform to increase the generalizability of findings. The non- naïvety of participants in MTurk samples is an additional concern with data collected using this method (Chandler et al., 2014), as previous exposure to research tasks has been empirically shown to reduce the effect size of associated findings (Chandler et al., 2015). An empirical comparison of MTurk, Prolific Academic, and Crowdflower samples revealed the participants from Prolific Academic and Crowdflower were more naïve than MTurk participants. However, the reliability and reproducibility of the data from Crowdflower was low, making Prolific Academic the preferred alternative to MTurk (Peer et al., 2015). In a replication of the Peer et al. (2015) study, Adams et al. (2020) found comparable levels of reliability, dishonest behavior, reproducibility, and response rate between Prolific Academic and MTurk. Prolific Academic participants were found to be more naïve, ethnically diverse and had lower rates of attention check failure than MTurk workers. While Prolific Academic would appear to be an appealing alternative to MTurk, the cost of the service is considerably higher (see Prolific, 2021) than MTurk, and was, therefore, not considered as a viable option for data collection. 
Finally, we were limited by only sampling 'men and 'women'. This study tested the validity of some of the assumptions of the common conceptualizations of control, which are predominantly gendered. This focus was necessitated due to the dominance of the gendered perspective in the literature. It is, however, acknowledged that the findings from this research do not generalize to gender or sexual minorities. A greater understanding of the etiology of IPA in gender and sexual minorities is necessary due to their increased rates of IPA, relative to heterosexual populations (see Dank et al., 2014; Walters et al., 2013). While research has identified some of the risk factors for IPA, which include a need for control, for gender and sexual minorities, are similar to those for heterosexual populations (see Edwards et al., 2015 for a review), future research needs to go beyond correlations to ascertain the relative importance of these risk factors. This will assist in determining whether specific interventions and treatment programs are necessary for these populations and if so, what the most appropriate treatment targets would be.

This thesis did not find evidence to support the assumptions that underlie the common conceptualizations of control. At the heart of this problem is the application and reliance on existing gendered theory of IPA and a lack of critical analysis of the constructs underpinning IPA. The current hypothetico-deductive approach emphasizes theory testing and views phenomena as predictors of a given theory (Haig, 2005). This approach has, therefore, limited our understanding and the development of control as a construct. In contrast, the abductive theory of method attempts to provide explanations for phenomena (Haig). Future research should, therefore, adopt abductive methods of theory generation to develop causal explanations (Haig) of control.

This thesis has focussed on control in the context of IPA. However, control motivations were not found to be uniquely associated with IPA and it was, thus, suggested IPA may not be substantially different from aggression in other types of relationships. Indeed, control dynamics 
and aggression co-exist in other dyadic relationships (e.g., caregiver/ patient; parent/ child; school bully/ victim). In constructing theories of control, future research should, therefore, broaden the scope of inquiry to consider control dynamics in other relationships. For example, elder abuse shares many similarities with how control in intimate relationships is currently conceptualized (e.g., social isolation, economic dependence, physical abuse: Lachs \& Pillemer, 2015). Increased longevity coupled with decreasing availability of resources to care for this aging population (Carney, 2019) emphasizes the importance of research in this area.

\section{Conclusion}

This thesis aimed to investigate how control could best be conceptualized and measured. Using current measurement, the results collectively show that control cannot accurately be identified as a unique construct using a particular type or subset of behaviors (Chapters 2, 4, 5) or outcomes of IPA (Chapter 5). Nor is control associated with an increased frequency of physical and psychological IPA (Chapters 4, 5), or gender (Chapters 3, 4, 5), as is commonly accepted. Indeed, the findings question the validity of the commonly accepted understanding of control in relation to IPA and therefore its appropriateness in guiding practice, policy, legislation, and research. This dearth of empirically derived knowledge is surprising given the theoretical and practical importance control has been afforded in the last 50 years. It is likely that control is a valid concept but that it has not been conceptualized accurately making measurement of the phenomena with the currently available tools/scales difficult. As conceptualization is regarded as the first step in measurement (DeCarlo, 2019), it is perhaps necessary then to clarify the conceptualization of control from which valid and reliable scales can be developed. It is therefore argued that there is a need to develop evidence-based theories and conceptualizations of control, which reflect the lived experiences of individuals, irrespective of their gender or sexuality. This will in turn influence the 
development of more effective gender-inclusive policies and interventions to ensure that the causes and effects of IPA are effectively addressed. 


\section{REFERENCES}

References marked with an asterisk indicate they were included in the systematic review

A'Court, A. (2020). Chivalry vs. patriarchy: Exploring the psychological mechanisms of physical intimate partner violence (IPV) [Masters thesis, Victoria University of Wellington]. NZResearch.org.nz.

https://nzresearch.org.nz/records?utf8=\%E2\%9C\%93\&text=a\%27court

Adams, T., Li, Y., \& Liu, H. (2020). A replication of beyond the Turk: Alternative platforms for crowdsourcing behavioral research - sometimes preferable to student groups. AIS Transactions on Replication Research, 6, 15. doi.org/10.17705/1atrr.00058

American Psychological Association. (2001). Publication manual of the American Psychological Association (5th ed.). American Psychological Association.

Anderson, K. L. (2008). Is partner violence worse in the context of control? Journal of Marriage and Family, 70(5), 1157-1168. https://doi.org/10.1111/j.17413737.2008.00557.xArbuckle, J. L. (2017). Amos (Version 25.0). [Computer software]. IBM SPSS.

Arbuckle, J. L. (2017). Amos (Version 25.0). [Computer software]. IBM SPSS

Archer, J. (2000). Sex differences in aggression between heterosexual partners: A metaanalytic review. Psychological bulletin, 126(5), 651-680. https://doi.org/10.1037/0033-2909.126.5.651

Archer, J. (2002). Sex differences in physically aggressive acts between heterosexual partners. Aggression and Violent Behavior, 7(4), 313-351. https://doi.org/10.1016/S13591789(01)00061-1

Archer, J. (2018). Violence to partners: Gender symmetry revisited. In J. L. Ireland, P. Birch, \& C. A. Ireland (Eds.), The Routledge international handbook of human aggression: Current issues and perspectives (pp. 155-169). Routledge.

Archer, J., \& Browne, K. (1989). Human aggression: Naturalistic approaches. Routledge.

Arias, I., \& Beach, S. R. H. (1987). Validity of self-reports of marital violence. Journal of Family Violence, 2, 139-149. https://doi.org/10.1007/BF00977038

Babcock, J. C., Green, C. E., \& Robie, C. (2004). Does batterers' treatment work? A metaanalytic review of domestic violence treatment. Clinical Psychology Review, 23(8), 1023-1053. https://doi.org/10.1016/j.cpr.2002.07.001

* Babcock, J. C., Miller, S., \& Siard, C. (2003). Toward a typology of abusive women: Differences between partner-only and generally violent women in the use of violence. Psychology of Women Quarterly, 27(2), 153-161. https://doi.org/10.1111/14716402.00095 
Babcock, J. C., Snead, A. L., Bennett, V. E., \& Armenti, N. A. (2019). Distinguishing subtypes of mutual violence in the context of self-defense: Classifying types of partner violent couples using a modified Conflict Tactics Scale. Journal of Family Violence, 34(7), 687-696. https://doi.org/10.1007/s10896-018-0012-2

Bair-Merritt, M. H., Crowne, S. S., Thompson, D. A., Sibinga, E., Trent, M., \& Campbell, J. (2010). Why do women use intimate partner violence? A systematic review of women's motivations. Trauma Violence Abuse, 11(4), 178-189. https://doi.org/10.1177/1524838010379003

Bandalos, D. L. (2002). The effects of item parceling on goodness-of-fit and parameter estimate bias in structural equation modeling. Structural Equation Modeling, 9, 78102. https://doi.org/10.1207/S15328007SEM0901_5

* Barnett, O. W., Lee, C. Y., \& Thelen, R. E. (1997). Gender differences in attributions of self-defense and control in interpartner aggression. Violence Against Women, 3(5), 462-481. https://doi.org/10.1177/1077801297003005002

Bates, E. A., \& Graham-Kevan, N. (2016). Is the presence of control related to help-seeking behavior? A test of Johnson's assumptions regarding sex differences and the role of control in intimate partner violence. Partner Abuse, 7(1), 3-25. https://doi.org/10.1891/1946-6560.7.1.3

Bates, E. A., Graham-Kevan, N., \& Archer, J. (2014). Testing predictions from the male control theory of men's partner violence. Aggressive Behavior, 40, 42-55. https://doi.org/10.1002/ab.21499

Beck, C. J. A., Menke, J. M., Brewster, K. O., \& Figueredo, A. J. (2009). Validation of a measure of intimate partner abuse with couples participating in divorce mediation. Journal of Divorce \& Remarriage, 50(5), 295-308. https://doi.org/10.1080/10502550902766399

Beck, C. J. A., Menke, J. M., \& Figueredo, A. J. (2013). Validation of a measure of intimate partner abuse (Relationship Behavior Rating Scale-Revised) using item response theory analysis. Journal of Divorce \& Remarriage, 54(1), 58-77. https://doi.org/10.1080/10502556.2012.743830

Bell, K. M., \& Naugle, A. E. (2008). Intimate partner violence theoretical considerations: Moving towards a contextual framework. Clinical Psychology Review, 28(7), 10961107. https://doi.org/10.1016/j.cpr.2008.03.003

* Belus, J., Wanklyn, S., Iverson, K., Pukay-Martin, N., Langhinrichsen-Rohling, J., \& Monson, C. (2014). Do anger and jealousy mediate the relationship between adult attachment styles and intimate violence perpetration? Partner Abuse, 5(4), 388-406. https://doi.org/10.1891/1946-6560.5.4.388

Bloomfield, S., \& Dixon, L. (2015). An outcome evaluation of the Integrated Domestic Abuse Programme (IDAP) and Community Domestic Violence Programme (CDVP) (Annual Summary). National Offender Management Service. https://www.gov.uk/government/uploads/system/uploads/attachment_data/file/449008 /outcomeevaluation-idap-cdvp.pdf

Bonta, J., \& Andrews, D. A. (2017). The psychology of criminal conduct (6th ed.). Routledge.https://doi.org/10.1111/bjop.12254 
Borenstein, M., Hedges, L., Higgins, J., \& Rothstein, H. (2009). Introduction to metaanalysis. https://doi.org/10.1002/9780470743386

Borenstein, M., Hedges, L. V., Higgins, J, \& Rothstein, H. (2010). A basic introduction to fixed-effect and random-effects models for meta-analysis. Research Synthesis Methods, 1(2), 97-111. https://doi.org/10.1002/jrsm.12

Borenstein, M., Hedges, L., Higgins, J., \& Rothstein, H. (2011). Comprehensive MetaAnalysis (Version 2.2.064). [Computer software]. Biostat, Inc.

Borsboom, D. (2008). Latent variable theory. Measurement: Interdisciplinary Research and Perspectives, 6(1-2), 25-53. https://doi.org/10.1080/15366360802035497

Braverman, M. T., \& Arnold, M. E. (2008). An evaluator's balancing act: Making decisions about methodological rigor. New Directions for Evaluation, 2008(120), 71-86. https://doi.org/10.1002/ev.277

Brown, T. (2014). Confirmatory factor analysis for applied research (2nd ed.) https://ebookcentral.proquest.com/lib/vuw/reader.action?docID=1768752

Browne, M., \& Cudeck, R. (1992). Alternative ways of assessing model fit. Sociological Methods \& Research, 21(2), 230-258. https://doi.org/10.1177/0049124192021002005

Buhrmester, M., Kwang, T., \& Gosling, S. D. (2011). Amazon's Mechanical Turk: A new source of inexpensive, yet high-quality, data? Perspectives on Psychological Science, 6(1), 3-5. https://doi.org/10.1177/1745691610393980

Bushman, B. J., \& Anderson, C. A. (2001). Is it time to pull the plug on the hostile versus instrumental aggression dichotomy? Psychological Review, 108(1), 273-279. https://doi.org/10.1037/0033-295X.108.1.273

Buss, A. H. (2002). Human mate guarding. Neuroendocrinology Letters, 4(23), 23-29.

Cabrera-Nguyen, P. (2010). Author guidelines for reporting scale development and validation results in the journal of the society for social work and research. Journal of the Society for Social Work and Research, 1(2), 99-103. https://doi.org/10.5243/jsswr.2010.8

* Caldwell, J. E., Swan, S. C., Allen, C. T., Sullivan, T. P., \& Snow, D. L. (2009). Why I hit him: Women's reasons for intimate partner violence. Journal of Aggression, Maltreatment \& Trauma, 18(7), 672-697. https://doi.org/10.1080/10926770903231783

Campbell, A., Muncer, S., \& Bibel, D. (1985). Taxonomies of aggressive behavior: A preliminary report. Aggressive Behavior, 11(3), 217-222. https://doi.org/10.1002/1098-2337(1985)11:3<217::AID-AB2480110304>3.0.CO;2-2

Campbell, J. C., Sharps, P., \& Glass, N. E. (2000). Risk assessment for intimate partner violence. In G. F. Pinard \& L. Pagani (Eds.), Clinical assessment of dangerousness: Empirical contributions (pp. 136-167). Cambridge University Press

Card, N. A. (2012). Applied meta-analysis for social science research. The Guildford Press.

Carlson, R. G., \& Jones, K. D. (2010). Continuum of conflict and control: A conceptualization of intimate partner violence typologies. The Family Journal, 18(3), 248-254. https://doi.org/10.1177/1066480710371795 
Carney, A. (2019). Elder abuse: Forensic, legal and medical aspects. ProQuest Ebook Central. https://ebookcentral-proquest-com.helicon.vuw.ac.nz

* Carrado, M., George, M., Loxam, E., Jones, L., \& Templar, D. (1996). Aggression in British heterosexual relationships: A descriptive analysis. Aggressive Behavior, 22(6), 401-415. https://doi.org/10.1002/(SICI)1098-2337(1996)22:63.0.CO;2-K

Cascardi, M., Blank, S., \& Dodani, V. (2019). Comparison of the CADRI and CTS2 for measuring psychological and physical dating violence perpetration and victimization. Journal of Interpersonal Violence, 34(16), 3466-3491. https://doi.org/10.1177/0886260516670182

Cascardi, M., \& Vivian, D. (1995). Context for specific episodes of marital violence: Gender and severity of violence differences. Journal of Family Violence, 10(3), 265-293. https://doi.org/10.1007/BF02110993

Cattell, R. B. (1966). The scree test for the number of factors. Multivariate Behavioral Research, 1(2), 245-276. https://doi.org/10.1207/s15327906mbr0102_10

Cattell, R. B., \& Burdsal, C. A., Jr. (1975). The radial parceling double factoring design: A solution to the item-vs.-parcel controversy. Multivariate Behavioral Research, 10, 165-179. https://doi.org/10.1207/s15327906mbr1002_3

Chandler, J., Mueller, P., \& Paolacci, G. (2014). Nonnaïveté among Amazon Mechanical Turk workers: Consequences and solutions for behavioral researchers. Behavior Research Methods, 46, 112-130. https://doi.org/10.3758/s13428-013-0365-7

Chandler, J., Paolacci, G., Peer, E., Mueller, P., \& Ratliff, K. (2015). Non-naïve participants can reduce effect sizes. Psychological Science, 26(7), 1131-1139. https://doi.org/10.1177/0956797615585115

Cohen, J. (1969). Statistical power analysis for the behavioral sciences. Academic Press.

Coker, A. L., Davis, K. E., Arias, I., Desai, S., Sanderson, M., Brandt, H. M., \& Smith, P. H. (2002). Physical and mental health effects of intimate partner violence for men and women. American Journal of Preventive Medicine, 23(4), 260-268. https://doi.org/10.1016/s0749-3797(02)00514-7

Coleman, D. H., \& Straus, M. A. (1986). Marital power, conflict, and violence in a nationally representative sample of American couples. Violence \& Victims, 1, 141-157. https://doi.org/10.4324/9781315126401-21

Collins, L. M., \& Lanza, S. T. (2010). Latent class and latent transition analysis with applications in the social behavioral, and health sciences. Wiley. https://doi.org/10.1002/9780470567333

Comrey, A. L. (1973). A first course in factor analysis. Academic Press.

Coser, L. A. (1957). Social conflict and the theory of social change. The British Journal of Sociology, 8(3), 197-207. https://doi.org/0.2307/586859

Cosmides, L., \& Tooby, J. (2000). Evolutionary psychology and the emotions. In M. Lewis \& J. M. Haviland-Jones (Eds.), Handbook of emotions (2nd ed., pp. 91-115). Guilford. 
Courtney, M. G. R. (2013). Determining the number of factors to retain in EFA: Using the SPSS R-Menu v2.0 to make more judicious estimations. Practical Assessment, Research \& Evaluation, 18(8), 1-14. https://doi.org/10.7275/9cf5-2m72

Cousins, A., \& Gangestad, S. (2007). Perceived threats of female infidelity, male proprietariness, and violence in college dating couples. Violence \& Victims, 22(6), 651-668. https://doi.org/10.1891/088667007782793156

Crossman, K. A., \& Hardesty, J. L. (2018). Placing coercive control at the center: what are the processes of coercive control and what makes control coercive? Psychology of Violence, 8(2), 196-206. https://doi.org/10.1037/vio0000094

Dank, M., Lachman, P., Zweig, J., \& Yahner, J. (2014). Dating violence experiences of lesbian, gay, bisexual, and transgender youth. J Youth Adolescence, 43(5), 846-857. https://doi.org/10.1007/s10964-013-9975-8

DeCarlo, M. (2019). Scientific inquiry in social work ( ${ }^{\text {rd }}$ ed.). https://scientificinquiryinsocialwork.pressbooks.com

* DeKeseredy, W. S., Saunders, D. G., Schwartz, M. D., \& Alvi, S. (1997). The meanings and motives for women's use of violence in Canadian college dating relationships: Results from a national survey. Sociological Spectrum, 17(2), 199-222. https://doi.org/10.1080/02732173.1997.9982160

Dempsey, F., Hammond, M. D., \& Dixon, L. (2020). Investigating whether controlling and aggressive relationship behaviors are discriminant. Aggressive Behavior(46), 437-448. https://doi.org/10.1002/ab.21905

Desmarais, S. L., Reeves, K. A., Nicholls, T. L., Telford, R. P., \& Fiebert, M. S. (2012). Prevalence of physical violence in intimate relationships: Part 2. Rates of male and female perpetration. Partner Abuse, 3(2), 1-10. https://doi.org/10.1891/19466560.3.2.e2

DeVellis, R. (2012). Scale development: Theory and applications (3rd ed.). SAGE.

Dichter, M. E., Thomas, K. A., Crits-Christoph, P., Ogden, S. N., \& Rhodes, K. V. (2018). Coercive control in intimate partner violence: Relationship with women's experience of violence, use of violence, and danger. Psychology of Violence, 8(5), 596-604. https://doi.org/10.1037/vio0000158

Dillon, G., Hussain, R., Loxton, D., Rahman, S., \& Sajaratulnisah, O. (2013). Mental and physical health and intimate partner violence against women: A review of the literature. International Journal of Family Medicine, 2013, 1-15. https://doi.org/10.1155/2013/313909

Dixon, L., Archer, J., \& Graham-Kevan, N. (2012). Perpetrator programmes for partner violence: Are they based on ideology or evidence? Legal and Criminological Psychology, 17(2), 196-215. https://doi.org/10.1111/j.2044-8333.2011.02029.x

Dixon, L., \& Graham-Kevan, N. (2011). Understanding the nature and etiology of intimate partner violence and implications for practice and policy. Clinical Psychology Review, 31(7), 1145-1155. https://doi.org/10.1016/j.cpr.2011.07.001 
Dixon, L., \& Graham-Kevan, N. (2020). Assessing the risk and treatment needs of people who perpetrate intimate partner violence. In J. S. Wormith, L. A. Craig, \& T. E. Hogue (Eds.), The Wiley handbook of what works in violence risk management: Theory, research and practice (pp. 297-314). John Wiley \& Sons Ltd. https://doi.org/10.1002/9781119315933

Dixon, L., Treharne, G. J., Celi, E. M., Hines, D. A., Lysova, A V., \& Douglas, E. M. (2020). Examining men's experiences of abuse from a female intimate partner in four Englishspeaking countries. Journal of Interpersonal Violence, 1-27. https://doi.org/10.1177/0886260520922342

Dixon, L., \& Wride, A. (2020). Classification of intimate partner aggression. Aggression \& Violent Behaviour. https://doi.org/10.1016/j.avb.2020.101437

Dobash, R. E., \& Dobash, R. P. (1979). Violence against wives. Free Press.

Dobash, R. E., \& Dobash, R. P. (1992). Women, violence and social change. Routledge.

Dobash, R. P., \& Dobash, R. E. (2004). Women's violence to men in intimate relationships: Working on a puzzle. The British Journal of Criminology, 44(3), 324-349. https://doi.org/10.1093/bjc/azh026

Domestic Abuse Act 2018 (Scotland). Retrieved from https://www.legislation.gov.uk/asp/2018/5

Domestic Violence Act 2018 (Ireland). Retrieved from http://www.irishstatutebook.ie/eli/2018/act/6/section/39/enacted/en/html.

Durant, L. E., Carey, M. P., \& Schroder, K. E. E. (2002). Effects of anonymity, gender and erotophilia on the quality of data obtained from self-reports of socially sensitive behaviors. Journal of Behavioral Medicine, 25, 439-467. https://doi.org/10.1023/A:1020419023766

Dutton, D. G. (1995a). Male abusiveness in intimate relationships. Clinical Psychology Review, 15, 567-581. https://doi.org/10.1016/0272-7358(95)00028-N

Dutton, D. G. (1995b). The domestic assault of women: Psychological and criminal justice perspectives. UBC Press.

Dutton, D. G. (2006a). Rethinking domestic violence. UBC Press. https://ebookcentralproquest-com.helicon.vuw.ac.nz/lib/vuw/detail.action?docID=3412393\&pqorigsite $=$ primo

Dutton, D. G. (2006b). On comparing apples with apples deemed nonexistent: A reply to Johnson. Journal of Child Custody, 2(4), 53-63. https://doi.org/10.1300/J190v02n04_04

Dutton, M. A. (1999). Multidimensional assessment of woman battering: Commentary on Smith, Smith, and Earp. Psychology of Women Quarterly, 23(1), 195-198. https://doi.org/10.1111/j.1471-6402.1999.tb00351.x

Dutton, M. A., Goodman, L., \& Schmidt, R. J. (2005). Development and validation of a coercive control measure for intimate partner violence: Final technical report. https://www.ncjrs.gov/App/Publications/abstract.aspx?ID=235977 
Edwards, K. M., Sylaska, K. M., \& Neal, A. M. (2015). Intimate partner violence among sexual minority populations: A critical review of the literature and agenda for future research. Psychology of Violence, 5(2), 112-121. https://doi.org/10.1037/a0038656

Egger, M., Smith, G., Schneider, M., \& Minder, C. (1997). Bias in meta-analysis detected by a simple, graphical test. BMJ, 315(7109), 629-634. https://doi.org/10.1136/bmj.315.7109.629

Ehrensaft, M. K., Langhinrichsen-Rohling, J., Heyman, R. E., O'Leary, K. D., \& Lawrence, E. (1999). Feeling controlled in marriage: A phenomenon specific to physically aggressive couples? Journal of Family Psychology, 13(1), 20-32.

Ehrensaft, M. K., Moffitt, T. E., \& Caspi, A. (2004). Clinically abusive relationships in an unselected birth cohort: men's and women's participation and developmental antecedents. Journal of Abnormal Psychology, 113(2), 258-270. https://doi.org/10.1037/0021-843X.113.2.258

* Elmquist, J., Hamel, J., Shorey, R., Labrecque, L., Ninnemann, A., \& Stuart, G. (2014). Motivations for intimate partner violence in men and women arrested for domestic violence and court referred to batterer intervention programs. Partner Abuse, 5(4), 359-374. https://doi.org/10.1891/1946-6560.5.4.359

Esquivel-Santoveña, E. E., \& Dixon, L. (2012). Investigating the true rate of physical intimate partner violence: A review of nationally representative surveys. Aggression and Violent Behavior, 17(3), 208-219. https://doi.org/10.1016/j.avb.2012.02.002

Family Violence Death Review Committee. (2016). Fifth Report: January 2014 to December 2015. Health Quality \& Safety Commission New Zealand. https://www.hqsc.govt.nz/our-programmes/mrc/fvdrc/publications-andresources/publication/2434/

Faul, F., Erdfelder, E., Buchner, A., \& Lang, A. G. (2009). Statistical power analyses using $\mathrm{G}^{*}$ Power 3.1: Tests for correlation and regression analyses. Behavior Research Methods, 41, 1149-1160. https://doi.org/10.3758/BRM.41.4.1149

Felson, R. B., \& Outlaw, M. C. (2007). The control motive and marital violence. Violence and Victims, 22(4), 387-407. https://doi.org/10.1891/088667007781553964

* Fernández-Fuertes, A. A., \& Fuertes, A. (2010). Physical and psychological aggression in dating relationships of Spanish adolescents: Motives and consequences. Child Abuse \& Neglect, 34, 183-191. https://doi.org/10.1016/j.chiabu.2010.01.002

* Fiebert, M., \& Gonzalez, D. (1997). College women who initiate assaults on their male partners and the reasons offered for such behavior. Psychological Reports, 80(2), 583590. https://doi.org/10.2466/pr0.1997.80.2.583

Field, A. (2009). Discovering statistics using SPSS (3rd ed.). SAGE Publications.

Follingstad, D. R. (2007). Rethinking current approaches to psychological abuse: Conceptual and methodological issues. Aggression and Violent Behavior, 12(4), 439-458. https://doi.org/10.1016/j.avb.2006.07.004

Follingstad, D. R., \& Rogers, M. (2013). Validity concerns in the measurement of women's and men's report of intimate partner violence. Sex Roles, 69(3-4), 149-167. https://doi.org/10.1007/s11199-013-0264-5 
* Follingstad, D. R., Wright, S., Lloyd, S., \& Sebastian, J. A. (1991). Sex differences in motivations and effects in dating violence. Family Relations, 40(1), 51-57. https://doi.org/10.2307/585658

Ford, D. A., \& Regoli, M. J. (1993). The criminal prosecution of wife batterers: Process, problems, and effects. In N. Z. Hilton (Ed.), Legal responses to wife assault (pp. 127164). SAGE.

Foshee, V. A. (1996). Gender differences in adolescent dating abuse prevalence, types, and injuries. Health Education Research, 11(3), 275-286. https://doi.org/10.1093/her/11.3.275-a

Gannon, T. A., Olver, M. E., Mallion, J. S., \& James, M. (2019). Does specialized psychological treatment for offending reduce recidivism? A meta-analysis examining staff and program variables as predictors of treatment effectiveness. Clinical Psychology Review, 73, 101752. https://doi.org/10.1016/j.cpr.2019.101752

Garcia-Moreno, C., \& Watts, C. (2011). Violence against women: An urgent public health priority. Bulletin of the World Health Organization, 89. https://doi.org/10.2471/BLT.10.085217

Garner, B., \& Black, H. (2014). Black's law dictionary (10th ed.). Thomson Reuters.

Gaskin, C. J., \& Happell, B. (2014). On exploratory factor analysis: A review of recent evidence, an assessment of current practice, and recommendations for future use. International Journal of Nursing Studies, 51(3), 511-521. https://doi.org/10.1016/j.ijnurstu.2013.10.005

Geiser, C. (2012). Data analysis with Mplus. https://doi.org/10.1177/0165025419881721

Giles, D. (2002). Advanced research methods in psychology https://ebookcentral.proquest.com/lib/vuw/detail.action?docID=1144492

Gottman, J. M., Jacobson, N. S., Rushe, R. H., Shortt, J. W., Babcock, J., La Taillade, J. J., \& Waltz, J. (1995). The relationship between heart rate reactivity, emotionally aggressive behavior, and general violence in batterers. Journal of Family Psychology, 9(3), 227-248. https://doi.org/10.1037/0893-3200.9.3.227

Graham-Kevan, N. (2007). Power and control in relationship aggression. In J. H. T. Nicholls (Ed.), Family interventions in domestic violence: A handbook of gender-inclusive theory and treatment (pp. 87-107). Springer Publishing.

Graham-Kevan, N., \& Archer, J. (2003). Intimate terrorism and common couple violence: A test of Johnson's predictions in four British samples. Journal of Interpersonal Violence, 18(11), 1247-1270. https://doi.org/10.1177/0886260503256656

Graham-Kevan, N., \& Archer, J. (2009). Control tactics and partner violence in heterosexual relationships. Evolution and Human Behavior, 30(6), 445-452. https://doi.org/10.1016/j.evolhumbehav.2009.06.007

Haig, B. D. (2005). An abductive theory of scientific method. Psychological Methods, 10(4), 371-388. https://doi.org/10.1037/1082-989X.10.4.371

Hair, J. F. (2010). Multivariate data analysis (7th ed.). Prentice Hall. 
Hamberger, L. K. (2005). Men's and women's use of intimate partner violence in clinical samples: Toward a gender-sensitive analysis. Violence and Victims, 20(2), 131-151. https://doi.org/10.1891/088667005780905632

Hamberger, L. K., \& Guse, C. E. (2002). Men's and women's use of intimate partner violence in clinical samples. Violence Against Women, 8(11), 1301-1331. https://doi.org/10.1177/107780102762478028

Hamberger, L. K., \& Guse, C. E. (2005). Typology of reactions to intimate partner violence among men and women arrested for partner violence. Violence and Victims, 20(3), 303-317. https://doi.org/10.1891/vivi.20.3.303

Hamberger, L., \& Larsen, K. (2015). Men's and women's experience of intimate partner violence: A review of ten years of comparative studies in clinical samples; Part I. Journal of Family Violence, 30(6), 699-717.

Hamberger, L. K., Larsen, S. E., \& Lehrner, A. (2017). Coercive control in intimate partner violence. Aggression and Violent Behavior, 37(Supplement C), 1-11. https://doi.org/10.1016/j.avb.2017.08.003

Hamel, J. (2007). Toward a gender-inclusive conception of intimate partner violence research and theory: Part 1-Traditional perspectives. International Journal of Men's Health, 6, 36-53. https://doi.org/10.3149/jmh.0601.36

Hamel, J. (2014). Gender-inclusive treatment of intimate partner abuse: A comprehensive approach. Springer Publishing.

Hamel, J., Jones, D. N., Dutton, D. G., \& Graham-Kevan, N. (2015). The CAT: A genderinclusive measure of Controlling and Abusive Tactics. Violence and Victims, 30(4), 547-580. https://doi.org/10.1891/0886-6708.VV-D-13-00027

Harden, A., \& Gough, D. (2012). Quality and relevance appraisal. In D. Gough, S. Oliver, \& J. Thomas (Eds.), An introduction to systematic reviews. SAGE.

Hardesty, J. L., Crossman, K. A., Haselschwerdt, M. L., Raffaelli, M., Ogolsky, B. G., \& Johnson, M. P. (2015). Toward a standard approach to operationalizing coercive control and classifying violence types. Journal of Marriage and Family, 77(4), 833843. https://doi.org/10.1111/jomf.12201

* Harned, M. (2001). Abused women or abused men? An examination of the context and outcomes of dating violence. Violence and Victims, 16(3), 269-285. https://doi.org/10.1891/0886-6708.16.3.269

Hart, C. M., Ritchie, T. D., Hepper, E. G., \& Gebauer, J. E. (2015). The Balanced Inventory of Desirable Responding Short Form (BIDR-16). SAGE Open, 5(4). https://doi.org/10.1177/2158244015621113

Hassouneh, D., \& Glass, N. (2008). The influence of gender role stereotyping on women's experiences of female same-sex intimate partner violence. Violence Against Women, 14(3), 310-325. https://doi.org/10.1177/1077801207313734

Hauser, D., \& Schwarz, N. (2016). Attentive Turkers: MTurk participants perform better on online attention checks than do subject pool participants. Behavior Research Methods, 48(1), 400-407. https://doi.org/10.3758/s13428-015-0578-z 
Hawker, S., Payne, S., Kerr, C., Hardey, M., \& Powell, J. (2002). Appraising the evidence: Reviewing disparate data systematically. Qualitative Health Research, 12(9), 12841299. https://doi.org/10.1177/1049732302238251

Heckert, D. A., \& Gondolf, E. W. (2000). Assessing assault self-reports by batterer program participants and their partners. Journal of Family Violence, 15, 181-197. https://doi:10.1023/a:1007594928605

Heffernan, R., Wegerhoff, D., \& Ward, T. (2019). Dynamic risk factors: Conceptualization, measurement, and evidence. Aggression and Violent Behavior, 48, 6-16. https://doi.org/10.1016/j.avb.2019.06.004

Hester, M., Jones, C., Williamson, E., Fahmy, E., \& Feder, G. (2017). Is it coercive controlling violence? A cross-sectional domestic violence and abuse survey of men attending general practice in England. Psychology of Violence, 7(3), 417-427. https://doi.org/10.1037/vio0000107

* Hettrich, E. L., \& O'Leary, K. D. (2007). Females' reasons for their physical aggression in dating relationships. Journal of Interpersonal Violence, 22(9), 1131-1143. https://doi.org/10.1177/0886260507303729

Higgins, J., Thompson, S., Deeks, J., \& Altman, D. (2003). Measuring inconsistency in metaanalyses. BMJ, 327(7414), 557-560. https://doi.org/10.1136/bmj.327.7414.557

Hines, D. A., Brown, J., \& Dunning, E. (2007). Characteristics of callers to the domestic abuse helpline for men. Journal of Family Violence, 22, 63-72. https://doi: 10.1007/s10896-006-9052-0

Hines, D. A., \& Douglas, E. M. (2010). Intimate terrorism by women towards men: Does it exist? Journal of Aggression, Conflict and Peace Research, 2(3), 36-56. https://doi.org/10.5042/jacpr.2010.0335

Hines, D. A., \& Douglas, E. M. (2018). Influence of intimate terrorism, situational couple violence, and mutual violent control on male victims. Psychology of Men \& Masculinity, 19(4), 612-623. https://doi.org/10.1037/men0000142

Hines, D. A., \& Douglas, E. M. (2019). An empirical test of Johnson's typology of intimate partner violence in two samples of men. Partner Abuse, 10(2), 180-198. https://doi.org/10.1891/1946-6560.10.2.180

Hinkin, T., R. (1998). A brief tutorial on the development of measures for use in survey questionnaires. Organizational Research Methods, 1(1), 104-121. https://doi.org/10.1177/109442819800100106

Holtzworth-Munroe, A., \& Stuart, G. L. (1994). Typologies of male batterers: Three subtypes and the differences among them. Psychological bulletin, 116(3), 476-497. https://doi.org/10.1037/0033-2909.116.3.476

Home Office (UK). (2013). Information for local areas on the change to the definition of domestic violence and abuse.

https://assets.publishing.service.gov.uk/government/uploads/system/uploads/attachme nt_data/file/142701/guide-on-definition-of-dv.pdf. 
Hong, Q. N., Pluye, P., Fàbregues, S., Bartlett, G., Boardman, F., Cargo, M., Dagenais, P., Gagnon, M.-P., Griffiths, F., Nicolau, B., O’Cathain, A., Rousseau, M.-C., \& I., V. Mixed Methods Appraisal Tool (MMAT) (Version 2018). Registration of Copyright (\#1148552). Canadian Intellectual Property Office, Industry Canada.

Horn, J. L. (1965). A rationale and test for the number of factors in factor analysis. Psychometrika, 30(2), 179-185. https://doi.org/10.1007/BF02289447

Hou, J., Yu, L., Ting, S., Sze, Y., \& Fang, X. (2011). The status and characteristics of couple violence in China. Journal of Family Violence, 26(2), 81-92. https://doi.org/10.1007/s10896-010-9343-3

Howard, M. C. (2016). A review of exploratory factor analysis decisions and overview of current practices: What we are doing and how can we improve? International Journal of Human-Computer Interaction, 32(1), 51-62. https://doi.org/10.1080/10447318.2015.1087664

IBM Corp. (2017). IBM SPSS Statistics for Windows (Version 25.0). [Computer software]. IBM Corp.

IBM Corp. (2019a). IBM SPSS Statistics for Windows (Version 26.0). [Computer software]. IBM Corp.

IBM Corp. (2019b). IBM SPSS Categories 26 [Computer software]. IBM Corp.

Ireland, J. L. (2009). Conducting individualised theory-driven assessments of violent offenders. In J. Ireland, C. Ireland, \& P. Birch (Eds.), Violent and sexual offenders: Assessment, treatment and management (pp. 68-96). Willan Publishing.

Jaccard, J., McDonald, R., Wan, C. K., Guilamo-Ramos, V., Dittus, P., \& Quinlan, S. (2004). Recalling sexual partners: The accuracy of self-reports. Journal of Health Psychology, 9, 699-712. https://doi:10.1177/1359105304045354

Johnson, M. P. (1995). Patriarchal terrorism and common couple violence: Two forms of violence against women. Journal of Marriage and the Family Relations, 57, 283-294. https://doi.org/10.2307/353683

Johnson, M. P. (1999, November). Two types of violence against women in the American family: Identifying patriarchal terrorism and common couple violence [Paper presentation]. National Council on Family Relations, Irvine, CA, United States.

Johnson, M. P. (2006). Conflict and control: Symmetry and asymmetry in domestic violence. Violence Against Women, 12, 1003-1018. https://doi.org/10.1177/1077801206293328

Johnson, M. P. (2008). A typology of domestic violence: Intimate terrorism, violent resistance, and situational couple violence. Northeastern University Press.

Joinson, A. N. (1999). Social desirability, anonymity, and Internet-based questionnaires. Behavior Research Methods, 31(3), 433-438. https://doi.org/10.3758/BF03200723

Kaiser, H. F. (1974). An index of factorial simplicity. Psychometrika, 39(1), 31-36. https://doi.org/10.1007/BF02291575 * Kelley, E. L., Edwards, K. M., Dardis, C. M., \& Gidycz, C. A. (2015). Motives for physical dating violence among college students: A gendered analysis. Psychology of Violence, 5(1), 56-65. https://doi.org/10.1037/a0036171 
Kelly, J. B., \& Johnson, M. P. (2008). Differentiation among types of intimate partner violence: Research update and implications for interventions. Family Court Review, 46, 746-499. https://doi.org/10.1111/j.1744-1617.2008.00215.x

* Kernsmith, P. (2005). Exerting power or striking back: A gendered comparison of motivations for domestic violence perpetration. Violence \& Victims, 20(2), 173-185. https://doi.org/10.1891/088667005780905605

Kernsmith, P. (2006). Gender differences in the impact of family of origin violence on perpetrators of domestic violence. Journal of Family Violence, 21(2), 163-171. https://doi.org/10.1007/s10896-005-9014-y

Kline, R. B. (2005). Principles and practice of structural equation modeling, (2nd ed.). Guilford Publications.

Koss, M. P., Goodman, L. A., Browne, A., Fitzgerald, L. F., Keita, G. P., \& Russo, N. F. (1994). Understanding the perpetrator and the victim: Who abuses and who is abused? In M. P. Koss, L. A. Goodman, \& A. Browne (Eds.), No safe haven: Male violence against women at home, at work, and in the community (pp. 19-38). American Psychological Association.

Krug, E. G., Dahlberg, L. L., Mercy, J. A., Zwi, A., \& Lozano-Ascencio, R. (2002). World report on violence and health. Organisation mondiale de la Santé. https://www.who.int/violence_injury_prevention/violence/world_report/en/

Kyriazos, T. A. (2018). Applied psychometrics: Sample size and sample power considerations in factor analysis (EFA, CFA) and SEM in general. Psychology, 9, 2207-2230. https://doi.org/10.4236/psych.2018.98126

Lachs, P., \& Pillemer, K. (2015). Elder abuse. The New England Journal of Medicine, 373(20), 1947-1956. https://doi.org/10.1056/nejmra1404688

Landenburger, K. (1989). A process of entrapment in and recovery from an abusive relationship. Issues in Mental Health Nursing, 10(3-4), 209-227. https://doi.org/10.3109/01612848909140846

Langhinrichsen-Rohling, J. (2010). Controversies involving gender and intimate partner violence in the United States. Sex Roles, 62(3), 179-193. https://doi.org/10.1007/s11199-009-9628-2

Langhinrichsen-Rohling, J., McCullars, A., \& Misra, T. A. (2012). Motivations for men and women's intimate partner violence perpetration: A comprehensive review. Partner Abuse, 3(4), 429-468. https://doi.org/10.1891/1946-6560.3.4.429

Langhinrichsen-Rohling, J., Neidig, P., \& Thorn, G. (1995). Violent marriages: Gender differences in levels of current violence and past abuse. Journal of Family Violence, 10, 159-176. https://doi:10.1007/ BF02110598

Lanza, S. T., Bray, B. C., \& Collins, L. M. (2013). An introduction to latent class and latent transition analysis. In I. Weiner (Ed.), Research methods in psychology (2nd ed., pp. 691-716). John Wiley \& Sons, Inc.

Laroche, D. (2005). Aspects of the context and consequences of domestic violence: Situational couple violence and intimate terrorism in Canada in 1999. Institut de la statistique du Québec. 
Lehmann, P., Simmons, C. A., \& Pillai, V. K. (2012). The validation of the Checklist of Controlling Behaviors (CCB): Assessing coercive control in abusive relationships. Violence Against Women, 18(8), 913-933. https://doi.org/10.1177/1077801212456522

Leisch, F., Weingessel, A., \& Hornik, K. (2012). Generation of artificial binary data (Version 0. 9-19). [R package]. https://CRAN.R-project.org/package=bindata

* Leisring, P. A. (2013). Physical and emotional abuse in romantic relationships: Motivation for perpetration among college women. Journal of Interpersonal Violence, 28(7), 1437-1454. https://doi.org/10.1177/0886260512468236

Leonard, K. E., \& Senchak, M. (1996). Prospective prediction of husband marital aggression within newlywed couples. Journal of Abnormal Psychology, 105, 369-380. https://doi.org/10.1037/0021-843X.105.3.369

Linting, M., Meulman, J. J., Groenen, P. J. F., \& Van Der Kooij, A. J. (2007). Nonlinear principal components analysis: Introduction and application. Psychological Methods, 12(3), 336-358. https://doi.org/10.1037/1082-989X.12.3.336

Linting, M., Os, B., \& Meulman, J. (2011). Statistical significance of the contribution of variables to the PCA solution: An alternative permutation strategy. Psychometrika, 76(3), 440-460. https://doi.org/10.1007/s11336-011-9216-6

Linting, M., \& van der Kooij, A. J. (2012). Nonlinear principal components analysis with CATPCA: A tutorial. Journal of Personality Assessment, 94(1), 12-25. https://doi.org/10.1080/00223891.2011.627965

Little, R. J. A., \& Rubin, D. B. (2002). Statistical analysis with missing data (2nd ed.). New Jersey: John Wiley \& Sons, Inc.

Lo, Y., Mendell, N. R., \& Rubin, D. B. (2001). Testing the number of components in a normal mixture. Biometrika, 88(3), 767-778. https://doi.org/10.1093/biomet/88.3.767

Loseke, D. R., Gelles, R. J., \& Cavanaugh, M. M. (2005). Section I: Controversies in conceptualisation. In D. R. Loseke, R. J. Gelles, \& M. M. Cavanaugh (Eds.), Current controversies on family violence (2nd ed., pp. 1-4). SAGE.

MacCallum, R., Browne, M., \& Sugawara, H. (1996). Power analysis and determination of sample size for covariance structure modeling. Psychological Methods, 1(2), 130-149. https://doi.org/10.1037/1082-989X.1.2.130

* Makepeace, J. M. (1986). Gender differences in courtship violence victimization. Family Relations, 35(3), 383-388. https://doi.org/10.2307/584365

Matsunaga, M. (2008). Item parceling in structural equation modeling: A primer. Communication Methods and Measures, 2(4), 260-293. https://doi.org/10.1080/19312450802458935

McHugh, M. C., Rakowski, S., \& Swiderski, C. (2013). Men's experience of psychological abuse: Conceptualization and measurement issues. Sex Roles, 69(3), 168-181. https://doi.org/10.1007/s11199-013-0274-3

McNeish, D. (2016). On using Bayesian methods to address small sample problems. Structural Equation Modeling: A Multidisciplinary Journal, 23(5), 750-773. https://doi.org/10.1080/10705511.2016.1186549 
Milfont, T., \& Fischer, R. (2010). Testing measurement invariance across groups: applications in cross-cultural research. International Journal of Psychological Research, 3(1), 111. https://doi.org/10.21500/20112084.857

Ministry of Justice. (2014). New Zealand Crime \& Safety Survey. https://www.justice.govt.nz/justice-sector-policy/research-data/nzcass/resources-anddownloads/

Ministry of Justice. (2020). New Zealand Crime and Victims Survey: Cycle 2 (October 2018 September 2019). https://www.justice.govt.nz/justice-sector-policy/researchdata/nzcvs/\#survey-results

Moher, D., Liberati, A., Tetzlaff, J., \& Altman, D. G. (2009). Preferred reporting items for systematic reviews and meta-analyses: The PRISMA statement. Physical Therapy, 89(9), 873-880. https://doi.org/10.1136/bmj.b2535

Muthén, L. K., \& Muthén, B. O. (1998-2017a). Mplus (Version 8). [Computer software]. Muthén \& Muthén.

Muthén, L. K., \& Muthén, B. O. (1998-2017b). Mplus User’s Guide (Eighth ed.). Muthén \& Muthén.

Myhill, A. (2015). Measuring coercive control. Violence Against Women, 21(3), 355-375. https://doi.org/10.1177/1077801214568032

National Center for Injury Prevention and Control. (2020). Preventing intimate partner violence. Centers for Disease Control and Prevention. https://www.cdc.gov/violenceprevention/intimatepartnerviolence/fastfact.html

* Neal, A. M., Dixon, K. J., Edwards, K. M., \& Gidycz, C. A. (2015). Why did she do it? College women's motives for intimate partner violence perpetration. Partner Abuse, 6(4), 425-441. https://doi.org/10.1891/1946-6560.6.4.425

Nunnally, J. C., \& Bernstein, I. H. (1994). Psychometric theory (3rd ed.). McGraw-Hill, Inc.

O'Leary, K. D. (1999). Psychological abuse: A variable deserving critical attention in domestic violence. Violence and Victims, 14(1), 3-23. https://doi.org/10.1891/08866708.14.1.3

O’Leary, K. D., \& Smith Slep, A. M. (2003). A dyadic longitudinal model of adolescent dating aggression. Journal of Clinical Child \& Adolescent Psychology, 32(3), 314327. https://doi.org/10.1207/S15374424JCCP3203_01

O'Leary, K. D., Smith Slep, A. M., \& O'Leary, S. G. (2007). Multivariate models of men's and women's partner aggression. J Consult Clin Psychol, 75(5), 752-764. https://doi.org/10.1037/0022-006X.75.5.752

O'Leary, K. D., Tintle, N., \& Bromet, E. (2014). Risk factors for physical violence against partners in the U.S. Psychology of Violence, 4(1), 65-77. https://doi.org/10.1037/a0034537

Oxford University Press. (2020a). Oxford English Dictionary (3rd ed.). Oxford University Press. https://www-oedcom.helicon.vuw.ac.nz/view/Entry/35725?redirectedFrom=coercion\#eid 
Oxford University Press. (2020b). Oxford English Dictionary (3rd ed.). Oxford University Press. https://www-oed-

com.helicon.vuw.ac.nz/search?searchType $=$ dictionary \&q=control\&_searchBtn=Searc $\mathrm{h}$

Paulhus, D. L. (1991). Measurement and control of response biases. In J. P. Robinson, P. Shaver, L. Wrightsman, \& F. Andrews. (Eds.), Measures of personality and social psychological attitudes (pp. 17-59). Academic Press.

Peer, E., Samat, S., Brandimarte, L., \& Acquisti, A. (2015). Beyond the Turk: An empirical comparison of alternative platforms for crowdsourcing online research. Journal of Experimental Social Psychology, 70, 153-163. https://doi.org/10.1016/j.jesp.2017.01.006

Pence, E., \& Paymar, M. (1993). Education groups for men who batter: The Duluth Model. Springer Publishing Company.

Perry, A. R., \& Fromuth, M. E. (2005). Courtship violence using couple data: Characteristics and perceptions. Journal of Interpersonal Violence, 20(9), 1078-1095. https://doi.org/10.1177/0886260505278106

Piatek, R. (2017). Bayesian inference for factor modeling (Version 0.1.2). [R package]. https://CRAN.R-project.org/package=BayesFM

Podsakoff, P. M., MacKenzie, S. B., \& Podsakoff, N. P. (2012). Sources of method bias in social science research and recommendations on how to control it. Annual Review of Psychology, 63(1), 539-569. https://doi.org/10.1146/annurev-psych-120710-100452

Porcu, M., \& Giambona, F. (2017). Introduction to latent class analysis with applications. The Journal of Early Adolescence, 37(1), 129-158. https://doi.org/10.1177/0272431616648452

Preacher, K., \& MacCallum, R. (2003). Repairing Tom Swift's electric factor analysis machine. Understanding Statistics, 2(1), 13-43. https://doi.org/10.1207/S15328031US0201_02

Prinz, J. (2004). Gut reactions: A perceptual theory of emotion. Oxford University Press.

Prolific (2019) Representative samples on Prolific. https://researcher-help.prolific.co/hc/engb/articles/360019236753-Representative-Samples-on-Prolific-limited-release-

Prolific. (2021) Pricing. https://www.prolific.co/pricing

Putnick, D. L., \& Bornstein, M. H. (2016). Measurement invariance conventions and reporting: The state of the art and future directions for psychological research. Developmental Review, 41, 71-90. https://doi.org/10.1016/j.dr.2016.06.004

R Core Team. (2013). R: A language and environment for statistical computing (Version 1.1.456). [Computer software]. R Foundation for Statistical Computing. http://www.R-project.org/

Raiche, G. (2020). Parallel analysis and other non-graphical solutions to the Cattell scree test (Version 2.4.1). [R package]. https://rdrr.io/cran/nFactors/ 
Raîche, G., Walls, T. A., Magis, D., Riopel, M., \& Blais, J.-G. (2013). Non-graphical solutions for Cattell's scree test. Methodology: European Journal of Research Methods for the Behavioral and Social Sciences, 9(1), 23-29. https://doi.org/10.1027/1614-2241/a000051

Robert, C. P., \& Casella, G. (2004). Monte Carlo statistical methods (2nd ed.). Springer.

Rogers, W. M., \& Schmitt, N. (2004). Parameter recovery and model fit using multidimensional composites: A comparison of four empirical parceling algorithms. Multivariate Behavioral Research, 39, 379-412. https://doi.org/10.1207/S15327906MBR3903_1

Rosenberg, M. L., O'Carroll, P. W., \& Powell, K. E. (1992). Let's be clear: Violence is a public health problem. JAMA, 267(22), 3071-3072. https://doi.org/10.1001/jama.1992.03480220089034

* Ross, J. M. (2011). Personality and situational correlates of self-reported reasons for intimate partner violence among women versus men referred for batterers' intervention. Behavioral Sciences \& the Law, 29(5), 711-727. https://doi.org/10.1002/bsl.1004

* Saunders, D. G. (1986). When battered women use violence: Husband-abuse or selfdefense. Victims and Violence, 1(1), 47-60. https://doi.org/10.1891/0886-6708.1.1.47

Schafer, J. L. (1999). Multiple imputation: A primer. Statistical Methods in Medical Research, 8(1), 3-15. https://doi.org/10.1177/096228029900800102

Scheer, R. (2001). Intentions, motives, and causation. Philosophy, 76(297), 397-413. www.jstor.org/stable/3751778

Scottish Government. (2015). A criminal offence of domestic abuse. A Scottish Government Consultation Paper. https://consult.gov.scot/criminal-law-and-sentencingteam/criminal-offence-domestic-abuse/user_uploads/00491481.pdf-1

Sears, H., Byers, S., \& Price, E. (2007). The co-occurrence of adolescent boys' and girls' use of psychologically, physically, and sexually abusive behaviours in their dating relationships. Journal of Adolescence, 30(3), 487-504. https://doi.org/10.1016/j.adolescence.2006.05.002

Sedgwick, P. (2013). Meta-analyses: How to read a funnel plot. British Medical Journal, 346. https://doi.org/10.1136/bmj.f1342

Serious Crimes Act 2015 (UK). Retrieved from http://www.legislation.gov.uk/ukpga/2015/9/section/76/enacted.

Shea, B. J., Hamel, C., Wells, G. A., Bouter, L. M., Kristjansson, E., Grimshaw, J., Henry, D. A., \& Boers, M. (2009). AMSTAR is a reliable and valid measurement tool to assess the methodological quality of systematic reviews. Journal of Clinical Epidemiology, 62(10), 1013-1020. https://doi.org/10.1016/j.jclinepi.2008.10.009

Shepard, M. F. (1992). Predicting batterer recidivism five years after community intervention. Journal of Family Violence, 7(3), 167-178. https://doi.org/10.1007/BF00979025

Shi, L., \& Lin, L. (2019). The trim-and-fill method for publication bias: Practical guidelines and recommendations based on a large database of meta-analyses. Medicine, 98(23), e15987. https://doi.org/10.1097/MD.0000000000015987 
* Shorey, R. C., Meltzer, C., \& Cornelius, T. L. (2010). Motivations for self-defensive aggression in dating relationships. Violence \& Victims, 25(5), 662-676. https://doi.org/10.1891/0886-6708.25.5.662

Smith, M. D. (1990). Patriarchal ideology and wife beating: A test of a feminist hypothesis. Violence \& Victims, 5, 257-273. https://doi.org/10.1891/0886-6708.5.4.257

Smith, P. H., Earp, J. A., \& DeVillis, R. (1995). Measuring battering: Development of the Women's Experience with Battering (WEB) Scale. Women's Health: Research on Gender, Behavior, and Policy, 1(4), 273-288.

Smith, P. H., Tessaro, I., \& Earp, J. A. (1995). Women's experiences with battering: A conceptualization from qualitative research. Women's Health Issues, 5(4), 173-182. https://doi.org/10.1016/1049-3867(95)00615-X

Stark, E. (2006). Commentary on Johnson's "conflict and control: Gender symmetry and asymmetry in domestic violence". Violence Against Women, 12(11), 1019-1025. https://doi.org/10.1177/1077801206293329

Stark, E. (2007). Coercive control: The entrapment of women in personal life. Oxford University. https://ebookcentral-proquestcom.helicon.vuw.ac.nz/lib/vuw/detail.action?docID=4704270\&pq-origsite=primo

Stark, E. (2010). Do violent acts equal abuse? Resolving the gender parity/asymmetry dilemma. Sex Roles, 62(3-4), 201-211. https://doi.org/10.1007/s11199-009-9717-2

Stark, E., \& Hester, M. (2019). Coercive control: Update and review. Violence Against Women, 25(1), 81-104. https://doi.org/10.1177/1077801218816191

Stets, J., \& Hammond, S. A. (2002). Gender, control, marital commitment. Journal of Family Issues, 23, 3-25. https://doi.org/10.1177/0192513X02023001001

Stevens, J. (1986). Applied multivariate statistics for the social sciences. New Jersey: L. Erlbaum Associates.

Stewart, L., Gabora, N., Allegri, N., \& Slavin-Stewart, M. (2014). Profile of female perpetrators of intimate partner violence in an offender population: Implications for treatment. Partner Abuse, 5(2), 168-188. https://doi.org/10.1891/1946-6560.5.2.168

Stewart, N., Chandler, J., \& Paolacci, G. (2017). Crowdsourcing samples in cognitive science. Trends in Cognitive Sciences, 21(10), 736-748. https://doi.org/10.1016/j.tics.2017.06.007

Stith, S. M., Jester, S. B., \& Bird, G. W. (1992). A typology of college students who use violence in their dating relationships. Journal of College Student Development, 33(5), 411-421.

Stith, S. M., Smith, D. B., Penn, C. E., Ward, D. B., \& Tritt, D. (2004). Intimate partner physical abuse perpetration and victimization risk factors: A meta-analytic review. Aggression and Violent Behavior, 10(1), 65-98. https://doi.org/10.1016/j.avb.2003.09.001

Strauchler, O., McCloskey, K., Malloy, K., Sitaker, M., Grigsby, N., \& Gillig, P. (2004). Humiliation, manipulation, and control: Evidence of centrality in domestic violence against an adult partner. Journal of Family Violence, 19(6), 339-346. https://doi.org/10.1007/s10896-004-0679-4 
Straus, M. A. (1979). Measuring intrafamily conflict and violence: The Conflict Tactics (CT) Scales. Journal of Marriage and the Family, 41(1), 75. https://doi.org/10.2307/351733

Straus, M. A. (1997). Physical assaults by women partners: A major social problem. In M. R. Walsh (Ed.), Women, Men and Gender: Ongoing Debates (pp. 210-221). Yale University Press.

Straus, M. A. (2004). Prevalence of violence against dating partners by male and female university students worldwide. Violence Against Women, 10(7), 790-811. https://doi.org/10.1177/1077801204265552

Straus, M. A., \& Douglas, E. M. (2004). A short form of the Revised Conflict Tactics Scales, and typologies for severity and mutuality. Violence and Victims, 19(5), 507-520. https://doi.org/10.1891/vivi.19.5.507.63686

Straus, M. A., \& Gelles, R. J. (1986). Societal change and change in family violence from 1975 to 1985 as revealed by two national surveys. Journal of Marriage and Family, 48(3), 465-479. https://doi.org/10.2307/352033

Straus, M. A., \& Gelles, R. J. (1999). Physical violence in American families, risk factors and adaptations to violence in 8145 families. Transaction publishers.

Straus, M. A., Gelles, R. J., \& Steinmetz, S. K. (1980). Behind closed doors: Violence in the American family. Anchor Press/Doubleday.

Straus, M. A., \& Gozjolko, K. L. (2014). "Intimate terrorism" and gender differences in injury of dating partners by male and female university students. Journal of Family Violence, 29(1), 51-65. https://doi.org/10.1007/s10896-013-9560-7

Straus, M., \& Gozjolko, K. (2016). Concordance between partners in "intimate terrorism": A comparison of two typologies. Aggression and Violent Behavior, 29, 55-60. https://doi.org/10.1016/j.avb.2016.06.003

Straus, M. A., Hamby, S. L., Boney-McCoy, S., \& Sugarman, D. (1996). The revised Conflict Tactics Scale (CTS2): Development and preliminary psychometric data. Journal of Family Issues, 17(3), 283-316. https://doi.org/10.1177/019251396017003001

* Stuart, G. L., Moore, T. M., Hellmuth, J. C., Ramsey, S. E., \& Kahler, C. W. (2006). Reasons for intimate partner violence perpetration among arrested women. Violence Against Women, 12(7), 609-621. https://doi.org/10.1177/1077801206290173

Sugarman, D., \& Frankel, S. (1996). Patriarchal ideology and wife-assault: A meta-analytic review. Journal of Family Violence, 11(1), 13-40. https://doi.org/10.1007/BF02333338

Sugarman, D. B., \& Hotaling, G. T. (1997). Intimate violence and social desirability: A metaanalytic review. Journal of Interpersonal Violence, 12, 275-290. https://doi.org/10.1177/088626097012002008

Sullivan, J. A. (2019). Achieving cumulative progress in understanding crime: Some insights from the philosophy of science. Psychology, Crime \& Law, 25(6), 561-576. https://doi.org/10.1080/1068316x.2019.1572755

* Swan, S., \& Snow, D. (2003). Behavioral and psychological differences among abused women who use violence in intimate relationships. Violence Against Women, 9(1), 75109. https://doi.org/10.1177/1077801202238431 
Thompson, M. P., Basile, K. C., Hertz, M. F., \& Sitterle, D. (2006). Measuring intimate partner violence victimization and perpetration: A compendium of assessment tools. National Center for Injury Prevention and Control of the Centers for Disease Control and Prevention.

Tinsley, H. E. A., \& Tinsley, D. J. (1987). Uses of factor analysis in counseling psychology research. Journal of Counseling Psychology, 34, 414-424. https://doi.org/10.1037/0022-0167.34.4.414

Tjaden, P., \& Thoennes, N. (2000). The extent, nature, and consequences of intimate partner violence: Findings from the National Violence Against Women Survey. National Institute of Justice/ Centres for Disease Control and Prevention.

Tolman, R. M. (1989). The development of a measure of psychological maltreatment of women by their male partners. Violence and Victims, 4(3), 159-177. https://doi.org/10.1891/0886-6708.4.3.159

Troisi, G. (2018). Measuring intimate partner violence and traumatic affect: Development of VITA, an Italian scale. Frontiers in Psychology, 9, 1-14. https://doi.org/10.3389/fpsyg.2018.01282

United States Census Bureau (2019). U.S Census Bureau quick facts: Unites States. https://www.census.gov/quickfacts/fact/table/US/PST045219

van Ravenzwaaij, D., Cassey, P., \& Brown, S. D. (2018). A simple introduction to Markov Chain Monte-Carlo sampling. Psychonomic Bulletin \& Review, 25(1), 143-154. https://doi.org/10.3758/s13423-016-1015-8

Wagers, S. (2015). Deconstructing the "power and control motive": Moving beyond a unidimensional view of power in domestic violence theory. Partner Abuse, 6(2), 230242. https://doi.org/10.1891/1946-6560.6.2.230

Walker, K., Sleath, E., \& Tramontano, C. (2017). The prevalence and typologies of controlling behaviors in a general population sample. Journal of Interpersonal Violence, 1-30. https://doi.org/10.1177/0886260517731785

* Walley-Jean, C., \& Swan, S. (2009). Motivations and justifications for partner aggression in a sample of African American college women. Journal of Aggression, Maltreatment \& Trauma, 18(7), 698-717. https://doi.org/10.1080/10926770903231759

Walters, M. L., Chen, J., \& Breiding, M. J. (2013). The National Intimate Partner and Sexual Violence Survey (NISVS): 2010 findings on victimization by sexual orientation. National Center for Injury Prevention and Control. https://www.cdc.gov/violenceprevention/pdf/nisvs_sofindings.pdf

Ward, T. (2017). Emotion, cognition and motivation: An enactive perspective. In T. A. Gannon \& T. Ward (Eds.), Sexual offending: Emotion, cognition and motivation (pp. 1-17). https://ebookcentral-proquestcom.helicon.vuw.ac.nz/lib/vuw/reader.action?docID=4773526

Ward, T., \& Carter, E. (2018). The classification of offending and crime related problems: A functional perspective. Psychology, Crime \& Law, 25(6), 542-560. https://doi.org/10.1080/1068316X.2018.1557182 
Ward, T., \& Hudson, S. M. (1998). The construction and development of theory in the sexual offending area: A meta-theoretical framework. Sexual Abuse: A Journal of Research and Treatment, 10(1), 47-63. https://doi.org/10.1177/107906329801000106

Watson, D., \& Clark, L. A. (1994). The PANAS-X: Manual for the Positive and Negative Affect Schedule - Expanded Form. https://doi.org/10.17077/48vt-m4t2

Whitaker, D. J., Haileyesus, T., Swahn, M., \& Saltzman, L. S. (2007). Differences in frequency of violence and reported injury between relationships with reciprocal and nonreciprocal IPV. American Journal of Public Health, 97, 941-947. https://doi:10.25105/AJPH.2005.079020

World Health Organization. (2010). Preventing intimate partner and sexual violence against women: Taking action and generating evidence.

https://www.who.int/violence_injury_prevention/publications/violence/978924156400 7_eng.pdf

World Health Organization. (2019). RESPECT women: Preventing violence against women. World Health Organization. https://www.who.int/publications/en/ 


\section{APPENDIX A}

$11 / 30 / 2020$

Rightslink Printable License

JOHN WILEY AND SONS LICENSE TERMS AND CONDITIONS

Nov 29,2020

This Agreement between Fiona Dempsey ("You") and John Wiley and Sons ("John Wiley and Sons") consists of your license details and the terms and conditions provided by John Wiley and Sons and Copyright Clearance Center.

License Number

License Date

Licensed Content Publisher

Licensed Content Title

Licensed Content Author

Licensed Content Date

Licensed Content Volume

Licensed Content Issue

Licensed Content Pages

Type of use

Requestor type

Format

Portion

Will you translating?

Title

Institution name

Expected presentation date

Requestor/location

Publisher Tax ID

Total

\section{9}

Oct 22,2020

Aggressive Behavior

Investigating whether controlling and aggressive relationship behaviors are discriminant

Fiona Dempsey, Matt Hammond, Louise Dixon

Jun 11, 2020

46

5

12

Dissertation/Thesis

Author of this Wiley article

Electronic

Full article

No

Control and Intimate Partner Aggression

Victoria University of Wellington

2021

Removed by author

EU826007151

0.00 USD 


\section{TERMS AND \\ CONDITIONS}

This copyrigbted material is owned by or exclusively licensed to John Wiley \& Sons, Inc. or one of its group companies (each a"Wiley Company") or handled on behalf of a society with which a Wiley Company has exclusive publishing rights in relation to a particular work (collectively "WILEY"). By clicking "accept" in connection with completing this licensing transaction, you agree that the following terms and conditions apply to this transaction (along with the billing and payment terms and conditions established by the Copyright Clearance Center Inc.., ("CCC's Billing and Payment terms and conditions"), at the time that you opened your RightsLink account (these are available at any time at http://mvaccount.copvright.com).

\section{Terms and Conditions}

- The materials you have requested permission to reproduce or reuse (the "Wiley Materials") are protected by copyright.

- You are hereby granted a personal, non-exclusive, non-sub licensable (on a stand- alone basis), non-transferable, worldwide, limited license to reproduce the Wiley materials for the purpose specified in the licensing process. This license, and any CONTENT (PDF or image file) purchased as part of your order, is for a one-time use only and limited to any maximum distribution number specified in the license. The first instance of republication or reuse granted by this license must be completed within two years of the date of the grant of this license (although copies prepared before the end date may be distributed thereafter). The Wiley Materials shall not be used in any other manner or for any other purpose, beyond what is granted in the license.

Permission is granted subject to an appropriate acknowledgement given to the author, title of the material/book /joumal and the publisher. You shall also duplicate the copyright notice that appears in the Wiley publication in your use of the Wiley Material. Permission is also granted on the understanding that nowhere in the text is a previously published source acknowledged for all or part of this Wiley Material. Any third party content is expressly excluded from this permission.

- With respect to the Wiley Materials, all rights are reserved. Except as expressly granted by the terms of the license, no part of the Wiley Materials may be copied, modified, adapted (except for minor reformatting required by the new Publication), translated, reproduced, transferred or distributed, in any form or by any means, and no derivative works may be made based on the Wiley Materials without the prior permission of the respective copyright owner. For STM Signatory Publishers clearing permission under the terms of the STM Permissions Ginidelines only, the terms of the license are extended to include subsequent editions and for editions in other languages, provided such editions are for the work as a whole in situ and does not involve the separate exploitation of the permitted figures or extracts, You may not alter, remove or suppress in any manner any copyright, trademark or other notices displayed by the Wiley Materials. You may not license, rent, sell, loan, lease, 
- pledge, offer as security, transfer or assign the Wiley Materials on a stand-alone basis, or any of the rights granted to you hereunder to any other person.

- The Wiley Materials and all of the intellectual property rights therein shall at all times remain the exclusive property of John Wiley \& Sons Inc, the Wiley Companies, or their respective licensors, and your interest therein is only that of having possession of and the right to reproduce the Wiley Materials pursuant to Section 2 herein during the continuance of this Agreement. You agree that you own no right, title or interest in or to the Wiley Materials or any of the intellectual property rights therein. You shall have no rights hereunder other than the license as provided for above in Section 2. No right, license or interest to any trademark, trade name, service mark or other branding ("Marks") of WILEY or its licensors is granted hereunder, and you agree that you shall not assert any such right, license or interest with respect thereto

- NEITHER WILEY NOR ITS LICENSORS MAKES ANY WARRANTY OR REPRESENTATION OF ANY KIND TO YOU OR ANY THIRD PARTY, EXPRESS, IMPLIED OR STATUTORY, WITHRESPECT TO THEMATERIALS OR THE ACCURACY OF ANY INFORMATION CONTAINED IN THE MATERIALS, INCLUDING, WITHOUT LIMITATION, ANY IMPLIED WARRANTY OF MERCHANTABILITY, ACCURACY, SATISFACTORY QUALITY, FITNESS FOR A PARTICULAR PURPOSE, USABILITY, INTEGRATION OR NONINFRINGEMENT AND ALI SUCH WARRANTIES ARE HEREBY EXCLUDED BY WILEY AND ITS LICENSORS AND WAIVED BYYOU.

- WILEY shall have the right to terminate this Agreement immediately upon breach of this Agreement by you.

- You shall indemnify, defend and hold harmless WILEY, its Licensors and their respective directors, officers, agents and employees, from and against any actual or threatened claims, demands, causes of action or proceedings arising from any breach of this Agreement by you.

- IN NO EVENT SHALL WILEY OR ITS LICENSORS BE LIABLE TO YOU OR ANY OTHER PARTY OR ANY OTHER PERSON OR ENTITY FOR ANY SPECIAL, CONSEQUENTIAL, INCIDENTAL, INDIRECT, EXEMPLARY OR PUNITIVE DAMAGES, HOWEVER CAUSED, ARISING OUT OF OR IN CONNECTION WITH THE DOWNLOADING, PROVISIONING, VIEWING OR USE OF THE MATERIALS REGARDLESS OF THE FORM OF ACTION, WHETHER FOR BREACH OF CONTRACT, BREACH OF WARRANTY, TORT, NEGLIGENCE, INFRINGEMENT OR OTHERWISE (INCLUDING, WITHOUT LIMITATION, DAMAGES BASED ON LOSS OF PROFITS, DATA, FILES, USE, BUSINESS OPPORTUNITY OR CLAIMS OF THIRD PARTIES), AND WHETHER OR NOT THE PARTY HAS BEEN ADVISED OF THE POSSIBILITY OF SUCH DAMAGES. THIS LIMITATION SHALL APPLY NOTWITHSTANDING ANY FAIIURE OF ESSENTIAL PURPOSE OF ANY LIMITED REMEDY PROVIDED HEREIN.

- Should any provision of this Agreement be held by a court of competent 
- jurisdiction to be illegal, invalid, or unenforceable, that provision shall be deemed amended to achieve as nearly as possible the same economic effect as the original provision, and the legality, validity and enforceability of the remaining provisions of this Agreement shall not be affected or impaired thereby.

- The failure of either party to enforce any term or condition of this Agreement shall not constitute a waiver of either party's right to enforce each and every term and condition of this Agreement. No breach under this agreement shall be deemed waived or excused by either party unless such waiver or consent is in writing signed by the party granting such waiver or consent. The waiver by or consent of a party to a breach of any provision of this Agreement shall not operate or be construed as a waiver of or consent to any other or subsequent breach by such other party.

- This Agreement may not be assigned (including by operation of law or otherwise) by you without WILEY's prior written consent.

- Any fee required for this permission shall be non-refundable after thirty (30) days from receipt by the CCC.

- These terms and conditions together with CCC's Billing and Payment terms and conditions (which are incorporated herein) form the entire agreement between you and WILEY concerning this licensing transaction and (in the absence of fraud) supersedes all prior agreements and representations of the parties, oral or written. This Agreement may not be amended except in writing signed by both parties. This Agreement shall be binding upon and inure to the benefit of the parties' successors, legal representatives, and authorized assigns.

- In the event of any conflict between your obligations established by these terms and conditions and those established by CCC's Billing and Payment terms and conditions, these terms and conditions shall prevail.

- WILEY expressly reserves all rights not specifically granted in the combination of(i) the license details provided by you and accepted in the course of this licensing transaction, (ii) these terms and conditions and (iii) CCC's Billing and Payment terms and conditions.

- This Agreement will be void if the Type of Use, Format, Circulation, or Requestor Type was misrepresented during the licensing process.

- This Agreement shall be governed by and construed in accordance with the laws of the State of New York, USA, without regards to such state's conflict of law rules. Any legal action, suit or proceeding arising out of or relating to these Terms and Conditions or the breach thereof shall be instituted in a court of competent jurisdiction in New York County in the State of New York in the United States of America and each party hereby consents and submits to the personal jurisdiction of such court, waives any objection to venue in such court and consents to service of process by registered or certified mail, return receipt requested, at the last known address of such party. 


\section{WILEY OPEN ACCESS TERMS AND CONDITIONS}

Wiley Publishes Open Access Articles in fully Open Access Journals and in Subscription journals offering Online Open. Although most of the fully Open Access journals publish open access articles under the terms of the Creative Commons Attribution (CC BY) License only, the subscription journals and a few of the Open Access Journals offer a choice of Creative Commons Licenses. The license type is clearly identified on the article.

The Creative Commons Attribution License

The Creative Commons Attribution License (CC-BY) allows users to copy, distribute and transmit an article, adapt the article and make commercial use of the article. The CC-BY license permits commercial and non-

\section{Creative Commons Attribution Non-Commercial License}

The Creative Commons Attribution Non-Commercial (CC-BY_NC)License permits use, distribution and reproduction in any medium, provided the original work is properly cited and is not used for commercial purposes.(see below)

\section{Creative Commons Attribution-Non-Commercial-NoDerivs License}

The Creative Commons Attribution Non-Commercial-NoDerivs License (CC-BYNC-ND) permits use, distribution and reproduction in any medium, provided the original work is properly cited, is not used for commercial purposes and no modifications or adaptations are made. (see below)

Use by commercial "for-profit" organizations

Use of Wiley Open Access articles for commercial, promotional, or marketing purposes requires further explicit permission from Wiley and will be subject to a fee.

Further details can be found on Wiley Online Library http://olabout.wiley com/WileyCDA/Section/id$\underline{410895 . \mathrm{html}}$

Other Terms and Conditions:

vl.10 Last updated September 2015

Questions? customercare@ copyright.com or +1-855-239-3415 (toll free in the US) or +1-978-646-2777. 


\section{APPENDIX B}

Pattern matrix for the experienced behavior scale

Item

Factor 1

Factor 2

Factor 3

Factor 4

My partner broke one or more of my bones

1.07

* My partner had me arrested

My partner ignored legal or court orders to stay away from me

1.05

My partner harmed members of my family or others close to me

1.02

My partner ruined my reputation at work or in the community

0.99

0.97

* My partner deliberately caused me legal trouble

0.96

* My partner used a weapon or something like a weapon against me

0.94

My partner made false accusations to authorities (e.g., Police, Health organisations) about physical or sexual abuse I committed

My partner attempted to harm or kill themselves

0.93

My partner threatened me with a weapon

0.93

My partner threatened to make false accusations to authorities (e.g., Police, Health organisations) about physical or sexual abuse I committed

* My partner threatened to hurt members of my family or someone I care about

0.92

0.92

0.92

* My partner choked, strangled, or suffocated me

0.90

* My partner threatened to commit suicide

0.90

My partner deliberately caused me to lose my job $\quad 0.90$

My partner harassed my family or friends in some way 0.89

$\begin{array}{ll}\text { My partner insisted I have sex in belittling or humiliating ways } & 0.87\end{array}$

My partner slammed me or held me against a wall 0.86

$\begin{array}{lr}\text { My partner kicked me } & 0.85\end{array}$ 
* My partner threatened to harm themselves $\quad 0.84$

$\begin{array}{ll}\text { My partner threatened to harm or kill me } & 0.81\end{array}$

$\begin{array}{ll}\text { My partner physically forced me to engage in sexual activities against my will } & 0.79\end{array}$

* My partner tried to convince my friends, family, or children that I was crazy $\quad 0.78$

* My partner embarrassed, insulted, or threatened me on social media $\quad 0.77$

* My partner kept me from going to work $\quad 0.77$

$\begin{array}{ll}\text { My partner hit or punched me } & 0.76\end{array}$

$\begin{array}{ll}\text { My partner threatened to ruin my reputation at work or in the community } & 0.75\end{array}$

My partner deliberately broke or destroyed something that was important to me $\quad 0.75$

* My partner tried to turn my family, friends, and children against me $\quad 0.75$

* My partner threatened to post inappropriate photos of me on social media $\quad 0.73$

My partner demanded or insisted that I engage in sexual activities against my will 0.73

* My partner restricted my use of the telephone 0.69

* My partner used technology (e.g., webcam, GPS, spyware) to monitor my activities 0.68

My partner slapped me $\quad 0.67$

* My partner threatened to withhold money from me 0.64

* My partner threatened to disclose damaging or embarrassing information 0.62

My partner threatened to harm or damage things I care about $\quad 0.61$

* My partner pressured me to have sex when I said 'No' 0.60

My partner denied me access to my family members or friends $\quad 0.56$

$\begin{array}{ll}\text { My partner denied incidents of abuse } & 0.55\end{array}$

* My partner limited my access to transportation $\quad 0.55$

* My partner kept me from leaving the house $\quad 0.54$

* My partner forbade me to go out without them $\quad 0.53$

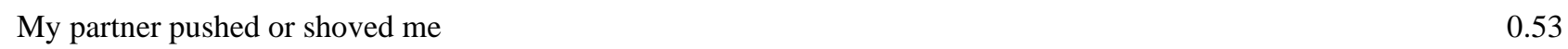


* My partner made me dependent on them for money

My partner deliberately kept me from sleeping

My partner was critical or unpleasant toward me

My partner screamed or yelled at me

My partner wanted to be left alone and/or to spend less time with me

* My partner deliberately ignored me

My partner tried to ignore things I did or said that irritated or upset them

My partner deliberately acted in a hurtful way towards me

* My partner told me my feelings were irrational or crazy

* My partner stomped out of the house or room during a disagreement with me 0.75

My partner withdrew from me and started doing their own thing 0.74

* My partner blamed me for the problems in our relationship 0.74

My partner pouted or acted upset when they didn't get their way 0.74

My partner changed the subject on purpose when I was trying to discuss a problem 0.71

* My partner tried to provoke an argument 0.71

* My partner treated me like I was stupid 0.67

* My partner treated me like I was inferior to them 0.63

* My partner told me I was lying or confused 0.62

* My partner purposely withheld affection or sex 0.59

* My partner threatened to leave the relationship 0.53

* My partner ordered me around 0.52

My partner criticized my friends, family, or co-workers 0.48

* My partner told me what I care about is unimportant 0.47

My partner made me feel guilty for not spending enough time together 0.47

My partner repeatedly reminded me of what they wanted until I gave in 0.46 
* My partner insisted on knowing where I went and who I spoke to when we were not together

* My partner kept track of my telephone/cell phone use 0.62

* My partner tried to restrict my activities 0.60

* My partner seemed to always be watching me 0.58

* My partner checked my social network page(s) to monitor me 0.58

* My partner called me or text messaged me constantly 0.53

* My partner accused me of being unfaithful or flirting with others 0.52

* My partner read my personal mail or email without my consent 0.51

* My partner listened to my telephone conversations 0.47

My partner focused on maintaining or improving the quality of our relationship -0.80

My partner showed concern about my feelings -0.78

My partner shared and discussed their feelings with me -0.72

My partner was willing to let me have things my way -0.48

$\begin{array}{lll}\text { Eigenvalue: } & 46.12 \quad 6.04 & 2.66\end{array}$

\begin{tabular}{lrl} 
\% of variance explained: & 55.57 & 7.23 \\
\hline
\end{tabular}

Note. ${ }^{*}$ Items are part of (sub)scales designed to measure control 


\section{APPENDIX C}

Pattern matrix for the perpetrated behavior scale

\begin{tabular}{lcc}
\hline & Item & Factor 1 \\
\hline
\end{tabular}

I broke one or more of my partner's bones

1.07

I harmed members of my partner's family or others close to them

1.02

* I deliberately caused my partner legal trouble

* I had my partner arrested

I ignored legal or court orders to stay away from my partner

I used a weapon or something like a weapon against my partner

I threatened to make false accusations to authorities (e.g., Police, Health organisations) about physical or sexual abuse my partner committed

I slammed my partner or held them against a wall

* I attempted to harm or kill myself

I ruined my partner's reputation at work or in the community

I made false accusations to authorities (e.g., Police, Health organisations) about physical or sexual abuse my

* I threatened to harm myself

* I deliberately caused my partner to lose their job

* I threatened to commit suicide

* I kept my partner from going to work

* I threatened to hurt members of my partner's family or someone they care about

* I threatened to harm or kill my partner 
* I tried to turn my partner's family, friends, and children against them 0.82

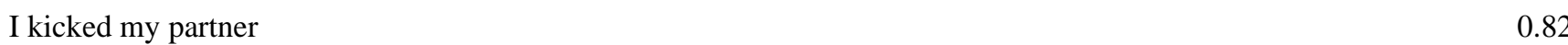

$\begin{array}{ll}\text { I hit or punched my partner } & 0.81\end{array}$

* I tried to convince my partner's friends, family, or children that my partner was crazy 0.81

I demanded or insisted that my partner engage in sexual activities against their will $\quad 0.81$

$\begin{array}{ll}\text { I physically forced my partner to engage in sexual activities against their will } & 0.81\end{array}$

I insisted my partner have sex in belittling or humiliating ways 0.76

* I embarrassed, insulted, or threatened my partner on social media 0.74

* I restricted my partner's use of the telephone $\quad 0.74$

I denied my partner access to their family members or friends 0.73

* I pressured my partner to have sex when they said 'No' 0.72

* I threatened to post inappropriate photos of my partner on social media 0.71

* I treated my partner like they were helpless or incapable $\quad 0.70$

I deliberately broke or destroyed something that was important to my partner 0.69

I pushed or shoved my partner $\quad 0.66$

* I kept my partner from leaving the house $\quad 0.66$

* I limited my partner's access to transportation $\quad 0.65$

I deliberately kept my partner from sleeping $\quad 0.63$

I threatened to harm or damage things my partner cares about $\quad 0.62$

I threatened to ruin my partner's reputation at work or in the community $\quad 0.61$

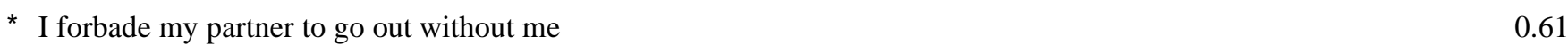

$\begin{array}{ll}\text { I slapped my partner } & 0.60\end{array}$

* I threatened to disclose damaging or embarrassing information 0.58

$\begin{array}{ll}\text { I denied incidents of abuse } & 0.57\end{array}$

I insulted or shamed my partner in front of others $\quad 0.56$ 
* I threatened to withhold money from my partner

* I made important financial decisions without talking to my partner about them first

* I deliberately made my partner feel sexually inadequate

* I complained that my partner spent too much time with friends

* I made my partner dependent on me for money

I hit or kicked a wall, door, or furniture in anger

I wanted to be left alone and/or to spend less time with my partner

I tried to ignore things my partner did or said that irritated or upset me

* I deliberately ignored my partner

I was critical or unpleasant toward my partner

I withdrew from my partner and started doing my own thing

* I stomped out of the house or room during a disagreement with my partner

I screamed or yelled at my partner

* I purposely withheld affection or sex

* I insisted on knowing where my partner went and who they spoke to when we were not together

* I checked my partner's social network page(s) to monitor them

* I accused my partner of being unfaithful or flirting with others

* I kept track of my partner's telephone/cell phone use

* I read my partner's personal mail or email without their consent

* I tried to provoke an argument 


\section{APPENDIX D}

List of parcelled items included in the confirmatory factor analysis for the experienced and perpetrated models

\section{Experienced Behavior}

\begin{tabular}{|c|c|c|}
\hline Item & Latent Factor & Parcel \\
\hline My partner deliberately broke or destroyed something that was important to me & Eclectic Aggression & EA1 \\
\hline My partner demanded or insisted that I engage in sexual activities against my will & Eclectic Aggression & EA1 \\
\hline My partner denied incidents of abuse & Eclectic Aggression & EA1 \\
\hline My partner physically forced me to engage in sexual activities against my will & Eclectic Aggression & EA1 \\
\hline My partner threatened to disclose damaging or embarrassing information & Eclectic Aggression & EA1 \\
\hline My partner threatened to post inappropriate photos of me on social media & Eclectic Aggression & EA1 \\
\hline My partner tried to turn my family, friends, and children against me & Eclectic Aggression & EA1 \\
\hline My partner denied me access to my family members or friends & Eclectic Aggression & EA2 \\
\hline My partner kept me from going to work & Eclectic Aggression & EA2 \\
\hline My partner limited my access to transportation & Eclectic Aggression & EA2 \\
\hline My partner restricted my use of the telephone & Eclectic Aggression & EA2 \\
\hline My partner threatened to commit suicide & Eclectic Aggression & EA2 \\
\hline My partner threatened to harm or damage things I care about & Eclectic Aggression & EA2 \\
\hline My partner threatened to harm themselves & Eclectic Aggression & EA2 \\
\hline My partner embarrassed, insulted, or threatened me on social media & Eclectic Aggression & EA3 \\
\hline My partner kicked me & Eclectic Aggression & EA3 \\
\hline My partner slapped me & Eclectic Aggression & EA3 \\
\hline
\end{tabular}


My partner threatened to hurt members of my family or someone I care about

My partner threatened to withhold money from me

My partner tried to convince my friends, family, or children that I was crazy

My partner ordered me around

My partner pouted or acted upset when they didn't get their way

My partner purposely withheld affection or sex

My partner screamed or yelled at me

My partner told me I was lying or confused

My partner wanted to be left alone and/or to spend less time with me

My partner withdrew from me and started doing their own thing

My partner blamed me for the problems in our relationship

My partner changed the subject on purpose when I was trying to discuss a problem

My partner deliberately acted in a hurtful way towards me

My partner deliberately ignored me

My partner threatened to leave the relationship

My partner treated me like I was stupid

My partner tried to ignore things I did or said that irritated or upset them

My partner criticized my friends, family, or co-workers

My partner stomped out of the house or room during a disagreement with me

My partner told me my feelings were irrational or crazy

My partner treated me like I was inferior to them

My partner tried to provoke an argument
Eclectic Aggression

Eclectic Aggression

Eclectic Aggression

Direct Psychological Aggression

Direct Psychological Aggression

Direct Psychological Aggression

Direct Psychological Aggression

Direct Psychological Aggression

Direct Psychological Aggression

Direct Psychological Aggression

Direct Psychological Aggression

Direct Psychological Aggression

Direct Psychological Aggression

Direct Psychological Aggression

Direct Psychological Aggression

Direct Psychological Aggression

Direct Psychological Aggression

Direct Psychological Aggression

Direct Psychological Aggression

Direct Psychological Aggression

Direct Psychological Aggression

Direct Psychological Aggression
EA3

EA3

EA3

DPA1

DPA1

DPA

DPA1

DPA1

DPA1

DPA1

DPA2

DPA2

DPA2

DPA2

DPA2

DPA2

DPA2

DPA3

DPA3

DPA3

DPA3

DPA3 
My partner was critical or unpleasant toward me

My partner called me or text messaged me constantly

My partner listened to my telephone conversations

My partner seemed to always be watching me

My partner accused me of being unfaithful or flirting with others

My partner kept track of my telephone/cell phone use

My partner read my personal mail or email without my consent

My partner checked my social network page(s) to monitor me

My partner insisted on knowing where I went and who I spoke to when we were not together

My partner tried to restrict my activities

My partner focused on maintaining or improving the quality of our relationship

My partner showed concern about my feelings

My partner shared and discussed their feelings with me

My partner was willing to let me have things my way
Direct Psychological Aggression

DPA3

Monitoring Acts

MA1

Monitoring Acts

MA1

Monitoring Acts

MA1

Monitoring Acts

MA2

Monitoring Acts

MA2

Monitoring Acts

MA2

Monitoring Acts

MA3

Monitoring Acts

MA3

Monitoring Acts

Prosocial Relationship Behavior

PR1

Prosocial Relationship Behavior

PR1

Prosocial Relationship Behavior

PR2

Prosocial Relationship Behavior

\begin{tabular}{lcc}
\hline \multicolumn{1}{c}{ Perpetrated Behavior } & \multicolumn{1}{c}{ Latent Factor } & Eclectic Aggression \\
\hline I deliberately broke or destroyed something that was important to my partner & Eclectic Aggression & EA1 \\
I demanded or insisted that my partner engage in sexual activities against their will & Eclectic Aggression & Eclectic Aggression \\
I denied incidents of abuse & Eclectic Aggression & EA1 \\
I physically forced my partner to engage in sexual activities against their will & EA1
\end{tabular}


I threatened to post inappropriate photos of my partner on social media

I tried to turn my partner's family, friends, and children against them

I denied my partner access to their family members or friends

I kept my partner from going to work

I limited my partner's access to transportation

I restricted my partner's use of the telephone

I threatened to commit suicide

I threatened to harm myself

I threatened to harm or damage things my partner cares about

I embarrassed, insulted, or threatened my partner on social media

I kicked my partner

I slapped my partner

I threatened to hurt members of my partner's family or someone they care about

I threatened to withhold money from my partner

I tried to convince my partner's friends, family, or children that my partner was crazy

I stomped out of the house or room during a disagreement with my partner

I tried to ignore things my partner did or said that irritated or upset me

I withdrew from my partner and started doing my own thing

I deliberately ignored my partner

I purposely withheld affection or sex

I was critical or unpleasant toward my partner

I screamed or yelled at my partner
Eclectic Aggression

EA1

Eclectic Aggression

EA1

Eclectic Aggression

EA2

Eclectic Aggression

EA2

Eclectic Aggression

EA2

Eclectic Aggression

EA2

Eclectic Aggression

EA2

Eclectic Aggression

EA2

Eclectic Aggression

EA2

Eclectic Aggression

EA3

Eclectic Aggression

EA3

Eclectic Aggression

EA3

Eclectic Aggression

EA3

Eclectic Aggression

EA3

Eclectic Aggression

EA3

Direct Psychological Aggression

DPA1

Direct Psychological Aggression

DPA1

Direct Psychological Aggression

DPA1

Direct Psychological Aggression

DPA2

Direct Psychological Aggression

DPA2

Direct Psychological Aggression

DPA2

Direct Psychological Aggression 
I wanted to be left alone and/or to spend less time with my partner

I accused my partner of being unfaithful or flirting with others

I criticized my partner's friends, family, or co-workers

I told my partner they were lying or confused

I checked my partner's social network page(s) to monitor them

I deliberately acted in a hurtful way towards my partner

I read my partner's personal mail or email without their consent

I insisted on knowing where my partner went and who they spoke to when we were not together

I kept track of my partner's telephone/cell phone use

I tried to provoke an argument
Direct Psychological Aggression DPA3

Monitoring Acts MA1

Monitoring Acts MA1

Monitoring Acts $\quad$ MA1

Monitoring Acts MA2

Monitoring Acts MA2

Monitoring Acts MA2

Monitoring Acts MA3

Monitoring Acts MA3

Monitoring Acts MA3 


\section{APPENDIX E}

Standardised and unstandardised coefficients for the four-factor model (experienced behavior)

\begin{tabular}{cccccc}
\hline Observed indicator & Latent Factor & $\beta$ & $B$ & $S . E$ & $p$ \\
\hline EA1 & Eclectic Aggression & .85 & 1.00 & & \\
EA2 & Eclectic Aggression & .90 & 1.30 & .06 & $<.001$ \\
EA3 & Eclectic Aggression & .77 & .80 & .04 & $<.001$ \\
DPA1 & Direct Psychological Aggression & .92 & 1.00 & & \\
DPA2 & Direct Psychological Aggression & .95 & 1.20 & .03 & $<.001$ \\
DPA3 & Direct Psychological Aggression & .95 & 1.14 & .03 & $<.001$ \\
MA1 & Monitoring Acts & .60 & 1.00 & & $<.001$ \\
MA2 & Monitoring Acts & .89 & 1.50 & .11 & $<.001$ \\
MA3 & Monitoring Acts & .90 & 1.78 & .13 & $<.001$ \\
PR1 & Prosocial Relationship Behavior & 1.08 & 1.00 & & $<.001$ \\
PR2 & Prosocial Relationship Behavior & .61 & .56 & .09 & .06 \\
PR3 & Prosocial Relationship Behavior & .31 & .30 & & \\
\hline
\end{tabular}




\section{APPENDIX F}

Standardised and unstandardised coefficients for the three-factor model (perpetrated behavior)

\begin{tabular}{cccccc}
\hline Observed indicator & Latent Factor & $\beta$ & $B$ & S.E & $p$ \\
\hline EA1 & Eclectic Aggression & .89 & 1.00 & & \\
EA2 & Eclectic Aggression & .88 & 1.16 & .05 & $<.001$ \\
EA3 & Eclectic Aggression & .76 & .79 & .04 & $<.001$ \\
DPA1 & Direct Psychological Aggression & .80 & 1.00 & & \\
DPA2 & Direct Psychological Aggression & .89 & 1.40 & .07 & $<.001$ \\
DPA3 & Direct Psychological Aggression & .78 & 1.04 & .06 & $<.001$ \\
MA1 & Monitoring Acts & .73 & 1.00 & & $<.001$ \\
MA2 & Monitoring Acts & .83 & 1.07 & .07 & .07 \\
MA3 & Monitoring Acts & .77 & .93 & .06 & $<.001$ \\
\hline
\end{tabular}




\section{APPENDIX G}

Factor correlations for the experienced behavior model

\begin{tabular}{lcccc}
\hline Factor & $\begin{array}{c}\text { Eclectic } \\
\text { Aggression }\end{array}$ & $\begin{array}{c}\text { Direct } \\
\text { Psychological } \\
\text { Aggression }\end{array}$ & $\begin{array}{c}\text { Monitoring } \\
\text { Acts }\end{array}$ & $\begin{array}{c}\text { Prosocial } \\
\text { Relationship } \\
\text { Behavior }\end{array}$ \\
\hline $\begin{array}{l}\text { Eclectic Aggression } \\
\begin{array}{l}\text { Direct Psychological } \\
\text { Aggression }\end{array}\end{array}$ & -74 & - & - & - \\
$\begin{array}{l}\text { Monitoring Acts } \\
\text { Prosocial Relationship }\end{array}$ & .80 & - & - & - \\
Behavior & -.21 & -.85 & - & - \\
& & -.18 & -.11 & - \\
\hline
\end{tabular}




\section{APPENDIX H}

Factor correlations for the perpetrated behavior model

\begin{tabular}{lccc}
\hline Factor & $\begin{array}{c}\text { Eclectic } \\
\text { Aggression }\end{array}$ & $\begin{array}{c}\text { Direct } \\
\text { Psychological } \\
\text { Aggression }\end{array}$ & $\begin{array}{c}\text { Monitoring } \\
\text { Acts }\end{array}$ \\
\hline $\begin{array}{l}\text { Eclectic Aggression } \\
\text { Direct Psychological }\end{array}$ & - & - & - \\
Aggression & .47 & - & - \\
Monitoring Acts & .67 & .79 & - \\
\hline
\end{tabular}




\section{APPENDIX I}

Factor loadings for the experienced behavior model

\begin{tabular}{ccc}
\hline Observed indicator & Factor & $\beta$ \\
\hline EA1 & Eclectic Aggression & .85 \\
EA2 & Eclectic Aggression & .90 \\
EA3 & Eclectic Aggression & .77 \\
DPA1 & Direct Psychological Aggression & .92 \\
DPA2 & Direct Psychological Aggression & .95 \\
DPA3 & Direct Psychological Aggression & .95 \\
MA1 & Monitoring Acts & .60 \\
MA2 & Monitoring Acts & .89 \\
MA3 & Monitoring Acts & .90 \\
PR1 & Prosocial Relationship Behavior & 1.08 \\
PR2 & Prosocial Relationship Behavior & .61 \\
PR3 & Prosocial Relationship Behavior & .31 \\
\hline
\end{tabular}




\section{APPENDIX J}

Factor loadings for the perpetrated behavior model

\begin{tabular}{ccc}
\hline Observed indicator & Factor & $\beta$ \\
\hline EA1 & Eclectic Aggression & .89 \\
EA2 & Eclectic Aggression & .88 \\
EA3 & Eclectic Aggression & .76 \\
DPA1 & Direct Psychological Aggression & .80 \\
DPA2 & Direct Psychological Aggression & .89 \\
DPA3 & Direct Psychological Aggression & .78 \\
MA1 & Monitoring Acts & .73 \\
MA2 & Monitoring Acts & .83 \\
MA3 & Monitoring Acts & .77 \\
& & \\
\hline
\end{tabular}




\section{APPENDIX K}

Data Extraction Form

\begin{tabular}{|c|c|}
\hline Data to be extracted & Study \# 1 \\
\hline Title & $\begin{array}{l}\text { Toward a typology of abusive women: Differences } \\
\text { between partner-only and generally violent women in the } \\
\text { use of violence }\end{array}$ \\
\hline Author/ Year & Babcock, J. C.; Miller, S. A.; Siard, C. (2003) \\
\hline Study Design & Cross sectional \\
\hline Sampling Method & Non-random, convenience \\
\hline Sample & $\begin{array}{l}52 \text { women engaged in treatment for IPA in Texas, USA. } M \\
\text { age }=31.54, S D=9.8 \text {. Comparison of partner-only }(n=26) \\
\text { and generally violent women }(n=26) .\end{array}$ \\
\hline Measures Used & $\begin{array}{l}\text { Reasons for Using Violence Scale: } 11 \text { items; 4-point Likert } \\
\text { scale; alpha }=.80 \text {. Developed based on clinical experience } \\
\text { of one of the authors. } \\
\text { Reasons for violence: Open ended question coded by } 2 \mathrm{x} \\
\text { coders } \\
\text { General violence: Questionnaire modified from previous } \\
\text { typology study of male batterers (Waltz et al., 2000). One } \\
\text { binary question. Used to differentiate generally violent } \\
\text { from partner only aggressors. } \\
\text { IPA and self-defense: Physical ( } 12 \text { items) and } \\
\text { psychological subscales ( } 8 \text { items) of the CTS2. A positive } \\
\text { response to one of the physical items would elicit a follow- } \\
\text { up question asking how many times this was in self- } \\
\text { defense }\end{array}$ \\
\hline Statistical Analysis & MANOVA, chi-square \\
\hline Results/ Findings & $\begin{array}{l}\text { Generally violent women more violent towards their } \\
\text { partners in terms of both frequency and severity. No group } \\
\text { differences in self-defense or victimisation. Generally } \\
\text { violent women more likely to endorse the following } \\
\text { motivations: "he was asking for it", "lost control", } \\
\text { "frustrated", "to push his buttons" }\end{array}$ \\
\hline Main Conclusions & $\begin{array}{l}\text { Generally violent women use more instrumental violence, } \\
\text { to control their partners. They use a greater variety of } \\
\text { aggressive acts and employ greater minimisation e.g., } \\
\text { victim blaming. }\end{array}$ \\
\hline Strengths/ Weaknesses & $\begin{array}{l}\text { No scale development or psychometrics reported for } \\
\text { Reasons for Using Violence Scale (except the alpha). } \\
\text { Conflation of reasons with motivations. These words are } \\
\text { used interchangeably in the text. } \\
\text { Self -report. } \\
\text { Small sample } \\
\text { Definition of self-defense didn't appear to be provided in } \\
\text { measure. } \\
\text { No definitions of IPA or motivations. }\end{array}$ \\
\hline Quality Assessment Score & $48 \%$ \\
\hline
\end{tabular}




\begin{tabular}{|l|l|}
\hline Data to be extracted & Study \# 2 \\
\hline Title & $\begin{array}{l}\text { Gender differences in attributions of self-defense and } \\
\text { control in inter-partner aggression }\end{array}$ \\
\hline Author/ Year & Barnett, O. W.; Lee, C. Y.; Thelen, R. E. (1997) \\
\hline Study Design & Cross sectional \\
\hline Sampling Method & Non-random, convenience \\
\hline Sample & $\begin{array}{l}\text { 30 women either living in a shelter or from shelter out- } \\
\text { reach groups. 34 men from court mandated batterer } \\
\text { programs. No information regarding how the participants } \\
\text { were recruited. }\end{array}$ \\
\hline Measures Used & $\begin{array}{l}\text { Relationship Abuse Questionnaire (Barnett, 1989): Based } \\
\text { on the CTS. 28 items; 4 subscales - verbal (4), } \\
\text { psychological (7), threat (6), physical (10). Additional } \\
\text { items on outcomes (4) and attributions for abuse (8). }\end{array}$ \\
\hline Statistical Analysis & Repeated measures ANOVA, One-way ANOVA (post hoc) \\
\hline Results/ Findings & $\begin{array}{l}\text { No significant gender differences for frequency of abuse } \\
\text { for the 4 violence categories. Significant main effect for } \\
\text { attributions and significant interaction for gender x } \\
\text { attributions. Post-hoc analyses showed males significantly } \\
\text { higher on 'show who was boss' and 'unaware of intention' } \\
\text { and females higher on 'protecting self'. }\end{array}$ \\
\hline Quality Assessment Score & $\begin{array}{l}\text { Support for gender differences in outcomes and } \\
\text { motivations for the use of violence. }\end{array}$ \\
\hline Main Conclusions & $\begin{array}{l}\text { Small and non-representative sample } \\
\text { Self-report } \\
\text { Although piloted, the Relationship Abuse Questionnaire } \\
\text { has not been validated. }\end{array}$ \\
\hline Strengths/ Weaknesses & \begin{tabular}{l}
$52 \%$ \\
\hline
\end{tabular} \\
\hline
\end{tabular}

\begin{tabular}{|l|l|}
\hline Data to be extracted & Study \# 3 \\
\hline Title & $\begin{array}{l}\text { Do anger and jealousy mediate the relationship between } \\
\text { adult attachment styles and intimate partner violence }\end{array}$ \\
\hline Author/ Year & $\begin{array}{l}\text { Belus, J. M.; Wanklyn, S. G.; Iverson, K. M; Pukay- } \\
\text { Martin, N. D.; Langhinrichsen-Rohling, J.; Monson, C. M. } \\
\text { (2014) }\end{array}$ \\
\hline Study Design & Cross sectional \\
\hline Sampling Method & $\begin{array}{l}\text { Randomly selected from a list of enrolled undergraduate } \\
\text { students. Convenience sample. }\end{array}$ \\
\hline Sample & $\begin{array}{l}\text { N=431 (125 males; 306 females) M age 21.25 years. } \\
94.6 \% \text { identified as White. }\end{array}$ \\
\hline Measures Used & $\begin{array}{l}\text { IPA: Physical aggression subscale of CTS2 (12 items). } \\
\text { Alphas in this study were .79 for females and .64 for males. } \\
\text { Multidimensional Jealousy Scale (Pfeiffer \& Wong, 1989): } \\
\text { Emotional subscale (8 items). 5-point Likert scale. High } \\
\text { internal consistency (females = .85; males = .88) in this } \\
\text { study and construct validity referenced. } \\
\text { Multidimensional Anger Inventory (Siegel, 1986): 38 } \\
\text { items. 5-point Likert scale. Construct validity and test- }\end{array}$ \\
\hline
\end{tabular}




\begin{tabular}{|l|l|}
\hline & $\begin{array}{l}\text { retest reliability referenced. Alphas in this study were } .87 \\
\text { for females and .84 for males. }\end{array}$ \\
\hline Statistical Analysis & Mediation \\
\hline Results/ Findings & $\begin{array}{l}\text { Anger mediated the relationship between all four } \\
\text { attachment styles and IPA for females. Neither anger nor } \\
\text { jealousy mediated the relationship between attachment and } \\
\text { IPA in males. }\end{array}$ \\
\hline Main Conclusions & $\begin{array}{l}\text { Perpetration of IPA in young females is related to } \\
\text { emotional states, specifically anger. This is not the case for } \\
\text { young men. }\end{array}$ \\
\hline Strengths/ Weaknesses & $\begin{array}{l}\text { Sample was randomly selected; however, participants were } \\
\text { not ethnically diverse, and no efforts were made to increase } \\
\text { the representativeness of the sample. } \\
\text { Emotions conflated with motivations for the use if IPA }\end{array}$ \\
\hline Quality Assessment Score & 84\% \\
\hline
\end{tabular}

\begin{tabular}{|c|c|}
\hline Data to be extracted & Study \# 4 \\
\hline Title & $\begin{array}{l}\text { Why I hit him: Women's reasons for intimate partner } \\
\text { violence }\end{array}$ \\
\hline Author/ Year & $\begin{array}{l}\text { Caldwell, J. E.; Swan, S. C.; Allen, C. T.; Sullivan, T. P.; } \\
\text { Snow, D. L. (2009) }\end{array}$ \\
\hline Study Design & Cross sectional \\
\hline Sampling Method & Non-random, convenience \\
\hline Sample & $\begin{array}{l}412 \text { females who have perpetrated IPA against a male } \\
\text { partner. Community sample recruited through brochures/ } \\
\text { posters. Screened via telephone to ensure they met study } \\
\text { criteria. Ethnically diverse. }\end{array}$ \\
\hline Measures Used & $\begin{array}{l}\text { Motives and Reasons for IPV Scale: Developed for this } \\
\text { study using EFA. } 5 \text { factors found - expression of negative } \\
\text { emotions ( } 5 \text { items; } \alpha=.86 \text { ), self-defense ( } 5 \text { items; } \alpha=.86 \text { ), } \\
\text { control ( } 6 \text { items; } \alpha=.78 \text { ), jealousy ( } 2 \text { items; } \alpha=.73 \text { ), tough } \\
\text { guise ( } 8 \text { items; } \alpha=.82 \text { ). } \\
\text { Physical aggression: CTS } 2 \text { physical subscale ( } 12 \text { items). } \\
\text { Alpha in the current study was } .87 \text {. } \\
\text { Psychological aggression: CTS } 2 \text { psychological subscale ( } 8 \\
\text { items) and the emotional/ verbal abuse subscale of the } \\
\text { Psychological Maltreatment of Women Inventory (Tolman, } \\
\text { 1989; } 13 \text { items). Alpha in the current study for the } \\
\text { combined measure was .76. }\end{array}$ \\
\hline Statistical Analysis & $\begin{array}{l}\text { EFA, C/EFA, t-tests, hierarchical regression, polynomial } \\
\text { regression, ANCOVA. }\end{array}$ \\
\hline Results/ Findings & $\begin{array}{l}\text { Most frequently endorsed motives related to the expression } \\
\text { of negative emotions. Motives in the control factor related } \\
\text { to both attempts to control their partner and resisting their } \\
\text { partner's attempts to control them. Self-defense and } \\
\text { jealousy also important motives for the women in this } \\
\text { study. }\end{array}$ \\
\hline
\end{tabular}




\begin{tabular}{|l|l|}
\hline Main Conclusions & $\begin{array}{l}\text { Women endorsed both instrumental and defensive motives } \\
\text { for perpetrating IPA. }\end{array}$ \\
\hline Strengths/ Weaknesses & $\begin{array}{l}\text { Use of EFA and C/EFA to develop measure. Ethnically } \\
\text { diverse sample. } \\
\text { Two of the items in the self-defense factor do not tap into } \\
\text { this construct well (e.g., because he became abusive when } \\
\text { he drank). }\end{array}$ \\
\hline Quality Assessment Score & $87 \%$ \\
\hline
\end{tabular}

\begin{tabular}{|c|c|}
\hline Data to be extracted & Study \# 5 \\
\hline Title & $\begin{array}{l}\text { Aggression in British heterosexual relationships: A } \\
\text { descriptive analysis }\end{array}$ \\
\hline Author/ Year & $\begin{array}{l}\text { Carrado, M.; George, M. J.; Loxam, E.; Jones, L.; Templar, } \\
\text { D. (1996) }\end{array}$ \\
\hline Study Design & Cross sectional \\
\hline Sampling Method & Non-random. \\
\hline Sample & $\begin{array}{l}1978 \text { heterosexual adults in UK. Sampling quotas used to } \\
\text { ensure sample was representative of the adult population of } \\
\text { the UK as determined by the most recent census ( } 1996 ; \\
\text { age, sex, SES, relationship status, geographical region). } \\
106 \text { women and } 85 \text { men }(N=191) \text { reported perpetrating } \\
\text { and their reasons for perpetrating IPA. }\end{array}$ \\
\hline Measures Used & $\begin{array}{l}\text { IPA: } 5 \text { items based on the CTS. } \\
\text { Motives: } 8 \text { items excluding 'other', 'I don't know'. }\end{array}$ \\
\hline Statistical Analysis & No mention of analysis conducted \\
\hline Results/ Findings & $\begin{array}{l}\text { The main reasons endorsed for the use of IPA for males } \\
\text { and females was to get through to their partner and } \\
\text { retaliation for verbal abuse. Percentages only. Some group } \\
\text { comparisons made, but only p values reported in text. }\end{array}$ \\
\hline Main Conclusions & $\begin{array}{l}\text { The findings regarding self-defense are contradictory to } \\
\text { other UK studies - women in this study did not endorse } \\
\text { this with any frequency. The authors attributed this to } \\
\text { married men withdrawing from confrontation, resulting in } \\
\text { an escalation in aggression from their female partner } \\
\text { (reference } 2 \text { x studies). }\end{array}$ \\
\hline Strengths/ Weaknesses & $\begin{array}{l}\text { Large, representative sample } \\
\text { Measures used have not been validated. No psychometric } \\
\text { information was provided about them. } \\
\text { Although they made group comparisons by sex, } \\
\text { relationship status and SES, no mention of the type of } \\
\text { analysis conducted was mentioned. } \\
\text { Group comparisons were not made on the reasons for IPA. } \\
\text { The self-defense item was poorly worded and may have } \\
\text { contributed to the low frequency with which it was } \\
\text { endorsed as a reason for IPA ("I thought he/ she was about } \\
\text { to use a physical action against me"). }\end{array}$ \\
\hline Quality Assessment Score & $37 \%$ \\
\hline
\end{tabular}




\begin{tabular}{|c|c|}
\hline Data to be extracted & Study \# 6 \\
\hline Title & $\begin{array}{l}\text { The meanings and motives for women's use of violence in } \\
\text { Canadian college dating relationships: Results from a } \\
\text { national survey }\end{array}$ \\
\hline Author/ Year & $\begin{array}{l}\text { DeKeseredy, W. S.; Saunders, D. G.; Schwartz, M. D.; } \\
\text { Alvi, S. (1997) }\end{array}$ \\
\hline Study Design & Cross sectional \\
\hline Sampling Method & Non-random, convenience \\
\hline Sample & $\begin{array}{l}1835 \text { female, college students. Median age } 20 \text { years. } \\
\text { Survey questions related to heterosexual, dating } \\
\text { relationships. } 1061 \text { had engaged in IPA }\end{array}$ \\
\hline Measures Used & $\begin{array}{l}\text { IPA: Physical subscale of the CTS (slightly modified; } 9 \\
\text { items). Motives: } 3 \text { x questions based on Saunders (1986). } \\
\text { These questions asked the primary motivation for } \\
\text { perpetrating IPA; self-defense; trying to fight back; } \\
\text { initiated the aggression. } \\
\text { No psychometric information regarding the measures used } \\
\text { or alphas provided for this study. }\end{array}$ \\
\hline Statistical Analysis & ANOVA, chi-square (post hoc) \\
\hline Results/ Findings & $\begin{array}{l}1 \% \text { difference between the percentage of women using } \\
\text { minor violence for self-defense or initiating the attack. } \\
0.2 \% \text { between the percentage of women using severe } \\
\text { violence for self-defense or initiating the attack. The most } \\
\text { common motive for both minor and severe violence was } \\
\text { fighting back. }\end{array}$ \\
\hline Main Conclusions & $\begin{array}{l}\text { Much of the violence perpetrated by this sample was in } \\
\text { self-defense. The majority of women did not initiate } \\
\text { attacks. }\end{array}$ \\
\hline Strengths/ Weaknesses & $\begin{array}{l}\text { Self-defense well defined. } \\
\text { Fighting back not well defined. It is unclear how this item } \\
\text { differs from self-defense, and whether it is restricted to } \\
\text { retaliation to a physical attack. } \\
\text { CTS is a well validated measure. } \\
\text { Lack of psychometric information. } \\
\text { Large sample size } \\
\text { Authors focus heavily on self-defense in the discussion } \\
\text { section. However, fighting back is a more frequently } \\
\text { endorsed motive. }\end{array}$ \\
\hline Quality Assessment Score & $50 \%$ \\
\hline
\end{tabular}

\begin{tabular}{|l|l|}
\hline Data to be extracted & Study \# 7 \\
\hline Title & $\begin{array}{l}\text { Motivations for intimate partner violence in men and } \\
\text { women arrested for domestic violence and court referred to } \\
\text { batterer intervention programs }\end{array}$ \\
\hline
\end{tabular}




\begin{tabular}{|c|c|}
\hline Author/ Year & $\begin{array}{l}\text { Elmquist, J.; Hamel, J.; Shorey, R. C.; Labrecque, L.; } \\
\text { Ninnemann, A.; Stuart, G. L. (2014) }\end{array}$ \\
\hline Study Design & Cross sectional \\
\hline Sampling Method & Non-random, convenience \\
\hline Sample & $\begin{array}{l}90 \text { men }(M \text { age }=40.30) \text { and } 87 \text { women }(N=177) \text { arrested } \\
\text { for IPA and referred to intervention programmes. }\end{array}$ \\
\hline Measures Used & $\begin{array}{l}\text { Reasons for Violence Scale (Stuart et al., 2006): } 29 \text { items. } \\
\text { Authors coded these into } 7 \text { categories as proposed by } \\
\text { Langhinrichsen-Rohling et al. (2012). No psychometrics } \\
\text { reported. } \\
\text { CTS2: Physical subscale: } 12 \text { items; } \alpha=.97 \text { for males and } \alpha \\
=.95 \text { for females. Psychological subscale: } 8 \text { items; } \alpha=.84 \\
\text { for males and } \alpha=.79 \text { for females. No other psychometric } \\
\text { information reported, except to state that the CTS2 has } \\
\text { good reliability and validity. }\end{array}$ \\
\hline Statistical Analysis & t-tests \\
\hline Results/ Findings & $\begin{array}{l}\text { Most frequently endorsed motives for males and females } \\
\text { were self-defense, expression of negative emotions, and } \\
\text { communication difficulties. No significant gender } \\
\text { differences in the motives for the use of physical } \\
\text { aggression reported, with the exceptions of expression of } \\
\text { negative emotions and retaliation (women higher). }\end{array}$ \\
\hline Main Conclusions & $\begin{array}{l}\text { Men and women endorse multiple motives for their use of } \\
\text { violence. These may be context specific. Women in } \\
\text { treatment programmes for IPA may have elevated } \\
\text { personality traits associated with emotional regulation } \\
\text { difficulties. As there was no significant gender difference } \\
\text { on } 5 \text { of the } 7 \text { motives, gender-neutral treatment } \\
\text { programmes are suggested. Treatment programmes should } \\
\text { target those motives most frequently endorsed. }\end{array}$ \\
\hline Strengths/ Weaknesses & $\begin{array}{l}\text { Validity of measure used } \\
\text { Lack of social desirability measure } \\
\text { Some data for females is not reported and the reader is } \\
\text { directed to another study. } \\
\text { No details regarding the coding of the items into the } 7 \\
\text { categories e.g., no inter-rater reliability score }\end{array}$ \\
\hline Quality Assessment Score & $56 \%$ \\
\hline
\end{tabular}

\begin{tabular}{|l|l|}
\hline Data to be extracted & Study \# 8 \\
\hline Title & $\begin{array}{l}\text { Physical and psychological aggression in dating } \\
\text { relationships of Spanish adolescents: Motives and } \\
\text { consequences }\end{array}$ \\
\hline Author/ Year & Fernandez-Fuertes, A. A.; Fuertes, A. (2010) \\
\hline Study Design & Cross sectional \\
\hline Sampling Method & Non-random, convenience \\
\hline
\end{tabular}




\begin{tabular}{|c|c|}
\hline Sample & $\begin{array}{l}567 \text { male }(n=236) \text { and female }(n=331) \text { Spanish high } \\
\text { school students }(15-19 \text { years }) . M \text { age }=16.6 \text { years. Survey } \\
\text { related to heterosexual relationships. }\end{array}$ \\
\hline Measures Used & $\begin{array}{l}\text { Conflict in Adolescent Dating Relationships Inventory } \\
\text { (CADRI; Spanish Version; Fernández-Fuertes, Fuertes, } \& \\
\text { Pulido, 2006): } 5 \text { items measure verbal-emotional } \\
\text { aggression; } \alpha=.78 \text {, and } 2 \text { items measure physical } \\
\text { aggression; } \alpha=.74 . \text {-point Likert scale. } \\
\text { Motives that cause arguments: Developed for this study } \\
\text { using EFA }-3 \text { factor solution: Dissatisfaction with partner } \\
\text { ( } 4 \text { items; } \alpha=.70), \text { relationship decline ( } 4 \text { items; } \alpha=.68 \text { ), } \\
\text { jealousy }(3 \text { items; } \alpha=.71) \text {. }\end{array}$ \\
\hline Statistical Analysis & EFA, correlation \\
\hline Results/ Findings & $\begin{array}{l}\text { Dissatisfaction with partner the most frequently reported } \\
\text { motive for males and females, followed by relationship } \\
\text { decline, and then jealousy. There was no significant gender } \\
\text { difference for dissatisfaction with partner or for jealousy. } \\
\text { Relationship decline was reported as a motive for } \\
\text { arguments more frequently by males, although the effect } \\
\text { size was small (.02) }\end{array}$ \\
\hline Main Conclusions & $\begin{array}{l}\text { Dissatisfaction with one's partner seemed to be the most } \\
\text { commonly noted motive for arguments between partners, } \\
\text { but jealousy was more central in explaining how often } \\
\text { aggressive acts were perpetrated }\end{array}$ \\
\hline Strengths/ Weaknesses & $\begin{array}{l}\text { Small number of items in each factor } \\
\text { None of the factors are motives, and neither a list of the } \\
\text { items, nor the pattern matrix was provided. No information } \\
\text { provided as to where the items were taken from or what } \\
\text { theory they might be informed by. }\end{array}$ \\
\hline Quality Assessment Score & $65 \%$ \\
\hline
\end{tabular}

\begin{tabular}{|l|l|}
\hline Data to be extracted & Study \# 9 \\
\hline Title & $\begin{array}{l}\text { College women who initiate assaults on their male partners } \\
\text { and the reasons offered for such behavior }\end{array}$ \\
\hline Author/ Year & Fiebert, M.; Gonzalez, D. (1997) \\
\hline Study Design & Cross sectional \\
\hline Sampling Method & Non-random, convenience \\
\hline Sample & $\begin{array}{l}\text { 978 female college students in the US. 285 had engaged in } \\
\text { physical IPA. }\end{array}$ \\
\hline Measures Used & $\begin{array}{l}\text { Domestic Behaviour and Analysis Form: This form } \\
\text { consisted of 5 questions relating to physical aggression, 5 } \\
\text { questions relating to immediate reasons for initiating } \\
\text { aggression, 10 questions relating to beliefs about the use of } \\
\text { women's aggression towards men, which the authors } \\
\text { referred to as "deeper reasons". This measure was not } \\
\text { piloted and no details regarding the source of the items or } \\
\text { psychometrics were provided. }\end{array}$ \\
\hline
\end{tabular}




\begin{tabular}{|l|l|}
\hline Statistical Analysis & ANOVA, chi-square (post hoc). \\
\hline Results/ Findings & $\begin{array}{l}\text { The most frequently endorsed reasons: partner not sensitive } \\
\text { to needs, gain partner's attention, partner not listening to } \\
\text { me, partner being verbally abusive, did not believe my } \\
\text { actions would hurt my partner. There appears to be little } \\
\text { difference in the frequency with which these were } \\
\text { endorsed (46-38\%), but this was never statistically tested. }\end{array}$ \\
\hline Main Conclusions & $\begin{array}{l}\text { The motives related to trying to engage their partner's } \\
\text { attention, particularly emotionally. }\end{array}$ \\
\hline Strengths/ Weaknesses & $\begin{array}{l}\text { Motives are conflated with reasons and beliefs } \\
\text { Small number of items and a lack of diversity in the } \\
\text { immediate reasons for initiating aggression } \\
\text { Measure used lacks reliability and validity }\end{array}$ \\
\hline Quality Assessment Score & $28 \%$ \\
\hline
\end{tabular}

\begin{tabular}{|l|l|}
\hline Data to be extracted & Study \# 10 \\
\hline Title & $\begin{array}{l}\text { Sex differences in motivations and effects in dating } \\
\text { violence }\end{array}$ \\
\hline Author/ Year & $\begin{array}{l}\text { Follingstad, D. R.; Wright, S; Lloyd, S; Sebastian, J. A } \\
\text { (1991) }\end{array}$ \\
\hline Study Design & Cross sectional \\
\hline Sampling Method & Non-random, convenience \\
\hline Sample & $\begin{array}{l}\text { 495 US college students (207 males and 288 females). } M \\
\text { age } \text { females - 20.2 years; males - 20.6 years. 115 had } \\
\text { engaged in IPA (24 males and 59 females) }\end{array}$ \\
\hline Measures Used & $\begin{array}{l}\text { CTS - Physical aggression subscale: 9 items; } \alpha=\text {.83 (cited } \\
\text { Straus, 1979). Likert scale. } \\
\text { Motivations and Effects Questionnaire: Developed for this } \\
\text { study. 13 motivation items. Choose the strongest } \\
\text { motivation for the use of aggression. }\end{array}$ \\
\hline Statistical Analysis & $\begin{array}{l}\text { Chi-square } \\
\text { Results/ Findings } \\
\text { motivations - Females more frequently reported the use of } \\
\text { force in retaliation for emotional hurt, to show anger, and } \\
\text { to get control. Males more frequently reported the use of } \\
\text { force in retaliation for being hit first and because of } \\
\text { jealousy. } \\
\text { The most frequently endorsed motivations by perpetrators } \\
\text { were (in order): wanting to show anger, retaliating for } \\
\text { emotional hurt, expressing jealousy, not knowing how to } \\
\text { express themselves verbally, retaliating for being hit, } \\
\text { needing to protect themselves (i.e., self- defence), wanting } \\
\text { to get control. }\end{array}$ \\
\hline Main Conclusions & $\begin{array}{l}\text { Due to the number of sex differences in motivations, } \\
\text { gender specific interventions may be more effective. As the } \\
\text { findings regarding control and self-defense were }\end{array}$ \\
\hline
\end{tabular}




\begin{tabular}{|l|l|}
\hline & $\begin{array}{l}\text { inconsistent with previous literature, more research is } \\
\text { required. }\end{array}$ \\
\hline Strengths/ Weaknesses & $\begin{array}{l}\text { Motivations and Effects Questionnaire not previously } \\
\text { validated or piloted. No information provided as to the } \\
\text { source of the items. } \\
\text { Self-defense item requires greater clarity (what are } \\
\text { respondents protecting themselves from) }\end{array}$ \\
\hline Quality Assessment Score & $44 \%$ \\
\hline
\end{tabular}

\begin{tabular}{|l|l|}
\hline Data to be extracted & Study \# 11 \\
\hline Title & $\begin{array}{l}\text { The intended function of domestic violence is different for } \\
\text { arrested male and female perpetrators }\end{array}$ \\
\hline Author/ Year & Hamberger, L. (1994) \\
\hline Study Design & Cross sectional \\
\hline Sampling Method & Non-random, convenience \\
\hline Sample & $\begin{array}{l}\text { 294 individuals court-referred to IPA treatment } \\
\text { programmes (75 women and 219 men). No additional } \\
\text { information regarding ethnicity etc. provided. }\end{array}$ \\
\hline Measures Used & $\begin{array}{l}\text { CTS: Not clear whether the entire CTS was administered, } \\
\text { or just the questions relating to physical force, which the } \\
\text { study is interested in. } \\
\text { Motivation was assessed with one question: "What is the } \\
\text { function, purpose or payoff of your violence?" }\end{array}$ \\
\hline Statistical Analysis & PCA \\
\hline Results/ Findings & N/A \\
\hline Main Conclusions & N/A \\
\hline Strengths/ Weaknesses & N/A \\
\hline Quality Assessment Score & $\begin{array}{l}\text { The study involved collecting participants motivations for } \\
\text { their use of IPA to conduct a principal components } \\
\text { analysis. No further analysis was conducted. Therefore, } \\
\text { this study had to be excluded from further analysis. }\end{array}$ \\
\hline
\end{tabular}

\begin{tabular}{|l|l|}
\hline Data to be extracted & Study \# 12 \\
\hline Title & $\begin{array}{l}\text { Abused women or abused men? An examination of the } \\
\text { context and outcomes of dating violence }\end{array}$ \\
\hline Author/ Year & Harned, M. S. (2001) \\
\hline Study Design & Cross sectional \\
\hline Sampling Method & Random, convenience \\
\hline Sample & $\begin{array}{l}\text { 874 US college students (489 women and 385 men). } \\
\text { Response rate reported (38\%). Sample ethnically } \\
\text { representative of the university. } M \text { age }=21.3 \text { years. } 134 \\
\text { had engaged in IPA (92 women and 42 men). }\end{array}$ \\
\hline
\end{tabular}




\begin{tabular}{|c|c|}
\hline Measures Used & $\begin{array}{l}\text { CTS2 - Physical aggression subscale: } 12 \text { items. } \\
\text { Demonstrated construct validity in student sample and } \alpha= \\
.86 \text { (Straus et al., 1996). } \\
\text { Motivations and Effects Questionnaire (Follingstad et al., } \\
\text { 1991): } 12 \text { item version. Used a 6-point Likert scale. }\end{array}$ \\
\hline Statistical Analysis & $\begin{array}{l}\text { Exploratory PCA with varimax rotation: } 2 \text { factors: anger/ } \\
\text { jealousy ( } 4 \text { items; } \alpha=.78 \text { for females and } \alpha=.72 \text { for } \\
\text { males) and self-defense ( } 2 \text { items; } \alpha=.68 \text { for females and } \alpha \\
=.86 \text { for males). The remaining } 6 \text { items did not reliably } \\
\text { load onto components for both males and females and so } \\
\text { were treated as single items. These items were: Inability to } \\
\text { express self verbally, to feel more powerful, to get control } \\
\text { over the person, to prove love, because it was sexually } \\
\text { arousing, to get attention. } \\
\text { t-tests. }\end{array}$ \\
\hline Results/ Findings & $\begin{array}{l}\text { Females were significantly higher on anger/ jealousy. No } \\
\text { other significant gender differences. For both sexes, anger/ } \\
\text { jealousy, self-defense and an inability to express self } \\
\text { verbally were the top three most frequently endorsed } \\
\text { motives. }\end{array}$ \\
\hline Main Conclusions & $\begin{array}{l}\text { Self-defense equally endorsed by males and females, } \\
\text { contrary to the hypothesis and previous research. }\end{array}$ \\
\hline Strengths/ Weaknesses & $\begin{array}{l}\text { Survey piloted } \\
\text { Sample representative of the population } \\
\text { Combining of male and female data in PCA - these should } \\
\text { have been done separately and then subjected to invariance } \\
\text { testing. } \\
\text { No measure of social desirability used. } \\
\text { The self-defense factor is comprised of two items, one of } \\
\text { which relates to retaliation. }\end{array}$ \\
\hline Quality Assessment Score & $70 \%$ \\
\hline
\end{tabular}

\begin{tabular}{|l|l|}
\hline Data to be extracted & Study \# 13 \\
\hline Title & $\begin{array}{l}\text { Females' reasons for their physical aggression in dating } \\
\text { relationships. }\end{array}$ \\
\hline Author/ Year & Hettrich, E. L.; O'Leary, K. D. (2007) \\
\hline Study Design & Cross sectional \\
\hline Sampling Method & Non-random, convenience \\
\hline Sample & $\begin{array}{l}\text { 127 US college women. Response rate reported. Ethnically } \\
\text { diverse sample. } M \text { age at time of relationship = 18.97 } \\
\text { years. }\end{array}$ \\
\hline Measures Used & $\begin{array}{l}\text { Modified CTS (Straus \& Gelles, 1990): 20 items (self and } \\
\text { partner and verbal and physical). 5-point Likert scale. No } \\
\text { additional of psychometric information provided. } \\
\text { Reasons for Aggression Scale: 1 x open ended question } \\
\text { and 12 x reasons based on Follingstad et al. (1991), Kelley }\end{array}$ \\
\hline
\end{tabular}




\begin{tabular}{|l|l|}
\hline & $\begin{array}{l}\text { (1995), and Harned (2001). 5-point Likert scale. No } \\
\text { psychometric information provided. }\end{array}$ \\
\hline Statistical Analysis & $\begin{array}{l}\text { Moscriptive data only } \\
\text { partner lied, poor communication, temper, embarrassed, } \\
\text { jealousy. Most frequently cited reasons that were "not a } \\
\text { cause" were partner forced sex, prevent partner from } \\
\text { committing an illegal act, drugs/ alcohol, self-defense. } \\
\text { Agreement between open and closed ended questions in } \\
\text { top three reasons reported. }\end{array}$ \\
\hline Results/ Findings & $\begin{array}{l}\text { Motives related to emotional responses as opposed to self- } \\
\text { defense. The primary reasons for engaging in IPA were } \\
\text { anger and communication difficulties. }\end{array}$ \\
\hline Strengths/ Weaknesses & $\begin{array}{l}\text { No psychometric information provided } \\
\text { No discussion of analysis conducted. }\end{array}$ \\
\hline Quality Assessment Score & 39\% \\
\hline
\end{tabular}

\begin{tabular}{|l|l|}
\hline Data to be extracted & Study \# 14 \\
\hline Title & $\begin{array}{l}\text { Motives for physical dating violence among college } \\
\text { students: A gendered analysis }\end{array}$ \\
\hline Author/ Year & $\begin{array}{l}\text { Kelley, E. L; Edwards, K. M.; Dardis, C. M.; Gidycz, C. A. } \\
\text { (2015) }\end{array}$ \\
\hline Study Design & Cross sectional \\
\hline Sampling Method & Non-random, convenience \\
\hline Sample & $\begin{array}{l}\text { 221 US college students (89 males and 132 females). } M \\
\text { age }=19.00 \text { years. Ethnically homogenous sample, } \\
\text { although this is likely to be representative of the university } \\
\text { population. }\end{array}$ \\
\hline Measures Used & $\begin{array}{l}\text { CTS2 - physical aggression subscale: 12 items. Construct } \\
\text { validity mentioned. No alphas reported for this study. } \\
\text { Motives and Reasons for IPV scale (Caldwell, Swan, } \\
\text { Allen, Sullivan, \& Snow, 2009): 19 items. 4-point Likert } \\
\text { scale. 3 factors: emotional expression/ dysregulation } \text { (9 } \\
\text { items, } \alpha=.89), \text { control/ tough guise (6 items, } \alpha=.80), \text { self- } \\
\text { defense (4 items, } \alpha=.83) .\end{array}$ \\
\hline Statistical Analysis & EFA (oblique rotation), correlations, linear regression \\
\hline Results/ Findings & $\begin{array}{l}\text { No significant sex differences for self-defense. Females } \\
\text { reported emotional expression/ dysregulation as a motive } \\
\text { with a greater frequency (sig). Males more frequently } \\
\text { reported control/ tough guise as a motive (not sig). Greater } \\
\text { frequency of IPA was associated with control/ tough guise } \\
\text { for males and females. }\end{array}$ \\
\hline Main Conclusions & $\begin{array}{l}\text { Treatment programmes for IPA should be gender-specific } \\
\text { Conducted an EFA as the Motives and Reasons for IPV } \\
\text { scale had not previously been used with a student sample. }\end{array}$ \\
\hline Strengths/ Weaknesses & \begin{tabular}{l} 
81\% \\
\hline
\end{tabular} \\
\hline
\end{tabular}




\begin{tabular}{|l|l|}
\hline Data to be extracted & Study \# 15 \\
\hline Title & $\begin{array}{l}\text { Exerting power or striking back: A gendered comparison } \\
\text { of motivations for domestic violence perpetration }\end{array}$ \\
\hline Author/ Year & Kernsmith, P. (2005) \\
\hline Study Design & Cross sectional \\
\hline Sampling Method & Non-random, convenience \\
\hline Sample & $\begin{array}{l}125 \text { individuals court-referred to IPA treatment } \\
\text { programmes (59 women and 66 men). M age = 34 years. }\end{array}$ \\
\hline Measures Used & $\begin{array}{l}\text { Motivations and Effects Questionnaire (Follingstad et al., } \\
\text { 1991): 16 items. 5-point Likert scale. No other information } \\
\text { provided. }\end{array}$ \\
\hline Statistical Analysis & "Factor analysis", t-tests \\
\hline Results/ Findings & $\begin{array}{l}\text { Retaliation for emotional hurt was the most frequently } \\
\text { cited motivation for males and females. }\end{array}$ \\
\hline Main Conclusions & $\begin{array}{l}\text { This study investigated whether power and control were } \\
\text { motivations for female perpetrated IPA. However, revenge } \\
\text { and retaliation were primary motivations for women. } \\
\text { Therefore, treatment programmes, which are based on } \\
\text { power and control may not be appropriate for them. }\end{array}$ \\
\hline Strengths/ Weaknesses & $\begin{array}{l}\text { Statistical analysis in this study is very poor - A factor } \\
\text { analysis was conducted, but there is no mention of what } \\
\text { type, no discussion of eigenvalues, factor loadings, cut-off } \\
\text { points, scree plots. There is no pattern matrix. In addition, } \\
\text { there is no mention of the types of the other analysis } \\
\text { conducted e.g., t-test, correlation, just the reporting of the } \\
\text { results. }\end{array}$ \\
\hline \begin{tabular}{l}
$33 \%$ \\
\hline
\end{tabular} \\
\hline
\end{tabular}

\begin{tabular}{|l|l|}
\hline Data to be extracted & Study \# 16 \\
\hline Title & $\begin{array}{l}\text { Physical and emotional abuse in romantic relationships: } \\
\text { Motivation for perpetration among college women }\end{array}$ \\
\hline Author/ Year & Leisring, P. A. (2013) \\
\hline Study Design & Cross sectional \\
\hline Sampling Method & Non-random, convenience \\
\hline Sample & $\begin{array}{l}\text { 348 US college women. } M \text { age }=18.8 \text { years. 125 had } \\
\text { engaged in physical IPA. Sample was not ethnically } \\
\text { diverse. }\end{array}$ \\
\hline Measures Used & $\begin{array}{l}\text { CTS2 - physical aggression subscale: 12 items. } \alpha=.72 \text { for } \\
\text { this study. } \\
\text { Modified version of Motivations and Effects Questionnaire } \\
\text { (Follingstad et al., 1991): 15 items. No psychometric } \\
\text { information reported. }\end{array}$ \\
\hline Statistical Analysis & $\begin{array}{l}\text { Chi-square } \\
\text { Anger, retaliation for emotional hurt and stress were the } \\
\text { three most frequently endorsed motives for women } \\
\text { perpetrating minor physical IPA. Anger, retaliation for } \\
\text { emotional hurt and an inability to express self verbally }\end{array}$ \\
\hline Results/ Findings &
\end{tabular}




\begin{tabular}{|l|l|}
\hline & $\begin{array}{l}\text { were the three most frequently endorsed motives for } \\
\text { women perpetrating severe physical IPA. }\end{array}$ \\
\hline Main Conclusions & $\begin{array}{l}\text { As anger is the main motivator for both minor and severe } \\
\text { IPA it is an important treatment target. Other treatment } \\
\text { targets include emotional regulation and stress } \\
\text { management skills. } \\
\text { The clear definition of self-defense may have attributed to } \\
\text { the low rates of endorsement in this study. }\end{array}$ \\
\hline Strengths/ Weaknesses & Self-defense item based on the legal definition \\
\hline Quality Assessment Score & $57 \%$ \\
\hline
\end{tabular}

\begin{tabular}{|c|c|}
\hline Data to be extracted & Study \# 17 \\
\hline Title & Gender differences in courtship violence victimization \\
\hline Author/ Year & Makepeace, J. M. (1986) \\
\hline Study Design & Cross sectional \\
\hline Sampling Method & Non-random, convenience \\
\hline Sample & $\begin{array}{l}2338 \text { US college students ( } 1278 \text { females and } 1060 \text { males). } \\
M \text { age }=21.5 \text { years. No details of the ethnic composition of } \\
\text { the sample provided. } 391 \text { had engaged in physical IPA } \\
\text { ( } 264 \text { females and } 127 \text { males) and their data was used in the } \\
\text { analysis for motives. }\end{array}$ \\
\hline Measures Used & $\begin{array}{l}\text { IPA measure designed for this study which asked about the } \\
\text { frequency, type, first and worst incident. Violence items } \\
\text { based on CTS. No psychometric information provided. No } \\
\text { mention of motives in the description of this measure, } \\
\text { therefore no information regarding the source of the } \\
\text { motives items. } 7 \text { motives were measured (including } \\
\text { 'other'), although these were only listed in one of the tables } \\
\text { in the results section. }\end{array}$ \\
\hline Statistical Analysis & $\mathrm{Z}$ test for differences in proportions \\
\hline Results/ Findings & $\begin{array}{l}\text { Uncontrollable anger, intimidate and self-defense were the } \\
\text { three most frequently endorsed motives for men } \\
\text { perpetrating physical IPA. Self-defense, uncontrollable } \\
\text { anger and retaliate were the three most frequently endorsed } \\
\text { motives for women perpetrating physical IPA. } \\
\text { Significant gender differences were found for self-defense, } \\
\text { to harm (females higher), and intimidate (males higher). }\end{array}$ \\
\hline Main Conclusions & $\begin{array}{l}\text { Female violence is self-defensive; male violence is } \\
\text { culpability reducing. }\end{array}$ \\
\hline Strengths/ Weaknesses & $\begin{array}{l}\text { Measure had not been validated or piloted } \\
\text { Items used in motives scale were too brief and not defined } \\
\text { e.g., self-defense, retaliate. } \\
\text { Lack of detail regarding analyses conducted }\end{array}$ \\
\hline Quality Assessment Score & $27 \%$ \\
\hline
\end{tabular}




\begin{tabular}{|c|c|}
\hline Data to be extracted & Study \# 18 \\
\hline Title & $\begin{array}{l}\text { Why did she do it? College women's motives for intimate } \\
\text { partner violence perpetration }\end{array}$ \\
\hline Author/ Year & $\begin{array}{l}\text { Neal, A. M.; Dixon, K. J.; Edwards, K. M.; Gidycz, C. A. } \\
(2015)\end{array}$ \\
\hline Study Design & Cross sectional \\
\hline Sampling Method & Non-random, convenience \\
\hline Sample & $\begin{array}{l}484 \text { US college women. } M \text { age }=18.7 \text { years. } \\
\text { Predominantly white sample. }\end{array}$ \\
\hline Measures Used & $\begin{array}{l}\text { CTS2 used to measure IPA } \\
\text { Modified version of the Motivations and Effects } \\
\text { Questionnaire (Follingstad et al., 1991): } 11 \text { items. } \\
\text { No psychometric information provided for either measure. }\end{array}$ \\
\hline Statistical Analysis & Chi-square \\
\hline Results/ Findings & $\begin{array}{l}\text { Anger, retaliation (for being hit first or for emotion hurt) } \\
\text { and an inability to express themselves verbally were the } \\
\text { three most frequently endorsed motives for women } \\
\text { perpetrating physical and psychological IPA. There were } \\
\text { significant differences in the frequency of motivation by } \\
\text { type of IPA: Inability to express themselves verbally, loss } \\
\text { of control, and retaliation were endorsed more frequently } \\
\text { by those engaging in physical IPA; Desire to get attention } \\
\text { and power and control were endorsed more frequently by } \\
\text { those engaging in psychological IPA. }\end{array}$ \\
\hline Main Conclusions & $\begin{array}{l}\text { Women's motivations for physical and psychological IPA } \\
\text { different to those for sexual IPA. This has implications for } \\
\text { treatment. }\end{array}$ \\
\hline Strengths/ Weaknesses & $\begin{array}{l}\text { The retaliation item was comprised of both physical and } \\
\text { emotional elements, which may have affected the results. }\end{array}$ \\
\hline Quality Assessment Score & $78 \%$ \\
\hline
\end{tabular}

\begin{tabular}{|l|l|}
\hline Data to be extracted & Study \# 19 \\
\hline Title & $\begin{array}{l}\text { Personality and situational correlates of self-reported } \\
\text { reasons for intimate partner violence among women versus } \\
\text { men referred for batterers' intervention }\end{array}$ \\
\hline Author/ Year & Ross, J. M. (2011) \\
\hline Study Design & Cross sectional \\
\hline Sampling Method & Non-random, convenience \\
\hline Sample & $\begin{array}{l}86(30 \text { women and 56 men) people referred to treatment for } \\
\text { IPA. } M \text { age = 30 years. Ethnic composition of sample } \\
\text { reported, however, no indication as to whether this is } \\
\text { representative of the population/ community. }\end{array}$ \\
\hline Measures Used & $\begin{array}{l}\text { CTS2 - physical aggression subscale: } 12 \text { items. Reliability } \\
\text { for this subscale for the current study } \alpha=.78 . \\
\text { Reasons for Violence (RFV) Scale (Stuart, Moore, Gordon, } \\
\text { Hellmuth, Ramsey, \& Kahler, 2006): 29 items. PCA } \\
\text { conducted, which yielded 4 factors: dominate-punish (a= }\end{array}$ \\
\hline
\end{tabular}




\begin{tabular}{|l|l|}
\hline & $\begin{array}{l}0.92), \text { emotion dysregulation }(\mathrm{a}=0.89), \text { retaliation }(\mathrm{a}= \\
0.86), \text { and defence } \\
(\mathrm{a}=0.82)\end{array}$ \\
\hline Statistical Analysis & $\begin{array}{l}\text { PCA, t-test } \\
\text { three most frequently endorsed motives for women } \\
\text { perpetrating IPA. Retaliation, emotion dysregulation, and } \\
\text { defence were the three most frequently endorsed motives } \\
\text { for men perpetrating IPA. } \\
\text { Women were significantly endorsed defence as a motive } \\
\text { more frequently than men. No other significant differences. }\end{array}$ \\
\hline Main Conclusions & $\begin{array}{l}\text { The same top three reasons for IPV were endorsed by } \\
\text { women and men. Dominate-punish was the least endorsed } \\
\text { reason for both males and females, although was endorsed } \\
\text { at a (non-sig) higher rate by females. }\end{array}$ \\
\hline Strengths/ Weaknesses & $\begin{array}{l}\text { Greater detail regarding the PCA should have been } \\
\text { provided. }\end{array}$ \\
\hline Quality Assessment Score & 52\% \\
\hline
\end{tabular}

\begin{tabular}{|l|l|}
\hline Data to be extracted & Study \# 20 \\
\hline Title & $\begin{array}{l}\text { When battered women use violence: Husband abuse or } \\
\text { self-defense? }\end{array}$ \\
\hline Author/ Year & Saunders, D. G. (1986) \\
\hline Study Design & Cross sectional \\
\hline Sampling Method & Non-random, convenience \\
\hline Sample & $\begin{array}{l}\text { 52 women from refuges/ help-seeking organisations in the } \\
\text { US. No mean age reported. Ethnic composition of sample } \\
\text { not reported. }\end{array}$ \\
\hline Measures Used & $\begin{array}{l}\text { CTS: Verbal and physical subscales were administered, but } \\
\text { only the physical aggression subscale was used for analysis } \\
\text { (9 items). The reliability and validity of this scale is } \\
\text { mentioned, although no psychometric information is } \\
\text { reported. } \\
\text { Motives: Following the minor and severe scales of the } \\
\text { CTS, the following questions were asked to assess motive: } \\
\text { (a) "What percentage of these times do you estimate that } \\
\text { you acted in self-defense, that is, protecting yourself from } \\
\text { immediate physical harm?"; (b) "What percentage of these } \\
\text { times were you trying to } \\
\text { fight back?"; and (c) "What percentage of these times did } \\
\text { you assault your partner before he actually attacked you, or } \\
\text { threatened you with a weapon?" } \\
\text { Marlowe-Crowne Social Desirability Scale (Crown \& } \\
\text { Marlowe, 1964): 18 items. Concurrent validity discussed. }\end{array}$ \\
\hline Correlation \\
\hline Results/ Findings & $\begin{array}{l}\text { Self-defense and fighting back are positively and } \\
\text { significantly correlated for minor and severe aggression. }\end{array}$ \\
\hline
\end{tabular}




\begin{tabular}{|l|l|}
\hline & $\begin{array}{l}\text { Self-defense and initiating an attack are negatively, but not } \\
\text { significantly, correlated for minor and severe aggression. } \\
\text { Fighting back and initiating an attack are negatively, but } \\
\text { not significantly, correlated for minor and severe } \\
\text { aggression. }\end{array}$ \\
\hline Main Conclusions & $\begin{array}{l}\text { The most frequently cited motive was self-defense. The } \\
\text { concepts of self-defense and fighting back were conflated } \\
\text { in this sample. }\end{array}$ \\
\hline Strengths/ Weaknesses & $\begin{array}{l}\text { Motive measure not validated or piloted prior to use in the } \\
\text { study } \\
\text { Self-defense item based on the legal definition } \\
\text { Use of social desirability scale } \\
\text { Limited number of motives assessed } \\
\text { "Fight back" was not defined. This may have led to the } \\
\text { women associating this item with self-defense, as opposed } \\
\text { to tapping into retaliation/ revenge as was intended by the } \\
\text { author. }\end{array}$ \\
\hline Quality Assessment Score & \begin{tabular}{l} 
44\% \\
\hline
\end{tabular} \\
\hline
\end{tabular}

\begin{tabular}{|l|l|}
\hline Data to be extracted & Study \# 21 \\
\hline Title & $\begin{array}{l}\text { Motivations for self-defensive aggression in dating } \\
\text { relationships }\end{array}$ \\
\hline Author/ Year & Shorey, R. C.; Meltzer, C.; Cornelius, T. L. (2010) \\
\hline Study Design & Cross sectional \\
\hline Sampling Method & Non-random, convenience \\
\hline Sample & $\begin{array}{l}\text { 193 US college students. } \text { M age = 18.73. The sample was > } \\
90 \% \text { white. } 60 \text { people had engaged in physical aggression } \\
\text { and were used in the analysis of motives (14 males and 46 } \\
\text { females). }\end{array}$ \\
\hline Measures Used & $\begin{array}{l}\text { CTS2 - physical, psychological aggression subscales: } \\
\text { Internal consistency and construct and discriminate validity } \\
\text { noted. } \\
\text { Reasons for Violence Scale (RVS: Stuart et al, 2006): 29 } \\
\text { items. } \alpha=.93 .\end{array}$ \\
\hline Statistical Analysis & $\begin{array}{l}\text { To get partners attention, jealousy, and because it was } \\
\text { sexually arousing were the three most frequently endorsed } \\
\text { motives for men perpetrating IPA. Because it was sexually } \\
\text { arousing, retaliation for emotional hurt, and to get partners } \\
\text { attention were the three most frequently endorsed motives } \\
\text { for women perpetrating IPA. No significant gender } \\
\text { differences were found. }\end{array}$ \\
\hline Results/ Findings & $\begin{array}{l}\text { Common motivations for males and females related to } \\
\text { emotional dysregulation and communication difficulties, } \\
\text { indicating an inability to resolve relationship conflict non- } \\
\text { violently. }\end{array}$ \\
\hline Strengths/ Weaknesses & Small sample size \\
\hline Main Conclusions &
\end{tabular}




\begin{tabular}{|l|l|}
\hline & $\begin{array}{l}\text { The authors designed a measure of self-defense. However, } \\
\text { none of the items were based on the legal definition. This } \\
\text { measure was not piloted prior to use in the study or subject } \\
\text { to scale development. }\end{array}$ \\
\hline Quality Assessment Score & $69 \%$ \\
\hline
\end{tabular}

\begin{tabular}{|l|l|}
\hline Data to be extracted & Study \# 22 \\
\hline Title & $\begin{array}{l}\text { Reasons for intimate partner violence perpetration among } \\
\text { arrested women }\end{array}$ \\
\hline Author/ Year & $\begin{array}{l}\text { Stuart, G. L.; Moore, T. M.; Gordon, K. C.; Hellmuth, J. } \\
\text { C.; Ramsey, S. E.; Kahler, C. W. (2006) }\end{array}$ \\
\hline Study Design & Cross sectional \\
\hline Sampling Method & Non-random, convenience \\
\hline Sample & $\begin{array}{l}\text { 87 women referred to treatment for IPA. M age = 31.2 } \\
\text { years. Ethnic composition predominantly white (76\%). } \\
\text { Response rate reported. }\end{array}$ \\
\hline Measures Used & $\begin{array}{l}\text { CTS2: Reliability and validity mentioned. No } \\
\text { psychometric information available. } \\
\text { Reasons for Violence Scale: Developed for this study on } \\
\text { the basis of a literature review. 29 items. Multiple items } \\
\text { could be endorsed for one act. }\end{array}$ \\
\hline Statistical Analysis & $\begin{array}{l}\text { Descriptive data only } \\
\text { anger, because your partner provoked you or pushed you } \\
\text { over the edge, and self-defense. }\end{array}$ \\
\hline Results/ Findings & $\begin{array}{l}\text { Self-defense was among one of the most common reasons } \\
\text { for IPA perpetration }\end{array}$ \\
\hline Main Conclusions & $\begin{array}{l}\text { One of the hypotheses relate to the use of violence in self- } \\
\text { defense. This seems to have clouded the way in which the } \\
\text { findings of the study have been reported and appears } \\
\text { intentionally misleading. In listing and discussing the most } \\
\text { common motivations, self-defense is always listed first }- \text { in } \\
\text { the abstract, results (text and in the table), and discussion, } \\
\text { despite it being the third most frequently reported motive. } \\
\text { This is suggestive of outcome reporting bias. } \\
\text { t-tests were conducted to test a second hypothesis, and } \\
\text { although not relevant to the research question of this } \\
\text { review, it is worth noting that the authors did not take into } \\
\text { account the skewness of the data when conducting this } \\
\text { analysis. } \\
\text { Measure developed for this study, but no scale } \\
\text { development done. Items were based on a literature review. }\end{array}$ \\
\hline Strengths/ Weaknesses & \begin{tabular}{l}
$58 \%$ \\
\hline
\end{tabular} \\
\hline Q &
\end{tabular}




\begin{tabular}{|c|c|}
\hline Data to be extracted & Study \# 23 \\
\hline Title & $\begin{array}{l}\text { Behavioural and psychological differences among abused } \\
\text { women who use violence in intimate relationships }\end{array}$ \\
\hline Author/ Year & Swan, S. C.; Snow, D. L. (2003) \\
\hline Study Design & Cross sectional \\
\hline Sampling Method & Non-random, convenience \\
\hline Sample & $\begin{array}{l}95 \text { women referred to treatment for IPA. Sample was } \\
\text { predominantly African American }(77 \%) \text {. Percentages } \\
\text { within age brackets reported, rather than mean age. }\end{array}$ \\
\hline Measures Used & $\begin{array}{l}\text { CTS } 2 \text { - physical aggression subscale: } 8 \text { items }-4 \text { minor } \\
\text { and } 4 \text { severe. Reliability and validity mentioned. A = .84 in } \\
\text { current study. } \\
\text { Motivations for Violence scale (Swan \& Gill, } 1998) \text { : } \\
\text { Developed for this study. 4-point Likert scale. The scale } \\
\text { measured } 3 \text { motives for the use of violence: In self-defense } \\
(2 \text { items, } \alpha=.78) \text {, for control ( } 1 \text { item), to get even ( } 1 \text { item). }\end{array}$ \\
\hline Statistical Analysis & MANOVA, ANOVA (post-hoc) \\
\hline Results/ Findings & $\begin{array}{l}\text { The most commonly reported motive was self-defense, } \\
\text { followed by get even, control. Victim reported the use of } \\
\text { self-defense more frequently, but this was only significant } \\
\text { in relation to the Mixed-Female Coercive type. The } \\
\text { Abused Aggressor type endorsed control as a motive } \\
\text { significantly more than the other groups. The Abused } \\
\text { Aggressor type endorsed get even as a motive significantly } \\
\text { more than the Mixed-Female Coercive type and Victim } \\
\text { type. }\end{array}$ \\
\hline Main Conclusions & $\begin{array}{l}\text { Self-defense was the common motive, with } 75 \% \text { endorsing } \\
\text { this motive. }\end{array}$ \\
\hline Strengths/ Weaknesses & $\begin{array}{l}\text { Small number of items in motivations scale. } \\
\text { Measure of motivations not piloted of validated } \\
\text { Small number of women in each group/ type. }\end{array}$ \\
\hline Quality Assessment Score & $56 \%$ \\
\hline
\end{tabular}

\begin{tabular}{|l|l|}
\hline Data to be extracted & Study \# 24 \\
\hline Title & $\begin{array}{l}\text { Motivations and justifications for partner aggression in a } \\
\text { sample of African American college women }\end{array}$ \\
\hline Author/ Year & Walley-Jean, J. C.; Swan, S. (2009) \\
\hline Study Design & Cross sectional \\
\hline Sampling Method & Non-random, convenience \\
\hline Sample & $\begin{array}{l}\text { 82 African American college students. } M \text { age }=20.13 \\
\text { years. 42 women had perpetrated physical IPA and so were } \\
\text { included in the analysis related to motives. }\end{array}$ \\
\hline Measures Used & $\begin{array}{l}\text { CTS2 }- \text { physical aggression subscale: (12 items, } \alpha=.70) . \\
\text { Internal consistency and construct and discriminant validity } \\
\text { mentioned. } \\
\text { Motivations and Effects Questionnaire (MEQ: Follingstad } \\
\text { et al., 1991): 13 items. PCA to investigate factor structure. }\end{array}$ \\
\hline
\end{tabular}




\begin{tabular}{|l|l|}
\hline & $\begin{array}{l}\text { Four factors: Expression of negative emotions (4 items), } \\
\text { aggression as response (4 items), communication (3 items), } \\
\text { expression of positive emotions (2 items). Internal } \\
\text { consistencies not reported. }\end{array}$ \\
\hline Statistical Analysis & PCA (varimax rotation), multiple regression \\
\hline Results/ Findings & $\begin{array}{l}\text { The most frequently endorsed motives were to show anger, } \\
\text { the inability to express self verbally, and (equally) in } \\
\text { retaliation for emotional hurt and to get attention. }\end{array}$ \\
\hline Main Conclusions & $\begin{array}{l}\text { Women in this sample used violence as a means of } \\
\text { communicating or expressing negative emotions }\end{array}$ \\
\hline Strengths/ Weaknesses & $\begin{array}{l}\text { Sample size is too small to conduct a PCA. } \\
\text { EFA should have been conducted as opposed to a PCA } \\
\text { Low number of items in the individual factors. } \\
\text { Incorrect (orthogonal) rotation method used in PCA. }\end{array}$ \\
\hline Quality Assessment Score & 52\% \\
\hline
\end{tabular}




\section{APPENDIX L}

Quality Assessment Form

\begin{tabular}{|c|c|c|c|}
\hline Study 1 - Title: & \multicolumn{3}{|c|}{$\begin{array}{l}\text { Toward a typology of abusive women: Differences between } \\
\text { partner-only and generally violent women in the use of violence }\end{array}$} \\
\hline Author (Year): & \multicolumn{3}{|c|}{ Babcock, J. C., Miller, S. A., \& Siard, C. (2003) } \\
\hline \multicolumn{4}{|l|}{ Sampling: } \\
\hline Questions & Description & Score & Comments \\
\hline \multirow[t]{4}{*}{$\begin{array}{l}\text { Was the sampling } \\
\text { strategy appropriate to } \\
\text { address the research } \\
\text { question/s? } \\
\text { Why was this group } \\
\text { targeted? } \\
\text { Were the participants } \\
\text { representative of the } \\
\text { target population? }\end{array}$} & $\begin{array}{l}\text { Good }=3=\text { Details (age/ } \\
\text { gender/ ethnicity /context) of } \\
\text { who was studied and how } \\
\text { they were recruited. } \\
\text { Rationale for studying this } \\
\text { population. Response rates } \\
\text { shown and explained (why } \\
\text { certain individuals chose not } \\
\text { to participate, and any } \\
\text { attempts to achieve a sample } \\
\text { that represents the target } \\
\text { population). }\end{array}$ & \multirow[t]{4}{*}{2.5} & \multirow[t]{4}{*}{$\begin{array}{l}\text { It is noted in the } \\
\text { limitations that the } \\
\text { sample may not be } \\
\text { representative of the } \\
\text { population. No attempts } \\
\text { were made to achieve } \\
\text { this. }\end{array}$} \\
\hline & $\begin{array}{l}\text { Fair }=2=\text { Most information } \\
\text { given, but some missing. }\end{array}$ & & \\
\hline & $\begin{array}{l}\text { Poor }=1=\text { Sampling } \\
\text { mentioned but few } \\
\text { descriptive details. }\end{array}$ & & \\
\hline & $\begin{array}{l}\text { Very Poor }=0=\text { No details } \\
\text { of sample. }\end{array}$ & & \\
\hline \multirow{3}{*}{$\begin{array}{l}\text { Reporting of power or a } \\
\text { priori power analysis } \\
\text { conducted? } \\
\text { Does the study have } \geq \\
80 \% \text { power to detect an } \\
\text { effect at } r=.20 \text { ? }\end{array}$} & $\begin{array}{l}\text { Good = } 3=\text { Two of the above } \\
\text { (State in comments). }\end{array}$ & \multirow[t]{3}{*}{0} & \multirow{3}{*}{$\begin{array}{l}\text { No reporting of power } \\
\text { or a priori power } \\
\text { analysis conducted. } \\
\text { This was not able to be } \\
\text { determined as } \\
\text { insufficient information } \\
\text { was supplied for a } \\
\text { MANOVA e.g., } \\
\text { correlation between } \\
\text { repeated measures. }\end{array}$} \\
\hline & $\begin{array}{l}\text { Poor }=1.5=\text { Only one of the } \\
\text { above (State in comments). }\end{array}$ & & \\
\hline & $\begin{array}{l}\text { Very Poor }=0=\text { None of the } \\
\text { above }\end{array}$ & & \\
\hline \multicolumn{4}{|l|}{ Method: } \\
\hline Questions & Description & Score & Comments \\
\hline \multirow{2}{*}{$\begin{array}{l}\text { Has IPA been clearly } \\
\text { defined? } \\
\text { Have motivations been } \\
\text { clearly defined? }\end{array}$} & $\begin{array}{l}\text { Good }=3=\text { Clear description } \\
\text { of both IPA and motivations. }\end{array}$ & \multirow[t]{2}{*}{0} & \multirow[t]{2}{*}{$\begin{array}{l}\text { Neither IPA nor } \\
\text { motivations were } \\
\text { defined }\end{array}$} \\
\hline & $\begin{array}{l}\text { Fair }=2=\text { Clear description } \\
\text { of one variable, but } \\
\text { inadequate description of the } \\
\text { other. }\end{array}$ & & \\
\hline
\end{tabular}




\begin{tabular}{|c|c|c|c|}
\hline & $\begin{array}{l}\text { Poor }=1=\text { Inadequate } \\
\text { descriptions of both } \\
\text { variables. } \\
\text { Very Poor }=0=\text { No } \\
\text { description of either variable. }\end{array}$ & & \\
\hline $\begin{array}{l}\text { Are the measurements } \\
\text { used appropriate? }\end{array}$ & $\begin{array}{l}\text { Good }=3=\text { Validated and } \\
\text { reliability tested measures of } \\
\text { the outcome are used, or } \\
\text { measures are pre-tested prior } \\
\text { to data collection. } \\
\text { Fair }=2=\text { Appropriate } \\
\text { measures have been used but } \\
\text { have not been described well. } \\
\text { Poor }=1=\text { Measures used are } \\
\text { inappropriate/ failure to } \\
\text { report validity and reliability } \\
\text { of measures. } \\
\text { Very Poor }=0=\text { No mention } \\
\text { of measures used. }\end{array}$ & 1 & $\begin{array}{l}\text { The only measure that } \\
\text { has been validated in the } \\
\text { CTS2. The Reasons for } \\
\text { Violence Scale has not } \\
\text { been properly developed } \\
\text { nor piloted prior to the } \\
\text { study. The inter-rater } \\
\text { reliability for this scale } \\
\text { seems overly high } \\
86.5 \% \text {. } \\
\text { Although there was a } \\
\text { question specifically } \\
\text { relating to self-defense, } \\
\text { this was not clearly or } \\
\text { consistently defined. }\end{array}$ \\
\hline \multicolumn{4}{|l|}{ Data Analysis: } \\
\hline Questions & Description & Score & Comments \\
\hline $\begin{array}{l}\text { Is the statistical analysis } \\
\text { appropriate to answer } \\
\text { the research question } \\
\text { and for the type of data } \\
\text { collected? }\end{array}$ & 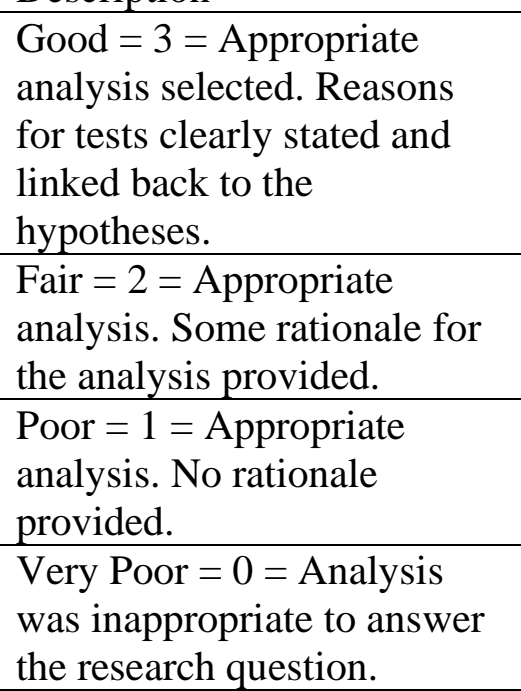 & 0 & $\begin{array}{l}\text { The analysis was not } \\
\text { appropriate to answer } \\
\text { the research question as } \\
\text { looking at the means } \\
\text { and SDs, it appears the } \\
\text { data was not normally } \\
\text { distributed and therefore } \\
\text { likely a General Linear } \\
\text { Model should not have } \\
\text { been used. }\end{array}$ \\
\hline $\begin{array}{l}\text { Was the description of } \\
\text { the analysis sufficiently } \\
\text { rigorous? }\end{array}$ & $\begin{array}{l}\text { Good }=3=\text { Clear description } \\
\text { of how analysis was done. } \\
\text { Statistical significance } \\
\text { discussed. } \\
\begin{array}{l}\text { Fair }=2=\text { Some details about } \\
\text { the analysis provided. }\end{array} \\
\begin{array}{l}\text { Poor }=1=\text { Minimal details } \\
\text { about analysis provided. }\end{array} \\
\begin{array}{l}\text { Very Poor }=0=\text { No } \\
\text { discussion of analysis }\end{array}\end{array}$ & 1.5 & $\begin{array}{l}\text { There was small section } \\
\text { describing the analytic } \\
\text { plan, which included } \\
\text { information regarding } \\
\text { the effect size. }\end{array}$ \\
\hline \multicolumn{4}{|l|}{ Results: } \\
\hline Questions & Description & Score & Comments \\
\hline
\end{tabular}




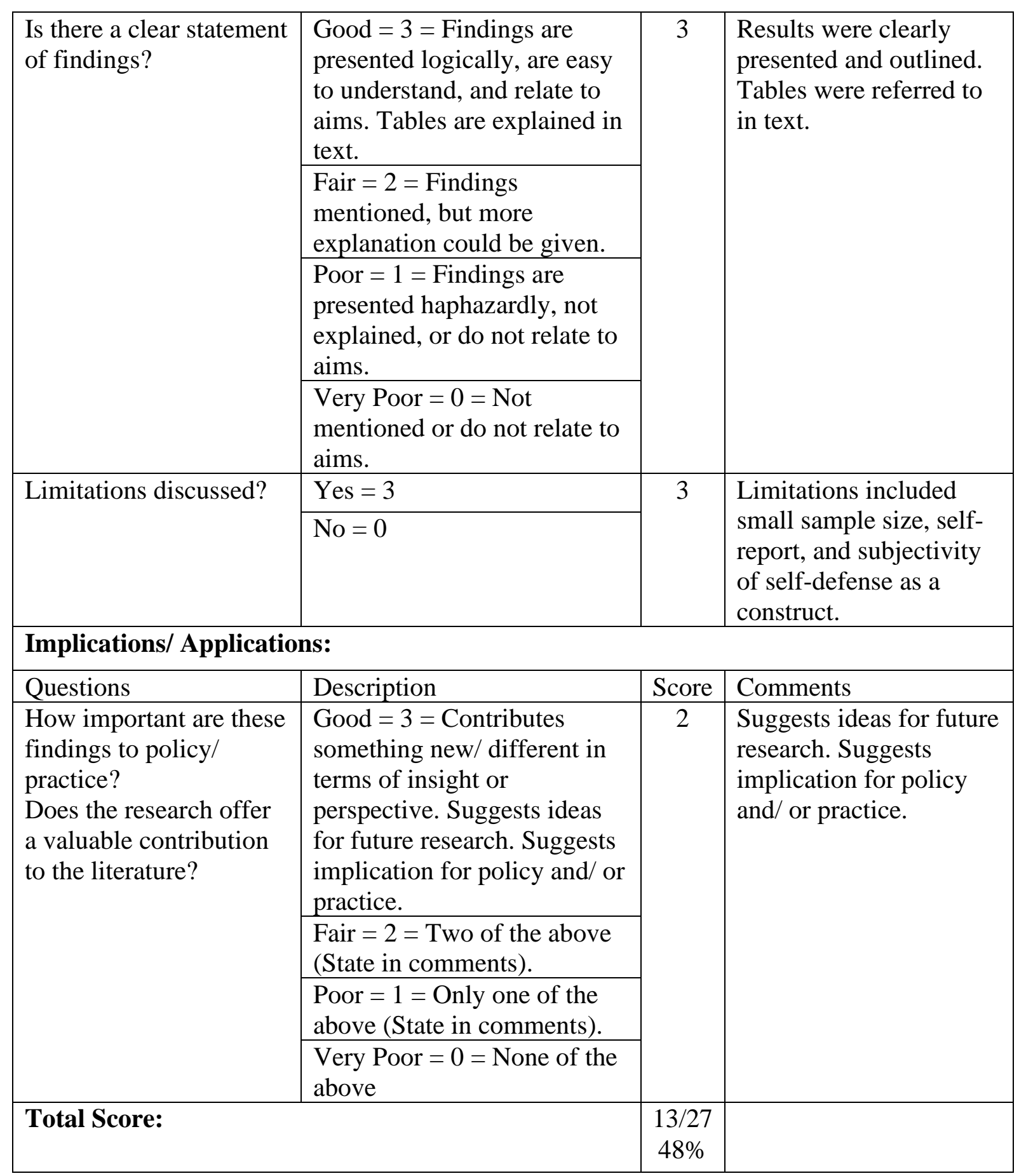




\begin{tabular}{|c|c|c|c|}
\hline Study 2 - Title: & \multicolumn{3}{|c|}{$\begin{array}{l}\text { Gender differences in attributions of self-defense and control in } \\
\text { interpartner aggression }\end{array}$} \\
\hline Author (Year): & \multicolumn{3}{|c|}{ Barnett, O. W., Lee, C. Y., \& Thelen, R. E. (1997) } \\
\hline \multicolumn{4}{|l|}{ Sampling: } \\
\hline Questions & Description & Score & Comments \\
\hline \multirow[t]{4}{*}{$\begin{array}{l}\text { Was the sampling } \\
\text { strategy appropriate to } \\
\text { address the research } \\
\text { question/s? } \\
\text { Why was this group } \\
\text { targeted? } \\
\text { Were the participants } \\
\text { representative of the } \\
\text { target population? }\end{array}$} & $\begin{array}{l}\text { Good }=3=\text { Details (age/ } \\
\text { gender/ ethnicity /context) of } \\
\text { who was studied and how } \\
\text { they were recruited. } \\
\text { Rationale for studying this } \\
\text { population. Response rates } \\
\text { shown and explained (why } \\
\text { certain individuals chose not } \\
\text { to participate, and any } \\
\text { attempts to achieve a sample } \\
\text { that represents the target } \\
\text { population). }\end{array}$ & \multirow[t]{4}{*}{1} & \multirow[t]{4}{*}{$\begin{array}{l}\text { No recruitment } \\
\text { information. Sample } \\
\text { consisted entirely of } \\
\text { participants who } \\
\text { identified as White. No } \\
\text { attempts to achieve a } \\
\text { representative sample. }\end{array}$} \\
\hline & $\begin{array}{l}\text { Fair }=2=\text { Most information } \\
\text { given, but some missing. }\end{array}$ & & \\
\hline & $\begin{array}{l}\text { Poor }=1=\text { Sampling } \\
\text { mentioned but few } \\
\text { descriptive details. }\end{array}$ & & \\
\hline & $\begin{array}{l}\text { Very Poor }=0=\text { No details } \\
\text { of sample. }\end{array}$ & & \\
\hline \multirow{3}{*}{$\begin{array}{l}\text { Reporting of power or a } \\
\text { priori power analysis } \\
\text { conducted? } \\
\text { Does the study have } \geq \\
80 \% \text { power to detect an } \\
\text { effect at } r=.20 \text { ? }\end{array}$} & $\begin{array}{l}\text { Good = } 3=\text { Two of the above } \\
\text { (State in comments). }\end{array}$ & \multirow[t]{3}{*}{0} & \multirow{3}{*}{$\begin{array}{l}\text { No reporting of power } \\
\text { or a priori power } \\
\text { analysis conducted. } \\
\text { This was not able to be } \\
\text { determined as } \\
\text { insufficient information } \\
\text { was supplied for a } \\
\text { repeated measures } \\
\text { ANOVA e.g., } \\
\text { correlations between } \\
\text { repeated measures }\end{array}$} \\
\hline & $\begin{array}{l}\text { Poor }=1.5=\text { Only one of the } \\
\text { above (State in comments). }\end{array}$ & & \\
\hline & $\begin{array}{l}\text { Very Poor }=0=\text { None of the } \\
\text { above }\end{array}$ & & \\
\hline \multicolumn{4}{|l|}{ Method: } \\
\hline Questions & Description & Score & Comments \\
\hline \multirow{3}{*}{$\begin{array}{l}\text { Has IPA been clearly } \\
\text { defined? } \\
\text { Have motivations been } \\
\text { clearly defined? }\end{array}$} & $\begin{array}{l}\text { Good }=3=\text { Clear description } \\
\text { of both IPA and motivations. }\end{array}$ & \multirow[t]{3}{*}{0} & \multirow[t]{3}{*}{$\begin{array}{l}\text { Neither IPA nor } \\
\text { motivations were } \\
\text { defined }\end{array}$} \\
\hline & $\begin{array}{l}\text { Fair }=2=\text { Clear description } \\
\text { of one variable, but } \\
\text { inadequate description of the } \\
\text { other. }\end{array}$ & & \\
\hline & $\begin{array}{l}\text { Poor }=1=\text { Inadequate } \\
\text { descriptions of both } \\
\text { variables. }\end{array}$ & & \\
\hline
\end{tabular}




\begin{tabular}{|c|c|c|c|}
\hline & $\begin{array}{l}\text { Very Poor }=0=\text { No } \\
\text { description of either variable. }\end{array}$ & & \\
\hline \multirow[t]{4}{*}{$\begin{array}{l}\text { Are the measurements } \\
\text { used appropriate? }\end{array}$} & $\begin{array}{l}\text { Good }=3=\text { Validated and } \\
\text { reliability tested measures of } \\
\text { the outcome are used, or } \\
\text { measures are pre-tested prior } \\
\text { to data collection. }\end{array}$ & \multirow[t]{4}{*}{3} & \multirow[t]{4}{*}{$\begin{array}{l}\text { Although piloted in } \\
\text { three previous studies, } \\
\text { the Relationship Abuse } \\
\text { Questionnaire has not } \\
\text { been validated. }\end{array}$} \\
\hline & $\begin{array}{l}\text { Fair }=2=\text { Appropriate } \\
\text { measures have been used but } \\
\text { have not been described well. }\end{array}$ & & \\
\hline & $\begin{array}{l}\text { Poor }=1=\text { Measures used are } \\
\text { inappropriate/ failure to } \\
\text { report validity and reliability } \\
\text { of measures. }\end{array}$ & & \\
\hline & $\begin{array}{l}\text { Very Poor }=0=\text { No mention } \\
\text { of measures used. }\end{array}$ & & \\
\hline \multicolumn{4}{|l|}{ Data Analysis: } \\
\hline Questions & Description & Score & Comments \\
\hline \multirow[t]{4}{*}{$\begin{array}{l}\text { Is the statistical analysis } \\
\text { appropriate to answer } \\
\text { the research question } \\
\text { and for the type of data } \\
\text { collected? }\end{array}$} & $\begin{array}{l}\text { Good }=3=\text { Appropriate } \\
\text { analysis selected. Reasons } \\
\text { for tests clearly stated and } \\
\text { linked back to the } \\
\text { hypotheses. }\end{array}$ & \multirow[t]{4}{*}{1} & \multirow[t]{4}{*}{$\begin{array}{l}\text { The analysis was } \\
\text { appropriate to answer } \\
\text { the research question, } \\
\text { but no rationale was } \\
\text { provided. }\end{array}$} \\
\hline & $\begin{array}{l}\text { Fair }=2=\text { Appropriate } \\
\text { analysis. Some rationale for } \\
\text { the analysis provided. }\end{array}$ & & \\
\hline & $\begin{array}{l}\text { Poor }=1=\text { Appropriate } \\
\text { analysis. No rationale } \\
\text { provided. }\end{array}$ & & \\
\hline & $\begin{array}{l}\text { Very Poor }=0=\text { Analysis } \\
\text { was inappropriate to answer } \\
\text { the research question. }\end{array}$ & & \\
\hline \multirow[t]{4}{*}{$\begin{array}{l}\text { Was the description of } \\
\text { the analysis sufficiently } \\
\text { rigorous? }\end{array}$} & $\begin{array}{l}\text { Good }=3=\text { Clear description } \\
\text { of how analysis was done. } \\
\text { Statistical significance } \\
\text { discussed. }\end{array}$ & \multirow[t]{4}{*}{3} & \multirow{4}{*}{$\begin{array}{l}\text { A separate section on } \\
\text { statistical analysis was } \\
\text { included that clearly } \\
\text { described the tests } \\
\text { conducted. }\end{array}$} \\
\hline & $\begin{array}{l}\text { Fair }=2=\text { Some details about } \\
\text { the analysis provided. }\end{array}$ & & \\
\hline & $\begin{array}{l}\text { Poor }=1=\text { Minimal details } \\
\text { about analysis provided. }\end{array}$ & & \\
\hline & $\begin{array}{l}\text { Very Poor }=0=\text { No } \\
\text { discussion of analysis }\end{array}$ & & \\
\hline \multicolumn{4}{|l|}{ Results: } \\
\hline Questions & Description & Score & Comments \\
\hline $\begin{array}{l}\text { Is there a clear statement } \\
\text { of findings? }\end{array}$ & $\begin{array}{l}\text { Good }=3=\text { Findings are } \\
\text { presented logically, are easy } \\
\text { to understand, and relate to } \\
\text { aims. Tables are explained in } \\
\text { text. }\end{array}$ & 2 & $\begin{array}{l}\text { Results for each analysis } \\
\text { were clearly described, } \\
\text { although were not } \\
\text { explicitly tied back to } \\
\text { the hypotheses. }\end{array}$ \\
\hline
\end{tabular}




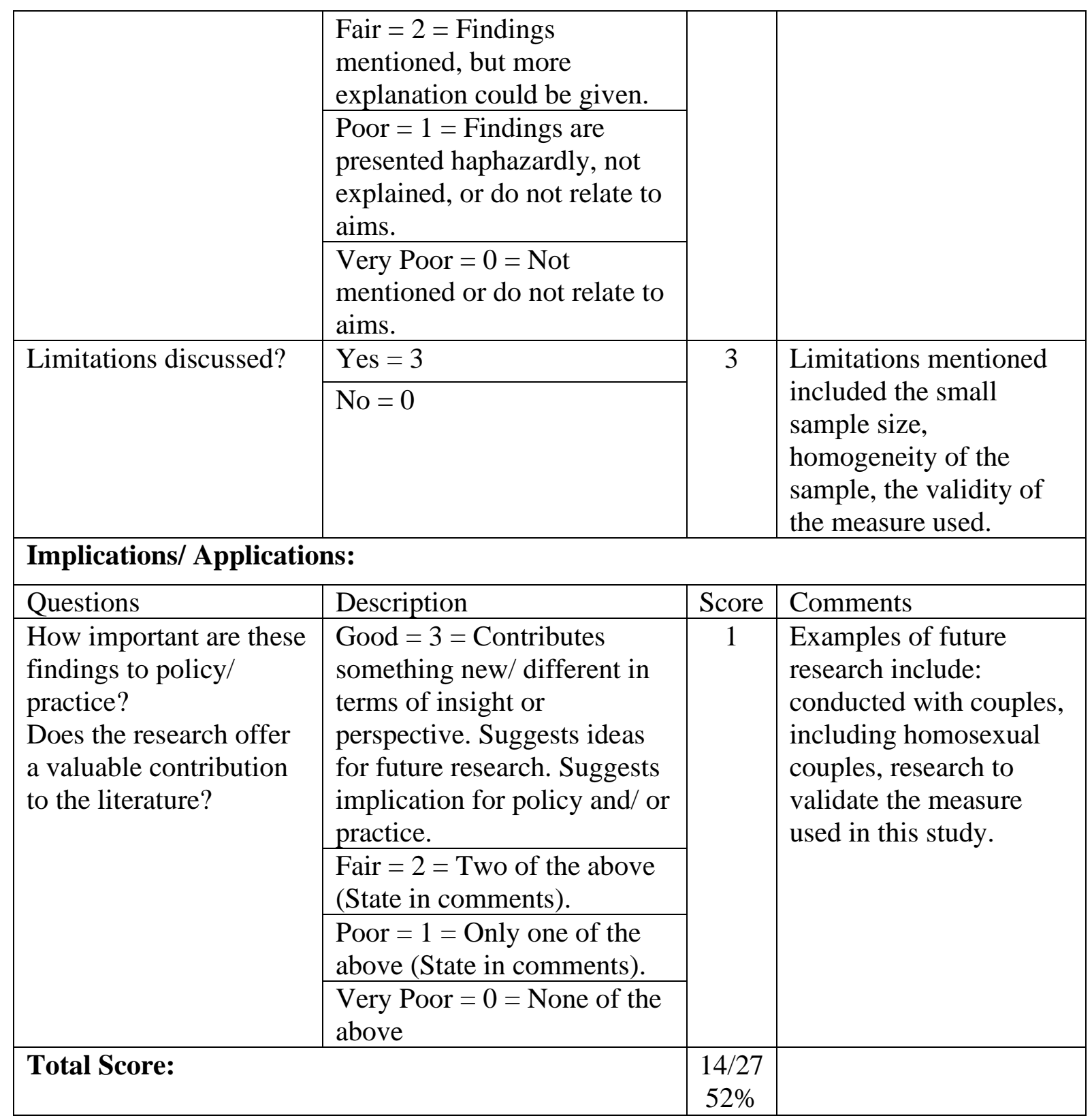

\begin{tabular}{|c|c|c|c|}
\hline Study 3 - Title: & \multicolumn{3}{|c|}{$\begin{array}{l}\text { Do anger and jealousy mediate the relationship between adult } \\
\text { attachment styles and intimate partner violence }\end{array}$} \\
\hline Author (Year): & \multicolumn{3}{|c|}{$\begin{array}{l}\text { Belus, J. M.; Wanklyn, S. G.; Iverson, K. M; Pukay-Martin, N. } \\
\text { D.; Langhinrichsen-Rohling, J.; \& Monson, C. M. (2014) }\end{array}$} \\
\hline \multicolumn{4}{|l|}{ Sampling: } \\
\hline Questions & Description & Score & Comments \\
\hline $\begin{array}{l}\text { Was the sampling } \\
\text { strategy appropriate to } \\
\text { address the research } \\
\text { question/s? } \\
\text { Why was this group } \\
\text { targeted? }\end{array}$ & $\begin{array}{l}\text { Good = } 3=\text { Details (age/ } \\
\text { gender/ ethnicity /context) } \\
\text { of who was studied and } \\
\text { how they were recruited. } \\
\text { Rationale for studying this } \\
\text { population. Response rates }\end{array}$ & 2 & $\begin{array}{l}94.6 \% \text { of the sample } \\
\text { consisted of } \\
\text { participants who } \\
\text { identified as White - } \\
\text { No attempts to achieve }\end{array}$ \\
\hline
\end{tabular}




\begin{tabular}{|c|c|c|c|}
\hline \multirow[t]{4}{*}{$\begin{array}{l}\text { Were the participants } \\
\text { representative of the } \\
\text { target population? }\end{array}$} & $\begin{array}{l}\text { shown and explained (why } \\
\text { certain individuals chose } \\
\text { not to participate, and any } \\
\text { attempts to achieve a } \\
\text { sample that represents the } \\
\text { target population). }\end{array}$ & & \multirow{4}{*}{$\begin{array}{l}\text { a more representative } \\
\text { sample. } \\
\text { Rationale for why a } \\
\text { student sample was } \\
\text { used is provided in the } \\
\text { discussion section. } \\
\text { As the survey was } \\
\text { administered via a mail } \\
\text { survey, no explanation } \\
\text { for the response rate } \\
(34 \%) \text { was provided. }\end{array}$} \\
\hline & $\begin{array}{l}\text { Fair }=2=\text { Most } \\
\text { information given, but } \\
\text { some missing. }\end{array}$ & & \\
\hline & $\begin{array}{l}\text { Poor = } 1=\text { Sampling } \\
\text { mentioned but few } \\
\text { descriptive details. }\end{array}$ & & \\
\hline & $\begin{array}{l}\text { Very Poor }=0=\text { No details } \\
\text { of sample. }\end{array}$ & & \\
\hline \multirow{3}{*}{$\begin{array}{l}\text { Reporting of power or } \\
\text { a priori power analysis } \\
\text { conducted? } \\
\text { Does the study have } \geq \\
80 \% \text { power to detect an } \\
\text { effect at } r=.20 \text { ? }\end{array}$} & $\begin{array}{l}\text { Good }=3=\text { Two of the } \\
\text { above (State in comments). }\end{array}$ & \multirow[t]{3}{*}{0} & \multirow{3}{*}{$\begin{array}{l}\text { No reporting of power } \\
\text { or a priori power } \\
\text { analysis conducted. } \\
\text { Due to the type of } \\
\text { analysis conducted this } \\
\text { was not able to be } \\
\text { calculated and } \\
\text { consequently this } \\
\text { section is scored out of } \\
1.5, \text { not } 3 \text {. }\end{array}$} \\
\hline & $\begin{array}{l}\text { Poor }=1.5=\text { Only one of } \\
\text { the above (State in } \\
\text { comments). }\end{array}$ & & \\
\hline & $\begin{array}{l}\text { Very Poor }=0=\text { None of } \\
\text { the above }\end{array}$ & & \\
\hline \multicolumn{4}{|l|}{ Method: } \\
\hline Questions & Description & Score & Comments \\
\hline \multirow{4}{*}{$\begin{array}{l}\text { Has IPA been clearly } \\
\text { defined? } \\
\text { Have motivations been } \\
\text { clearly defined? }\end{array}$} & $\begin{array}{l}\text { Good }=3=\text { Clear } \\
\text { description of both IPA and } \\
\text { motivations. }\end{array}$ & \multirow[t]{4}{*}{1.5} & \multirow[t]{4}{*}{$\begin{array}{l}\text { Description of IPA } \\
\text { only }\end{array}$} \\
\hline & $\begin{array}{l}\text { Fair }=2=\text { Clear description } \\
\text { of one variable, but } \\
\text { inadequate description of } \\
\text { the other. }\end{array}$ & & \\
\hline & $\begin{array}{l}\text { Poor }=1=\text { Inadequate } \\
\text { descriptions of both } \\
\text { variables. }\end{array}$ & & \\
\hline & $\begin{array}{l}\text { Very Poor }=0=\text { No } \\
\text { description of either } \\
\text { variable. }\end{array}$ & & \\
\hline \multirow[t]{2}{*}{$\begin{array}{l}\text { Are the measurements } \\
\text { used appropriate? }\end{array}$} & $\begin{array}{l}\text { Good }=3=\text { Validated and } \\
\text { reliability tested measures } \\
\text { of the outcome are used, or } \\
\text { measures are pre-tested } \\
\text { prior to data collection. }\end{array}$ & 3 & \multirow{2}{*}{$\begin{array}{l}\text { Measures used are } \\
\text { reliable and well } \\
\text { validated. } \\
\text { Psychometrics have } \\
\text { been provided for all } \\
\text { measures. }\end{array}$} \\
\hline & $\begin{array}{l}\text { Fair }=2=\text { Appropriate } \\
\text { measures have been used } \\
\text { but have not been described } \\
\text { well. }\end{array}$ & & \\
\hline
\end{tabular}




\begin{tabular}{|c|c|c|c|}
\hline & $\begin{array}{l}\text { Poor }=1=\text { Measures used } \\
\text { are inappropriate/ failure to } \\
\text { report validity and } \\
\text { reliability of measures. } \\
\text { Very Poor }=0=\text { No } \\
\text { mention of measures used. }\end{array}$ & & \\
\hline \multicolumn{4}{|l|}{ Data Analysis: } \\
\hline Questions & Description & Score & Comments \\
\hline \multirow[t]{4}{*}{$\begin{array}{l}\text { Is the statistical } \\
\text { analysis appropriate to } \\
\text { answer the research } \\
\text { question and for the } \\
\text { type of data collected? }\end{array}$} & $\begin{array}{l}\text { Good }=3=\text { Appropriate } \\
\text { analysis selected. Reasons } \\
\text { for tests clearly stated and } \\
\text { linked back to the } \\
\text { hypotheses. }\end{array}$ & \multirow[t]{4}{*}{3} & \multirow[t]{4}{*}{$\begin{array}{l}\text { Appropriate analyses } \\
\text { that were explicitly } \\
\text { linked back to the aims } \\
\text { of the study. }\end{array}$} \\
\hline & $\begin{array}{l}\text { Fair }=2=\text { Appropriate } \\
\text { analysis. Some rationale for } \\
\text { the analysis provided. }\end{array}$ & & \\
\hline & $\begin{array}{l}\text { Poor }=1=\text { Appropriate } \\
\text { analysis. No rationale } \\
\text { provided. }\end{array}$ & & \\
\hline & $\begin{array}{l}\text { Very Poor }=0=\text { Analysis } \\
\text { was inappropriate to answer } \\
\text { the research question. }\end{array}$ & & \\
\hline \multirow[t]{4}{*}{$\begin{array}{l}\text { Was the description of } \\
\text { the analysis sufficiently } \\
\text { rigorous? }\end{array}$} & $\begin{array}{l}\text { Good }=3=\text { Clear } \\
\text { description of how analysis } \\
\text { was done. Statistical } \\
\text { significance discussed. }\end{array}$ & \multirow[t]{4}{*}{3} & \multirow[t]{4}{*}{$\begin{array}{l}\text { The separate section on } \\
\text { statistical analysis was } \\
\text { very thorough. }\end{array}$} \\
\hline & $\begin{array}{l}\text { Fair }=2=\text { Some details } \\
\text { about the analysis provided. }\end{array}$ & & \\
\hline & $\begin{array}{l}\text { Poor }=1=\text { Minimal details } \\
\text { about analysis provided. }\end{array}$ & & \\
\hline & $\begin{array}{l}\text { Very Poor }=0=\text { No } \\
\text { discussion of analysis }\end{array}$ & & \\
\hline \multicolumn{4}{|l|}{ Results: } \\
\hline Questions & Description & Score & Comments \\
\hline \multirow[t]{4}{*}{$\begin{array}{l}\text { Is there a clear } \\
\text { statement of findings? }\end{array}$} & $\begin{array}{l}\text { Good }=3=\text { Findings are } \\
\text { presented logically, are } \\
\text { easy to understand, and } \\
\text { relate to aims. Tables are } \\
\text { explained in text. }\end{array}$ & \multirow[t]{4}{*}{3} & \multirow[t]{4}{*}{$\begin{array}{l}\text { Results from the } \\
\text { separate analyses } \\
\text { clearly and concisely } \\
\text { explained. }\end{array}$} \\
\hline & $\begin{array}{l}\text { Fair }=2=\text { Findings } \\
\text { mentioned, but more } \\
\text { explanation could be given. }\end{array}$ & & \\
\hline & $\begin{array}{l}\text { Poor }=1=\text { Findings are } \\
\text { presented haphazardly, not } \\
\text { explained, or do not relate } \\
\text { to aims. }\end{array}$ & & \\
\hline & $\begin{array}{l}\text { Very Poor }=0=\text { Not } \\
\text { mentioned or do not relate } \\
\text { to aims. }\end{array}$ & & \\
\hline
\end{tabular}




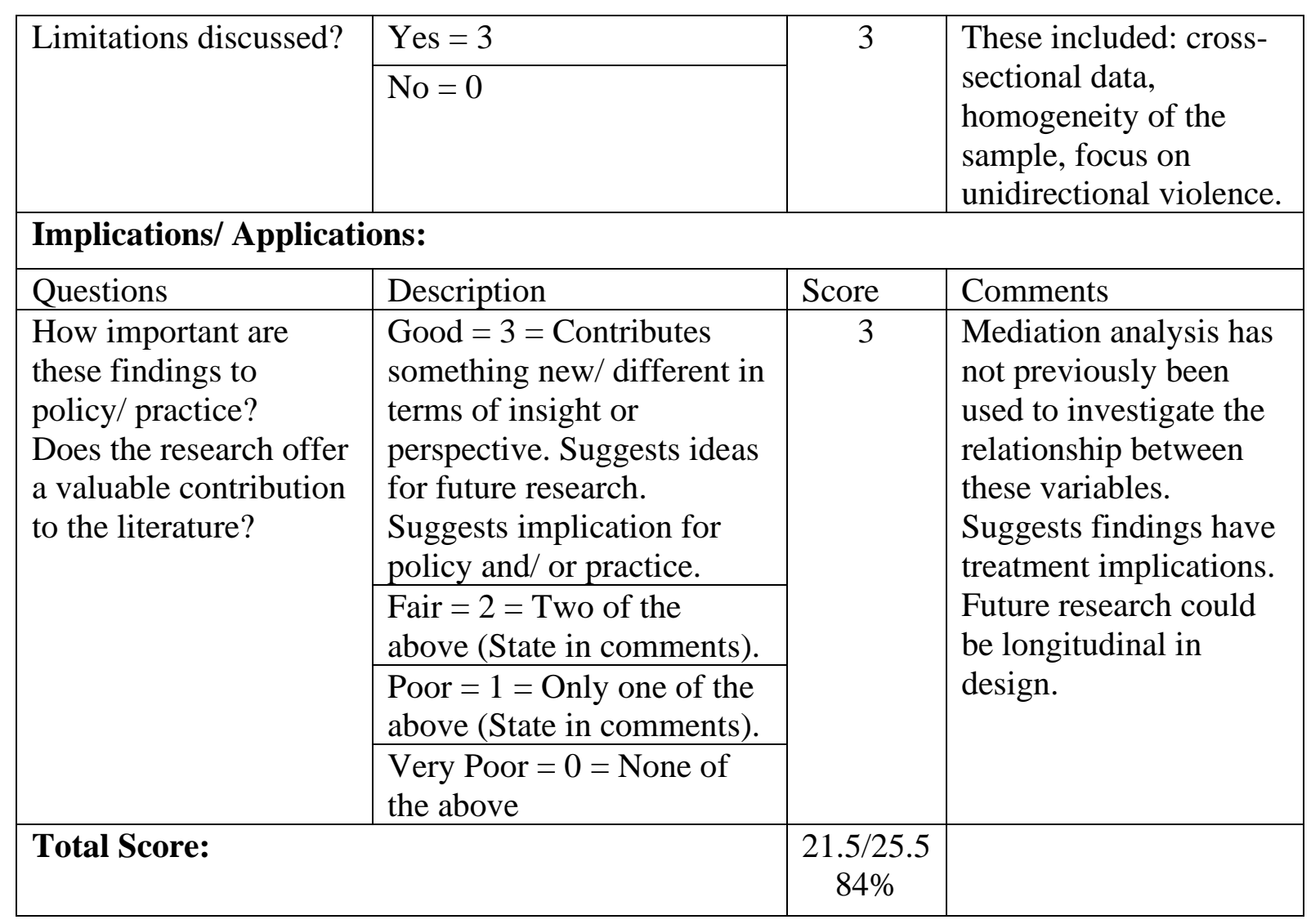

\begin{tabular}{|c|c|c|c|}
\hline Study 4 - Title: & \multicolumn{3}{|c|}{ Why I hit him: Women's reasons for intimate partner violence } \\
\hline Author (Year): & \multicolumn{3}{|c|}{$\begin{array}{l}\text { Caldwell, J. E., Swan, S. C., Allen, C. T., Sullivan, T. P., \& } \\
\text { Snow, D. L. (2009) }\end{array}$} \\
\hline \multicolumn{4}{|l|}{ Sampling: } \\
\hline Questions & Description & Score & Comments \\
\hline $\begin{array}{l}\text { Was the sampling } \\
\text { strategy appropriate to } \\
\text { address the research } \\
\text { question/s? } \\
\text { Why was this group } \\
\text { targeted? } \\
\text { Were the participants } \\
\text { representative of the } \\
\text { target population? }\end{array}$ & $\begin{array}{l}\text { Good = } 3=\text { Details (age/ } \\
\text { gender/ ethnicity /context) } \\
\text { of who was studied and how } \\
\text { they were recruited. } \\
\text { Rationale for studying this } \\
\text { population. Response rates } \\
\text { shown and explained (why } \\
\text { certain individuals chose not } \\
\text { to participate, and any } \\
\text { attempts to achieve a sample } \\
\text { that represents the target } \\
\text { population). } \\
\text { Fair }=2=\text { Most information } \\
\text { given, but some missing. } \\
\text { Poor = } 1=\text { Sampling } \\
\text { mentioned but few } \\
\text { descriptive details. }\end{array}$ & 3 & $\begin{array}{l}\text { Details of sample, } \\
\text { recruitment and } \\
\text { rationale provided. } \\
\text { Sample was ethnically } \\
\text { diverse, perhaps more } \\
\text { so than the general } \\
\text { population. }\end{array}$ \\
\hline
\end{tabular}




\begin{tabular}{|c|c|c|c|}
\hline & $\begin{array}{l}\text { Very Poor }=0=\text { No details } \\
\text { of sample. }\end{array}$ & & \\
\hline \multirow{3}{*}{$\begin{array}{l}\text { Reporting of power or a } \\
\text { priori power analysis } \\
\text { conducted? } \\
\text { Does the study have } \geq \\
80 \% \text { power to detect an } \\
\text { effect at } r=.20 \text { ? }\end{array}$} & $\begin{array}{l}\text { Good }=3=\text { Two of the } \\
\text { above (State in comments). }\end{array}$ & \multirow[t]{3}{*}{1.5} & \multirow{3}{*}{$\begin{array}{l}\text { No reporting of power } \\
\text { or a priori power } \\
\text { analysis conducted. } \\
\text { Study has } \geq 80 \% \text { power } \\
\text { to detect an effect at } r= \\
.20\end{array}$} \\
\hline & $\begin{array}{l}\text { Poor }=1.5=\text { Only one of the } \\
\text { above (State in comments). }\end{array}$ & & \\
\hline & $\begin{array}{l}\text { Very Poor }=0=\text { None of the } \\
\text { above }\end{array}$ & & \\
\hline \multicolumn{4}{|l|}{ Method: } \\
\hline Questions & Description & Score & Comments \\
\hline \multirow{4}{*}{$\begin{array}{l}\text { Has IPA been clearly } \\
\text { defined? } \\
\text { Have motivations been } \\
\text { clearly defined? }\end{array}$} & $\begin{array}{l}\text { Good }=3=\text { Clear } \\
\text { description of both IPA and } \\
\text { motivations. }\end{array}$ & \multirow[t]{4}{*}{2} & \multirow[t]{4}{*}{$\begin{array}{l}\text { Clear definition of } \\
\text { motivations }\end{array}$} \\
\hline & $\begin{array}{l}\text { Fair }=2=\text { Clear description } \\
\text { of one variable, but } \\
\text { inadequate description of the } \\
\text { other. }\end{array}$ & & \\
\hline & $\begin{array}{l}\text { Poor }=1=\text { Inadequate } \\
\text { descriptions of both } \\
\text { variables. }\end{array}$ & & \\
\hline & $\begin{array}{l}\text { Very Poor }=0=\text { No } \\
\text { description of either } \\
\text { variable. }\end{array}$ & & \\
\hline \multirow[t]{4}{*}{$\begin{array}{l}\text { Are the measurements } \\
\text { used appropriate? }\end{array}$} & $\begin{array}{l}\text { Good }=3=\text { Validated and } \\
\text { reliability tested measures of } \\
\text { the outcome are used, or } \\
\text { measures are pre-tested prior } \\
\text { to data collection. }\end{array}$ & \multirow[t]{4}{*}{3} & \multirow{4}{*}{$\begin{array}{l}\text { Scale developed for this } \\
\text { study using appropriate } \\
\text { statistical techniques. } \\
\text { These were well and } \\
\text { thoroughly described. } \\
\text { Existing measures used } \\
\text { have proved reliability } \\
\text { and validity. }\end{array}$} \\
\hline & $\begin{array}{l}\text { Fair }=2=\text { Appropriate } \\
\text { measures have been used but } \\
\text { have not been described } \\
\text { well. }\end{array}$ & & \\
\hline & $\begin{array}{l}\text { Poor }=1=\text { Measures used } \\
\text { are inappropriate/ failure to } \\
\text { report validity and reliability } \\
\text { of measures. }\end{array}$ & & \\
\hline & $\begin{array}{l}\text { Very Poor }=0=\text { No mention } \\
\text { of measures used. }\end{array}$ & & \\
\hline \multicolumn{4}{|l|}{ Data Analysis: } \\
\hline Questions & Description & Score & Comments \\
\hline $\begin{array}{l}\text { Is the statistical analysis } \\
\text { appropriate to answer } \\
\text { the research question } \\
\text { and for the type of data } \\
\text { collected? }\end{array}$ & $\begin{array}{l}\text { Good }=3=\text { Appropriate } \\
\text { analysis selected. Reasons } \\
\text { for tests clearly stated and } \\
\text { linked back to the } \\
\text { hypotheses. }\end{array}$ & 3 & $\begin{array}{l}\text { Appropriate analyses } \\
\text { conducted and } \\
\text { explanations as to what } \\
\text { each was testing }\end{array}$ \\
\hline
\end{tabular}




\begin{tabular}{|c|c|c|c|}
\hline & $\begin{array}{l}\text { Fair }=2=\text { Appropriate } \\
\text { analysis. Some rationale for } \\
\text { the analysis provided. }\end{array}$ & & \\
\hline & $\begin{array}{l}\text { Poor }=1=\text { Appropriate } \\
\text { analysis. No rationale } \\
\text { provided. }\end{array}$ & & \\
\hline & $\begin{array}{l}\text { Very Poor }=0=\text { Analysis } \\
\text { was inappropriate to answer } \\
\text { the research question. }\end{array}$ & & \\
\hline \multirow[t]{4}{*}{$\begin{array}{l}\text { Was the description of } \\
\text { the analysis sufficiently } \\
\text { rigorous? }\end{array}$} & $\begin{array}{l}\text { Good }=3=\text { Clear } \\
\text { description of how analysis } \\
\text { was done. Statistical } \\
\text { significance discussed. }\end{array}$ & \multirow[t]{4}{*}{3} & \multirow[t]{4}{*}{$\begin{array}{l}\text { Thorough description } \\
\text { of the analyses } \\
\text { conducted, including } \\
\text { significance. }\end{array}$} \\
\hline & $\begin{array}{l}\text { Fair }=2=\text { Some details } \\
\text { about the analysis provided. }\end{array}$ & & \\
\hline & $\begin{array}{l}\text { Poor }=1=\text { Minimal details } \\
\text { about analysis provided. }\end{array}$ & & \\
\hline & $\begin{array}{l}\text { Very Poor }=0=\text { No } \\
\text { discussion of analysis }\end{array}$ & & \\
\hline \multicolumn{4}{|l|}{ Results: } \\
\hline Questions & Description & Score & Comments \\
\hline \multirow[t]{4}{*}{$\begin{array}{l}\text { Is there a clear } \\
\text { statement of findings? }\end{array}$} & $\begin{array}{l}\text { Good }=3=\text { Findings are } \\
\text { presented logically, are easy } \\
\text { to understand, and relate to } \\
\text { aims. Tables are explained } \\
\text { in text. }\end{array}$ & \multirow[t]{4}{*}{3} & \multirow[t]{4}{*}{$\begin{array}{l}\text { Results were clearly } \\
\text { and logically presented. }\end{array}$} \\
\hline & $\begin{array}{l}\text { Fair }=2=\text { Findings } \\
\text { mentioned, but more } \\
\text { explanation could be given. }\end{array}$ & & \\
\hline & $\begin{array}{l}\text { Poor }=1=\text { Findings are } \\
\text { presented haphazardly, not } \\
\text { explained, or do not relate to } \\
\text { aims. }\end{array}$ & & \\
\hline & $\begin{array}{l}\text { Very Poor }=0=\text { Not } \\
\text { mentioned or do not relate to } \\
\text { aims. }\end{array}$ & & \\
\hline \multirow[t]{2}{*}{ Limitations discussed? } & Yes $=3$ & \multirow[t]{2}{*}{3} & \multirow{2}{*}{$\begin{array}{l}\text { These included: self- } \\
\text { report measures, } \\
\text { retrospective reporting, } \\
\text { scale developed for this } \\
\text { study may require } \\
\text { revision. }\end{array}$} \\
\hline & No $=0$ & & \\
\hline \multicolumn{4}{|c|}{ Implications/ Applications: } \\
\hline Questions & Description & Score & Comments \\
\hline $\begin{array}{l}\text { How important are } \\
\text { these findings to policy/ } \\
\text { practice? }\end{array}$ & $\begin{array}{l}\text { Good = } 3=\text { Contributes } \\
\text { something new/ different in } \\
\text { terms of insight or } \\
\text { perspective. Suggests ideas } \\
\text { for future research. Suggests }\end{array}$ & 2 & $\begin{array}{l}\text { Development of a new } \\
\text { scale for measuring the } \\
\text { motives for IPA. } \\
\text { Implications for } \\
\text { treatment. }\end{array}$ \\
\hline
\end{tabular}




\begin{tabular}{|l|l|l|l|}
\hline $\begin{array}{l}\text { Does the research offer } \\
\text { a valuable contribution } \\
\text { to the literature? }\end{array}$ & $\begin{array}{l}\text { implication for policy and/ } \\
\text { or practice. }\end{array}$ & \multirow{3}{*}{$\begin{array}{l}\text { No suggestions for } \\
\text { future research. } \\
\text { (State in comments). }\end{array}$} & \\
\cline { 2 - 3 } & $\begin{array}{l}\text { Poor =1 = Only one of the } \\
\text { above (State in comments). }\end{array}$ & & \\
\cline { 1 - 2 } & $\begin{array}{l}\text { Very Poor = 0 None of the } \\
\text { above }\end{array}$ & & \\
\hline Total Score: & $87.5 / 27$ & \\
\hline
\end{tabular}

\begin{tabular}{|c|c|c|c|}
\hline Study 5 - Title: & \multicolumn{3}{|c|}{$\begin{array}{l}\text { Aggression in British heterosexual relationships: A descriptive } \\
\text { analysis }\end{array}$} \\
\hline Author (Year): & \multicolumn{3}{|c|}{$\begin{array}{l}\text { Carrado, M., George, M. J., Loxam, E., Jones, L., \& Templar, } \\
\text { D. (1996) }\end{array}$} \\
\hline \multicolumn{4}{|l|}{ Sampling: } \\
\hline Questions & Description & Score & Comments \\
\hline \multirow[t]{4}{*}{$\begin{array}{l}\text { Was the sampling } \\
\text { strategy appropriate to } \\
\text { address the research } \\
\text { question/s? } \\
\text { Why was this group } \\
\text { targeted? } \\
\text { Were the participants } \\
\text { representative of the } \\
\text { target population? }\end{array}$} & $\begin{array}{l}\text { Good }=3=\text { Details (age/ } \\
\text { gender/ ethnicity /context) of } \\
\text { who was studied and how } \\
\text { they were recruited. } \\
\text { Rationale for studying this } \\
\text { population. Response rates } \\
\text { shown and explained (why } \\
\text { certain individuals chose not } \\
\text { to participate, and any } \\
\text { attempts to achieve a sample } \\
\text { that represents the target } \\
\text { population). }\end{array}$ & \multirow[t]{4}{*}{3} & \multirow[t]{2}{*}{$\begin{array}{l}\text { This was a nationally } \\
\text { representative sample. } \\
\text { Sampling quotas used to } \\
\text { ensure sample was } \\
\text { representative of the } \\
\text { adult population of the } \\
\text { UK as determined by } \\
\text { the most recent census } \\
\text { (1996; age, sex, SES, } \\
\text { relationship status, } \\
\text { geographical region) }\end{array}$} \\
\hline & $\begin{array}{l}\text { Fair }=2=\text { Most information } \\
\text { given, but some missing. }\end{array}$ & & \\
\hline & $\begin{array}{l}\text { Poor }=1=\text { Sampling } \\
\text { mentioned but few } \\
\text { descriptive details. }\end{array}$ & & \\
\hline & $\begin{array}{l}\text { Very Poor }=0=\text { No details } \\
\text { of sample. }\end{array}$ & & \\
\hline \multirow{3}{*}{$\begin{array}{l}\text { Reporting of power or a } \\
\text { priori power analysis } \\
\text { conducted? } \\
\text { Does the study have } \geq \\
80 \% \text { power to detect an } \\
\text { effect at } r=.20 \text { ? }\end{array}$} & $\begin{array}{l}\text { Good }=3=\text { Two of the above } \\
\text { (State in comments). }\end{array}$ & \multirow[t]{3}{*}{0} & \multirow{3}{*}{$\begin{array}{l}\text { No reporting of power } \\
\text { or a priori power } \\
\text { analysis conducted. } \\
\text { This was not able to be } \\
\text { determined as no } \\
\text { information regarding } \\
\text { the type of analysis was } \\
\text { supplied or results } \\
\text { reported. }\end{array}$} \\
\hline & $\begin{array}{l}\text { Poor }=1.5=\text { Only one of the } \\
\text { above (State in comments). }\end{array}$ & & \\
\hline & $\begin{array}{l}\text { Very Poor }=0=\text { None of the } \\
\text { above }\end{array}$ & & \\
\hline \multicolumn{4}{|l|}{ Method: } \\
\hline
\end{tabular}




\begin{tabular}{|c|c|c|c|}
\hline Questions & Description & Score & Comments \\
\hline \multirow{4}{*}{$\begin{array}{l}\text { Has IPA been clearly } \\
\text { defined? } \\
\text { Have motivations been } \\
\text { clearly defined? }\end{array}$} & $\begin{array}{l}\text { Good }=3=\text { Clear description } \\
\text { of both IPA and motivations. }\end{array}$ & \multirow[t]{4}{*}{0} & \multirow{4}{*}{$\begin{array}{l}\text { No description of either } \\
\text { IPA or motivation. This } \\
\text { paper does not explicitly } \\
\text { claim to measure } \\
\text { motives but talks about } \\
\text { attempting to assess the } \\
\text { motive of self-defense in } \\
\text { the discussion section. }\end{array}$} \\
\hline & $\begin{array}{l}\text { Fair }=2=\text { Clear description } \\
\text { of one variable, but } \\
\text { inadequate description of the } \\
\text { other. }\end{array}$ & & \\
\hline & $\begin{array}{l}\text { Poor }=1=\text { Inadequate } \\
\text { descriptions of both } \\
\text { variables. }\end{array}$ & & \\
\hline & $\begin{array}{l}\text { Very Poor }=0=\text { No } \\
\text { description of either variable. }\end{array}$ & & \\
\hline \multirow[t]{4}{*}{$\begin{array}{l}\text { Are the measurements } \\
\text { used appropriate? }\end{array}$} & $\begin{array}{l}\text { Good }=3=\text { Validated and } \\
\text { reliability tested measures of } \\
\text { the outcome are used, or } \\
\text { measures are pre-tested prior } \\
\text { to data collection. }\end{array}$ & \multirow[t]{4}{*}{1} & \multirow[t]{4}{*}{$\begin{array}{l}\text { Measures have not been } \\
\text { validated. No } \\
\text { psychometric } \\
\text { information was } \\
\text { reported. }\end{array}$} \\
\hline & $\begin{array}{l}\text { Fair }=2=\text { Appropriate } \\
\text { measures have been used but } \\
\text { have not been described well. }\end{array}$ & & \\
\hline & $\begin{array}{l}\text { Poor }=1=\text { Measures used are } \\
\text { inappropriate/ failure to } \\
\text { report validity and reliability } \\
\text { of measures. }\end{array}$ & & \\
\hline & $\begin{array}{l}\text { Very Poor }=0=\text { No mention } \\
\text { of measures used. }\end{array}$ & & \\
\hline \multicolumn{4}{|l|}{ Data Analysis: } \\
\hline Questions & Description & Score & Comments \\
\hline \multirow[t]{4}{*}{$\begin{array}{l}\text { Is the statistical analysis } \\
\text { appropriate to answer } \\
\text { the research question } \\
\text { and for the type of data } \\
\text { collected? }\end{array}$} & $\begin{array}{l}\text { Good }=3=\text { Appropriate } \\
\text { analysis selected. Reasons } \\
\text { for tests clearly stated and } \\
\text { linked back to the } \\
\text { hypotheses. }\end{array}$ & \multirow[t]{4}{*}{1} & \multirow[t]{4}{*}{$\begin{array}{l}\text { Type of analysis } \\
\text { conducted was never } \\
\text { mentioned, however, it } \\
\text { was limited to frequency } \\
\text { data and some group } \\
\text { comparisons. }\end{array}$} \\
\hline & $\begin{array}{l}\text { Fair }=2=\text { Appropriate } \\
\text { analysis. Some rationale for } \\
\text { the analysis provided. }\end{array}$ & & \\
\hline & $\begin{array}{l}\text { Poor }=1=\text { Appropriate } \\
\text { analysis. No rationale } \\
\text { provided. }\end{array}$ & & \\
\hline & $\begin{array}{l}\text { Very Poor }=0=\text { Analysis } \\
\text { was inappropriate to answer } \\
\text { the research question. }\end{array}$ & & \\
\hline \multirow[t]{2}{*}{$\begin{array}{l}\text { Was the description of } \\
\text { the analysis sufficiently } \\
\text { rigorous? }\end{array}$} & $\begin{array}{l}\text { Good }=3=\text { Clear description } \\
\text { of how analysis was done. } \\
\text { Statistical significance } \\
\text { discussed. }\end{array}$ & \multirow[t]{2}{*}{0} & \multirow[t]{2}{*}{$\begin{array}{l}\text { There was no } \\
\text { description of the } \\
\text { analysis, nor of } \\
\text { statistical significance. }\end{array}$} \\
\hline & $\begin{array}{l}\text { Fair }=2=\text { Some details about } \\
\text { the analysis provided. }\end{array}$ & & \\
\hline
\end{tabular}




\begin{tabular}{|c|c|c|c|}
\hline & $\begin{array}{l}\text { Poor }=1=\text { Minimal details } \\
\text { about analysis provided. } \\
\text { Very Poor }=0=\text { No } \\
\text { discussion of analysis }\end{array}$ & & \\
\hline \multicolumn{4}{|l|}{ Results: } \\
\hline Questions & Description & Score & Comments \\
\hline $\begin{array}{l}\text { Is there a clear statement } \\
\text { of findings? }\end{array}$ & $\begin{array}{l}\text { Good }=3=\text { Findings are } \\
\text { presented logically, are easy } \\
\text { to understand, and relate to } \\
\text { aims. Tables are explained in } \\
\text { text. } \\
\text { Fair }=2=\text { Findings } \\
\text { mentioned, but more } \\
\text { explanation could be given. } \\
\text { Poor }=1=\text { Findings are } \\
\text { presented haphazardly, not } \\
\text { explained, or do not relate to } \\
\text { aims. } \\
\text { Very Poor }=0=\text { Not } \\
\text { mentioned or do not relate to } \\
\text { aims. }\end{array}$ & 2 & $\begin{array}{l}\text { This paper did not have } \\
\text { clear hypotheses or } \\
\text { aims. However, the } \\
\text { tables were explained in } \\
\text { text and the results } \\
\text { section presented } \\
\text { logically. }\end{array}$ \\
\hline Limitations discussed? & $\begin{array}{l}\text { Yes }=3 \\
\text { No }=0\end{array}$ & 3 & $\begin{array}{l}\text { These include: Failure to } \\
\text { use the CTS, lack of } \\
\text { information on } \\
\text { chronicity of assaults }\end{array}$ \\
\hline \multicolumn{4}{|c|}{ Implications/ Applications: } \\
\hline Questions & Description & Score & Comments \\
\hline $\begin{array}{l}\text { How important are these } \\
\text { findings to policy/ } \\
\text { practice? } \\
\text { Does the research offer } \\
\text { a valuable contribution } \\
\text { to the literature? }\end{array}$ & $\begin{array}{l}\text { Good }=3=\text { Contributes } \\
\text { something new/ different in } \\
\text { terms of insight or } \\
\text { perspective. Suggests ideas } \\
\text { for future research. Suggests } \\
\text { implication for policy and/ or } \\
\text { practice. } \\
\text { Fair }=2=\text { Two of the above } \\
\text { (State in comments). } \\
\text { Poor = 1 = Only one of the } \\
\text { above (State in comments). } \\
\text { Very Poor = } 0=\text { None of the } \\
\text { above }\end{array}$ & 0 & \\
\hline Total Score: & & $\begin{array}{c}10 / 27 \\
37 \%\end{array}$ & \\
\hline
\end{tabular}




\begin{tabular}{|c|c|c|c|}
\hline Study 6 - Title: & \multicolumn{3}{|c|}{$\begin{array}{l}\text { The meanings and motives for women's use of violence in } \\
\text { Canadian college dating relationships: Results from a national } \\
\text { survey }\end{array}$} \\
\hline Author (Year): & \multicolumn{3}{|c|}{$\begin{array}{l}\text { DeKeseredy, W. S., Saunders, D. G., Schwartz, M. D., \& Alvi, } \\
\text { S. (1997) }\end{array}$} \\
\hline \multicolumn{4}{|l|}{ Sampling: } \\
\hline Questions & Description & Score & Comments \\
\hline \multirow[t]{4}{*}{$\begin{array}{l}\text { Was the sampling } \\
\text { strategy appropriate to } \\
\text { address the research } \\
\text { question/s? } \\
\text { Why was this group } \\
\text { targeted? } \\
\text { Were the participants } \\
\text { representative of the } \\
\text { target population? }\end{array}$} & $\begin{array}{l}\text { Good }=3=\text { Details (age/ } \\
\text { gender/ ethnicity /context) } \\
\text { of who was studied and how } \\
\text { they were recruited. } \\
\text { Rationale for studying this } \\
\text { population. Response rates } \\
\text { shown and explained (why } \\
\text { certain individuals chose not } \\
\text { to participate, and any } \\
\text { attempts to achieve a sample } \\
\text { that represents the target } \\
\text { population). }\end{array}$ & \multirow[t]{4}{*}{2} & \multirow[t]{4}{*}{$\begin{array}{l}\text { Median age reported. } \\
\text { No details regarding the } \\
\text { ethnic composition of } \\
\text { the sample. Rationale } \\
\text { for studying this } \\
\text { population discussed. } \\
\text { No attempts to ensure } \\
\text { the sample was } \\
\text { representative. } \\
\text { Response rate reported. }\end{array}$} \\
\hline & $\begin{array}{l}\text { Fair }=2=\text { Most information } \\
\text { given, but some missing. }\end{array}$ & & \\
\hline & $\begin{array}{l}\text { Poor }=1=\text { Sampling } \\
\text { mentioned but few } \\
\text { descriptive details. }\end{array}$ & & \\
\hline & $\begin{array}{l}\text { Very Poor }=0=\text { No details } \\
\text { of sample. }\end{array}$ & & \\
\hline \multirow{3}{*}{$\begin{array}{l}\text { Reporting of power or a } \\
\text { priori power analysis } \\
\text { conducted? } \\
\text { Does the study have } \geq \\
80 \% \text { power to detect an } \\
\text { effect at } r=.20 \text { ? }\end{array}$} & $\begin{array}{l}\text { Good }=3=\text { Two of the } \\
\text { above (State in comments). }\end{array}$ & \multirow[t]{3}{*}{1.5} & \multirow{3}{*}{$\begin{array}{l}\text { No reporting of power } \\
\text { or a priori power } \\
\text { analysis conducted. } \\
\text { Study has } \geq 80 \% \text { power } \\
\text { to detect an effect at } r= \\
.20\end{array}$} \\
\hline & $\begin{array}{l}\text { Poor }=1.5=\text { Only one of the } \\
\text { above (State in comments). }\end{array}$ & & \\
\hline & $\begin{array}{l}\text { Very Poor }=0=\text { None of the } \\
\text { above }\end{array}$ & & \\
\hline \multicolumn{4}{|l|}{ Method: } \\
\hline Questions & Description & Score & Comments \\
\hline \multirow[t]{3}{*}{$\begin{array}{l}\text { Has IPA been clearly } \\
\text { defined? } \\
\text { Have motivations been } \\
\text { clearly defined? }\end{array}$} & $\begin{array}{l}\text { Good }=3=\text { Clear } \\
\text { description of both IPA and } \\
\text { motivations. }\end{array}$ & \multirow[t]{3}{*}{1} & \multirow[t]{3}{*}{$\begin{array}{l}\text { Physical violence is } \\
\text { discussed in some detail } \\
\text { in the introduction but } \\
\text { is not defined. }\end{array}$} \\
\hline & $\begin{array}{l}\text { Fair }=2=\text { Clear description } \\
\text { of one variable, but } \\
\text { inadequate description of } \\
\text { the other. }\end{array}$ & & \\
\hline & $\begin{array}{l}\text { Poor }=1=\text { Inadequate } \\
\text { descriptions of both } \\
\text { variables. }\end{array}$ & & \\
\hline
\end{tabular}




\begin{tabular}{|c|c|c|c|}
\hline & $\begin{array}{l}\text { Very Poor }=0=\text { No } \\
\text { description of either } \\
\text { variable. }\end{array}$ & & \\
\hline \multirow[t]{4}{*}{$\begin{array}{l}\text { Are the measurements } \\
\text { used appropriate? }\end{array}$} & $\begin{array}{l}\text { Good }=3=\text { Validated and } \\
\text { reliability tested measures of } \\
\text { the outcome are used, or } \\
\text { measures are pre-tested } \\
\text { prior to data collection. }\end{array}$ & \multirow[t]{4}{*}{1} & \multirow{4}{*}{$\begin{array}{l}\text { CTS is a well validated } \\
\text { measure, but } \\
\text { psychometrics were not } \\
\text { reported. There was no } \\
\text { information provided as } \\
\text { to how the questions for } \\
\text { the motives were } \\
\text { developed, or internal } \\
\text { consistency reported. }\end{array}$} \\
\hline & $\begin{array}{l}\text { Fair }=2=\text { Appropriate } \\
\text { measures have been used } \\
\text { but have not been described } \\
\text { well. }\end{array}$ & & \\
\hline & $\begin{array}{l}\text { Poor }=1=\text { Measures used } \\
\text { are inappropriate/ failure to } \\
\text { report validity and reliability } \\
\text { of measures. }\end{array}$ & & \\
\hline & $\begin{array}{l}\text { Very Poor }=0=\text { No mention } \\
\text { of measures used. }\end{array}$ & & \\
\hline \multicolumn{4}{|l|}{ Data Analysis: } \\
\hline Questions & Description & Score & Comments \\
\hline \multirow[t]{4}{*}{$\begin{array}{l}\text { Is the statistical analysis } \\
\text { appropriate to answer } \\
\text { the research question } \\
\text { and for the type of data } \\
\text { collected? }\end{array}$} & $\begin{array}{l}\text { Good }=3=\text { Appropriate } \\
\text { analysis selected. Reasons } \\
\text { for tests clearly stated and } \\
\text { linked back to the } \\
\text { hypotheses. }\end{array}$ & \multirow[t]{4}{*}{2} & \multirow[t]{4}{*}{$\begin{array}{l}\text { Appropriate analysis } \\
\text { with some rationale for } \\
\text { the tests selected. }\end{array}$} \\
\hline & $\begin{array}{l}\text { Fair }=2=\text { Appropriate } \\
\text { analysis. Some rationale for } \\
\text { the analysis provided. }\end{array}$ & & \\
\hline & $\begin{array}{l}\text { Poor }=1=\text { Appropriate } \\
\text { analysis. No rationale } \\
\text { provided. }\end{array}$ & & \\
\hline & $\begin{array}{l}\text { Very Poor }=0=\text { Analysis } \\
\text { was inappropriate to answer } \\
\text { the research question. }\end{array}$ & & \\
\hline \multirow[t]{4}{*}{$\begin{array}{l}\text { Was the description of } \\
\text { the analysis sufficiently } \\
\text { rigorous? }\end{array}$} & $\begin{array}{l}\text { Good }=3=\text { Clear } \\
\text { description of how analysis } \\
\text { was done. Statistical } \\
\text { significance discussed. }\end{array}$ & \multirow[t]{4}{*}{0} & \multirow{4}{*}{$\begin{array}{l}\text { No discussion regarding } \\
\text { the analysis beyond } \\
\text { stating what tests were } \\
\text { conducted. } \\
\text { No discussion of } \\
\text { statistical significance. }\end{array}$} \\
\hline & $\begin{array}{l}\text { Fair }=2=\text { Some details } \\
\text { about the analysis provided. }\end{array}$ & & \\
\hline & $\begin{array}{l}\text { Poor }=1=\text { Minimal details } \\
\text { about analysis provided. }\end{array}$ & & \\
\hline & $\begin{array}{l}\text { Very Poor }=0=\text { No } \\
\text { discussion of analysis }\end{array}$ & & \\
\hline \multicolumn{4}{|l|}{ Results: } \\
\hline Questions & Description & Score & Comments \\
\hline $\begin{array}{l}\text { Is there a clear } \\
\text { statement of findings? }\end{array}$ & $\begin{array}{l}\text { Good }=3=\text { Findings are } \\
\text { presented logically, are easy } \\
\text { to understand, and relate to }\end{array}$ & 3 & $\begin{array}{l}\text { Tables referenced in } \\
\text { text. Hypotheses } \\
\text { referred to in text. }\end{array}$ \\
\hline
\end{tabular}




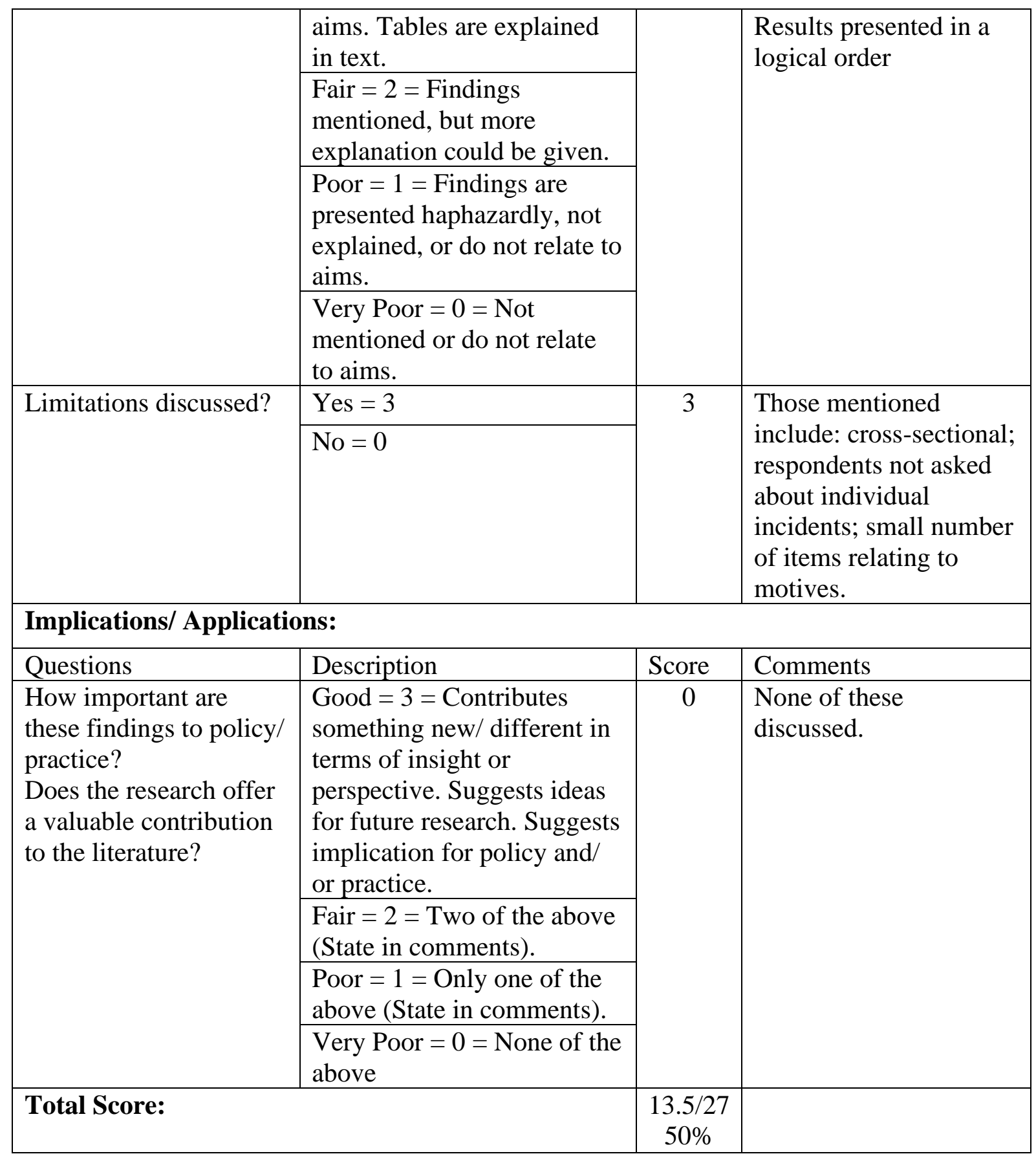

\begin{tabular}{|l|l|c|l|}
\hline Study 7 - Title: & $\begin{array}{l}\text { Motivations for intimate partner violence in men and women } \\
\text { arrested for domestic violence and court referred to batterer } \\
\text { intervention programs }\end{array}$ \\
\hline Author (Year): & $\begin{array}{l}\text { Elmquist, J., Hamel, J., Shorey, R. C., Labrecque, L., } \\
\text { Ninnemann, A., \& Stuart, G. L. (2014) }\end{array}$ \\
\hline Sampling: & Description & Score & Comments \\
\hline Questions & $\begin{array}{l}\text { Good = 3 = Details (age/ } \\
\text { gender/ ethnicity /context) of }\end{array}$ & 1 & $\begin{array}{l}\text { Details of male sample } \\
\text { reported. Reader is }\end{array}$ \\
\hline $\begin{array}{l}\text { Was the sampling } \\
\text { strategy appropriate to }\end{array}$
\end{tabular}




\begin{tabular}{|c|c|c|c|}
\hline \multirow[t]{4}{*}{$\begin{array}{l}\text { address the research } \\
\text { question/s? } \\
\text { Why was this group } \\
\text { targeted? } \\
\text { Were the participants } \\
\text { representative of the } \\
\text { target population? }\end{array}$} & $\begin{array}{l}\text { who was studied and how } \\
\text { they were recruited. } \\
\text { Rationale for studying this } \\
\text { population. Response rates } \\
\text { shown and explained (why } \\
\text { certain individuals chose not } \\
\text { to participate, and any } \\
\text { attempts to achieve a sample } \\
\text { that represents the target } \\
\text { population). }\end{array}$ & & \multirow{4}{*}{$\begin{array}{l}\text { directed to another study } \\
\text { for the description of the } \\
\text { female sample. No } \\
\text { rationale for studying } \\
\text { this population. No } \\
\text { attempts to ensure } \\
\text { sample was } \\
\text { representative of the } \\
\text { population. Although the } \\
\text { authors said participants } \\
\text { were recruited through } \\
\text { intervention } \\
\text { programmes, there is no } \\
\text { further detail as to how, } \\
\text { and no response rates } \\
\text { were provided. }\end{array}$} \\
\hline & $\begin{array}{l}\text { Fair }=2=\text { Most information } \\
\text { given, but some missing. }\end{array}$ & & \\
\hline & $\begin{array}{l}\text { Poor }=1=\text { Sampling } \\
\text { mentioned but few } \\
\text { descriptive details. }\end{array}$ & & \\
\hline & $\begin{array}{l}\text { Very Poor }=0=\text { No details } \\
\text { of sample. }\end{array}$ & & \\
\hline \multirow{3}{*}{$\begin{array}{l}\text { Reporting of power or a } \\
\text { priori power analysis } \\
\text { conducted? } \\
\text { Does the study have } \geq \\
80 \% \text { power to detect an } \\
\text { effect at } r=.20 ?\end{array}$} & $\begin{array}{l}\text { Good }=3=\text { Two of the } \\
\text { above (State in comments). }\end{array}$ & \multirow[t]{4}{*}{0} & \multirow{3}{*}{$\begin{array}{l}\text { No reporting of power or } \\
\text { a priori power analysis } \\
\text { conducted. } \\
\text { Study has }<80 \% \text { power } \\
\text { to detect an effect at } r= \\
.20\end{array}$} \\
\hline & $\begin{array}{l}\text { Poor }=1.5=\text { Only one of the } \\
\text { above (State in comments). }\end{array}$ & & \\
\hline & $\begin{array}{l}\text { Very Poor }=0=\text { None of the } \\
\text { above }\end{array}$ & & \\
\hline \multicolumn{3}{|l|}{ Method: } & \\
\hline Questions & Description & Score & Comments \\
\hline \multirow{4}{*}{$\begin{array}{l}\text { Has IPA been clearly } \\
\text { defined? } \\
\text { Have motivations been } \\
\text { clearly defined? }\end{array}$} & $\begin{array}{l}\text { Good }=3=\text { Clear description } \\
\text { of both IPA and motivations. }\end{array}$ & \multirow[t]{4}{*}{1.5} & \multirow[t]{4}{*}{$\begin{array}{l}\text { IPA is defined, motives } \\
\text { are not. }\end{array}$} \\
\hline & $\begin{array}{l}\text { Fair }=2=\text { Clear description } \\
\text { of one variable, but } \\
\text { inadequate description of the } \\
\text { other. }\end{array}$ & & \\
\hline & $\begin{array}{l}\text { Poor }=1=\text { Inadequate } \\
\text { descriptions of both } \\
\text { variables. }\end{array}$ & & \\
\hline & $\begin{array}{l}\text { Very Poor }=0=\text { No } \\
\text { description of either variable. }\end{array}$ & & \\
\hline \multirow[t]{2}{*}{$\begin{array}{l}\text { Are the measurements } \\
\text { used appropriate? }\end{array}$} & $\begin{array}{l}\text { Good }=3=\text { Validated and } \\
\text { reliability tested measures of } \\
\text { the outcome are used, or } \\
\text { measures are pre-tested prior } \\
\text { to data collection. }\end{array}$ & \multirow[t]{2}{*}{1.5} & \multirow{2}{*}{$\begin{array}{l}\text { CTS is a reliable and } \\
\text { well validated tool. The } \\
\text { authors have reported } \\
\text { alphas for the current, } \\
\text { but no additional } \\
\text { psychometric } \\
\text { information. }\end{array}$} \\
\hline & $\begin{array}{l}\text { Fair }=2=\text { Appropriate } \\
\text { measures have been used but } \\
\text { have not been described well. }\end{array}$ & & \\
\hline
\end{tabular}




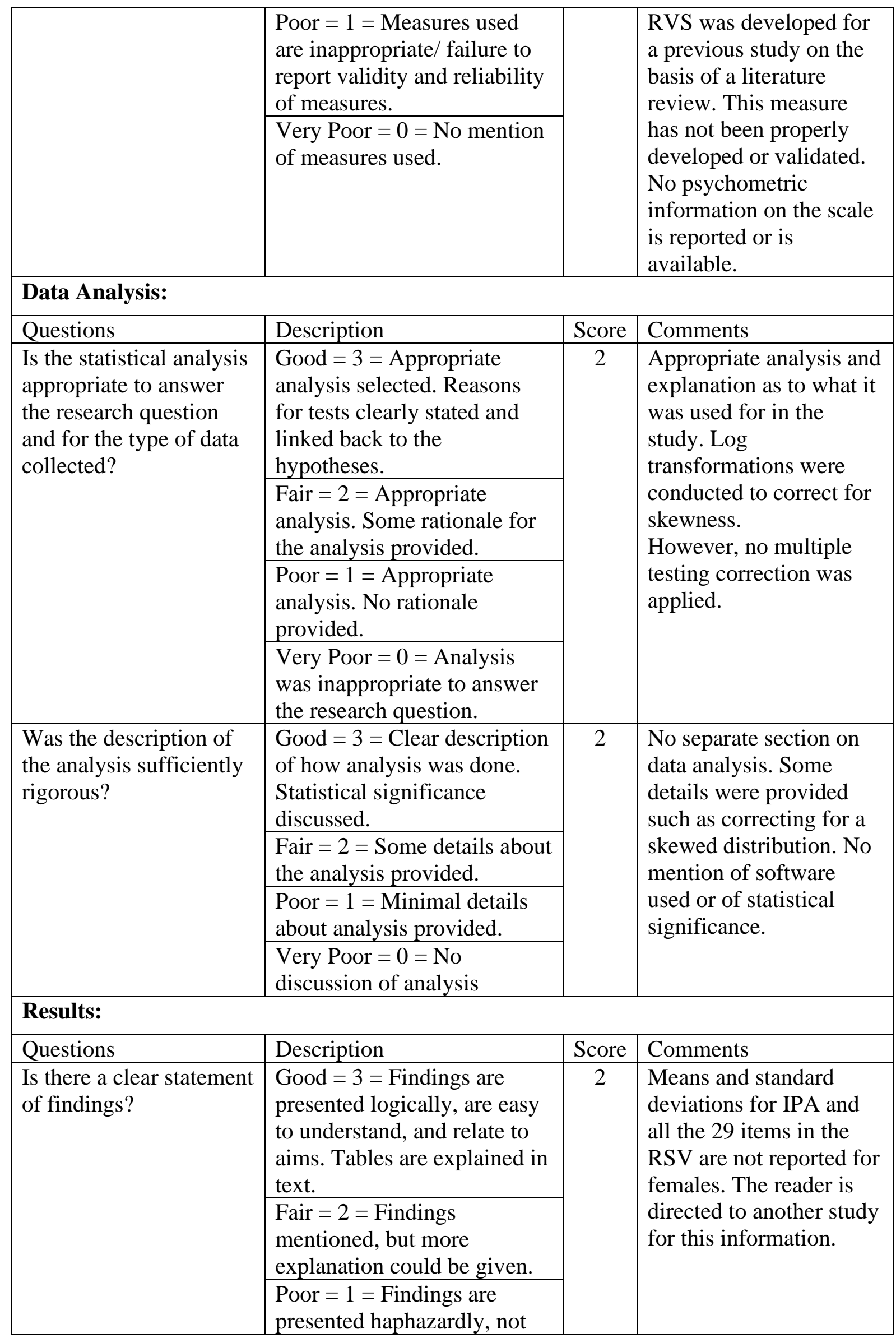




\begin{tabular}{|c|c|c|c|}
\hline & $\begin{array}{l}\text { explained, or do not relate to } \\
\text { aims. } \\
\text { Very Poor }=0=\text { Not } \\
\text { mentioned or do not relate to } \\
\text { aims. }\end{array}$ & & \\
\hline \multirow[t]{2}{*}{ Limitations discussed? } & Yes $=3$ & \multirow[t]{2}{*}{3} & \multirow{2}{*}{$\begin{array}{l}\text { These include: failure to } \\
\text { use a measure of social } \\
\text { desirability; the } \\
\text { measures used were } \\
\text { retrospective and } \\
\text { acontextual; } \\
\text { homogeneity of the } \\
\text { sample }\end{array}$} \\
\hline & $\mathrm{No}=0$ & & \\
\hline \multicolumn{4}{|c|}{ Implications/ Applications: } \\
\hline Questions & Description & Score & Comments \\
\hline \multirow[t]{4}{*}{$\begin{array}{l}\text { How important are these } \\
\text { findings to policy/ } \\
\text { practice? } \\
\text { Does the research offer } \\
\text { a valuable contribution } \\
\text { to the literature? }\end{array}$} & $\begin{array}{l}\text { Good }=3=\text { Contributes } \\
\text { something new/ different in } \\
\text { terms of insight or } \\
\text { perspective. Suggests ideas } \\
\text { for future research. Suggests } \\
\text { implication for policy and/ or } \\
\text { practice. }\end{array}$ & \multirow[t]{4}{*}{2} & \multirow[t]{4}{*}{$\begin{array}{l}\text { Suggests ideas for future } \\
\text { research. Suggests } \\
\text { implication for policy } \\
\text { and/ or practice. }\end{array}$} \\
\hline & $\begin{array}{l}\text { Fair }=2=\text { Two of the above } \\
\text { (State in comments). }\end{array}$ & & \\
\hline & $\begin{array}{l}\text { Poor }=1=\text { Only one of the } \\
\text { above (State in comments). }\end{array}$ & & \\
\hline & $\begin{array}{l}\text { Very Poor }=0=\text { None of the } \\
\text { above }\end{array}$ & & \\
\hline \multicolumn{2}{|l|}{ Total Score: } & $\begin{array}{c}15 / 27 \\
56 \%\end{array}$ & \\
\hline
\end{tabular}

\begin{tabular}{|l|l|c|l|}
\hline Study 8 - Title: & \multicolumn{2}{|l|}{$\begin{array}{l}\text { Physical and psychological aggression in dating relationships of } \\
\text { Spanish adolescents: Motives and consequences }\end{array}$} \\
\hline Author (Year): & Fernandez-Fuertes, A. A., \& Fuertes, A. (2010) \\
\hline Sampling: & Description & Score & Comments \\
\hline Questions & $\begin{array}{l}\text { Good = 3 = Details (age/ } \\
\text { gender/ ethnicity /context) } \\
\text { of who was studied and how } \\
\text { they were recruited. } \\
\text { Rationale for studying this } \\
\text { population. Response rates } \\
\text { shown and explained (why } \\
\text { certain individuals chose not } \\
\text { to participate, and any } \\
\text { attempts to achieve a sample }\end{array}$ & 2 & $\begin{array}{l}\text { No ethnic breakdown of } \\
\text { sample provided. } \\
\text { Rationale provided for } \\
\text { studying this } \\
\text { strategy appropriate to } \\
\text { address the research } \\
\text { question/s? } \\
\begin{array}{l}\text { Why was this group } \\
\text { targeted? } \\
\text { Were the participants } \\
\text { representative of the } \\
\text { target population? }\end{array}\end{array}$ \\
\hline
\end{tabular}




\begin{tabular}{|c|c|c|c|}
\hline & $\begin{array}{l}\text { that represents the target } \\
\text { population). }\end{array}$ & & \\
\hline & $\begin{array}{l}\text { Fair }=2=\text { Most information } \\
\text { given, but some missing. }\end{array}$ & & \\
\hline & $\begin{array}{l}\text { Poor }=1=\text { Sampling } \\
\text { mentioned but few } \\
\text { descriptive details. }\end{array}$ & & \\
\hline & $\begin{array}{l}\text { Very Poor }=0=\text { No details } \\
\text { of sample. }\end{array}$ & & \\
\hline \multirow{3}{*}{$\begin{array}{l}\text { Reporting of power or a } \\
\text { priori power analysis } \\
\text { conducted? } \\
\text { Does the study have } \geq \\
80 \% \text { power to detect an } \\
\text { effect at } r=.20 \text { ? }\end{array}$} & $\begin{array}{l}\text { Good }=3=\text { Two of the } \\
\text { above (State in comments). }\end{array}$ & \multirow[t]{3}{*}{0} & \multirow{3}{*}{$\begin{array}{l}\text { No reporting of power } \\
\text { or a priori power } \\
\text { analysis conducted. } \\
\text { Study has }<80 \% \text { power } \\
\text { to detect an effect at } r= \\
.20\end{array}$} \\
\hline & $\begin{array}{l}\text { Poor }=1.5=\text { Only one of the } \\
\text { above (State in comments). }\end{array}$ & & \\
\hline & $\begin{array}{l}\text { Very Poor }=0=\text { None of the } \\
\text { above }\end{array}$ & & \\
\hline \multicolumn{4}{|l|}{ Method: } \\
\hline Questions & Description & Score & Comments \\
\hline \multirow{4}{*}{$\begin{array}{l}\text { Has IPA been clearly } \\
\text { defined? } \\
\text { Have motivations been } \\
\text { clearly defined? }\end{array}$} & $\begin{array}{l}\text { Good }=3=\text { Clear } \\
\text { description of both IPA and } \\
\text { motivations. }\end{array}$ & \multirow[t]{4}{*}{1.5} & \multirow[t]{4}{*}{ Only IPA defined. } \\
\hline & $\begin{array}{l}\text { Fair }=2=\text { Clear description } \\
\text { of one variable, but } \\
\text { inadequate description of } \\
\text { the other. }\end{array}$ & & \\
\hline & $\begin{array}{l}\text { Poor }=1=\text { Inadequate } \\
\text { descriptions of both } \\
\text { variables. }\end{array}$ & & \\
\hline & $\begin{array}{l}\text { Very Poor }=0=\text { No } \\
\text { description of either } \\
\text { variable. }\end{array}$ & & \\
\hline \multirow[t]{4}{*}{$\begin{array}{l}\text { Are the measurements } \\
\text { used appropriate? }\end{array}$} & $\begin{array}{l}\text { Good }=3=\text { Validated and } \\
\text { reliability tested measures of } \\
\text { the outcome are used, or } \\
\text { measures are pre-tested } \\
\text { prior to data collection. }\end{array}$ & \multirow[t]{4}{*}{2.5} & \multirow{4}{*}{$\begin{array}{l}\text { CADRI is a well } \\
\text { validated and age } \\
\text { appropriate tool. } \\
\text { Spanish version has } \\
\text { been used in a previous } \\
\text { study. } \\
\text { Motive measure } \\
\text { developed for this study } \\
\text { used EFA, however, it } \\
\text { would have been useful } \\
\text { to have more } \\
\text { information e.g., pattern } \\
\text { matrix, items, for this } \\
\text { scale. }\end{array}$} \\
\hline & $\begin{array}{l}\text { Fair }=2=\text { Appropriate } \\
\text { measures have been used } \\
\text { but have not been described } \\
\text { well. }\end{array}$ & & \\
\hline & $\begin{array}{l}\text { Poor }=1=\text { Measures used } \\
\text { are inappropriate/ failure to } \\
\text { report validity and reliability } \\
\text { of measures. }\end{array}$ & & \\
\hline & $\begin{array}{l}\text { Very Poor }=0=\text { No mention } \\
\text { of measures used. }\end{array}$ & & \\
\hline \multicolumn{4}{|l|}{ Data Analysis: } \\
\hline Questions & Description & Score & Comments \\
\hline
\end{tabular}




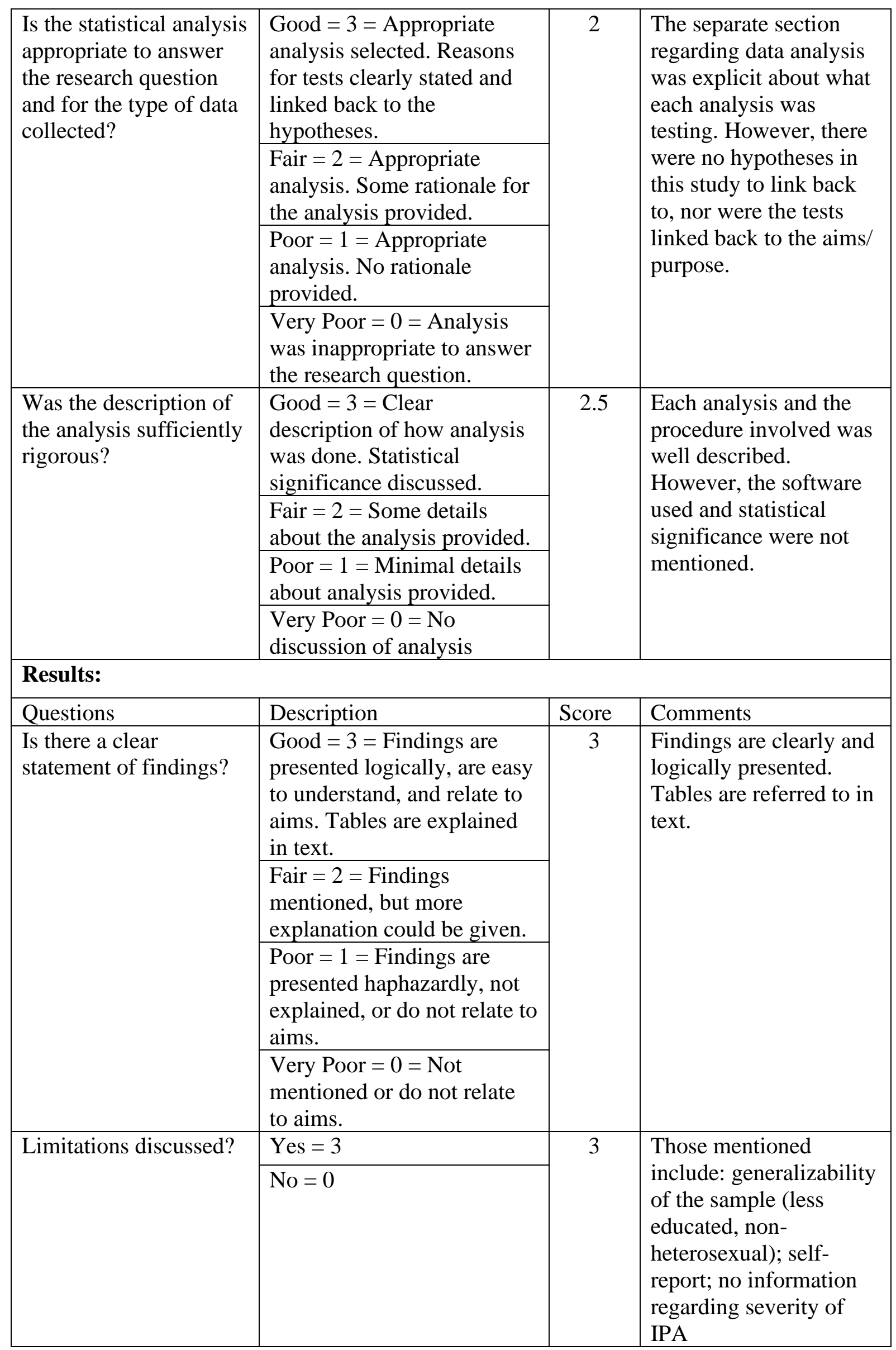




\begin{tabular}{|c|c|c|c|}
\hline \multicolumn{4}{|c|}{ Implications/ Applications: } \\
\hline Questions & Description & Score & Comments \\
\hline \multirow[t]{4}{*}{$\begin{array}{l}\text { How important are } \\
\text { these findings to policy/ } \\
\text { practice? } \\
\text { Does the research offer } \\
\text { a valuable contribution } \\
\text { to the literature? }\end{array}$} & $\begin{array}{l}\text { Good = } 3=\text { Contributes } \\
\text { something new/ different in } \\
\text { terms of insight or } \\
\text { perspective. Suggests ideas } \\
\text { for future research. Suggests } \\
\text { implication for policy and/ } \\
\text { or practice. }\end{array}$ & \multirow[t]{4}{*}{1} & \multirow[t]{4}{*}{$\begin{array}{l}\text { Implications for practice } \\
\text { only - the importance of } \\
\text { early intervention. }\end{array}$} \\
\hline & $\begin{array}{l}\text { Fair }=2=\text { Two of the above } \\
\text { (State in comments). }\end{array}$ & & \\
\hline & $\begin{array}{l}\text { Poor }=1=\text { Only one of the } \\
\text { above (State in comments). }\end{array}$ & & \\
\hline & $\begin{array}{l}\text { Very Poor }=0=\text { None of the } \\
\text { above }\end{array}$ & & \\
\hline \multicolumn{2}{|l|}{ Total Score: } & $\begin{array}{c}17.5 / 27 \\
65 \%\end{array}$ & \\
\hline
\end{tabular}

\begin{tabular}{|c|c|c|c|}
\hline Study 9 - Title: & \multicolumn{3}{|c|}{$\begin{array}{l}\text { College women who initiate assaults on their male partners and } \\
\text { the reasons offered for such behavior }\end{array}$} \\
\hline Author (Year): & \multicolumn{3}{|c|}{ Fiebert, M., \& Gonzalez, D. (1997) } \\
\hline \multicolumn{4}{|l|}{ Sampling: } \\
\hline Questions & Description & Score & Comments \\
\hline \multirow[t]{4}{*}{$\begin{array}{l}\text { Was the sampling } \\
\text { strategy appropriate to } \\
\text { address the research } \\
\text { question/s? } \\
\text { Why was this group } \\
\text { targeted? } \\
\text { Were the participants } \\
\text { representative of the } \\
\text { target population? }\end{array}$} & $\begin{array}{l}\text { Good }=3=\text { Details (age/ } \\
\text { gender/ ethnicity /context) of } \\
\text { who was studied and how } \\
\text { they were recruited. } \\
\text { Rationale for studying this } \\
\text { population. Response rates } \\
\text { shown and explained (why } \\
\text { certain individuals chose not } \\
\text { to participate, and any } \\
\text { attempts to achieve a sample } \\
\text { that represents the target } \\
\text { population). }\end{array}$ & \multirow[t]{4}{*}{2} & \multirow[t]{4}{*}{$\begin{array}{l}\text { Details of age ranges } \\
\text { and ethnic breakdown } \\
\text { provided. No rationale } \\
\text { for why a student } \\
\text { sample was studied. } \\
\text { Response rates } \\
\text { mentioned, but due to } \\
\text { the design of the study } \\
\text { were not available. }\end{array}$} \\
\hline & $\begin{array}{l}\text { Fair }=2=\text { Most information } \\
\text { given, but some missing. }\end{array}$ & & \\
\hline & $\begin{array}{l}\text { Poor }=1=\text { Sampling } \\
\text { mentioned but few } \\
\text { descriptive details. }\end{array}$ & & \\
\hline & $\begin{array}{l}\text { Very Poor }=0=\text { No details } \\
\text { of sample. }\end{array}$ & & \\
\hline \multirow{2}{*}{$\begin{array}{l}\text { Reporting of power or a } \\
\text { priori power analysis } \\
\text { conducted? }\end{array}$} & $\begin{array}{l}\text { Good }=3=\text { Two of the } \\
\text { above (State in comments). }\end{array}$ & \multirow[t]{2}{*}{1.5} & \multirow{2}{*}{$\begin{array}{l}\text { No reporting of power } \\
\text { or a priori power } \\
\text { analysis conducted. }\end{array}$} \\
\hline & $\begin{array}{l}\text { Poor }=1.5=\text { Only one of the } \\
\text { above (State in comments). }\end{array}$ & & \\
\hline
\end{tabular}




\begin{tabular}{|c|c|c|c|}
\hline $\begin{array}{l}\text { Does the study have } \geq \\
80 \% \text { power to detect an } \\
\text { effect at } r=.20 ?\end{array}$ & $\begin{array}{l}\text { Very Poor }=0=\text { None of the } \\
\text { above }\end{array}$ & & $\begin{array}{l}\text { The study has } \geq 80 \% \\
\text { power to detect an effect } \\
\text { at } r=.20 \text {. }\end{array}$ \\
\hline \multicolumn{4}{|l|}{ Method: } \\
\hline Questions & Description & Score & Comments \\
\hline \multirow{4}{*}{$\begin{array}{l}\text { Has IPA been clearly } \\
\text { defined? } \\
\text { Have motivations been } \\
\text { clearly defined? }\end{array}$} & $\begin{array}{l}\text { Good }=3=\text { Clear description } \\
\text { of both IPA and motivations. }\end{array}$ & \multirow[t]{4}{*}{0} & \multirow[t]{4}{*}{$\begin{array}{l}\text { Neither IPA nor } \\
\text { motivations were } \\
\text { defined. }\end{array}$} \\
\hline & $\begin{array}{l}\text { Fair }=2=\text { Clear description } \\
\text { of one variable, but } \\
\text { inadequate description of the } \\
\text { other. }\end{array}$ & & \\
\hline & $\begin{array}{l}\text { Poor }=1=\text { Inadequate } \\
\text { descriptions of both } \\
\text { variables. }\end{array}$ & & \\
\hline & $\begin{array}{l}\text { Very Poor }=0=\text { No } \\
\text { description of either variable. }\end{array}$ & & \\
\hline \multirow[t]{4}{*}{$\begin{array}{l}\text { Are the measurements } \\
\text { used appropriate? }\end{array}$} & $\begin{array}{l}\text { Good }=3=\text { Validated and } \\
\text { reliability tested measures of } \\
\text { the outcome are used, or } \\
\text { measures are pre-tested prior } \\
\text { to data collection. }\end{array}$ & \multirow[t]{4}{*}{1} & \multirow{4}{*}{$\begin{array}{l}\text { Measure used appears to } \\
\text { have been developed for } \\
\text { this study. No } \\
\text { information regarding } \\
\text { the source of the items } \\
\text { is provided. No formal } \\
\text { scale development took } \\
\text { place. The measure was } \\
\text { not piloted prior to use } \\
\text { in the study. No } \\
\text { psychometrics were } \\
\text { reported. }\end{array}$} \\
\hline & $\begin{array}{l}\text { Fair }=2=\text { Appropriate } \\
\text { measures have been used but } \\
\text { have not been described } \\
\text { well. }\end{array}$ & & \\
\hline & $\begin{array}{l}\text { Poor }=1=\text { Measures used } \\
\text { are inappropriate/ failure to } \\
\text { report validity and reliability } \\
\text { of measures. }\end{array}$ & & \\
\hline & $\begin{array}{l}\text { Very Poor }=0=\text { No mention } \\
\text { of measures used. }\end{array}$ & & \\
\hline \multicolumn{4}{|l|}{ Data Analysis: } \\
\hline Questions & Description & Score & Comments \\
\hline \multirow[t]{4}{*}{$\begin{array}{l}\text { Is the statistical analysis } \\
\text { appropriate to answer } \\
\text { the research question } \\
\text { and for the type of data } \\
\text { collected? }\end{array}$} & $\begin{array}{l}\text { Good = } 3=\text { Appropriate } \\
\text { analysis selected. Reasons } \\
\text { for tests clearly stated and } \\
\text { linked back to the } \\
\text { hypotheses. }\end{array}$ & \multirow[t]{4}{*}{1} & \multirow[t]{4}{*}{$\begin{array}{l}\text { Appropriate analyses. } \\
\text { Stated which tests were } \\
\text { conducted, but no } \\
\text { rationale provided. No } \\
\text { hypotheses to link back } \\
\text { to. }\end{array}$} \\
\hline & $\begin{array}{l}\text { Fair }=2=\text { Appropriate } \\
\text { analysis. Some rationale for } \\
\text { the analysis provided. }\end{array}$ & & \\
\hline & $\begin{array}{l}\text { Poor }=1=\text { Appropriate } \\
\text { analysis. No rationale } \\
\text { provided. }\end{array}$ & & \\
\hline & $\begin{array}{l}\text { Very Poor }=0=\text { Analysis } \\
\text { was inappropriate to answer } \\
\text { the research question. }\end{array}$ & & \\
\hline
\end{tabular}




\begin{tabular}{|c|c|c|c|}
\hline \multirow[t]{4}{*}{$\begin{array}{l}\text { Was the description of } \\
\text { the analysis sufficiently } \\
\text { rigorous? }\end{array}$} & $\begin{array}{l}\text { Good }=3=\text { Clear description } \\
\text { of how analysis was done. } \\
\text { Statistical significance } \\
\text { discussed. }\end{array}$ & \multirow[t]{4}{*}{1} & \multirow{4}{*}{$\begin{array}{l}\text { No discussion of } \\
\text { analysis besides stating } \\
\text { what was conducted } \\
\text { with which variables. } \\
\text { No mention of software } \\
\text { used or of statistical } \\
\text { significance. }\end{array}$} \\
\hline & $\begin{array}{l}\text { Fair }=2=\text { Some details } \\
\text { about the analysis provided. }\end{array}$ & & \\
\hline & $\begin{array}{l}\text { Poor }=1=\text { Minimal details } \\
\text { about analysis provided. }\end{array}$ & & \\
\hline & $\begin{array}{l}\text { Very Poor }=0=\text { No } \\
\text { discussion of analysis }\end{array}$ & & \\
\hline \multicolumn{4}{|l|}{ Results: } \\
\hline Questions & Description & Score & Comments \\
\hline \multirow[t]{4}{*}{$\begin{array}{l}\text { Is there a clear } \\
\text { statement of findings? }\end{array}$} & $\begin{array}{l}\text { Good }=3=\text { Findings are } \\
\text { presented logically, are easy } \\
\text { to understand, and relate to } \\
\text { aims. Tables are explained in } \\
\text { text. }\end{array}$ & \multirow[t]{4}{*}{1} & \multirow{4}{*}{$\begin{array}{l}\text { It was not clear which } \\
\text { variables had been used } \\
\text { in the ANOVA - results } \\
\text { regarding ethnicity were } \\
\text { reported, but this was } \\
\text { not included in the list } \\
\text { of variables or } \\
\text { tabulated. Greater detail } \\
\text { could have been } \\
\text { provided, such as how } \\
\text { the frequency data was } \\
\text { analysed. }\end{array}$} \\
\hline & $\begin{array}{l}\text { Fair }=2=\text { Findings } \\
\text { mentioned, but more } \\
\text { explanation could be given. }\end{array}$ & & \\
\hline & $\begin{array}{l}\text { Poor }=1=\text { Findings are } \\
\text { presented haphazardly, not } \\
\text { explained, or do not relate to } \\
\text { aims. }\end{array}$ & & \\
\hline & $\begin{array}{l}\text { Very Poor }=0=\text { Not } \\
\text { mentioned or do not relate to } \\
\text { aims. }\end{array}$ & & \\
\hline \multirow[t]{2}{*}{ Limitations discussed? } & Yes $=3$ & \multirow[t]{2}{*}{0} & \multirow{2}{*}{$\begin{array}{l}\text { No limitations } \\
\text { mentioned }\end{array}$} \\
\hline & $\mathrm{No}=0$ & & \\
\hline \multicolumn{4}{|c|}{ Implications/ Applications: } \\
\hline Questions & Description & Score & Comments \\
\hline \multirow[t]{4}{*}{$\begin{array}{l}\text { How important are these } \\
\text { findings to policy/ } \\
\text { practice? } \\
\text { Does the research offer } \\
\text { a valuable contribution } \\
\text { to the literature? }\end{array}$} & $\begin{array}{l}\text { Good }=3=\text { Contributes } \\
\text { something new/ different in } \\
\text { terms of insight or } \\
\text { perspective. Suggests ideas } \\
\text { for future research. Suggests } \\
\text { implication for policy and/ or } \\
\text { practice. }\end{array}$ & \multirow[t]{4}{*}{0} & \multirow[t]{4}{*}{ None of these } \\
\hline & $\begin{array}{l}\text { Fair }=2=\text { Two of the above } \\
\text { (State in comments). }\end{array}$ & & \\
\hline & $\begin{array}{l}\text { Poor }=1=\text { Only one of the } \\
\text { above (State in comments). }\end{array}$ & & \\
\hline & $\begin{array}{l}\text { Very Poor }=0=\text { None of the } \\
\text { above }\end{array}$ & & \\
\hline \multicolumn{2}{|l|}{ Total Score: } & $\begin{array}{l}7.5 / 27 \\
28 \%\end{array}$ & \\
\hline
\end{tabular}




\begin{tabular}{|c|c|c|c|}
\hline Study 10 - Title: & \multicolumn{3}{|c|}{ Sex differences in motivations and effects in dating violence } \\
\hline Author (Year): & \multicolumn{3}{|c|}{$\begin{array}{l}\text { Follingstad, D. R., Wright, S., Lloyd, S., \& Sebastian, J. A. } \\
\text { (1991) }\end{array}$} \\
\hline \multicolumn{4}{|l|}{ Sampling: } \\
\hline Questions & Description & Score & Comments \\
\hline \multirow[t]{4}{*}{$\begin{array}{l}\text { Was the sampling } \\
\text { strategy appropriate to } \\
\text { address the research } \\
\text { question/s? } \\
\text { Why was this group } \\
\text { targeted? } \\
\text { Were the participants } \\
\text { representative of the } \\
\text { target population? }\end{array}$} & $\begin{array}{l}\text { Good }=3=\text { Details (age/ } \\
\text { gender/ ethnicity /context) of } \\
\text { who was studied and how } \\
\text { they were recruited. } \\
\text { Rationale for studying this } \\
\text { population. Response rates } \\
\text { shown and explained (why } \\
\text { certain individuals chose not } \\
\text { to participate, and any } \\
\text { attempts to achieve a sample } \\
\text { that represents the target } \\
\text { population). }\end{array}$ & \multirow[t]{4}{*}{2} & \multirow[t]{4}{*}{$\begin{array}{l}\text { Details of sample, but } \\
\text { not of how they were } \\
\text { recruited, or of the } \\
\text { response rates. Any } \\
\text { attempts to ensure the } \\
\text { sample was } \\
\text { representative were not } \\
\text { reported. Very good } \\
\text { rationale for studying } \\
\text { this population. }\end{array}$} \\
\hline & $\begin{array}{l}\text { Fair }=2=\text { Most information } \\
\text { given, but some missing. }\end{array}$ & & \\
\hline & $\begin{array}{l}\text { Poor }=1=\text { Sampling } \\
\text { mentioned but few } \\
\text { descriptive details. }\end{array}$ & & \\
\hline & $\begin{array}{l}\text { Very Poor }=0=\text { No details } \\
\text { of sample. }\end{array}$ & & \\
\hline \multirow{3}{*}{$\begin{array}{l}\text { Reporting of power or a } \\
\text { priori power analysis } \\
\text { conducted? } \\
\text { Does the study have } \geq \\
80 \% \text { power to detect an } \\
\text { effect at } r=.20 \text { ? }\end{array}$} & $\begin{array}{l}\text { Good }=3=\text { Two of the above } \\
\text { (State in comments). }\end{array}$ & \multirow[t]{3}{*}{0} & \multirow{3}{*}{$\begin{array}{l}\text { No reporting of power } \\
\text { or a priori power } \\
\text { analysis conducted. } \\
\text { The study has }<80 \% \\
\text { power to detect an effect } \\
\text { at } r=.20 \text {. }\end{array}$} \\
\hline & $\begin{array}{l}\text { Poor }=1.5=\text { Only one of the } \\
\text { above (State in comments). }\end{array}$ & & \\
\hline & $\begin{array}{l}\text { Very Poor }=0=\text { None of the } \\
\text { above }\end{array}$ & & \\
\hline \multicolumn{4}{|l|}{ Method: } \\
\hline Questions & Description & Score & Comments \\
\hline \multirow{4}{*}{$\begin{array}{l}\text { Has IPA been clearly } \\
\text { defined? } \\
\text { Have motivations been } \\
\text { clearly defined? }\end{array}$} & $\begin{array}{l}\text { Good }=3=\text { Clear description } \\
\text { of both IPA and motivations. }\end{array}$ & \multirow[t]{4}{*}{0} & \multirow[t]{4}{*}{$\begin{array}{l}\text { No description of either } \\
\text { IPA or motivations }\end{array}$} \\
\hline & $\begin{array}{l}\text { Fair }=2=\text { Clear description } \\
\text { of one variable, but } \\
\text { inadequate description of the } \\
\text { other. }\end{array}$ & & \\
\hline & $\begin{array}{l}\text { Poor }=1=\text { Inadequate } \\
\text { descriptions of both } \\
\text { variables. }\end{array}$ & & \\
\hline & $\begin{array}{l}\text { Very Poor }=0=\text { No } \\
\text { description of either variable. }\end{array}$ & & \\
\hline $\begin{array}{l}\text { Are the measurements } \\
\text { used appropriate? }\end{array}$ & $\begin{array}{l}\text { Good }=3=\text { Validated and } \\
\text { reliability tested measures of }\end{array}$ & 1.5 & $\begin{array}{l}\text { The CTS is a well } \\
\text { validated and reliable }\end{array}$ \\
\hline
\end{tabular}




\begin{tabular}{|c|c|c|c|}
\hline & $\begin{array}{l}\text { the outcome are used, or } \\
\text { measures are pre-tested prior } \\
\text { to data collection. } \\
\text { Fair }=2=\text { Appropriate } \\
\text { measures have been used but } \\
\text { have not been described well. } \\
\text { Poor }=1=\text { Measures used are } \\
\text { inappropriate/ failure to } \\
\text { report validity and reliability } \\
\text { of measures. } \\
\begin{array}{l}\text { Very Poor }=0=\text { No mention } \\
\text { of measures used. }\end{array}\end{array}$ & & $\begin{array}{l}\text { tool and psychometric } \\
\text { information regarding } \\
\text { this was provided. The } \\
\text { MEQ was not piloted } \\
\text { prior to this study and } \\
\text { was not developed } \\
\text { appropriately. No details } \\
\text { as to the source of the } \\
\text { items were provided and } \\
\text { no psychometric } \\
\text { information was } \\
\text { available. }\end{array}$ \\
\hline \multicolumn{4}{|l|}{ Data Analysis: } \\
\hline Questions & Description & Score & Comments \\
\hline $\begin{array}{l}\text { Is the statistical analysis } \\
\text { appropriate to answer } \\
\text { the research question } \\
\text { and for the type of data } \\
\text { collected? }\end{array}$ & $\begin{array}{l}\text { Good }=3=\text { Appropriate } \\
\text { analysis selected. Reasons } \\
\text { for tests clearly stated and } \\
\text { linked back to the } \\
\text { hypotheses. } \\
\text { Fair }=2=\text { Appropriate } \\
\text { analysis. Some rationale for } \\
\text { the analysis provided. } \\
\text { Poor }=1=\text { Appropriate } \\
\text { analysis. No rationale } \\
\text { provided. } \\
\text { Very Poor = } 0=\text { Analysis } \\
\text { was inappropriate to answer } \\
\text { the research question. }\end{array}$ & 1 & $\begin{array}{l}\text { The analysis was } \\
\text { appropriate, but no } \\
\text { rationale was provided. }\end{array}$ \\
\hline $\begin{array}{l}\text { Was the description of } \\
\text { the analysis sufficiently } \\
\text { rigorous? }\end{array}$ & $\begin{array}{l}\text { Good }=3=\text { Clear description } \\
\text { of how analysis was done. } \\
\text { Statistical significance } \\
\text { discussed. } \\
\text { Fair }=2=\text { Some details about } \\
\text { the analysis provided. } \\
\text { Poor }=1=\text { Minimal details } \\
\text { about analysis provided. } \\
\begin{array}{l}\text { Very Poor }=0=\text { No } \\
\text { discussion of analysis }\end{array}\end{array}$ & 1 & $\begin{array}{l}\text { An ANOVA was } \\
\text { conducted, however, } \\
\text { this was not stated, only } \\
\text { the were results reported } \\
\text { (this analysis does not } \\
\text { directly relate to the } \\
\text { research question of this } \\
\text { review). No mention of } \\
\text { software used or of } \\
\text { statistical significance. }\end{array}$ \\
\hline \multicolumn{4}{|l|}{ Results: } \\
\hline Questions & Description & Score & Comments \\
\hline $\begin{array}{l}\text { Is there a clear statement } \\
\text { of findings? }\end{array}$ & $\begin{array}{l}\text { Good }=3=\text { Findings are } \\
\text { presented logically, are easy } \\
\text { to understand, and relate to } \\
\text { aims. Tables are explained in } \\
\text { text. } \\
\text { Fair }=2=\text { Findings } \\
\text { mentioned, but more } \\
\text { explanation could be given. }\end{array}$ & 2 & $\begin{array}{l}\text { Greater clarity could } \\
\text { have improved the } \\
\text { results section e.g., } \\
\text { reporting the most } \\
\text { frequently endorsed } \\
\text { motives in descending, } \\
\text { as opposed to random, } \\
\text { order. }\end{array}$ \\
\hline
\end{tabular}




\begin{tabular}{|c|c|c|c|}
\hline & $\begin{array}{l}\text { Poor }=1=\text { Findings are } \\
\text { presented haphazardly, not } \\
\text { explained, or do not relate to } \\
\text { aims. } \\
\text { Very Poor }=0=\text { Not } \\
\text { mentioned or do not relate to } \\
\text { aims. }\end{array}$ & & \\
\hline \multirow[t]{2}{*}{ Limitations discussed? } & Yes $=3$ & \multirow[t]{2}{*}{1.5} & \multirow{2}{*}{$\begin{array}{l}\text { These were not } \\
\text { explicitly mentioned as } \\
\text { limitations of the study, } \\
\text { but areas that future } \\
\text { research could expand } \\
\text { on. }\end{array}$} \\
\hline & $\mathrm{No}=0$ & & \\
\hline \multicolumn{4}{|c|}{ Implications/ Applications: } \\
\hline Questions & Description & Score & Comments \\
\hline \multirow[t]{4}{*}{$\begin{array}{l}\text { How important are these } \\
\text { findings to policy/ } \\
\text { practice? } \\
\text { Does the research offer } \\
\text { a valuable contribution } \\
\text { to the literature? }\end{array}$} & $\begin{array}{l}\text { Good = } 3=\text { Contributes } \\
\text { something new/ different in } \\
\text { terms of insight or } \\
\text { perspective. Suggests ideas } \\
\text { for future research. Suggests } \\
\text { implication for policy and/ or } \\
\text { practice. }\end{array}$ & \multirow[t]{4}{*}{3} & \multirow{4}{*}{$\begin{array}{l}\text { This is the first study to } \\
\text { investigate both the } \\
\text { motivations and effects } \\
\text { of IPA and design a tool } \\
\text { for this use. There are } \\
\text { some ideas for future } \\
\text { research and } \\
\text { implications for } \\
\text { treatment. }\end{array}$} \\
\hline & $\begin{array}{l}\text { Fair }=2=\text { Two of the above } \\
\text { (State in comments). }\end{array}$ & & \\
\hline & $\begin{array}{l}\text { Poor }=1=\text { Only one of the } \\
\text { above (State in comments). }\end{array}$ & & \\
\hline & $\begin{array}{l}\text { Very Poor }=0=\text { None of the } \\
\text { above }\end{array}$ & & \\
\hline \multicolumn{2}{|l|}{ Total Score: } & $\begin{array}{l}12 / 27 \\
44 \%\end{array}$ & \\
\hline
\end{tabular}

\begin{tabular}{|c|c|c|c|}
\hline Study 11 - Title: & \multicolumn{3}{|c|}{$\begin{array}{l}\text { The intended function of domestic violence is different for } \\
\text { arrested male and female perpetrators }\end{array}$} \\
\hline Author (Year): & \multicolumn{3}{|l|}{ Hamberger, L. (1994) } \\
\hline \multicolumn{4}{|l|}{ Sampling: } \\
\hline Questions & Description & Score & Comments \\
\hline $\begin{array}{l}\text { Was the sampling } \\
\text { strategy appropriate to } \\
\text { address the research } \\
\text { question/s? } \\
\text { Why was this group } \\
\text { targeted? } \\
\text { Were the participants } \\
\text { representative of the } \\
\text { target population? }\end{array}$ & $\begin{array}{l}\text { Good }=3=\text { Details (age/ } \\
\text { gender/ ethnicity /context) of } \\
\text { who was studied and how } \\
\text { they were recruited. } \\
\text { Rationale for studying this } \\
\text { population. Response rates } \\
\text { shown and explained (why } \\
\text { certain individuals chose not } \\
\text { to participate, and any } \\
\text { attempts to achieve a sample }\end{array}$ & N/A & $\begin{array}{l}\text { This study was } \\
\text { excluded from the } \\
\text { review. Refer to the } \\
\text { data extraction form for } \\
\text { additional details. }\end{array}$ \\
\hline
\end{tabular}




\begin{tabular}{|c|c|c|c|}
\hline & $\begin{array}{l}\text { that represents the target } \\
\text { population). }\end{array}$ & & \\
\hline & $\begin{array}{l}\text { Fair }=2=\text { Most information } \\
\text { given, but some missing. }\end{array}$ & & \\
\hline & $\begin{array}{l}\text { Poor }=1=\text { Sampling } \\
\text { mentioned but few } \\
\text { descriptive details. }\end{array}$ & & \\
\hline & $\begin{array}{l}\text { Very Poor }=0=\text { No details of } \\
\text { sample. }\end{array}$ & & \\
\hline \multirow{3}{*}{$\begin{array}{l}\text { Reporting of power or a } \\
\text { priori power analysis } \\
\text { conducted? } \\
\text { Does the study have } \geq \\
80 \% \text { power to detect an } \\
\text { effect at } r=.20 \text { ? }\end{array}$} & $\begin{array}{l}\text { Good = } 3=\text { Two of the above } \\
\text { (State in comments). }\end{array}$ & \multirow[t]{3}{*}{ N/A } & \\
\hline & $\begin{array}{l}\text { Poor }=1.5=\text { Only one of the } \\
\text { above (State in comments). }\end{array}$ & & \\
\hline & $\begin{array}{l}\text { Very Poor }=0=\text { None of the } \\
\text { above }\end{array}$ & & \\
\hline \multicolumn{4}{|l|}{ Method: } \\
\hline Questions & Description & Score & Comments \\
\hline \multirow{4}{*}{$\begin{array}{l}\text { Has IPA been clearly } \\
\text { defined? } \\
\text { Have motivations been } \\
\text { clearly defined? }\end{array}$} & $\begin{array}{l}\text { Good }=3=\text { Clear description } \\
\text { of both IPA and motivations. }\end{array}$ & \multirow[t]{4}{*}{ N/A } & \\
\hline & $\begin{array}{l}\text { Fair }=2=\text { Clear description } \\
\text { of one variable, but } \\
\text { inadequate description of the } \\
\text { other. }\end{array}$ & & \\
\hline & $\begin{array}{l}\text { Poor }=1=\text { Inadequate } \\
\text { descriptions of both } \\
\text { variables. }\end{array}$ & & \\
\hline & $\begin{array}{l}\text { Very Poor }=0=\text { No } \\
\text { description of either variable. }\end{array}$ & & \\
\hline \multirow[t]{4}{*}{$\begin{array}{l}\text { Are the measurements } \\
\text { used appropriate? }\end{array}$} & $\begin{array}{l}\text { Good }=3=\text { Validated and } \\
\text { reliability tested measures of } \\
\text { the outcome are used, or } \\
\text { measures are pre-tested prior } \\
\text { to data collection. }\end{array}$ & \multirow[t]{4}{*}{ N/A } & \\
\hline & $\begin{array}{l}\text { Fair }=2=\text { Appropriate } \\
\text { measures have been used but } \\
\text { have not been described well. }\end{array}$ & & \\
\hline & $\begin{array}{l}\text { Poor }=1=\text { Measures used are } \\
\text { inappropriate/ failure to } \\
\text { report validity and reliability } \\
\text { of measures. }\end{array}$ & & \\
\hline & $\begin{array}{l}\text { Very Poor }=0=\text { No mention } \\
\text { of measures used. }\end{array}$ & & \\
\hline \multicolumn{4}{|l|}{ Data Analysis: } \\
\hline Questions & Description & Score & Comments \\
\hline $\begin{array}{l}\text { Is the statistical analysis } \\
\text { appropriate to answer }\end{array}$ & $\begin{array}{l}\text { Good = } 3=\text { Appropriate } \\
\text { analysis selected. Reasons for }\end{array}$ & N/A & \\
\hline
\end{tabular}




\begin{tabular}{|c|c|c|c|}
\hline \multirow{4}{*}{$\begin{array}{l}\text { the research question } \\
\text { and for the type of data } \\
\text { collected? }\end{array}$} & $\begin{array}{l}\text { tests clearly stated and linked } \\
\text { back to the hypotheses. }\end{array}$ & & \\
\hline & $\begin{array}{l}\text { Fair }=2=\text { Appropriate } \\
\text { analysis. Some rationale for } \\
\text { the analysis provided. }\end{array}$ & & \\
\hline & $\begin{array}{l}\text { Poor }=1=\text { Appropriate } \\
\text { analysis. No rationale } \\
\text { provided. }\end{array}$ & & \\
\hline & $\begin{array}{l}\text { Very Poor }=0=\text { Analysis } \\
\text { was inappropriate to answer } \\
\text { the research question. }\end{array}$ & & \\
\hline \multirow[t]{4}{*}{$\begin{array}{l}\text { Was the description of } \\
\text { the analysis sufficiently } \\
\text { rigorous? }\end{array}$} & $\begin{array}{l}\text { Good }=3=\text { Clear description } \\
\text { of how analysis was done. } \\
\text { Statistical significance } \\
\text { discussed. }\end{array}$ & \multirow[t]{4}{*}{ N/A } & \\
\hline & $\begin{array}{l}\text { Fair }=2=\text { Some details about } \\
\text { the analysis provided. }\end{array}$ & & \\
\hline & $\begin{array}{l}\text { Poor }=1=\text { Minimal details } \\
\text { about analysis provided. }\end{array}$ & & \\
\hline & $\begin{array}{l}\text { Very Poor }=0=\text { No } \\
\text { discussion of analysis }\end{array}$ & & \\
\hline \multicolumn{4}{|l|}{ Results: } \\
\hline Questions & Description & Score & Comments \\
\hline \multirow[t]{4}{*}{$\begin{array}{l}\text { Is there a clear statement } \\
\text { of findings? }\end{array}$} & $\begin{array}{l}\text { Good }=3=\text { Findings are } \\
\text { presented logically, are easy } \\
\text { to understand, and relate to } \\
\text { aims. Tables are explained in } \\
\text { text. }\end{array}$ & \multirow[t]{4}{*}{$\mathrm{N} / \mathrm{A}$} & \\
\hline & $\begin{array}{l}\text { Fair }=2=\text { Findings } \\
\text { mentioned, but more } \\
\text { explanation could be given. }\end{array}$ & & \\
\hline & $\begin{array}{l}\text { Poor }=1=\text { Findings are } \\
\text { presented haphazardly, not } \\
\text { explained, or do not relate to } \\
\text { aims. }\end{array}$ & & \\
\hline & $\begin{array}{l}\text { Very Poor }=0=\text { Not } \\
\text { mentioned or do not relate to } \\
\text { aims. }\end{array}$ & & \\
\hline \multirow[t]{2}{*}{ Limitations discussed? } & Yes $=3$ & \multirow[t]{2}{*}{ N/A } & \\
\hline & $\mathrm{No}=0$ & & \\
\hline \multicolumn{4}{|c|}{ Implications/ Applications: } \\
\hline Questions & Description & Score & Comments \\
\hline $\begin{array}{l}\text { How important are these } \\
\text { findings to policy/ } \\
\text { practice? }\end{array}$ & $\begin{array}{l}\text { Good = } 3=\text { Contributes } \\
\text { something new/ different in } \\
\text { terms of insight or } \\
\text { perspective. Suggests ideas } \\
\text { for future research. Suggests }\end{array}$ & N/A & \\
\hline
\end{tabular}




\begin{tabular}{|c|c|c|c|}
\hline \multirow{4}{*}{$\begin{array}{l}\text { Does the research offer a } \\
\text { valuable contribution to } \\
\text { the literature? }\end{array}$} & $\begin{array}{l}\text { implication for policy and/ or } \\
\text { practice. }\end{array}$ & & \\
\hline & $\begin{array}{l}\text { Fair }=2=\text { Two of the above } \\
\text { (State in comments). }\end{array}$ & & \\
\hline & $\begin{array}{l}\text { Poor }=1=\text { Only one of the } \\
\text { above (State in comments). }\end{array}$ & & \\
\hline & $\begin{array}{l}\text { Very Poor }=0=\text { None of the } \\
\text { above }\end{array}$ & & \\
\hline \multicolumn{2}{|l|}{ Total Score: } & N/A & \\
\hline
\end{tabular}

\begin{tabular}{|c|c|c|c|}
\hline Study 12 - Title: & \multicolumn{3}{|c|}{$\begin{array}{l}\text { Abused women or abused men? An examination of the context } \\
\text { and outcomes of dating violence }\end{array}$} \\
\hline Author (Year): & \multicolumn{3}{|c|}{ Harned, M. S. (2001) } \\
\hline \multicolumn{4}{|l|}{ Sampling: } \\
\hline Questions & Description & Score & Comments \\
\hline \multirow[t]{4}{*}{$\begin{array}{l}\text { Was the sampling } \\
\text { strategy appropriate to } \\
\text { address the research } \\
\text { question/s? } \\
\text { Why was this group } \\
\text { targeted? } \\
\text { Were the participants } \\
\text { representative of the } \\
\text { target population? }\end{array}$} & $\begin{array}{l}\text { Good }=3=\text { Details (age/ } \\
\text { gender/ ethnicity /context) of } \\
\text { who was studied and how } \\
\text { they were recruited. } \\
\text { Rationale for studying this } \\
\text { population. Response rates } \\
\text { shown and explained (why } \\
\text { certain individuals chose not } \\
\text { to participate, and any } \\
\text { attempts to achieve a sample } \\
\text { that represents the target } \\
\text { population). }\end{array}$ & \multirow[t]{4}{*}{3} & \multirow[t]{4}{*}{$\begin{array}{l}\text { Details of sample, } \\
\text { recruitment, and } \\
\text { response rate. Rationale } \\
\text { for studying this } \\
\text { population provided. } \\
\text { Breakdown of } \\
\text { population and sample } \\
\text { by ethnicity shows } \\
\text { sample is representative. }\end{array}$} \\
\hline & $\begin{array}{l}\text { Fair }=2=\text { Most information } \\
\text { given, but some missing. }\end{array}$ & & \\
\hline & $\begin{array}{l}\text { Poor }=1=\text { Sampling } \\
\text { mentioned but few } \\
\text { descriptive details. }\end{array}$ & & \\
\hline & $\begin{array}{l}\text { Very Poor }=0=\text { No details } \\
\text { of sample. }\end{array}$ & & \\
\hline \multirow{3}{*}{$\begin{array}{l}\text { Reporting of power or a } \\
\text { priori power analysis } \\
\text { conducted? } \\
\text { Does the study have } \geq \\
80 \% \text { power to detect an } \\
\text { effect at } r=.20 \text { ? }\end{array}$} & $\begin{array}{l}\text { Good = } 3=\text { Two of the above } \\
\text { (State in comments). }\end{array}$ & \multirow[t]{3}{*}{0} & \multirow{3}{*}{$\begin{array}{l}\text { No reporting of power } \\
\text { or a priori power } \\
\text { analysis conducted. } \\
\text { The study has }<80 \% \\
\text { power to detect an effect } \\
\text { at } r=.20 \text {. }\end{array}$} \\
\hline & $\begin{array}{l}\text { Poor }=1.5=\text { Only one of the } \\
\text { above (State in comments). }\end{array}$ & & \\
\hline & $\begin{array}{l}\text { Very Poor }=0=\text { None of the } \\
\text { above }\end{array}$ & & \\
\hline \multicolumn{4}{|l|}{ Method: } \\
\hline Questions & Description & Score & Comments \\
\hline $\begin{array}{l}\text { Has IPA been clearly } \\
\text { defined? }\end{array}$ & $\begin{array}{l}\text { Good = } 3=\text { Clear description } \\
\text { of both IPA and motivations. }\end{array}$ & 1.5 & $\begin{array}{l}\text { Clear definition of IPA. } \\
\text { No definition of } \\
\text { motivations }\end{array}$ \\
\hline
\end{tabular}




\begin{tabular}{|c|c|c|c|}
\hline \multirow[t]{3}{*}{$\begin{array}{l}\text { Have motivations been } \\
\text { clearly defined? }\end{array}$} & $\begin{array}{l}\text { Fair }=2=\text { Clear description } \\
\text { of one variable, but } \\
\text { inadequate description of the } \\
\text { other. }\end{array}$ & & \\
\hline & $\begin{array}{l}\text { Poor }=1=\text { Inadequate } \\
\text { descriptions of both } \\
\text { variables. }\end{array}$ & & \\
\hline & $\begin{array}{l}\text { Very Poor }=0=\text { No } \\
\text { description of either variable. }\end{array}$ & & \\
\hline \multirow[t]{4}{*}{$\begin{array}{l}\text { Are the measurements } \\
\text { used appropriate? }\end{array}$} & $\begin{array}{l}\text { Good }=3=\text { Validated and } \\
\text { reliability tested measures of } \\
\text { the outcome are used, or } \\
\text { measures are pre-tested prior } \\
\text { to data collection. }\end{array}$ & \multirow[t]{4}{*}{3} & \multirow{4}{*}{$\begin{array}{l}\text { CTS2 is a reliable and } \\
\text { well validated tool. } \\
\text { Psychometric } \\
\text { information provided in } \\
\text { support of this. } \\
\text { MEQ not previously } \\
\text { validated, however, an } \\
\text { EPCA was conducted in } \\
\text { this study. The survey } \\
\text { was piloted prior to } \\
\text { administration in the } \\
\text { study. }\end{array}$} \\
\hline & $\begin{array}{l}\text { Fair }=2=\text { Appropriate } \\
\text { measures have been used but } \\
\text { have not been described well. }\end{array}$ & & \\
\hline & $\begin{array}{l}\text { Poor }=1=\text { Measures used } \\
\text { are inappropriate/ failure to } \\
\text { report validity and reliability } \\
\text { of measures. }\end{array}$ & & \\
\hline & $\begin{array}{l}\text { Very Poor }=0=\text { No mention } \\
\text { of measures used. }\end{array}$ & & \\
\hline \multicolumn{4}{|l|}{ Data Analysis: } \\
\hline Questions & Description & Score & Comments \\
\hline \multirow[t]{4}{*}{$\begin{array}{l}\text { Is the statistical analysis } \\
\text { appropriate to answer } \\
\text { the research question } \\
\text { and for the type of data } \\
\text { collected? }\end{array}$} & $\begin{array}{l}\text { Good }=3=\text { Appropriate } \\
\text { analysis selected. Reasons } \\
\text { for tests clearly stated and } \\
\text { linked back to the } \\
\text { hypotheses. }\end{array}$ & \multirow[t]{4}{*}{2} & \multirow{4}{*}{$\begin{array}{l}\text { Appropriate analysis. } \\
\text { Although not explicitly } \\
\text { stated, the EPCA is } \\
\text { conducted to provide } \\
\text { psychometric } \\
\text { information for the } \\
\text { MEQ, which was not } \\
\text { formally developed. }\end{array}$} \\
\hline & $\begin{array}{l}\text { Fair }=2=\text { Appropriate } \\
\text { analysis. Some rationale for } \\
\text { the analysis provided. }\end{array}$ & & \\
\hline & $\begin{array}{l}\text { Poor }=1=\text { Appropriate } \\
\text { analysis. No rationale } \\
\text { provided. }\end{array}$ & & \\
\hline & $\begin{array}{l}\text { Very Poor }=0=\text { Analysis } \\
\text { was inappropriate to answer } \\
\text { the research question. }\end{array}$ & & \\
\hline \multirow[t]{4}{*}{$\begin{array}{l}\text { Was the description of } \\
\text { the analysis sufficiently } \\
\text { rigorous? }\end{array}$} & $\begin{array}{l}\text { Good }=3=\text { Clear description } \\
\text { of how analysis was done. } \\
\text { Statistical significance } \\
\text { discussed. }\end{array}$ & \multirow[t]{4}{*}{2} & \multirow{4}{*}{$\begin{array}{l}\text { More details regarding } \\
\text { the EPCA would have } \\
\text { been beneficial. } \\
\text { Statistical significance } \\
\text { regarding Bonferroni } \\
\text { correction mentioned. }\end{array}$} \\
\hline & $\begin{array}{l}\text { Fair }=2=\text { Some details about } \\
\text { the analysis provided. }\end{array}$ & & \\
\hline & $\begin{array}{l}\text { Poor }=1=\text { Minimal details } \\
\text { about analysis provided. }\end{array}$ & & \\
\hline & $\begin{array}{l}\text { Very Poor }=0=\text { No } \\
\text { discussion of analysis }\end{array}$ & & \\
\hline
\end{tabular}




\begin{tabular}{|c|c|c|c|}
\hline \multicolumn{4}{|l|}{ Results: } \\
\hline Questions & Description & Score & Comments \\
\hline \multirow[t]{4}{*}{$\begin{array}{l}\text { Is there a clear statement } \\
\text { of findings? }\end{array}$} & $\begin{array}{l}\text { Good }=3=\text { Findings are } \\
\text { presented logically, are easy } \\
\text { to understand, and relate to } \\
\text { aims. Tables are explained in } \\
\text { text. }\end{array}$ & \multirow[t]{4}{*}{2.5} & \multirow[t]{4}{*}{$\begin{array}{l}\text { Findings and clear and } \\
\text { tables are explained in } \\
\text { text, though not linked } \\
\text { back to the hypotheses. }\end{array}$} \\
\hline & $\begin{array}{l}\text { Fair }=2=\text { Findings } \\
\text { mentioned, but more } \\
\text { explanation could be given. }\end{array}$ & & \\
\hline & $\begin{array}{l}\text { Poor }=1=\text { Findings are } \\
\text { presented haphazardly, not } \\
\text { explained, or do not relate to } \\
\text { aims. }\end{array}$ & & \\
\hline & $\begin{array}{l}\text { Very Poor }=0=\text { Not } \\
\text { mentioned or do not relate to } \\
\text { aims. }\end{array}$ & & \\
\hline \multirow[t]{2}{*}{ Limitations discussed? } & Yes $=3$ & \multirow[t]{2}{*}{3} & \multirow{2}{*}{$\begin{array}{l}\text { These include: self- } \\
\text { report, lack of } \\
\text { anonymity, use of } \\
\text { college sample, limited } \\
\text { number of motivations. }\end{array}$} \\
\hline & $\mathrm{No}=0$ & & \\
\hline \multicolumn{4}{|c|}{ Implications/ Applications: } \\
\hline Questions & Description & Score & Comments \\
\hline \multirow[t]{4}{*}{$\begin{array}{l}\text { How important are these } \\
\text { findings to policy/ } \\
\text { practice? } \\
\text { Does the research offer } \\
\text { a valuable contribution } \\
\text { to the literature? }\end{array}$} & $\begin{array}{l}\text { Good }=3=\text { Contributes } \\
\text { something new/ different in } \\
\text { terms of insight or } \\
\text { perspective. Suggests ideas } \\
\text { for future research. Suggests } \\
\text { implication for policy and/ or } \\
\text { practice. }\end{array}$ & \multirow[t]{4}{*}{2} & \multirow[t]{4}{*}{$\begin{array}{l}\text { The study suggests ideas } \\
\text { for future research and } \\
\text { implications for } \\
\text { practice. }\end{array}$} \\
\hline & $\begin{array}{l}\text { Fair }=2=\text { Two of the above } \\
\text { (State in comments). }\end{array}$ & & \\
\hline & $\begin{array}{l}\text { Poor }=1=\text { Only one of the } \\
\text { above (State in comments). }\end{array}$ & & \\
\hline & $\begin{array}{l}\text { Very Poor }=0=\text { None of the } \\
\text { above }\end{array}$ & & \\
\hline \multicolumn{2}{|l|}{ Total Score: } & $\begin{array}{l}19 / 27 \\
70 \%\end{array}$ & \\
\hline
\end{tabular}




\begin{tabular}{|c|c|c|c|}
\hline Study 13 - Title: & \multicolumn{3}{|c|}{$\begin{array}{l}\text { Females' reasons for their physical aggression in dating } \\
\text { relationships. }\end{array}$} \\
\hline Author (Year): & \multicolumn{3}{|c|}{ Hettrich, E. L., \& O'Leary, K. D. (2007) } \\
\hline \multicolumn{4}{|l|}{ Sampling: } \\
\hline Questions & Description & Score & Comments \\
\hline \multirow[t]{4}{*}{$\begin{array}{l}\text { Was the sampling } \\
\text { strategy appropriate to } \\
\text { address the research } \\
\text { question/s? } \\
\text { Why was this group } \\
\text { targeted? } \\
\text { Were the participants } \\
\text { representative of the } \\
\text { target population? }\end{array}$} & $\begin{array}{l}\text { Good }=3=\text { Details (age/ } \\
\text { gender/ ethnicity /context) of } \\
\text { who was studied and how } \\
\text { they were recruited. } \\
\text { Rationale for studying this } \\
\text { population. Response rates } \\
\text { shown and explained (why } \\
\text { certain individuals chose not } \\
\text { to participate, and any } \\
\text { attempts to achieve a sample } \\
\text { that represents the target } \\
\text { population). }\end{array}$ & \multirow[t]{4}{*}{3} & \multirow[t]{4}{*}{$\begin{array}{l}\text { Details of sample and } \\
\text { rationale, and response } \\
\text { rate provided. Sample } \\
\text { was ethnically diverse, } \\
\text { perhaps more so than } \\
\text { would be expected of } \\
\text { the student population. }\end{array}$} \\
\hline & $\begin{array}{l}\text { Fair }=2=\text { Most information } \\
\text { given, but some missing. }\end{array}$ & & \\
\hline & $\begin{array}{l}\text { Poor }=1=\text { Sampling } \\
\text { mentioned but few } \\
\text { descriptive details. }\end{array}$ & & \\
\hline & $\begin{array}{l}\text { Very Poor }=0=\text { No details } \\
\text { of sample. }\end{array}$ & & \\
\hline \multirow{3}{*}{$\begin{array}{l}\text { Reporting of power or a } \\
\text { priori power analysis } \\
\text { conducted? } \\
\text { Does the study have } \geq \\
80 \% \text { power to detect an } \\
\text { effect at } r=.20 \text { ? }\end{array}$} & $\begin{array}{l}\text { Good }=3=\text { Two of the above } \\
\text { (State in comments). }\end{array}$ & \multirow[t]{3}{*}{ N/A } & \multirow{3}{*}{$\begin{array}{l}\text { No reporting of power } \\
\text { or a priori power } \\
\text { analysis conducted. } \\
\text { A power analysis was } \\
\text { unable to be calculated } \\
\text { as the study is } \\
\text { descriptive. } \\
\text { Consequently, this } \\
\text { section is not counted in } \\
\text { the total score. }\end{array}$} \\
\hline & $\begin{array}{l}\text { Poor }=1.5=\text { Only one of the } \\
\text { above (State in comments). }\end{array}$ & & \\
\hline & $\begin{array}{l}\text { Very Poor }=0=\text { None of the } \\
\text { above }\end{array}$ & & \\
\hline \multicolumn{4}{|l|}{ Method: } \\
\hline Questions & Description & Score & Comments \\
\hline \multirow{4}{*}{$\begin{array}{l}\text { Has IPA been clearly } \\
\text { defined? } \\
\text { Have motivations been } \\
\text { clearly defined? }\end{array}$} & $\begin{array}{l}\text { Good = } 3=\text { Clear description } \\
\text { of both IPA and motivations. }\end{array}$ & \multirow[t]{4}{*}{0} & \multirow[t]{4}{*}{$\begin{array}{l}\text { IPA was discussed but } \\
\text { not defined. }\end{array}$} \\
\hline & $\begin{array}{l}\text { Fair }=2=\text { Clear description } \\
\text { of one variable, but } \\
\text { inadequate description of the } \\
\text { other. }\end{array}$ & & \\
\hline & $\begin{array}{l}\text { Poor }=1=\text { Inadequate } \\
\text { descriptions of both } \\
\text { variables. }\end{array}$ & & \\
\hline & $\begin{array}{l}\text { Very Poor }=0=\text { No } \\
\text { description of either variable. }\end{array}$ & & \\
\hline
\end{tabular}




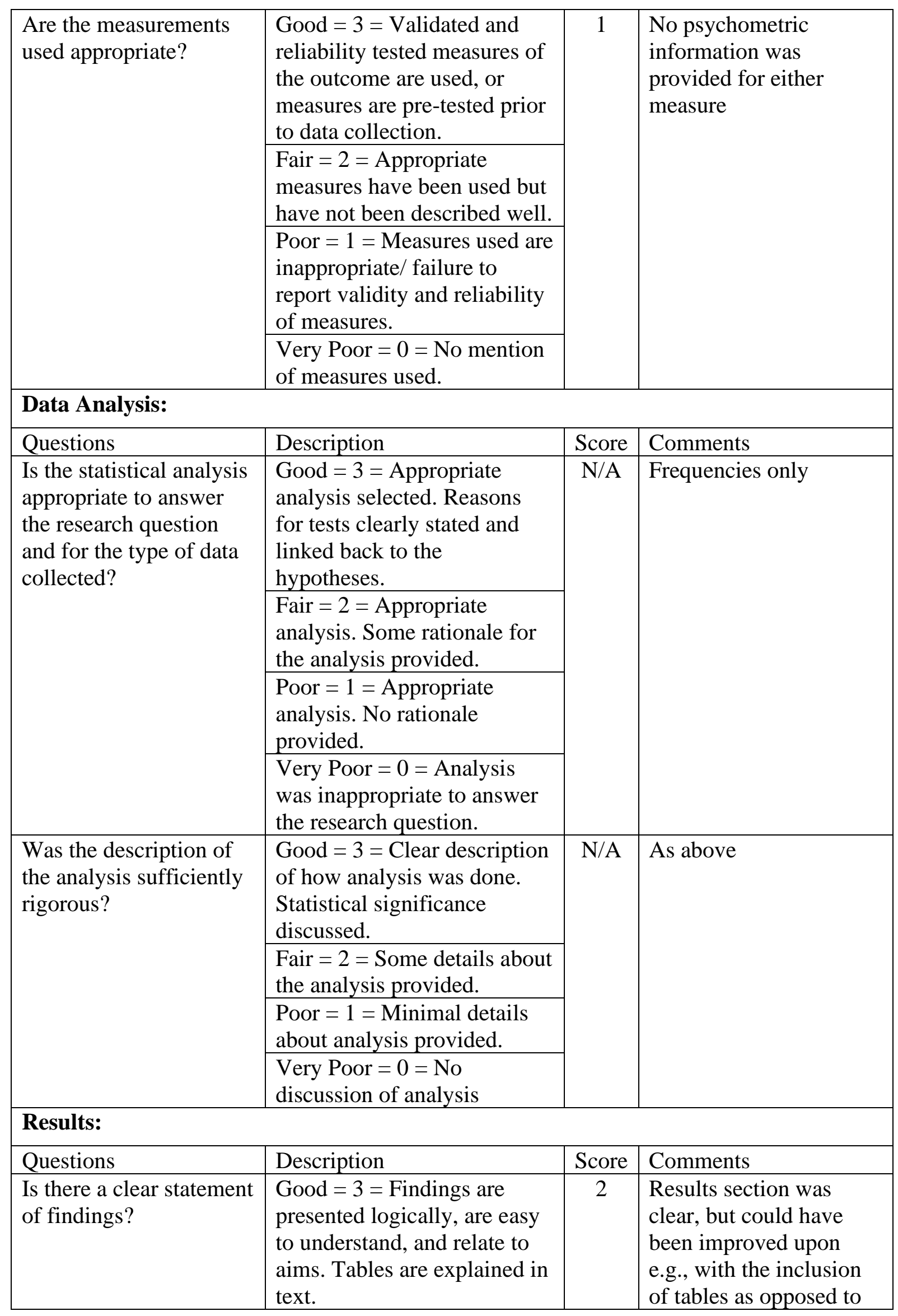




\begin{tabular}{|c|c|c|c|}
\hline & $\begin{array}{l}\text { Fair }=2=\text { Findings } \\
\text { mentioned, but more } \\
\text { explanation could be given. }\end{array}$ & & \multirow{3}{*}{$\begin{array}{l}\text { reporting all the results } \\
\text { in text. Findings were } \\
\text { not linked back to the } \\
\text { hypotheses of aims of } \\
\text { the study. }\end{array}$} \\
\hline & $\begin{array}{l}\text { Poor }=1=\text { Findings are } \\
\text { presented haphazardly, not } \\
\text { explained, or do not relate to } \\
\text { aims. }\end{array}$ & & \\
\hline & $\begin{array}{l}\text { Very Poor }=0=\text { Not } \\
\text { mentioned or do not relate to } \\
\text { aims. }\end{array}$ & & \\
\hline \multirow[t]{2}{*}{ Limitations discussed? } & Yes $=3$ & \multirow[t]{2}{*}{0} & \\
\hline & No $=0$ & & \\
\hline \multicolumn{4}{|c|}{ Implications/ Applications: } \\
\hline Questions & Description & Score & Comments \\
\hline \multirow[t]{4}{*}{$\begin{array}{l}\text { How important are these } \\
\text { findings to policy/ } \\
\text { practice? } \\
\text { Does the research offer } \\
\text { a valuable contribution } \\
\text { to the literature? }\end{array}$} & $\begin{array}{l}\text { Good }=3=\text { Contributes } \\
\text { something new/ different in } \\
\text { terms of insight or } \\
\text { perspective. Suggests ideas } \\
\text { for future research. Suggests } \\
\text { implication for policy and/ or } \\
\text { practice. }\end{array}$ & \multirow[t]{4}{*}{1} & \multirow[t]{4}{*}{ Future research only } \\
\hline & $\begin{array}{l}\text { Fair }=2=\text { Two of the above } \\
\text { (State in comments). }\end{array}$ & & \\
\hline & $\begin{array}{l}\text { Poor }=1=\text { Only one of the } \\
\text { above (State in comments). }\end{array}$ & & \\
\hline & $\begin{array}{l}\text { Very Poor }=0=\text { None of the } \\
\text { above }\end{array}$ & & \\
\hline \multicolumn{2}{|l|}{ Total Score: } & $\begin{array}{l}7 / 18 \\
39 \%\end{array}$ & \\
\hline
\end{tabular}

\begin{tabular}{|c|c|c|c|}
\hline Study 14 - Title: & \multicolumn{3}{|c|}{$\begin{array}{l}\text { Motives for physical dating violence among college students: A } \\
\text { gendered analysis }\end{array}$} \\
\hline Author (Year): & \multicolumn{3}{|c|}{$\begin{array}{l}\text { Kelley, E. L., Edwards, K. M., Dardis, C. M., \& Gidycz, C. A. } \\
(2015)\end{array}$} \\
\hline \multicolumn{4}{|l|}{ Sampling: } \\
\hline Questions & Description & Score & Comments \\
\hline $\begin{array}{l}\text { Was the sampling } \\
\text { strategy appropriate to } \\
\text { address the research } \\
\text { question/s? } \\
\text { Why was this group } \\
\text { targeted? } \\
\text { Were the participants } \\
\text { representative of the } \\
\text { target population? }\end{array}$ & $\begin{array}{l}\text { Good }=3=\text { Details (age/ } \\
\text { gender/ ethnicity /context) of } \\
\text { who was studied and how } \\
\text { they were recruited. } \\
\text { Rationale for studying this } \\
\text { population. Response rates } \\
\text { shown and explained (why } \\
\text { certain individuals chose not } \\
\text { to participate, and any } \\
\text { attempts to achieve a sample }\end{array}$ & 2 & $\begin{array}{l}\text { Details and rationale } \\
\text { provided. More } \\
\text { information on } \\
\text { recruitment would have } \\
\text { been beneficial. No } \\
\text { mention of response } \\
\text { rate of attempts to } \\
\text { achieve a representative } \\
\text { sample. }\end{array}$ \\
\hline
\end{tabular}




\begin{tabular}{|c|c|c|c|}
\hline & $\begin{array}{l}\text { that represents the target } \\
\text { population). }\end{array}$ & & \\
\hline & $\begin{array}{l}\text { Fair }=2=\text { Most information } \\
\text { given, but some missing. }\end{array}$ & & \\
\hline & $\begin{array}{l}\text { Poor }=1=\text { Sampling } \\
\text { mentioned but few } \\
\text { descriptive details. }\end{array}$ & & \\
\hline & $\begin{array}{l}\text { Very Poor }=0=\text { No details } \\
\text { of sample. }\end{array}$ & & \\
\hline \multirow{3}{*}{$\begin{array}{l}\text { Reporting of power or a } \\
\text { priori power analysis } \\
\text { conducted? } \\
\text { Does the study have } \geq \\
80 \% \text { power to detect an } \\
\text { effect at } r=.20 \text { ? }\end{array}$} & $\begin{array}{l}\text { Good }=3=\text { Two of the } \\
\text { above (State in comments). }\end{array}$ & \multirow[t]{3}{*}{1.5} & \multirow{3}{*}{$\begin{array}{l}\text { No reporting of power } \\
\text { or a priori power } \\
\text { analysis conducted. } \\
\text { The study has } \geq 80 \% \\
\text { power to detect an } \\
\text { effect at } r=.20\end{array}$} \\
\hline & $\begin{array}{l}\text { Poor }=1.5=\text { Only one of the } \\
\text { above (State in comments). }\end{array}$ & & \\
\hline & $\begin{array}{l}\text { Very Poor }=0=\text { None of the } \\
\text { above }\end{array}$ & & \\
\hline \multicolumn{4}{|l|}{ Method: } \\
\hline Questions & Description & Score & Comments \\
\hline \multirow{4}{*}{$\begin{array}{l}\text { Has IPA been clearly } \\
\text { defined? } \\
\text { Have motivations been } \\
\text { clearly defined? }\end{array}$} & $\begin{array}{l}\text { Good }=3=\text { Clear } \\
\text { description of both IPA and } \\
\text { motivations. }\end{array}$ & \multirow[t]{4}{*}{1.5} & \multirow[t]{4}{*}{$\begin{array}{l}\text { Good description of } \\
\text { motivation. No } \\
\text { description of IPA. }\end{array}$} \\
\hline & $\begin{array}{l}\text { Fair }=2=\text { Clear description } \\
\text { of one variable, but } \\
\text { inadequate description of the } \\
\text { other. }\end{array}$ & & \\
\hline & $\begin{array}{l}\text { Poor }=1=\text { Inadequate } \\
\text { descriptions of both } \\
\text { variables. }\end{array}$ & & \\
\hline & $\begin{array}{l}\text { Very Poor }=0=\text { No } \\
\text { description of either } \\
\text { variable. }\end{array}$ & & \\
\hline \multirow[t]{4}{*}{$\begin{array}{l}\text { Are the measurements } \\
\text { used appropriate? }\end{array}$} & $\begin{array}{l}\text { Good }=3=\text { Validated and } \\
\text { reliability tested measures of } \\
\text { the outcome are used, or } \\
\text { measures are pre-tested prior } \\
\text { to data collection. }\end{array}$ & \multirow[t]{4}{*}{3} & \multirow{4}{*}{$\begin{array}{l}\text { Measures were } \\
\text { appropriate. The } \\
\text { measure for assessing } \\
\text { motive had not been } \\
\text { used with a student } \\
\text { sample, so an EFA was } \\
\text { performed. }\end{array}$} \\
\hline & $\begin{array}{l}\text { Fair }=2=\text { Appropriate } \\
\text { measures have been used but } \\
\text { have not been described } \\
\text { well. }\end{array}$ & & \\
\hline & $\begin{array}{l}\text { Poor }=1=\text { Measures used } \\
\text { are inappropriate/ failure to } \\
\text { report validity and reliability } \\
\text { of measures. }\end{array}$ & & \\
\hline & $\begin{array}{l}\text { Very Poor }=0=\text { No mention } \\
\text { of measures used. }\end{array}$ & & \\
\hline
\end{tabular}




\begin{tabular}{|c|c|c|c|}
\hline Questions & Description & Score & Comments \\
\hline \multirow[t]{4}{*}{$\begin{array}{l}\text { Is the statistical analysis } \\
\text { appropriate to answer } \\
\text { the research question } \\
\text { and for the type of data } \\
\text { collected? }\end{array}$} & $\begin{array}{l}\text { Good = } 3=\text { Appropriate } \\
\text { analysis selected. Reasons } \\
\text { for tests clearly stated and } \\
\text { linked back to the } \\
\text { hypotheses. }\end{array}$ & \multirow[t]{4}{*}{2} & \multirow[t]{4}{*}{$\begin{array}{l}\text { Appropriate analyses } \\
\text { conducted and rationale } \\
\text { for these provided. }\end{array}$} \\
\hline & $\begin{array}{l}\text { Fair }=2=\text { Appropriate } \\
\text { analysis. Some rationale for } \\
\text { the analysis provided. }\end{array}$ & & \\
\hline & $\begin{array}{l}\text { Poor }=1=\text { Appropriate } \\
\text { analysis. No rationale } \\
\text { provided. }\end{array}$ & & \\
\hline & $\begin{array}{l}\text { Very Poor }=0=\text { Analysis } \\
\text { was inappropriate to answer } \\
\text { the research question. }\end{array}$ & & \\
\hline \multirow[t]{4}{*}{$\begin{array}{l}\text { Was the description of } \\
\text { the analysis sufficiently } \\
\text { rigorous? }\end{array}$} & $\begin{array}{l}\text { Good }=3=\text { Clear } \\
\text { description of how analysis } \\
\text { was done. Statistical } \\
\text { significance discussed. }\end{array}$ & \multirow[t]{4}{*}{3} & \multirow[t]{4}{*}{$\begin{array}{l}\text { Very thorough } \\
\text { description of analysis } \\
\text { and treatment of data }\end{array}$} \\
\hline & $\begin{array}{l}\text { Fair }=2=\text { Some details } \\
\text { about the analysis provided. }\end{array}$ & & \\
\hline & $\begin{array}{l}\text { Poor }=1=\text { Minimal details } \\
\text { about analysis provided. }\end{array}$ & & \\
\hline & $\begin{array}{l}\text { Very Poor }=0=\text { No } \\
\text { discussion of analysis }\end{array}$ & & \\
\hline \multicolumn{4}{|l|}{ Results: } \\
\hline Questions & Description & Score & Comments \\
\hline \multirow[t]{4}{*}{$\begin{array}{l}\text { Is there a clear } \\
\text { statement of findings? }\end{array}$} & $\begin{array}{l}\text { Good }=3=\text { Findings are } \\
\text { presented logically, are easy } \\
\text { to understand, and relate to } \\
\text { aims. Tables are explained } \\
\text { in text. }\end{array}$ & \multirow[t]{4}{*}{3} & \multirow[t]{4}{*}{$\begin{array}{l}\text { Findings are clear and } \\
\text { logical and explicitly } \\
\text { related back to the aim } \\
\text { of the study. Tables are } \\
\text { explained in text. }\end{array}$} \\
\hline & $\begin{array}{l}\text { Fair }=2=\text { Findings } \\
\text { mentioned, but more } \\
\text { explanation could be given. }\end{array}$ & & \\
\hline & $\begin{array}{l}\text { Poor }=1=\text { Findings are } \\
\text { presented haphazardly, not } \\
\text { explained, or do not relate to } \\
\text { aims. }\end{array}$ & & \\
\hline & $\begin{array}{l}\text { Very Poor }=0=\text { Not } \\
\text { mentioned or do not relate to } \\
\text { aims. }\end{array}$ & & \\
\hline \multirow[t]{2}{*}{ Limitations discussed? } & Yes $=3$ & \multirow[t]{2}{*}{3} & \multirow{2}{*}{$\begin{array}{l}\text { These included: } \\
\text { Retrospective, cross- } \\
\text { sectional, did not relate } \\
\text { to specific incidents, not } \\
\text { able to select multiple } \\
\text { motives, self-defense } \\
\text { items do not reflect the }\end{array}$} \\
\hline & No $=0$ & & \\
\hline
\end{tabular}




\begin{tabular}{|c|c|c|c|}
\hline & & & $\begin{array}{l}\text { legal definition, lack of } \\
\text { diversity in sample. }\end{array}$ \\
\hline \multicolumn{4}{|c|}{ Implications/ Applications: } \\
\hline Questions & Description & Score & Comments \\
\hline \multirow[t]{4}{*}{$\begin{array}{l}\text { How important are } \\
\text { these findings to policy/ } \\
\text { practice? } \\
\text { Does the research offer } \\
\text { a valuable contribution } \\
\text { to the literature? }\end{array}$} & $\begin{array}{l}\text { Good = } 3=\text { Contributes } \\
\text { something new/ different in } \\
\text { terms of insight or } \\
\text { perspective. Suggests ideas } \\
\text { for future research. Suggests } \\
\text { implication for policy and/ } \\
\text { or practice. }\end{array}$ & \multirow[t]{4}{*}{3} & \multirow[t]{4}{*}{$\begin{array}{l}\text { Theoretically based. } \\
\text { Suggests ideas for } \\
\text { future research and } \\
\text { includes clinical and } \\
\text { policy implications. }\end{array}$} \\
\hline & $\begin{array}{l}\text { Fair }=2=\text { Two of the above } \\
\text { (State in comments). }\end{array}$ & & \\
\hline & $\begin{array}{l}\text { Poor }=1=\text { Only one of the } \\
\text { above (State in comments). }\end{array}$ & & \\
\hline & $\begin{array}{l}\text { Very Poor }=0=\text { None of the } \\
\text { above }\end{array}$ & & \\
\hline \multicolumn{2}{|l|}{ Total Score: } & $\begin{array}{l}22 / 27 \\
81 \%\end{array}$ & \\
\hline
\end{tabular}

\begin{tabular}{|c|c|c|c|}
\hline Study 15 - Title: & \multicolumn{3}{|c|}{$\begin{array}{l}\text { Exerting power or striking back: A gendered comparison of } \\
\text { motivations for domestic violence perpetration }\end{array}$} \\
\hline Author (Year): & \multicolumn{3}{|l|}{ Kernsmith, P. (2005) } \\
\hline \multicolumn{4}{|l|}{ Sampling: } \\
\hline Questions & Description & Score & Comments \\
\hline \multirow[t]{4}{*}{$\begin{array}{l}\text { Was the sampling } \\
\text { strategy appropriate to } \\
\text { address the research } \\
\text { question/s? } \\
\text { Why was this group } \\
\text { targeted? } \\
\text { Were the participants } \\
\text { representative of the } \\
\text { target population? }\end{array}$} & $\begin{array}{l}\text { Good }=3=\text { Details (age/ } \\
\text { gender/ ethnicity /context) of } \\
\text { who was studied and how } \\
\text { they were recruited. } \\
\text { Rationale for studying this } \\
\text { population. Response rates } \\
\text { shown and explained (why } \\
\text { certain individuals chose not } \\
\text { to participate, and any } \\
\text { attempts to achieve a sample } \\
\text { that represents the target } \\
\text { population). }\end{array}$ & \multirow[t]{4}{*}{2} & \multirow[t]{4}{*}{$\begin{array}{l}\text { Details of sample } \\
\text { provided. Attempt to } \\
\text { ensure the sample was } \\
\text { demographically similar, } \\
\text { in terms of race and } \\
\text { class, to L.A county. } \\
\text { Response rate and } \\
\text { rationale for studying } \\
\text { this population not } \\
\text { provided. }\end{array}$} \\
\hline & $\begin{array}{l}\text { Fair }=2=\text { Most information } \\
\text { given, but some missing. }\end{array}$ & & \\
\hline & $\begin{array}{l}\text { Poor }=1 \text { = Sampling } \\
\text { mentioned but few } \\
\text { descriptive details. }\end{array}$ & & \\
\hline & $\begin{array}{l}\text { Very Poor }=0=\text { No details } \\
\text { of sample. }\end{array}$ & & \\
\hline
\end{tabular}




\begin{tabular}{|c|c|c|c|}
\hline \multirow{3}{*}{$\begin{array}{l}\text { Reporting of power or a } \\
\text { priori power analysis } \\
\text { conducted? } \\
\text { Does the study have } \geq \\
80 \% \text { power to detect an } \\
\text { effect at } r=.20 ?\end{array}$} & $\begin{array}{l}\text { Good }=3=\text { Two of the } \\
\text { above (State in comments). }\end{array}$ & \multirow[t]{3}{*}{0} & \multirow{3}{*}{$\begin{array}{l}\text { No reporting of power or } \\
\text { a priori power analysis } \\
\text { conducted. } \\
\text { The study has }<80 \% \\
\text { power to detect an effect } \\
\text { at } r=.20 ?\end{array}$} \\
\hline & $\begin{array}{l}\text { Poor }=1.5=\text { Only one of the } \\
\text { above (State in comments). }\end{array}$ & & \\
\hline & $\begin{array}{l}\text { Very Poor }=0=\text { None of the } \\
\text { above }\end{array}$ & & \\
\hline \multicolumn{4}{|l|}{ Method: } \\
\hline Questions & Description & Score & Comments \\
\hline \multirow{4}{*}{$\begin{array}{l}\text { Has IPA been clearly } \\
\text { defined? } \\
\text { Have motivations been } \\
\text { clearly defined? }\end{array}$} & $\begin{array}{l}\text { Good }=3=\text { Clear description } \\
\text { of both IPA and motivations. }\end{array}$ & \multirow[t]{4}{*}{0} & \multirow[t]{4}{*}{$\begin{array}{l}\text { Neither IPA nor } \\
\text { motivations defined. }\end{array}$} \\
\hline & $\begin{array}{l}\text { Fair }=2=\text { Clear description } \\
\text { of one variable, but } \\
\text { inadequate description of the } \\
\text { other. }\end{array}$ & & \\
\hline & $\begin{array}{l}\text { Poor }=1=\text { Inadequate } \\
\text { descriptions of both } \\
\text { variables. }\end{array}$ & & \\
\hline & $\begin{array}{l}\text { Very Poor }=0=\text { No } \\
\text { description of either variable. }\end{array}$ & & \\
\hline \multirow[t]{4}{*}{$\begin{array}{l}\text { Are the measurements } \\
\text { used appropriate? }\end{array}$} & $\begin{array}{l}\text { Good }=3=\text { Validated and } \\
\text { reliability tested measures of } \\
\text { the outcome are used, or } \\
\text { measures are pre-tested prior } \\
\text { to data collection. }\end{array}$ & \multirow[t]{4}{*}{1} & \multirow[t]{4}{*}{$\begin{array}{l}\text { Very little information } \\
\text { provided regarding the } \\
\text { measures used, including } \\
\text { a lack of psychometric } \\
\text { information. }\end{array}$} \\
\hline & $\begin{array}{l}\text { Fair }=2=\text { Appropriate } \\
\text { measures have been used but } \\
\text { have not been described } \\
\text { well. }\end{array}$ & & \\
\hline & $\begin{array}{l}\text { Poor }=1=\text { Measures used } \\
\text { are inappropriate/ failure to } \\
\text { report validity and reliability } \\
\text { of measures. }\end{array}$ & & \\
\hline & $\begin{array}{l}\text { Very Poor }=0=\text { No mention } \\
\text { of measures used. }\end{array}$ & & \\
\hline \multicolumn{4}{|l|}{ Data Analysis: } \\
\hline Questions & Description & Score & Comments \\
\hline \multirow[t]{2}{*}{$\begin{array}{l}\text { Is the statistical analysis } \\
\text { appropriate to answer } \\
\text { the research question } \\
\text { and for the type of data } \\
\text { collected? }\end{array}$} & $\begin{array}{l}\text { Good }=3=\text { Appropriate } \\
\text { analysis selected. Reasons } \\
\text { for tests clearly stated and } \\
\text { linked back to the } \\
\text { hypotheses. }\end{array}$ & \multirow[t]{2}{*}{.5} & \multirow{2}{*}{$\begin{array}{l}\text { An EFA was conducted, } \\
\text { but there was no } \\
\text { rationale for why this } \\
\text { was done. The t-tests } \\
\text { were appropriate, but } \\
\text { reasons for their use } \\
\text { were not stated. }\end{array}$} \\
\hline & $\begin{array}{l}\text { Fair }=2=\text { Appropriate } \\
\text { analysis. Some rationale for } \\
\text { the analysis provided. }\end{array}$ & & \\
\hline
\end{tabular}




\begin{tabular}{|c|c|c|c|}
\hline & $\begin{array}{l}\text { Poor }=1=\text { Appropriate } \\
\text { analysis. No rationale } \\
\text { provided. }\end{array}$ & & \\
\hline & $\begin{array}{l}\text { Very Poor }=0=\text { Analysis } \\
\text { was inappropriate to answer } \\
\text { the research question. }\end{array}$ & & \\
\hline \multirow[t]{4}{*}{$\begin{array}{l}\text { Was the description of } \\
\text { the analysis sufficiently } \\
\text { rigorous? }\end{array}$} & $\begin{array}{l}\text { Good }=3=\text { Clear description } \\
\text { of how analysis was done. } \\
\text { Statistical significance } \\
\text { discussed. }\end{array}$ & \multirow[t]{4}{*}{0} & \multirow[t]{4}{*}{$\begin{array}{l}\text { No discussion of } \\
\text { analysis }\end{array}$} \\
\hline & $\begin{array}{l}\text { Fair }=2=\text { Some details } \\
\text { about the analysis provided. }\end{array}$ & & \\
\hline & $\begin{array}{l}\text { Poor }=1=\text { Minimal details } \\
\text { about analysis provided. }\end{array}$ & & \\
\hline & $\begin{array}{l}\text { Very Poor }=0=\text { No } \\
\text { discussion of analysis }\end{array}$ & & \\
\hline \multicolumn{4}{|l|}{ Results: } \\
\hline Questions & Description & Score & Comments \\
\hline \multirow[t]{4}{*}{$\begin{array}{l}\text { Is there a clear } \\
\text { statement of findings? }\end{array}$} & $\begin{array}{l}\text { Good }=3=\text { Findings are } \\
\text { presented logically, are easy } \\
\text { to understand, and relate to } \\
\text { aims. Tables are explained in } \\
\text { text. }\end{array}$ & \multirow[t]{4}{*}{1} & \multirow{4}{*}{$\begin{array}{l}\text { Insufficient results e.g., } \\
\text { the aim of the study was } \\
\text { to compare male's and } \\
\text { female's motivations for } \\
\text { IPA and to investigate } \\
\text { whether female IPA was } \\
\text { motivated by power and } \\
\text { control. However, the } \\
\text { only results presented } \\
\text { are the three "factor" } \\
\text { means and the t-test } \\
\text { results in text. }\end{array}$} \\
\hline & $\begin{array}{l}\text { Fair }=2=\text { Findings } \\
\text { mentioned, but more } \\
\text { explanation could be given. }\end{array}$ & & \\
\hline & $\begin{array}{l}\text { Poor }=1=\text { Findings are } \\
\text { presented haphazardly, not } \\
\text { explained, or do not relate to } \\
\text { aims. }\end{array}$ & & \\
\hline & $\begin{array}{l}\text { Very Poor }=0=\text { Not } \\
\text { mentioned or do not relate to } \\
\text { aims. }\end{array}$ & & \\
\hline \multirow[t]{2}{*}{ Limitations discussed? } & Yes $=3$ & \multirow[t]{2}{*}{3} & \multirow{2}{*}{$\begin{array}{l}\text { These include: Influence } \\
\text { of treatment on attitudes/ } \\
\text { behaviour, } \\
\text { generalisability of } \\
\text { sample, self-report }\end{array}$} \\
\hline & $\mathrm{No}=0$ & & \\
\hline \multicolumn{4}{|c|}{ Implications/ Applications: } \\
\hline Questions & Description & Score & Comments \\
\hline \multirow[t]{2}{*}{$\begin{array}{l}\text { How important are these } \\
\text { findings to policy/ } \\
\text { practice? } \\
\text { Does the research offer } \\
\text { a valuable contribution } \\
\text { to the literature? }\end{array}$} & $\begin{array}{l}\text { Good }=3=\text { Contributes } \\
\text { something new/ different in } \\
\text { terms of insight or } \\
\text { perspective. Suggests ideas } \\
\text { for future research. Suggests } \\
\text { implication for policy and/ or } \\
\text { practice. }\end{array}$ & \multirow[t]{2}{*}{1.5} & \multirow[t]{2}{*}{$\begin{array}{l}\text { Suggests implication for } \\
\text { policy and practice and } \\
\text { some future research. }\end{array}$} \\
\hline & $\begin{array}{l}\text { Fair }=2=\text { Two of the above } \\
\text { (State in comments). }\end{array}$ & & \\
\hline
\end{tabular}




\begin{tabular}{|l|l|l|l|}
\hline & $\begin{array}{l}\text { Poor =1 = Only one of the } \\
\text { above (State in comments). }\end{array}$ & \multirow{3}{*}{$\begin{array}{l}\text { Very Poor = 0 None of the } \\
\text { above }\end{array}$} & \\
\hline Total Score: & $9 / 27$ & $33 \%$ & \\
\hline
\end{tabular}

\begin{tabular}{|c|c|c|c|}
\hline Study 16 - Title: & \multicolumn{3}{|c|}{$\begin{array}{l}\text { Physical and emotional abuse in romantic relationships: } \\
\text { Motivation for perpetration among college women }\end{array}$} \\
\hline Author (Year): & \multicolumn{3}{|l|}{ Leisring, P. A. (2013) } \\
\hline \multicolumn{4}{|l|}{ Sampling: } \\
\hline Questions & Description & Score & Comments \\
\hline \multirow[t]{4}{*}{$\begin{array}{l}\text { Was the sampling } \\
\text { strategy appropriate to } \\
\text { address the research } \\
\text { question/s? } \\
\text { Why was this group } \\
\text { targeted? } \\
\text { Were the participants } \\
\text { representative of the } \\
\text { target population? }\end{array}$} & $\begin{array}{l}\text { Good = } 3=\text { Details (age/ } \\
\text { gender/ ethnicity /context) } \\
\text { of who was studied and how } \\
\text { they were recruited. } \\
\text { Rationale for studying this } \\
\text { population. Response rates } \\
\text { shown and explained (why } \\
\text { certain individuals chose } \\
\text { not to participate, and any } \\
\text { attempts to achieve a } \\
\text { sample that represents the } \\
\text { target population). }\end{array}$ & \multirow[t]{4}{*}{2.5} & \multirow[t]{4}{*}{$\begin{array}{l}\text { Details of sample } \\
\text { provided. Rational for } \\
\text { studying this population } \\
\text { explained. No discussion } \\
\text { of representativeness of } \\
\text { sample. }\end{array}$} \\
\hline & $\begin{array}{l}\text { Fair }=2=\text { Most information } \\
\text { given, but some missing. }\end{array}$ & & \\
\hline & $\begin{array}{l}\text { Poor }=1=\text { Sampling } \\
\text { mentioned but few } \\
\text { descriptive details. }\end{array}$ & & \\
\hline & $\begin{array}{l}\text { Very Poor }=0=\text { No details } \\
\text { of sample. }\end{array}$ & & \\
\hline \multirow{3}{*}{$\begin{array}{l}\text { Reporting of power or a } \\
\text { priori power analysis } \\
\text { conducted? } \\
\text { Does the study have } \geq \\
80 \% \text { power to detect an } \\
\text { effect at } r=.20 \text { ? }\end{array}$} & $\begin{array}{l}\text { Good }=3=\text { Two of the } \\
\text { above (State in comments). }\end{array}$ & \multirow[t]{3}{*}{0} & \multirow{3}{*}{$\begin{array}{l}\text { No reporting of power } \\
\text { or a priori power } \\
\text { analysis conducted. } \\
\text { The study has }<80 \% \\
\text { power to detect an effect } \\
\text { at } r=.20\end{array}$} \\
\hline & $\begin{array}{l}\text { Poor }=1.5=\text { Only one of } \\
\text { the above (State in } \\
\text { comments). }\end{array}$ & & \\
\hline & $\begin{array}{l}\text { Very Poor }=0=\text { None of } \\
\text { the above }\end{array}$ & & \\
\hline \multicolumn{4}{|l|}{ Method: } \\
\hline Questions & Description & Score & Comments \\
\hline $\begin{array}{l}\text { Has IPA been clearly } \\
\text { defined? } \\
\text { Have motivations been } \\
\text { clearly defined? }\end{array}$ & $\begin{array}{l}\text { Good }=3=\text { Clear } \\
\text { description of both IPA and } \\
\text { motivations. }\end{array}$ & 0 & $\begin{array}{l}\text { IPA and motivations } \\
\text { discussed but neither } \\
\text { defined. }\end{array}$ \\
\hline
\end{tabular}




\begin{tabular}{|c|c|c|c|}
\hline & $\begin{array}{l}\text { Fair }=2=\text { Clear description } \\
\text { of one variable, but } \\
\text { inadequate description of } \\
\text { the other. }\end{array}$ & & \\
\hline & $\begin{array}{l}\text { Poor }=1=\text { Inadequate } \\
\text { descriptions of both } \\
\text { variables. }\end{array}$ & & \\
\hline & $\begin{array}{l}\text { Very Poor }=0=\text { No } \\
\text { description of either } \\
\text { variable. }\end{array}$ & & \\
\hline $\begin{array}{l}\text { Are the measurements } \\
\text { used appropriate? }\end{array}$ & $\begin{array}{l}\text { Good }=3=\text { Validated and } \\
\text { reliability tested measures } \\
\text { of the outcome are used, or } \\
\text { measures are pre-tested } \\
\text { prior to data collection. }\end{array}$ & 1 & $\begin{array}{l}\text { The CTS2 is a reliable } \\
\text { and well validated tool. } \\
\text { The alpha reported for } \\
\text { this study was above the } \\
\text { recommended level. }\end{array}$ \\
\hline & $\begin{array}{l}\text { Fair }=2=\text { Appropriate } \\
\text { measures have been used } \\
\text { but have not been described } \\
\text { well. }\end{array}$ & & $\begin{array}{l}\text { The MEQ has been used } \\
\text { in several previous } \\
\text { studies, however, no } \\
\text { psychometric }\end{array}$ \\
\hline & $\begin{array}{l}\text { Poor }=1=\text { Measures used } \\
\text { are inappropriate/ failure to } \\
\text { report validity and } \\
\text { reliability of measures. }\end{array}$ & & $\begin{array}{l}\text { information was } \\
\text { reported. }\end{array}$ \\
\hline & $\begin{array}{l}\text { Very Poor }=0=\text { No } \\
\text { mention of measures used. }\end{array}$ & & \\
\hline Data Analysis: & & & \\
\hline Questions & Description & Score & Comments \\
\hline $\begin{array}{l}\text { Is the statistical } \\
\text { analysis appropriate to } \\
\text { answer the research } \\
\text { question and for the } \\
\text { type of data collected? }\end{array}$ & $\begin{array}{l}\text { Good }=3=\text { Appropriate } \\
\text { analysis selected. Reasons } \\
\text { for tests clearly stated and } \\
\text { linked back to the } \\
\text { hypotheses. }\end{array}$ & 2 & $\begin{array}{l}\text { Mostly frequencies. Chi- } \\
\text { square analysis was } \\
\text { appropriate. Statement } \\
\text { of what this was testing. } \\
\text { No hypotheses to link }\end{array}$ \\
\hline & $\begin{array}{l}\text { Fair }=2=\text { Appropriate } \\
\text { analysis. Some rationale for } \\
\text { the analysis provided. }\end{array}$ & & back to. \\
\hline & $\begin{array}{l}\text { Poor }=1=\text { Appropriate } \\
\text { analysis. No rationale } \\
\text { provided. }\end{array}$ & & \\
\hline & $\begin{array}{l}\text { Very Poor }=0=\text { Analysis } \\
\text { was inappropriate to answer } \\
\text { the research question. }\end{array}$ & & \\
\hline $\begin{array}{l}\text { Was the description of } \\
\text { the analysis sufficiently } \\
\text { rigorous? }\end{array}$ & $\begin{array}{l}\text { Good }=3=\text { Clear } \\
\text { description of how analysis } \\
\text { was done. Statistical } \\
\text { significance discussed. }\end{array}$ & 1 & $\begin{array}{l}\text { No details other than } \\
\text { stating what tests were } \\
\text { performed. Neither the } \\
\text { software used nor }\end{array}$ \\
\hline & $\begin{array}{l}\text { Fair }=2=\text { Some details } \\
\text { about the analysis provided. }\end{array}$ & & $\begin{array}{l}\text { statistical significance } \\
\text { was discussed }\end{array}$ \\
\hline & $\begin{array}{l}\text { Poor }=1=\text { Minimal details } \\
\text { about analysis provided. }\end{array}$ & & \\
\hline
\end{tabular}




\begin{tabular}{|c|c|c|c|}
\hline & $\begin{array}{l}\text { Very Poor }=0=\text { No } \\
\text { discussion of analysis }\end{array}$ & & \\
\hline \multicolumn{4}{|l|}{ Results: } \\
\hline Questions & Description & Score & Comments \\
\hline \multirow[t]{4}{*}{$\begin{array}{l}\text { Is there a clear } \\
\text { statement of findings? }\end{array}$} & $\begin{array}{l}\text { Good }=3=\text { Findings are } \\
\text { presented logically, are easy } \\
\text { to understand, and relate to } \\
\text { aims. Tables are explained } \\
\text { in text. }\end{array}$ & \multirow[t]{4}{*}{3} & \multirow[t]{4}{*}{$\begin{array}{l}\text { The findings are } \\
\text { presented logically, } \\
\text { although there were no } \\
\text { explicit aims in this } \\
\text { study. The tables are } \\
\text { explained in text. }\end{array}$} \\
\hline & $\begin{array}{l}\text { Fair }=2=\text { Findings } \\
\text { mentioned, but more } \\
\text { explanation could be given. }\end{array}$ & & \\
\hline & $\begin{array}{l}\text { Poor }=1=\text { Findings are } \\
\text { presented haphazardly, not } \\
\text { explained, or do not relate } \\
\text { to aims. }\end{array}$ & & \\
\hline & $\begin{array}{l}\text { Very Poor }=0=\text { Not } \\
\text { mentioned or do not relate } \\
\text { to aims. }\end{array}$ & & \\
\hline \multirow[t]{2}{*}{ Limitations discussed? } & Yes $=3$ & \multirow[t]{2}{*}{3} & \multirow{2}{*}{$\begin{array}{l}\text { These include: The } \\
\text { omission of one of the } \\
\text { items from the original } \\
\text { MEQ scale, low alpha } \\
\text { on one of the subscales } \\
\text { of the MMEA (not } \\
\text { relevant to this review). }\end{array}$} \\
\hline & $\mathrm{No}=0$ & & \\
\hline \multicolumn{4}{|c|}{ Implications/ Applications: } \\
\hline Questions & Description & Score & Comments \\
\hline \multirow[t]{4}{*}{$\begin{array}{l}\text { How important are } \\
\text { these findings to policy/ } \\
\text { practice? } \\
\text { Does the research offer } \\
\text { a valuable contribution } \\
\text { to the literature? }\end{array}$} & $\begin{array}{l}\text { Good = } 3=\text { Contributes } \\
\text { something new/ different in } \\
\text { terms of insight or } \\
\text { perspective. Suggests ideas } \\
\text { for future research. } \\
\text { Suggests implication for } \\
\text { policy and/ or practice. }\end{array}$ & \multirow[t]{4}{*}{3} & \multirow[t]{4}{*}{$\begin{array}{l}\text { Suggests areas for future } \\
\text { research and } \\
\text { implications for } \\
\text { treatment. The } \\
\text { measurement of IPA, in } \\
\text { particular self-defense } \\
\text { and psychological } \\
\text { aggression, is novel. }\end{array}$} \\
\hline & $\begin{array}{l}\text { Fair }=2=\text { Two of the above } \\
\text { (State in comments). }\end{array}$ & & \\
\hline & $\begin{array}{l}\text { Poor }=1=\text { Only one of the } \\
\text { above (State in comments). }\end{array}$ & & \\
\hline & $\begin{array}{l}\text { Very Poor }=0=\text { None of } \\
\text { the above }\end{array}$ & & \\
\hline \multicolumn{2}{|l|}{ Total Score: } & $\begin{array}{c}15.5 / 27 \\
57 \%\end{array}$ & \\
\hline
\end{tabular}




\begin{tabular}{|c|c|c|c|}
\hline Study 17 - Title: & \multicolumn{3}{|c|}{ Gender differences in courtship violence victimization } \\
\hline Author (Year): & \multicolumn{3}{|l|}{ Makepeace, J. M. (1986) } \\
\hline \multicolumn{4}{|l|}{ Sampling: } \\
\hline Questions & Description & Score & Comments \\
\hline \multirow[t]{4}{*}{$\begin{array}{l}\text { Was the sampling } \\
\text { strategy appropriate to } \\
\text { address the research } \\
\text { question/s? } \\
\text { Why was this group } \\
\text { targeted? } \\
\text { Were the participants } \\
\text { representative of the } \\
\text { target population? }\end{array}$} & $\begin{array}{l}\text { Good }=3=\text { Details (age/ } \\
\text { gender/ ethnicity /context) of } \\
\text { who was studied and how } \\
\text { they were recruited. } \\
\text { Rationale for studying this } \\
\text { population. Response rates } \\
\text { shown and explained (why } \\
\text { certain individuals chose not } \\
\text { to participate, and any } \\
\text { attempts to achieve a sample } \\
\text { that represents the target } \\
\text { population). }\end{array}$ & \multirow[t]{4}{*}{1.5} & \multirow[t]{4}{*}{$\begin{array}{l}\text { Age, gender, and } \\
\text { context reported. No } \\
\text { details regarding the } \\
\text { ethnic composition of } \\
\text { the sample. No attempts } \\
\text { to achieve a } \\
\text { representative sample } \\
\text { reported. No mention of } \\
\text { response rates. } \\
\text { Rationale for studying } \\
\text { this population } \\
\text { provided. }\end{array}$} \\
\hline & $\begin{array}{l}\text { Fair }=2=\text { Most information } \\
\text { given, but some missing. }\end{array}$ & & \\
\hline & $\begin{array}{l}\text { Poor }=1=\text { Sampling } \\
\text { mentioned but few } \\
\text { descriptive details. }\end{array}$ & & \\
\hline & $\begin{array}{l}\text { Very Poor }=0=\text { No details } \\
\text { of sample. }\end{array}$ & & \\
\hline \multirow{3}{*}{$\begin{array}{l}\text { Reporting of power or a } \\
\text { priori power analysis } \\
\text { conducted? } \\
\text { Does the study have } \geq \\
80 \% \text { power to detect an } \\
\text { effect at } r=.20 \text { ? }\end{array}$} & $\begin{array}{l}\text { Good }=3=\text { Two of the } \\
\text { above (State in comments). }\end{array}$ & \multirow[t]{3}{*}{0} & \multirow{3}{*}{$\begin{array}{l}\text { No reporting of power } \\
\text { or a priori power } \\
\text { analysis conducted. } \\
\text { Due to the type of } \\
\text { analysis conducted this } \\
\text { was not able to be } \\
\text { calculated a priori and } \\
\text { consequently this } \\
\text { section is scored out of } \\
1.5, \text { not } 3 \text {. }\end{array}$} \\
\hline & $\begin{array}{l}\text { Poor }=1.5=\text { Only one of the } \\
\text { above (State in comments). }\end{array}$ & & \\
\hline & $\begin{array}{l}\text { Very Poor }=0=\text { None of the } \\
\text { above }\end{array}$ & & \\
\hline \multicolumn{4}{|l|}{ Method: } \\
\hline Questions & Description & Score & Comments \\
\hline \multirow{4}{*}{$\begin{array}{l}\text { Has IPA been clearly } \\
\text { defined? } \\
\text { Have motivations been } \\
\text { clearly defined? }\end{array}$} & $\begin{array}{l}\text { Good }=3=\text { Clear description } \\
\text { of both IPA and motivations. }\end{array}$ & \multirow[t]{4}{*}{0} & \multirow[t]{4}{*}{$\begin{array}{l}\text { Neither IPA nor } \\
\text { motivations defined }\end{array}$} \\
\hline & $\begin{array}{l}\text { Fair }=2=\text { Clear description } \\
\text { of one variable, but } \\
\text { inadequate description of the } \\
\text { other. }\end{array}$ & & \\
\hline & $\begin{array}{l}\text { Poor }=1=\text { Inadequate } \\
\text { descriptions of both } \\
\text { variables. }\end{array}$ & & \\
\hline & $\begin{array}{l}\text { Very Poor }=0=\text { No } \\
\text { description of either variable. }\end{array}$ & & \\
\hline
\end{tabular}




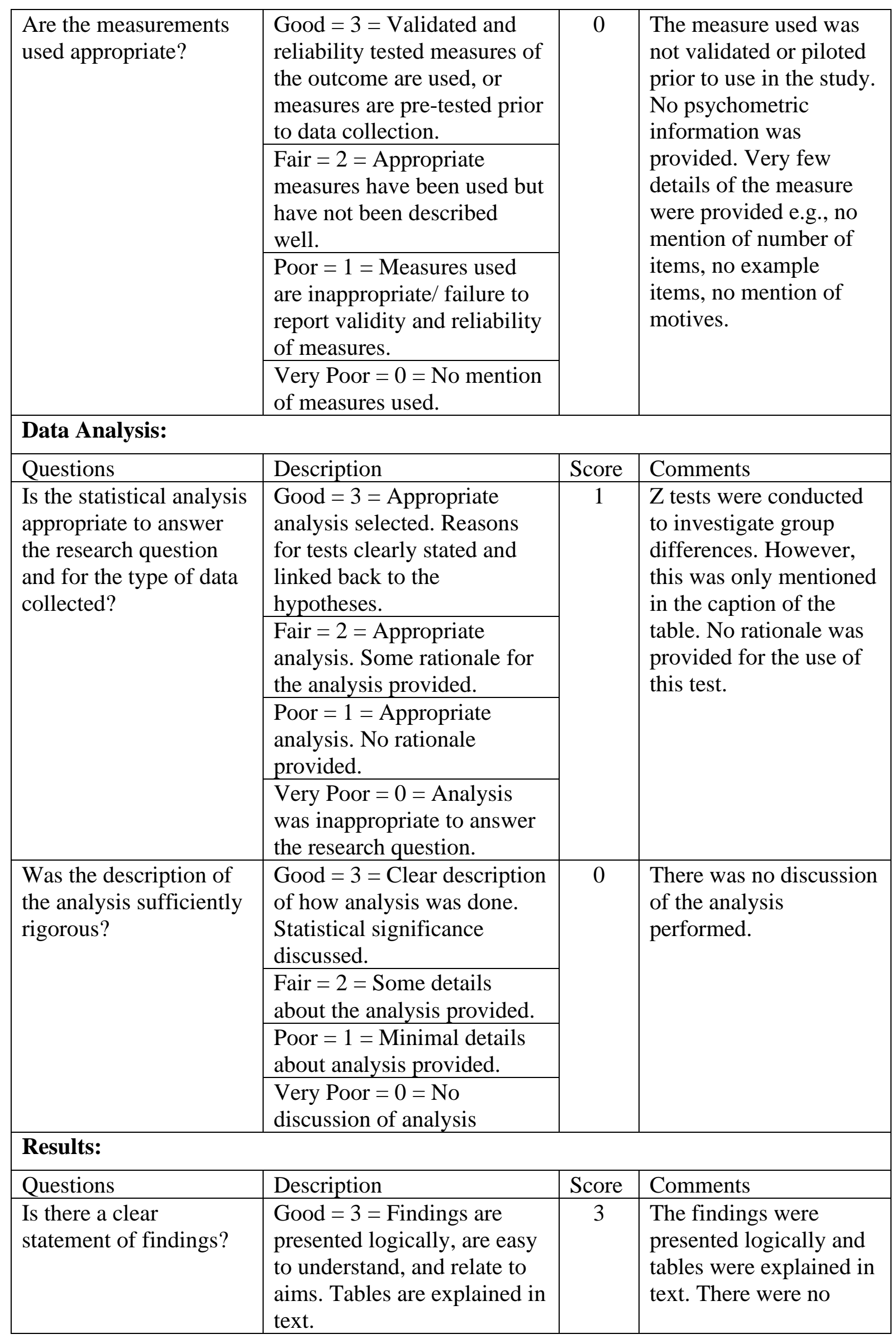




\begin{tabular}{|c|c|c|c|}
\hline & $\begin{array}{l}\text { Fair }=2=\text { Findings } \\
\text { mentioned, but more } \\
\text { explanation could be given. }\end{array}$ & & $\begin{array}{l}\text { aims for the findings to } \\
\text { relate back to. }\end{array}$ \\
\hline & $\begin{array}{l}\text { Poor }=1=\text { Findings are } \\
\text { presented haphazardly, not } \\
\text { explained, or do not relate to } \\
\text { aims. }\end{array}$ & & \\
\hline & $\begin{array}{l}\text { Very Poor }=0=\text { Not } \\
\text { mentioned or do not relate to } \\
\text { aims. }\end{array}$ & & \\
\hline Limitations discussed? & Yes $=3$ & 0 & No discussion of study \\
\hline & $\mathrm{No}=0$ & & limitations \\
\hline Implications/ Applicatio & & & \\
\hline Questions & Description & Score & Comments \\
\hline $\begin{array}{l}\text { How important are these } \\
\text { findings to policy/ } \\
\text { practice? } \\
\text { Does the research offer } \\
\text { a valuable contribution } \\
\text { to the literature? }\end{array}$ & $\begin{array}{l}\text { Good }=3=\text { Contributes } \\
\text { something new/ different in } \\
\text { terms of insight or } \\
\text { perspective. Suggests ideas } \\
\text { for future research. Suggests } \\
\text { implication for policy and/ or } \\
\text { practice. }\end{array}$ & 1.5 & $\begin{array}{l}\text { Some discussion } \\
\text { regarding treatment } \\
\text { implications. A very } \\
\text { brief suggestion for } \\
\text { future research that } \\
\text { could have been } \\
\text { elaborated on. }\end{array}$ \\
\hline & $\begin{array}{l}\text { Fair }=2=\text { Two of the above } \\
\text { (State in comments). }\end{array}$ & & \\
\hline & $\begin{array}{l}\text { Poor }=1=\text { Only one of the } \\
\text { above (State in comments). }\end{array}$ & & \\
\hline & $\begin{array}{l}\text { Very Poor }=0=\text { None of the } \\
\text { above }\end{array}$ & & \\
\hline Total Score: & & $\begin{array}{l}7 / 25.5 \\
27 \% \\
\end{array}$ & \\
\hline
\end{tabular}

\begin{tabular}{|l|l|c|l|}
\hline Study 18 - Title: & $\begin{array}{l}\text { Why did she do it? College women's motives for intimate partner } \\
\text { violence perpetration }\end{array}$ \\
\hline Author (Year): & $\begin{array}{l}\text { Neal, A. M., Dixon, K. J., Edwards, K. M., \& Gidycz, C. A. } \\
\text { (2015) }\end{array}$ \\
\hline Sampling: & Description & Score & Comments \\
\hline Questions & $\begin{array}{l}\text { Good = 3 = Details (age/ } \\
\text { gender/ ethnicity /context) of } \\
\text { who was studied and how } \\
\text { they were recruited. } \\
\text { Rationale for studying this } \\
\text { population. Response rates } \\
\text { shown and explained (why } \\
\text { certain individuals chose not } \\
\text { to participate, and any } \\
\text { attempts to achieve a sample }\end{array}$ & 1 & $\begin{array}{l}\text { Details of sample } \\
\text { provided. No rationale } \\
\text { for studying this } \\
\text { population given. No } \\
\text { strategy appropriate to } \\
\text { address the research } \\
\text { question/s? } \\
\begin{array}{l}\text { Why was this group } \\
\text { targeted? } \\
\text { Were the participants } \\
\text { representative of the } \\
\text { target population? }\end{array}\end{array}$ \\
\hline
\end{tabular}




\begin{tabular}{|c|c|c|c|}
\hline & $\begin{array}{l}\text { that represents the target } \\
\text { population). }\end{array}$ & & \\
\hline & $\begin{array}{l}\text { Fair }=2=\text { Most information } \\
\text { given, but some missing. }\end{array}$ & & \\
\hline & $\begin{array}{l}\text { Poor }=1=\text { Sampling } \\
\text { mentioned but few } \\
\text { descriptive details. }\end{array}$ & & \\
\hline & $\begin{array}{l}\text { Very Poor }=0=\text { No details } \\
\text { of sample. }\end{array}$ & & \\
\hline \multirow{3}{*}{$\begin{array}{l}\text { Reporting of power or a } \\
\text { priori power analysis } \\
\text { conducted? } \\
\text { Does the study have } \geq \\
80 \% \text { power to detect an } \\
\text { effect at } r=.20 ?\end{array}$} & $\begin{array}{l}\text { Good }=3=\text { Two of the } \\
\text { above (State in comments). }\end{array}$ & \multirow[t]{3}{*}{1.5} & \multirow{3}{*}{$\begin{array}{l}\text { No reporting of power } \\
\text { or a priori power } \\
\text { analysis conducted. } \\
\text { The study has } \geq 80 \% \\
\text { power to detect an } \\
\text { effect at } r=.20\end{array}$} \\
\hline & $\begin{array}{l}\text { Poor }=1.5=\text { Only one of the } \\
\text { above (State in comments). }\end{array}$ & & \\
\hline & $\begin{array}{l}\text { Very Poor }=0=\text { None of the } \\
\text { above }\end{array}$ & & \\
\hline \multicolumn{4}{|l|}{ Method: } \\
\hline Questions & Description & Score & Comments \\
\hline \multirow{4}{*}{$\begin{array}{l}\text { Has IPA been clearly } \\
\text { defined? } \\
\text { Have motivations been } \\
\text { clearly defined? }\end{array}$} & $\begin{array}{l}\text { Good }=3=\text { Clear } \\
\text { description of both IPA and } \\
\text { motivations. }\end{array}$ & \multirow[t]{4}{*}{3} & \multirow[t]{4}{*}{$\begin{array}{l}\text { IPA and motives } \\
\text { defined }\end{array}$} \\
\hline & $\begin{array}{l}\text { Fair }=2=\text { Clear description } \\
\text { of one variable, but } \\
\text { inadequate description of the } \\
\text { other. }\end{array}$ & & \\
\hline & $\begin{array}{l}\text { Poor }=1=\text { Inadequate } \\
\text { descriptions of both } \\
\text { variables. }\end{array}$ & & \\
\hline & $\begin{array}{l}\text { Very Poor }=0=\text { No } \\
\text { description of either } \\
\text { variable. }\end{array}$ & & \\
\hline \multirow[t]{4}{*}{$\begin{array}{l}\text { Are the measurements } \\
\text { used appropriate? }\end{array}$} & $\begin{array}{l}\text { Good }=3=\text { Validated and } \\
\text { reliability tested measures of } \\
\text { the outcome are used, or } \\
\text { measures are pre-tested prior } \\
\text { to data collection. }\end{array}$ & \multirow[t]{4}{*}{1} & \multirow{4}{*}{$\begin{array}{l}\text { Failure to report } \\
\text { validity and reliability } \\
\text { of CTS2 and MEQ. } \\
\text { A "modified" version of } \\
\text { the MEQ was used, but } \\
\text { no details as to what } \\
\text { was modified were } \\
\text { provided. }\end{array}$} \\
\hline & $\begin{array}{l}\text { Fair }=2=\text { Appropriate } \\
\text { measures have been used but } \\
\text { have not been described } \\
\text { well. }\end{array}$ & & \\
\hline & $\begin{array}{l}\text { Poor }=1=\text { Measures used } \\
\text { are inappropriate/ failure to } \\
\text { report validity and reliability } \\
\text { of measures. }\end{array}$ & & \\
\hline & $\begin{array}{l}\text { Very Poor }=0=\text { No mention } \\
\text { of measures used. }\end{array}$ & & \\
\hline
\end{tabular}




\begin{tabular}{|c|c|c|c|}
\hline Questions & Description & Score & Comments \\
\hline \multirow[t]{4}{*}{$\begin{array}{l}\text { Is the statistical analysis } \\
\text { appropriate to answer } \\
\text { the research question } \\
\text { and for the type of data } \\
\text { collected? }\end{array}$} & $\begin{array}{l}\text { Good = } 3=\text { Appropriate } \\
\text { analysis selected. Reasons } \\
\text { for tests clearly stated and } \\
\text { linked back to the } \\
\text { hypotheses. }\end{array}$ & \multirow[t]{4}{*}{3} & \multirow{4}{*}{$\begin{array}{l}\text { The type of analysis } \\
\text { was appropriate and an } \\
\text { explanation for it's use } \\
\text { was provided. The } \\
\text { authors consider the } \\
\text { study to be exploratory } \\
\text { in nature and thus, no } \\
\text { hypotheses are stated. }\end{array}$} \\
\hline & $\begin{array}{l}\text { Fair }=2=\text { Appropriate } \\
\text { analysis. Some rationale for } \\
\text { the analysis provided. }\end{array}$ & & \\
\hline & $\begin{array}{l}\text { Poor }=1=\text { Appropriate } \\
\text { analysis. No rationale } \\
\text { provided. }\end{array}$ & & \\
\hline & $\begin{array}{l}\text { Very Poor }=0=\text { Analysis } \\
\text { was inappropriate to answer } \\
\text { the research question. }\end{array}$ & & \\
\hline \multirow[t]{4}{*}{$\begin{array}{l}\text { Was the description of } \\
\text { the analysis sufficiently } \\
\text { rigorous? }\end{array}$} & $\begin{array}{l}\text { Good }=3=\text { Clear } \\
\text { description of how analysis } \\
\text { was done. Statistical } \\
\text { significance discussed. }\end{array}$ & \multirow[t]{5}{*}{2.5} & \multirow{4}{*}{$\begin{array}{l}\text { Analysis described and } \\
\text { statistical significance } \\
\text { discussed. Software } \\
\text { used to conduct the } \\
\text { analysis was not stated. }\end{array}$} \\
\hline & $\begin{array}{l}\text { Fair }=2=\text { Some details } \\
\text { about the analysis provided. }\end{array}$ & & \\
\hline & $\begin{array}{l}\text { Poor }=1=\text { Minimal details } \\
\text { about analysis provided. }\end{array}$ & & \\
\hline & $\begin{array}{l}\text { Very Poor }=0=\text { No } \\
\text { discussion of analysis }\end{array}$ & & \\
\hline \multicolumn{3}{|l|}{ Results: } & \\
\hline Questions & Description & Score & Comments \\
\hline \multirow[t]{4}{*}{$\begin{array}{l}\text { Is there a clear } \\
\text { statement of findings? }\end{array}$} & $\begin{array}{l}\text { Good }=3=\text { Findings are } \\
\text { presented logically, are easy } \\
\text { to understand, and relate to } \\
\text { aims. Tables are explained } \\
\text { in text. }\end{array}$ & \multirow[t]{4}{*}{3} & \multirow[t]{4}{*}{$\begin{array}{l}\text { Findings are clear and } \\
\text { directly relate to the aim } \\
\text { of the study. The tables } \\
\text { are referred to in text. }\end{array}$} \\
\hline & $\begin{array}{l}\text { Fair }=2=\text { Findings } \\
\text { mentioned, but more } \\
\text { explanation could be given. }\end{array}$ & & \\
\hline & $\begin{array}{l}\text { Poor }=1=\text { Findings are } \\
\text { presented haphazardly, not } \\
\text { explained, or do not relate to } \\
\text { aims. }\end{array}$ & & \\
\hline & $\begin{array}{l}\text { Very Poor }=0=\text { Not } \\
\text { mentioned or do not relate to } \\
\text { aims. }\end{array}$ & & \\
\hline \multirow[t]{2}{*}{ Limitations discussed? } & Yes $=3$ & \multirow[t]{2}{*}{3} & \multirow{2}{*}{$\begin{array}{l}\text { These include: Focus on } \\
\text { the individual's most } \\
\text { severe behaviour/ type } \\
\text { of IPA, as opposed to }\end{array}$} \\
\hline & $\mathrm{No}=0$ & & \\
\hline
\end{tabular}




\begin{tabular}{|c|c|c|c|}
\hline & & & $\begin{array}{l}\text { investigating different } \\
\text { motives for different } \\
\text { behaviours. }\end{array}$ \\
\hline \multicolumn{4}{|c|}{ Implications/ Applications: } \\
\hline Questions & Description & Score & Comments \\
\hline \multirow[t]{4}{*}{$\begin{array}{l}\text { How important are } \\
\text { these findings to policy/ } \\
\text { practice? } \\
\text { Does the research offer } \\
\text { a valuable contribution } \\
\text { to the literature? }\end{array}$} & $\begin{array}{l}\text { Good }=3=\text { Contributes } \\
\text { something new/ different in } \\
\text { terms of insight or } \\
\text { perspective. Suggests ideas } \\
\text { for future research. Suggests } \\
\text { implication for policy and/ } \\
\text { or practice. }\end{array}$ & \multirow[t]{4}{*}{3} & \multirow[t]{3}{*}{$\begin{array}{l}\text { Investigates motivations } \\
\text { by type of IPA. } \\
\text { Future research and } \\
\text { treatment implications } \\
\text { are discussed in detail. }\end{array}$} \\
\hline & $\begin{array}{l}\text { Fair }=2=\text { Two of the above } \\
\text { (State in comments). }\end{array}$ & & \\
\hline & $\begin{array}{l}\text { Poor }=1=\text { Only one of the } \\
\text { above (State in comments). }\end{array}$ & & \\
\hline & $\begin{array}{l}\text { Very Poor }=0=\text { None of the } \\
\text { above }\end{array}$ & & \\
\hline \multicolumn{2}{|l|}{ Total Score: } & $\begin{array}{l}21 / 27 \\
78 \%\end{array}$ & \\
\hline
\end{tabular}

\begin{tabular}{|c|c|c|c|}
\hline Study 19 - Title: & \multicolumn{3}{|c|}{$\begin{array}{l}\text { Personality and situational correlates of self-reported reasons for } \\
\text { intimate partner violence among women versus men referred for } \\
\text { batterers' intervention }\end{array}$} \\
\hline Author (Year): & \multicolumn{3}{|l|}{ Ross, J. M. (2011) } \\
\hline \multicolumn{4}{|l|}{ Sampling: } \\
\hline Questions & Description & Score & Comments \\
\hline $\begin{array}{l}\text { Was the sampling } \\
\text { strategy appropriate to } \\
\text { address the research } \\
\text { question/s? } \\
\text { Why was this group } \\
\text { targeted? } \\
\text { Were the participants } \\
\text { representative of the } \\
\text { target population? }\end{array}$ & $\begin{array}{l}\text { Good }=3=\text { Details (age/ } \\
\text { gender/ ethnicity /context) of } \\
\text { who was studied and how } \\
\text { they were recruited. } \\
\text { Rationale for studying this } \\
\text { population. Response rates } \\
\text { shown and explained (why } \\
\text { certain individuals chose not } \\
\text { to participate, and any } \\
\text { attempts to achieve a sample } \\
\text { that represents the target } \\
\text { population). } \\
\text { Fair }=2=\text { Most information } \\
\text { given, but some missing. } \\
\text { Poor = } 1=\text { Sampling } \\
\text { mentioned but few } \\
\text { descriptive details. } \\
\text { Very Poor = } 0=\text { No details } \\
\text { of sample. }\end{array}$ & 1 & $\begin{array}{l}\text { Details of sample } \\
\text { provided. No rationale } \\
\text { for studying this } \\
\text { population given. No } \\
\text { response rate provided. } \\
\text { No attempt to ensure a } \\
\text { representative sample } \\
\text { reported. }\end{array}$ \\
\hline
\end{tabular}




\begin{tabular}{|c|c|c|c|}
\hline \multirow{3}{*}{$\begin{array}{l}\text { Reporting of power or a } \\
\text { priori power analysis } \\
\text { conducted? } \\
\text { Does the study have } \geq \\
80 \% \text { power to detect an } \\
\text { effect at } r=.20 \text { ? }\end{array}$} & $\begin{array}{l}\text { Good }=3=\text { Two of the } \\
\text { above (State in comments). }\end{array}$ & \multirow[t]{3}{*}{0} & \multirow{3}{*}{$\begin{array}{l}\text { Low power was } \\
\text { discussed as a limitation } \\
\text { of the study. However, } \\
\text { an a priori power } \\
\text { analysis was not } \\
\text { conducted. } \\
\text { The study has }<80 \% \\
\text { power to detect an effect } \\
\text { at } r=.20\end{array}$} \\
\hline & $\begin{array}{l}\text { Poor }=1.5=\text { Only one of the } \\
\text { above (State in comments). }\end{array}$ & & \\
\hline & $\begin{array}{l}\text { Very Poor }=0=\text { None of the } \\
\text { above }\end{array}$ & & \\
\hline \multicolumn{4}{|l|}{ Method: } \\
\hline Questions & Description & Score & Comments \\
\hline \multirow{4}{*}{$\begin{array}{l}\text { Has IPA been clearly } \\
\text { defined? } \\
\text { Have motivations been } \\
\text { clearly defined? }\end{array}$} & $\begin{array}{l}\text { Good = } 3=\text { Clear description } \\
\text { of both IPA and motivations. }\end{array}$ & \multirow[t]{4}{*}{0} & \multirow[t]{4}{*}{ Neither variable defined } \\
\hline & $\begin{array}{l}\text { Fair }=2=\text { Clear description } \\
\text { of one variable, but } \\
\text { inadequate description of the } \\
\text { other. }\end{array}$ & & \\
\hline & $\begin{array}{l}\text { Poor }=1=\text { Inadequate } \\
\text { descriptions of both } \\
\text { variables. }\end{array}$ & & \\
\hline & $\begin{array}{l}\text { Very Poor }=0=\text { No } \\
\text { description of either variable. }\end{array}$ & & \\
\hline \multirow[t]{4}{*}{$\begin{array}{l}\text { Are the measurements } \\
\text { used appropriate? }\end{array}$} & $\begin{array}{l}\text { Good }=3=\text { Validated and } \\
\text { reliability tested measures of } \\
\text { the outcome are used, or } \\
\text { measures are pre-tested prior } \\
\text { to data collection. }\end{array}$ & \multirow[t]{4}{*}{3} & \multirow{4}{*}{$\begin{array}{l}\text { The psychometric } \\
\text { properties of the CTS2 } \\
\text { were discussed and the } \\
\text { internal consistencies } \\
\text { provided for the current } \\
\text { study. } \\
\text { No psychometric } \\
\text { information was } \\
\text { provided for the RVS, } \\
\text { however, a PCA was } \\
\text { conducted. }\end{array}$} \\
\hline & $\begin{array}{l}\text { Fair }=2=\text { Appropriate } \\
\text { measures have been used but } \\
\text { have not been described well. }\end{array}$ & & \\
\hline & $\begin{array}{l}\text { Poor }=1=\text { Measures used } \\
\text { are inappropriate/ failure to } \\
\text { report validity and reliability } \\
\text { of measures. }\end{array}$ & & \\
\hline & $\begin{array}{l}\text { Very Poor }=0=\text { No mention } \\
\text { of measures used. }\end{array}$ & & \\
\hline \multicolumn{4}{|l|}{ Data Analysis: } \\
\hline Questions & Description & Score & Comments \\
\hline \multirow[t]{2}{*}{$\begin{array}{l}\text { Is the statistical analysis } \\
\text { appropriate to answer } \\
\text { the research question } \\
\text { and for the type of data } \\
\text { collected? }\end{array}$} & $\begin{array}{l}\text { Good = } 3=\text { Appropriate } \\
\text { analysis selected. Reasons } \\
\text { for tests clearly stated and } \\
\text { linked back to the } \\
\text { hypotheses. }\end{array}$ & \multirow[t]{2}{*}{1} & \multirow{2}{*}{$\begin{array}{l}\text { PCA is an appropriate } \\
\text { technique considering } \\
\text { the measure used has } 29 \\
\text { items. However, no } \\
\text { rationale was given for } \\
\text { performing this analysis. } \\
\text { T- test was appropriate } \\
\text { for group comparison. }\end{array}$} \\
\hline & $\begin{array}{l}\text { Fair }=2=\text { Appropriate } \\
\text { analysis. Some rationale for } \\
\text { the analysis provided. }\end{array}$ & & \\
\hline
\end{tabular}




\begin{tabular}{|c|c|c|c|}
\hline & $\begin{array}{l}\text { Poor }=1=\text { Appropriate } \\
\text { analysis. No rationale } \\
\text { provided. } \\
\text { Very Poor }=0=\text { Analysis } \\
\text { was inappropriate to answer } \\
\text { the research question. }\end{array}$ & & \\
\hline $\begin{array}{l}\text { Was the description of } \\
\text { the analysis sufficiently } \\
\text { rigorous? }\end{array}$ & $\begin{array}{l}\text { Good }=3=\text { Clear description } \\
\text { of how analysis was done. } \\
\text { Statistical significance } \\
\text { discussed. } \\
\text { Fair }=2=\text { Some details about } \\
\text { the analysis provided. } \\
\begin{array}{l}\text { Poor }=1=\text { Minimal details } \\
\text { about analysis provided. }\end{array} \\
\begin{array}{l}\text { Very Poor }=0=\text { No } \\
\text { discussion of analysis }\end{array}\end{array}$ & \multirow[t]{2}{*}{1} & $\begin{array}{l}\text { Insufficient detail } \\
\text { regarding the PCA e.g., } \\
\text { rotation method. } \\
\text { No mention of t-tests - } \\
\text { the reporting of the } \\
\text { statistic in the text was } \\
\text { the only indication of } \\
\text { what analysis was } \\
\text { conducted. } \\
\text { Statistical significance } \\
\text { was not discussed and a } \\
\text { multi test correction was } \\
\text { not employed. }\end{array}$ \\
\hline \multicolumn{3}{|l|}{ Results: } & \\
\hline Questions & Description & Score & Comments \\
\hline $\begin{array}{l}\text { Is there a clear statement } \\
\text { of findings? }\end{array}$ & $\begin{array}{l}\text { Good = } 3=\text { Findings are } \\
\text { presented logically, are easy } \\
\text { to understand, and relate to } \\
\text { aims. Tables are explained in } \\
\text { text. } \\
\text { Fair }=2=\text { Findings } \\
\text { mentioned, but more } \\
\text { explanation could be given. } \\
\text { Poor }=1=\text { Findings are } \\
\text { presented haphazardly, not } \\
\text { explained, or do not relate to } \\
\text { aims. } \\
\text { Very Poor }=0=\text { Not } \\
\text { mentioned or do not relate to } \\
\text { aims. }\end{array}$ & 3 & $\begin{array}{l}\text { Results relate to the } \\
\text { aims of the study and are } \\
\text { presented in a logical } \\
\text { order. Tables are } \\
\text { referred to in text. }\end{array}$ \\
\hline Limitations discussed? & $\begin{array}{l}\text { Yes }=3 \\
\text { No }=0\end{array}$ & 3 & $\begin{array}{l}\text { These include: small } \\
\text { sample size, } \\
\text { generalisability of } \\
\text { findings from a forensic } \\
\text { sample }\end{array}$ \\
\hline \multicolumn{4}{|c|}{ Implications/ Applications: } \\
\hline Questions & Description & Score & Comments \\
\hline $\begin{array}{l}\text { How important are these } \\
\text { findings to policy/ } \\
\text { practice? }\end{array}$ & $\begin{array}{l}\text { Good = } 3=\text { Contributes } \\
\text { something new/ different in } \\
\text { terms of insight or } \\
\text { perspective. Suggests ideas }\end{array}$ & 2 & $\begin{array}{l}\text { Discusses clinical } \\
\text { implications and } \\
\text { suggests ideas for future } \\
\text { research. }\end{array}$ \\
\hline
\end{tabular}




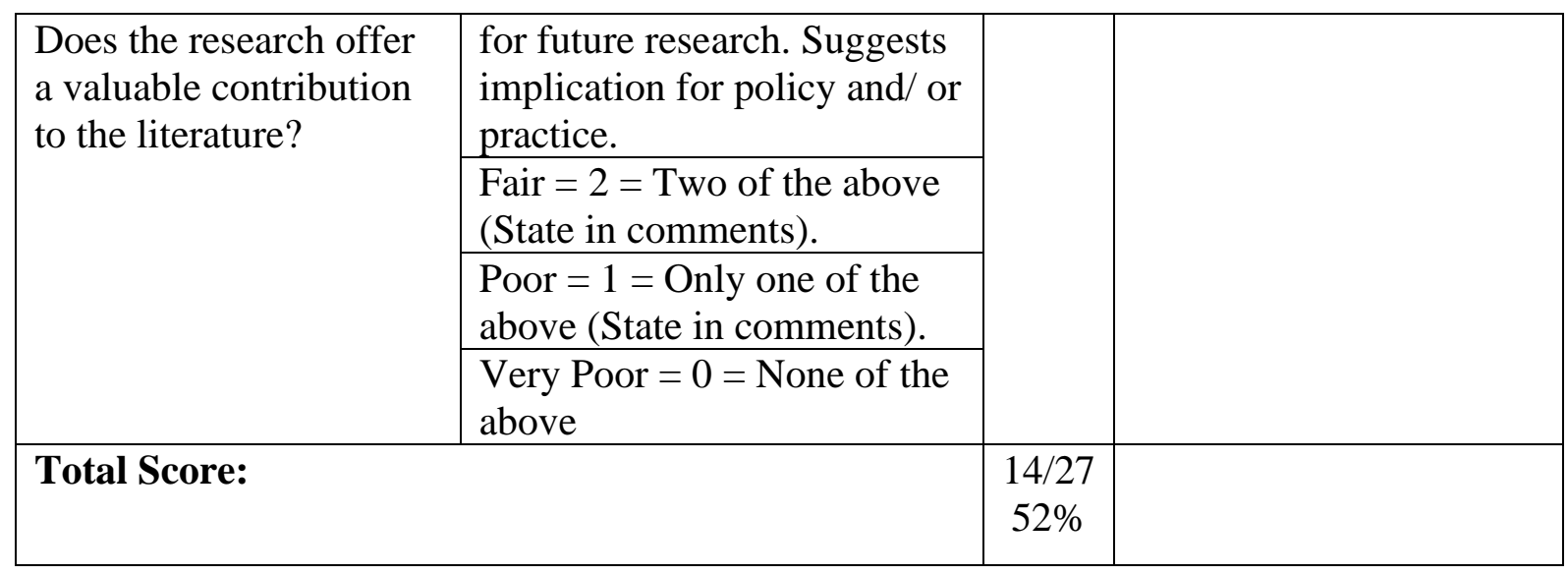

\begin{tabular}{|c|c|c|c|}
\hline Study 20 - Title: & \multicolumn{3}{|c|}{$\begin{array}{l}\text { When battered women use violence: Husband abuse or self- } \\
\text { defense? }\end{array}$} \\
\hline Author (Year): & \multicolumn{3}{|l|}{ Saunders, D. G. (1986) } \\
\hline \multicolumn{4}{|l|}{ Sampling: } \\
\hline Questions & Description & Score & Comments \\
\hline \multirow[t]{4}{*}{$\begin{array}{l}\text { Was the sampling } \\
\text { strategy appropriate to } \\
\text { address the research } \\
\text { question/s? } \\
\text { Why was this group } \\
\text { targeted? } \\
\text { Were the participants } \\
\text { representative of the } \\
\text { target population? }\end{array}$} & $\begin{array}{l}\text { Good }=3=\text { Details (age/ } \\
\text { gender/ ethnicity /context) of } \\
\text { who was studied and how } \\
\text { they were recruited. } \\
\text { Rationale for studying this } \\
\text { population. Response rates } \\
\text { shown and explained (why } \\
\text { certain individuals chose not } \\
\text { to participate, and any } \\
\text { attempts to achieve a sample } \\
\text { that represents the target } \\
\text { population). }\end{array}$ & \multirow[t]{4}{*}{0} & \multirow[t]{4}{*}{$\begin{array}{l}\text { Age and ethnic } \\
\text { composition of sample } \\
\text { not reported. No } \\
\text { response rate reported. } \\
\text { No attempt to ensure } \\
\text { sample was } \\
\text { representative reported. } \\
\text { No rationale for } \\
\text { studying this population } \\
\text { given. }\end{array}$} \\
\hline & $\begin{array}{l}\text { Fair }=2=\text { Most information } \\
\text { given, but some missing. }\end{array}$ & & \\
\hline & $\begin{array}{l}\text { Poor }=1=\text { Sampling } \\
\text { mentioned but few } \\
\text { descriptive details. }\end{array}$ & & \\
\hline & $\begin{array}{l}\text { Very Poor }=0=\text { No details } \\
\text { of sample. }\end{array}$ & & \\
\hline \multirow{3}{*}{$\begin{array}{l}\text { Reporting of power or a } \\
\text { priori power analysis } \\
\text { conducted? } \\
\text { Does the study have } \geq \\
80 \% \text { power to detect an } \\
\text { effect at } r=.20 \text { ? }\end{array}$} & $\begin{array}{l}\text { Good = } 3=\text { Two of the above } \\
\text { (State in comments). }\end{array}$ & \multirow[t]{3}{*}{0} & \multirow{3}{*}{$\begin{array}{l}\text { No reporting of power } \\
\text { or a priori power } \\
\text { analysis conducted. } \\
\text { The study has }<80 \% \\
\text { power to detect an effect } \\
\text { at } r=.20\end{array}$} \\
\hline & $\begin{array}{l}\text { Poor }=1.5=\text { Only one of the } \\
\text { above (State in comments). }\end{array}$ & & \\
\hline & $\begin{array}{l}\text { Very Poor }=0=\text { None of the } \\
\text { above }\end{array}$ & & \\
\hline \multicolumn{4}{|l|}{ Method: } \\
\hline Questions & Description & Score & Comments \\
\hline
\end{tabular}




\begin{tabular}{|c|c|c|c|}
\hline \multirow{4}{*}{$\begin{array}{l}\text { Has IPA been clearly } \\
\text { defined? } \\
\text { Have motivations been } \\
\text { clearly defined? }\end{array}$} & $\begin{array}{l}\text { Good }=3=\text { Clear description } \\
\text { of both IPA and motivations. }\end{array}$ & \multirow[t]{4}{*}{0} & \multirow[t]{4}{*}{$\begin{array}{l}\text { Neither IPA nor } \\
\text { motivations defined }\end{array}$} \\
\hline & $\begin{array}{l}\text { Fair }=2=\text { Clear description } \\
\text { of one variable, but } \\
\text { inadequate description of the } \\
\text { other. }\end{array}$ & & \\
\hline & $\begin{array}{l}\text { Poor }=1=\text { Inadequate } \\
\text { descriptions of both } \\
\text { variables. }\end{array}$ & & \\
\hline & $\begin{array}{l}\text { Very Poor }=0=\text { No } \\
\text { description of either variable. }\end{array}$ & & \\
\hline \multirow[t]{4}{*}{$\begin{array}{l}\text { Are the measurements } \\
\text { used appropriate? }\end{array}$} & $\begin{array}{l}\text { Good }=3=\text { Validated and } \\
\text { reliability tested measures of } \\
\text { the outcome are used, or } \\
\text { measures are pre-tested prior } \\
\text { to data collection. }\end{array}$ & \multirow[t]{4}{*}{2} & \multirow{4}{*}{$\begin{array}{l}\text { CTS2 and social } \\
\text { desirability measure } \\
\text { well validated and } \\
\text { psychometric } \\
\text { information was } \\
\text { provided. The motive } \\
\text { measure has not been } \\
\text { validated and was not } \\
\text { piloted prior to use in } \\
\text { the study. }\end{array}$} \\
\hline & $\begin{array}{l}\text { Fair }=2=\text { Appropriate } \\
\text { measures have been used but } \\
\text { have not been described well. }\end{array}$ & & \\
\hline & $\begin{array}{l}\text { Poor }=1=\text { Measures used are } \\
\text { inappropriate/ failure to } \\
\text { report validity and reliability } \\
\text { of measures. }\end{array}$ & & \\
\hline & $\begin{array}{l}\text { Very Poor }=0=\text { No mention } \\
\text { of measures used. }\end{array}$ & & \\
\hline \multicolumn{4}{|l|}{ Data Analysis: } \\
\hline Questions & Description & Score & Comments \\
\hline \multirow[t]{4}{*}{$\begin{array}{l}\text { Is the statistical analysis } \\
\text { appropriate to answer } \\
\text { the research question } \\
\text { and for the type of data } \\
\text { collected? }\end{array}$} & $\begin{array}{l}\text { Good }=3=\text { Appropriate } \\
\text { analysis selected. Reasons } \\
\text { for tests clearly stated and } \\
\text { linked back to the } \\
\text { hypotheses. }\end{array}$ & \multirow[t]{4}{*}{1} & \multirow[t]{4}{*}{$\begin{array}{l}\text { Appropriate analysis } \\
\text { conducted. Reasons for } \\
\text { the tests not stated or } \\
\text { linked back to the } \\
\text { hypotheses. }\end{array}$} \\
\hline & $\begin{array}{l}\text { Fair }=2=\text { Appropriate } \\
\text { analysis. Some rationale for } \\
\text { the analysis provided. }\end{array}$ & & \\
\hline & $\begin{array}{l}\text { Poor }=1=\text { Appropriate } \\
\text { analysis. No rationale } \\
\text { provided. }\end{array}$ & & \\
\hline & $\begin{array}{l}\text { Very Poor }=0=\text { Analysis } \\
\text { was inappropriate to answer } \\
\text { the research question. }\end{array}$ & & \\
\hline \multirow[t]{2}{*}{$\begin{array}{l}\text { Was the description of } \\
\text { the analysis sufficiently } \\
\text { rigorous? }\end{array}$} & $\begin{array}{l}\text { Good = } 3=\text { Clear description } \\
\text { of how analysis was done. } \\
\text { Statistical significance } \\
\text { discussed. }\end{array}$ & \multirow[t]{2}{*}{2} & \multirow{2}{*}{$\begin{array}{l}\text { A more explicit } \\
\text { description would have } \\
\text { been beneficial e.g., } \\
\text { type of correlation, } \\
\text { software used. Statistical } \\
\text { significance was }\end{array}$} \\
\hline & $\begin{array}{l}\text { Fair }=2=\text { Some details about } \\
\text { the analysis provided. }\end{array}$ & & \\
\hline
\end{tabular}




\begin{tabular}{|c|c|c|c|}
\hline & $\begin{array}{l}\text { Poor }=1=\text { Minimal details } \\
\text { about analysis provided. } \\
\text { Very Poor }=0=\text { No } \\
\text { discussion of analysis } \\
\end{array}$ & & $\begin{array}{l}\text { discussed and a multi } \\
\text { test correct was applied. }\end{array}$ \\
\hline \multicolumn{4}{|l|}{ Results: } \\
\hline Questions & Description & Score & Comments \\
\hline \multirow[t]{4}{*}{$\begin{array}{l}\text { Is there a clear statement } \\
\text { of findings? }\end{array}$} & $\begin{array}{l}\text { Good }=3=\text { Findings are } \\
\text { presented logically, are easy } \\
\text { to understand, and relate to } \\
\text { aims. Tables are explained in } \\
\text { text. }\end{array}$ & \multirow[t]{4}{*}{3} & \multirow[t]{4}{*}{$\begin{array}{l}\text { The findings are } \\
\text { presented in a logical } \\
\text { order and relate to the } \\
\text { hypotheses. Tables } \\
\text { explained well in text. }\end{array}$} \\
\hline & $\begin{array}{l}\text { Fair }=2=\text { Findings } \\
\text { mentioned, but more } \\
\text { explanation could be given. }\end{array}$ & & \\
\hline & $\begin{array}{l}\text { Poor }=1=\text { Findings are } \\
\text { presented haphazardly, not } \\
\text { explained, or do not relate to } \\
\text { aims. }\end{array}$ & & \\
\hline & $\begin{array}{l}\text { Very Poor }=0=\text { Not } \\
\text { mentioned or do not relate to } \\
\text { aims. }\end{array}$ & & \\
\hline \multirow[t]{2}{*}{ Limitations discussed? } & Yes $=3$ & \multirow[t]{2}{*}{3} & \multirow{2}{*}{$\begin{array}{l}\text { These include: } \\
\text { Participants not asked } \\
\text { about motives for } \\
\text { specific incidents, } \\
\text { sample not } \\
\text { representative }\end{array}$} \\
\hline & $\mathrm{No}=0$ & & \\
\hline \multicolumn{4}{|c|}{ Implications/ Applications: } \\
\hline Questions & Description & Score & Comments \\
\hline \multirow[t]{4}{*}{$\begin{array}{l}\text { How important are these } \\
\text { findings to policy/ } \\
\text { practice? } \\
\text { Does the research offer } \\
\text { a valuable contribution } \\
\text { to the literature? }\end{array}$} & $\begin{array}{l}\text { Good }=3=\text { Contributes } \\
\text { something new/ different in } \\
\text { terms of insight or } \\
\text { perspective. Suggests ideas } \\
\text { for future research. Suggests } \\
\text { implication for policy and/ or } \\
\text { practice. }\end{array}$ & \multirow[t]{4}{*}{1} & \multirow[t]{4}{*}{$\begin{array}{l}\text { Some very vague ideas } \\
\text { regarding future } \\
\text { research }\end{array}$} \\
\hline & $\begin{array}{l}\text { Fair }=2=\text { Two of the above } \\
\text { (State in comments). }\end{array}$ & & \\
\hline & $\begin{array}{l}\text { Poor }=1=\text { Only one of the } \\
\text { above (State in comments). }\end{array}$ & & \\
\hline & $\begin{array}{l}\text { Very Poor }=0=\text { None of the } \\
\text { above }\end{array}$ & & \\
\hline \multicolumn{2}{|l|}{ Total Score: } & $\begin{array}{l}12 / 27 \\
44 \%\end{array}$ & \\
\hline
\end{tabular}




\begin{tabular}{|c|c|c|c|}
\hline Study 21 - Title: & \multicolumn{3}{|c|}{ Motivations for self-defensive aggression in dating relationships } \\
\hline Author (Year): & \multicolumn{3}{|c|}{ Shorey, R. C., Meltzer, C., \& Cornelius, T. L. (2010) } \\
\hline \multicolumn{4}{|l|}{ Sampling: } \\
\hline Questions & Description & Score & Comments \\
\hline \multirow[t]{4}{*}{$\begin{array}{l}\text { Was the sampling } \\
\text { strategy appropriate to } \\
\text { address the research } \\
\text { question/s? } \\
\text { Why was this group } \\
\text { targeted? } \\
\text { Were the participants } \\
\text { representative of the } \\
\text { target population? }\end{array}$} & $\begin{array}{l}\text { Good = } 3=\text { Details (age/ } \\
\text { gender/ ethnicity /context) } \\
\text { of who was studied and how } \\
\text { they were recruited. } \\
\text { Rationale for studying this } \\
\text { population. Response rates } \\
\text { shown and explained (why } \\
\text { certain individuals chose not } \\
\text { to participate, and any } \\
\text { attempts to achieve a sample } \\
\text { that represents the target } \\
\text { population). }\end{array}$ & \multirow[t]{4}{*}{2} & \multirow[t]{4}{*}{$\begin{array}{l}\text { Number of males and } \\
\text { females in sample only } \\
\text { supplied in tables in the } \\
\text { results section. } \\
\text { Discussion on dating } \\
\text { violence, but not } \\
\text { explicit why a college } \\
\text { sample was selected. } \\
\text { Response rates not } \\
\text { provided. No attempts } \\
\text { to ensure the sample } \\
\text { was representative were } \\
\text { reported. }\end{array}$} \\
\hline & $\begin{array}{l}\text { Fair }=2=\text { Most information } \\
\text { given, but some missing. }\end{array}$ & & \\
\hline & $\begin{array}{l}\text { Poor }=1=\text { Sampling } \\
\text { mentioned but few } \\
\text { descriptive details. }\end{array}$ & & \\
\hline & $\begin{array}{l}\text { Very Poor }=0=\text { No details } \\
\text { of sample. }\end{array}$ & & \\
\hline \multirow{3}{*}{$\begin{array}{l}\text { Reporting of power or a } \\
\text { priori power analysis } \\
\text { conducted? } \\
\text { Does the study have } \geq \\
80 \% \text { power to detect an } \\
\text { effect at } r=.20 \text { ? }\end{array}$} & $\begin{array}{l}\text { Good }=3=\text { Two of the } \\
\text { above (State in comments). }\end{array}$ & \multirow[t]{3}{*}{0} & \multirow{3}{*}{$\begin{array}{l}\text { No reporting of power } \\
\text { or a priori power } \\
\text { analysis conducted. } \\
\text { The study has }<80 \% \\
\text { power to detect an } \\
\text { effect at } r=.20\end{array}$} \\
\hline & $\begin{array}{l}\text { Poor }=1.5=\text { Only one of the } \\
\text { above (State in comments). }\end{array}$ & & \\
\hline & $\begin{array}{l}\text { Very Poor }=0=\text { None of the } \\
\text { above }\end{array}$ & & \\
\hline \multicolumn{4}{|l|}{ Method: } \\
\hline Questions & Description & Score & Comments \\
\hline \multirow{4}{*}{$\begin{array}{l}\text { Has IPA been clearly } \\
\text { defined? } \\
\text { Have motivations been } \\
\text { clearly defined? }\end{array}$} & $\begin{array}{l}\text { Good }=3=\text { Clear } \\
\text { description of both IPA and } \\
\text { motivations. }\end{array}$ & \multirow[t]{4}{*}{0} & \multirow[t]{4}{*}{$\begin{array}{l}\text { Both were discussed, } \\
\text { but not defined. }\end{array}$} \\
\hline & $\begin{array}{l}\text { Fair }=2=\text { Clear description } \\
\text { of one variable, but } \\
\text { inadequate description of the } \\
\text { other. }\end{array}$ & & \\
\hline & $\begin{array}{l}\text { Poor }=1=\text { Inadequate } \\
\text { descriptions of both } \\
\text { variables. }\end{array}$ & & \\
\hline & $\begin{array}{l}\text { Very Poor }=0=\text { No } \\
\text { description of either } \\
\text { variable. }\end{array}$ & & \\
\hline
\end{tabular}




\begin{tabular}{|c|c|c|c|}
\hline \multirow[t]{3}{*}{$\begin{array}{l}\text { Are the measurements } \\
\text { used appropriate? }\end{array}$} & $\begin{array}{l}\text { Good }=3=\text { Validated and } \\
\text { reliability tested measures of } \\
\text { the outcome are used, or } \\
\text { measures are pre-tested prior } \\
\text { to data collection. } \\
\text { Fair }=2=\text { Appropriate } \\
\text { measures have been used } \\
\text { but have not been described } \\
\text { well. }\end{array}$ & \multirow[t]{3}{*}{3} & \multirow[t]{3}{*}{$\begin{array}{l}\text { Measures were } \\
\text { appropriate and } \\
\text { psychometric } \\
\text { information was } \\
\text { supplied for both the } \\
\text { CTS2 and the RVS. }\end{array}$} \\
\hline & $\begin{array}{l}\text { Poor }=1=\text { Measures used } \\
\text { are inappropriate/ failure to } \\
\text { report validity and reliability } \\
\text { of measures. }\end{array}$ & & \\
\hline & $\begin{array}{l}\text { Very Poor }=0=\text { No mention } \\
\text { of measures used. }\end{array}$ & & \\
\hline \multicolumn{4}{|l|}{ Data Analysis: } \\
\hline Questions & Description & Score & Comments \\
\hline \multirow[t]{4}{*}{$\begin{array}{l}\text { Is the statistical analysis } \\
\text { appropriate to answer } \\
\text { the research question } \\
\text { and for the type of data } \\
\text { collected? }\end{array}$} & $\begin{array}{l}\text { Good }=3=\text { Appropriate } \\
\text { analysis selected. Reasons } \\
\text { for tests clearly stated and } \\
\text { linked back to the } \\
\text { hypotheses. }\end{array}$ & \multirow[t]{4}{*}{3} & \multirow{4}{*}{$\begin{array}{l}\text { Appropriate analysis } \\
\text { and reasons for its } \\
\text { selection reported - } \\
\text { Mann-Whitney U tests } \\
\text { used to account for } \\
\text { skewness in data. Each } \\
\text { analysis was clearly } \\
\text { linked back to the } \\
\text { relevant hypothesis. }\end{array}$} \\
\hline & $\begin{array}{l}\text { Fair }=2=\text { Appropriate } \\
\text { analysis. Some rationale for } \\
\text { the analysis provided. }\end{array}$ & & \\
\hline & $\begin{array}{l}\text { Poor }=1=\text { Appropriate } \\
\text { analysis. No rationale } \\
\text { provided. }\end{array}$ & & \\
\hline & $\begin{array}{l}\text { Very Poor }=0=\text { Analysis } \\
\text { was inappropriate to answer } \\
\text { the research question. }\end{array}$ & & \\
\hline \multirow[t]{4}{*}{$\begin{array}{l}\text { Was the description of } \\
\text { the analysis sufficiently } \\
\text { rigorous? }\end{array}$} & $\begin{array}{l}\text { Good }=3=\text { Clear } \\
\text { description of how analysis } \\
\text { was done. Statistical } \\
\text { significance discussed. }\end{array}$ & \multirow[t]{4}{*}{2.5} & \multirow{4}{*}{$\begin{array}{l}\text { The analyses were } \\
\text { clearly described. } \\
\text { However, the software } \\
\text { used to conduct this } \\
\text { was not mentioned. }\end{array}$} \\
\hline & $\begin{array}{l}\text { Fair }=2=\text { Some details } \\
\text { about the analysis provided. }\end{array}$ & & \\
\hline & $\begin{array}{l}\text { Poor }=1=\text { Minimal details } \\
\text { about analysis provided. }\end{array}$ & & \\
\hline & $\begin{array}{l}\text { Very Poor }=0=\text { No } \\
\text { discussion of analysis }\end{array}$ & & \\
\hline \multicolumn{4}{|l|}{ Results: } \\
\hline Questions & Description & Score & Comments \\
\hline $\begin{array}{l}\text { Is there a clear } \\
\text { statement of findings? }\end{array}$ & $\begin{array}{l}\text { Good }=3=\text { Findings are } \\
\text { presented logically, are easy } \\
\text { to understand, and relate to } \\
\text { aims. Tables are explained } \\
\text { in text. }\end{array}$ & 3 & $\begin{array}{l}\text { Findings were } \\
\text { presented answering } \\
\text { each of the hypotheses } \\
\text { in turn. Tables were } \\
\text { explained in text. }\end{array}$ \\
\hline
\end{tabular}




\begin{tabular}{|c|c|c|c|}
\hline & $\begin{array}{l}\text { Fair }=2=\text { Findings } \\
\text { mentioned, but more } \\
\text { explanation could be given. }\end{array}$ & & \\
\hline & $\begin{array}{l}\text { Poor }=1=\text { Findings are } \\
\text { presented haphazardly, not } \\
\text { explained, or do not relate to } \\
\text { aims. }\end{array}$ & & \\
\hline & $\begin{array}{l}\text { Very Poor }=0=\text { Not } \\
\text { mentioned or do not relate to } \\
\text { aims. }\end{array}$ & & \\
\hline \multirow[t]{2}{*}{ Limitations discussed? } & Yes $=3$ & \multirow[t]{2}{*}{3} & \multirow{2}{*}{$\begin{array}{l}\text { These include: Small } \\
\text { number of male } \\
\text { participants, ethnic } \\
\text { homogeneity of sample } \\
\text { retrospective self-report } \\
\text { measures. }\end{array}$} \\
\hline & $\mathrm{No}=0$ & & \\
\hline \multicolumn{4}{|c|}{ Implications/ Applications: } \\
\hline Questions & Description & Score & Comments \\
\hline \multirow[t]{4}{*}{$\begin{array}{l}\text { How important are } \\
\text { these findings to policy/ } \\
\text { practice? } \\
\text { Does the research offer } \\
\text { a valuable contribution } \\
\text { to the literature? }\end{array}$} & $\begin{array}{l}\text { Good }=3=\text { Contributes } \\
\text { something new/ different in } \\
\text { terms of insight or } \\
\text { perspective. Suggests ideas } \\
\text { for future research. Suggests } \\
\text { implication for policy and/ } \\
\text { or practice. }\end{array}$ & \multirow[t]{4}{*}{2} & \multirow[t]{4}{*}{$\begin{array}{l}\text { Suggests ideas for } \\
\text { future research and } \\
\text { implications for } \\
\text { practice. }\end{array}$} \\
\hline & $\begin{array}{l}\text { Fair }=2=\text { Two of the above } \\
\text { (State in comments). }\end{array}$ & & \\
\hline & $\begin{array}{l}\text { Poor }=1=\text { Only one of the } \\
\text { above (State in comments). }\end{array}$ & & \\
\hline & $\begin{array}{l}\text { Very Poor }=0=\text { None of the } \\
\text { above }\end{array}$ & & \\
\hline \multicolumn{2}{|l|}{ Total Score: } & $\begin{array}{c}18.5 / 27 \\
69 \%\end{array}$ & \\
\hline
\end{tabular}

\begin{tabular}{|c|c|c|c|}
\hline Study 22 - Title: & \multicolumn{3}{|c|}{$\begin{array}{l}\text { Reasons for intimate partner violence perpetration among } \\
\text { arrested women }\end{array}$} \\
\hline Author (Year): & \multicolumn{3}{|c|}{$\begin{array}{l}\text { Stuart, G. L., Moore, T. M., Gordon, K. C., Hellmuth, J. C., } \\
\text { Ramsey, S. E., \& Kahler, C. W. (2006) }\end{array}$} \\
\hline \multicolumn{4}{|l|}{ Sampling: } \\
\hline Questions & Description & Score & Comments \\
\hline $\begin{array}{l}\text { Was the sampling } \\
\text { strategy appropriate to } \\
\text { address the research } \\
\text { question/s? } \\
\text { Why was this group } \\
\text { targeted? }\end{array}$ & $\begin{array}{l}\text { Good = } 3=\text { Details (age/ } \\
\text { gender/ ethnicity /context) } \\
\text { of who was studied and how } \\
\text { they were recruited. } \\
\text { Rationale for studying this } \\
\text { population. Response rates }\end{array}$ & 2.5 & $\begin{array}{l}\text { Details of sample } \\
\text { provided. Response rate } \\
\text { given, though not } \\
\text { explained. No attempt } \\
\text { to ensure the sample } \\
\text { was representative. No }\end{array}$ \\
\hline
\end{tabular}




\begin{tabular}{|c|c|c|c|}
\hline \multirow[t]{4}{*}{$\begin{array}{l}\text { Were the participants } \\
\text { representative of the } \\
\text { target population? }\end{array}$} & $\begin{array}{l}\text { shown and explained (why } \\
\text { certain individuals chose not } \\
\text { to participate, and any } \\
\text { attempts to achieve a sample } \\
\text { that represents the target } \\
\text { population). }\end{array}$ & & \multirow[t]{4}{*}{$\begin{array}{l}\text { rationale given for } \\
\text { studying this } \\
\text { population, though this } \\
\text { is probably assumed. }\end{array}$} \\
\hline & $\begin{array}{l}\text { Fair }=2=\text { Most information } \\
\text { given, but some missing. }\end{array}$ & & \\
\hline & $\begin{array}{l}\text { Poor }=1=\text { Sampling } \\
\text { mentioned but few } \\
\text { descriptive details. }\end{array}$ & & \\
\hline & $\begin{array}{l}\text { Very Poor }=0=\text { No details } \\
\text { of sample. }\end{array}$ & & \\
\hline \multirow{3}{*}{$\begin{array}{l}\text { Reporting of power or a } \\
\text { priori power analysis } \\
\text { conducted? } \\
\text { Does the study have } \geq \\
80 \% \text { power to detect an } \\
\text { effect at } r=.20 \text { ? }\end{array}$} & $\begin{array}{l}\text { Good }=3=\text { Two of the } \\
\text { above (State in comments). }\end{array}$ & \multirow[t]{3}{*}{ N/A } & \multirow{3}{*}{$\begin{array}{l}\text { No reporting of power } \\
\text { or a priori power } \\
\text { analysis conducted. } \\
\text { A power analysis was } \\
\text { unable to be calculated } \\
\text { as the study is } \\
\text { descriptive. } \\
\text { Consequently, this } \\
\text { section is not counted in } \\
\text { the total score. }\end{array}$} \\
\hline & $\begin{array}{l}\text { Poor }=1.5=\text { Only one of the } \\
\text { above (State in comments). }\end{array}$ & & \\
\hline & $\begin{array}{l}\text { Very Poor }=0=\text { None of the } \\
\text { above }\end{array}$ & & \\
\hline \multicolumn{4}{|l|}{ Method: } \\
\hline Questions & Description & Score & Comments \\
\hline \multirow{4}{*}{$\begin{array}{l}\text { Has IPA been clearly } \\
\text { defined? } \\
\text { Have motivations been } \\
\text { clearly defined? }\end{array}$} & $\begin{array}{l}\text { Good }=3=\text { Clear } \\
\text { description of both IPA and } \\
\text { motivations. }\end{array}$ & \multirow[t]{4}{*}{0} & \multirow[t]{4}{*}{$\begin{array}{l}\text { Neither IPA nor } \\
\text { motivations defined }\end{array}$} \\
\hline & $\begin{array}{l}\text { Fair }=2=\text { Clear description } \\
\text { of one variable, but } \\
\text { inadequate description of } \\
\text { the other. }\end{array}$ & & \\
\hline & $\begin{array}{l}\text { Poor }=1=\text { Inadequate } \\
\text { descriptions of both } \\
\text { variables. }\end{array}$ & & \\
\hline & $\begin{array}{l}\text { Very Poor }=0=\text { No } \\
\text { description of either } \\
\text { variable. }\end{array}$ & & \\
\hline \multirow[t]{2}{*}{$\begin{array}{l}\text { Are the measurements } \\
\text { used appropriate? }\end{array}$} & $\begin{array}{l}\text { Good }=3=\text { Validated and } \\
\text { reliability tested measures of } \\
\text { the outcome are used, or } \\
\text { measures are pre-tested } \\
\text { prior to data collection. }\end{array}$ & \multirow[t]{2}{*}{1.5} & \multirow{2}{*}{$\begin{array}{l}\text { It was stated the CTS2 } \\
\text { is a valid and reliable } \\
\text { measure, though no } \\
\text { psychometric } \\
\text { information was } \\
\text { provided. The RSV was } \\
\text { developed for this study } \\
\text { and was not piloted }\end{array}$} \\
\hline & $\begin{array}{l}\text { Fair }=2=\text { Appropriate } \\
\text { measures have been used } \\
\text { but have not been described } \\
\text { well. }\end{array}$ & & \\
\hline
\end{tabular}




\begin{tabular}{|c|c|c|c|}
\hline & $\begin{array}{l}\text { Poor }=1=\text { Measures used } \\
\text { are inappropriate/ failure to } \\
\text { report validity and reliability } \\
\text { of measures. } \\
\text { Very Poor }=0=\text { No mention } \\
\text { of measures used. }\end{array}$ & & $\begin{array}{l}\text { prior to use, nor } \\
\text { developed as a scale. }\end{array}$ \\
\hline \multicolumn{4}{|l|}{ Data Analysis: } \\
\hline Questions & Description & Score & Comments \\
\hline \multirow[t]{4}{*}{$\begin{array}{l}\text { Is the statistical analysis } \\
\text { appropriate to answer } \\
\text { the research question } \\
\text { and for the type of data } \\
\text { collected? }\end{array}$} & $\begin{array}{l}\text { Good }=3=\text { Appropriate } \\
\text { analysis selected. Reasons } \\
\text { for tests clearly stated and } \\
\text { linked back to the } \\
\text { hypotheses. }\end{array}$ & \multirow[t]{4}{*}{ N/A } & \multirow[t]{4}{*}{ Frequencies only. } \\
\hline & $\begin{array}{l}\text { Fair }=2=\text { Appropriate } \\
\text { analysis. Some rationale for } \\
\text { the analysis provided. }\end{array}$ & & \\
\hline & $\begin{array}{l}\text { Poor }=1=\text { Appropriate } \\
\text { analysis. No rationale } \\
\text { provided. }\end{array}$ & & \\
\hline & $\begin{array}{l}\text { Very Poor }=0=\text { Analysis } \\
\text { was inappropriate to answer } \\
\text { the research question. }\end{array}$ & & \\
\hline \multirow[t]{4}{*}{$\begin{array}{l}\text { Was the description of } \\
\text { the analysis sufficiently } \\
\text { rigorous? }\end{array}$} & $\begin{array}{l}\text { Good }=3=\text { Clear } \\
\text { description of how analysis } \\
\text { was done. Statistical } \\
\text { significance discussed. }\end{array}$ & \multirow[t]{4}{*}{ N/A } & \\
\hline & $\begin{array}{l}\text { Fair }=2=\text { Some details } \\
\text { about the analysis provided. }\end{array}$ & & \\
\hline & $\begin{array}{l}\text { Poor }=1=\text { Minimal details } \\
\text { about analysis provided. }\end{array}$ & & \\
\hline & $\begin{array}{l}\text { Very Poor }=0=\text { No } \\
\text { discussion of analysis }\end{array}$ & & \\
\hline \multicolumn{4}{|l|}{ Results: } \\
\hline Questions & Description & Score & Comments \\
\hline \multirow[t]{3}{*}{$\begin{array}{l}\text { Is there a clear } \\
\text { statement of findings? }\end{array}$} & $\begin{array}{l}\text { Good }=3=\text { Findings are } \\
\text { presented logically, are easy } \\
\text { to understand, and relate to } \\
\text { aims. Tables are explained } \\
\text { in text. }\end{array}$ & \multirow[t]{3}{*}{1.5} & \multirow{3}{*}{$\begin{array}{l}\text { The study was } \\
\text { investigating the most } \\
\text { common motivations } \\
\text { for perpetration. Thus, } \\
\text { findings should have } \\
\text { been presented in the } \\
\text { order of most to least } \\
\text { common. This was not } \\
\text { done in text or in the } \\
\text { table. }\end{array}$} \\
\hline & $\begin{array}{l}\text { Fair }=2=\text { Findings } \\
\text { mentioned, but more } \\
\text { explanation could be given. }\end{array}$ & & \\
\hline & $\begin{array}{l}\text { Poor }=1=\text { Findings are } \\
\text { presented haphazardly, not } \\
\text { explained, or do not relate to } \\
\text { aims. }\end{array}$ & & \\
\hline
\end{tabular}




\begin{tabular}{|c|c|c|c|}
\hline & $\begin{array}{l}\text { Very Poor }=0=\text { Not } \\
\text { mentioned or do not relate } \\
\text { to aims. }\end{array}$ & & \\
\hline \multirow[t]{2}{*}{ Limitations discussed? } & Yes $=3$ & \multirow[t]{2}{*}{3} & \multirow{2}{*}{$\begin{array}{l}\text { These include: } \\
\text { Retrospective reporting } \\
\text { self-report, use of } \\
\text { acontextual tool (CTS2) }\end{array}$} \\
\hline & No $=0$ & & \\
\hline \multicolumn{4}{|c|}{ Implications/ Applications: } \\
\hline Questions & Description & Score & Comments \\
\hline \multirow[t]{4}{*}{$\begin{array}{l}\text { How important are } \\
\text { these findings to policy/ } \\
\text { practice? } \\
\text { Does the research offer } \\
\text { a valuable contribution } \\
\text { to the literature? }\end{array}$} & $\begin{array}{l}\text { Good }=3=\text { Contributes } \\
\text { something new/ different in } \\
\text { terms of insight or } \\
\text { perspective. Suggests ideas } \\
\text { for future research. Suggests } \\
\text { implication for policy and/ } \\
\text { or practice. }\end{array}$ & \multirow[t]{4}{*}{2} & \multirow[t]{4}{*}{$\begin{array}{l}\text { Treatment implications } \\
\text { and suggestions for } \\
\text { future research } \\
\text { discussed. }\end{array}$} \\
\hline & $\begin{array}{l}\text { Fair }=2=\text { Two of the above } \\
\text { (State in comments). }\end{array}$ & & \\
\hline & $\begin{array}{l}\text { Poor }=1=\text { Only one of the } \\
\text { above (State in comments). }\end{array}$ & & \\
\hline & $\begin{array}{l}\text { Very Poor }=0=\text { None of the } \\
\text { above }\end{array}$ & & \\
\hline \multicolumn{2}{|l|}{ Total Score: } & $\begin{array}{c}10.5 / 18 \\
58 \%\end{array}$ & \\
\hline
\end{tabular}

\begin{tabular}{|c|c|c|c|}
\hline Study 23 - Title: & \multicolumn{3}{|c|}{$\begin{array}{l}\text { Behavioral and psychological differences among abused women } \\
\text { who use violence in intimate relationships }\end{array}$} \\
\hline Author (Year): & \multicolumn{3}{|c|}{ Swan, S. C., \& Snow, D. L. (2003) } \\
\hline \multicolumn{4}{|l|}{ Sampling: } \\
\hline Questions & Description & Score & Comments \\
\hline \multirow[t]{2}{*}{$\begin{array}{l}\text { Was the sampling } \\
\text { strategy appropriate to } \\
\text { address the research } \\
\text { question/s? } \\
\text { Why was this group } \\
\text { targeted? } \\
\text { Were the participants } \\
\text { representative of the } \\
\text { target population? }\end{array}$} & $\begin{array}{l}\text { Good }=3=\text { Details (age/ } \\
\text { gender/ ethnicity /context) of } \\
\text { who was studied and how } \\
\text { they were recruited. } \\
\text { Rationale for studying this } \\
\text { population. Response rates } \\
\text { shown and explained (why } \\
\text { certain individuals chose not } \\
\text { to participate, and any } \\
\text { attempts to achieve a sample } \\
\text { that represents the target } \\
\text { population). }\end{array}$ & \multirow[t]{2}{*}{1.5} & \multirow[t]{2}{*}{$\begin{array}{l}\text { Details of sample } \\
\text { provided. No attempt to } \\
\text { ensure the sample was } \\
\text { representative. Response } \\
\text { rate not reported. } \\
\text { Rationale for studying } \\
\text { this population not } \\
\text { provided. }\end{array}$} \\
\hline & $\begin{array}{l}\text { Fair }=2=\text { Most information } \\
\text { given, but some missing. }\end{array}$ & & \\
\hline
\end{tabular}




\begin{tabular}{|c|c|c|c|}
\hline & $\begin{array}{l}\text { Poor }=1=\text { Sampling } \\
\text { mentioned but few } \\
\text { descriptive details. } \\
\text { Very Poor }=0=\text { No details } \\
\text { of sample. }\end{array}$ & & \\
\hline \multirow{3}{*}{$\begin{array}{l}\text { Reporting of power or a } \\
\text { priori power analysis } \\
\text { conducted? } \\
\text { Does the study have } \geq \\
80 \% \text { power to detect an } \\
\text { effect at } r=.20 \text { ? }\end{array}$} & $\begin{array}{l}\text { Good }=3=\text { Two of the above } \\
\text { (State in comments). }\end{array}$ & \multirow[t]{3}{*}{0} & \multirow{3}{*}{$\begin{array}{l}\text { Low power was } \\
\text { discussed as a limitation } \\
\text { of the study. However, } \\
\text { an a priori power } \\
\text { analysis was not } \\
\text { conducted. } \\
\text { The study has }<80 \% \\
\text { power to detect an effect } \\
\text { at } r=.20\end{array}$} \\
\hline & $\begin{array}{l}\text { Poor }=1.5=\text { Only one of the } \\
\text { above (State in comments). }\end{array}$ & & \\
\hline & $\begin{array}{l}\text { Very Poor }=0=\text { None of the } \\
\text { above }\end{array}$ & & \\
\hline \multicolumn{4}{|l|}{ Method: } \\
\hline Questions & Description & Score & Comments \\
\hline \multirow{4}{*}{$\begin{array}{l}\text { Has IPA been clearly } \\
\text { defined? } \\
\text { Have motivations been } \\
\text { clearly defined? }\end{array}$} & $\begin{array}{l}\text { Good = } 3=\text { Clear description } \\
\text { of both IPA and motivations. }\end{array}$ & \multirow[t]{4}{*}{0} & \multirow[t]{4}{*}{$\begin{array}{l}\text { Neither IPA nor } \\
\text { motivations defined }\end{array}$} \\
\hline & $\begin{array}{l}\text { Fair }=2=\text { Clear description } \\
\text { of one variable, but } \\
\text { inadequate description of the } \\
\text { other. }\end{array}$ & & \\
\hline & $\begin{array}{l}\text { Poor }=1=\text { Inadequate } \\
\text { descriptions of both } \\
\text { variables. }\end{array}$ & & \\
\hline & $\begin{array}{l}\text { Very Poor }=0=\text { No } \\
\text { description of either variable. }\end{array}$ & & \\
\hline \multirow[t]{4}{*}{$\begin{array}{l}\text { Are the measurements } \\
\text { used appropriate? }\end{array}$} & $\begin{array}{l}\text { Good }=3=\text { Validated and } \\
\text { reliability tested measures of } \\
\text { the outcome are used, or } \\
\text { measures are pre-tested prior } \\
\text { to data collection. }\end{array}$ & \multirow[t]{4}{*}{1.5} & \multirow{4}{*}{$\begin{array}{l}\text { The reliability and } \\
\text { validity of the CTS } 2 \text { was } \\
\text { mentioned and the } \\
\text { internal consistency } \\
\text { score for the current } \\
\text { study provided. } \\
\text { The measure used to } \\
\text { assess motivations was } \\
\text { not validated or piloted } \\
\text { prior to its use in the } \\
\text { study. No information } \\
\text { was provided as to the } \\
\text { source of the items. The } \\
\text { number of items in the } \\
\text { scale was low. }\end{array}$} \\
\hline & $\begin{array}{l}\text { Fair }=2=\text { Appropriate } \\
\text { measures have been used but } \\
\text { have not been described well. }\end{array}$ & & \\
\hline & $\begin{array}{l}\text { Poor }=1=\text { Measures used } \\
\text { are inappropriate/ failure to } \\
\text { report validity and reliability } \\
\text { of measures. }\end{array}$ & & \\
\hline & $\begin{array}{l}\text { Very Poor }=0=\text { No mention } \\
\text { of measures used. }\end{array}$ & & \\
\hline \multicolumn{4}{|l|}{ Data Analysis: } \\
\hline Questions & Description & Score & Comments \\
\hline $\begin{array}{l}\text { Is the statistical analysis } \\
\text { appropriate to answer } \\
\text { the research question }\end{array}$ & $\begin{array}{l}\text { Good }=3=\text { Appropriate } \\
\text { analysis selected. Reasons } \\
\text { for tests clearly stated and }\end{array}$ & 3 & $\begin{array}{l}\text { Studies of this nature } \\
\text { often yield skewed data } \\
\text { making a GLM }\end{array}$ \\
\hline
\end{tabular}




\begin{tabular}{|c|c|c|c|}
\hline \multirow[t]{4}{*}{$\begin{array}{l}\text { and for the type of data } \\
\text { collected? }\end{array}$} & $\begin{array}{l}\text { linked back to the } \\
\text { hypotheses. }\end{array}$ & & \multirow{4}{*}{$\begin{array}{l}\text { inappropriate. However, } \\
\text { as none of the standard } \\
\text { deviations were reported } \\
\text { in this study, it is not } \\
\text { possible to say whether } \\
\text { this is the case. } \\
\text { Assuming the data is } \\
\text { normally distributed, the } \\
\text { analyses are appropriate } \\
\text { and were linked back to } \\
\text { the relevant hypotheses. }\end{array}$} \\
\hline & $\begin{array}{l}\text { Fair }=2=\text { Appropriate } \\
\text { analysis. Some rationale for } \\
\text { the analysis provided. }\end{array}$ & & \\
\hline & $\begin{array}{l}\text { Poor }=1=\text { Appropriate } \\
\text { analysis. No rationale } \\
\text { provided. }\end{array}$ & & \\
\hline & $\begin{array}{l}\text { Very Poor }=0=\text { Analysis } \\
\text { was inappropriate to answer } \\
\text { the research question. }\end{array}$ & & \\
\hline \multirow[t]{4}{*}{$\begin{array}{l}\text { Was the description of } \\
\text { the analysis sufficiently } \\
\text { rigorous? }\end{array}$} & $\begin{array}{l}\text { Good }=3=\text { Clear description } \\
\text { of how analysis was done. } \\
\text { Statistical significance } \\
\text { discussed. }\end{array}$ & \multirow[t]{4}{*}{2} & \multirow{4}{*}{$\begin{array}{l}\text { Greater detail could } \\
\text { have been provided e.g., } \\
\text { the name of the } \\
\text { univariate tests } \\
\text { performed, the software } \\
\text { used to conduct } \\
\text { analyses. Statistical } \\
\text { significance was } \\
\text { discussed. }\end{array}$} \\
\hline & $\begin{array}{l}\text { Fair }=2=\text { Some details about } \\
\text { the analysis provided. }\end{array}$ & & \\
\hline & $\begin{array}{l}\text { Poor }=1=\text { Minimal details } \\
\text { about analysis provided. }\end{array}$ & & \\
\hline & $\begin{array}{l}\text { Very Poor }=0=\text { No } \\
\text { discussion of analysis }\end{array}$ & & \\
\hline \multicolumn{4}{|l|}{ Results: } \\
\hline Questions & Description & Score & Comments \\
\hline \multirow[t]{4}{*}{$\begin{array}{l}\text { Is there a clear statement } \\
\text { of findings? }\end{array}$} & $\begin{array}{l}\text { Good }=3=\text { Findings are } \\
\text { presented logically, are easy } \\
\text { to understand, and relate to } \\
\text { aims. Tables are explained in } \\
\text { text. }\end{array}$ & \multirow[t]{4}{*}{3} & \multirow[t]{4}{*}{$\begin{array}{l}\text { Findings are presented } \\
\text { in relation the } \\
\text { hypotheses. Tables are } \\
\text { explained in text. }\end{array}$} \\
\hline & $\begin{array}{l}\text { Fair }=2=\text { Findings } \\
\text { mentioned, but more } \\
\text { explanation could be given. }\end{array}$ & & \\
\hline & $\begin{array}{l}\text { Poor }=1=\text { Findings are } \\
\text { presented haphazardly, not } \\
\text { explained, or do not relate to } \\
\text { aims. }\end{array}$ & & \\
\hline & $\begin{array}{l}\text { Very Poor }=0=\text { Not } \\
\text { mentioned or do not relate to } \\
\text { aims. }\end{array}$ & & \\
\hline \multirow[t]{2}{*}{ Limitations discussed? } & Yes $=3$ & \multirow[t]{2}{*}{3} & \multirow{2}{*}{$\begin{array}{l}\text { These include: Small } \\
\text { number of participants } \\
\text { in each group, ambiguity } \\
\text { of motive items. }\end{array}$} \\
\hline & $\mathrm{No}=0$ & & \\
\hline \multicolumn{4}{|c|}{ Implications/ Applications: } \\
\hline Questions & Description & Score & Comments \\
\hline $\begin{array}{l}\text { How important are these } \\
\text { findings to policy/ } \\
\text { practice? }\end{array}$ & $\begin{array}{l}\text { Good }=3=\text { Contributes } \\
\text { something new/ different in } \\
\text { terms of insight or } \\
\text { perspective. Suggests ideas }\end{array}$ & 1 & $\begin{array}{l}\text { Future research briefly } \\
\text { alluded to. }\end{array}$ \\
\hline
\end{tabular}




\begin{tabular}{|c|c|c|c|}
\hline \multirow[t]{4}{*}{$\begin{array}{l}\text { Does the research offer } \\
\text { a valuable contribution } \\
\text { to the literature? }\end{array}$} & $\begin{array}{l}\text { for future research. Suggests } \\
\text { implication for policy and/ or } \\
\text { practice. }\end{array}$ & & \\
\hline & $\begin{array}{l}\text { Fair }=2=\text { Two of the above } \\
\text { (State in comments). }\end{array}$ & & \\
\hline & $\begin{array}{l}\text { Poor }=1=\text { Only one of the } \\
\text { above (State in comments). }\end{array}$ & & \\
\hline & $\begin{array}{l}\text { Very Poor }=0=\text { None of the } \\
\text { above }\end{array}$ & & \\
\hline \multicolumn{2}{|l|}{ Total Score: } & $\begin{array}{l}15 / 27 \\
56 \%\end{array}$ & \\
\hline
\end{tabular}

\begin{tabular}{|c|c|c|c|}
\hline Study 24 - Title: & \multicolumn{3}{|c|}{$\begin{array}{l}\text { Motivations and justifications for partner aggression in a sample } \\
\text { of African American college women }\end{array}$} \\
\hline Author (Year): & \multicolumn{3}{|c|}{ Walley-Jean, J. C., \& Swan, S. (2009) } \\
\hline \multicolumn{4}{|l|}{ Sampling: } \\
\hline Questions & Description & Score & Comments \\
\hline \multirow[t]{4}{*}{$\begin{array}{l}\text { Was the sampling } \\
\text { strategy appropriate to } \\
\text { address the research } \\
\text { question/s? } \\
\text { Why was this group } \\
\text { targeted? } \\
\text { Were the participants } \\
\text { representative of the } \\
\text { target population? }\end{array}$} & $\begin{array}{l}\text { Good }=3=\text { Details (age/ } \\
\text { gender/ ethnicity /context) of } \\
\text { who was studied and how } \\
\text { they were recruited. } \\
\text { Rationale for studying this } \\
\text { population. Response rates } \\
\text { shown and explained (why } \\
\text { certain individuals chose not } \\
\text { to participate, and any } \\
\text { attempts to achieve a sample } \\
\text { that represents the target } \\
\text { population). }\end{array}$ & \multirow[t]{4}{*}{3} & \multirow[t]{4}{*}{$\begin{array}{l}\text { The focus of this study } \\
\text { was on African } \\
\text { Americans. Thus, } \\
\text { ethnicity and } \\
\text { representativeness are } \\
\text { not applicable here. } \\
\text { Good rationale for } \\
\text { studying this population. } \\
\text { Response rate not } \\
\text { reported. }\end{array}$} \\
\hline & $\begin{array}{l}\text { Fair }=2=\text { Most information } \\
\text { given, but some missing. }\end{array}$ & & \\
\hline & $\begin{array}{l}\text { Poor }=1=\text { Sampling } \\
\text { mentioned but few } \\
\text { descriptive details. }\end{array}$ & & \\
\hline & $\begin{array}{l}\text { Very Poor }=0=\text { No details } \\
\text { of sample. }\end{array}$ & & \\
\hline \multirow{3}{*}{$\begin{array}{l}\text { Reporting of power or a } \\
\text { priori power analysis } \\
\text { conducted? } \\
\text { Does the study have } \geq \\
80 \% \text { power to detect an } \\
\text { effect at } r=.20 \text { ? }\end{array}$} & $\begin{array}{l}\text { Good }=3=\text { Two of the } \\
\text { above (State in comments). }\end{array}$ & \multirow[t]{3}{*}{0} & \multirow{3}{*}{$\begin{array}{l}\text { No reporting of power or } \\
\text { a priori power analysis } \\
\text { conducted. } \\
\text { The study has }<80 \% \\
\text { power to detect an effect } \\
\text { at } r=.20\end{array}$} \\
\hline & $\begin{array}{l}\text { Poor }=1.5=\text { Only one of the } \\
\text { above (State in comments). }\end{array}$ & & \\
\hline & $\begin{array}{l}\text { Very Poor }=0=\text { None of the } \\
\text { above }\end{array}$ & & \\
\hline
\end{tabular}




\begin{tabular}{|c|c|c|c|}
\hline Questions & Description & Score & Comments \\
\hline \multirow{4}{*}{$\begin{array}{l}\text { Has IPA been clearly } \\
\text { defined? } \\
\text { Have motivations been } \\
\text { clearly defined? }\end{array}$} & $\begin{array}{l}\text { Good }=3=\text { Clear description } \\
\text { of both IPA and motivations. }\end{array}$ & \multirow[t]{4}{*}{0} & \multirow[t]{4}{*}{ Neither variable defined. } \\
\hline & $\begin{array}{l}\text { Fair }=2=\text { Clear description } \\
\text { of one variable, but } \\
\text { inadequate description of the } \\
\text { other. }\end{array}$ & & \\
\hline & $\begin{array}{l}\text { Poor }=1=\text { Inadequate } \\
\text { descriptions of both } \\
\text { variables. }\end{array}$ & & \\
\hline & $\begin{array}{l}\text { Very Poor }=0=\text { No } \\
\text { description of either } \\
\text { variable. }\end{array}$ & & \\
\hline \multirow[t]{4}{*}{$\begin{array}{l}\text { Are the measurements } \\
\text { used appropriate? }\end{array}$} & $\begin{array}{l}\text { Good }=3=\text { Validated and } \\
\text { reliability tested measures of } \\
\text { the outcome are used, or } \\
\text { measures are pre-tested prior } \\
\text { to data collection. }\end{array}$ & \multirow[t]{4}{*}{2} & \multirow{4}{*}{$\begin{array}{l}\text { The reliability and } \\
\text { validity of the CTS } 2 \text { was } \\
\text { mentioned and the } \\
\text { internal consistency } \\
\text { score for the current } \\
\text { study provided. } \\
\text { No psychometric } \\
\text { information was } \\
\text { provided for the MEQ. }\end{array}$} \\
\hline & $\begin{array}{l}\text { Fair }=2=\text { Appropriate } \\
\text { measures have been used but } \\
\text { have not been described } \\
\text { well. }\end{array}$ & & \\
\hline & $\begin{array}{l}\text { Poor }=1=\text { Measures used } \\
\text { are inappropriate/ failure to } \\
\text { report validity and reliability } \\
\text { of measures. }\end{array}$ & & \\
\hline & $\begin{array}{l}\text { Very Poor }=0=\text { No mention } \\
\text { of measures used. }\end{array}$ & & \\
\hline \multicolumn{4}{|l|}{ Data Analysis: } \\
\hline Questions & Description & Score & Comments \\
\hline \multirow[t]{4}{*}{$\begin{array}{l}\text { Is the statistical analysis } \\
\text { appropriate to answer } \\
\text { the research question } \\
\text { and for the type of data } \\
\text { collected? }\end{array}$} & $\begin{array}{l}\text { Good = } 3=\text { Appropriate } \\
\text { analysis selected. Reasons } \\
\text { for tests clearly stated and } \\
\text { linked back to the } \\
\text { hypotheses. }\end{array}$ & \multirow[t]{4}{*}{0} & \multirow{4}{*}{$\begin{array}{l}\text { PCA was conducted to } \\
\text { investigate the factor } \\
\text { structure. This is a } \\
\text { technique for data } \\
\text { reduction. An EFA } \\
\text { should have been } \\
\text { performed. The means } \\
\text { and standard deviations } \\
\text { suggest the data is } \\
\text { skewed. Thus, a Poisson } \\
\text { regression would have } \\
\text { been more appropriate }\end{array}$} \\
\hline & $\begin{array}{l}\text { Fair }=2=\text { Appropriate } \\
\text { analysis. Some rationale for } \\
\text { the analysis provided. }\end{array}$ & & \\
\hline & $\begin{array}{l}\text { Poor }=1=\text { Appropriate } \\
\text { analysis. No rationale } \\
\text { provided. }\end{array}$ & & \\
\hline & $\begin{array}{l}\text { Very Poor }=0=\text { Analysis } \\
\text { was inappropriate to answer } \\
\text { the research question. }\end{array}$ & & \\
\hline $\begin{array}{l}\text { Was the description of } \\
\text { the analysis sufficiently } \\
\text { rigorous? }\end{array}$ & $\begin{array}{l}\text { Good }=3=\text { Clear description } \\
\text { of how analysis was done. } \\
\text { Statistical significance } \\
\text { discussed. }\end{array}$ & 2 & $\begin{array}{l}\text { More information } \\
\text { regarding the PCA } \\
\text { would have been }\end{array}$ \\
\hline
\end{tabular}




\begin{tabular}{|c|c|c|c|}
\hline & $\begin{array}{l}\text { Fair }=2=\text { Some details } \\
\text { about the analysis provided. }\end{array}$ & & \multirow[t]{3}{*}{$\begin{array}{l}\text { beneficial e.g., pattern } \\
\text { matrix }\end{array}$} \\
\hline & $\begin{array}{l}\text { Poor }=1=\text { Minimal details } \\
\text { about analysis provided. }\end{array}$ & & \\
\hline & $\begin{array}{l}\text { Very Poor }=0=\text { No } \\
\text { discussion of analysis }\end{array}$ & & \\
\hline \multicolumn{4}{|l|}{ Results: } \\
\hline Questions & Description & Score & Comments \\
\hline \multirow[t]{4}{*}{$\begin{array}{l}\text { Is there a clear } \\
\text { statement of findings? }\end{array}$} & $\begin{array}{l}\text { Good }=3=\text { Findings are } \\
\text { presented logically, are easy } \\
\text { to understand, and relate to } \\
\text { aims. Tables are explained in } \\
\text { text. }\end{array}$ & \multirow[t]{4}{*}{3} & \multirow[t]{4}{*}{$\begin{array}{l}\text { The findings are clearly } \\
\text { described and the tables } \\
\text { are referred to in the text. }\end{array}$} \\
\hline & $\begin{array}{l}\text { Fair }=2=\text { Findings } \\
\text { mentioned, but more } \\
\text { explanation could be given. }\end{array}$ & & \\
\hline & $\begin{array}{l}\text { Poor }=1=\text { Findings are } \\
\text { presented haphazardly, not } \\
\text { explained, or do not relate to } \\
\text { aims. }\end{array}$ & & \\
\hline & $\begin{array}{l}\text { Very Poor }=0=\text { Not } \\
\text { mentioned or do not relate to } \\
\text { aims. }\end{array}$ & & \\
\hline \multirow[t]{2}{*}{ Limitations discussed? } & Yes $=3$ & \multirow[t]{2}{*}{3} & \multirow{2}{*}{$\begin{array}{l}\text { These include: } \\
\text { Reliability of the } \\
\text { measures used, self- } \\
\text { selection }\end{array}$} \\
\hline & $\mathrm{No}=0$ & & \\
\hline \multicolumn{4}{|c|}{ Implications/ Applications: } \\
\hline Questions & Description & Score & Comments \\
\hline \multirow[t]{4}{*}{$\begin{array}{l}\text { How important are these } \\
\text { findings to policy/ } \\
\text { practice? } \\
\text { Does the research offer } \\
\text { a valuable contribution } \\
\text { to the literature? }\end{array}$} & $\begin{array}{l}\text { Good }=3=\text { Contributes } \\
\text { something new/ different in } \\
\text { terms of insight or } \\
\text { perspective. Suggests ideas } \\
\text { for future research. Suggests } \\
\text { implication for policy and/ } \\
\text { or practice. }\end{array}$ & \multirow[t]{4}{*}{1} & \multirow[t]{4}{*}{$\begin{array}{l}\text { Investigated motivations } \\
\text { for IPA in African } \\
\text { American women. }\end{array}$} \\
\hline & $\begin{array}{l}\text { Fair }=2=\text { Two of the above } \\
\text { (State in comments). }\end{array}$ & & \\
\hline & $\begin{array}{l}\text { Poor }=1=\text { Only one of the } \\
\text { above (State in comments). }\end{array}$ & & \\
\hline & $\begin{array}{l}\text { Very Poor }=0=\text { None of the } \\
\text { above }\end{array}$ & & \\
\hline \multicolumn{2}{|l|}{ Total Score: } & $\begin{array}{l}14 / 27 \\
52 \% \\
\end{array}$ & \\
\hline
\end{tabular}




\section{APPENDIX M}

\section{Coding of Motivations}

\begin{tabular}{|c|c|c|c|c|c|c|}
\hline Study & Study Label & Item & Coder 1 & Coder 2 & Coder 3 & Review/ Meta Label \\
\hline Babcock et al., 2003 & Self-defense & $\begin{array}{l}\text { My partner is } \\
\text { physically aggressive } \\
\text { towards me first }\end{array}$ & Self-defense & Self-defense & Self-defense & Self-defense \\
\hline \multirow[t]{7}{*}{ Barnett et al., 1997} & Teasing your partner & $\begin{array}{l}\text { You were teasing } \\
\text { your partner }\end{array}$ & Teasing your partner & Playfulness & Out of playfulness & Playfulness \\
\hline & $\begin{array}{l}\text { Letting out violent } \\
\text { feelings }\end{array}$ & $\begin{array}{l}\text { You were letting out } \\
\text { your violent feelings }\end{array}$ & $\begin{array}{l}\text { Expressing negative } \\
\text { emotion/Emotional } \\
\text { regulation difficulties }\end{array}$ & $\begin{array}{l}\text { Expression of } \\
\text { negative emotions }\end{array}$ & $\begin{array}{l}\text { Unable to } \\
\text { communicate feelings }\end{array}$ & $\begin{array}{l}\text { Emotional regulation } \\
\text { difficulties }\end{array}$ \\
\hline & $\begin{array}{l}\text { Teaching partner a } \\
\text { lesson }\end{array}$ & $\begin{array}{l}\text { You were teaching } \\
\text { your partner a lesson }\end{array}$ & $\begin{array}{l}\text { Teaching partner a } \\
\text { lesson }\end{array}$ & Asserting dominance & Punish partner & Punish partner \\
\hline & $\begin{array}{l}\text { Trying to upset } \\
\text { partner emotionally }\end{array}$ & $\begin{array}{l}\text { You were trying to } \\
\text { upset your partner } \\
\text { emotionally }\end{array}$ & Emotional abuse & Asserting dominance & $\begin{array}{l}\text { Emotional } \\
\text { manipulation/trying to } \\
\text { upset partner }\end{array}$ & $\begin{array}{l}\text { Emotionally hurt } \\
\text { partner }\end{array}$ \\
\hline & $\begin{array}{l}\text { Showing partner who } \\
\text { was boss }\end{array}$ & $\begin{array}{l}\text { You were showing } \\
\text { partner who was } \\
\text { boss }\end{array}$ & Dominance & Asserting dominance & Dominance & Asserting dominance \\
\hline & $\begin{array}{l}\text { Protecting yourself } \\
\text { from physical harm }\end{array}$ & $\begin{array}{l}\text { You were protecting } \\
\text { yourself from } \\
\text { physical harm }\end{array}$ & Self-defense & Self-defense & Self-defense & Self-defense \\
\hline & $\begin{array}{l}\text { Trying to get } \\
\text { partners attention }\end{array}$ & $\begin{array}{l}\text { You were trying to } \\
\text { get your partner's } \\
\text { attention }\end{array}$ & Attention seeking & Attention seeking & To get attention & To get attention \\
\hline Belus et al., 2014 & Jealousy & $\begin{array}{l}\text { X works very closely } \\
\text { with a member of the } \\
\text { opposite sex (in } \\
\text { school or office). } \\
\text { Scale is from very } \\
\text { pleased to very } \\
\text { displeased }\end{array}$ & Jealousy & Jealousy & Jealousy & Jealousy \\
\hline
\end{tabular}




\begin{tabular}{|c|c|c|c|c|c|c|}
\hline Study & Study Label & Item & Coder 1 & Coder 2 & Coder 3 & Review/ Meta Label \\
\hline $\begin{array}{c}\text { Belus et al.cont., } \\
2014\end{array}$ & Anger & $\begin{array}{l}\text { I get angry when } \\
\text { someone lets me } \\
\text { down }\end{array}$ & Anger & Anger & Anger & Expression of anger \\
\hline \multirow[t]{5}{*}{ Caldwell et al., 2009} & $\begin{array}{l}\text { Expression of } \\
\text { negative emotions }\end{array}$ & $\begin{array}{l}\text { Because you were } \\
\text { frustrated }\end{array}$ & $\begin{array}{l}\text { Expression of negative } \\
\text { emotions/ Emotional } \\
\text { regulation difficulties }\end{array}$ & $\begin{array}{l}\text { Expression of } \\
\text { negative emotions }\end{array}$ & $\begin{array}{l}\text { Expression of negative } \\
\text { emotions }\end{array}$ & $\begin{array}{l}\text { Emotional regulation } \\
\text { difficulties }\end{array}$ \\
\hline & Self-defense & $\begin{array}{l}\text { To defend yourself } \\
\text { from your partner }\end{array}$ & Self-defense & Self-defense & Self-defense & Self-defense \\
\hline & Control & To feel in control & Control & Control & Control & Control \\
\hline & Jealousy & $\begin{array}{l}\text { Because you thought } \\
\text { your partner was } \\
\text { unfaithful }\end{array}$ & Jealousy & Jealousy & Jealousy & Jealousy \\
\hline & Tough guise & $\begin{array}{l}\text { To intimidate your } \\
\text { partner }\end{array}$ & $\begin{array}{l}\text { Dominance/ status/ } \\
\text { empowerment }\end{array}$ & Asserting dominance & Dominance & Asserting dominance \\
\hline \multirow[t]{6}{*}{ Carrado et al., 1996} & $\begin{array}{l}\text { Get through to my } \\
\text { partner }\end{array}$ & $\begin{array}{l}\text { I thought it was the } \\
\text { only way to get } \\
\text { through to him/ her }\end{array}$ & $\begin{array}{l}\text { Communication } \\
\text { difficulties }\end{array}$ & $\begin{array}{l}\text { Communication } \\
\text { difficulties }\end{array}$ & $\begin{array}{l}\text { Communication } \\
\text { difficulties }\end{array}$ & $\begin{array}{l}\text { Communication } \\
\text { difficulties }\end{array}$ \\
\hline & $\begin{array}{l}\text { Retaliation for verbal } \\
\text { abuse/ threat }\end{array}$ & $\begin{array}{l}\text { I was getting back at } \\
\text { him/ her for } \\
\text { something nasty he/ } \\
\text { she said }\end{array}$ & Retaliation & Retaliation & $\begin{array}{l}\text { Retaliation (against } \\
\text { verbal abuse) }\end{array}$ & Retaliation (verbal) \\
\hline & $\begin{array}{l}\text { Retaliation for } \\
\text { physical abuse }\end{array}$ & $\begin{array}{l}\text { I was getting back at } \\
\text { him/ her for some } \\
\text { physical action he/ } \\
\text { she had used against } \\
\text { me }\end{array}$ & $\begin{array}{l}\text { Retaliation (against } \\
\text { physical violence) }\end{array}$ & $\begin{array}{l}\text { Retaliation for } \\
\text { physical abuse }\end{array}$ & $\begin{array}{l}\text { Retaliation (against } \\
\text { physical abuse) }\end{array}$ & Retaliation (physical) \\
\hline & $\begin{array}{l}\text { Stop my partner } \\
\text { doing something }\end{array}$ & $\begin{array}{l}\text { To stop him/ her } \\
\text { doing something }\end{array}$ & Control & Control & Control & Control \\
\hline & $\begin{array}{l}\text { Make my partner do } \\
\text { what I wanted }\end{array}$ & $\begin{array}{l}\text { To make him/ her do } \\
\text { what I wanted }\end{array}$ & Control & Control & Asserting dominance & Control \\
\hline & Self-defense & $\begin{array}{l}\text { I thought he/ she was } \\
\text { about to use a } \\
\text { physical action } \\
\text { against me }\end{array}$ & Self-defense & Self-defense & Self-defense & Self-defense \\
\hline
\end{tabular}




\begin{tabular}{|c|c|c|c|c|c|c|}
\hline Study & Study Label & Item & Coder 1 & Coder 2 & Coder 3 & Review/ Meta Label \\
\hline \multirow[t]{2}{*}{$\begin{array}{l}\text { DeKeseredy et al., } \\
1997\end{array}$} & Self-defense & $\begin{array}{l}\text { What \% of these } \\
\text { times were you } \\
\text { acting in self- } \\
\text { defense, that is } \\
\text { protecting yourself } \\
\text { from immediate } \\
\text { harm? }\end{array}$ & Self-defense & Self-defense & Self-defense & Self-defense \\
\hline & Fighting back & $\begin{array}{l}\text { What } \% \text { of these } \\
\text { times were you } \\
\text { trying to fight back } \\
\text { in a situation where } \\
\text { you were not the } \\
\text { first to use these or } \\
\text { similar tactics? }\end{array}$ & Retaliation & Retaliation & Retaliation & Retaliation (physical) \\
\hline \multirow[t]{6}{*}{ Elmquist et al., 2014} & Power/ control & $\begin{array}{l}\text { To get your partner } \\
\text { to do something or } \\
\text { to stop your partner } \\
\text { from doing } \\
\text { something (e.g., } \\
\text { going out with } \\
\text { friends) }\end{array}$ & Control & Control & Control & Control \\
\hline & Self-defense & $\begin{array}{l}\text { To protect yourself } \\
\text { (e.g., self-defense) }\end{array}$ & Self-defense & Self-defense & Self-defense & Self-defense \\
\hline & Jealousy & $\begin{array}{l}\text { Because you were } \\
\text { jealous }\end{array}$ & Jealousy & Jealousy & \begin{tabular}{|l} 
Jealousy \\
\end{tabular} & Jealousy \\
\hline & $\begin{array}{l}\text { Communication } \\
\text { difficulties }\end{array}$ & $\begin{array}{l}\text { To get your } \\
\text { partner's attention }\end{array}$ & $\begin{array}{l}\text { Communication } \\
\text { difficulties }\end{array}$ & $\begin{array}{l}\text { Unable to } \\
\text { communicate } \\
\text { feelings }\end{array}$ & $\begin{array}{l}\text { Communication } \\
\text { difficulties }\end{array}$ & $\begin{array}{l}\text { Communication } \\
\text { difficulties }\end{array}$ \\
\hline & $\begin{array}{l}\text { Expression of } \\
\text { negative emotions }\end{array}$ & $\begin{array}{l}\text { Because you were } \\
\text { afraid your partner } \\
\text { was going to leave } \\
\text { you }\end{array}$ & $\begin{array}{l}\text { Expression of negative } \\
\text { emotions }\end{array}$ & $\begin{array}{l}\text { Expression of } \\
\text { negative emotions }\end{array}$ & $\begin{array}{l}\text { Expression of } \\
\text { negative emotions }\end{array}$ & $\begin{array}{l}\text { Emotional regulation } \\
\text { difficulties }\end{array}$ \\
\hline & Retaliation & $\begin{array}{l}\text { To get back at or to } \\
\text { retaliate for being } \\
\text { emotionally hurt by } \\
\text { your partner }\end{array}$ & Retaliation & Retaliation & Retaliation & $\begin{array}{l}\text { Retaliation } \\
\text { (emotional) }\end{array}$ \\
\hline
\end{tabular}




\begin{tabular}{|c|c|c|c|c|c|c|}
\hline Study & Study Label & Item & Coder 1 & Coder 2 & Coder 3 & Review/ Meta Label \\
\hline $\begin{array}{l}\text { Fernandez-Fuertes } \\
\& \text { Fuertes, } 2010\end{array}$ & Jealousy & $\begin{array}{l}\text { I had a fit of } \\
\text { jealousy }\end{array}$ & Jealousy & Jealousy & Jealousy & Jealousy \\
\hline \multirow[t]{4}{*}{$\begin{array}{l}\text { Fiebert \& Gonzalez, } \\
1997\end{array}$} & $\begin{array}{l}\text { My partner wasn't } \\
\text { sensitive to my needs }\end{array}$ & $\begin{array}{l}\text { My partner wasn't } \\
\text { sensitive to my } \\
\text { needs }\end{array}$ & $\begin{array}{l}\text { Expressing negative } \\
\text { emotion }\end{array}$ & Retaliation & $\begin{array}{l}\text { Communication } \\
\text { difficulties }\end{array}$ & $\begin{array}{l}\text { Emotional regulation } \\
\text { difficulties }\end{array}$ \\
\hline & $\begin{array}{l}\text { Get partner's } \\
\text { attention }\end{array}$ & $\begin{array}{l}\text { I wished to gain my } \\
\text { partner's attention }\end{array}$ & Attention seeking & Attention seeking & Attention seeking & To get attention \\
\hline & $\begin{array}{l}\text { Partner was verbally } \\
\text { abusive }\end{array}$ & $\begin{array}{l}\text { My partner was } \\
\text { being verbally } \\
\text { abusive to me }\end{array}$ & Retaliation & Retaliation & $\begin{array}{l}\text { Retaliation (against } \\
\text { verbal abuse) }\end{array}$ & Retaliation (verbal) \\
\hline & $\begin{array}{l}\text { Partner wasn't } \\
\text { listening to me }\end{array}$ & $\begin{array}{l}\text { My partner was not } \\
\text { listening to me }\end{array}$ & Communication difficulties & $\begin{array}{l}\text { Communication } \\
\text { difficulties }\end{array}$ & Control & $\begin{array}{l}\text { Communication } \\
\text { difficulties }\end{array}$ \\
\hline \multirow{12}{*}{$\begin{array}{l}\text { Follingstad et al., } \\
1991\end{array}$} & To show anger & To show anger & Anger & Express anger & Anger & Expression of anger \\
\hline & $\begin{array}{l}\text { Due to an inability to } \\
\text { express self verbally }\end{array}$ & $\begin{array}{l}\text { Due to an inability } \\
\text { to express self } \\
\text { verbally }\end{array}$ & Communication difficulties & $\begin{array}{l}\text { Communication } \\
\text { difficulties }\end{array}$ & $\begin{array}{l}\text { Communication } \\
\text { difficulties }\end{array}$ & $\begin{array}{l}\text { Communication } \\
\text { difficulties }\end{array}$ \\
\hline & $\begin{array}{l}\text { To feel more } \\
\text { powerful }\end{array}$ & $\begin{array}{l}\text { To feel more } \\
\text { powerful }\end{array}$ & Dominance/empowerment & Asserting dominance & Asserting dominance & Asserting dominance \\
\hline & $\begin{array}{l}\text { To get control over } \\
\text { other person }\end{array}$ & $\begin{array}{l}\text { To get control over } \\
\text { other person }\end{array}$ & Control & Control & Control & Control \\
\hline & $\begin{array}{l}\text { In retaliation for } \\
\text { being hit first }\end{array}$ & $\begin{array}{l}\text { In retaliation for } \\
\text { being hit first }\end{array}$ & $\begin{array}{l}\text { Retaliation (against } \\
\text { physical violence) }\end{array}$ & $\begin{array}{l}\text { Retaliation to } \\
\text { physical abuse }\end{array}$ & $\begin{array}{l}\text { Retaliation (against } \\
\text { physical abuse) }\end{array}$ & $\begin{array}{l}\text { Retaliation } \\
\text { (physical) }\end{array}$ \\
\hline & $\begin{array}{l}\text { To protect self (self- } \\
\text { defense) }\end{array}$ & $\begin{array}{l}\text { To protect self } \\
\text { (self-defense) }\end{array}$ & Self-defense & Self-defense & Self-defense & Self-defense \\
\hline & $\begin{array}{l}\text { In retaliation for } \\
\text { emotional hurt }\end{array}$ & $\begin{array}{l}\text { In retaliation for } \\
\text { emotional hurt }\end{array}$ & Retaliation & Retaliation & $\begin{array}{l}\text { Retaliation (against } \\
\text { verbal abuse) }\end{array}$ & $\begin{array}{l}\text { Retaliation } \\
\text { (emotional) }\end{array}$ \\
\hline & $\begin{array}{l}\text { To punish person for } \\
\text { wrong behaviour }\end{array}$ & $\begin{array}{l}\text { To punish person } \\
\text { for wrong } \\
\text { behaviour }\end{array}$ & Punish partner & Asserting dominance & Punishment/ control & Punish partner \\
\hline & To prove love & To prove love & To prove love & To prove love & To prove love & To prove love \\
\hline & $\begin{array}{l}\text { Because it was } \\
\text { sexually arousing }\end{array}$ & $\begin{array}{l}\text { Because it was } \\
\text { sexually arousing }\end{array}$ & For sexual pleasure & Sexually arousing & Sexual arousal & Sexually arousing \\
\hline & To get attention & To get attention & Attention seeking & Attention seeking & Attention seeking & To get attention \\
\hline & $\begin{array}{l}\text { Anger displaced onto } \\
\text { partner }\end{array}$ & $\begin{array}{l}\text { Anger displaced } \\
\text { onto partner }\end{array}$ & $\begin{array}{l}\text { Anger/Emotion regulation } \\
\text { difficulties }\end{array}$ & $\begin{array}{l}\text { Emotional regulation } \\
\text { difficulties }\end{array}$ & $\begin{array}{l}\text { Anger/Emotion } \\
\text { regulation difficulties }\end{array}$ & $\begin{array}{l}\text { Emotional regulation } \\
\text { difficulties }\end{array}$ \\
\hline
\end{tabular}




\begin{tabular}{|c|c|c|c|c|c|c|}
\hline Study & Study Label & Item & Coder 1 & Coder 2 & Coder 3 & Review/ Meta Label \\
\hline $\begin{array}{l}\text { Follingstad et al., } \\
1991 \text { cont. }\end{array}$ & Because of jealousy & Because of jealousy & Jealousy & Jealousy & Jealousy & Jealousy \\
\hline \multirow[t]{8}{*}{ Harned, 2001} & Anger/ jealousy & To show anger & Anger & Anger & Anger & Expression of anger \\
\hline & Self-defense & To protect self & Retaliation/ self-defense & Self-defense & Self-defense & Self-defense \\
\hline & $\begin{array}{l}\text { Inability to express } \\
\text { self verbally }\end{array}$ & $\begin{array}{l}\text { Inability to express } \\
\text { self verbally }\end{array}$ & $\begin{array}{l}\text { Communication } \\
\text { difficulties }\end{array}$ & $\begin{array}{l}\text { Communication } \\
\text { difficulties }\end{array}$ & $\begin{array}{l}\text { Anger/Emotional } \\
\text { regulation difficulties }\end{array}$ & $\begin{array}{l}\text { Communication } \\
\text { difficulties }\end{array}$ \\
\hline & $\begin{array}{l}\text { To feel more } \\
\text { powerful }\end{array}$ & $\begin{array}{l}\text { To feel more } \\
\text { powerful }\end{array}$ & $\begin{array}{l}\text { Dominance/ } \\
\text { empowerment }\end{array}$ & Asserting dominance & Asserting dominance & Asserting dominance \\
\hline & $\begin{array}{l}\text { To get control over } \\
\text { the other person }\end{array}$ & $\begin{array}{l}\text { To get control over } \\
\text { the other person }\end{array}$ & Control & Control & Control & Control \\
\hline & To prove love & To prove love & To prove love & To prove love & To prove love & To prove love \\
\hline & $\begin{array}{l}\text { Because it is sexually } \\
\text { arousing }\end{array}$ & $\begin{array}{l}\text { Because it is sexually } \\
\text { arousing }\end{array}$ & For sexual pleasure & Sexually arousing & Sexual arousal & Sexually arousing \\
\hline & To get attention & To get attention & Attention seeking & $\begin{array}{l}\text { Communication } \\
\text { difficulties }\end{array}$ & To get attention & To get attention \\
\hline \multirow{6}{*}{$\begin{array}{l}\text { Hettrich \& O’Leary, } \\
2007\end{array}$} & Anger & Anger & Anger & Express anger & Anger & Expression of anger \\
\hline & Partner lied & Partner lied & $\begin{array}{l}\text { Retaliation (against } \\
\text { perceived injustice) }\end{array}$ & $\begin{array}{l}\text { Retaliation for } \\
\text { emotional hurt }\end{array}$ & Punish/Retaliation & $\begin{array}{l}\text { Retaliation } \\
\text { (emotional) }\end{array}$ \\
\hline & Poor communication & Poor communication & $\begin{array}{l}\text { Communication } \\
\text { difficulties }\end{array}$ & $\begin{array}{l}\text { Communication } \\
\text { difficulties }\end{array}$ & $\begin{array}{l}\text { Communication } \\
\text { difficulties }\end{array}$ & $\begin{array}{l}\text { Communication } \\
\text { difficulties }\end{array}$ \\
\hline & Temper & Temper & Anger & Express anger & $\begin{array}{l}\text { Emotional regulation } \\
\text { difficulties }\end{array}$ & Expression of anger \\
\hline & Embarrassed & Embarrassed & Embarrassment & Asserting dominance & Embarrassment & Embarrassed \\
\hline & Jealousy & Jealousy & Jealousy & Jealousy & Jealousy & Jealousy \\
\hline \multirow[t]{3}{*}{ Kelley et al., 2015} & $\begin{array}{l}\text { Emotional } \\
\text { expression/ } \\
\text { dysregulation }\end{array}$ & $\begin{array}{l}\text { Because you were } \\
\text { frustrated }\end{array}$ & $\begin{array}{l}\text { Emotional regulation } \\
\text { difficulties/retaliation to } \\
\text { perceived injustice }\end{array}$ & $\begin{array}{l}\text { Expression of } \\
\text { negative emotions }\end{array}$ & $\begin{array}{l}\text { Emotional } \\
\text { dysregulation }\end{array}$ & $\begin{array}{l}\text { Emotional regulation } \\
\text { difficulties }\end{array}$ \\
\hline & Control/tough guise & $\begin{array}{l}\text { To intimidate your } \\
\text { partner }\end{array}$ & Control/ dominance & Asserting dominance & Intimidation & Asserting dominance \\
\hline & Self-defense & $\begin{array}{l}\text { To get your partner } \\
\text { to stop hitting or } \\
\text { hurting you }\end{array}$ & Self-defense & Self-defense & Self-defense & Self-defense \\
\hline
\end{tabular}




\begin{tabular}{|c|c|c|c|c|c|c|}
\hline Study & Study Label & Item & Coder 1 & Coder 2 & Coder 3 & Review/ Meta Label \\
\hline \multirow[t]{3}{*}{ Kernsmith, 2005} & $\begin{array}{l}\text { Striking back for } \\
\text { abuse }\end{array}$ & $\begin{array}{l}\text { In retaliation for } \\
\text { emotional hurt }\end{array}$ & Retaliation & Retaliation & Retaliation & $\begin{array}{l}\text { Retaliation } \\
\text { (emotional) }\end{array}$ \\
\hline & Discipline partner & To punish partner & Control & Asserting dominance & Punishment/ control & Punish partner \\
\hline & Exerting power & $\begin{array}{l}\text { To feel more } \\
\text { powerful }\end{array}$ & $\begin{array}{l}\text { Dominance/ } \\
\text { empowerment }\end{array}$ & Asserting dominance & Asserting dominance & Asserting dominance \\
\hline \multirow[t]{15}{*}{ Leisring, 2013} & To show anger & To show anger & Anger & Express anger & $\begin{array}{l}\text { Anger/ emotional } \\
\text { regulation difficulties }\end{array}$ & Expression of anger \\
\hline & $\begin{array}{l}\text { Due to an inability to } \\
\text { express self verbally }\end{array}$ & $\begin{array}{l}\text { Due to an inability to } \\
\text { express self verbally }\end{array}$ & $\begin{array}{l}\text { Communication } \\
\text { difficulties }\end{array}$ & $\begin{array}{l}\text { Communication } \\
\text { difficulties }\end{array}$ & $\begin{array}{l}\text { Communication } \\
\text { difficulties }\end{array}$ & $\begin{array}{l}\text { Communication } \\
\text { difficulties }\end{array}$ \\
\hline & $\begin{array}{l}\text { To feel more } \\
\text { powerful }\end{array}$ & $\begin{array}{l}\text { To feel more } \\
\text { powerful }\end{array}$ & Empowerment & Asserting dominance & Asserting dominance & Asserting dominance \\
\hline & $\begin{array}{l}\text { To get control over } \\
\text { the other person }\end{array}$ & $\begin{array}{l}\text { To get control over } \\
\text { the other person }\end{array}$ & Control & Control & Control & Control \\
\hline & $\begin{array}{l}\text { In retaliation for } \\
\text { being hit first }\end{array}$ & $\begin{array}{l}\text { In retaliation for } \\
\text { being hit first }\end{array}$ & Retaliation & $\begin{array}{l}\text { Retaliation to } \\
\text { physical abuse }\end{array}$ & $\begin{array}{l}\text { Retaliation (against } \\
\text { physical abuse) }\end{array}$ & Retaliation (physical) \\
\hline & $\begin{array}{l}\text { To protect self from } \\
\text { immediate physical } \\
\text { harm }\end{array}$ & $\begin{array}{l}\text { To protect self from } \\
\text { immediate physical } \\
\text { harm }\end{array}$ & Self-defense & Self-defense & Self-defense & Self-defense \\
\hline & $\begin{array}{l}\text { In retaliation for } \\
\text { emotional hurt }\end{array}$ & $\begin{array}{l}\text { In retaliation for } \\
\text { emotional hurt }\end{array}$ & Retaliation & $\begin{array}{l}\text { Retaliation for } \\
\text { emotional hurt }\end{array}$ & $\begin{array}{l}\text { Retaliation (against } \\
\text { verbal abuse) }\end{array}$ & $\begin{array}{l}\text { Retaliation } \\
\text { (emotional) }\end{array}$ \\
\hline & $\begin{array}{l}\text { Anger displaced onto } \\
\text { partner }\end{array}$ & $\begin{array}{l}\text { Anger displaced onto } \\
\text { partner }\end{array}$ & $\begin{array}{l}\text { Anger/ emotion } \\
\text { regulation difficulties }\end{array}$ & $\begin{array}{l}\text { Emotional regulation } \\
\text { difficulties }\end{array}$ & $\begin{array}{l}\text { Anger/ emotional } \\
\text { regulation difficulties }\end{array}$ & $\begin{array}{l}\text { Emotional regulation } \\
\text { difficulties }\end{array}$ \\
\hline & $\begin{array}{l}\text { To punish person for } \\
\text { wrong behavior }\end{array}$ & $\begin{array}{l}\text { To punish person for } \\
\text { wrong behavior }\end{array}$ & Punish partner & Asserting dominance & Punishment/ control & Punish partner \\
\hline & To prove love & To prove love & To prove love & To prove love & To prove love & To prove love \\
\hline & $\begin{array}{l}\text { To get partner's } \\
\text { attention }\end{array}$ & $\begin{array}{l}\text { To get partner's } \\
\text { attention }\end{array}$ & Attention seeking & Attention seeking & Attention seeking & To get attention \\
\hline & Because of jealousy & Because of jealousy & Jealousy & Jealousy & Jealousy & Jealousy \\
\hline & Because of stress & Because of stress & Stress & $\begin{array}{l}\text { Emotional regulation } \\
\text { difficulties }\end{array}$ & $\begin{array}{l}\text { Emotional regulation } \\
\text { difficulties }\end{array}$ & $\begin{array}{l}\text { Emotional regulation } \\
\text { difficulties }\end{array}$ \\
\hline & To win an argument & To win an argument & Dominance & Asserting dominance & Asserting dominance & Asserting dominance \\
\hline & To get my way & To get my way & Dominance/ control & Asserting dominance & Control & Control \\
\hline \multirow[t]{2}{*}{ Makepeace, 1986} & Self-defense & Self-defense & Self-defense & Self-defense & Self-defense & Self-defense \\
\hline & To harm & To harm & To harm & To harm & Harm & To harm \\
\hline
\end{tabular}




\begin{tabular}{|c|c|c|c|c|c|c|}
\hline Study & Study Label & Item & Coder 1 & Coder 2 & Coder 3 & Review/ Meta Label \\
\hline \multirow[t]{4}{*}{$\begin{array}{l}\text { Makepeace, } 1986 \\
\text { cont. }\end{array}$} & Retaliate & Retaliate & Retaliation & Retaliation & Retaliate & $\begin{array}{l}\text { Retaliation (not } \\
\text { specified) }\end{array}$ \\
\hline & Intimidate & Intimidate & Asserting dominance & $\begin{array}{l}\text { Intimidation / } \\
\text { asserting dominance }\end{array}$ & Intimidation & Asserting dominance \\
\hline & "Get" something & "Get" something & Instrumental & Control & Control & Control \\
\hline & Uncontrollable anger & Uncontrollable anger & $\begin{array}{l}\text { Anger/Emotion } \\
\text { regulation difficulties }\end{array}$ & $\begin{array}{l}\text { Emotional regulation } \\
\text { difficulties }\end{array}$ & $\begin{array}{l}\text { Emotion regulation } \\
\text { difficulties }\end{array}$ & $\begin{array}{l}\text { Emotional regulation } \\
\text { difficulties }\end{array}$ \\
\hline \multirow[t]{11}{*}{ Neal et al., 2015} & Anger & To show anger & $\begin{array}{l}\text { Anger/ emotion } \\
\text { regulation difficulties }\end{array}$ & Anger & Anger & Expression of anger \\
\hline & Retaliation & $\begin{array}{l}\text { Retaliation for being } \\
\text { hit }\end{array}$ & Retaliation & Retaliation & Retaliate & Retaliation (physical) \\
\hline & Power and control & $\begin{array}{l}\text { To feel more } \\
\text { powerful }\end{array}$ & $\begin{array}{l}\text { Dominance/ } \\
\text { empowerment/ control }\end{array}$ & Asserting dominance & Control & Asserting dominance \\
\hline & Self-defense & Self-defense & Self-defense & Self-defense & Self-defense & Self-defense \\
\hline & Punish partner & Punish partner & $\begin{array}{l}\text { Punish partner/ control/ } \\
\text { retaliation }\end{array}$ & Asserting dominance & Punish partner & Punish partner \\
\hline & To prove love & To prove love & To prove love & To prove love & To prove love & To prove love \\
\hline & Sexual arousal & $\begin{array}{l}\text { Because it is sexually } \\
\text { arousing }\end{array}$ & For sexual pleasure & Sexually arousing & Sexual arousal & Sexually arousing \\
\hline & To get attention & To get attention & $\begin{array}{l}\text { Attention seeking/ } \\
\text { communication } \\
\text { difficulties }\end{array}$ & $\begin{array}{l}\text { Communication } \\
\text { difficulties }\end{array}$ & To get attention & To get attention \\
\hline & Jealousy & Jealousy & Jealousy & Jealousy & Jealousy & Jealousy \\
\hline & $\begin{array}{l}\text { Inability to express } \\
\text { oneself verbally }\end{array}$ & $\begin{array}{l}\text { Inability to express } \\
\text { oneself verbally }\end{array}$ & $\begin{array}{l}\text { Communication } \\
\text { difficulties }\end{array}$ & $\begin{array}{l}\text { Communication } \\
\text { difficulties }\end{array}$ & $\begin{array}{l}\text { Unable to } \\
\text { communicate feelings }\end{array}$ & $\begin{array}{l}\text { Communication } \\
\text { difficulties }\end{array}$ \\
\hline & Loss of control & Loss of control & $\begin{array}{l}\text { Self-regulation } \\
\text { difficulties }\end{array}$ & Loss of control & Loss of control & Loss of control \\
\hline \multirow[t]{3}{*}{ Ross, 2011} & Dominate-Punish & $\begin{array}{l}\text { To feel more } \\
\text { powerful }\end{array}$ & $\begin{array}{l}\text { Domination/ } \\
\text { empowerment/ control/ } \\
\text { retaliation }\end{array}$ & Asserting dominance & Intimidation & Asserting dominance \\
\hline & $\begin{array}{l}\text { Emotion } \\
\text { dysregulation }\end{array}$ & Because of stress & $\begin{array}{l}\text { Emotional regulation } \\
\text { difficulties }\end{array}$ & $\begin{array}{l}\text { Expression of } \\
\text { negative emotions }\end{array}$ & $\begin{array}{l}\text { Emotion } \\
\text { dysregulation }\end{array}$ & $\begin{array}{l}\text { Emotional regulation } \\
\text { difficulties }\end{array}$ \\
\hline & Defence & To protect self & Self-defense & Self-defense & Self-defense & Self-defense \\
\hline
\end{tabular}




\begin{tabular}{|c|c|c|c|c|c|c|}
\hline Study & Study Label & Item & Coder 1 & Coder 2 & Coder 3 & Review/ Meta Label \\
\hline Ross, 2011 cont. & Retaliate & $\begin{array}{l}\text { To get back at or to } \\
\text { retaliate for being } \\
\text { emotionally hurt by } \\
\text { your partner }\end{array}$ & $\begin{array}{l}\text { Retaliation/ emotional } \\
\text { regulation difficulties }\end{array}$ & Retaliation & Retaliate & $\begin{array}{l}\text { Retaliation } \\
\text { (emotional) }\end{array}$ \\
\hline \multirow[t]{2}{*}{ Saunders, 1986} & Self-defense & $\begin{array}{l}\text { What \% of these } \\
\text { times were you } \\
\text { acting in self- } \\
\text { defense, that is, } \\
\text { protecting yourself } \\
\text { from immediate } \\
\text { physical harm? }\end{array}$ & Self-defense & Self-defense & Self-defense & Self-defense \\
\hline & Fighting back & $\begin{array}{l}\text { What } \% \text { of these } \\
\text { times were you } \\
\text { trying to fight back? }\end{array}$ & Retaliation & Retaliation & Retaliation & Retaliation (physical) \\
\hline \multirow[t]{12}{*}{ Shorey et al., 2010} & To protect self & To protect self & Self-defense & Self-defense & Self-defense & Self-defense \\
\hline & Anger & Anger & Anger & Anger & Anger & Expression of anger \\
\hline & $\begin{array}{l}\text { Inability to express } \\
\text { verbally }\end{array}$ & $\begin{array}{l}\text { Show feelings that } \\
\text { cannot be expressed } \\
\text { in words }\end{array}$ & $\begin{array}{l}\text { Communication } \\
\text { difficulties }\end{array}$ & $\begin{array}{l}\text { Communication } \\
\text { difficulties }\end{array}$ & $\begin{array}{l}\text { Unable to } \\
\text { communicate feelings }\end{array}$ & $\begin{array}{l}\text { Communication } \\
\text { difficulties }\end{array}$ \\
\hline & Feel more powerful & Feel more powerful & Asserting dominance & Asserting dominance & Dominance & Asserting dominance \\
\hline & Control partner & Control partner & Control/ dominance & Control & Control & Control \\
\hline & $\begin{array}{l}\text { Get back/ revenge for } \\
\text { being hit first }\end{array}$ & $\begin{array}{l}\text { Get back/ revenge for } \\
\text { being hit first }\end{array}$ & $\begin{array}{l}\text { Retaliation (against } \\
\text { physical violence) }\end{array}$ & Retaliation & Retaliate & Retaliation (physical) \\
\hline & $\begin{array}{l}\text { Punish partner for } \\
\text { wrong behaviour }\end{array}$ & $\begin{array}{l}\text { Punish partner for } \\
\text { wrong behaviour }\end{array}$ & $\begin{array}{l}\text { Dominance/ retaliation/ } \\
\text { control }\end{array}$ & Asserting dominance & Punish partner & Punish partner \\
\hline & Prove love & Prove love to partner & To prove love & To prove love & To prove love & To prove love \\
\hline & $\begin{array}{l}\text { Retaliate for } \\
\text { emotional hurt }\end{array}$ & $\begin{array}{l}\text { Get back/ retaliate } \\
\text { for emotional hurt }\end{array}$ & $\begin{array}{l}\text { Retaliation (against } \\
\text { perceived injustice) }\end{array}$ & Retaliation & Retaliate & $\begin{array}{l}\text { Retaliation } \\
\text { (emotional) }\end{array}$ \\
\hline & Sexually arousing & $\begin{array}{l}\text { It was sexually } \\
\text { arousing }\end{array}$ & For sexual pleasure & Sexually arousing & Sexual arousal & Sexually arousing \\
\hline & $\begin{array}{l}\text { Get partner's } \\
\text { attention }\end{array}$ & $\begin{array}{l}\text { Get partner's } \\
\text { attention }\end{array}$ & Attention seeking & $\begin{array}{l}\text { Communication } \\
\text { difficulties }\end{array}$ & To get attention & To get attention \\
\hline & $\begin{array}{l}\text { Get partner to do/ } \\
\text { stop something }\end{array}$ & $\begin{array}{l}\text { Get partner to do/ } \\
\text { stop something }\end{array}$ & Control & Asserting dominance & Control & Control \\
\hline
\end{tabular}




\begin{tabular}{|c|c|c|c|c|c|c|}
\hline Study & Study Label & Example Item & Coder 1 & Coder 2 & Coder 3 & Review/ Meta Label \\
\hline \multirow{15}{*}{$\begin{array}{l}\text { Shorey et al., } 2010 \\
\text { cont. }\end{array}$} & Jealousy & Jealousy & Jealousy & Jealousy & Jealousy & Jealousy \\
\hline & $\begin{array}{l}\text { Angry at someone } \\
\text { else }\end{array}$ & $\begin{array}{l}\text { Angry at someone } \\
\text { else }\end{array}$ & $\begin{array}{l}\text { Anger/ emotional } \\
\text { regulation difficulties }\end{array}$ & Anger & Anger & $\begin{array}{l}\text { Emotional regulation } \\
\text { difficulties }\end{array}$ \\
\hline & $\begin{array}{l}\text { Partner was going to } \\
\text { walk away/ leave } \\
\text { conflict }\end{array}$ & $\begin{array}{l}\text { Partner was going to } \\
\text { walk away/ leave } \\
\text { conflict }\end{array}$ & Control & Asserting dominance & $\begin{array}{l}\text { To prevent partner } \\
\text { ending conflict }\end{array}$ & Control \\
\hline & $\begin{array}{l}\text { Afraid partner would } \\
\text { leave you }\end{array}$ & $\begin{array}{l}\text { Afraid partner would } \\
\text { leave you }\end{array}$ & $\begin{array}{l}\text { Fear/emotion regulation } \\
\text { difficulties }\end{array}$ & Asserting dominance & $\begin{array}{l}\text { Expression of } \\
\text { negative emotions }\end{array}$ & $\begin{array}{l}\text { Emotional regulation } \\
\text { difficulties }\end{array}$ \\
\hline & $\begin{array}{l}\text { Partner did not care } \\
\text { about you }\end{array}$ & $\begin{array}{l}\text { Partner did not care } \\
\text { about you }\end{array}$ & $\begin{array}{l}\text { Partner did not care } \\
\text { about you }\end{array}$ & Asserting dominance & $\begin{array}{l}\text { Perceived lack of care } \\
\text { or attention from } \\
\text { partner }\end{array}$ & $\begin{array}{l}\text { Emotional regulation } \\
\text { difficulties }\end{array}$ \\
\hline & $\begin{array}{l}\text { Did not know what } \\
\text { else to do with } \\
\text { feelings }\end{array}$ & $\begin{array}{l}\text { Did not know what } \\
\text { else to do with } \\
\text { feelings }\end{array}$ & $\begin{array}{l}\text { Emotion regulation } \\
\text { difficulties }\end{array}$ & $\begin{array}{l}\text { Expression of } \\
\text { negative emotions }\end{array}$ & $\begin{array}{l}\text { Unable to } \\
\text { communicate feelings }\end{array}$ & $\begin{array}{l}\text { Emotional regulation } \\
\text { difficulties }\end{array}$ \\
\hline & $\begin{array}{l}\text { Make partner agree } \\
\text { with you }\end{array}$ & $\begin{array}{l}\text { Make partner agree } \\
\text { with you }\end{array}$ & Dominance/ control & Asserting dominance & Control & Control \\
\hline & Shut partner up & Shut partner up & $\begin{array}{l}\text { Control/ dominance/ } \\
\text { avoidance }\end{array}$ & Asserting dominance & Control/dominance & Control \\
\hline & $\begin{array}{l}\text { Get away from } \\
\text { partner }\end{array}$ & $\begin{array}{l}\text { Get away from } \\
\text { partner }\end{array}$ & $\begin{array}{l}\text { Escape/ avoidance/ self- } \\
\text { defense }\end{array}$ & Self-defense & $\begin{array}{l}\text { To get away from } \\
\text { partner }\end{array}$ & Self-defense \\
\hline & $\begin{array}{l}\text { Hurt partner's } \\
\text { feelings }\end{array}$ & $\begin{array}{l}\text { Hurt partner's } \\
\text { feelings }\end{array}$ & Harm & $\begin{array}{l}\text { Emotionally hurt } \\
\text { partner }\end{array}$ & $\begin{array}{l}\text { Emotional } \\
\text { manipulation/trying to } \\
\text { upset partner }\end{array}$ & $\begin{array}{l}\text { Emotionally hurt } \\
\text { partner }\end{array}$ \\
\hline & Provoked & Provoked & Retaliation & Retaliation & Provocation & $\begin{array}{l}\text { Retaliation (not } \\
\text { specified) }\end{array}$ \\
\hline & Stress & Stress & $\begin{array}{l}\text { Stress/emotional } \\
\text { regulation difficulties }\end{array}$ & $\begin{array}{l}\text { Expression of } \\
\text { negative emotions }\end{array}$ & Stress & $\begin{array}{l}\text { Emotional regulation } \\
\text { difficulties }\end{array}$ \\
\hline & $\begin{array}{l}\text { Make partner scared/ } \\
\text { afraid }\end{array}$ & $\begin{array}{l}\text { Make partner scared/ } \\
\text { afraid }\end{array}$ & Control/dominance & Asserting dominance & Intimidation & Asserting dominance \\
\hline & Wanted to have sex & Wanted to have sex & For sexual pleasure & Asserting dominance & Sexual arousal & Sexually arousing \\
\hline & $\begin{array}{l}\text { Partner cheated on } \\
\text { you }\end{array}$ & $\begin{array}{l}\text { Partner cheated on } \\
\text { you }\end{array}$ & $\begin{array}{l}\text { Retaliation against } \\
\text { perceived injustice }\end{array}$ & Jealousy & Infidelity & Infidelity \\
\hline Stuart et al., 2006 & Self-defense & Self-defense & Self-defense & Self-defense & Self-defense & Self-defense \\
\hline
\end{tabular}




\begin{tabular}{|c|c|c|c|c|c|c|}
\hline Study & Study Label & Item & Coder 1 & Coder 2 & Coder 3 & Review/ Meta Label \\
\hline \multirow[t]{16}{*}{$\begin{array}{l}\text { Stuart et al., } 2006 \\
\text { cont. }\end{array}$} & $\begin{array}{l}\text { To get away from } \\
\text { your partner }\end{array}$ & $\begin{array}{l}\text { Get away from } \\
\text { partner }\end{array}$ & Escape/ self-defense & Self-defense & $\begin{array}{l}\text { To get away from } \\
\text { partner }\end{array}$ & Self-defense \\
\hline & $\begin{array}{l}\text { Get back/ revenge for } \\
\text { being hit first }\end{array}$ & $\begin{array}{l}\text { Get back/ revenge for } \\
\text { being hit first }\end{array}$ & $\begin{array}{l}\text { Retaliation against } \\
\text { physical violence }\end{array}$ & Retaliation & Retaliate & Retaliation (physical) \\
\hline & Show anger & To show anger & $\begin{array}{l}\text { Communication } \\
\text { difficulties/ emotion } \\
\text { regulation difficulties }\end{array}$ & Anger & Anger & Expression of anger \\
\hline & $\begin{array}{l}\text { Inability to express } \\
\text { verbally }\end{array}$ & $\begin{array}{l}\text { Show feelings that } \\
\text { cannot be expressed } \\
\text { in words }\end{array}$ & $\begin{array}{l}\text { Communication } \\
\text { difficulties/ difficulty } \\
\text { expressing negative } \\
\text { emotion }\end{array}$ & $\begin{array}{l}\text { Communication } \\
\text { difficulties }\end{array}$ & $\begin{array}{l}\text { Unable to } \\
\text { communicate feelings }\end{array}$ & $\begin{array}{l}\text { Communication } \\
\text { difficulties }\end{array}$ \\
\hline & Stress & Stress & Stress & $\begin{array}{l}\text { Expression of } \\
\text { negative emotions }\end{array}$ & Stress & $\begin{array}{l}\text { Emotional regulation } \\
\text { difficulties }\end{array}$ \\
\hline & $\begin{array}{l}\text { Did not know what } \\
\text { else to do with } \\
\text { feelings }\end{array}$ & $\begin{array}{l}\text { Did not know what } \\
\text { else to do with } \\
\text { feelings }\end{array}$ & $\begin{array}{l}\text { Emotional regulation } \\
\text { difficulties }\end{array}$ & $\begin{array}{l}\text { Expression of } \\
\text { negative emotions }\end{array}$ & $\begin{array}{l}\text { Unable to } \\
\text { communicate feelings }\end{array}$ & $\begin{array}{l}\text { Emotional regulation } \\
\text { difficulties }\end{array}$ \\
\hline & Prove love & Prove love to partner & $\begin{array}{l}\text { Prove love/ } \\
\text { communication } \\
\text { difficulties }\end{array}$ & To prove love & To prove love & To prove love \\
\hline & Jealousy & Jealousy & Jealousy & Jealousy & Jealousy & Jealousy \\
\hline & $\begin{array}{l}\text { Angry at someone } \\
\text { else }\end{array}$ & $\begin{array}{l}\text { Angry at someone } \\
\text { else }\end{array}$ & $\begin{array}{l}\text { Anger/Emotional } \\
\text { regulation difficulties }\end{array}$ & Anger & Anger & $\begin{array}{l}\text { Emotional regulation } \\
\text { difficulties }\end{array}$ \\
\hline & Provoked & Provoked & Retaliation & Retaliation & Provocation & $\begin{array}{l}\text { Retaliation (not } \\
\text { specified) }\end{array}$ \\
\hline & $\begin{array}{l}\text { Partner cheated on } \\
\text { you }\end{array}$ & $\begin{array}{l}\text { Partner cheated on } \\
\text { you }\end{array}$ & Retaliation & Jealousy & Infidelity & Infidelity \\
\hline & $\begin{array}{l}\text { Partner was going to } \\
\text { walk away/ leave } \\
\text { conflict }\end{array}$ & $\begin{array}{l}\text { Partner was going to } \\
\text { walk away/ leave } \\
\text { conflict }\end{array}$ & Control/dominance & Asserting dominance & $\begin{array}{l}\text { To prevent partner } \\
\text { ending conflict }\end{array}$ & Control \\
\hline & Feel more powerful & Feel more powerful & $\begin{array}{l}\text { Dominance/ } \\
\text { empowerment }\end{array}$ & Asserting dominance & Dominance & Asserting dominance \\
\hline & Control partner & Control partner & Control & Control & Control & Control \\
\hline & Shut partner up & Shut partner up & Control/dominance & Asserting dominance & Control & Control \\
\hline & $\begin{array}{l}\text { Get partner to do } \\
\text { something }\end{array}$ & $\begin{array}{l}\text { Get partner to do } \\
\text { something }\end{array}$ & Control/dominance & Asserting dominance & $\begin{array}{l}\text { Instrumental } \\
\text { aggression }\end{array}$ & Control \\
\hline
\end{tabular}




\begin{tabular}{|c|c|c|c|c|c|c|}
\hline Study Label & Example Item & Coder 1 & Coder 2 & Coder 3 & Review/ Meta Label & \\
\hline \multirow[t]{10}{*}{$\begin{array}{l}\text { Stuart et al., } 2006 \\
\text { cont. }\end{array}$} & $\begin{array}{l}\text { Hurt partner's } \\
\text { feelings }\end{array}$ & $\begin{array}{l}\text { Hurt partner's } \\
\text { feelings }\end{array}$ & Harm & $\begin{array}{l}\text { Emotionally hurt } \\
\text { partner }\end{array}$ & $\begin{array}{l}\text { Emotional } \\
\text { manipulation/trying to } \\
\text { upset partner }\end{array}$ & $\begin{array}{l}\text { Emotionally hurt } \\
\text { partner }\end{array}$ \\
\hline & $\begin{array}{l}\text { Retaliate for } \\
\text { emotional hurt }\end{array}$ & $\begin{array}{l}\text { Retaliate for } \\
\text { emotional hurt }\end{array}$ & Retaliation & Retaliation & Retaliate & $\begin{array}{l}\text { Retaliation } \\
\text { (emotional) }\end{array}$ \\
\hline & $\begin{array}{l}\text { Make partner agree } \\
\text { with you }\end{array}$ & $\begin{array}{l}\text { Make partner agree } \\
\text { with you }\end{array}$ & Control/dominance & Asserting dominance & Control & Control \\
\hline & Make partner scared & Make partner scared & Dominance & Asserting dominance & Intimidation & Asserting dominance \\
\hline & $\begin{array}{l}\text { Afraid partner would } \\
\text { leave you }\end{array}$ & $\begin{array}{l}\text { Afraid partner would } \\
\text { leave you }\end{array}$ & $\begin{array}{l}\text { Fear/ emotion } \\
\text { regulation difficulties/ } \\
\text { communication } \\
\text { difficulties }\end{array}$ & Asserting dominance & $\begin{array}{l}\text { Expression of } \\
\text { negative emotions }\end{array}$ & $\begin{array}{l}\text { Emotional regulation } \\
\text { difficulties }\end{array}$ \\
\hline & $\begin{array}{l}\text { Punish partner for } \\
\text { wrong behaviour }\end{array}$ & $\begin{array}{l}\text { Punish partner for } \\
\text { wrong behaviour }\end{array}$ & $\begin{array}{l}\text { Punish partner/ control/ } \\
\text { retaliation }\end{array}$ & Asserting dominance & Punish partner & Punish partner \\
\hline & $\begin{array}{l}\text { Partner did not care } \\
\text { about you }\end{array}$ & $\begin{array}{l}\text { Partner did not care } \\
\text { about you }\end{array}$ & $\begin{array}{l}\text { Partner did not care } \\
\text { about you }\end{array}$ & Asserting dominance & $\begin{array}{l}\text { Perceived lack of care } \\
\text { or attention from } \\
\text { partner }\end{array}$ & $\begin{array}{l}\text { Emotional regulation } \\
\text { difficulties }\end{array}$ \\
\hline & $\begin{array}{l}\text { Get partner's } \\
\text { attention }\end{array}$ & $\begin{array}{l}\text { Get partner's } \\
\text { attention }\end{array}$ & Attention seeking & $\begin{array}{l}\text { Communication } \\
\text { difficulties }\end{array}$ & To get attention & To get attention \\
\hline & Sexually arousing & Sexually arousing & For sexual pleasure & Sexually arousing & Sexual arousal & Sexually arousing \\
\hline & Wanted to have sex & Wanted to have sex & For sexual pleasure & Asserting dominance & Sexual arousal & Sexually arousing \\
\hline \multirow[t]{2}{*}{ Swan \& Snow, 2003} & Self-defense & $\begin{array}{l}\text { How often do you } \\
\text { use violence to } \\
\text { defend yourself from } \\
\text { your partner? }\end{array}$ & Self-defense & Self-defense & Self-defense & Self-defense \\
\hline & $\begin{array}{l}\text { To control their } \\
\text { partner }\end{array}$ & $\begin{array}{l}\text { How often do you } \\
\text { threaten to use } \\
\text { violence to make } \\
\text { your partner do the } \\
\text { things you want him } \\
\text { to do? }\end{array}$ & Control & Control & Control & Control \\
\hline
\end{tabular}




\begin{tabular}{|c|c|c|c|c|c|c|}
\hline Study & Study Label & Example Item & Coder 1 & Coder 2 & Coder 3 & Review/ Meta Label \\
\hline $\begin{array}{l}\text { Swan \& Snow, } 2003 \\
\text { cont. }\end{array}$ & Retribution & $\begin{array}{l}\text { How often do you } \\
\text { use violence to get } \\
\text { even with your } \\
\text { partner for something } \\
\text { he has done? }\end{array}$ & Punish partner/ to harm & Retaliation & Retaliate & Retaliation \\
\hline \multirow[t]{4}{*}{$\begin{array}{l}\text { Walley-Jean \& } \\
\text { Swan, 2009 }\end{array}$} & Show anger & Show anger & $\begin{array}{l}\text { Anger/ communication } \\
\text { difficulties }\end{array}$ & Anger & Anger & Expression of anger \\
\hline & $\begin{array}{l}\text { Inability to express } \\
\text { self verbally }\end{array}$ & $\begin{array}{l}\text { Inability to express } \\
\text { self verbally }\end{array}$ & $\begin{array}{l}\text { Communication } \\
\text { difficulties }\end{array}$ & \begin{tabular}{|l|}
$\begin{array}{l}\text { Communication } \\
\text { difficulties }\end{array}$ \\
\end{tabular} & $\begin{array}{l}\text { Unable to } \\
\text { communicate feelings }\end{array}$ & $\begin{array}{l}\text { Communication } \\
\text { difficulties }\end{array}$ \\
\hline & $\begin{array}{l}\text { Retaliation for } \\
\text { emotional hurt }\end{array}$ & $\begin{array}{l}\text { Retaliation for } \\
\text { emotional hurt }\end{array}$ & Retaliation & Retaliation & Retaliate & $\begin{array}{l}\text { Retaliation } \\
\text { (emotional) }\end{array}$ \\
\hline & To get attention & To get attention & Attention seeking & \begin{tabular}{|l} 
Communication \\
difficulties
\end{tabular} & To get attention & To get attention \\
\hline
\end{tabular}




\section{APPENDIX N}

Summary statistics and forest plot for asserting dominance

$\begin{array}{cccccccc}\text { Study name } & \text { Hedges' g } & \text { Standard error } & \text { Variance } & \text { Lower limit } & \text { Upper limit } & \text { p-value } & \text { Weighting } \\ \text { Barnett et al., 1997 } & .66 & .25 & .07 & .16 & 1.15 & .010 & 15.3 \\ \quad \text { Harned, 2001 } & -.34 & .19 & .04 & -.70 & .03 & .072 & 18.3 \\ \text { Kelley et al., 2015 } & .20 & .14 & .02 & -.07 & .47 & .151 & 20.5 \\ \quad \text { Ross, 2011 } & -.18 & .23 & .05 & -.62 & .26 & .420 & 16.58 \\ \text { Shorey et al., 2010 } & .40 & .50 & .25 & -.58 & 1.40 & .419 & 7.6 \\ \text { Caldwell et al., 2008 } & .52 & .10 & .01 & . .32 & .72 & <.001 & 21.8 \\ & .19 & .17 & .03 & -.13 & .52 & .248 & \end{array}$

Note. Effect sizes in the negative direction indicate the relationship between this motivational-category and physical IPA was stronger for women relative to men.

Hedge's g and 95\% Cl

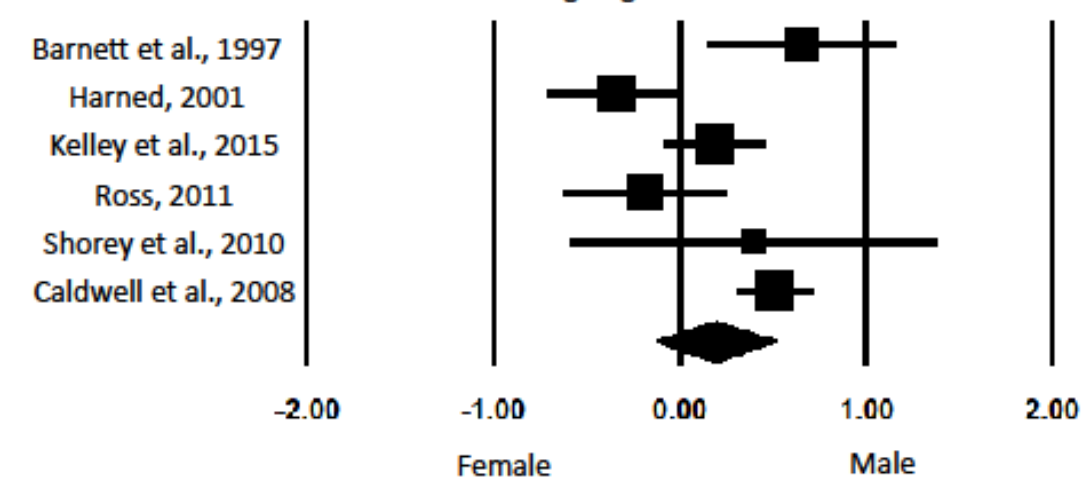




\section{APPENDIX O}

Summary statistics and forest plot for communication difficulties

$\begin{array}{cccccccc}\text { Study name } & \text { Hedges' g } & \text { Standard error } & \text { Variance } & \text { Lower limit } & \text { Upper limit } & \text { p-value } & \text { Weighting } \\ \text { Elmquist et al., 2014 } & -.25 & .15 & .02 & -0.54 & .05 & .097 & 47.0 \\ \text { Harned, 2001 } & -.33 & .19 & .04 & -.69 & .04 & .080 & 30.6 \\ \text { Shorey et al., 2010 } & .00 & .30 & .09 & -.59 & .59 & 1.000 & 11.7 \\ \text { Walley-Jean \& Swan, 2009 } & -.18 & .32 & .10 & -.80 & .44 & .574 & 10.7 \\ & -.24 & .10 & .01 & -.44 & -.03 & .022\end{array}$

Note. Effect sizes in the negative direction indicate the relationship between this motivational-category and physical IPA was stronger for women relative to men

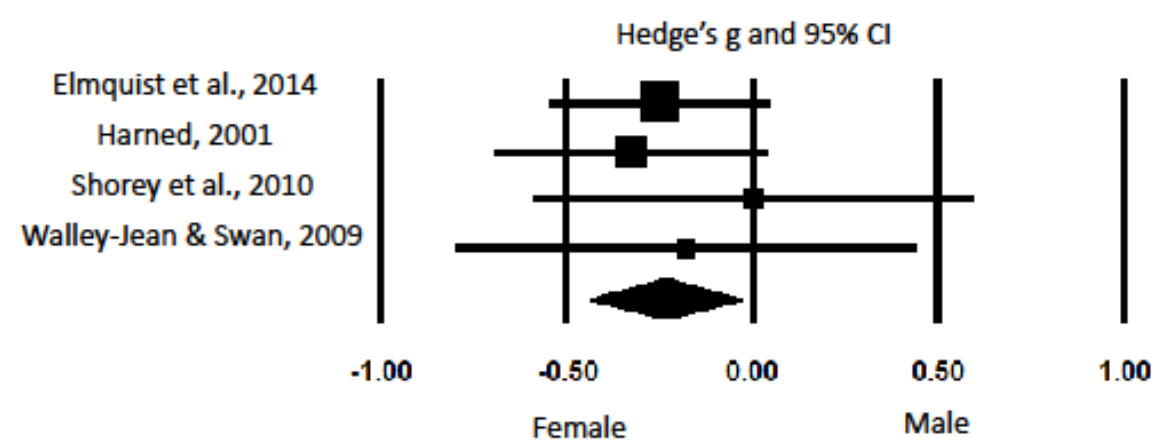




\section{APPENDIX P}

Summary statistics and forest plot for control

$\begin{array}{cccccccc}\text { Study name } & \text { Hedges' g } & \text { Standard error } & \text { Variance } & \text { Lower limit } & \text { Upper limit } & \text { p-value } & \text { Weighting } \\ \text { Elmquist et al., } 2014 & -.07 & .15 & .02 & -.36 & .23 & .654 & 26.3 \\ \text { Harned, 2001 } & .23 & .19 & .03 & -.13 & .60 & .212 & 17.7 \\ \text { Shorey et al., 2010 } & .34 & .49 & .24 & -.63 & 1.31 & .493 & 2.65 \\ \text { Caldwell et al., 2008 } & .24 & .10 & .01 & .05 & .44 & .015 & 53.3 \\ & .16 & .08 & .01 & .00 & .32 & .046 & \end{array}$

Note. Effect sizes in the negative direction indicate the relationship between this motivational-category and physical IPA was stronger for women relative to men

Hedge's $g$ and $95 \% \mathrm{Cl}$

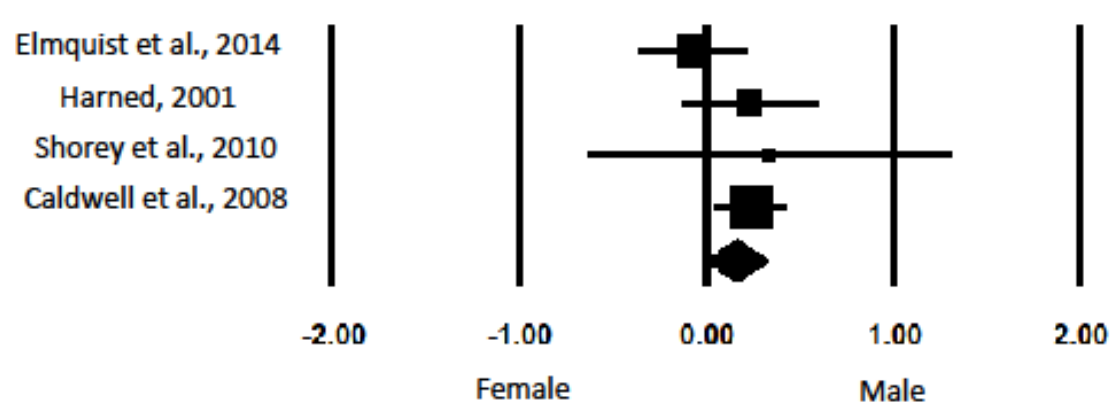




\section{APPENDIX Q}

Summary statistics and forest plot for emotional regulation difficulties

\begin{tabular}{|c|c|c|c|c|c|c|c|}
\hline Study name & Hedges' g & Standard error & Variance & Lower limit & Upper limit & p-value & Weighting \\
\hline Barnett et al., 1997 & .28 & .25 & .06 & -.21 & .76 & .270 & 16.1 \\
\hline Elmquist et al., 2014 & -.24 & .15 & .02 & -.53 & .06 & .111 & 18.7 \\
\hline Kelley et al., 2015 & -.50 & .14 & .02 & -.77 & -.23 & $<.001$ & 19.0 \\
\hline Ross, 2011 & -.28 & .23 & .05 & -.73 & .16 & .207 & 16.8 \\
\hline Shorey et al., 2010 & .39 & .50 & .25 & -.59 & 1.37 & .437 & 9.6 \\
\hline \multirow[t]{2}{*}{ Caldwell et al., 2008} & .49 & .10 & .01 &. .29 & .69 & $<.001$ & 19.6 \\
\hline & -.01 & .21 & .05 & -.42 & .41 & .969 & \\
\hline
\end{tabular}

Note. Effect sizes in the negative direction indicate the relationship between this motivational-category and physical IPA was stronger for women relative to men

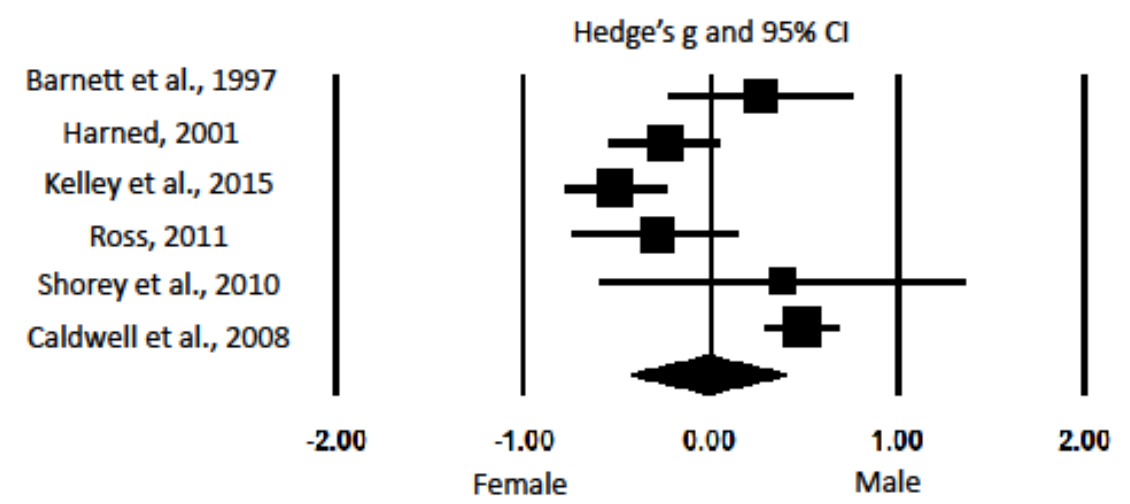




\section{APPENDIX R}

Summary statistics and forest plot for jealousy

$\begin{array}{cccccccc}\text { Study name } & \text { Hedges' g } & \begin{array}{c}\text { Standard } \\ \text { error }\end{array} & \text { Variance } & \text { Lower limit } & \text { Upper limit } & \text { p-value } & \text { Weighting } \\ \text { Belus et al., 2014 } & -.20 & .11 & .01 & -.41 & .01 & .060 & 21.8 \\ \text { Elmquist et al., 2014 } & -.23 & .15 & .02 & -.52 & .07 & .130 & 20.9 \\ \text { Shorey et al., 2010 } & .40 & .30 & .09 & -.20 & 1.00 & .189 & 13.5 \\ \text { Caldwell et al., 2008 } & .20 & .10 & .01 & .01 & .40 & .043 & 22.1 \\ \text { andez-Fuertes \& Fuertes, 2010 } & .53 & .08 & .01 & .37 & .70 & <.001 & 22.5 \\ & .13 & .17 & .03 & -.20 & .46 & .448 & \end{array}$

Note. Effect sizes in the negative direction indicate the relationship between this motivational-category and physical IPA was stronger for women relative to men

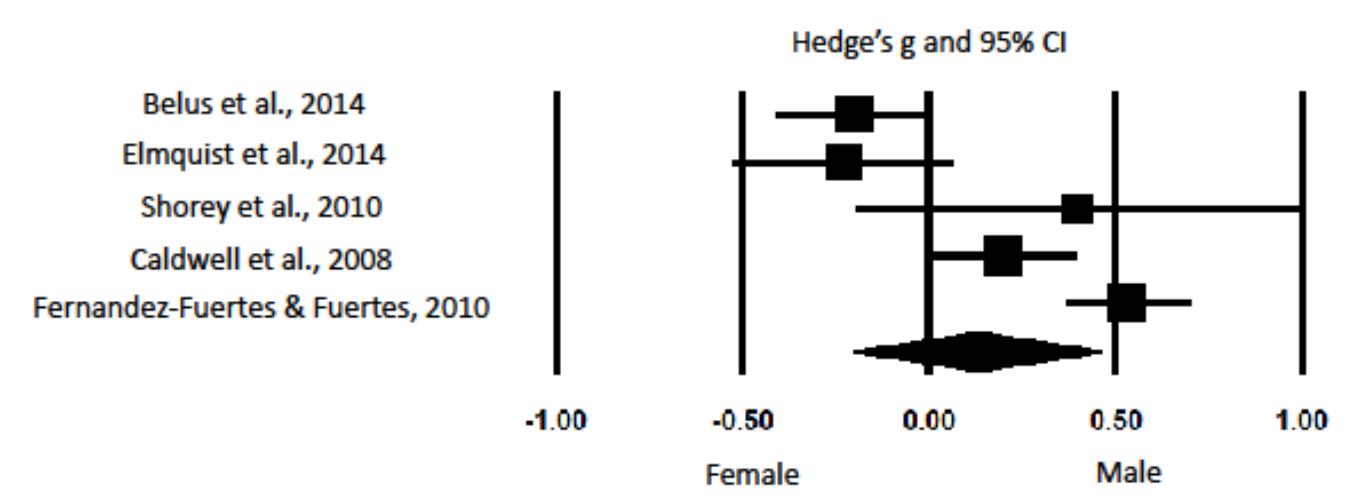




\section{APPENDIX S}

Summary statistics and forest plot for retaliation for emotional hurt

$\begin{array}{cccccccc}\text { Study name } & \text { Hedges' g } & \text { Standard error } & \text { Variance } & \text { Lower limit } & \text { Upper limit } & \text { p-value } & \text { Weighting } \\ \text { Elmquist et al., 2014 } & -.28 & .15 & .02 & -.57 & .02 & .067 & 52.1 \\ \quad \text { Ross, 2011 } & -.41 & .23 & .05 & -.85 & .04 & .073 & 23.0 \\ \text { Shorey et al., 2010 } & -.22 & .30 & .09 & -.81 & .37 & .465 & 12.9 \\ \text { Walley-Jean \& Swan, 2009 } & -.10 & .32 & .10 & -.72 & .52 & .755 & 11.9 \\ & -.28 & .11 & .01 & -.49 & -.06 & .011\end{array}$

Note. Effect sizes in the negative direction indicate the relationship between this motivational-category and physical IPA was stronger for women relative to men

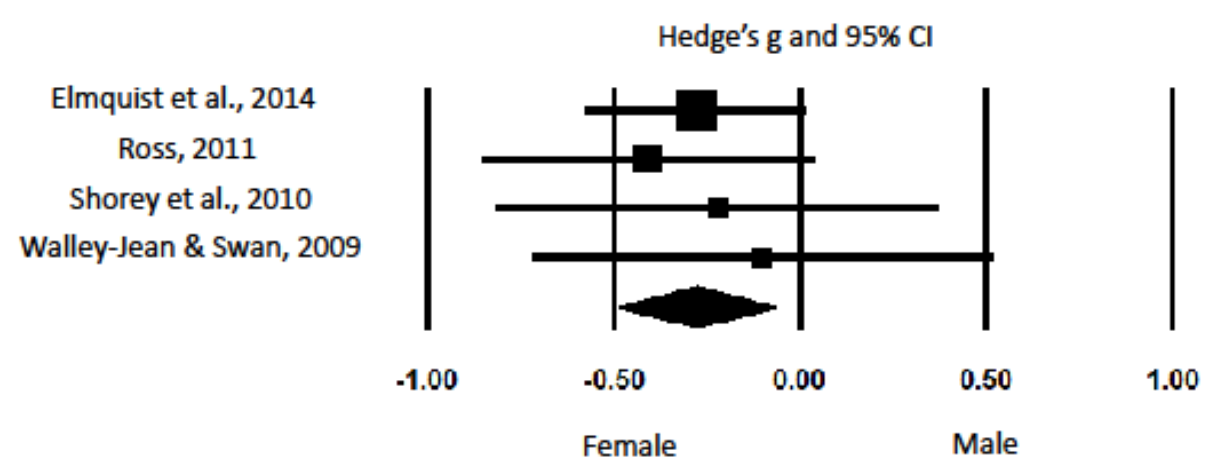




\section{APPENDIX T}

Summary statistics and forest plot for retaliation for self-defense

$\begin{array}{cccccccc}\text { Study name } & \text { Hedges' g } & \text { Standard error } & \text { Variance } & \text { Lower limit } & \text { Upper limit } & \text { p-value } & \text { Weighting } \\ \text { Barnett et al., 1997 } & -.50 & .25 & .06 & -.99 & -.00 & .049 & 13.2 \\ \text { Elmquist et al., 2014 } & -.27 & .15 & .02 & -.56 & .03 & .077 & 16.7 \\ \quad \text { Harned, 2001 } & .18 & .19 & .03 & -.18 & .54 & .333 & 15.5 \\ \text { Kelley et al., 2015 } & -.18 & .14 & .02 & -.45 & .09 & .187 & 17.1 \\ \quad \text { Ross, 2011 } & -1.16 & .24 & .06 & -1.63 & -.69 & <.001 & 13.5 \\ \text { Shorey et al., 2010 } & .78 & .54 & .29 & -.28 & 1.84 & .149 & 6.0 \\ \text { Caldwell et al., 2008 } & -.61 & .10 & .01 & -.81 & -.40 & <.001 & 18.1 \\ & -.33 & .16 & .03 & -.64 & -.02 & .037 & \end{array}$

Note. Effect sizes in the negative direction indicate the relationship between this motivational-category and physical IPA was stronger for women relative to men

Hedge's g and 95\% Cl

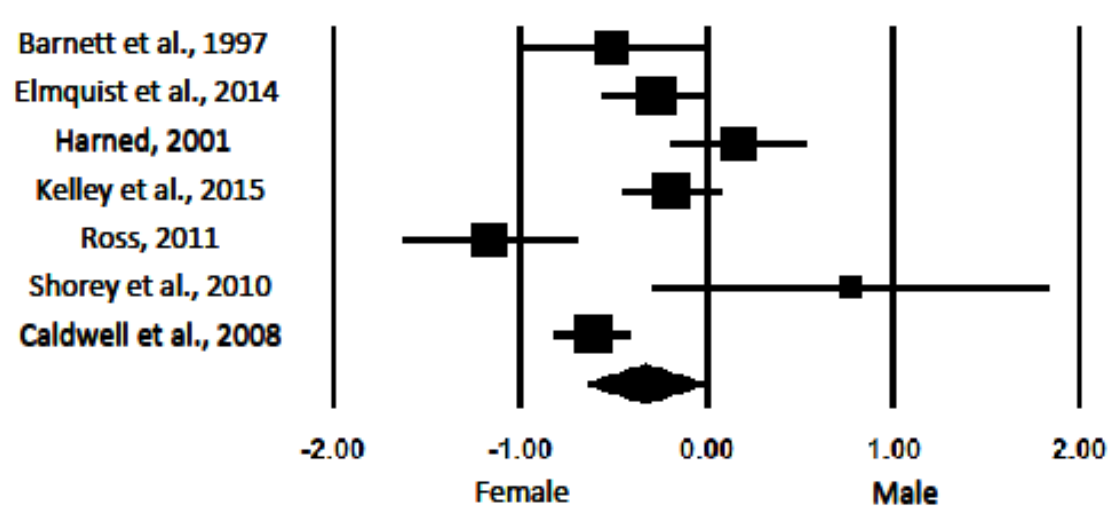




\section{APPENDIX U}

Summary statistics and forest plot for to express anger

$\begin{array}{cccccccc}\text { Study name } & \text { Hedges' g } & \text { Standard error } & \text { Variance } & \text { Lower limit } & \text { Upper limit } & \text { p-value } & \text { Weighting } \\ \text { Belus et al., 2014 } & -.12 & .11 & .01 & -.33 & .09 & .263 & 34.4 \\ \text { Harned, 2001 } & -.38 & .19 & .04 & -.75 & -.02 & .039 & 28.2 \\ \text { Shorey et al., 2010 } & .23 & .30 & .09 & -.37 & .82 & .452 & 19.7 \\ \text { Walley-Jean \& Swan, 2009 } & .76 & .34 & .11 & .10 & 1.42 & .025 & 17.7 \\ & .03 & .19 & .04 & -.35 & .41 & .880\end{array}$

Note. Effect sizes in the negative direction indicate the relationship between this motivational-category and physical IPA was stronger for women relative to men

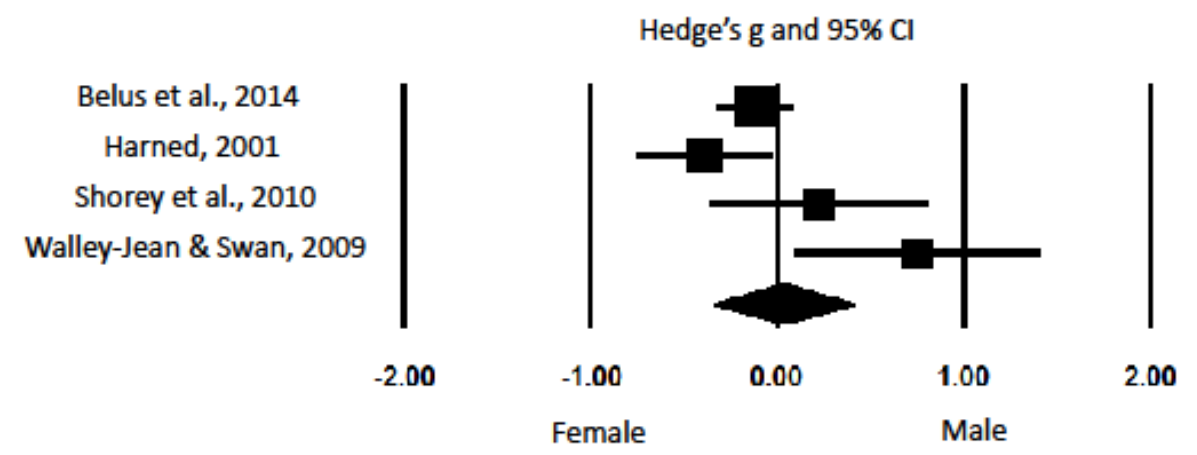




\section{APPENDIX V}

Summary statistics and forest plot for to get attention

$\begin{array}{ccccccccc}\text { Study name } & \text { Hedges' g } & \text { Standard error } & \text { Variance } & \text { Lower limit } & \text { Upper limit } & \text { p-value } & \text { Weighting } \\ \text { Barnett et al., 1997 } & .27 & .25 & .06 & -.22 & .75 & .282 & 24.4 \\ \text { Harned, 2001 } & .06 & .19 & .03 & -.30 & .42 & .751 & 43.9 \\ \text { Shorey et al., 2010 } & .12 & .30 & .09 & -.47 & .71 & .691 & 16.6 \\ \text { Walley-Jean \& Swan, 2009 } & -.10 & .32 & .10 & -.72 & .52 & .755 & 15.2 \\ & .10 & .12 & .02 & -.15 & .34 & .435 & \end{array}$

Note. Effect sizes in the negative direction indicate females were higher on this motivational-category.

Hedge's $\mathrm{g}$ and $95 \% \mathrm{Cl}$

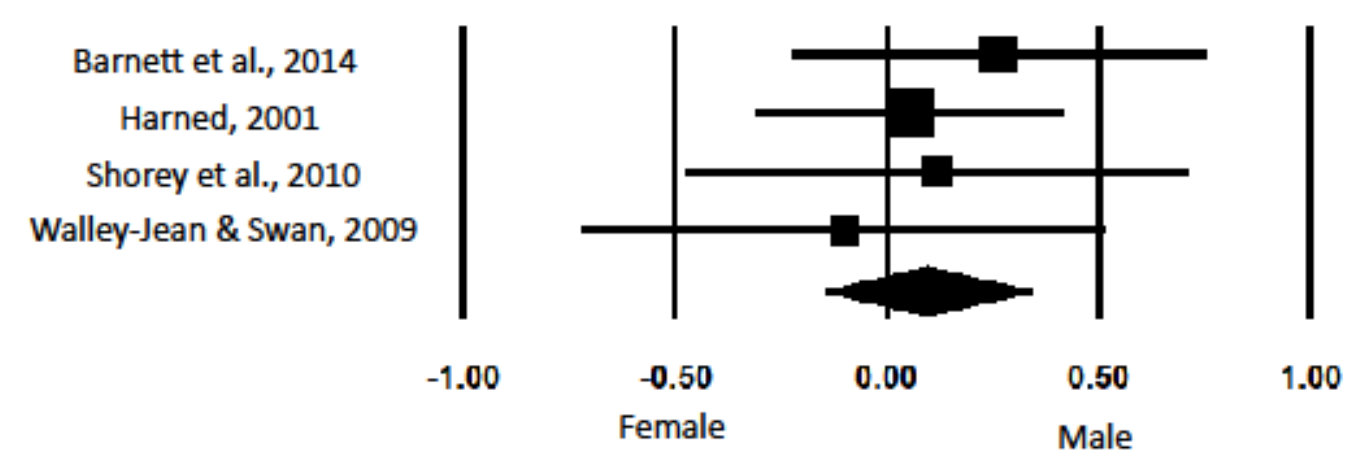


APPENDIX W

Funnel plot of Standard Error by Standard difference in means for asserting dominance

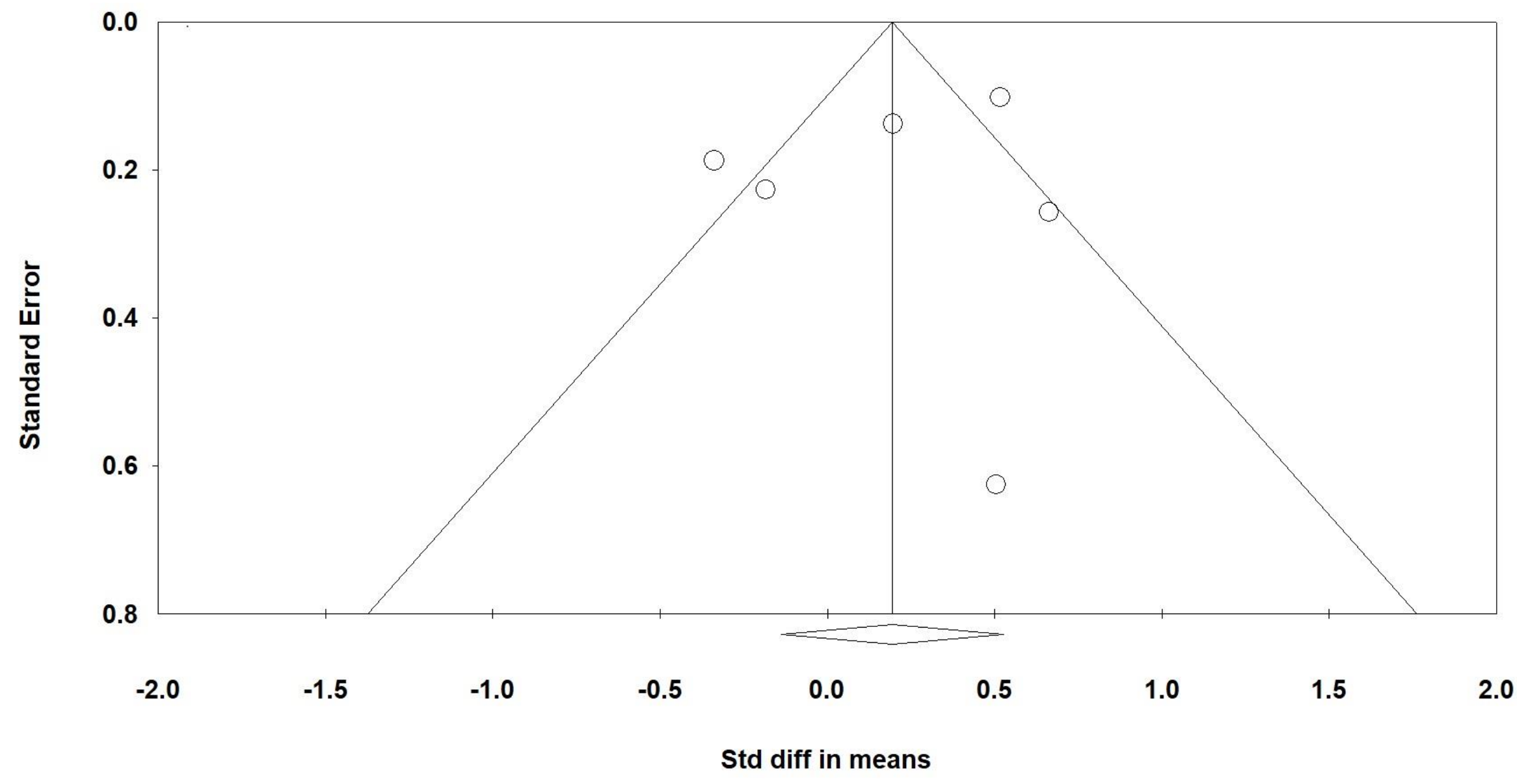




\section{APPENDIX X}

Funnel plot of Standard Error by Standard difference in means for communication difficulties

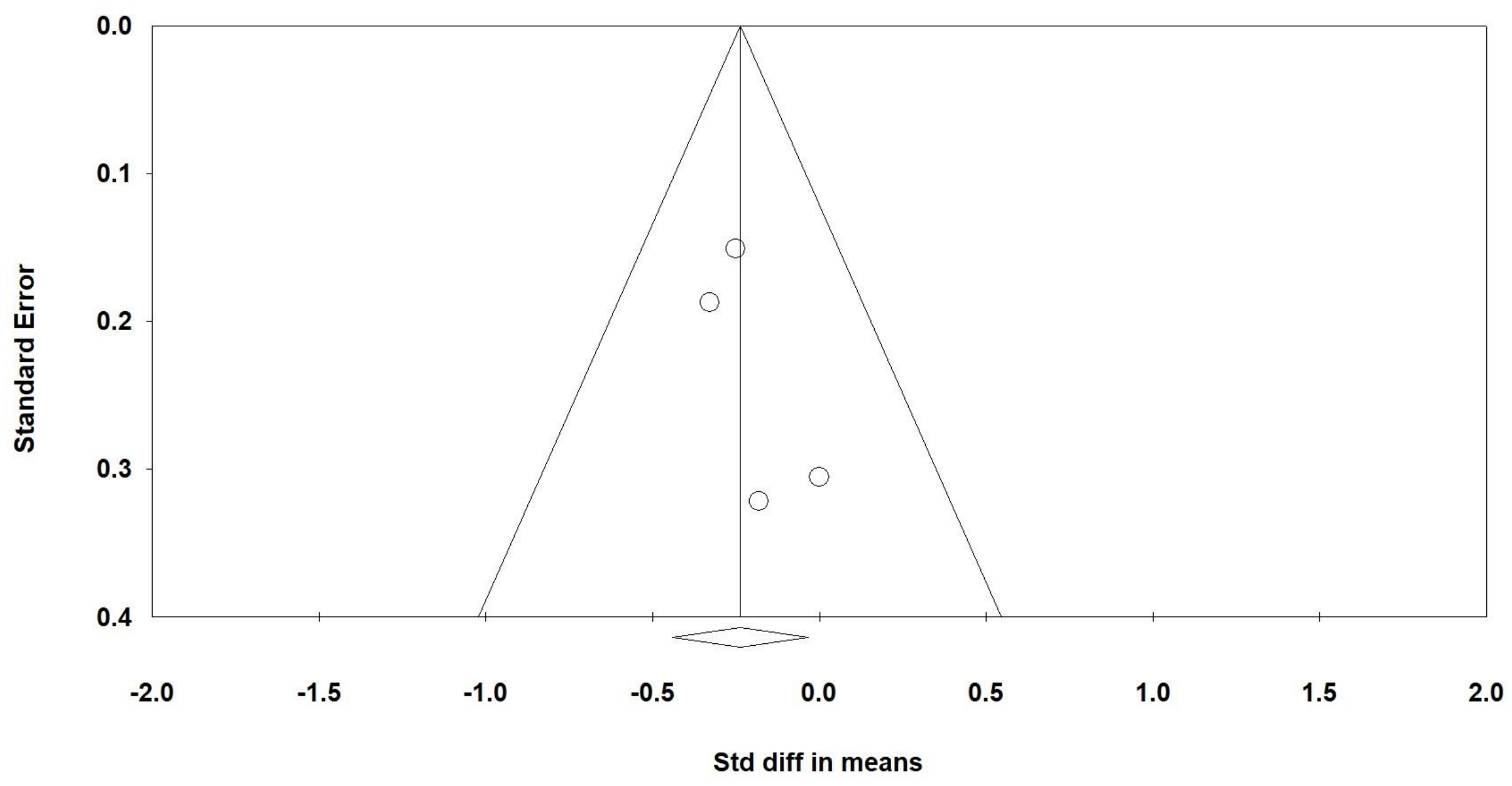


APPENDIX Y

Funnel plot of Standard Error by Standard difference in means for control

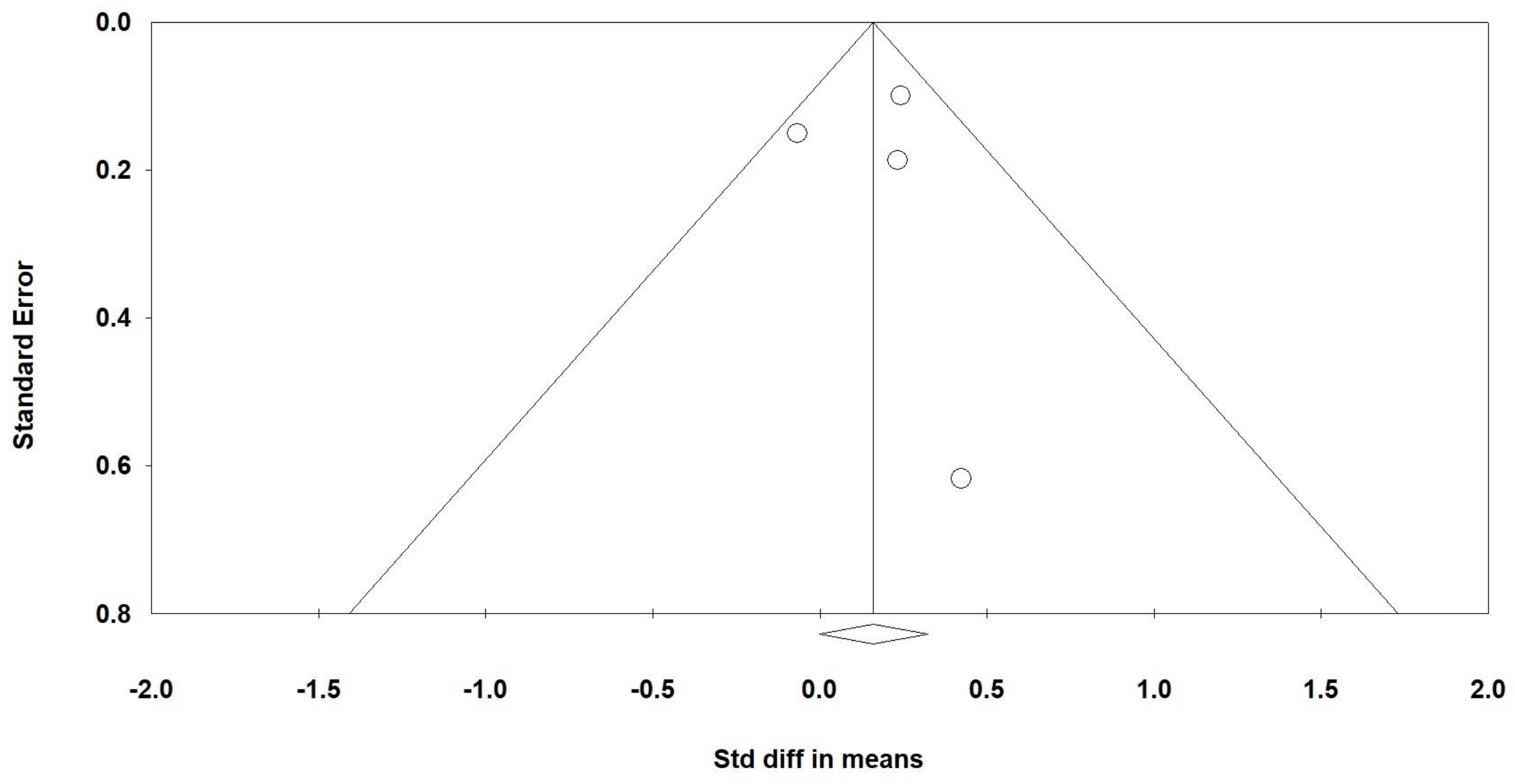




\section{APPENDIX Z}

Funnel plot of Standard Error by Standard difference in means for emotional regulation difficulties

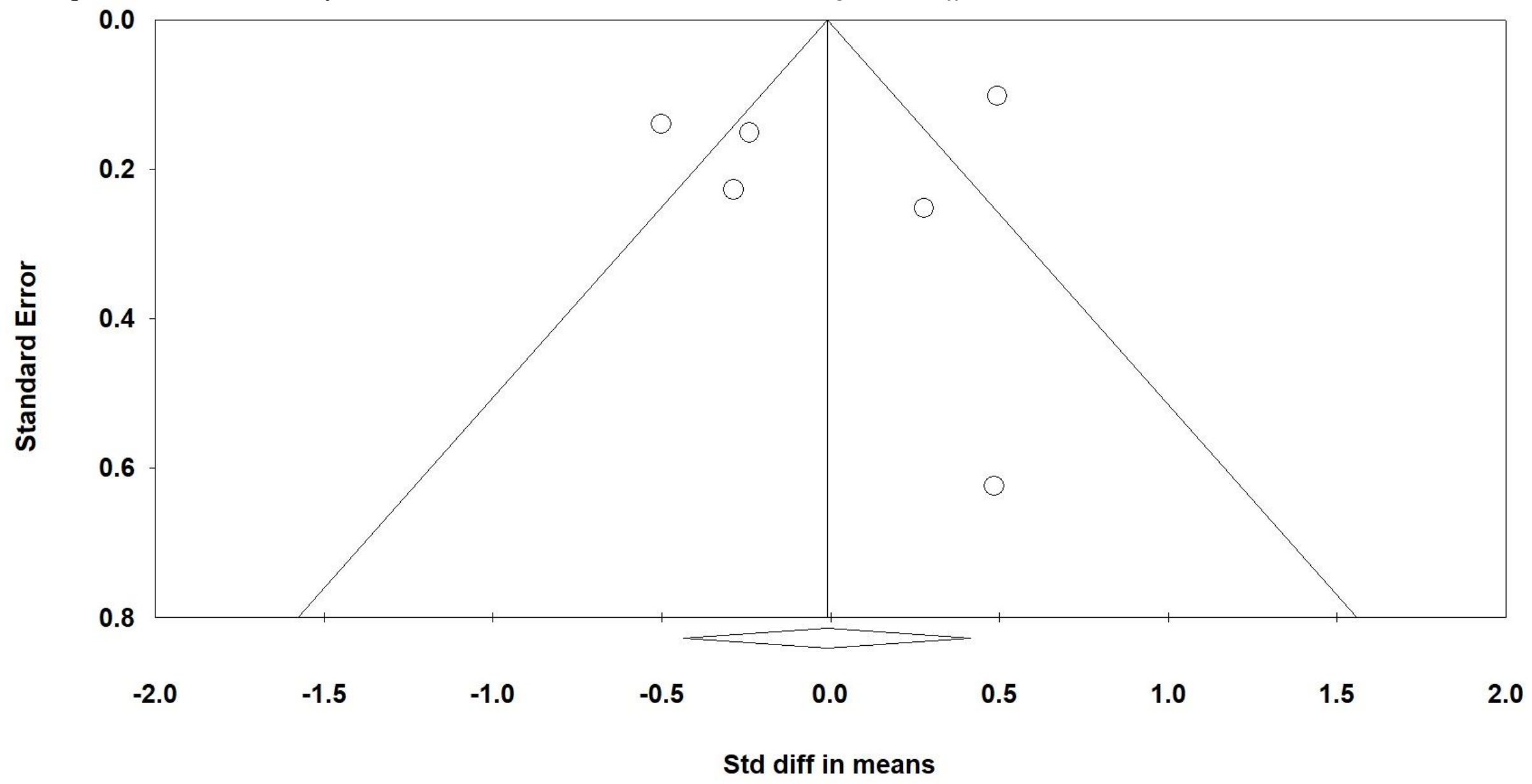




\section{APPENDIX AA}

Funnel plot of Standard Error by Standard difference in means for jealousy

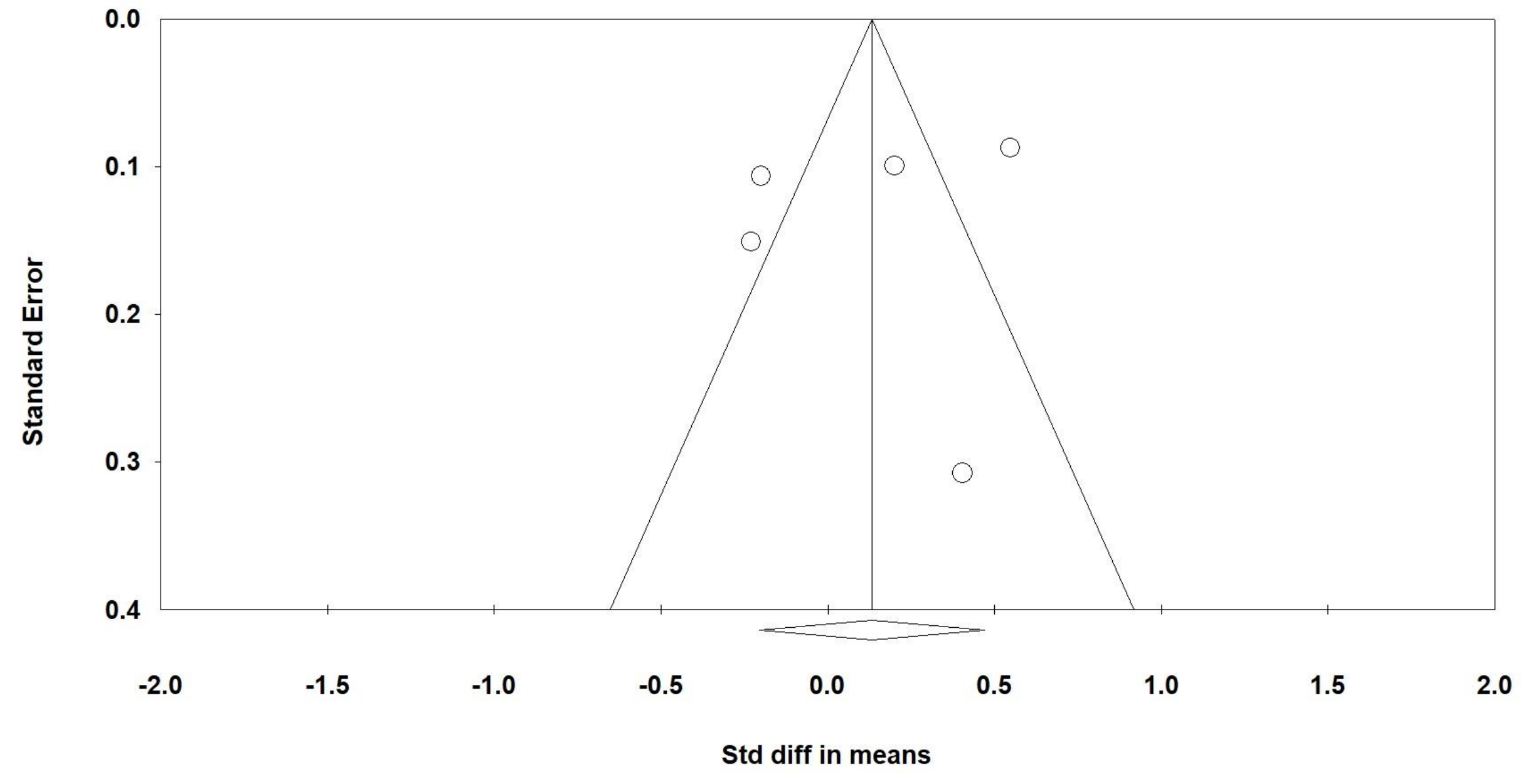




\section{APPENDIX BB}

Funnel plot of Standard Error by Standard difference in means for retaliation for emotional hurt

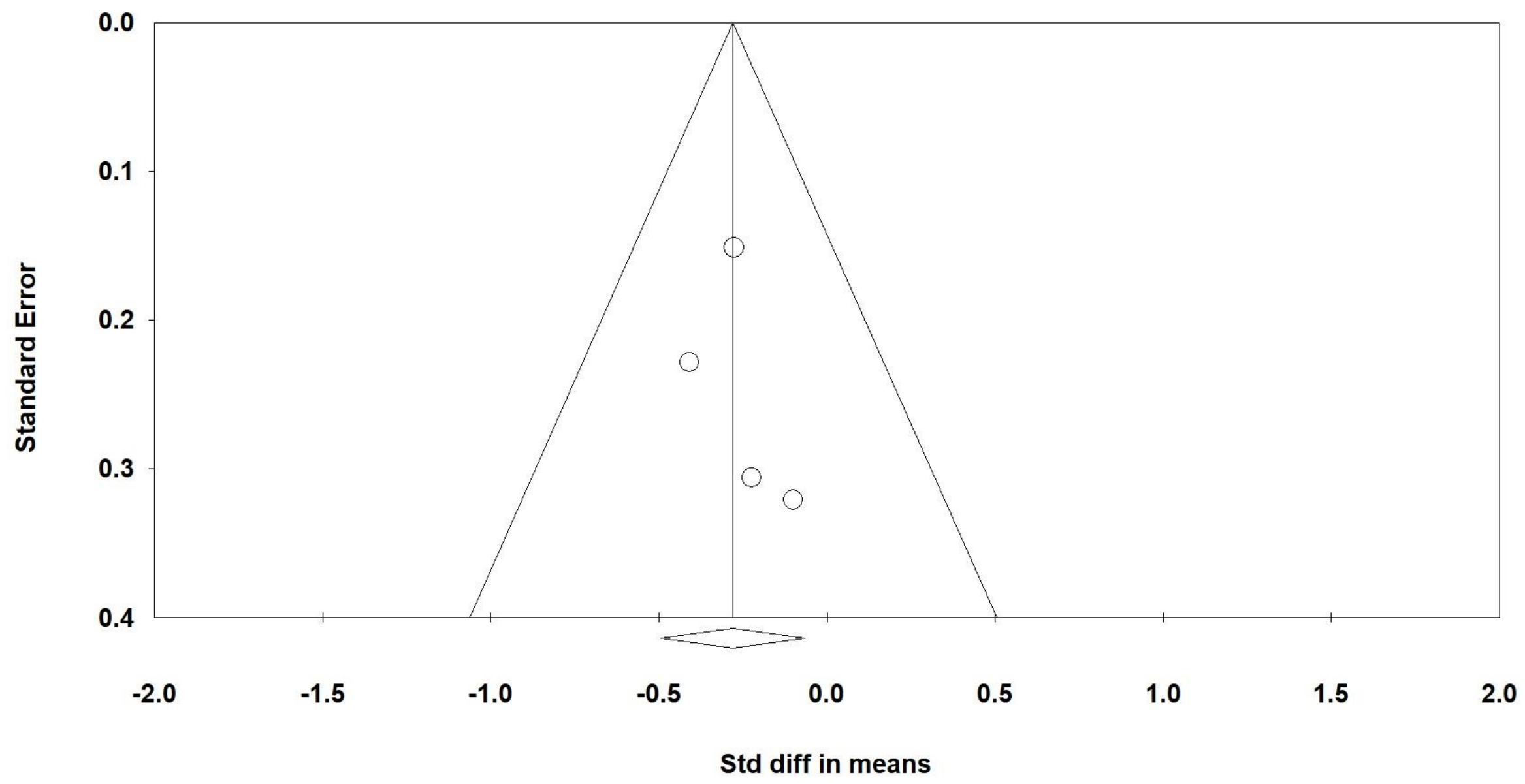




\section{APPENDIX CC}

Funnel plot of Standard Error by Standard difference in means for self-defense

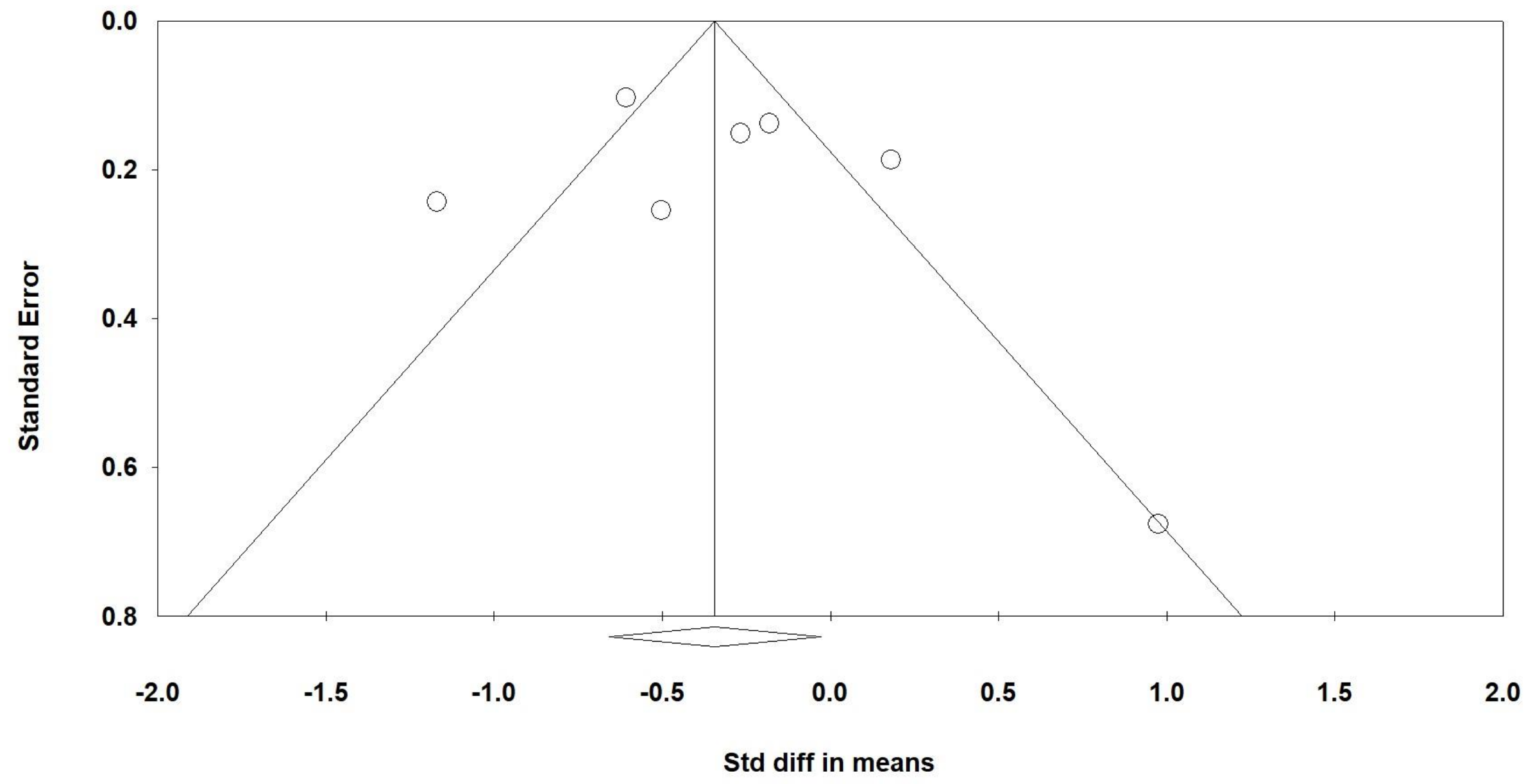




\section{APPENDIX DD}

Funnel plot of Standard Error by Standard difference in means for to express anger

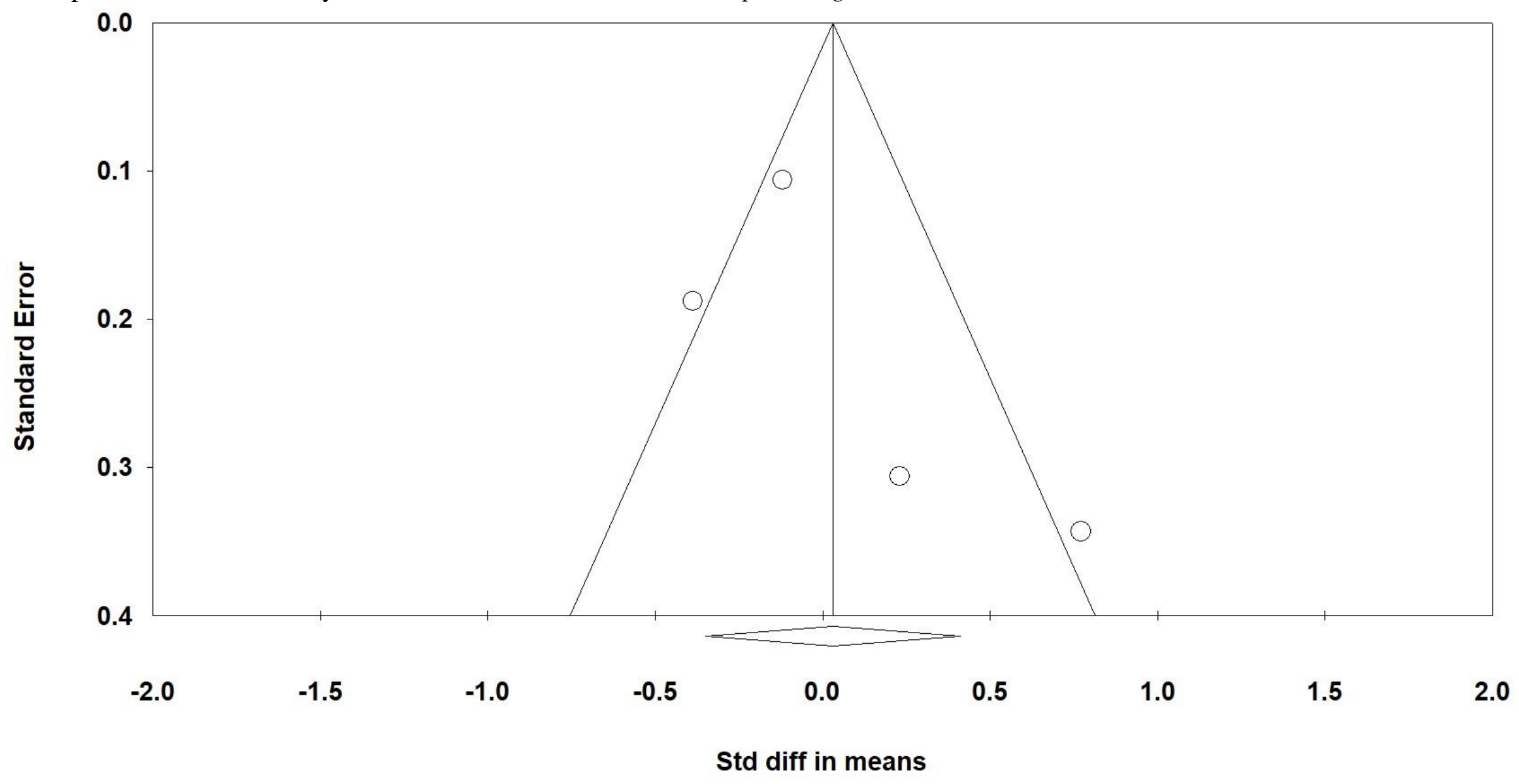




\section{APPENDIX EE}

Funnel plot of Standard Error by Standard difference in means for to get attention

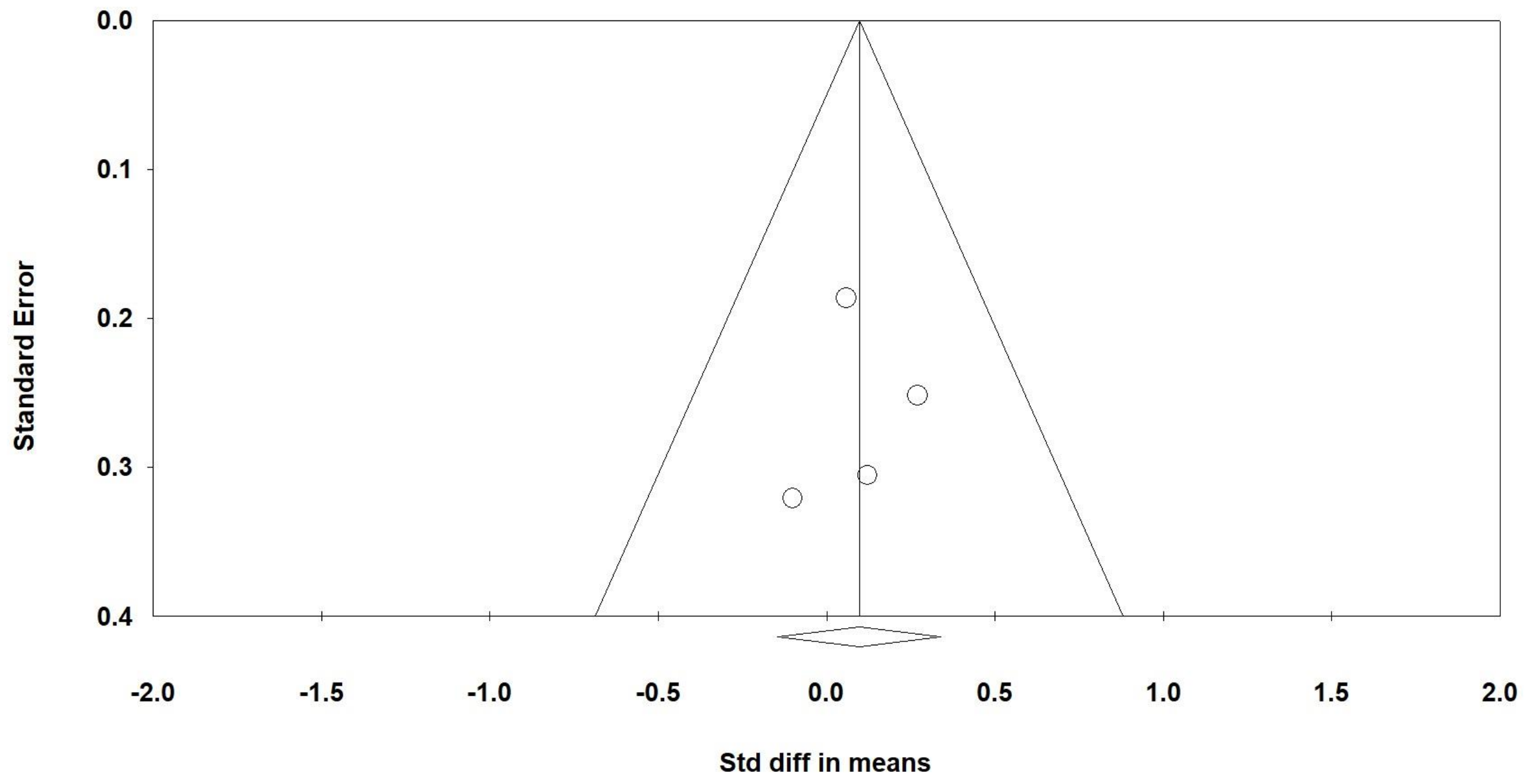




\section{APPENDIX FF}

Items included in the categorical principal components and latent class analyses in Chapter 4

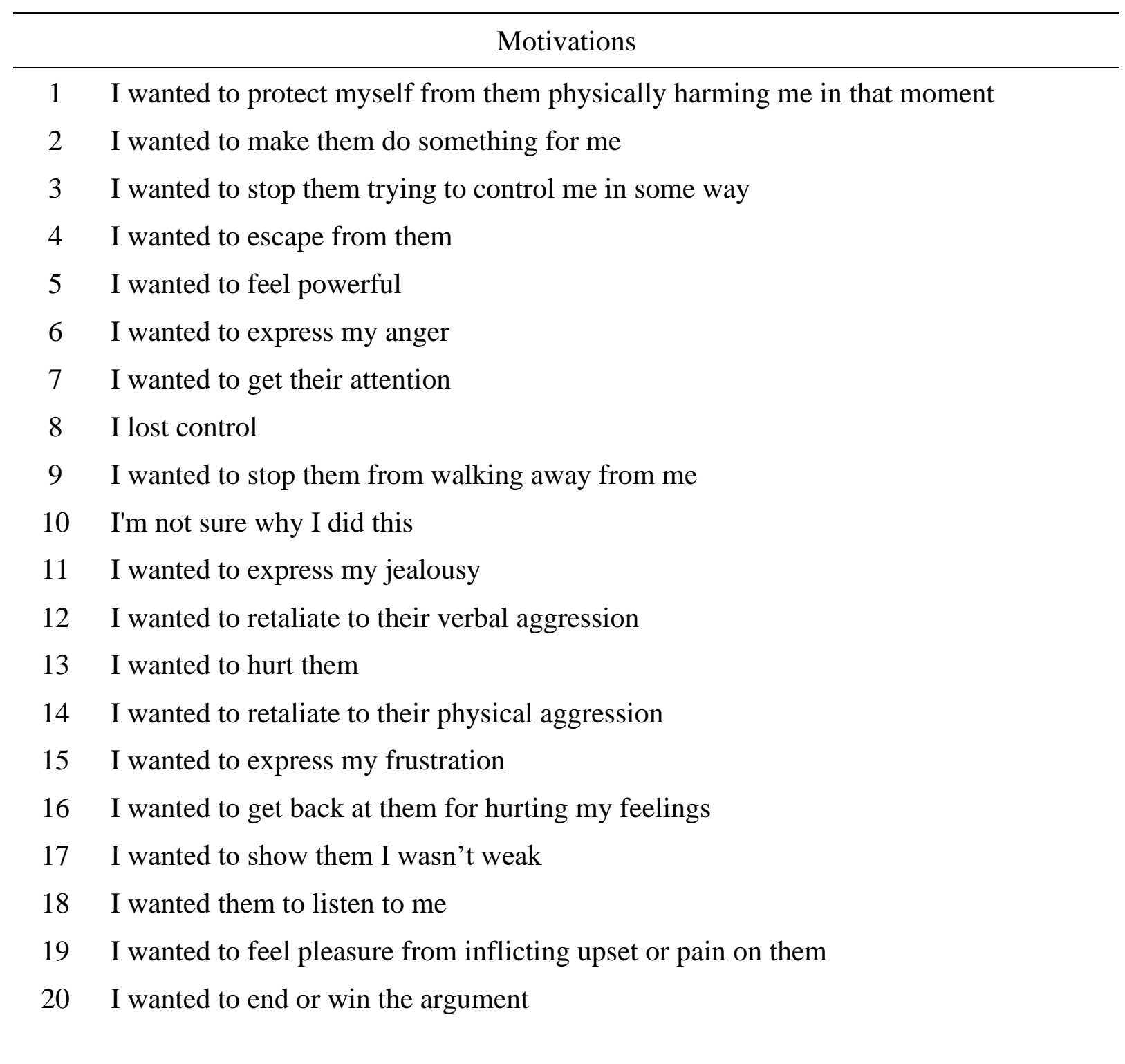

Behavioural Items

EA I made my partner dependent on me for money

EA I threatened my partner with a weapon

EA I threatened to disclose damaging or embarrassing information

EA I deliberately broke or destroyed something that was important to my partner

EA I threatened to withhold money from my partner

EA I kicked my partner

EA I kept my partner from leaving the house

EA I pressured my partner to have sex when they said 'No' 
EA I hit or punched my partner

EA I slapped my partner

EA I denied incidents of abuse

DPA I deliberately ignored my partner

DPA I made my partner feel guilty for not spending enough time together

DPA I pouted or acted upset when I didn't get my way

DPA I purposely withheld affection or sex

DPA I screamed or yelled at my partner

DPA I stomped out of the house or room during a disagreement with my partner

DPA I threatened to leave the relationship

DPA I was critical or unpleasant toward my partner

MA I checked my partner's social network page(s)

MA I criticized my partner's friends, family, or co-workers

MA I deliberately acted in a hurtful way towards my partner

MA I insisted on knowing where my partner went and who they spoke to when we were not together

MA I kept track of my partner's telephone/cell phone use

MA I told my partner they were lying or confused

MA I tried to provoke an argument with my partner

MA I called my partner or text messaged them constantly 


\section{APPENDIX GG}

Intercorrelations for the three-component solution produced by the CATPCA

\begin{tabular}{lccc}
\hline Component & Eclectic Aggression & $\begin{array}{c}\text { Direct Psychological } \\
\text { Aggression }\end{array}$ & Monitoring Acts \\
\hline Eclectic Aggression & - & - & - \\
$\begin{array}{l}\text { Direct Psychological } \\
\text { Aggression }\end{array}$ & $.42 *$ & - & - \\
Monitoring Acts & $.52 *$ & $.37 *$ & - \\
\hline Note. ${ }^{*} p=<0.01$ & &
\end{tabular}




\section{APPENDIX HH}

Non-significant and cross-loading items that were removed from the CATPCA

\section{Non-significant items}

I made my partner dependent on me for money

I changed the subject on purpose when my partner was trying to discuss a problem

I deliberately ignored my partner

I deliberately broke or destroyed something that was important to my partner

I insisted on knowing where my partner went and who they spoke to when we were not together
I wanted to retaliate to their verbal aggression

I wanted to hurt them

I wanted to retaliate to their physical aggression

I wanted to express my frustration

I wanted to get back at them for hurting my feelings

I wanted to feel pleasure from inflicting upset or pain on them

I wanted to end or win the argument

I'm not sure why I did this

I wanted to express my jealousy

I'm not sure why I did this

I wanted to express my jealousy

I wanted to express my frustration

I wanted to show them I wasn't weak

I wanted to feel pleasure from inflicting upset or pain on them

I wanted to end or win the argument

I'm not sure why I did this

I wanted to express my jealousy

I wanted to retaliate to their verbal aggression

I wanted them to listen to me 
I threatened to withhold money from my partner

I purposely withheld affection or sex

I told my partner they were lying or confused

I kept my partner from leaving the house
I wanted to express my jealousy

I wanted to retaliate to their verbal aggression

I wanted to hurt them

I wanted to retaliate to their physical aggression

I wanted to express my frustration

I wanted to get back at them for hurting my feelings

I wanted to show them I wasn't weak

I wanted them to listen to me

I wanted to feel pleasure from inflicting upset or pain on them

I wanted to end or win the argument

I wanted to express my jealousy

I wanted to retaliate to their verbal aggression

I wanted to hurt them

I wanted to retaliate to their physical aggression

I wanted to get back at them for hurting my feelings

I wanted to show them I wasn't weak

I wanted to feel pleasure from inflicting upset or pain on them

I wanted to end or win the argument

I'm not sure why I did this

I wanted to express my frustration

I wanted them to listen to me

I'm not sure why I did this 
I wanted to express my jealousy

I wanted to retaliate to their verbal aggression

I wanted to show them I wasn't weak

I wanted them to listen to me

I wanted to feel pleasure from inflicting upset or pain on them

I stomped out of the house or room during a disagreement with my partner

I slapped my partner
I wanted to express my frustration

I wanted to feel pleasure from inflicting upset or pain on them

I'm not sure why I did this

I wanted to express my jealousy

I wanted to retaliate to their verbal aggression

I wanted to show them I wasn't weak

I wanted them to listen to me

I wanted to feel pleasure from inflicting upset or pain on them

\section{Cross-loading items}

I deliberately ignored my partner

I insisted on knowing where my partner went and who they spoke to when we were not together

I made my partner feel guilty for not spending enough time together
I wanted to retaliate to their physical aggression

I wanted to retaliate to their physical aggression

I wanted to protect myself from them physically harming me in that moment

I wanted to make them do something for me

I wanted to stop them trying to control me in some way

I wanted to escape from them

I wanted to feel powerful

I wanted to express my anger 
I wanted to get their attention

\section{I lost control}

I wanted to stop them from walking away from me

I'm not sure why I did this

I wanted to express my jealousy

I wanted to retaliate to their verbal aggression

I wanted to hurt them

I wanted to retaliate to their physical aggression

I wanted to express my frustration

I wanted to get back at them for hurting my feelings

I wanted to show them I wasn't weak

I wanted them to listen to me

I wanted to feel pleasure from inflicting upset or pain on them

I wanted to end or win the argument

I wanted to protect myself from them physically harming me in that moment

I wanted to make them do something for me

I wanted to stop them trying to control me in some way

I wanted to escape from them

I wanted to feel powerful

I wanted to express my anger

I wanted to get their attention

I lost control 
I wanted to stop them from walking away from me

I'm not sure why I did this

I wanted to express my jealousy

I wanted to retaliate to their verbal aggression

I wanted to hurt them

I wanted to retaliate to their physical aggression

I wanted to express my frustration

I wanted to get back at them for hurting my feelings

I wanted to show them I wasn't weak

I wanted them to listen to me

I wanted to feel pleasure from inflicting upset or pain on them

I wanted to end or win the argument

I wanted to protect myself from them physically harming me in that moment

I wanted to make them do something for me

I wanted to stop them trying to control me in some way

I wanted to escape from them

I wanted to feel powerful

I wanted to express my anger

I wanted to get their attention

I lost control

I wanted to stop them from walking away from me

I'm not sure why I did this 
I told my partner they were lying or confused

I tried to provoke an argument with my partner
I wanted to express my frustration

\section{I wanted them to listen to me}

I wanted to protect myself from them physically harming me in that moment

I wanted to make them do something for me

I wanted to stop them trying to control me in some way

I wanted to escape from them

I wanted to feel powerful

I wanted to express my anger

I wanted to get their attention

I lost control

I wanted to stop them from walking away from me

I wanted to express my jealousy

I wanted to retaliate to their verbal aggression

I wanted to hurt them

I wanted to retaliate to their physical aggression

I wanted to get back at them for hurting my feelings

I wanted to show them I wasn't weak

I wanted to feel pleasure from inflicting upset or pain on them

I wanted to end or win the argument

I wanted to protect myself from them physically harming me in that moment

I wanted to make them do something for me

I wanted to stop them trying to control me in some way 
I wanted to escape from them

I wanted to feel powerful

I wanted to express my anger

I wanted to get their attention

I lost control

I wanted to stop them from walking away from me

I'm not sure why I did this

I wanted to express my jealousy

I wanted to retaliate to their verbal aggression

I wanted to hurt them

I wanted to retaliate to their physical aggression

I wanted to express my frustration

I wanted to get back at them for hurting my feelings

I wanted to show them I wasn't weak

I wanted them to listen to me

I wanted to feel pleasure from inflicting upset or pain on them

I wanted to end or win the argument

I called my partner or text messaged them constantly

I wanted to protect myself from them physically harming me in that moment

I wanted to make them do something for me

I wanted to stop them trying to control me in some way

I wanted to escape from them

I wanted to feel powerful 
I wanted to express my anger

I wanted to get their attention

I lost control

I wanted to stop them from walking away from me

I'm not sure why I did this

I wanted to express my jealousy

I wanted to retaliate to their verbal aggression

I wanted to hurt them

I wanted to retaliate to their physical aggression

I wanted to express my frustration

I wanted to get back at them for hurting my feelings

I wanted to show them I wasn't weak

I wanted them to listen to me

I wanted to feel pleasure from inflicting upset or pain on them

I wanted to end or win the argument

I wanted to protect myself from them physically harming me in that moment

I wanted to make them do something for me

I wanted to stop them trying to control me in some way

I wanted to escape from them

I wanted to feel powerful

I wanted to express my anger

I wanted to get their attention 
I lost control

I wanted to stop them from walking away from me

I'm not sure why I did this

I wanted to express my jealousy

I wanted to retaliate to their verbal aggression

I wanted to hurt them

I wanted to retaliate to their physical aggression

I wanted to express my frustration

I wanted to get back at them for hurting my feelings

I wanted to show them I wasn't weak

I wanted them to listen to me

I wanted to feel pleasure from inflicting upset or pain on them

I wanted to end or win the argument 


\section{APPENDIX II}

Pattern matrix for the three-component solution

\begin{tabular}{|c|c|c|c|c|}
\hline Behavior & Motive & $\begin{array}{c}\text { Component } \\
1\end{array}$ & $\begin{array}{c}\text { Component } \\
2\end{array}$ & $\begin{array}{c}\text { Component } \\
3\end{array}$ \\
\hline \multirow[t]{18}{*}{ I kicked my partner } & I wanted to make them do something for me & .903 & & \\
\hline & I wanted to end or win the argument & .903 & & \\
\hline & I wanted to hurt them & .902 & & \\
\hline & I wanted to express my frustration & .902 & & \\
\hline & I wanted to feel powerful & .902 & & \\
\hline & I wanted to retaliate to their verbal aggression & .901 & & \\
\hline & I wanted to feel pleasure from inflicting upset or pain on them & .901 & & \\
\hline & I wanted to stop them from walking away from me & .901 & & \\
\hline & I wanted to show them I wasn't weak & .901 & & \\
\hline & I wanted them to listen to me & .901 & & \\
\hline & I wanted to express my jealousy & .901 & & \\
\hline & I wanted to get their attention & .901 & & \\
\hline & I wanted to escape from them & .901 & & \\
\hline & I wanted to retaliate to their physical aggression & .901 & & \\
\hline & I wanted to express my anger & .901 & & \\
\hline & $\begin{array}{l}\text { I wanted to protect myself from them physically harming me in that } \\
\text { moment }\end{array}$ & .901 & & \\
\hline & I wanted to get back at them for hurting my feelings & .901 & & \\
\hline & I wanted to stop them trying to control me in some way & .901 & & \\
\hline
\end{tabular}


I lost control

I wanted to hurt them

I'm not sure why I did this

I wanted to end or win the argument

I wanted to get their attention

I wanted to feel pleasure from inflicting upset or pain on them

I lost control

I wanted to express my frustration

I wanted to make them do something for me

I wanted to stop them trying to control me in some way

I wanted to express my anger

I wanted to show them I wasn't weak

I wanted them to listen to me

I wanted to retaliate to their physical aggression

I wanted to get back at them for hurting my feelings

I wanted to protect myself from them physically harming me in that moment

I wanted to stop them from walking away from me 
I wanted to make them do something for me

I wanted to stop them trying to control me in some way

I'm not sure why I did this

$-.822$

I wanted to express my anger

I wanted to get their attention

I wanted to escape from them $\quad .820$

I wanted to feel powerful $\quad .820$

I wanted to hurt them $\quad .818$

I wanted to get back at them for hurting my feelings $\quad .818$

I wanted to retaliate to their physical aggression $\quad .817$

I wanted to end or win the argument $\quad .817$

I wanted to express my frustration $\quad .817$

I wanted to protect myself from them physically harming me in that moment

I wanted to show them I wasn't weak

I wanted to feel pleasure from inflicting upset or pain on them

I wanted them to listen to me

I wanted to stop them from walking away from me

I wanted to retaliate to their verbal aggression 
I pressured my partner to have sex when they said 'No'

I kept my partner from leaving the house

I threatened to withhold money from my partner

I pressured my partner to have sex when they said 'No'

I threatened to withhold money from my partner

I kept my partner from leaving the house

I threatened to withhold money from my partner

I pressured my partner to have sex when they said 'No'
I wanted to stop them trying to control me in some way

I wanted to retaliate to their physical aggression

I wanted to retaliate to their physical aggression

I wanted to protect myself from them physically harming me in that moment

I wanted to hurt them

I wanted to get back at them for hurting my feelings

I wanted to get their attention

I wanted to get back at them for hurting my feelings

I wanted to stop them trying to control me in some way

I wanted to hurt them

I wanted to end or win the argument

I wanted to retaliate to their verbal aggression

I wanted to escape from them

I wanted to escape from them

I wanted to protect myself from them physically harming me in that moment

I wanted to protect myself from them physically harming me in that moment

I wanted to show them I wasn't weak

I wanted to stop them from walking away from me

I wanted to express my anger

I wanted to get their attention

I wanted to make them do something for me 
I pressured my partner to have sex when they said 'No'

I kept my partner from leaving the house

I threatened to withhold money from my partner

I pressured my partner to have sex when they said 'No'

I kept my partner from leaving the house

I threatened to withhold money from my partner

I kept my partner from leaving the house

I threatened to withhold money from my partner
I'm not sure why I did this

$-.797$

I wanted to feel powerful

I wanted to express my frustration

I wanted to express my frustration

I wanted to feel powerful

.797

I wanted them to listen to me

I lost control

I wanted to feel pleasure from inflicting upset or pain on them

I wanted to express my jealousy

I lost control

I wanted to escape from them

$-.796$

.796

I wanted to express my frustration

.796

I wanted to make them do something for me

I wanted to express my anger $\quad .794$

I wanted to get their attention $\quad .793$

I wanted to end or win the argument $\quad .793$

I wanted to express my anger $\quad .792$

I wanted to stop them trying to control me in some way $\quad .792$

I'm not sure why I did this $\quad .792$

I lost control $\quad .791$

I wanted to stop them from walking away from me $\quad .791$

I wanted to make them do something for me $\quad .791$ 
I slapped my partner

I denied incidents of abuse

I slapped my partner

I denied incidents of abuse

I slapped my partner

I denied incidents of abuse

I slapped my partner
I lost control

I wanted to retaliate to their physical aggression

I wanted to express my frustration

I wanted to make them do something for me

I'm not sure why I did this

.783

$-.783$

I wanted to hurt them

I wanted to express my anger

I wanted to escape from them

I wanted to feel pleasure from inflicting upset or pain on them

I wanted to get their attention

.780

I wanted to escape from them

.779

I wanted to retaliate to their verbal aggression

I wanted to feel powerful

I wanted to stop them from walking away from me

I wanted to stop them trying to control me in some way

I wanted to stop them trying to control me in some way

I wanted to express my anger $\quad .777$

$\begin{array}{ll}\text { I wanted to end or win the argument } & -.777\end{array}$

I wanted to show them I wasn't weak $\quad .777$

I wanted to get their attention $\quad .777$

I wanted to make them do something for me $\quad .777$

I wanted to retaliate to their physical aggression $\quad .776$ 
I denied incidents of abuse

I slapped my partner

\section{I denied incidents of abuse}

I deliberately broke or destroyed something that was important to my partner
I wanted to protect myself from them physically harming me in that moment

I wanted to get back at them for hurting my feelings

I wanted to protect myself from them physically harming me in that moment

I wanted them to listen to me

I wanted to stop them from walking away from me

I lost control

I wanted to feel powerful

I wanted to hurt them

I wanted to end or win the argument

I wanted to get back at them for hurting my feelings

I wanted to express my frustration

I wanted to express my jealousy

I wanted to make them do something for me

I wanted to protect myself from them physically harming me in that moment

I wanted to retaliate to their physical aggression

I wanted to express my anger

I wanted to escape from them

I wanted to stop them trying to control me in some way

I wanted to retaliate to their verbal aggression 
I threatened to disclose damaging or embarrassing information

I wanted to stop them from walking away from me

I'm not sure why I did this

I wanted them to listen to me

I wanted to get their attention

I wanted to hurt them

I wanted to feel powerful

I wanted to get back at them for hurting my feelings

I wanted to express my jealousy

I wanted to retaliate to their physical aggression

I wanted to express my anger

I wanted to make them do something for me

I wanted to protect myself from them physically harming me in that moment

I wanted to stop them from walking away from me

I wanted to escape from them

I wanted to stop them trying to control me in some way

I wanted to express my frustration

I'm not sure why I did this

I wanted to show them I wasn't weak

I wanted to feel powerful

I wanted to end or win the argument

I wanted to retaliate to their verbal aggression

I lost control 
I choked, strangled, or suffocated my partner

I made my partner dependent on me for money

I choked, strangled, or suffocated my partner

I made my partner dependent on me for money

I choked, strangled, or suffocated my partner
I wanted to feel pleasure from inflicting upset or pain on them

I wanted to hurt them

I wanted to get back at them for hurting my feelings

I wanted to express my jealousy

I wanted to get their attention

I wanted them to listen to me

I'm not sure why I did this

I wanted them to listen to me

I wanted to get back at them for hurting my feelings

I lost control

I wanted to protect myself from them physically harming me in that moment

I wanted to feel powerful

I wanted to make them do something for me

I wanted to get their attention

I wanted to protect myself from them physically harming me in that moment

I wanted to stop them from walking away from me

I wanted to escape from them

I wanted to stop them trying to control me in some way

I wanted to express my jealousy

I wanted to feel powerful

I wanted to end or win the argument 
I made my partner dependent on me for money

I was critical or unpleasant toward my partner
I wanted to express my frustration

I wanted to feel pleasure from inflicting upset or pain on them

I wanted to retaliate to their verbal aggression

I wanted to express my anger

I wanted to hurt them

I wanted to retaliate to their physical aggression

I wanted to show them I wasn't weak

I wanted to express my anger

I wanted to stop them from walking away from me

I wanted to escape from them $\quad .634$

I wanted to get their attention $\quad .633$

I lost control

I wanted them to listen to me

I'm not sure why I did this $\quad .630$

I wanted to show them I wasn't weak $\quad .629$

I wanted to express my jealousy $\quad .629$

I wanted to stop them trying to control me in some way $\quad 628$

I wanted to make them do something for me $\quad .628$

I wanted to express my frustration

I wanted to hurt them

I wanted to stop them trying to control me in some way

I wanted to express my anger 
I wanted to feel pleasure from inflicting upset or pain on them

I wanted to get back at them for hurting my feelings

I wanted to get their attention

I wanted to end or win the argument

I wanted to show them I wasn't weak

I lost control

I wanted to express my jealousy

I wanted them to listen to me

I'm not sure why I did this

I wanted to stop them from walking away from me

I wanted to feel powerful

I wanted to retaliate to their verbal aggression

I wanted to escape from them

I wanted to protect myself from them physically harming me in that moment

I wanted to retaliate to their physical aggression

I wanted to make them do something for me

I lost control

I wanted to express my frustration 
I pouted or acted upset when I didn't get my way

I wanted to express my jealousy

.758

I'm not sure why I did this

.757

I wanted to get their attention

I wanted to stop them from walking away from me

I wanted to feel powerful

I wanted to stop them trying to control me in some way

I wanted to show them I wasn't weak

I wanted to get back at them for hurting my feelings

I wanted to hurt them

I wanted to feel pleasure from inflicting upset or pain on them

I wanted to protect myself from them physically harming me in that moment

I wanted to escape from them

I wanted to retaliate to their physical aggression

I wanted to make them do something for me

I wanted to express my anger

I wanted to get their attention

I wanted to express my frustration

I wanted to end or win the argument

I'm not sure why I did this

I wanted to make them do something for me

I wanted to feel powerful

I wanted to express my jealousy 
I deliberately acted in a hurtful way towards my partner

I lost control

I wanted them to listen to me

I wanted to stop them trying to control me in some way

I wanted to get back at them for hurting my feelings

I wanted to stop them from walking away from me

I wanted to retaliate to their verbal aggression

I wanted to feel pleasure from inflicting upset or pain on them

I wanted to show them I wasn't weak

I wanted to protect myself from them physically harming me in that moment

I wanted to hurt them

I wanted to escape from them

.643

.643

.643

.642

.641

.641

.641

.638

.634

I wanted to retaliate to their physical aggression

.626

I wanted to retaliate to their verbal aggression

.617

I'm not sure why I did this

$-.616$

I wanted to get back at them for hurting my feelings

.615

I wanted to express my jealousy

.615

I wanted to hurt them

.615

I wanted them to listen to me

I wanted to show them I wasn't weak

I lost control

.614

I wanted to end or win the argument

.614

I wanted to stop them from walking away from me 
I wanted to express my frustration

I wanted to get their attention

I wanted to feel powerful

I wanted to escape from them

I wanted to feel pleasure from inflicting upset or pain on them

I wanted to express my anger

I wanted to stop them trying to control me in some way

I wanted to protect myself from them physically harming me in that moment

I wanted to retaliate to their physical aggression

I stomped out of the house or room during a disagreement with my partner

I lost control

I wanted them to listen to me

I wanted to express my anger

I wanted to show them I wasn't weak

I wanted to make them do something for me

I wanted to escape from them

I wanted to stop them from walking away from me

I wanted to get back at them for hurting my feelings

I wanted to protect myself from them physically harming me in that moment

I wanted to stop them trying to control me in some way 
I wanted to feel powerful

I wanted to retaliate to their verbal aggression

I wanted to feel pleasure from inflicting upset or pain on them

I wanted to hurt them

I wanted to make them do something for me

I wanted to retaliate to their physical aggression

I wanted to express my anger

I wanted to get back at them for hurting my feelings

I wanted to retaliate to their verbal aggression

I wanted them to listen to me

I wanted to stop them trying to control me in some way

I wanted to protect myself from them physically harming me in that moment

I wanted to get their attention

I wanted to feel powerful

I wanted to escape from them

I lost control 
I wanted to make them do something for me

I wanted to express my jealousy

I wanted to get their attention

I wanted them to listen to me

I wanted to end or win the argument

I lost control

I wanted to stop them from walking away from me

I wanted to show them I wasn't weak

I wanted to express my frustration

I wanted to feel powerful

I wanted to stop them trying to control me in some way

I wanted to feel pleasure from inflicting upset or pain on them

I wanted to express my anger

I wanted to escape from them

I wanted to get back at them for hurting my feelings

I wanted to hurt them

I wanted to protect myself from them physically harming me in that moment

I wanted to retaliate to their physical aggression

I'm not sure why I did this

I wanted to retaliate to their verbal aggression

I wanted to make them do something for me

.524

.866

.866

.865

.864

.864

.863

.863

.862

.862

.860

.860

.860

.859

.859

.859

.857

.854

.853

.853

.853 
I wanted them to listen to me

I wanted to end or win the argument

I wanted to stop them from walking away from me

I wanted to get their attention

I lost control

I wanted to retaliate to their verbal aggression

I'm not sure why I did this

I wanted to express my anger

I wanted to express my frustration

I wanted to escape from them

I wanted to get back at them for hurting my feelings

I wanted to feel pleasure from inflicting upset or pain on them

I wanted to protect myself from them physically harming me in that moment

I wanted to feel powerful

I wanted to express my jealousy

I wanted to stop them trying to control me in some way

I wanted to retaliate to their physical aggression 
I wanted to show them I wasn't weak

I wanted to express my anger

I wanted to express my frustration

I lost control

I wanted to get back at them for hurting my feelings

I wanted to feel powerful

I wanted to stop them from walking away from me

I wanted to get their attention

I wanted to feel pleasure from inflicting upset or pain on them

I wanted to stop them trying to control me in some way

I wanted to escape from them

I wanted to protect myself from them physically harming me in that

I wanted to hurt them

I wanted to make them do something for me

\begin{tabular}{|c|c|c|c|}
\hline Eigenvalue: & 149.88 & 84.40 & 78.54 \\
\hline$\%$ of variance explained: & 39.76 & 22.39 & 20.83 \\
\hline
\end{tabular}




\section{APPENDIX JJ}

Comparison of the fit statistics between the freely estimated and constrained models

\begin{tabular}{|c|c|c|c|c|c|c|}
\hline \multirow[b]{2}{*}{ Classes } & \multicolumn{3}{|c|}{ Freely estimated model } & \multicolumn{3}{|c|}{ Constrained model } \\
\hline & AIC & $\mathrm{BIC}$ & Entropy & AIC & $\mathrm{BIC}$ & Entropy \\
\hline Two classes & 42577.06 & 42890.86 & 1.000 & 40889.83 & 41193.51 & 1.000 \\
\hline Three classes & 38504.08 & 38959.60 & 1.000 & 36816.85 & 37262.25 & 1.000 \\
\hline Four classes & 34399.77 & 34997.01 & .989 & 32712.55 & 33299.66 & .989 \\
\hline Five classes & 31445.13 & 32184.08 & .989 & 29757.91 & 30486.74 & .989 \\
\hline Six classes & 30042.64 & 30923.31 & .994 & 28355.48 & 29226.03 & .994 \\
\hline Seven classes & 28968.28 & 29990.67 & .995 & 27281.01 & 28293.28 & .995 \\
\hline Eight classes & 27568.16 & 28732.26 & .989 & 25881.32 & 27035.30 & .989 \\
\hline Nine classes & 26350.30 & 27656.13 & .992 & 24663.42 & 25959.12 & .992 \\
\hline Ten classes & 25609.31 & 27056.85 & .995 & 23922.44 & 25359.86 & .995 \\
\hline Eleven classes & 24683.27 & 26272.53 & .995 & 22996.50 & 24575.64 & .995 \\
\hline Twelve classes & 23952.35 & 25683.32 & .994 & 22265.57 & 23986.42 & .994 \\
\hline
\end{tabular}

Note: AIC = Akaike Information Criterion; BIC = Bayesian Information Criterion. 


\section{APPENDIX KK}

Items included in the categorical principal components, latent class, and manifest variable path analyses in Chapter 5

\begin{tabular}{ll}
\hline & \\
\hline 1 & I felt intimidated \\
2 & I felt ashamed \\
3 & I felt afraid for my physical safety \\
4 & I felt isolated from friends and family \\
5 & I felt frustrated \\
6 & I felt controlled \\
7 & It didn't affect me in any way \\
8 & I found it funny \\
9 & I felt trapped \\
10 & I felt angry \\
11 & I felt like I wasn't able to make decisions for myself \\
12 & I felt guilty \\
13 & I felt like I wasn't able to do what I wanted to do \\
14 & I felt afraid I was going to make them angry \\
15 & I felt sad \\
16 & I felt afraid I was going to set them off shouting or criticizing me \\
17 & I felt sexually aroused \\
18 & I felt helpless \\
19 & I felt dependent on my partner \\
20 & I felt emotionally hurt \\
& \\
\hline
\end{tabular}

Behavioural Items

EA I made my partner dependent on me for money

EA I threatened my partner with a weapon

EA I threatened to disclose damaging or embarrassing information

EA I deliberately broke or destroyed something that was important to my partner

EA I threatened to withhold money from my partner

EA I kicked my partner

EA I kept my partner from leaving the house

EA I pressured my partner to have sex when they said 'No' 
EA I hit or punched my partner

EA I slapped my partner

EA I denied incidents of abuse

DPA I deliberately ignored my partner

DPA I made my partner feel guilty for not spending enough time together

DPA I pouted or acted upset when I didn't get my way

DPA I purposely withheld affection or sex

DPA I screamed or yelled at my partner

DPA I stomped out of the house or room during a disagreement with my partner

DPA I threatened to leave the relationship

DPA I was critical or unpleasant toward my partner

MA I checked my partner's social network page(s)

MA I criticized my partner's friends, family, or co-workers

MA I deliberately acted in a hurtful way towards my partner

MA I insisted on knowing where my partner went and who they spoke to when we were not together

MA I kept track of my partner's telephone/cell phone use

MA I told my partner they were lying or confused

MA I tried to provoke an argument with my partner

MA I called my partner or text messaged them constantly 


\section{APPENDIX LL}

Non-significant and cross-loading items that were removed from the CATPCA

\section{Non-significant items}

My partner denied incidents of abuse

My partner accused me of being unfaithful or flirting with others

My partner made me dependent on them for money

My partner changed the subject on purpose when I was trying to discuss a problem
I felt like I wasn't able to make decisions for myself

I felt afraid I was going to set them off shouting or criticizing me

I felt guilty

I felt like I wasn't able to do what I wanted to do

I felt afraid I was going to make them angry

I felt afraid I was going to set them off shouting or criticizing me

I felt sexually aroused

I felt helpless

I felt dependent on my partner

I felt guilty

I felt like I wasn't able to do what I wanted to do

I felt afraid I was going to make them angry

I felt sad

I felt afraid I was going to set them off shouting or criticizing me

I felt sexually aroused

I felt helpless

I felt dependent on my partner

I felt emotionally hurt

I felt guilty 
I felt sexually aroused

I felt dependent on my partner

My partner checked my social network page(s)

My partner threatened me with a weapon

My partner criticized my friends, family, or co-workers
I felt guilty

I felt like I wasn't able to do what I wanted to do

I felt afraid I was going to make them angry

I felt sad

I felt afraid I was going to set them off shouting or criticizing me

I felt sexually aroused

\section{I felt helpless}

I felt dependent on my partner

I felt emotionally hurt

I felt guilty

I felt like I wasn't able to do what I wanted to do

I felt afraid I was going to make them angry

I felt sad

I felt afraid I was going to set them off shouting or criticizing me

I felt sexually aroused

\section{I felt helpless}

I felt dependent on my partner

I felt emotionally hurt

I felt guilty

I felt like I wasn't able to do what I wanted to do 
I felt afraid I was going to make them angry

I felt sad

I felt afraid I was going to set them off shouting or criticizing me

I felt sexually aroused

I felt helpless

I felt dependent on my partner

I felt emotionally hurt

I felt sexually aroused

I felt dependent on my partner

I felt like I wasn't able to make decisions for myself

I felt guilty

I felt like I wasn't able to do what I wanted to do

I felt afraid I was going to make them angry

I felt sad

I felt afraid I was going to set them off shouting or criticizing me

I felt sexually aroused

I felt helpless

I felt dependent on my partner

My partner ordered me around

I felt guilty

I felt dependent on my partner

I felt emotionally hurt

I felt like I wasn't able to make decisions for myself 
I felt guilty

I felt like I wasn't able to do what I wanted to do

I felt afraid I was going to make them angry

I felt sad

I felt afraid I was going to set them off shouting or criticizing me

I felt sexually aroused

I felt helpless

I felt dependent on my partner

I felt emotionally hurt

My partner threatened to withhold money from me

My partner pouted or acted upset when they didn't get their way
I felt guilty

I felt like I wasn't able to do what I wanted to do

I felt afraid I was going to make them angry

I felt sad

I felt afraid I was going to set them off shouting or criticizing me

I felt sexually aroused

I felt helpless

I felt dependent on my partner

I felt emotionally hurt

I felt like I wasn't able to make decisions for myself

I felt guilty

I felt like I wasn't able to do what I wanted to do

I felt afraid I was going to make them angry 
My partner told me I was lying or confused

My partner tried to provoke an argument with me
I felt sad

I felt afraid I was going to set them off shouting or criticizing me

I felt sexually aroused

I felt helpless

I felt dependent on my partner

I felt emotionally hurt

I felt like I wasn't able to make decisions for myself

I felt guilty

I felt like I wasn't able to do what I wanted to do

I felt afraid I was going to make them angry

I felt sad

I felt afraid I was going to set them off shouting or criticizing me

I felt sexually aroused

I felt helpless

I felt dependent on my partner

I felt emotionally hurt

I felt like I wasn't able to make decisions for myself

I felt guilty

I felt like I wasn't able to do what I wanted to do

I felt afraid I was going to make them angry

I felt sad

I felt afraid I was going to set them off shouting or criticizing me 
I felt sexually aroused

\section{I felt helpless}

I felt dependent on my partner

I felt emotionally hurt

My partner stomped out of the house or room during a disagreement with me

My partner called or text messaged me constantly

\section{My partner pressured me to have sex when I said 'No'}

My partner tried to restrict my activities
I felt like I wasn't able to make decisions for myself

I felt like I wasn't able to do what I wanted to do

I felt sexually aroused

I felt dependent on my partner

I felt like I wasn't able to make decisions for myself

I felt guilty

I felt like I wasn't able to do what I wanted to do

I felt afraid I was going to make them angry

I felt sad

I felt afraid I was going to set them off shouting or criticizing me

I felt sexually aroused

I felt helpless

I felt dependent on my partner

I felt emotionally hurt

I felt sad

I felt afraid I was going to set them off shouting or criticizing me

I felt emotionally hurt

I felt like I wasn't able to make decisions for myself 
My partner slapped me

My partner was critical or unpleasant toward me
I felt guilty

I felt like I wasn't able to do what I wanted to do

I felt afraid I was going to make them angry

I felt sad

I felt afraid I was going to set them off shouting or criticizing me

I felt sexually aroused

I felt helpless

I felt dependent on my partner

I felt emotionally hurt

I felt like I wasn't able to make decisions for myself

I felt guilty

I felt like I wasn't able to do what I wanted to do

I felt afraid I was going to make them angry

I felt sad

I felt afraid I was going to set them off shouting or criticizing me

I felt sexually aroused

I felt helpless

I felt dependent on my partner

I felt like I wasn't able to make decisions for myself

I felt guilty

I felt like I wasn't able to do what I wanted to do

I felt afraid I was going to make them angry 
I felt sad

I felt afraid I was going to set them off shouting or criticizing me

I felt sexually aroused

I felt helpless

I felt dependent on my partner

I felt emotionally hurt

\begin{tabular}{ll}
\hline Cross-loading items \\
\hline My partner made me dependent on them for money & I felt intimidated \\
& I felt ashamed \\
& I felt afraid for my physical safety \\
& I felt isolated from friends and family \\
& I felt frustrated \\
& I felt controlled \\
It didn't affect me in any way & I found it funny \\
I felt trapped \\
I felt angry \\
I felt like I wasn't able to make decisions for myself \\
I felt emotionally hurt \\
I felt intimidated \\
I felt ashamed \\
\hline
\end{tabular}


I felt afraid for my physical safety

I felt isolated from friends and family

I felt frustrated

I felt controlled

It didn't affect me in any way

I found it funny

I felt trapped

I felt angry

I felt like I wasn't able to make decisions for myself

My partner deliberately acted in a hurtful way towards me

My partner ordered me around
I felt intimidated

I felt ashamed

I felt afraid for my physical safety

I felt isolated from friends and family

I felt frustrated

I felt controlled

It didn't affect me in any way

I found it funny

I felt trapped

I felt angry

I felt emotionally hurt

I felt intimidated

I felt ashamed 
I felt afraid for my physical safety

I felt isolated from friends and family

I felt frustrated

I felt controlled

It didn't affect me in any way

I found it funny

I felt trapped

I felt angry

I felt like I wasn't able to make decisions for myself

I felt like I wasn't able to do what I wanted to do

I felt afraid I was going to make them angry

I felt sad

I felt afraid I was going to set them off shouting or criticizing me

I felt sexually aroused

\section{I felt helpless}

My partner made me feel guilty for not spending enough time together

I felt intimidated

I felt ashamed

I felt afraid for my physical safety

I felt isolated from friends and family

I felt frustrated

I felt controlled

It didn't affect me in any way 
I found it funny

I felt trapped

I felt angry

My partner pouted or acted upset when they didn't get their way

I felt intimidated

I felt ashamed

I felt afraid for my physical safety

I felt isolated from friends and family

I felt frustrated

I felt controlled

It didn't affect me in any way

I found it funny

I felt trapped

I felt angry

I felt intimidated

I felt ashamed

I felt afraid for my physical safety

I felt isolated from friends and family

I felt frustrated

I felt controlled

It didn't affect me in any way

I found it funny

I felt trapped 
My partner tried to provoke an argument with me

partner stomped out of the house or room during a disagreement with me
I felt angry

I felt intimidated

I felt ashamed

I felt afraid for my physical safety

I felt isolated from friends and family

I felt frustrated

I felt controlled

It didn't affect me in any way

I found it funny

I felt trapped

I felt angry

I felt intimidated

I felt ashamed

I felt afraid for my physical safety

I felt isolated from friends and family

I felt frustrated

I felt controlled

It didn't affect me in any way

I found it funny

I felt trapped

I felt angry

I felt guilty 
My partner called or text messaged me constantly

My partner tried to restrict my activities

My partner was critical or unpleasant toward me
I felt afraid I was going to make them angry

I felt sad

I felt afraid I was going to set them off shouting or criticizing me

I felt helpless

I felt emotionally hurt

I felt afraid for my physical safety

I felt intimidated

I felt ashamed

I felt afraid for my physical safety

I felt isolated from friends and family

I felt frustrated

\section{I felt controlled}

It didn't affect me in any way

I found it funny

I felt trapped

I felt angry

I felt intimidated

I felt ashamed

I felt afraid for my physical safety

I felt isolated from friends and family

I felt frustrated

I felt controlled 
It didn't affect me in any way

I found it funny

I felt trapped

I felt angry 


\section{APPENDIX MM}

Pattern matrix for the three-component solution

\begin{tabular}{|c|c|c|c|c|}
\hline Behavior & Outcome & $\begin{array}{l}\text { Component } \\
1\end{array}$ & $\begin{array}{l}\text { Component } \\
2\end{array}$ & $\begin{array}{l}\text { Component } \\
3\end{array}$ \\
\hline \multirow[t]{17}{*}{ My partner slammed or held me against a wall } & I felt angry & -.912 & & \\
\hline & I felt trapped & .910 & & \\
\hline & It didn't affect me in any way & .909 & & \\
\hline & I felt sexually aroused & .909 & & \\
\hline & $\begin{array}{l}\text { I felt afraid I was going to set them off shouting or } \\
\text { criticizing me }\end{array}$ & .909 & & \\
\hline & I felt isolated from friends and family & .909 & & \\
\hline & I felt afraid for my physical safety & .909 & & \\
\hline & I found it funny & .909 & & \\
\hline & I felt like I wasn't able to do what I wanted to do & .908 & & \\
\hline & I felt dependent on my partner & .908 & & \\
\hline & I felt afraid I was going to make them angry & .908 & & \\
\hline & I felt guilty & .908 & & \\
\hline & I felt ashamed & .908 & & \\
\hline & I felt helpless & .908 & & \\
\hline & I felt like I wasn't able to make decisions for myself & .908 & & \\
\hline & I felt frustrated & .908 & & \\
\hline & I felt emotionally hurt & .908 & & \\
\hline
\end{tabular}


I felt intimidated

.907

I felt sad

.907

I felt controlled

.907

I felt trapped

.853

I felt controlled

.848

I felt afraid for my physical safety

.848

I felt afraid I was going to set them off shouting or criticizing me

I felt angry

$-.844$

I felt afraid I was going to make them angry

.844

I felt ashamed

.842

I felt sexually aroused

838

I felt like I wasn't able to do what I wanted to do

.838

I felt isolated from friends and family

.838

I felt guilty

.838

It didn't affect me in any way

.837

I felt helpless

.837

I found it funny

I felt sad

.836

I felt dependent on my partner

.836

I felt frustrated

.836

I felt emotionally hurt

.836

I felt intimidated 
My partner hit or punched me

I felt like I wasn't able to make decisions for myself

I felt angry

.830

I felt emotionally hurt

I felt frustrated

.814

I felt isolated from friends and family

.814

I felt afraid I was going to make them angry

.809

It didn't affect me in any way

.807

I felt afraid I was going to set them off shouting or criticizing me

I felt like I wasn't able to do what I wanted to do

I felt trapped

.805

I felt intimidated

.804

I felt sexually aroused

.804

I felt ashamed

.804

I felt afraid for my physical safety

.803

I felt guilty

.803

I felt like I wasn't able to make decisions for myself

$-.803$

I felt dependent on my partner

.803

I felt controlled

.803

I felt helpless

.803

I felt sad

.803

I found it funny

.802 
My partner tried to turn my family, friends, and children against me

My partner slapped me

My partner tried to turn my family, friends, and children against me

\section{My partner slapped me}

My partner tried to turn my family, friends, and children against me
I felt angry

I felt isolated from friends and family

I felt afraid for my physical safety

I felt afraid I was going to set them off shouting or criticizing me

I felt sexually aroused

I felt afraid I was going to make them angry

.769

I felt emotionally hurt

$-.766$

I felt afraid for my physical safety

.764

I found it funny

.764

I felt dependent on my partner

.763

I felt like I wasn't able to do what I wanted to do

It didn't affect me in any way

I felt frustrated

.762

I felt intimidated

.762

I felt helpless

.760

I felt ashamed

.760

I felt sad

.760

I felt trapped

.760

I felt guilty

I felt controlled 
My partner slapped me

My partner tried to turn my family, friends, and children against me

My partner slapped me

My partner denied incidents of abuse

My partner slapped me

My partner denied incidents of abuse

My partner pressured me to have sex when I said 'No'

My partner denied incidents of abuse

My partner threatened me with a weapon

My partner denied incidents of abuse
I felt isolated from friends and family

I felt emotionally hurt

$-.759$

I felt like I wasn't able to make decisions for myself

It didn't affect me in any way

I felt intimidated

I felt controlled

.756

I found it funny

I felt angry

I felt trapped

.755

I felt ashamed

I felt frustrated

I felt like I wasn't able to do what I wanted to do

I felt afraid for my physical safety

.750

I felt sexually aroused

I felt afraid I was going to make them angry

I felt isolated from friends and family

I felt emotionally hurt

$-.742$

I felt angry

I felt dependent on my partner $\quad .739$

I felt frustrated $\quad .739$

It didn't affect me in any way $\quad .739$

I felt isolated from friends and family $\quad .738$ 
My partner threatened me with a weapon

My partner pressured me to have sex when I said ' $\mathrm{No}^{\prime}$

My partner threatened me with a weapon

My partner deliberately broke or destroyed something that was important to me

My partner pressured me to have sex when I said 'No'
I felt trapped

$-.737$

I felt helpless

.736

I found it funny

.734

I felt guilty

.733

I felt intimidated

.732

I felt controlled

.730

I felt sad

.730

I felt ashamed

.730

I felt isolated from friends and family $\quad .726$

I felt controlled

.725

It didn't affect me in any way

I felt intimidated

.722

I felt afraid for my physical safety

I found it funny

I felt frustrated

I felt trapped

.720

I felt ashamed

I felt dependent on my partner

.718

I felt like I wasn't able to make decisions for myself

$-.718$

I felt angry

.709

I felt like I wasn't able to make decisions for myself

$-.704$

I felt afraid for my physical safety 
I felt afraid I was going to make them angry

I felt trapped

$-.698$

I felt guilty

$-.698$

I felt like I wasn't able to do what I wanted to do

.698

I felt controlled

.697

I found it funny

.696

I felt sexually aroused

.696

It didn't affect me in any way

.696

I felt frustrated

.695

I felt ashamed

.694

I felt helpless

.694

I felt intimidated

.693

I felt angry

My partner deliberately broke or destroyed something that was important to me

It didn’t affect me in any way

I felt afraid I was going to set them off shouting or criticizing me

I felt sexually aroused

I felt afraid I was going to make them angry

I felt like I wasn't able to do what I wanted to do

I found it funny

I felt isolated from friends and family

I felt frustrated 
My partner threatened to withhold money from me

My partner threatened to disclose damaging or embarrassing information

My partner threatened to withhold money from me

My partner deliberately broke or destroyed something that was important to me

My partner threatened to withhold money from me

My partner threatened to disclose damaging or embarrassing information

My partner threatened to withhold money from me

My partner threatened to disclose damaging or embarrassing information

My partner threatened to withhold money from me
I felt emotionally hurt

I felt dependent on my partner

I felt ashamed

I felt guilty

I felt intimidated

.648

I felt afraid for my physical safety

.648

I felt sad

.648

I felt helpless

.648

I felt trapped

I felt controlled

I felt afraid for my physical safety

I felt emotionally hurt

$-.636$

I felt trapped

I felt angry

$-.628$

I felt like I wasn't able to make decisions for myself

$-.627$

I felt controlled

I felt afraid for my physical safety

I felt isolated from friends and family

I felt afraid I was going to make them angry

I felt like I wasn't able to make decisions for myself 
I felt frustrated

My partner threatened to disclose damaging or embarrassing information

I felt isolated from friends and family

My partner threatened to withhold money from me

It didn't affect me in any way

I felt ashamed

I felt intimidated

.611

I found it funny

My partner threatened to disclose damaging or embarrassing information

I felt trapped

I felt angry

$-.608$

I felt like I wasn't able to do what I wanted to do

It didn't affect me in any way

.606

I felt like I wasn't able to make decisions for myself

I felt afraid I was going to set them off shouting or criticizing me

I felt intimidated

I found it funny

I felt guilty

I felt controlled

.600

I felt helpless

.600

I felt ashamed

.600

I felt sad

.600

I felt frustrated 
My partner insisted on knowing where I went and who I spoke to when we were not together
I felt trapped

.931

I felt emotionally hurt

I found it funny

I felt frustrated

I felt like I wasn't able to do what I wanted to do

I felt like I wasn't able to make decisions for myself

I felt afraid I was going to make them angry

It didn't affect me in any way

I felt controlled

I felt guilty

I felt sad

I felt intimidated

I felt ashamed

I felt afraid I was going to set them off shouting or criticizing me

I felt angry

I felt isolated from friends and family

I felt dependent on my partner

I felt helpless

I felt sexually aroused

I felt afraid for my physical safety 
My partner accused me of being unfaithful or flirting with others

My partner checked my social network page(s)
I felt trapped

.751

I felt controlled

I found it funny

I felt intimidated

I felt sad

.749

It didn't affect me in any way

I felt ashamed

I felt like I wasn't able to make decisions for myself

$-.746$

I felt frustrated

I felt emotionally hurt

I felt angry

I felt isolated from friends and family

I felt afraid for my physical safety

I felt controlled

It didn't affect me in any way

$-.608$

I felt frustrated

I found it funny

I felt like I wasn't able to make decisions for myself

$-.605$

I felt trapped

.604

I felt intimidated

I felt angry

.601

I felt ashamed 
My partner called or text messaged me constantly

My partner deliberately ignored me
I felt isolated from friends and family

I felt afraid for my physical safety

I felt controlled

I found it funny

I felt frustrated

I felt angry

It didn't affect me in any way

$-.464$

I felt trapped

I felt intimidated

I felt ashamed

.452

I felt isolated from friends and family

.428

I felt helpless

I felt emotionally hurt

I felt guilty

I felt like I wasn't able to make decisions for myself

I felt trapped

I felt sad

I felt intimidated

I found it funny

I felt sexually aroused

$-.856$

I felt angry

.856

I felt ashamed 
My partner treated me like I was inferior to them

I felt afraid I was going to make them angry

.856

I felt like I wasn't able to do what I wanted to do

.856

I felt controlled

I felt dependent on my partner

.855

I felt afraid I was going to set them off shouting or criticizing me

.855

It didn't affect me in any way

.854

.854

I felt frustrated

.854

I felt isolated from friends and family

I felt afraid for my physical safety

.853

.850

I felt intimidated

I felt trapped

.686

.686

I felt controlled

.686

I felt ashamed

.686

I felt like I wasn't able to make decisions for myself

I felt dependent on my partner

I felt guilty

.686

.685

.685

I felt like I wasn't able to do what I wanted to do

I felt afraid I was going to set them off shouting or criticizing me

.684

.684

I found it funny

.682

I felt afraid I was going to make them angry

.682

I felt frustrated 
I felt isolated from friends and family

I felt sexually aroused

I felt angry

My partner changed the subject on purpose when I was trying to discuss a problem

I felt sad

It didn't affect me in any way

My partner treated me like I was inferior to them

I felt emotionally hurt

My partner changed the subject on purpose when I was trying to discuss a problem

I felt trapped

I felt controlled

I felt helpless

I felt frustrated

I felt like I wasn't able to make decisions for myself

I felt angry

I felt like I wasn't able to do what I wanted to do

I felt intimidated

I felt afraid for my physical safety

criticizing me

I felt afraid I was going to make them angry 
I felt isolated from friends and family

.663

I felt afraid for my physical safety

Eigenvalue:

127.97

79.64

59.94

$\%$ of variance explained:

43.23

26.90

25.20 


\section{APPENDIX NN}

Comparison of the fit statistics between the freely estimated and constrained models

\begin{tabular}{|c|c|c|c|c|c|c|}
\hline \multirow[b]{2}{*}{ Classes } & \multicolumn{3}{|c|}{ Freely estimated model } & \multicolumn{3}{|c|}{$\underline{\text { Constrained model }}$} \\
\hline & AIC & $\mathrm{BIC}$ & Entropy & AIC & BIC & Entropy \\
\hline Two classes & 60801.39 & 61115.62 & .93 & 59105.16 & 59409.25 & .93 \\
\hline Three classes & 582.70 .85 & 58726.99 & .93 & 56574.00 & 57020.65 & .93 \\
\hline Four classes & 57249.90 & 57847.95 & .91 & 55553.73 & 56141.64 & .91 \\
\hline Five classes & 56723.64 & 57463.59 & .93 & 55027.50 & 55757.32 & .93 \\
\hline Six classes & 56214.35 & 57096.22 & .92 & 54517.19 & 55389.21 & .92 \\
\hline
\end{tabular}

Note. AIC = Akaike Information Criterion; BIC = Bayesian Information Criterion. 


\section{APPENDIX 00}

Parameter estimates of the manifest variable path model

\begin{tabular}{|c|c|c|c|c|c|c|c|}
\hline Variables & B & $S E$ & $95 \% \mathrm{CI}$ & $\beta$ & $z$ & $p$ & $R^{2}$ \\
\hline Control on & & & & & & & .608 \\
\hline Gender & -.138 & .062 & $-.24,-.04$ & -.04 & -2.23 & .026 & \\
\hline EA & .083 & .008 & $.07, .10$ & .60 & 10.81 & $<.001$ & \\
\hline DPA & .034 & .008 & $.02, .05$ & .26 & 4.34 & $<.001$ & \\
\hline MA & .002 & .010 & $-.02, .02$ & .02 & .22 & .824 & \\
\hline Control x Gender x EA & -.001 & .011 & $-.02, .02$ & -.004 & -.08 & .936 & \\
\hline Control x Gender x DPA & -.014 & .009 & $-.03, .001$ & -.08 & -1.53 & .126 & \\
\hline Control x Gender x MA & .010 & .012 & $-.01, .03$ & .05 & .84 & .398 & \\
\hline Hostility on & & & & & & & .392 \\
\hline Gender & .097 & .149 & $-.15, .34$ & .015 & .65 & .513 & \\
\hline EA & -.108 & .016 & $-.14,-.08$ & -.40 & -6.60 & $<.001$ & \\
\hline DPA & .167 & .020 & $.13, .20$ & .64 & 8.45 & $<.001$ & \\
\hline MA & .053 & .028 & $.01, .03$ & .18 & 1.91 & .056 & \\
\hline Hostility x Gender x EA & .009 & .023 & $-.05, .03$ & -.02 & -.40 & 687 & \\
\hline Hostility x Gender x DPA & .008 & .025 & $-.05, .03$ & -.02 & -.33 & .738 & \\
\hline Hostility x Gender x MA & .026 & .034 & $-.03, .08$ & .06 & .76 & .445 & \\
\hline Guilt on & & & & & & & .256 \\
\hline Gender & -3.12 & .102 & $-.48,-.14$ & -.08 & -3.06 & $<.05$ & \\
\hline EA & .091 & .015 & $.06, .12$ & .54 & 5.97 & $<.001$ & \\
\hline DPA & .014 & .010 & $-.002, .03$ & .09 & 1.45 & .148 & \\
\hline MA & -.009 & .018 & $-.03, .016$ & -.05 & -.57 & .57 & \\
\hline Guilt $x$ Gender x EA & -.034 & .020 & $-.07,-.001$ & -.14 & -1.70 & .089 & \\
\hline Guilt x Gender x DPA & .007 & .012 & $-.01, .03$ & .03 & .61 & .543 & \\
\hline Guilt x Gender x MA & .007 & .018 & $-.02, .04$ & .03 & .37 & .715 & \\
\hline$\underline{\text { Sadness on }}$ & & & & & & & .227 \\
\hline Gender & -.009 & .125 & $-.22, .20$ & -.002 & -.07 & .944 & \\
\hline EA & -.004 & .013 & $-.03, .02$ & -.02 & -31 & .754 & \\
\hline DPA & .009 & .018 & $.07, .13$ & .52 & 5.47 & $<.001$ & \\
\hline MA & -.005 & .022 & $-.04, .03$ & .02 & -.23 & .805 & \\
\hline Sadness x Gender x EA & -.018 & .017 & $-.05, .01$ & .02 & -1.04 & .298 & \\
\hline Sadness x Gender x DPA & .004 & .022 & $-.03, .04$ & -.06 & .20 & .845 & \\
\hline Sadness x Gender x MA & .002 & .026 & $-.04, .05$ & .01 & .08 & .938 & \\
\hline
\end{tabular}

